Supporting Information

\title{
Ru-Catalyzed Migratory Geminal Semi-Hydrogenation of Internal Alkynes to Terminal Olefins
}

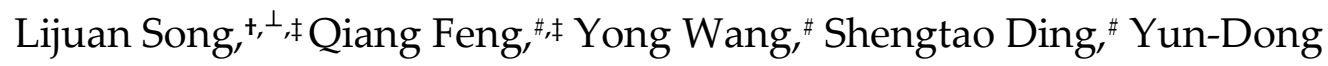
Wu, ${ }^{t, \$, \mathbb{I}}$ Xinhao Zhang, ${ }^{t, \mathbb{I}}$ Lung Wa Chung, ${ }^{\|, *}$ and Jianwei Sun ${ }^{\sharp, *}$

+ Lab of Computational Chemistry and Drug Design, Laboratory of Chemical Genomics, Peking University Shenzhen Graduate School, Shenzhen 518055, China

* Department of Chemistry, The Hong Kong University of Science and Technology, Clear Water Bay, Kowloon, Hong Kong SAR, China

" Department of Chemistry and Shenzhen Grubbs Institute, Southern University of Science and Technology, Shenzhen 518055, China

II Shenzhen Bay Laboratory, Shenzhen 518055, China

$\S$ College of Chemistry, Peking University, Beijing 100871, China

${ }^{\perp}$ Institute of Organic Chemistry, Justus Liebig University, Heinrich-Buff-Ring 17, D-35392 Giessen, Germany

Table of Contents

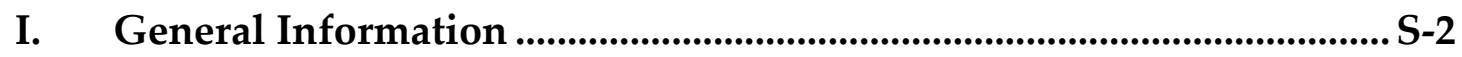

II. Optimization of the Reaction Conditions …............................................ S-3

III. Synthesis of 1-Silyl Alkynes …................................................................. S-5

IV. Ru(II)-Catalyzed gem-Hydrogenation of 1-Silyl Alkynes .................. S-19

V. Ru(II)-Catalyzed trans-Hydrogenation of 1-Silyl Alkynes ................. S-34

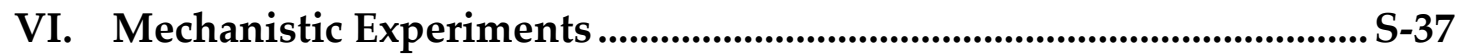

VII. Theoretical Details and Results ............................................................ S-39

${ }^{1} \mathrm{H}$ NMR and ${ }^{13} \mathrm{C}$ NMR Spectra 


\section{General Information}

All air or moisture sensitive reactions were conducted in oven-dried glassware under nitrogen atmosphere using dry solvents. Flash column chromatography was performed over silica gel (230-400 mesh) purchased from Qingdao Puke Co., China. Silanes and common organic chemicals were purchased from commercial suppliers, such as Sigma-Aldrich ${ }^{\circledR}$ and J\&K ${ }^{\circledR}$ Scientific Ltd., and used as received. $\left[\mathrm{CpRu}(\mathrm{MeCN})_{3}\right] \mathrm{PF}_{6}$ was purchased from Strem ${ }^{\circledR}$ Chemicals, Inc. Anhydrous dichloromethane, tetrahydrofuran and diethyl ether was purified by Innovative ${ }^{\circledR}$ solvent purification system. ${ }^{1} \mathrm{H}$ and ${ }^{13} \mathrm{C}$ NMR spectra were collected on a Bruker AV $400 \mathrm{MHz}$ NMR spectrometer using residue solvent peaks as an internal standard $\left({ }^{1} \mathrm{H} \mathrm{NMR}: \mathrm{CDCl}_{3}\right.$ at 7.28 ppm, ${ }^{13} \mathrm{C} \mathrm{NMR:} \mathrm{CDCl}_{3}$ at $\left.77.0 \mathrm{ppm}\right)$. IR spectra were recorded on Bruker TENSOR 27 spectrometer and are reported in terms of frequency of absorption $\left(\mathrm{cm}^{-1}\right)$. Mass spectra were collected on an Agilent GC/MS 5975C system, or a MALDI Micro MX mass spectrometer, or an API QSTAR XL System. 


\section{Optimization of the Reaction Conditions}

Table S1. Gem-Hydrogenation of Aromatic Substituted 1-Silyl Alkynes. ${ }^{a}$

\begin{tabular}{|c|c|c|c|c|c|c|}
\hline \multirow{2}{*}{$\begin{array}{r}\mathrm{Ph}-\overline{=} \\
1\end{array}$} & \multirow{2}{*}{\multicolumn{2}{|c|}{$\begin{array}{c}{\left[\mathrm{CpRu}(\mathrm{MeCN})_{3}\right] \mathrm{PF}_{6}} \\
\text { additive }(20 \mathrm{~mol} \%), \mathrm{H}_{2} \\
\text { solvent }(0.1 \mathrm{M}), \mathrm{rt}\end{array}$}} & \multirow{2}{*}{\multicolumn{2}{|c|}{$\mathrm{PhMe}_{2} \mathrm{Si}_{2(\mathrm{~B})}^{\mathrm{Ph}}$}} & \multirow{2}{*}{$+\sum_{\mathrm{H}(\mathrm{L}}^{\mathrm{Ph}}=\underbrace{\mathrm{r}}_{\mathrm{S}}$} & \multirow{2}{*}{ iMe ${ }_{2} \mathrm{Ph}$} \\
\hline & & & & & & \\
\hline entry & additive & $P-\mathrm{H}_{2}$ (bar) & solvent & time(h) & yield $(\%)^{b}$ & $\mathrm{~B} / \mathrm{L}^{b}$ \\
\hline 1 & - & 10 & $\mathrm{CHCl}_{3}$ & 5 & 95 & $1: 2$ \\
\hline 2 & proton sponge & 10 & $\mathrm{CHCl}_{3}$ & 5 & 72 & 1:11 \\
\hline 3 & DABCO & 10 & $\mathrm{CHCl}_{3}$ & 5 & 77 & $1: 12$ \\
\hline 4 & - & 1 & $\mathrm{CHCl}_{3}$ & 25 & 31 & $7: 1$ \\
\hline 5 & $\mathrm{NaBAr}_{4}$ & 1 & $\mathrm{CHCl}_{3}$ & 25 & 45 & $10: 1$ \\
\hline 6 & $\mathrm{NaBAr}_{4}$ & 1 & $\mathrm{PhF}$ & 25 & 23 & $22: 1$ \\
\hline 7 & $\mathrm{NaBAr}_{4}$ & 1 & $\mathrm{DCM}^{\mathrm{C}}$ & 48 & 52 & $>30: 1$ \\
\hline 8 & $\mathrm{NaBAr}_{4}$ & 10 & $\mathrm{DCM}^{c}$ & 2 & 52 & $1: 1.2$ \\
\hline 9 & - & 1 & $\mathrm{DCM}^{\mathrm{c}}$ & 48 & 18 & $18: 1$ \\
\hline 10 & $\mathrm{KBAr}_{4}{ }_{4}$ & 1 & $\mathrm{DCM}^{c}$ & 48 & $<5 \%$ & - \\
\hline 11 & $\mathrm{NaBF}_{4}$ & 1 & $\mathrm{DCM}^{\mathrm{c}}$ & 48 & 64 & $16: 1$ \\
\hline 12 & NaOTf & 1 & $\mathrm{DCM}^{c}$ & 48 & 46 & $23: 1$ \\
\hline 13 & ${ }^{n} \mathrm{Bu}_{4} \mathrm{NCl}$ & 1 & $\mathrm{DCM}^{\mathrm{c}}$ & 48 & 41 & $3: 1$ \\
\hline
\end{tabular}

${ }^{a}$ Reaction scale: 1 (0.05 mmol). ${ }^{b}$ Yield and selectivity were determined by analysis of the crude ${ }^{1} \mathrm{H}$ NMR spectroscopy. ${ }^{c}$ Concentration: $0.05 \mathrm{M}$. 
Table S2. Trans-Hydrogenation of Aromatic Substituted 1-Silyl Alkynes. ${ }^{a}$

\begin{tabular}{|c|c|c|c|c|c|c|}
\hline \multicolumn{2}{|c|}{$\begin{array}{c}\mathrm{Ph}=\mathrm{SiMe}_{2} \mathrm{Ph} \\
\mathbf{1}(0.1 \mathrm{mmol})\end{array}$} & $\begin{array}{c}\begin{array}{c}{\left[\mathrm{CpRu}(\mathrm{MeCN})_{3}\right] \mathrm{PF}_{6}} \\
(20 \mathrm{~mol} \%)\end{array} \\
\underset{\mathrm{H}_{2} \text {, additive }}{\text { solvent, rt, } 6.5 \mathrm{~h}}\end{array}$ & \multirow{2}{*}{$\underbrace{\mathrm{Ph} P h}_{\text {SiMe }}$} & $\overbrace{\text { 3s }}^{\mathrm{Ph}}=\mathrm{SiMe}_{2}^{\mathrm{H} P h}$ & \multicolumn{2}{|c|}{$+\sum_{\mathrm{Ph}}^{\mathrm{H}} \sum_{\mathrm{SiMe}_{2} \mathrm{Ph}}^{\mathrm{H}}$} \\
\hline entry & additive & solvent & & conv. $(\%)^{b}$ & yield $(\%)^{b}$ & $2 s / 3 s / 4 s^{b}$ \\
\hline 1 & DBU & $\mathrm{CHCl}_{3}$ & 10 & $>99$ & 71 & 1:6:0 \\
\hline 2 & pyridine & $\mathrm{CHCl}_{3}$ & 10 & 79 & 58 & $1: 29: 0$ \\
\hline 3 & 2,6-lutidine & $\mathrm{CHCl}_{3}$ & 10 & 94 & 66 & 1:3:0 \\
\hline 4 & 2,4,6-collidine & $\mathrm{CHCl}_{3}$ & 10 & 79 & 62 & 1:3:0 \\
\hline 5 & proton sponge & $\mathrm{CHCl}_{3}$ & 10 & $>99$ & 72 & $1: 11: 0$ \\
\hline 6 & DABCO & $\mathrm{CHCl}_{3}$ & 10 & $>99$ & 78 & $1: 12: 0$ \\
\hline 7 & 2,6-( $\left.{ }^{t} \mathrm{Bu}\right)_{2} \mathrm{Py}$ & $\mathrm{CHCl}_{3}$ & 10 & $>99$ & 77 & 1:2:0 \\
\hline 8 & $1,2,2,6,6-\mathrm{PMP}^{d}$ & $\mathrm{CHCl}_{3}$ & 10 & 39 & 46 & 1:3:0 \\
\hline 9 & ${ }^{i} \mathrm{Pr}_{2} \mathrm{NH}$ & $\mathrm{CHCl}_{3}$ & 10 & 35 & 21 & $1: 3: 0$ \\
\hline 10 & DABCO & DCM & 15 & 89 & 72 & $1: 5: 4$ \\
\hline 11 & DABCO & DCE & 15 & 78 & 54 & $1: 5: 5$ \\
\hline 12 & DABCO & $\mathrm{CHCl}_{3}$ & 15 & $>99$ & 87 & $1: 28: 0$ \\
\hline 13 & DABCO & THF & 15 & 64 & 54 & $1: 6: 11$ \\
\hline 14 & DABCO & 1,4-dioxane & 15 & 95 & 78 & $1: 20: 18$ \\
\hline 15 & DABCO & EtOAc & 15 & 60 & 46 & $1: 5: 9$ \\
\hline $16^{c}$ & DABCO & $\mathrm{CHCl}_{3}$ & 15 & 88 & 82 & $1: 15: 5$ \\
\hline
\end{tabular}

${ }^{a}$ Reaction scake: $1(0.1 \mathrm{mmol}),\left[\mathrm{CpRu}(\mathrm{MeCN})_{3}\right] \mathrm{PF}_{6}(20 \mathrm{~mol} \%) .{ }^{b}$ Yield and selectivity were determined by analysis of the crude ${ }^{1} \mathrm{H}$ NMR spectroscopy. Catalyst loading: $10 \mathrm{~mol} \% .{ }^{d} 1,2,2,6,6-\mathrm{PMP}=1,2,2,6,6$-pentamethylpiperidine. 


\section{Synthesis of 1-Silyl Alkynes}

\section{General Procedure A.}

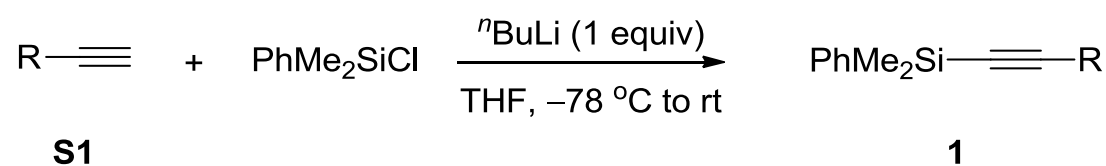

This procedure was modified from a literature procedure. ${ }^{1}$ At $-78{ }^{\circ} \mathrm{C}$, to solution of terminal alkynes S1 (1.0 equiv) in THF (0.5 M) under $\mathrm{N}_{2}$ atmosphere was slowly added ${ }^{n} \mathrm{BuLi}(2.4 \mathrm{M}$ solution in hexane, 1.0 equiv). The reaction mixture was stirred at the same temperature for $1 \mathrm{~h}$, then chlorodimethyl(phenyl)silane (1.0 equiv) was added dropwise. Next, the reaction mixture was allowed to warm to room temperature and stirred for 7 h before a saturated aqueous $\mathrm{NH}_{4} \mathrm{Cl}$ was added. The aqueous phase was separated and extracted with diethyl ether twice. The combined organic layers were washed with brine and dried over anhydrous $\mathrm{Na}_{2} \mathrm{SO}_{4}$. The solvent was removed on a rotary evaporator, and the residue was purified by silica gel flash column chromatography to give the desired 1-silyl alkynes $\mathbf{1}$.

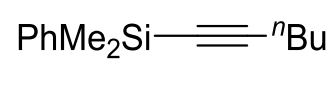

$1 \mathbf{a}$

Hex-1-yn-1-yldimethyl(phenyl)silane (1a) was prepared as colorless oil according to the General Procedure A.

${ }^{13} \mathrm{C}$ NMR $\left(100 \mathrm{MHz}, \mathrm{CDCl}_{3}\right) \delta 137.7,133.6,129.2,127.8,109.6,82.1,30.7,21.9$, $19.7,13.6,-0.6$.

${ }^{1} \mathbf{H}$ NMR $\left(400 \mathrm{MHz}, \mathrm{CDCl}_{3}\right) \delta 7.67-7.64(\mathrm{~m}, 2 \mathrm{H}), 7.40-7.37(\mathrm{~m}, 3 \mathrm{H}), 2.30(\mathrm{t}, J$ $=7.0 \mathrm{~Hz}, 2 \mathrm{H}), 1.60-1.52(\mathrm{~m}, 2 \mathrm{H}), 1.50-1.42(\mathrm{~m}, 2 \mathrm{H}), 0.94(\mathrm{t}, J=6.9 \mathrm{~Hz}, 3 \mathrm{H})$, $0.41(\mathrm{~s}, 6 \mathrm{H})$

The spectral data are identical to those reported in the literature. ${ }^{2}$ 


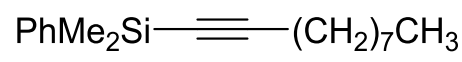

1b

Dec-1-yn-1-yldimethyl(phenyl)silane (1b) was prepared as colorless oil according to the General Procedure A.

${ }^{1} \mathbf{H}$ NMR $\left(400 \mathrm{MHz}, \mathrm{CDCl}_{3}\right) \delta 7.73-7.66(\mathrm{~m}, 2 \mathrm{H}), 7.45-7.38(\mathrm{~m}, 3 \mathrm{H}), 2.32(\mathrm{t}, J$ $=7.1 \mathrm{~Hz}, 2 \mathrm{H}), 1.66-1.56(\mathrm{~m}, 2 \mathrm{H}), 1.51-1.43(\mathrm{~m}, 2 \mathrm{H}), 1.35(\mathrm{dd}, J=10.6,7.1 \mathrm{~Hz}$, $8 \mathrm{H}), 0.95(\mathrm{t}, J=6.9 \mathrm{~Hz}, 3 \mathrm{H}), 0.45(\mathrm{~s}, 6 \mathrm{H})$.

${ }^{13} \mathrm{C}$ NMR $\left(100 \mathrm{MHz}, \mathrm{CDCl}_{3}\right) \delta 137.7,133.6,129.1,127.8,109.7,82.1,31.8,29.2$, $29.0,28.8,28.6,22.7,20.0,14.1,-0.6$.

The spectral data are identical to those reported in the literature. ${ }^{3}$

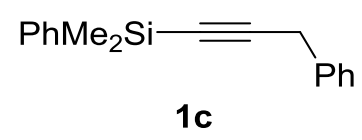

Dimethyl(phenyl)(3-phenylprop-1-yn-1-yl)silane (1c) was prepared was prepared as colorless oil according to the General Procedure A.

${ }^{1} \mathbf{H}$ NMR $\left(400 \mathrm{MHz} \mathrm{CDCl}_{3}\right) \delta 7.72-7.66(\mathrm{~m}, 2 \mathrm{H}), 7.42-7.33(\mathrm{~m}, 7 \mathrm{H}), 7.28(\mathrm{~d}, J$ $=7.1 \mathrm{~Hz}, 1 \mathrm{H}), 3.74(\mathrm{~s}, 2 \mathrm{H}), 0.48-0.46(\mathrm{~m}, 6 \mathrm{H})$.

${ }^{13} \mathrm{C}$ NMR $\left(100 \mathrm{MHz} \mathrm{CDCl}_{3}\right) \delta 136.8,135.6,133.1,128.8,128.0,127.33,127.29$, $126.1,105.6,84.3,25.7,-1.2$.

The spectral data are identical to those reported in the literature. ${ }^{4}$

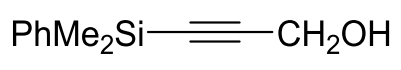

1d

3-(Dimethyl(phenyl)silyl)prop-2-yn-1-ol (1d) was prepared as colorless oil according to the reported procedure. ${ }^{5}$

${ }^{1} \mathrm{H}$ NMR $\left(400 \mathrm{MHz}, \mathrm{CDCl}_{3}\right) \delta 7.66(\mathrm{dd}, J=5.0,2.1 \mathrm{~Hz}, 2 \mathrm{H}), 7.45-7.38(\mathrm{~m}, 3 \mathrm{H})$, $4.30(\mathrm{~s}, 2 \mathrm{H}), 2.44(\mathrm{~s}, 1 \mathrm{H}), 0.48(\mathrm{~s}, 6 \mathrm{H})$.

${ }^{13} \mathrm{C}$ NMR $\left(100 \mathrm{MHz}, \mathrm{CDCl}_{3}\right) \delta 136.4,133.6,129.5,127.9,105.6,88.5,51.4,-1.2$.

The spectral data are identical to those reported in the literature. ${ }^{5}$ 


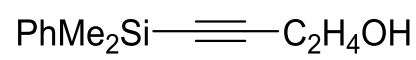

$1 \mathrm{e}$

4-(Dimethyl(phenyl)silyl)but-3-yn-1-ol (1e) was prepared as colorless oil according to the reported procedure. ${ }^{6}$

${ }^{1}$ H NMR $\left(400 \mathrm{MHz} \mathrm{CDCl}_{3}\right) \delta 7.70(\mathrm{dd}, J=6.5,2.9 \mathrm{~Hz}, 2 \mathrm{H}), 7.49-7.40(\mathrm{~m}, 3 \mathrm{H})$,

$3.75(\mathrm{t}, J=6.6 \mathrm{~Hz}, 2 \mathrm{H}), 2.80(\mathrm{~s}, 1 \mathrm{H}), 2.57(\mathrm{t}, J=6.6 \mathrm{~Hz}, 2 \mathrm{H}), 0.49(\mathrm{~s}, 6 \mathrm{H})$.

${ }^{13} \mathrm{C}$ NMR $\left(100 \mathrm{MHz} \mathrm{CDCl}_{3}\right) \delta 136.6,133.1,128.9,127.4,105.0,84.0,60.3,23.7,-$ 1.3.

The spectral data are identical to those reported in the literature. ${ }^{6}$

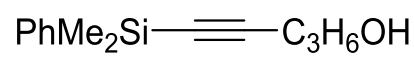

$1 f$

5-(Dimethyl(phenyl)silyl)pent-4-yn-1-ol (1f) was prepared as colorless oil according to the reported procedure. ${ }^{6}$

${ }^{1} \mathrm{H}$ NMR $\left(400 \mathrm{MHz}, \mathrm{CDCl}_{3}\right) \delta 7.67-7.60(\mathrm{~m}, 2 \mathrm{H}), 7.41-7.36(\mathrm{~m}, 3 \mathrm{H}), 3.76(\mathrm{t}, J$ $=6.1 \mathrm{~Hz}, 2 \mathrm{H}), 2.41(\mathrm{t}, J=7.0 \mathrm{~Hz}, 2 \mathrm{H}), 1.91(\mathrm{~s}, 1 \mathrm{H}), 1.83-1.77(\mathrm{~m}, 2 \mathrm{H}), 0.42-0.39$ (m, 6H).

${ }^{13} \mathrm{C}$ NMR $\left(100 \mathrm{MHz}, \mathrm{CDCl}_{3}\right) \delta 137.3,133.6,129.3,127.8,108.5,83.1,61.7,31.1$, $16.5,-0.8$.

The spectral data are identical to those reported in the literature. ${ }^{6}$

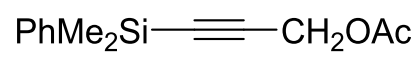

$1 \mathrm{~g}$

3-(Dimethyl(phenyl)silyl)prop-2-yn-1-yl acetate (1g) was prepared as colorless oil from 3-(Dimethyl(phenyl)silyl)prop-2-yn-1-ol (1d) according to reported procedure. ${ }^{7}$

${ }^{1}$ H NMR (400 MHz, $\left.\mathrm{CDCl}_{3}\right) \delta 7.66-7.63(\mathrm{~m}, 2 \mathrm{H}), 7.44-7.40(\mathrm{~m}, 3 \mathrm{H}), 4.88(\mathrm{~s}$, $2 \mathrm{H}), 2.14$ (s, $3 \mathrm{H}), 0.47$ (s, $6 \mathrm{H})$. 
${ }^{13} \mathrm{C}$ NMR $\left(100 \mathrm{MHz}, \mathrm{CDCl}_{3}\right) \delta 170.1,136.2,133.6,129.6,127.9,100.6,90.1,52.7$, $20.7,-1.2$.

IR (thin film) 2960, 2183, 1480, 1256, $1190 \mathrm{~cm}^{-1}$.

HRMS $m / z$ (CI) calcd. for $\mathrm{C}_{13} \mathrm{H}_{15} \mathrm{O}_{2} \mathrm{Si}[\mathrm{M}-\mathrm{H}]^{+}$231.0842, found 231.0846.

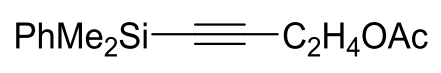

$1 \mathrm{~h}$

4-(Dimethyl(phenyl)silyl)but-3-yn-1-yl acetate (1h) was prepared as colorless oil from 4-(Dimethyl(phenyl)silyl)but-3-yn-1-ol (1e) according to reported procedure. ${ }^{7}$

${ }^{1} \mathrm{H}$ NMR $\left(400 \mathrm{MHz} \mathrm{CDCl}_{3}\right) \delta 7.67-7.64(\mathrm{~m}, 2 \mathrm{H}), 7.42-7.39(\mathrm{~m}, 3 \mathrm{H}), 4.23(\mathrm{t}, J$ $=6.8 \mathrm{~Hz}, 2 \mathrm{H}), 2.65(\mathrm{t}, J=6.8 \mathrm{~Hz}, 2 \mathrm{H}), 2.10(\mathrm{~s}, 3 \mathrm{H}), 0.43(\mathrm{~s}, 6 \mathrm{H})$.

${ }^{13} \mathrm{C}$ NMR $\left(100 \mathrm{MHz}, \mathrm{CDCl}_{3}\right) \delta 170.8,137.1,133.6,129.3,127.8,104.1,84.5,62.1$, $20.8,20.4,-0.9$.

IR (thin film) 2965, 2179, 1749, 1452, $1249 \mathrm{~cm}^{-1}$.

HRMS $m / z$ (CI) calcd. for $\mathrm{C}_{14} \mathrm{H}_{18} \mathrm{O}_{2} \mathrm{Si}\left[\mathrm{M}^{+}\right]$246.1076, found 246.1025.

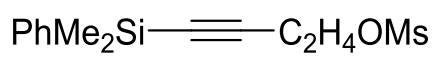

$1 \mathrm{i}$

4-(Dimethyl(phenyl)silyl)but-3-yn-1-yl methanesulfonate (1i) was prepared as colorless oil from 4-(dimethyl(phenyl)silyl)but-3-yn-1-ol (1e) according to reported procedure. ${ }^{8}$

${ }^{1} \mathbf{H}$ NMR (400 MHz, $\left.\mathrm{CDCl}_{3}\right) \delta 7.66$ - $7.63(\mathrm{~m}, 2 \mathrm{H}), 7.42-7.39$ (m, $\left.3 \mathrm{H}\right), 4.36$ $4.33(\mathrm{t}, J=6.8 \mathrm{~Hz}, 2 \mathrm{H}), 3.01(\mathrm{~s}, 3 \mathrm{H}), 2.77(\mathrm{t}, J=6.8 \mathrm{~Hz}, 2 \mathrm{H}), 0.44(\mathrm{~s}, 6 \mathrm{H})$.

${ }^{13} \mathrm{C}$ NMR $\left(100 \mathrm{MHz}, \mathrm{CDCl}_{3}\right) \delta 136.6,133.6,129.5,127.9,102.4,85.8,67.2,37.5$, 21.1, -1.0.

IR (thin film) 2961, 2181, 1429, 1360, $1175 \mathrm{~cm}^{-1}$.

HRMS m/z (CI) calcd. for $\mathrm{C}_{13} \mathrm{H}_{18} \mathrm{O}_{3} S S i\left[\mathrm{M}^{+}\right]$282.0746, found 282.0742. 


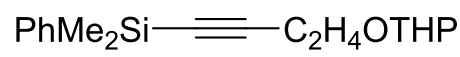

$1 \mathrm{j}$

Dimethyl(phenyl)(4-((tetrahydro-2H-pyran-2-yl)oxy)but-1-yn-1-yl)silane (1j) was prepared as colorless oil according to the reported procedure. ${ }^{9}$

${ }^{1}$ H NMR (400 MHz, $\left.\mathrm{CDCl}_{3}\right) \delta 7.65-7.63(\mathrm{~m}, 2 \mathrm{H}), 7.39-7.36(\mathrm{~m}, 3 \mathrm{H}), 4.68(\mathrm{t}, J$ $=3.4 \mathrm{~Hz}, 1 \mathrm{H}), 3.93-3.84(\mathrm{~m}, 2 \mathrm{H}), 3.62-3.56(\mathrm{~m}, 1 \mathrm{H}), 3.53-3.48(\mathrm{~m}, 1 \mathrm{H}), 2.60$ $(\mathrm{t}, J=7.1 \mathrm{~Hz}, 2 \mathrm{H}), 1.90-1.79(\mathrm{~m}, 1 \mathrm{H}), 1.76-1.68(\mathrm{~m}, 1 \mathrm{H}), 1.65-1.49(\mathrm{~m}, 4 \mathrm{H})$, $0.40(\mathrm{~s}, 6 \mathrm{H})$.

${ }^{13} \mathrm{C}$ NMR $\left(100 \mathrm{MHz}, \mathrm{CDCl}_{3}\right) \delta 137.3,133.6,129.2,127.8,105.9,98.6,83.5,65.4$, $61.9,30.5,25.4,21.4,19.2,-0.78$.

The spectral data are identical to those reported in the literature. ${ }^{9}$

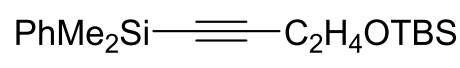

$1 \mathrm{k}$

tert-Butyl((4-(dimethyl(phenyl)silyl)but-3-yn-1-yl)oxy)dimethylsilane (1k) was prepared as colorless oil from 4-(dimethyl(phenyl)silyl)but-3-yn-1-ol (1e) according to reported procedure. ${ }^{10}$

${ }^{1} \mathrm{H}$ NMR $\left(400 \mathrm{MHz}, \mathrm{CDCl}_{3}\right) \delta 7.64-7.62(\mathrm{~m}, 2 \mathrm{H}), 7.39-7.35(\mathrm{~m}, 3 \mathrm{H}), 3.76(\mathrm{t}, J$ $=7.0 \mathrm{~Hz}, 2 \mathrm{H}), 2.50(\mathrm{t}, J=7.0 \mathrm{~Hz}, 2 \mathrm{H}), 0.90(\mathrm{~s}, 9 \mathrm{H}), 0.39(\mathrm{~s}, 6 \mathrm{H}), 0.08(\mathrm{~s}, 6 \mathrm{H})$.

${ }^{13} \mathrm{C}$ NMR $\left(100 \mathrm{MHz}_{2} \mathrm{CDCl}_{3}\right) \delta 137.4,133.7,129.3,127.8,106.1,83.5,61.7,25.9$, $24.4,18.3,-0.7,-5.3$.

IR (thin film) 2953, 2891, 2860, 2358, 2333, 2176, 1467, 1422, 1255, $1106 \mathrm{~cm}^{-1}$. HRMS $m / z$ (CI) calcd. for $\mathrm{C}_{18} \mathrm{H}_{31} \mathrm{OSi}_{2}[\mathrm{M}+\mathrm{H}]^{+}$319.1908, found 319.1919.

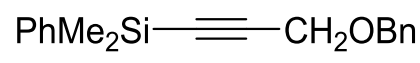

11

(3-(Benzyloxy)prop-1-yn-1-yl)dimethyl(phenyl)silane (11) was prepared as colorless oil according to the reported procedure. ${ }^{11}$ 
${ }^{1} \mathbf{H}$ NMR $\left(400 \mathrm{MHz}, \mathrm{CDCl}_{3}\right) \delta 7.86-7.79(\mathrm{~m}, 2 \mathrm{H}), 7.56-7.43(\mathrm{~m}, 8 \mathrm{H}), 4.77$ (d, J $=1.6 \mathrm{~Hz}, 2 \mathrm{H}), 4.36(\mathrm{~d}, J=1.5 \mathrm{~Hz}, 2 \mathrm{H}), 0.63(\mathrm{~s}, 6 \mathrm{H})$.

${ }^{13} \mathrm{C}$ NMR $\left(100 \mathrm{MHz}, \mathrm{CDCl}_{3}\right) \delta 137.3,136.5,133.6,129.4,128.3,127.9,127.8$, $127.7,103.3,89.5,71.4,57.8,-1.1$.

The spectral data are identical to those reported in the literature. ${ }^{11}$

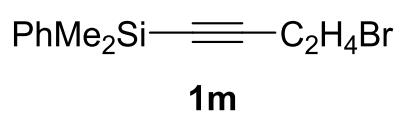

(4-Bromobut-1-yn-1-yl)dimethyl(phenyl)silane (1m) was prepared as colorless oil from 4-(Dimethyl(phenyl)silyl)but-3-yn-1-yl methanesulfonate (1i) according to reported procedure. ${ }^{12}$

${ }^{1} \mathrm{H}$ NMR (400 MHz, $\left.\mathrm{CDCl}_{3}\right) \delta 7.70-7.67(\mathrm{~m}, 2 \mathrm{H}), 7.44-7.42(\mathrm{~m}, 3 \mathrm{H}), 3.50(\mathrm{t}, J$ $=7.2 \mathrm{~Hz}, 2 \mathrm{H}), 2.88(\mathrm{t}, J=7.2 \mathrm{~Hz}, 2 \mathrm{H}), 0.46(\mathrm{~s}, 6 \mathrm{H})$.

${ }^{13} \mathrm{C}$ NMR $\left(100 \mathrm{MHz}, \mathrm{CDCl}_{3}\right) \delta 136.9,133.6,129.4,127.8,105.0,85.0,29.1,24.3$, 0.9 .

IR (thin film) 2962, 2178, 1428, 1270, $1116 \mathrm{~cm}^{-1}$.

HRMS $m / z$ (CI) calcd. for $\mathrm{C}_{12} \mathrm{H}_{14} \mathrm{BrSi}[\mathrm{M}-\mathrm{H}]^{+} 265.0048$, found 265.0053.

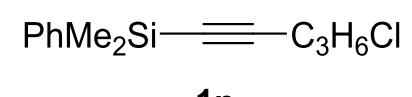

1n

(5-Chloropent-1-yn-1-yl)dimethyl(phenyl)silane (1n) was prepared as colorless oil according to the General Procedure A.

${ }^{1}$ H NMR (400 MHz, $\left.\mathrm{CDCl}_{3}\right) \delta$ 7.71-7.63 (m, 2H), $7.42(\mathrm{dd}, J=4.2,2.2 \mathrm{~Hz}, 3 \mathrm{H})$, $3.69(\mathrm{t}, J=6.4 \mathrm{~Hz}, 2 \mathrm{H}), 2.51(\mathrm{t}, J=6.8 \mathrm{~Hz}, 2 \mathrm{H}), 2.03(\mathrm{p}, J=6.6 \mathrm{~Hz}, 2 \mathrm{H}), 0.45(\mathrm{~s}$, $6 \mathrm{H})$.

${ }^{13} \mathrm{C} \mathrm{NMR}\left(100 \mathrm{MHz}, \mathrm{CDCl}_{3}\right) \delta 137.3,133.6,129.3,127.8,107.0,83.6,43.5,31.2$, $17.3,-0.7$.

The spectral data are identical to those reported in the literature. ${ }^{13}$ 


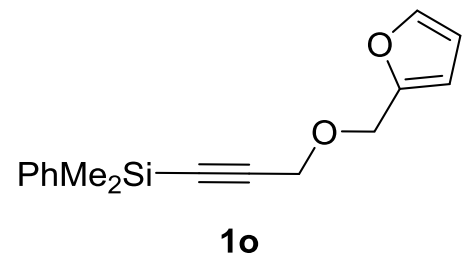

(3-(Furan-2-ylmethoxy)prop-1-yn-1-yl)dimethyl(phenyl)silane (1o) was prepared as light yellow oil according to the General Procedure A.

${ }^{1} \mathbf{H}$ NMR $\left(400 \mathrm{MHz}, \mathrm{CDCl}_{3}\right) \delta 7.71-7.68(\mathrm{~m}, 2 \mathrm{H}), 7.45-7.40(\mathrm{~m}, 4 \mathrm{H}), 6.40-$ $6.38(\mathrm{~m}, 2 \mathrm{H}), 4.61(\mathrm{~s}, 2 \mathrm{H}), 4.25(\mathrm{~s}, 2 \mathrm{H}), 0.50(\mathrm{~s}, 6 \mathrm{H})$.

${ }^{13} \mathrm{C}$ NMR $\left(100 \mathrm{MHz}, \mathrm{CDCl}_{3}\right) \delta 150.8,143.0,136.5,133.6,129.4,127.8,110.2$, $110.0,102.8,89.8,63.0,57.5,-1.0$.

IR (thin film) 3060, 2960, 2901, 2852, 2359, 2332, 2176, 1350, 1256, 1149, 1111, $1073 \mathrm{~cm}^{-1}$.

HRMS m/z (CI) calcd. for $\mathrm{C}_{16} \mathrm{H}_{18} \mathrm{O}_{2} \mathrm{Si}\left[\mathrm{M}^{+}\right] 270.1076$, found 270.1081 .

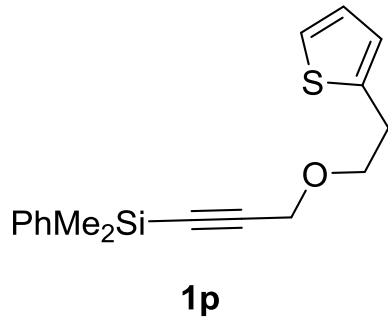

Dimethyl(phenyl)(3-(2-(thiophen-2-yl)ethoxy)prop-1-yn-1-yl)silane was prepared as light yellow oil according to the General Procedure A.

${ }^{1} \mathrm{H}$ NMR $\left(400 \mathrm{MHz}, \mathrm{CDCl}_{3}\right) \delta 7.66-7.64(\mathrm{~m}, 2 \mathrm{H}), 7.41-7.38(\mathrm{~m}, 3 \mathrm{H}), 7.16(\mathrm{~d}, J$ $=5.1 \mathrm{~Hz}, 1 \mathrm{H}), 6.96-6.94(\mathrm{~m}, 1 \mathrm{H}), 6.89(\mathrm{~s}, 1 \mathrm{H}), 4.25(\mathrm{~s}, 2 \mathrm{H}), 3.81(\mathrm{t}, J=6.9 \mathrm{~Hz}$, $2 \mathrm{H}), 3.16(\mathrm{t}, J=6.8 \mathrm{~Hz}, 2 \mathrm{H}), 0.46(\mathrm{~s}, 6 \mathrm{H})$.

${ }^{13} \mathrm{C}$ NMR $\left(100 \mathrm{MHz}, \mathrm{CDCl}_{3}\right) \delta 140.9,136.6,133.6,129.5,127.9,126.7,125.1$, 123.6, 103.1, 89.5, 70.4, 58.9, 30.2, -1.0 .

IR (thin film) 3097, 3060, 3010, 2857, 2360, 2331, 2174, 1429, 1402, 1352, 1254, $1099 \mathrm{~cm}^{-1}$.

HRMS $m / z$ (CI) calcd. for $\mathrm{C}_{17} \mathrm{H}_{21} \mathrm{OSSi}[\mathrm{M}+\mathrm{H}]^{+}$301.1077, found 301.1086. 


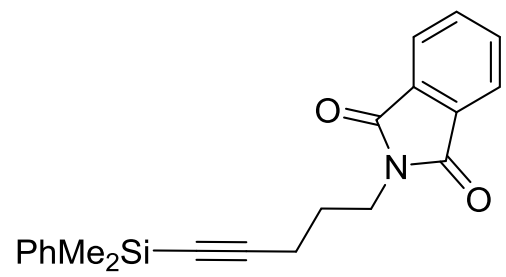

$1 q$

2-(5-(Dimethyl(phenyl)silyl)pent-4-yn-1-yl)isoindoline-1,3-dione (1q) was prepared as colorless oil from (5-chloropent-1-yn-1-yl)dimethyl(phenyl)silane (1n) according to the reported procedure. ${ }^{14}$

${ }^{1} \mathbf{H}$ NMR $\left(400 \mathrm{MHz}, \mathrm{CDCl}_{3}\right) \delta 7.83-7.79(\mathrm{~m}, 2 \mathrm{H}), 7.71-7.67$ (m, 2H), $7.62-$ $7.59(\mathrm{~m}, 2 \mathrm{H}), 7.38-7.35(\mathrm{~m}, 3 \mathrm{H}), 3.80(\mathrm{t}, J=7.0 \mathrm{~Hz}, 2 \mathrm{H}), 2.38(\mathrm{t}, J=7.2 \mathrm{~Hz}, 2 \mathrm{H})$, $2.01-1.93(\mathrm{~m}, 2 \mathrm{H}), 0.33(\mathrm{~s}, 6 \mathrm{H})$.

${ }^{13} \mathrm{C}$ NMR (100 MHz, $\left.\mathrm{CDCl}_{3}\right) \delta 168.2,137.3,133.8,133.6,132.0,129.2,127.7$, $123.2,107.6,83.2,37.3,27.4,17.8,-0.8$.

IR (thin film) 3059, 2952, 2359, 2332, 2173, 1771, 1708, 1432, 1394, 1363, 1251, $1113,1022 \mathrm{~cm}^{-1}$.

HRMS $m / z$ (CI) calcd. for $\mathrm{C}_{21} \mathrm{H}_{22} \mathrm{NO}_{2} \mathrm{Si}[\mathrm{M}+\mathrm{H}]^{+} 348.1414$, found 348.1418.

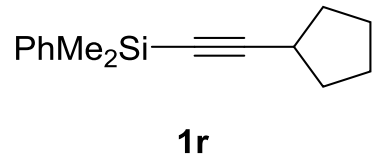

(Cyclopentylethynyl)dimethyl(phenyl)silane (1r)was prepared as colorless oil according to the General Procedure A.

${ }^{1} \mathrm{H}$ NMR (400 MHz, $\left.\mathrm{CDCl}_{3}\right) \delta 7.66-7.64(\mathrm{~m}, 2 \mathrm{H}), 7.39-7.38(\mathrm{~m}, 3 \mathrm{H}), 2.74-$ $2.67(\mathrm{~m}, 1 \mathrm{H}), 1.98-1.92(\mathrm{~m}, 2 \mathrm{H}), 1.78-1.55(\mathrm{~m}, 6 \mathrm{H}), 0.40(\mathrm{~s}, 6 \mathrm{H})$.

${ }^{13} \mathrm{C}$ NMR $\left(100 \mathrm{MHz}, \mathrm{CDCl}_{3}\right) \delta 137.9,133.7,129.1,127.7,114.0,81.2,33.9,31.1$, $25.1,-0.5$.

IR (thin film) 3056, 2960, 2873, 2165, 1425, 1261, 1114, $1007 \mathrm{~cm}^{-1}$. HRMS m/z (CI) calcd. for $\mathrm{C}_{15} \mathrm{H}_{20} \mathrm{Si}\left[\mathrm{M}^{+}\right]$228.1334, found 228.1330. 


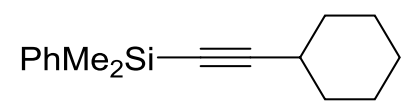

$1 \mathrm{~s}$

(Cyclohexylethynyl)dimethyl(phenyl)silane (1s)was prepared as colorless oil according to the General Procedure A.

${ }^{1} \mathrm{H}$ NMR $\left(400 \mathrm{MHz}, \mathrm{CDCl}_{3}\right) \delta 7.67(\mathrm{dd}, J=4.1,2.2 \mathrm{~Hz}, 2 \mathrm{H}), 7.39(\mathrm{dd}, J=3.8,1.9$ $\mathrm{Hz}, 3 \mathrm{H}), 2.48(\mathrm{t}, J=8.5 \mathrm{~Hz}, 1 \mathrm{H}), 1.87-1.84(\mathrm{~m}, 2 \mathrm{H}), 1.76-1.73(\mathrm{~m}, 2 \mathrm{H}), 1.56-$ $1.49(\mathrm{~m}, 3 \mathrm{H}), 1.34(\mathrm{q}, J=8.8 \mathrm{~Hz}, 3 \mathrm{H}), 0.41(\mathrm{~d}, J=1.8 \mathrm{~Hz}, 6 \mathrm{H})$.

${ }^{13} \mathrm{C}$ NMR $\left(100 \mathrm{MHz}_{2} \mathrm{CDCl}_{3}\right) \delta 137.9,133.7,129.1,127.7,81.6,32.5,30.1,25.8$, $24.8,-0.47$.

The spectral data are identical to those reported in the literature. ${ }^{13}$

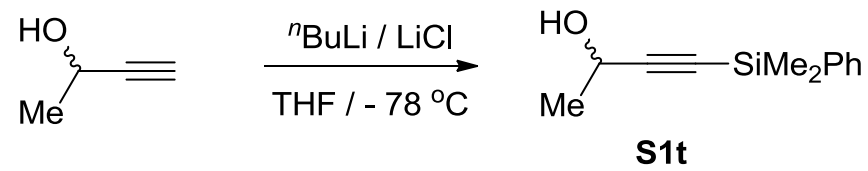

4-(Dimethyl(phenyl)silyl)but-3-yn-2-ol (S1t) was prepared according to the reported procedure. ${ }^{15}$

${ }^{1} \mathbf{H}$ NMR $\left(400 \mathrm{MHz}, \mathrm{CDCl}_{3}\right) \delta 7.67-7.65(\mathrm{~m}, 2 \mathrm{H}), 7.42-7.40(\mathrm{~m}, 3 \mathrm{H}), 4.57(\mathrm{q}, J$ $=6.6 \mathrm{~Hz}, 1 \mathrm{H}), 2.38(\mathrm{~s}, 1 \mathrm{H}), 1.49(\mathrm{~d}, J=6.6 \mathrm{~Hz}, 3 \mathrm{H}), 0.46(\mathrm{~s}, 6 \mathrm{H})$.

${ }^{13} \mathrm{C}$ NMR $\left(100 \mathrm{MHz}_{2} \mathrm{CDCl}_{3}\right) \delta 136.7,133.7,129.5,128.0,109.5,86.4,58.8,24.2,-$ 0.9 .

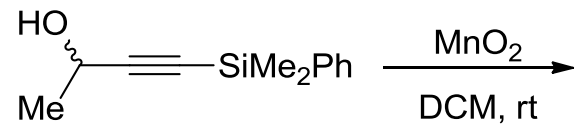

S1t

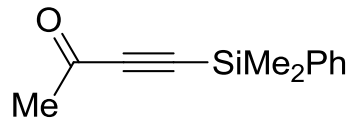

$1 \mathbf{t}$

4-(Dimethyl(phenyl)silyl)but-3-yn-2-one (1t) was prepared according to the reported procedure. ${ }^{16}$

${ }^{1} \mathbf{H}$ NMR (400 MHz, $\left.\mathrm{CDCl}_{3}\right) \delta 7.62(\mathrm{~d}, J=6.5 \mathrm{~Hz}, 2 \mathrm{H}), 7.45-7.39$ (m, 3H), 2.37 $(\mathrm{s}, 3 \mathrm{H}), 0.50(\mathrm{~s}, 6 \mathrm{H})$.

${ }^{13} \mathrm{C}$ NMR (100 MHz, $\left.\mathrm{CDCl}_{3}\right) \delta 184.3,134.7,133.7,130.0,128.1,103.5,95.3,32.5$, 
-1.7 .

The spectral data are identical to those reported in the literature. ${ }^{17}$

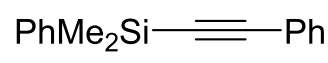

$1 \mathrm{u}$

Dimethyl(phenyl)(phenylethynyl)silane (1u) was prepared as colorless oil according to the General Procedure A.

${ }^{1}$ H NMR $\left(400 \mathrm{MHz} \mathrm{CDCl}_{3}\right) \delta 7.84-7.76(\mathrm{~m}, 2 \mathrm{H})$, 7.62-7.57 (m, 2H), 7.52 - 7.46 (m, 3H), $7.41-7.35(\mathrm{~m}, 3 \mathrm{H}), 0.60(\mathrm{~s}, 6 \mathrm{H})$.

${ }^{13} \mathrm{C}$ NMR (100 MHz, $\left.\mathrm{CDCl}_{3}\right) \delta 137.0,133.7,132.0,129.4,128.7,128.2,127.9$, $122.9,106.8,92.0,-0.8$.

The spectral data are identical to those reported in the literature. ${ }^{13}$

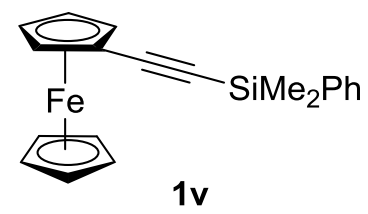

(Ferrocenylethynyl)dimethyl(phenyl)silane (1v) was prepared as orange yellow solid according to the reported procedure. ${ }^{13}$

${ }^{1}$ H NMR (400 MHz, $\left.\mathrm{CDCl}_{3}\right) \delta 7.77-7.69(\mathrm{~m}, 2 \mathrm{H}), 7.46-7.37$ (m, 3H), 4.50 (s, $2 \mathrm{H}), 4.23(\mathrm{~d}, J=4.4 \mathrm{~Hz}, 7 \mathrm{H}), 0.49(\mathrm{~s}, 6 \mathrm{H})$.

${ }^{13} \mathrm{C}$ NMR $\left(100 \mathrm{MHz}, \mathrm{CDCl}_{3}\right) \delta 137.6,133.7,129.3,127.8,106.2,88.4,71.9,70.1$, $68.9,64.5,-0.6$.

The spectral data are identical to those reported in the literature. ${ }^{13}$

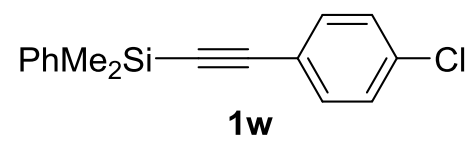

((4-Chlorophenyl)ethynyl)dimethyl(phenyl)silane (1w) was prepared as white solid according to the General Procedure A. 
${ }^{1}$ H NMR $\left(400 \mathrm{MHz}^{\mathrm{CDCl}} 3\right) \delta 7.71-7.69(\mathrm{~m}, 2 \mathrm{H}), 7.45-7.42(\mathrm{~m}, 5 \mathrm{H}), 7.30(\mathrm{~d}, \mathrm{~J}$ $=8.2 \mathrm{~Hz}, 2 \mathrm{H}), 0.52(\mathrm{~s}, 6 \mathrm{H})$.

${ }^{13} \mathrm{C}$ NMR $\left(100 \mathrm{MHz}, \mathrm{CDCl}_{3}\right) \delta 136.7,134.7,133.7,133.3,129.5,128.6,127.9$, $121.4,105.4,93.3,-0.9$.

IR (thin film) 2059, 3012, 2961, 2900, 2358, 2158, 1482, 1424, 1255, 1226, 1088, $1013 \mathrm{~cm}^{-1}$.

HRMS $m / z$ (CI) calcd. for $\mathrm{C}_{16} \mathrm{H}_{15} \mathrm{ClSi}\left[\mathrm{M}^{+}\right]$270.0632, found 270.0637.

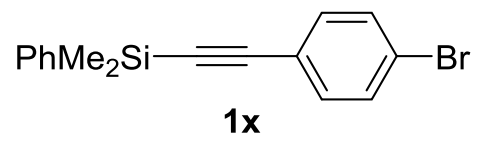

((4-Bromophenyl)ethynyl)dimethyl(phenyl)silane (1x) was prepared as white solid according to the General Procedure A.

${ }^{1} \mathbf{H}$ NMR $\left(400 \mathrm{MHz}, \mathrm{CDCl}_{3}\right) \delta 7.72-7.69(\mathrm{~m}, 2 \mathrm{H}), 7.48-7.36(\mathrm{~m}, 7 \mathrm{H}), 0.52(\mathrm{~s}$, $6 \mathrm{H})$.

${ }^{13} \mathrm{C}$ NMR (100 MHz, $\left.\mathrm{CDCl}_{3}\right) \delta 136.7,133.7,133.4,131.5,129.5,127.9,123.0$, $121.9,105.4,93.5,-0.9$.

The spectral data are identical to those reported in the literature. ${ }^{13}$

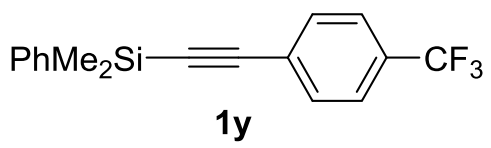

Dimethyl(phenyl)((4-(trifluoromethyl)phenyl)ethynyl)silane (1y) was prepared as colorless oil according to the General Procedure A.

${ }^{1} \mathbf{H}$ NMR $\left(400 \mathrm{MHz}, \mathrm{CDCl}_{3}\right) \delta 7.77-7.73(\mathrm{~m}, 2 \mathrm{H}), 7.66-7.60(\mathrm{~m}, 4 \mathrm{H}), 7.47$ $7.46(\mathrm{~m}, 3 \mathrm{H}), 0.58(\mathrm{~s}, 6 \mathrm{H})$.

${ }^{13} \mathrm{C}$ NMR (100 MHz, $\left.\mathrm{CDCl}_{3}\right) \delta$ 136.5, 133.7, 132.3, 130.3 (q, J = 326.2 Hz), 129.6, $128.0,126.7,125.1(q, J=3.7 \mathrm{~Hz}), 122.5,105.0,95.1,-1.0$.

${ }^{19}$ F NMR $\left(376 \mathrm{MHz} \mathrm{CDCl}_{3}\right) \delta-62.8$. 
IR (thin film) 3061, 3013, 2963, 2357, 2161, 1612, 1414, 1320, 1257, 1167, 1119, $1064 \mathrm{~cm}^{-1}$.

HRMS $m / z$ (CI) calcd. for $\mathrm{C}_{17} \mathrm{H}_{15} \mathrm{~F}_{3} \mathrm{Si}\left[\mathrm{M}^{+}\right]$384.0895, found 384.0900.

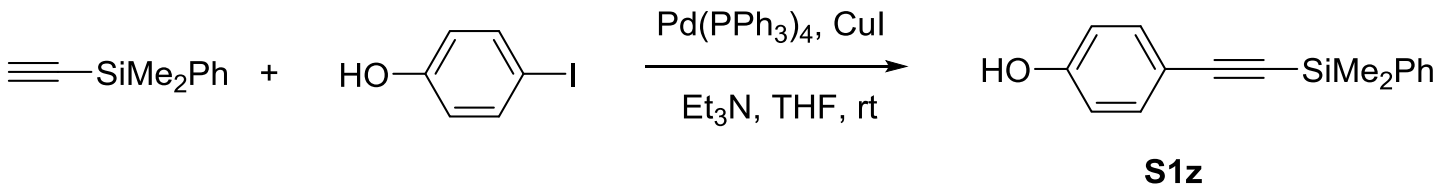

4-((Dimethyl(phenyl)silyl)ethynyl)phenol (S1z) was prepared as colorless oil according to the reported procedure. ${ }^{18}$

${ }^{1} \mathbf{H}$ NMR $\left(400 \mathrm{MHz}, \mathrm{CDCl}_{3}\right) \delta 7.76-7.72(\mathrm{~m}, 2 \mathrm{H}), 7.45-7.41(\mathrm{~m}, 5 \mathrm{H}), 6.77(\mathrm{~d}, J$ $=8.7 \mathrm{~Hz}, 2 \mathrm{H}), 5.32(\mathrm{~s}, 1 \mathrm{H}), 0.53(\mathrm{~s}, 6 \mathrm{H})$.

${ }^{13} \mathrm{C}$ NMR $\left(100 \mathrm{MHz}, \mathrm{CDCl}_{3}\right) \delta 155.9,137.2,133.8,133.7,129.4,127.9,115.4$, $115.2,106.8,90.5,-0.8$.

IR (thin film) 3379, 3058, 3012, 2960, 2898, 2358, 2151, 1602, 1505, 1430, 1256, $1222,1172,1111 \mathrm{~cm}^{-1}$.

HRMS m/z (CI) calcd. for $\mathrm{C}_{16} \mathrm{H}_{16} \mathrm{OSi}\left[\mathrm{M}^{+}\right]$252.0966, found 252.0970.

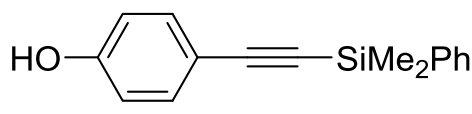

S1z

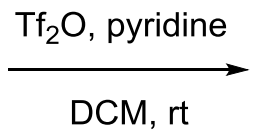

$\mathrm{DCM}, \mathrm{rt}$

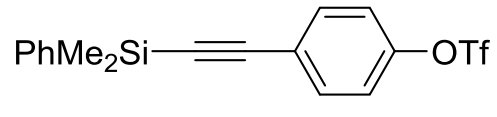

$1 z$

4-((Dimethyl(phenyl)silyl)ethynyl)phenyl trifluoromethanesulfonate (1z) was prepared as brown oil according to the reported procedures. ${ }^{18}$ ${ }^{1}$ H NMR $\left(400 \mathrm{MHz}, \mathrm{CDCl}_{3}\right) \delta 7.71-7.70(\mathrm{~m}, 2 \mathrm{H}), 7.59$ (d, J = 8.6 Hz, 2H), $7.44-$ $7.43(\mathrm{~m}, 3 \mathrm{H}), 7.25(\mathrm{~d}, J=8.7 \mathrm{~Hz}, 2 \mathrm{H}), 0.54(\mathrm{~s}, 6 \mathrm{H})$.

${ }^{13} \mathrm{C}$ NMR (100 MHz, $\left.\mathrm{CDCl}_{3}\right) \delta 149.2,136.4,133.9,133.7,129.6,128.0,123.6$, $121.4,118.7(q, J=319.0 \mathrm{~Hz}), 104.3,94.7,-1.04$.

${ }^{19}$ F NMR $\left(376 \mathrm{MHz}, \mathrm{CDCl}_{3}\right) \delta-72.7$.

IR (thin film) 3061, 2965, 2358, 2333, 1261, 1494, 1422, 1256, 1214, $1138 \mathrm{~cm}^{-1}$.

HRMS m/z (CI) calcd. for $\mathrm{C}_{17} \mathrm{H}_{15} \mathrm{~F}_{3} \mathrm{O}_{3} S S i\left[\mathrm{M}^{+}\right]$384.0463, found 384.0448. 


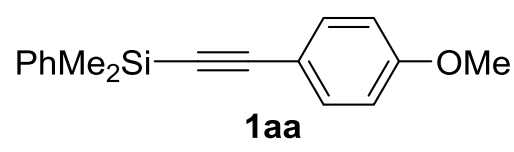

((4-Methoxyphenyl)ethynyl)dimethyl(phenyl)silane (1aa) was prepared as colorless oil according to the General Procedure A.

${ }^{1} \mathrm{H}$ NMR (400 MHz, $\left.\mathrm{CDCl}_{3}\right) \delta 7.72-7.69(\mathrm{~m}, 2 \mathrm{H}), 7.47-7.44(\mathrm{~m}, 1 \mathrm{H}), 7.41-$ $7.39(\mathrm{~m}, 4 \mathrm{H}), 6.86-6.82(\mathrm{~m}, 2 \mathrm{H}), 3.82(\mathrm{~s}, 3 \mathrm{H}), 0.49(\mathrm{~s}, 6 \mathrm{H})$.

${ }^{13} \mathrm{C}$ NMR (100 MHz, $\left.\mathrm{CDCl}_{3}\right) \delta 159.9,137.3,133.7,133.6,129.3,127.9,115.0$, $113.8,106.9,90.3,55.3,-0.73$.

The spectral data are identical to those reported in the literature. ${ }^{13}$

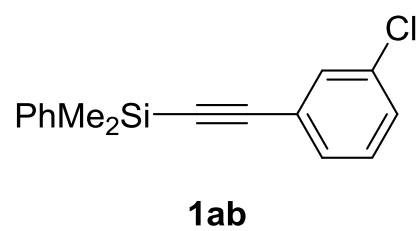

((3-Chlorophenyl)ethynyl)dimethyl(phenyl)silane was prepared as colorless oil according to the General Procedure A.

${ }^{1} \mathbf{H}$ NMR $\left(400 \mathrm{MHz}_{\mathrm{CDCl}}\right) \delta 7.75-7.72(\mathrm{~m}, 2 \mathrm{H}), 7.55(\mathrm{t}, J=1.6 \mathrm{~Hz}, 1 \mathrm{H}), 7.48-$ $7.41(\mathrm{~m}, 4 \mathrm{H}), 7.37-7.34(\mathrm{~m}, 1 \mathrm{H}), 7.30-7.28(\mathrm{~m}, 1 \mathrm{H}), 0.56(\mathrm{~s}, 6 \mathrm{H})$.

${ }^{13} \mathrm{C}$ NMR $\left(100 \mathrm{MHz}, \mathrm{CDCl}_{3}\right) \delta 136.6,134.1,133.7,131.9,130.1,129.5,129.4$, $128.9,127.9,124.6,105.0,93.7,-1.0$.

The spectral data are identical to those reported in the literature. ${ }^{13}$

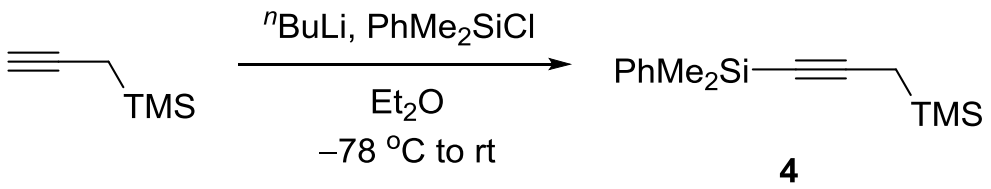

(3-(Dimethyl(phenyl)silyl)prop-2-yn-1-yl)trimethylsilane (4) was prepared as colorless oil according to the General Procedure A.

${ }^{1} \mathbf{H}$ NMR (400 MHz, $\left.\mathrm{CDCl}_{3}\right) \delta 7.65-7.63(\mathrm{~m}, 2 \mathrm{H}), 7.37$ - $7.35(\mathrm{~m}, 3 \mathrm{H}), 1.62(\mathrm{~s}$, 2H), $0.38(\mathrm{~s}, 6 \mathrm{H}), 0.13(\mathrm{~s}, 9 \mathrm{H})$. 
${ }^{13} \mathrm{C}$ NMR $\left(100 \mathrm{MHz}, \mathrm{CDCl}_{3}\right) \delta 138.1,133.6,129.1,127.7,107.8,80.9,8.9,-0.5,-$ 2.1.

IR (thin film) 3059, 3011, 2956, 2895, 2358, 2332, 2155, 1420, 1251, 1114, 1038 $\mathrm{cm}^{-1}$.

HRMS $m / z(\mathrm{CI})$ calcd. for $\mathrm{C}_{14} \mathrm{H}_{22} \mathrm{Si}_{2}\left[\mathrm{M}^{+}\right] 246.1260$, found 246.1265 .

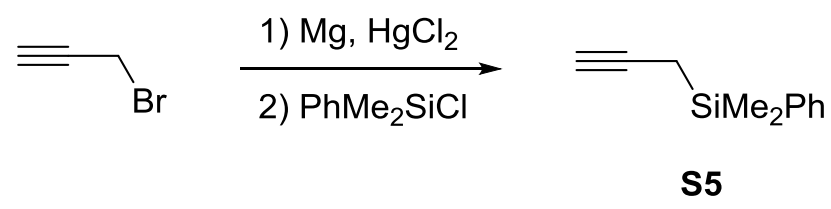

Ethynyldimethyl(phenyl)silane was prepared as colorless oil according to the reported procedure. ${ }^{19}$

${ }^{1} \mathrm{H}$ NMR $\left(400 \mathrm{MHz}, \mathrm{CDCl}_{3}\right) \delta 7.59-7.56(\mathrm{~m}, 2 \mathrm{H}), 7.40-7.35(\mathrm{~m}, 3 \mathrm{H}), 1.87(\mathrm{t}, J$ $=2.9 \mathrm{~Hz}, 2 \mathrm{H}), 1.73(\mathrm{~d}, J=2.9 \mathrm{~Hz}, 1 \mathrm{H}), 0.42(\mathrm{~s}, 6 \mathrm{H})$.

${ }^{13} \mathrm{C}$ NMR $\left(100 \mathrm{MHz} \mathrm{CDCl}_{3}\right) \delta 137.3,133.5,129.4,127.9,82.1,67.4,6.2,-3.6$.

The spectral data are identical to those reported in the literature. ${ }^{19}$

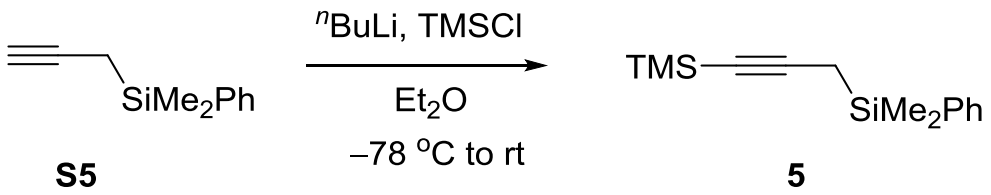

(3-(Dimethyl(phenyl)silyl)prop-1-yn-1-yl)trimethylsilane (5) was prepared as colorless oil according to the General Procedure A.

${ }^{1} \mathbf{H}$ NMR $\left(400 \mathrm{MHz} \mathrm{CDCl}_{3}\right) \delta 7.59$ - 7.569 (m, 2H), 7.39 - $7.36(\mathrm{~m}, 3 \mathrm{H}), 1.78(\mathrm{~s}$, $2 \mathrm{H}), 0.40(\mathrm{~s}, 6 \mathrm{H}), 0.13(\mathrm{~s}, 9 \mathrm{H})$.

${ }^{13} \mathrm{C}$ NMR $\left(100 \mathrm{MHz}_{2} \mathrm{CDCl}_{3}\right) \delta 137.4,133.6,129.4,127.7,105.2,83.9,8.3,0.2,-$ 3.6.

The spectral data are identical to those reported in the literature. ${ }^{20}$ 


\section{Ru(II)-Catalyzed gem-Hydrogenation of 1-Silyl Alkynes}

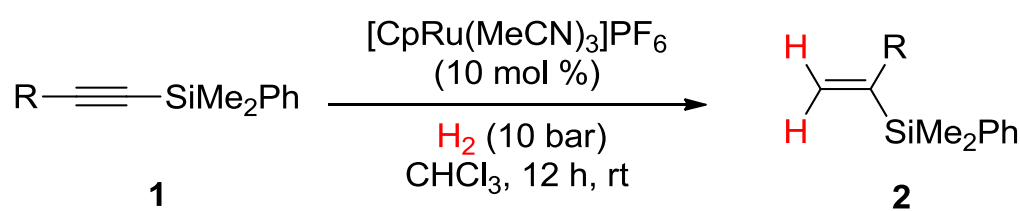

\section{General Procedure B.}

In a glove box, to an oven-dried 4-mL vial with a magnetic stirring bar were added the silyl alkyne $(0.20 \mathrm{mmol}),\left[\mathrm{CpRu}(\mathrm{MeCN})_{3}\right] \mathrm{PF}_{6}(8.7 \mathrm{mg}, 10 \mathrm{~mol} \%)$, and $\mathrm{CHCl}_{3}(2.0 \mathrm{~mL}, 0.1 \mathrm{M})$. The vial was then placed in a stainless autoclave (Parr ${ }^{\circledR}$ reactor) and purged with $\mathrm{H}_{2}$ for three cycles before it was filled with $\mathrm{H}_{2}$ at the required pressure (10 bar). Next, the reaction mixture was allowed to stir at room temperature for $12 \mathrm{~h}$ before it was concentrated under reduced pressure. The residue was purified by silica gel flash column chromatography to give the desired product.

\section{General Procedure C.}

In a glove box, to an oven-dried 10-mL schlenk tube with a magnetic stirring bar were added $\left[\mathrm{CpRu}(\mathrm{MeCN})_{3}\right] \mathrm{PF}_{6}(17.2 \mathrm{mg}, 0.04 \mathrm{mmol}, 20 \mathrm{~mol} \%), \mathrm{NaBAr}_{4}$ (28 mg, $0.04 \mathrm{mmol}, 20 \mathrm{~mol} \%$ ), and DCM (4.0 mL). The schlenk tube was sealed with a rubber stopper and removed from the glove box. A hydrogen balloon was then installed, and then rapid and careful evacuation and refill five times was performed. Next, a solution of the silyl alkyne $(0.20 \mathrm{mmol})$ was injected into the schlenk tube. The reaction mixture was then stirred at room temperature. The reaction progress was monitored by TLC. Upon completion, the mixture was concentrated under reduced pressure. The residue was purified by silica gel flash column chromatography to give the desired product. 


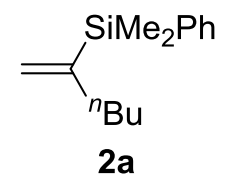

Hex-1-en-2-yldimethyl(phenyl)silane (2a) was prepared as colorless oil according to the General Procedure B in $90 \%$ yield $(B / L>30: 1)$.

${ }^{1} \mathbf{H}$ NMR $\left(400 \mathrm{MHz}, \mathrm{CDCl}_{3}\right) \delta 7.59-7.55(\mathrm{~m}, 2 \mathrm{H}), 7.41-7.38(\mathrm{~m}, 3 \mathrm{H}), 5.75$ $5.72(\mathrm{~m}, 1 \mathrm{H}), 5.46-5.44(\mathrm{~m}, 1 \mathrm{H}), 2.19(\mathrm{t}, J=7.6 \mathrm{~Hz}, 2 \mathrm{H}), 1.43-1.24(\mathrm{~m}, 4 \mathrm{H})$, $0.89(\mathrm{t}, J=7.2 \mathrm{~Hz}, 3 \mathrm{H}), 0.43(\mathrm{~s}, 6 \mathrm{H})$.

${ }^{13} \mathrm{C}$ NMR (100 MHz, $\left.\mathrm{CDCl}_{3}\right) \delta 150.5,138.5,133.9,128.9,127.7,125.6,35.7,31.1$, $22.5,13.9,-2.9$.

The spectral data are identical to those reported in the literature. ${ }^{21}$

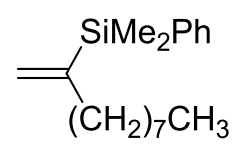

2b

Dec-1-en-2-yldimethyl(phenyl)silane (2b) was prepared as colorless oil according to the General Procedure B in $97 \%$ yield $(B / L>30: 1)$.

${ }^{1} \mathrm{H}$ NMR $\left(400 \mathrm{MHz}, \mathrm{CDCl}_{3}\right) \delta 7.60-7.55(\mathrm{~m}, 2 \mathrm{H}), 7.40-7.38(\mathrm{~m}, 3 \mathrm{H}), 5.75-$ $5.72(\mathrm{~m}, 1 \mathrm{H}), 5.46(\mathrm{~d}, J=3.0 \mathrm{~Hz}, 1 \mathrm{H}), 2.17(\mathrm{t}, J=7.6 \mathrm{~Hz}, 2 \mathrm{H}), 1.40-1.27(\mathrm{~m}$, $12 \mathrm{H}), 0.94(\mathrm{t}, J=7.0 \mathrm{~Hz}, 3 \mathrm{H}), 0.43(\mathrm{~s}, 6 \mathrm{H})$.

${ }^{13} \mathrm{C}$ NMR (100 MHz, $\left.\mathrm{CDCl}_{3}\right) \delta$ 150.0, 138.0, 133.3, 128.3, 127.1, 125.1, 35.5, 31.3, 28.89, 28.86, 28.7, 28.3, 22.1, 13.5, -3.5.

IR (thin film) 3056, 2953, 2923, 2855, 1458, 1427, 1250, 1110, 924, $819 \mathrm{~cm}^{-1}$. HRMS $m / z$ (CI) calcd. for $\mathrm{C}_{18} \mathrm{H}_{30} \mathrm{Si}\left[\mathrm{M}^{+}\right]$274.2117, found 274.2113. 


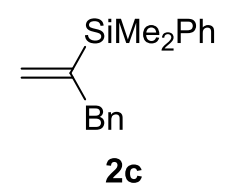

Dimethyl(phenyl)(3-phenylprop-1-en-2-yl)silane (2c) was prepared as colorless oil according to the General Procedure B in 80\% yield ( $B / L>30: 1)$. ${ }^{1} \mathbf{H}$ NMR (400 MHz, $\left.\mathrm{CDCl}_{3}\right) \delta 7.57$ - $7.52(\mathrm{~m}, 2 \mathrm{H}), 7.43-7.38(\mathrm{~m}, 3 \mathrm{H}), 7.32$ $7.27(\mathrm{~m}, 2 \mathrm{H}), 7.25-7.20(\mathrm{~m}, 1 \mathrm{H}), 7.16-7.10(\mathrm{~m}, 2 \mathrm{H}), 5.63(\mathrm{dt}, J=3.1,1.6 \mathrm{~Hz}$, $1 \mathrm{H}), 5.58-5.55(\mathrm{~m}, 1 \mathrm{H}), 3.50(\mathrm{~s}, 2 \mathrm{H}), 0.33(\mathrm{~s}, 6 \mathrm{H})$.

${ }^{13} \mathrm{C}$ NMR (100 MHz, $\left.\mathrm{CDCl}_{3}\right) \delta 149.6,139.8,137.9,134.0,129.3,129.0,128.1$, $127.8,127.7,125.9,42.6,-3.0$.

IR (thin film) 3057, 2955, 1596, 1493, 1426, 1251, 1110, 929, 818, $696 \mathrm{~cm}^{-1}$.

HRMS m/z (CI) calcd. for $\mathrm{C}_{17} \mathrm{H}_{20} \mathrm{Si}\left[\mathrm{M}^{+}\right]$252.1334, found 252.1331.

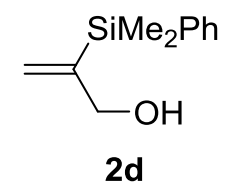

2-(Dimethyl(phenyl)silyl)prop-2-en-1-ol (2d) was prepared as colorless oil according to the General Procedure B in 85\% yield (B/L $>30: 1)$.

${ }^{1} \mathrm{H}$ NMR $\left(400 \mathrm{MHz}, \mathrm{CDCl}_{3}\right) \delta 7.57-7.54(\mathrm{~m}, 2 \mathrm{H}), 7.40-7.37(\mathrm{~m}, 3 \mathrm{H}), 5.95-$ $5.92(\mathrm{~m}, 1 \mathrm{H}), 5.52-5.50(\mathrm{~m}, 1 \mathrm{H}), 4.26(\mathrm{t}, J=1.6 \mathrm{~Hz}, 2 \mathrm{H}), 1.53$ (brms, 1H), $0.44(\mathrm{~s}$, $6 \mathrm{H})$.

${ }^{13} \mathrm{C}$ NMR $\left(100 \mathrm{MHz}, \mathrm{CDCl}_{3}\right) \delta 149.9,137.6,133.8,129.2,127.9,124.7,66.5,-3.1$. IR (thin film) 3333, 2957, 1428, 1250, $1111 \mathrm{~cm}^{-1}$. HRMS $m / z$ (CI) calcd. for $\mathrm{C}_{11} \mathrm{H}_{15} \mathrm{OSi}[\mathrm{M}-\mathrm{H}]^{+}$191.0892, found 191.0898. 


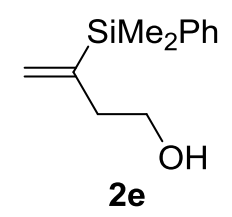

3-(Dimethyl(phenyl)silyl)but-3-en-1-ol (2e) was prepared as colorless oil according to the General Procedure B in 91\% yield $(B / L>30: 1)$.

${ }^{1} \mathbf{H}$ NMR (400 MHz, $\left.\mathrm{CDCl}_{3}\right) \delta 7.56$ - $7.53(\mathrm{~m}, 2 \mathrm{H}), 7.43$ - $7.38(\mathrm{~m}, 3 \mathrm{H}), 5.82$ $5.80(\mathrm{~m}, 1 \mathrm{H}), 5.60-5.58(\mathrm{~m}, 1 \mathrm{H}), 3.59(\mathrm{t}, J=6.8 \mathrm{~Hz}, 2 \mathrm{H}), 2.44(\mathrm{t}, J=6.8 \mathrm{~Hz}, 2$ H), 0.43 (s, $6 \mathrm{H})$.

${ }^{13} \mathrm{C}$ NMR $\left(100 \mathrm{MHz}, \mathrm{CDCl}_{3}\right) \delta 146.8,137.7,133.8,129.2,128.8,127.9,61.4,39.3$, -3.1 .

IR (thin film) 3384, 2956, 1428, 1250, $1112 \mathrm{~cm}^{-1}$.

HRMS $m / z$ (CI) calcd. for $\mathrm{C}_{12} \mathrm{H}_{17} \mathrm{OSi}[\mathrm{M}-\mathrm{H}]^{+}$205.1049, found 205.1050.

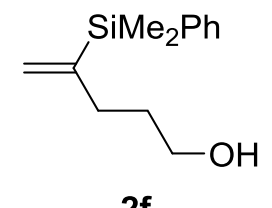

4-(Dimethyl(phenyl)silyl)pent-4-en-1-ol (2f) was prepared as colorless oil according to the General Procedure B in 93\% yield (B/L>30:1).

${ }^{1} \mathbf{H}$ NMR $\left(400 \mathrm{MHz}, \mathrm{CDCl}_{3}\right) \delta 7.52(\mathrm{dd}, J=6.5,3.0 \mathrm{~Hz}, 2 \mathrm{H}), 7.37-7.34(\mathrm{~m}, 3 \mathrm{H})$, $5.72(\mathrm{dd}, J=2.6,1.4 \mathrm{~Hz}, 1 \mathrm{H}), 5.47-5.43(\mathrm{~m}, 1 \mathrm{H}), 3.56(\mathrm{t}, J=6.5 \mathrm{~Hz}, 2 \mathrm{H}), 2.22-2.15$ (m, 2H), $1.66-1.58(\mathrm{~m}, 2 \mathrm{H}), 1.44(\mathrm{~s}, 1 \mathrm{H}), 0.39(\mathrm{~s}, 6 \mathrm{H})$.

${ }^{13} \mathrm{C}$ NMR (100 MHz, $\left.\mathrm{CDCl}_{3}\right) \delta$ 149.7, 138.1, 133.9, 129.0, 127.7, 126.1, 62.1, 31.9, $31.7,-3.0$.

IR (thin film) 3334, 3056, 2946, 2866, 1733, 1424, 1251, 1110, 1052, $922 \mathrm{~cm}^{-1}$.

HRMS $m / z$ (CI) calcd. for $\mathrm{C}_{13} \mathrm{H}_{24} \mathrm{ONSi}\left[\mathrm{M}+\mathrm{NH}_{4}\right]^{+}$238.1622, found 238.1624. 


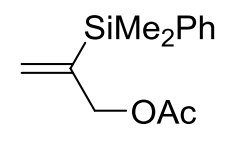

$2 \mathrm{~g}$

2-(Dimethyl(phenyl)silyl)allyl acetate (2g) was prepared as colorless oil according to the General Procedure B in $87 \%$ yield $(B / L>30: 1)$.

${ }^{1} \mathbf{H}$ NMR (400 MHz, $\left.\mathrm{CDCl}_{3}\right) \delta 7.56$ - $7.53(\mathrm{~m}, 2 \mathrm{H}), 7.41$ - 7.37 (m, $\left.3 \mathrm{H}\right), 5.94-$ $5.92(\mathrm{~m}, 1 \mathrm{H}), 5.58-5.56(\mathrm{~m}, 1 \mathrm{H}), 4.70(\mathrm{t}, J=1.6 \mathrm{~Hz}, 2 \mathrm{H}), 1.98(\mathrm{~s}, 3 \mathrm{H}), 0.45(\mathrm{~s}$, $6 \mathrm{H})$.

${ }^{13} \mathrm{C}$ NMR $\left(100 \mathrm{MHz}, \mathrm{CDCl}_{3}\right) \delta 170.6,144.7,137.1,133.8,129.2,127.8,127.4,67.9$, $20.8,-3.2$.

IR (thin film) 2959, 1750, 1428, 1373, $1230 \mathrm{~cm}^{-1}$.

HRMS $m / z$ (CI) calcd. for $\mathrm{C}_{13} \mathrm{H}_{17} \mathrm{O}_{2} \mathrm{Si}[\mathrm{M}-\mathrm{H}]^{+}$233.0998, found 233.0998.

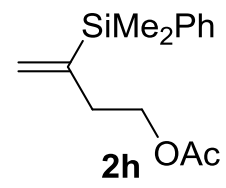

3-(Dimethyl(phenyl)silyl)but-3-en-1-yl acetate (2h) was prepared as colorless oil according to the General Procedure B in 99\% yield ( $B / L>30: 1)$. ${ }^{1} \mathbf{H}$ NMR (400 MHz, $\left.\mathrm{CDCl}_{3}\right) \delta 7.55-7.53(\mathrm{~m}, 2 \mathrm{H}), 7.43-7.38(\mathrm{~m}, 3 \mathrm{H}), 5.82$ $5.79(\mathrm{~m}, 1 \mathrm{H}), 5.58-5.55(\mathrm{~m}, 1 \mathrm{H}), 4.08(\mathrm{t}, J=7.6 \mathrm{~Hz}, 2 \mathrm{H}), 2.46(\mathrm{t}, J=7.6 \mathrm{~Hz}, 2$ H), 2.01 (s, $3 \mathrm{H}), 0.43$ (s, $6 \mathrm{H})$.

${ }^{13} \mathrm{C}$ NMR $\left(100 \mathrm{MHz}, \mathrm{CDCl}_{3}\right) \delta 171.0,145.9,137.6,133.8,129.1,128.4,127.8,63.7$, $34.4,20.9,-3.2$.

IR (thin film) 2958, 1760, 1428, 1364, $1247 \mathrm{~cm}^{-1}$.

HRMS $m / z$ (CI) calcd. for $\mathrm{C}_{14} \mathrm{H}_{18} \mathrm{O}_{2} \mathrm{Si}[\mathrm{M}-\mathrm{H}]^{+}$247.1155, found 247.1160. 


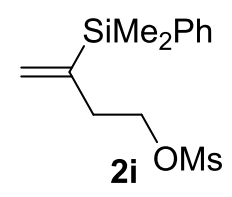

3-(Dimethyl(phenyl)silyl)but-3-en-1-yl methanesulfonate (2i) was prepared as colorless oil according to the General Procedure B in 95\% yield $(B / L>30: 1)$. ${ }^{1} \mathbf{H}$ NMR (400 MHz, $\left.\mathrm{CDCl}_{3}\right) \delta 7.56$ - $7.52(\mathrm{~m}, 2 \mathrm{H}), 7.41$ - 7.37 (m, $\left.3 \mathrm{H}\right), 5.83$ $5.82(\mathrm{~m}, 1 \mathrm{H}), 5.62-5.61(\mathrm{~m}, 1 \mathrm{H}), 4.15(\mathrm{t}, J=7.6 \mathrm{~Hz}, 2 \mathrm{H}), 2.87(\mathrm{~s}, 3 \mathrm{H}), 2.57(\mathrm{t}, J$ $=7.6 \mathrm{~Hz}, 2 \mathrm{H}), 0.44(\mathrm{~s}, 6 \mathrm{H})$.

${ }^{13} \mathrm{C}$ NMR $\left(100 \mathrm{MHz}, \mathrm{CDCl}_{3}\right) \delta 144.6,137.2,133.8,129.33,129.28,127.9,68.8$, $37.2,34.9,-3.3$.

IR (thin film) 2957, 1428, 1356, 1250, $1175 \mathrm{~cm}^{-1}$.

HRMS $m / z$ (CI) calcd. for $\mathrm{C}_{13} \mathrm{H}_{19} \mathrm{O}_{3} \mathrm{SSi}[\mathrm{M}-\mathrm{H}]^{+}$284.0824, found 283.0823.

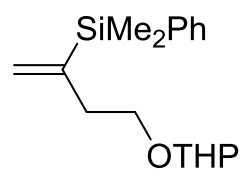

2j

Dimethyl(phenyl)(4-((tetrahydro-2H-pyran-2-yl)oxy)but-1-en-2-yl)silane (2j) was prepared as colorless oil according to the General Procedure B in $96 \%$ yield $(B / L>30: 1)$.

${ }^{1} \mathbf{H}$ NMR (400 MHz, $\left.\mathrm{CDCl}_{3}\right) \delta 7.56$ - $7.53(\mathrm{~m}, 2 \mathrm{H}), 7.38$ - 7.35 (m, $\left.3 \mathrm{H}\right), 5.80$ $5.78(\mathrm{~m}, 1 \mathrm{H}), 5.51-5.49(\mathrm{~m}, 1 \mathrm{H}), 4.51(\mathrm{t}, J=2.8 \mathrm{~Hz}, 1 \mathrm{H}), 3.84-3.73(\mathrm{~m}, 2 \mathrm{H})$, 3.50 - $3.37(\mathrm{~m}, 2 \mathrm{H}), 2.45(\mathrm{t}, J=7.6 \mathrm{~Hz}, 2 \mathrm{H}), 1.59-1.49(\mathrm{~m}, 6 \mathrm{H}), 0.41(\mathrm{~s}, 6 \mathrm{H})$.

${ }^{13} \mathrm{C}$ NMR $\left(100 \mathrm{MHz}, \mathrm{CDCl}_{3}\right) \delta 146.8,138.0,133.9,128.9,127.7,98.7,67.0,62.2$, $35.5,30.6,25.4,19.5,-3.09,-3.11$.

IR (thin film) 2944, 1428, 1352, 1249, $1032 \mathrm{~cm}^{-1}$.

HRMS $m / z$ (CI) calcd. for $\mathrm{C}_{17} \mathrm{H}_{25} \mathrm{O}_{2} \mathrm{Si}[\mathrm{M}-\mathrm{H}]^{+}$289.1624, found 289.1620. 


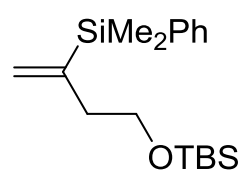

2k

tert-Butyl((3-(dimethyl(phenyl)silyl)but-3-en-1-yl)oxy)dimethylsilane (2k) was prepared as colorless oil according to the General Procedure B in $92 \%$ yield $(B / L>30: 1)$.

${ }^{1} \mathbf{H}$ NMR (400 MHz, $\left.\mathrm{CDCl}_{3}\right) \delta 7.56$ - $7.53(\mathrm{~m}, 2 \mathrm{H}), 7.40$ - $7.36(\mathrm{~m}, 3 \mathrm{H}), 5.79$ $5.77(\mathrm{~m}, 1 \mathrm{H}), 5.52-5.50(\mathrm{~m}, 1 \mathrm{H}), 3.58(\mathrm{t}, J=7.6 \mathrm{~Hz}, 2 \mathrm{H}), 2.39(\mathrm{t}, J=7.6 \mathrm{~Hz}, 2$ $\mathrm{H}), 0.89(\mathrm{~s}, 9 \mathrm{H}), 0.41(\mathrm{~s}, 6 \mathrm{H}), 0.02(\mathrm{~s}, 6 \mathrm{H})$.

${ }^{13} \mathrm{C}$ NMR $\left(100 \mathrm{MHz}, \mathrm{CDCl}_{3}\right) \delta 146.7,138.1,133.9,129.0,128.1,127.7,63.1,39.2$, $26.0,18.3,-3.1,-5.3$.

IR (thin film) 2956, 1471, 1428, 1254, $1096 \mathrm{~cm}^{-1}$.

HRMS $m / z$ (CI) calcd. for $\mathrm{C}_{18} \mathrm{H}_{31} \mathrm{Si}_{2}[\mathrm{M}-\mathrm{H}]^{+}$319.1914, found 319.1920.

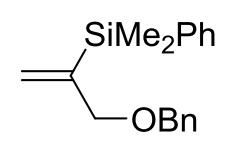

2)

(3-(Benzyloxy)prop-1-en-2-yl)dimethyl(phenyl)silane (21) was prepared as colorless oil according to the General Procedure B in 85\% yield $(B / L>30: 1)$.

${ }^{1} \mathbf{H}$ NMR $\left(400 \mathrm{MHz}, \mathrm{CDCl}_{3}\right) \delta 7.63-7.57(\mathrm{~m}, 2 \mathrm{H}), 7.43-7.29(\mathrm{~m}, 8 \mathrm{H}), 6.02(\mathrm{dt}, J$ = 3.1, 1.6 Hz, 1H), $5.61-5.57(\mathrm{~m}, 1 \mathrm{H}), 4.48(\mathrm{~s}, 2 \mathrm{H}), 4.18(\mathrm{t}, J=1.3 \mathrm{~Hz}, 2 \mathrm{H}), 0.48$ $(\mathrm{s}, 6 \mathrm{H})$.

${ }^{13} \mathrm{C}$ NMR (100 MHz, $\left.\mathrm{CDCl}_{3}\right) \delta 147.1,138.3,137.7,133.9,129.0,128.2,127.7$, 127.6, 127.4, 127.1, 74.3, 72.1, -3.0.

IR (thin film) 3059, 2955, 1421, 1353, 1250, 1205, 1101, 1022, 937, $820 \mathrm{~cm}^{-1}$. HRMS $m / z$ (CI) calcd. for $\mathrm{C}_{18} \mathrm{H}_{26} \mathrm{ONSi}\left[\mathrm{M}+\mathrm{NH}_{4}\right]^{+}$300.1778, found 300.1788 . 


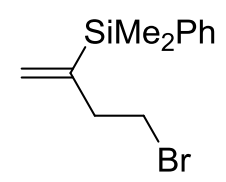

$2 \mathrm{~m}$

(4-Bromobut-1-en-2-yl)dimethyl(phenyl)silane $\mathbf{( 2 \mathrm { m } )}$ was prepared as colorless oil according to the General Procedure B in 93\% yield ( $B / L>30: 1)$. ${ }^{1} \mathbf{H}$ NMR (400 MHz, $\left.\mathrm{CDCl}_{3}\right) \delta 7.56$ - $7.53(\mathrm{~m}, 2 \mathrm{H}), 7.43$ - 7.38 (m, $\left.3 \mathrm{H}\right), 5.82$ $5.79(\mathrm{~m}, 1 \mathrm{H}), 5.60-5.58(\mathrm{~m}, 1 \mathrm{H}), 3.35(\mathrm{t}, J=7.6 \mathrm{~Hz}, 2 \mathrm{H}), 2.70(\mathrm{t}, J=7.6 \mathrm{~Hz}, 2$ $\mathrm{H}), 0.44(\mathrm{~s}, 6 \mathrm{H})$.

${ }^{13} \mathrm{C}$ NMR $\left(100 \mathrm{MHz}, \mathrm{CDCl}_{3}\right) \delta 147.4,137.4,133.8,129.2,128.5,127.9,39.1,31.7$, -3.2 .

IR (thin film) 2958, 1428, 1250, 1112, $817 \mathrm{~cm}^{-1}$.

HRMS $m / z$ (CI) calcd. for $\mathrm{C}_{12} \mathrm{H}_{16} \mathrm{BrSi}[\mathrm{M}-\mathrm{H}]^{+}$267.0205, found 267.0210.

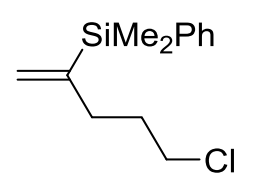

2n

(5-Chloropent-1-en-2-yl)dimethyl(phenyl)silane (2n) was prepared as colorless oil according to the General Procedure B in $68 \%$ yield $(B / L>30: 1)$.

${ }^{1}$ H NMR (400 MHz, $\left.\mathrm{CDCl}_{3}\right) \delta 7.55$ - $7.51(\mathrm{~m}, 2 \mathrm{H}), 7.39$ - $7.35(\mathrm{~m}, 3 \mathrm{H}), 5.74(\mathrm{dt}, J$ $=2.8,1.5 \mathrm{~Hz}, 1 \mathrm{H}), 5.50-5.48(\mathrm{~m}, 1 \mathrm{H}), 3.46(\mathrm{t}, J=6.6 \mathrm{~Hz}, 2 \mathrm{H}), 2.31-2.25(\mathrm{~m}$, $2 \mathrm{H}), 1.86-1.78(\mathrm{~m}, 2 \mathrm{H}), 0.42-0.39(\mathrm{~m}, 6 \mathrm{H})$.

${ }^{13} \mathrm{C} \mathrm{NMR}\left(100 \mathrm{MHz}_{2} \mathrm{CDCl}_{3}\right) \delta 148.8,138.0,133.8,129.1,127.8,126.8,44.5,32.9$ $31.6,-3.0$.

The spectral data are identical to those reported in the literature. ${ }^{21}$ 


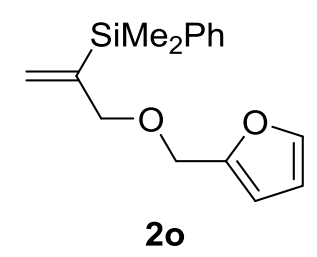

(3-(Furan-2-ylmethoxy)prop-1-en-2-yl)dimethyl(phenyl)silane (2o) was prepared as colorless oil according to the General Procedure B in 94\% yield $(B / L>30: 1)$.

${ }^{1}$ H NMR (400 MHz, $\left.\mathrm{CDCl}_{3}\right) \delta 7.57$ - $7.54(\mathrm{~m}, 2 \mathrm{H}), 7.41-7.35(\mathrm{~m}, 4 \mathrm{H}), 6.34(\mathrm{dd}$, $J=3.1,1.9 \mathrm{~Hz}, 1 \mathrm{H}), 6.24(\mathrm{~d}, J=3.1 \mathrm{~Hz}, 1 \mathrm{H}), 5.94-5.93(\mathrm{~m}, 1 \mathrm{H}), 5.54-5.53(\mathrm{~m}$, $1 \mathrm{H}), 4.35(\mathrm{~s}, 2 \mathrm{H}), 4.11(\mathrm{~s}, 2 \mathrm{H}), 0.43(\mathrm{~s}, 6 \mathrm{H})$.

${ }^{13} \mathrm{C}$ NMR (100 MHz, $\left.\mathrm{CDCl}_{3}\right) \delta 151.8,146.9,142.6,137.7,133.9,129.0,127.7$, $127.4,110.1,109.1,73.9,63.7,-3.1$.

IR (thin film) 3056, 2957, 2901, 2850, 2361, 2331, 1726, 1422, 1352, 1150, 1107, $1071 \mathrm{~cm}^{-1}$.

HRMS m/z (CI) calcd. for $\mathrm{C}_{16} \mathrm{H}_{24} \mathrm{NO}_{2} \mathrm{Si}\left[\mathrm{M}+\mathrm{NH}_{4}\right]^{+} 290.1576$, found 290.1584 .

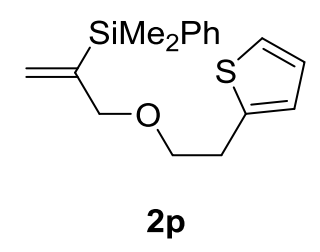

Dimethyl(phenyl)(3-(2-(thiophen-2-yl)ethoxy)prop-1-en-2-yl)silane (2p) was prepared as colorless oil according to the General Procedure B in 62\% yield $(B / L>30: 1)$.

${ }^{1}$ H NMR $\left(400 \mathrm{MHz} \mathrm{CDCl}_{3}\right) \delta 7.54-7.52(\mathrm{~m}, 2 \mathrm{H}), 7.38-7.34(\mathrm{~m}, 3 \mathrm{H}), 7.13(\mathrm{dd}$, $J=5.1,1.1 \mathrm{~Hz}, 1 \mathrm{H}), 6.92(\mathrm{dd}, J=5.1,3.4 \mathrm{~Hz}, 1 \mathrm{H}), 6.81-6.80(\mathrm{~m}, 1 \mathrm{H}), 5.92-5.90$ $(\mathrm{m}, 1 \mathrm{H}), 5.50(\mathrm{t}, J=1.5 \mathrm{~Hz}, 1 \mathrm{H}), 4.07(\mathrm{t}, J=1.4 \mathrm{~Hz}, 2 \mathrm{H}), 3.56(\mathrm{t}, J=7.0 \mathrm{~Hz}, 2 \mathrm{H})$, $3.03(\mathrm{t}, J=6.9 \mathrm{~Hz}, 2 \mathrm{H}), 0.40(\mathrm{~s}, 6 \mathrm{H})$.

${ }^{13} \mathrm{C}$ NMR $\left(100 \mathrm{MHz}, \mathrm{CDCl}_{3}\right) \delta 147.1,141.3,137.8,133.9,129.0,127.7,126.8$, $126.7,125.1,123.5,74.9,70.9,30.4,-3.1$.

IR (thin film) 3055, 2954, 2854, 2358, 2333, 1426, 1260, 1101. 
HRMS $m / z$ (CI) calcd. for $\mathrm{C}_{17} \mathrm{H}_{23} \mathrm{OSSi}[\mathrm{M}+\mathrm{H}]^{+}$303.1233, found 303.1234.

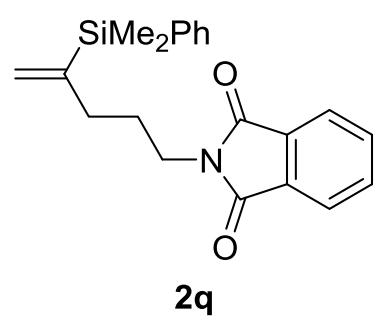

2-(4-(Dimethyl(phenyl)silyl)pent-4-en-1-yl)isoindoline-1,3-dione (2q) was prepared as colorless oil according to the General Procedure B in 94\% yield $(B / L>30: 1)$.

${ }^{1} \mathbf{H}$ NMR $\left(400 \mathrm{MHz}, \mathrm{CDCl}_{3}\right) \delta 7.83(\mathrm{dd}, J=4.3,2.4 \mathrm{~Hz}, 2 \mathrm{H}), 7.70(\mathrm{dd}, J=4.4,2.4$ $\mathrm{Hz}, 2 \mathrm{H}), 7.50-7.48(\mathrm{~m}, 2 \mathrm{H}), 7.32-7.30(\mathrm{~m}, 3 \mathrm{H}), 5.73(\mathrm{~m}, 1 \mathrm{H}), 5.44(\mathrm{~m}, 1 \mathrm{H})$, $3.62(\mathrm{t}, J=5.8 \mathrm{~Hz}, 2 \mathrm{H}), 2.17(\mathrm{t}, J=6.1 \mathrm{~Hz}, 2 \mathrm{H}), 1.78-1.72(\mathrm{~m}, 2 \mathrm{H}), 0.38(\mathrm{~s}, 6 \mathrm{H})$. ${ }^{13} \mathrm{C}$ NMR $\left(100 \mathrm{MHz}, \mathrm{CDCl}_{3}\right) \delta 168.3,148.9,137.9,133.8,133.7,132.1,128.9$, $127.8,126.3,123.1,37.8,32.9,27.5,-3.0$.

IR (thin film) 3055, 2950, 2359, 2332, 1771, 1709, 1397, 1363, 1262, 1108, 1023 $\mathrm{cm}^{-1}$.

HRMS $m / z$ (CI) calcd. for $\mathrm{C}_{21} \mathrm{H}_{24} \mathrm{NO}_{2} \mathrm{Si}[\mathrm{M}+\mathrm{H}]^{+} 350.1571$, found 350.1558 .

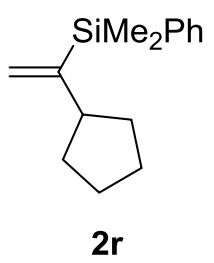

(1-Cyclopentylvinyl)dimethyl(phenyl)silane (2r) was prepared as colorless oil according to the General Procedure B in $92 \%$ yield $(B / L>30: 1)$. ${ }^{1} \mathrm{H}$ NMR $\left(400 \mathrm{MHz}, \mathrm{CDCl}_{3}\right) \delta 7.58$ - $7.54(\mathrm{~m}, 2 \mathrm{H}), 7.38$ - $7.36(\mathrm{~m}, 3 \mathrm{H}), 5.79(\mathrm{dd}$, $J=2.5,1.5 \mathrm{~Hz}, 1 \mathrm{H}), 5.44(\mathrm{dd}, J=2.6,0.6 \mathrm{~Hz}, 1 \mathrm{H}), 2.58-2.49(\mathrm{~m}, 1 \mathrm{H}), 1.77-1.62$ (m, 4H), $1.58-1.47(\mathrm{~m}, 2 \mathrm{H}), 1.40-1.30(\mathrm{~m}, 2 \mathrm{H}), 0.42(\mathrm{~s}, 6 \mathrm{H})$.

${ }^{13} \mathrm{C}$ NMR $\left(100 \mathrm{MHz}, \mathrm{CDCl}_{3}\right) \delta 154.1,138.9,134.0,128.8,127.6,123.7,45.5,32.8$, $24.9,-2.3$. 
IR (thin film) 3056, 2953, 2868, 1422, 1257, $1110 \mathrm{~cm}^{-1}$.

HRMS m/z (CI) calcd. for $\mathrm{C}_{15} \mathrm{H}_{22} \mathrm{Si}\left[\mathrm{M}^{+}\right]$230.1491, found 230.1495.

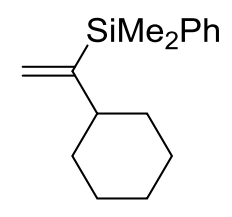

2s

(1-Cyclohexylvinyl)dimethyl(phenyl)silane (2s) was prepared as colorless oil according to the General Procedure B in 90\% yield (B/L>30:1).

${ }^{1} \mathrm{H}$ NMR $\left(400 \mathrm{MHz}, \mathrm{CDCl}_{3}\right) \delta 7.58$ - $7.55(\mathrm{~m}, 2 \mathrm{H}), 7.39$ - $7.36(\mathrm{~m}, 3 \mathrm{H}), 5.77(\mathrm{dd}$, $J=2.5,1.1 \mathrm{~Hz}, 1 \mathrm{H}), 5.45(\mathrm{~d}, J=2.5 \mathrm{~Hz}, 1 \mathrm{H}), 2.07(\mathrm{dd}, J=15.5,6.6 \mathrm{~Hz}, 1 \mathrm{H}), 1.75-$ $1.65(\mathrm{~m}, 5 \mathrm{H}), 1.29-1.12(\mathrm{~m}, 5 \mathrm{H}), 0.42(\mathrm{~s}, 6 \mathrm{H})$.

${ }^{13} \mathrm{C}$ NMR $\left(100 \mathrm{MHz} \mathrm{CDCl}_{3}\right) \delta 155.8,138.9,133.9,128.8,127.6,124.3,43.3,33.5$, $26.9,26.3,-2.4$.

The spectral data are identical to those reported in the literature. ${ }^{22}$

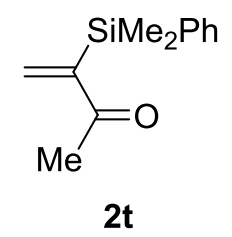

3-(Dimethyl(phenyl)silyl)but-3-en-2-one (2t) was prepared as colorless oil according to the General Procedure B in $88 \%$ yield $(B / L>5.6: 1)$. ${ }^{1} \mathrm{H}$ NMR (400 MHz, $\left.\mathrm{CDCl}_{3}\right) \delta 7.56-7.54(\mathrm{~m}, 2 \mathrm{H}), 7.37-7.33(\mathrm{~m}, 3 \mathrm{H}), 6.58(\mathrm{~s}$, $1 \mathrm{H}), 6.13(\mathrm{~s}, 1 \mathrm{H}), 2.29(\mathrm{~s}, 3 \mathrm{H}), 0.47(\mathrm{~s}, 6 \mathrm{H})$.

${ }^{13} \mathrm{C}$ NMR (100 MHz, $\left.\mathrm{CDCl}_{3}\right) \delta$ 203.6, 152.5, 138.7, 137.3, 134.0, 129.1, 127.7, 26.3, -2.8 .

The spectral data are identical to those reported in the literature. ${ }^{23}$ 


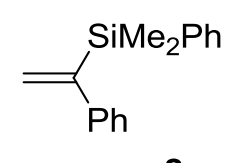

$2 \mathbf{u}$

Dimethyl(phenyl)(1-phenylvinyl)silane (2u) was prepared as colorless oil according to the General Procedure $C$ in $46 \%$ yield $(B / L>30: 1)$.

${ }^{1} \mathbf{H}$ NMR $\left(400 \mathrm{MHz}, \mathrm{CDCl}_{3}\right) \delta 7.58-7.56(\mathrm{~m}, 2 \mathrm{H}), 7.38-7.36(\mathrm{~m}, 3 \mathrm{H}), 7.24-$ $7.19(\mathrm{~m}, 3 \mathrm{H}), 7.14-7.12(\mathrm{~m}, 2 \mathrm{H}), 6.01(\mathrm{~d}, J=2.9 \mathrm{~Hz}, 1 \mathrm{H}), 5.69(\mathrm{~d}, J=2.8 \mathrm{~Hz}, 1 \mathrm{H})$, $0.43(\mathrm{~s}, 6 \mathrm{H})$.

${ }^{13} \mathrm{C}$ NMR (100 MHz, $\left.\mathrm{CDCl}_{3}\right) \delta 151.0,144.1,138.3,134.0,129.1,129.0,128.1$, $127.8,126.8,126.4,-2.34$.

IR (thin film) 3059, 2956, 2946, 1602, 1490, 1431, 1250, 1111, 988, $834 \mathrm{~cm}^{-1}$. HRMS $m / z$ (CI) calcd. for $\mathrm{C}_{16} \mathrm{H}_{19} \mathrm{Si}[\mathrm{M}+\mathrm{H}]^{+} 239.1251$, found 239.1259 .

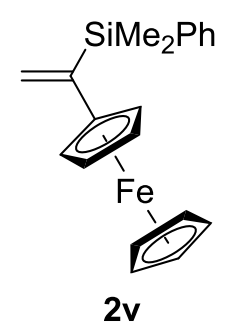

Ferrocenyldimethyl(phenyl)silane (2v) was prepared as red brown oil according to the General Procedure $C$ in $69 \%$ yield $(B / L>30: 1)$.

${ }^{1} \mathrm{H}$ NMR $\left(400 \mathrm{MHz}, \mathrm{CDCl}_{3}\right) \delta 7.61-7.58(\mathrm{~m}, 2 \mathrm{H}), 7.39-7.38(\mathrm{~m}, 3 \mathrm{H}), 6.16(\mathrm{~d}, J$ $=2.0 \mathrm{~Hz}, 1 \mathrm{H}), 5.53(\mathrm{~d}, J=2.0 \mathrm{~Hz}, 1 \mathrm{H}), 4.33(\mathrm{~s}, 2 \mathrm{H}), 4.20(\mathrm{~s}, 2 \mathrm{H}), 3.99(\mathrm{~s}, 5 \mathrm{H}), 0.49$ $(\mathrm{s}, 6 \mathrm{H})$.

${ }^{13} \mathrm{C}$ NMR (100 MHz, $\left.\mathrm{CDCl}_{3}\right) \delta$ 146.0, 138.7, 134.0, 129.0, 127.7, 125.3, 69.9, 68.4, $67.6,-1.90$.

IR (thin film) 3061, 2956, 1597, 1457, 1251, 1109, 815, 776, 731, $696 \mathrm{~cm}^{-1}$.

HRMS m/z (CI) calcd. for $\mathrm{C}_{20} \mathrm{H}_{23} \mathrm{FeSi}[\mathrm{M}+\mathrm{H}]^{+} 347.0913$, found 347.0923. 


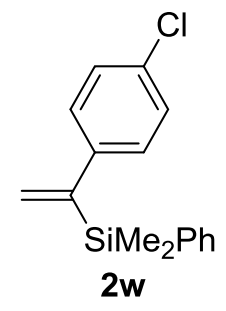

(1-(4-Chlorophenyl)vinyl)dimethyl(phenyl)silane (2w) was prepared as colorless oil according to the General Procedure $C$ in $71 \%$ yield $(B / L>30: 1)$. ${ }^{1} \mathbf{H}$ NMR $\left(400 \mathrm{MHz}, \mathrm{CDCl}_{3}\right) \delta 7.56-7.54(\mathrm{~m}, 2 \mathrm{H}), 7.40-7.36(\mathrm{~m}, 3 \mathrm{H}), 7.22$ $7.19(\mathrm{~m}, 2 \mathrm{H}), 7.06-7.03(\mathrm{~m}, 2 \mathrm{H}), 5.99(\mathrm{~d}, J=2.7 \mathrm{~Hz}, 1 \mathrm{H}), 5.71(\mathrm{~d}, J=2.7 \mathrm{~Hz}, 1 \mathrm{H})$, $0.43(\mathrm{~s}, 6 \mathrm{H})$.

${ }^{13} \mathrm{C}$ NMR (100 MHz, $\left.\mathrm{CDCl}_{3}\right) \delta 150.0,142.6,137.8,134.0,132.2,129.5,129.2$, $128.2,128.1,127.9,-2.5$.

IR (thin film) 3058, 2959, 2358, 2332, 1486, 1422, 1399, 1257, $1104 \mathrm{~cm}^{-1}$. HRMS $m / z$ (CI) calcd. for $\mathrm{C}_{16} \mathrm{H}_{17} \mathrm{ClSi}\left[\mathrm{M}^{+}\right]$272.0788, found 272.0786.

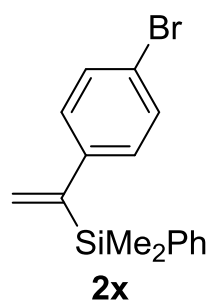

(1-(4-Bromophenyl)vinyl)dimethyl(phenyl)silane $\mathbf{( 2 x )}$ was prepared as colorless oil according to the General Procedure C in 73\% yield ( $B / L>30: 1)$. ${ }^{1} \mathbf{H}$ NMR (400 MHz, $\left.\mathrm{CDCl}_{3}\right) \delta 7.57$ - $7.54(\mathrm{~m}, 2 \mathrm{H}), 7.40-7.34(\mathrm{~m}, 5 \mathrm{H}), 7.00$ $6.97(\mathrm{~m}, 2 \mathrm{H}), 5.99(\mathrm{~d}, J=2.7 \mathrm{~Hz}, 1 \mathrm{H}), 5.71(\mathrm{~d}, J=2.7 \mathrm{~Hz}, 1 \mathrm{H}), 0.43(\mathrm{~s}, 6 \mathrm{H})$. ${ }^{13} \mathrm{C}$ NMR $\left(100 \mathrm{MHz}, \mathrm{CDCl}_{3}\right) \delta 150.1,143.1,137.8,134.0,131.2,129.5,129.2$, $128.5,127.9,120.4,-2.5$.

IR (thin film) 3057, 3010, 2959, 2360, 2332, 1482, 1442, 1396, 1257, 1108, 1007 $\mathrm{cm}^{-1}$.

HRMS $m / z$ (CI) calcd. for $\mathrm{C}_{16} \mathrm{H}_{17} \mathrm{BrSi}\left[\mathrm{M}^{+}\right]$316.0283, found 316.0280. 


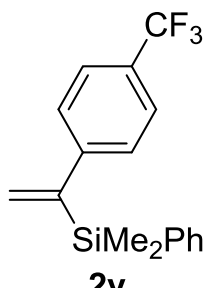

$2 y$

Dimethyl(phenyl)(1-(4-(trifluoromethyl)phenyl)vinyl)silane $\quad(2 y)$ was prepared as colorless oil according to the General Procedure $\mathrm{C}$ in $78 \%$ yield $(B / L>30: 1)$.

${ }^{1}$ H NMR (400 MHz, $\left.\mathrm{CDCl}_{3}\right) \delta 7.57$ - $7.55(\mathrm{~m}, 2 \mathrm{H}), 7.49(\mathrm{~d}, \mathrm{~J}=8.2 \mathrm{~Hz}, 2 \mathrm{H}), 7.41-$ $7.37(\mathrm{~m}, 3 \mathrm{H}), 7.20(\mathrm{~d}, J=8.0 \mathrm{~Hz}, 2 \mathrm{H}), 6.02(\mathrm{~d}, J=2.7 \mathrm{~Hz}, 1 \mathrm{H}), 5.78(\mathrm{~d}, J=2.7 \mathrm{~Hz}$, $1 \mathrm{H}), 0.44(\mathrm{~s}, 6 \mathrm{H})$.

${ }^{13} \mathrm{C}$ NMR (100 MHz, $\left.\mathrm{CDCl}_{3}\right) \delta 150.5,148.0,137.5,134.0,130.5,129.3,128.5(\mathrm{~d}, J$ $=32.2 \mathrm{~Hz}), 128.0,127.1,125.1(\mathrm{q}, J=3.8 \mathrm{~Hz}), 124.39(\mathrm{q}, J=270.3 \mathrm{~Hz}),-2.6$.

The spectral data are identical to those reported in the literature. ${ }^{24}$

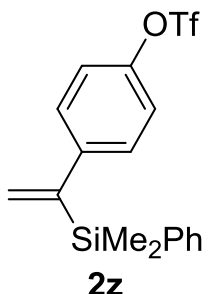

4-(1-(Dimethyl(phenyl)silyl)vinyl)phenyl trifluoromethanesulfonate (2z) was prepared as colorless oil according to the General Procedure C in $72 \%$ yield $(B / L>30: 1)$.

${ }^{1} \mathrm{H}$ NMR (400 MHz, $\left.\mathrm{CDCl}_{3}\right) \delta 7.55$ - $7.53(\mathrm{~m}, 2 \mathrm{H}), 7.42-7.35(\mathrm{~m}, 3 \mathrm{H}), 7.17$ $7.12(\mathrm{~m}, 4 \mathrm{H}), 6.00(\mathrm{~d}, J=2.3 \mathrm{~Hz}, 1 \mathrm{H}), 5.77(\mathrm{~d}, J=2.4 \mathrm{~Hz}, 1 \mathrm{H}), 0.43(\mathrm{~s}, 6 \mathrm{H})$.

${ }^{13} \mathrm{C}$ NMR (100 MHz, $\left.\mathrm{CDCl}_{3}\right) \delta 149.7,148.2,144.7,137.4,133.9,130.4,129.1$, $128.5,128.0,120.9,118.7(q, J=318.9 \mathrm{~Hz}),-2.6$.

${ }^{19}$ F NMR $\left(376 \mathrm{MHz}, \mathrm{CDCl}_{3}\right) \delta-72.9$.

IR (thin film) 3060, 2962, 2359, 2332, 1496, 1420, 1256, 1212, $1137 \mathrm{~cm}^{-1}$. HRMS $m / z$ (CI) calcd. for $\mathrm{C}_{17} \mathrm{H}_{17} \mathrm{~F}_{3} \mathrm{O}_{3} S S i\left[\mathrm{M}^{+}\right]$386.0620, found 386.0609. 


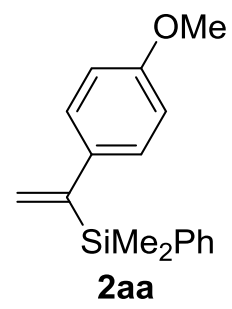

(1-(4-Methoxyphenyl)vinyl)dimethyl(phenyl)silane (2aa) was prepared as colorless oil according to the General Procedure $C$ in $30 \%$ yield $(B / L>30: 1)$.

${ }^{1}$ H NMR (400 MHz, $\left.\mathrm{CDCl}_{3}\right) \delta 7.58$ - $7.56(\mathrm{~m}, 2 \mathrm{H}), 7.37-7.36(\mathrm{~m}, 3 \mathrm{H}), 7.09(\mathrm{~d}, J$ $=8.3 \mathrm{~Hz}, 2 \mathrm{H}), 6.79(\mathrm{~d}, J=8.4 \mathrm{~Hz}, 2 \mathrm{H}), 5.99(\mathrm{~d}, J=2.4 \mathrm{~Hz}, 1 \mathrm{H}), 5.63(\mathrm{~d}, J=2.4 \mathrm{~Hz}$, 1H), 3.78 (s. 3H), 0.43 (s, 6H).

${ }^{13} \mathrm{C}$ NMR $\left(100 \mathrm{MHz}, \mathrm{CDCl}_{3}\right) \delta 158.4,149.8,138.5,136.4,134.0,129.0,127.9$ (2C), $127.8,113.5,55.2,-2.2$.

The spectral data are identical to those reported in the literature. ${ }^{24}$

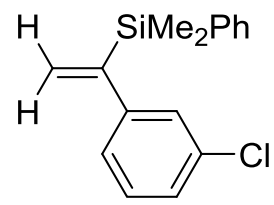

$2 a b$

(1-(3-Chlorophenyl)vinyl)dimethyl(phenyl)silane was prepared as colorless oil according to the General Procedure $C$ in $53 \%$ yield $(B / L>30: 1)$.

${ }^{1} \mathbf{H}$ NMR (400 MHz, $\left.\mathrm{CDCl}_{3}\right) \delta 7.57$ - $7.54(\mathrm{~m}, 2 \mathrm{H}), 7.41$ - $7.36(\mathrm{~m}, 3 \mathrm{H}), 7.19$ $7.12(\mathrm{~m}, 3 \mathrm{H}), 6.97-6.95(\mathrm{~m}, 1 \mathrm{H}), 5.98(\mathrm{~d}, J=2.6 \mathrm{~Hz}, 1 \mathrm{H}), 5.70(\mathrm{~d}, J=2.7 \mathrm{~Hz}, 1 \mathrm{H})$, $0.44(\mathrm{~s}, 6 \mathrm{H})$.

${ }^{13} \mathrm{C}$ NMR (100 MHz, $\left.\mathrm{CDCl}_{3}\right) \delta$ 150.2, 146.1, 137.6, 134.0, 133.9, 130.0 (2C), 129.2, $127.9,126.8,126.4,125.1,-2.5$.

IR (thin film) 3060, 2959, 1589, 1560, 1473, 1418, 1402, 1305, 1256, 1110, 1082 $\mathrm{cm}^{-1}$.

HRMS m/z (CI) calcd. for $\mathrm{C}_{16} \mathrm{H}_{17} \mathrm{ClSi}\left[\mathrm{M}^{+}\right]$272.0788, found 272.0785. 


\section{Ru(II)-Catalyzed trans-Hydrogenation of 1-Silyl Alkynes}

\section{General Procedure D.}

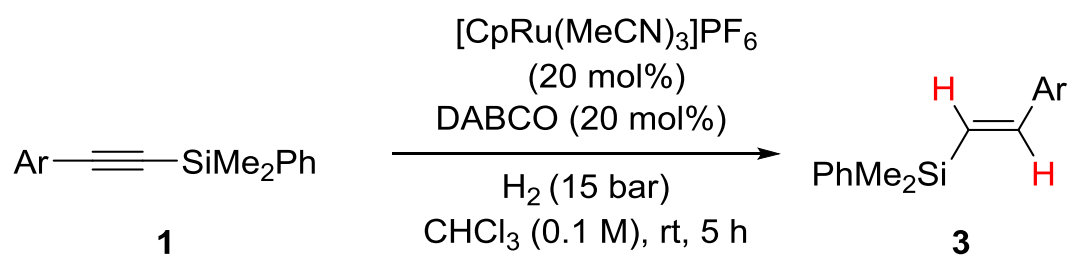

In a glove box, to an oven-dried 8-mL vial with a magnetic stirring bar were added the silyl alkyne $(0.20 \mathrm{mmol}),\left[\mathrm{CpRu}(\mathrm{MeCN})_{3}\right] \mathrm{PF}_{6}(17.3 \mathrm{mg}, 0.04 \mathrm{mmol}$, $20 \mathrm{~mol} \%)$, DABCO (4.5 mg, $0.04 \mathrm{mmol}, 20 \mathrm{~mol} \%)$, and $\mathrm{CHCl}_{3}(2.0 \mathrm{~mL})$. The vial was then moved into a stainless autoclave (Parr ${ }^{\circledR}$ reactor) and purged with $\mathrm{H}_{2}$ for three cycles before it was filled with $\mathrm{H}_{2}$ at the required pressure (15 bar). Next, the reaction mixture was allowed to stir at room temperature for $5 \mathrm{~h}$ before it was concentrated under reduced pressure. The residue was purified by silica gel flash column chromatography to give the desired product.

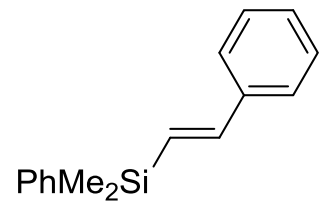

$3 \mathbf{u}$

(E)-Dimethyl(phenyl)(styryl)silane (3u) was prepared as colorless oil according to the General Procedure D in $81 \%$ yield $(B / L=1: 16)$.

${ }^{1} \mathbf{H}$ NMR (400 MHz, $\left.\mathrm{CDCl}_{3}\right) \delta 7.60-7.58(\mathrm{~m}, 2 \mathrm{H}), 7.47$ - $7.45(\mathrm{~m}, 2 \mathrm{H}), 7.38$ $7.24(\mathrm{~m}, 6 \mathrm{H}), 6.96(\mathrm{~d}, J=19.1 \mathrm{~Hz}, 1 \mathrm{H}), 6.61(\mathrm{~d}, J=19.1 \mathrm{~Hz}, 1 \mathrm{H}), 0.45(\mathrm{~s}, 6 \mathrm{H})$.

${ }^{13} \mathrm{C}$ NMR (100 MHz, $\left.\mathrm{CDCl}_{3}\right) \delta 145.3,138.5,138.1,133.9,129.0,128.5,128.1$, $127.8,127.1,126.5,-2.5$.

The spectral data are identical to those reported in the literature. ${ }^{25}$ 


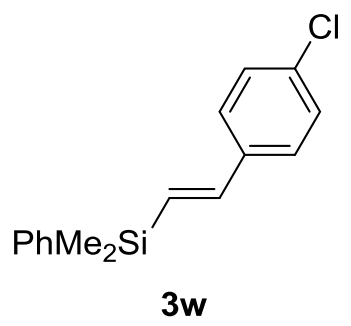

(E)-(4-Chlorostyryl)dimethyl(phenyl)silane (3w) was prepared as colorless oil according to the General Procedure $D$ in $73 \%$ yield $(B / L=1: 17)$.

${ }^{1}$ H NMR (400 MHz, $\left.\mathrm{CDCl}_{3}\right) \delta 7.62-7.60(\mathrm{~m}, 2 \mathrm{H}), 7.42$ - 7.39 (m, 5H), 7.33 $7.31(\mathrm{~m}, 2 \mathrm{H}), 6.92(\mathrm{~d}, J=20.6 \mathrm{~Hz}, 1 \mathrm{H}), 6.60(\mathrm{~d}, J=19.1 \mathrm{~Hz}, 1 \mathrm{H}), 0.48(\mathrm{~s}, 6 \mathrm{H})$.

${ }^{13} \mathrm{C}$ NMR $\left(100 \mathrm{MHz}, \mathrm{CDCl}_{3}\right) \delta 143.8,138.2,136.6,133.9,133.7,129.1,128.7$, $128.1,127.9,127.7,-2.6$.

The spectral data are identical to those reported in the literature. ${ }^{26}$

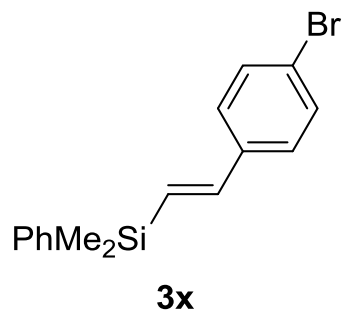

(E)-(4-Bromostyryl)dimethyl(phenyl)silane $\mathbf{( 3 x )}$ was prepared as colorless oil according to the General Procedure D in $75 \%$ yield $(B / L=1: 17)$.

${ }^{1}$ H NMR (400 MHz, $\left.\mathrm{CDCl}_{3}\right) \delta 7.62-7.60$ (m, 2H), $7.48(\mathrm{~d}, J=8.1 \mathrm{~Hz}, 2 \mathrm{H}), 7.42$ $7.41(\mathrm{~m}, 3 \mathrm{H}), 7.33(\mathrm{~d}, J=7.9 \mathrm{~Hz}, 2 \mathrm{H}), 6.90(\mathrm{~d}, J=19.1 \mathrm{~Hz}, 1 \mathrm{H}), 6.62(\mathrm{~d}, J=19.1$ $\mathrm{Hz}, 1 \mathrm{H}), 0.48(\mathrm{~s}, 6 \mathrm{H})$.

${ }^{13} \mathrm{C}$ NMR $\left(100 \mathrm{MHz}, \mathrm{CDCl}_{3}\right) \delta 143.9,138.2,137.0,133.9,131.6,129.1,128.3$, $128.0,127.8,122.0,-2.6$.

The spectral data are identical to those reported in the literature. ${ }^{25}$ 


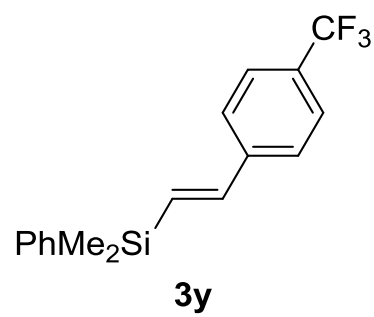

(E)-Dimethyl(phenyl)(4-(trifluoromethyl)styryl)silane (3y) was prepared as colorless oil according to the General Procedure D in $85 \%$ yield $(B / L<1: 30)$. ${ }^{1} \mathrm{H}$ NMR $\left(400 \mathrm{MHz}^{\mathrm{CDCl}} 3\right) \delta 7.61-7.54(\mathrm{~m}, 6 \mathrm{H}), 7.42-7.41(\mathrm{~m}, 3 \mathrm{H}), 6.98(\mathrm{~d}, J$ $=19.1 \mathrm{~Hz}, 1 \mathrm{H}), 6.74(\mathrm{~d}, J=19.1 \mathrm{~Hz}, 1 \mathrm{H}), 0.49(\mathrm{~s}, 6 \mathrm{H})$.

${ }^{13} \mathrm{C}$ NMR $\left(100 \mathrm{MHz} \mathrm{CDCl}_{3}\right) \delta 143.6,141.4,137.9,133.9,130.8,129.2,129.8(\mathrm{q}, J$ $=32.2 \mathrm{~Hz}), 127.9,126.6,125.5(\mathrm{q}, J=3.6 \mathrm{~Hz}), 122.8,-2.7$.

The spectral data are identical to those reported in the literature. ${ }^{25}$

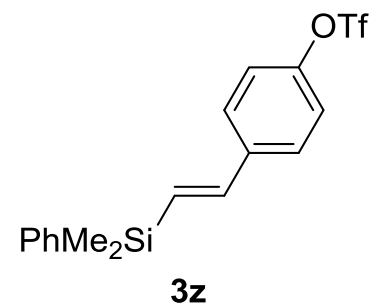

(E)-4-(2-(Dimethyl(phenyl)silyl)vinyl)phenyl trifluoromethanesulfonate (3z) was prepared as colorless oil according to the General Procedure D in $78 \%$ yield $(B / L<1: 30)$.

${ }^{1} \mathrm{H}$ NMR $\left(400 \mathrm{MHz} \mathrm{CDCl}_{3}\right) \delta 7.61-7.59(\mathrm{~m}, 2 \mathrm{H}), 7.52(\mathrm{~d}, J=8.5 \mathrm{~Hz}, 2 \mathrm{H}), 7.42-$ $7.41(\mathrm{~m}, 3 \mathrm{H}), 7.26(\mathrm{~d}, J=8.4 \mathrm{~Hz}, 2 \mathrm{H}), 6.94(\mathrm{~d}, J=19.1 \mathrm{~Hz}, 1 \mathrm{H}), 6.65(\mathrm{~d}, J=19.1$ $\mathrm{Hz}, 1 \mathrm{H}), 0.48$ (s, 6H).

${ }^{13} \mathrm{C} \mathrm{NMR}\left(100 \mathrm{MHz}, \mathrm{CDCl}_{3}\right) \delta 149.0,143.0,138.4,137.9,133.9,130.3,129.2$, $128.1,127.9,120.9(\mathrm{q}, J=108.5 \mathrm{~Hz}), 117.4,-2.7$.

${ }^{19}$ F NMR $\left(376 \mathrm{MHz} \mathrm{CDCl}_{3}\right) \delta-72.8$.

IR (thin film) 3060, 3001, 2960, 2358, 1606, 1497, 1419, 1252, 1209, $1135 \mathrm{~cm}^{-1}$.

HRMS $m / z$ (CI) calcd. for $\mathrm{C}_{17} \mathrm{H}_{17} \mathrm{~F}_{3} \mathrm{O}_{3} S S i\left[\mathrm{M}^{+}\right]$386.0620, found 386.0616. 


\section{Mechanistic Experiments}
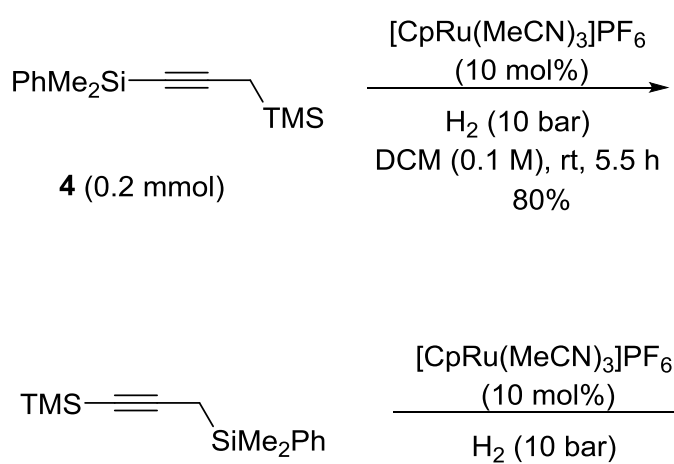

$5(0.2 \mathrm{mmol})$

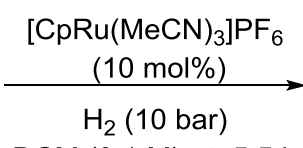

$\operatorname{DCM}(0.1 \mathrm{M}), \mathrm{rt}, 5.5 \mathrm{~h}$

$74 \%$

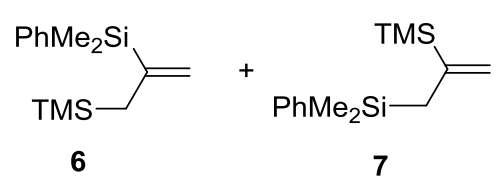

$6 / 7=1.54: 1$

$$
6 / 7=1.55: 1
$$

In two separate flasks, alkynes 4 and 5 were subjected to the same reaction conditions according the General Procedure B. After completion, the two reactions both afforded a mixture of 6 and 7. Notably, the ratios of 6 and 7 from both reactions are essentially the same on the basis of the ${ }^{1} \mathrm{H}$ NMR spectra of the crude mixture: $6 / 7=1.54: 1$ (from 4 ); $6 / 7=1.55: 1$ (from 5).

${ }^{1} \mathbf{H}$ NMR $\left(400 \mathrm{MHz} \mathrm{CDCl}_{3}\right) \delta 7.57$ - $7.53(\mathrm{~m}, 2 \mathrm{H}), 7.40-7.35(\mathrm{~m}, 3 \mathrm{H}), 5.60$ $5.59(\mathrm{~m}, 0.55 \mathrm{H}), 5.44-5.43(\mathrm{~m}, 0.37 \mathrm{H}), 5.36(\mathrm{~d}, J=2.8 \mathrm{~Hz}, 0.55 \mathrm{H}), 5.28(\mathrm{~d}, J=2.8$ $\mathrm{Hz}, 0.37 \mathrm{H}), 1.89$ (d, J = 0.7 Hz, 0.78H), $1.67(\mathrm{~d}, J=0.8 \mathrm{~Hz}, 1.14 \mathrm{H}), 0.38(\mathrm{~s}, 3.34 \mathrm{H})$, $0.32(\mathrm{~s}, 2.24 \mathrm{H}), 0.02(\mathrm{~s}, 3.26 \mathrm{H}),-0.04(\mathrm{~s}, 4.92 \mathrm{H})$. (eq.1)

The spectral data are identical to those reported in the literature. ${ }^{27}$
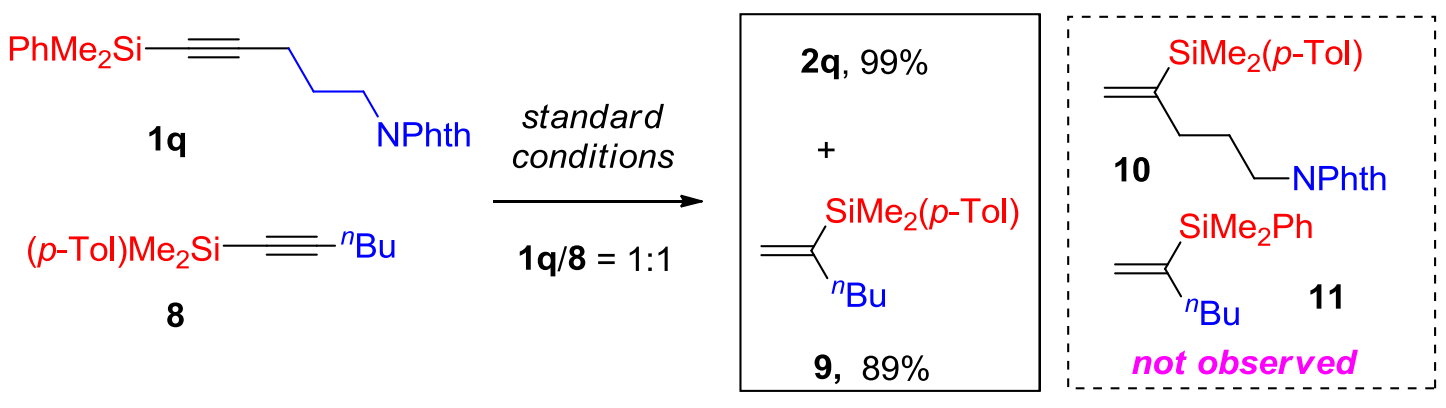

In a glove box, to an oven-dried 4-mL vial with a magnetic stirring bar were added alkyne 8 (46.0 mg, $0.20 \mathrm{mmol})$, alkyne 1q (69.4 mg, $0.20 \mathrm{mmol})$, 
$\left[\mathrm{CpRu}(\mathrm{MeCN})_{3}\right] \mathrm{PF}_{6}(17.6 \mathrm{mg}, 20 \mathrm{~mol} \%)$, and $\mathrm{CHCl}_{3}(2.0 \mathrm{~mL}, 0.1 \mathrm{M})$. The vial was then placed in a stainless autoclave (Parr ${ }^{\circledR}$ reactor) and purged with $\mathrm{H}_{2}$ for three cycles before it was filled with $\mathrm{H}_{2}$ at the required pressure (10 bar). Next, the reaction mixture was allowed to stir at room temperature for $12 \mathrm{~h}$ before it was concentrated under reduced pressure. The residue was purified by silica gel flash column chromatography to give the products $2 \mathbf{2}$ (99\% yield, $B / L>30: 1)$ and 9 (89\% yield, $B / L>30: 1)$.

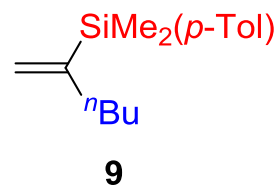

${ }^{1}$ H NMR $\left(400 \mathrm{MHz}, \mathrm{CDCl}_{3}\right) \delta 7.44(\mathrm{~d}, J=7.9 \mathrm{~Hz}, 2 \mathrm{H}), 7.19(\mathrm{~d}, J=7.5 \mathrm{~Hz}, 2 \mathrm{H})$, $5.70-5.69(\mathrm{~m}, 1 \mathrm{H}), 5.41-5.40(\mathrm{~m}, 1 \mathrm{H}), 2.37(\mathrm{~s}, 3 \mathrm{H}), 2.13(\mathrm{t}, J=7.3 \mathrm{~Hz}, 2 \mathrm{H}), 1.40$ $-1.23(\mathrm{~m}, 4 \mathrm{H}), 0.87(\mathrm{t}, J=7.2 \mathrm{~Hz}, 3 \mathrm{H}), 0.37(\mathrm{~s}, 6 \mathrm{H})$.

${ }^{13} \mathrm{C}$ NMR $\left(100 \mathrm{MHz}, \mathrm{CDCl}_{3}\right) \delta 150.7,138.7,134.8,134.0,128.5,125.5,35.6,31.1$, $22.5,21.4,13.9,-2.8$.

IR (thin film) 2958, 2926, 2866, 1602, 1456, 1392, 1257, $1104 \mathrm{~cm}^{-1}$. HRMS m/z (CI) calcd. for $\mathrm{C}_{15} \mathrm{H}_{24} \mathrm{Si}\left[\mathrm{M}^{+}\right]$232.1647, found 232.1648. 


\section{Theoretical Details and Results}

\section{Computational methods}

All calculations were performed with the Gaussian 09 Programs. ${ }^{28}$ Geometries were optimized in dichloromethane (DCM) by SMD model in the level of M06. ${ }^{29}$ The 6-31G(d) basis set was employed for $\mathrm{H}, \mathrm{C}, \mathrm{N}, \mathrm{O}$ and $\mathrm{Si}$ atoms, while the Lanl2dz basis set (augmented with $\mathrm{f}$ functions) and ECP were used for $\mathrm{Ru}{ }^{30}$ Frequency calculation was performed for each optimized structure to verify the stationary points as either minima or saddle point at the same level of theory. All the energies discussed are Gibbs free energies at $298 \mathrm{~K}$ and $1 \mathrm{~atm}$ in $\mathrm{kcal} / \mathrm{mol}$. Effect of zero-point energy (ZPE) correction is included in the relative electronic energies.
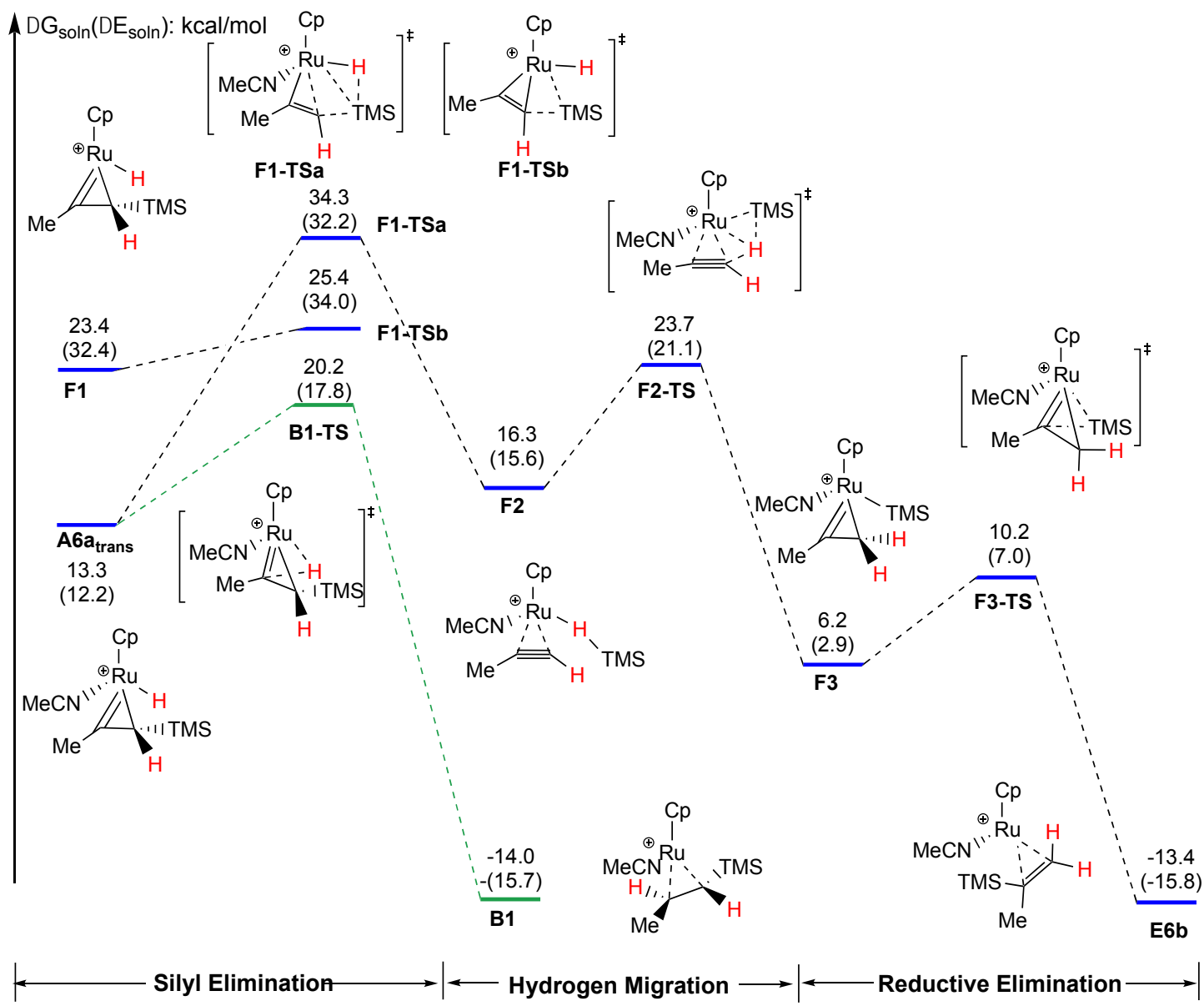

Figure S1. Free energy profile of the $\beta$-Si elimination pathway mechanism in solution by the SMD M06 method. 


\section{References}

(1) Ding, S.; Song, L.-J.; Chung, L. W.; Zhang, X.; Sun, J.; Wu, Y.-D. Ligand-Controlled Remarkable Regio- and Stereodivergence in Intermolecular Hydrosilylation of Internal Alkynes: Experimental and Theoretical Studies. J. Am. Chem. Soc. 2013, 135, 13835-13842.

(2) Lhermet, R.; Fürstner, A. Cross-Metathesis of Terminal Alkynes. Chem. Eur. J. 2014, 20, 13188-13193.

(3) Sugita, H.; Hatanaka, Y.; Hiyama, T. Silylation of 1-alkynes with chlorosilanes. promoted by zinc: Preparation of alkynylsilanes in a single step. Tetrahedron Lett. 1995, 36, 2769-2772..

(4) Fleming, I.; M. Mwaniki, J. A Synthesis of enantiomerically enriched propargyl silanes. J. Chem. Soc., Perkin Trans. 1 1998, 1237-1248.

(5) Kacprzynski, M. A.; May, T. L.; Kazane, S. A.; Hoveyda, A. H. Enantioselective Synthesis of Allylsilanes Bearing Tertiary and Quaternary Si-Substituted Carbons through Cu-Catalyzed Allylic Alkylations with Alkylzinc and Arylzinc Reagents. Angew. Chem. Int. Ed. 2007, 46, 4554-4558.

(6) Trost, B. M.; Machacek, M. R.; Faulk, B. D. Sequential Ru-Pd Catalysis: A Two-Catalyst One-Pot Protocol for the Synthesis of $\mathrm{N}$ - and O-Heterocycles. J. Am. Chem. Soc. 2006, 128, 6745-6754.

(7) Nogi, K.; Fujihara, T.; Terao, J.; Tsuji, Y. Cobalt-catalyzed carboxylation of. propargyl acetates with carbon dioxide. Chem. Commun. 2014, 50, 13052-13055.

(8) Wu, S.; Zeng, R.; Fu, C.; Yu, Y.; Zhang, X.; Ma, S. Rhodium-catalyzed C$\mathrm{H}$ functionalization-based approach to eight-membered lactams. Chem. Sci. 2015, 6, 2275-2285.

(9) Miura, K.; Hondo, T.; Okajima, S.; Nakagawa, T.; Takahashi, T.; Hosomi, A. 1,2-Silyl-Migrative Cyclization of Vinylsilanes Bearing a Hydroxy Group: Stereoselective Synthesis of Multisubstituted Tetrahydropyrans and Tetrashydrofurans1. J. Org. Chem. 2002, 67, 6082-6090.

(10) Smith, A. B.; Foley, M. A.; Dong, S.; Orbin, A. (+)-Rimocidin Synthetic Studies: Construction of the C(1-27) Aglycone Skeleton. J. Org. Chem. 2009, 74, 5987-6001.

(11) Ikeda, Z.; Oshima, K.; Matsubara, S. Preparation and Reaction of 2-Aryl-3-silyl-1,3-butadiene. Org. Lett. 2005, 7, 4859-4861.

(12) Leitner, C.; Gaich, T. Total synthesis of

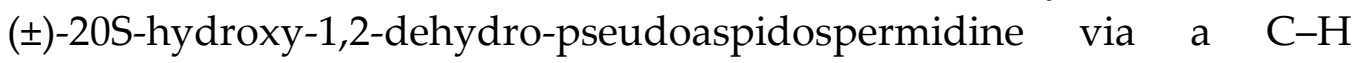
activation/transannular cyclization strategy. Chem. Commun. 2017, 53, 7451-7453. 
(13) Toutov, A. A.; Betz, K. N.; Schuman, D. P.; Liu, W.-B.; Fedorov, A.; Stoltz, B. M.; Grubbs, R. H. Alkali Metal-Hydroxide-Catalyzed C(sp)-H Bond silylation. J. Am. Chem. Soc. 2017, 139, 1668-1674.

(14) Yu, T.-B.; Bai, J. Z.; Guan, Z. Cycloaddition-Promoted Self-Assembly of a. Polymer into Well-Defined $\beta$ Sheets and Hierarchical Nanofibrils. Angew. Chem. Int. Ed. 2009, 48, 1097-1101.

(15) Brawn, R. A.; Panek, J. S. Preparation and Use of Enantioenriched Allenylsilanes for the Stereoselective Synthesis of Homopropargylic Ethers. Org. Lett. 2007, 9, 2689-2692.

(16) Mukherjee, S.; Kontokosta, D.; Patil, A.; Rallapalli, S.; Lee, D. Improved Method for the Synthesis of $\beta$-Carbonyl Silyl-1,3-Dithianes by the Double Conjugate Addition of 1,3-Dithiol to Propargylic Carbonyl Compounds. J. Org. Chem. 2009, 74, 9206-9209.

(17) Schubert, T.; Hummel, W.; Kula, M.-R.; Müller, M. Enantioselective Synthesis of Both Enantiomers of Various Propargylic Alcohols by Use of Two Oxidoreductases. Eur. J. Org. Chem. 2001, 2001, 4181-4187.

(18) Benedetti, E.; Delcourt, M. L.; Gatin-Fraudet, B.; Turcaud, S.; Micouin, L. Synthesis and photophysical studies of through-space conjugated [2.2]paracyclophane-based naphthalene fluorophores. RSC Advances 2017, 7, 50472-50476.

(19) Le Menez, P.; Fargeas, V.; Berque, I.; Poisson, J.; Ardisson, J.; Lallemand, J.-Y.; Pancrazi, A. Sequential Homoaldolization. Cuprate Rearrangement in a Stereoselective Synthesis of Stannyl Dienes: Application to the Synthesis of the Western C10-C15 Subunit of (.+-.)-Tylosin Aglycon. J. Org. Chem. 1995, 60, 3592-3599.

(20) Vyas, D. J.; Hazra, C. K.; Oestreich, M. Copper(I)-Catalyzed Regioselective Propargylic Substitution Involving Si-B Bond Activation. Org. Lett. 2011, 13, 4462-4465.

(21) Wang, P.; Yeo, X.-L.; Loh, T.-P. Copper-Catalyzed Highly Regioselective Silylcupration of Terminal Alkynes to Form $\alpha$-Vinylsilanes. J. Am. Chem. Soc. 2011, 133, 1254-1256.

(22) Nakamura, S.; Uchiyama, M.; Ohwada, T. Chemoselective Silylzincation of. Functionalized Terminal Alkynes Using Dianion-Type Zincate (SiBNOL-Zn-ate): Regiocontrolled Synthesis of Vinylsilanes. J. Am. Chem. Soc. 2004, 126, 11146-11147.

(23) Adam, W.; Richter, M. J. One-Pot Synthesis of $\alpha$-Trimethylsilyl Enones from. Vinylsilanes. Synthesis 1994, 1994, 176-180. Adam, W.; Richter, M. J. Synthesis 1994, 1994, 176.

(24) Iglesias, M.; Aliaga-Lavrijsen, M.; Miguel, P. J. S.; Fernández-Alvarez, F. J.; Pérez-Torrente, J. J.; Oro, L. A. Preferential $\alpha$-Hydrosilylation of Terminal Alkynes by Bis-N-Heterocyclic Carbene Rhodium(III) Catalysts. Adv. Synth. Catal. 2015, 357, 350-354. 
(25) Berthon-Gelloz, G.; Schumers, J.-M.; De Bo, G.; Markó, I. E. Highly $\beta$-(E)-Selective Hydrosilylation of Terminal and Internal Alkynes Catalyzed by a (IPr)Pt(diene) Complex. J. Org. Chem. 2008, 73, 4190-4197.

(26) Maruyama, Y.; Yamamura, K.; Sagawa, T.; Katayama, H.; Ozawa, F. Mechanisms of $\mathrm{C}-\mathrm{Si}$ and $\mathrm{C}-\mathrm{H}$ Bond Formation on the Reactions of Alkenylruthenium(II) Complexes with Hydrosilanes. Organometallics 2000, 19, 1308-1318.

(27) Angeles Cubillo de Dios, M.; Fleming, I.; Friedhoff, W.; Woode, P. D. W. Silyl-cupration of a propargylsilane as a test for the reversibility of a metallo-metallation. J. Organomet. Chem. 2001, 624, 69-72.

(28) Frisch, M. J.; Trucks, G. W.; Schlegel, H. B.; Scuseria, G. E.; Robb, M. A.; Cheeseman, J. R.; Scalmani, G.; Barone, V.; Mennucci, B.; Petersson, G. A.; Nakatsuji, H.; Caricato, M.; Li, X.; Hratchian, H. P.; Izmaylov, A. F.; Bloino, J.; Zheng, G.; Sonnenberg, J. L.; Hada, M.; Ehara, M.; Toyota, K.; Fukuda, R.; Hasegawa, J.; Ishida, M.; Nakajima, T.; Honda, Y.; Kitao, O.; Nakai, H.; Vreven, T.; Jr; Peralta, J. E.; Ogliaro, F.; Bearpark, M.; Heyd, J. J.; Brothers, E.; Kudin, K. N.; Staroverov, V. N.; Kobayashi, R.; Normand, J.; Raghavachari, K.; Rendell, A.; Burant, J. C.; Iyengar, S. S.; Tomasi, J.; Cossi, M.; Rega, N.; Millam, J. M.; Klene, M.; Knox, J. E.; Cross, J. B.; Bakken, V.; Adamo, C.; Jaramillo, J.; Gomperts, R.; Stratmann, R. E.; Yazyev, O.; Austin, A. J.; Cammi, R.; Pomelli, C.; Ochterski, J. W.; Martin, R. L.; Morokuma, K.; Zakrzewski, V. G.; Voth, G. A.; Salvador, P.; Dannenberg, J. J.; Dapprich, S.; Daniels, A. D.; Farkas; Foresman, J. B.; Ortiz, J. V.; Cioslowski, J.; Fox, D. J. Gaussian 09 Revision A.02; Gaussian Inc. Wallingford CT 2009.

(29) (a) Marenich, A. V.; Cramer, C. J.; Truhlar, D. G. Universal Solvation Model Based on Solute Electron Density and on a Continuum Model of the Solvent Defined by the Bulk Dielectric Constant and Atomic Surface Tensions. J. Phys. Chem. B 2009, 113, 6378-6396. (b) Zhao, Y.; Truhlar, D. G. The M06 suite of density functionals for main group thermochemistry, thermochemical kinetics, noncovalent interactions, excited states, and transition elements: two new functionals and systematic testing of four M06-class functionals and 12 other functionals. Theor. Chem. Acc. 2008, 120, 215-241.

(30) (a) Dunning, T. H., Jr.; Hay, P. J. In Methods of Electronic Structure Theory; Schaefer, H., III, Ed.; Springer US: 1977; Vol. 3, pp 1-27. (b) Hay, P. J.; Wadt, W. R. Ab initio effective core potentials for molecular calculations. Potentials for $\mathrm{K}$ to $\mathrm{Au}$ including the outermost core orbitals. J. Chem. Phys. 1985, 82, 299-310. (c) Ehlers, A. W.; Böhme, M.; Dapprich, S.; Gobbi, A.; Höllwarth, A.; Jonas, V.; Köhler, K. F.; Stegmann, R.; Veldkamp, A.; Frenking, G. A set of f-polarization functions for pseudo-potential basis 
sets of the transition metals $\mathrm{Sc}-\mathrm{Cu}, \mathrm{Y}-\mathrm{Ag}$ and La-Au. Chem. Phys. Lett. 1993, 208, 111-114.

\section{Energies}

Table S3. Absolute electronic energies, thermal corrections to energies and free energies at $298 \mathrm{~K}$ (in Hartree) of all stationary points for hydrogenation of alkyne $\mathrm{Me}-\mathrm{C} \equiv \mathrm{C}-\mathrm{TMS}$ catalyzed by $\left[\mathrm{CpRu}\left(\mathrm{NCM}_{3}\right)_{3}\right]^{+}$calculated at the SMD B3LYP method.

\begin{tabular}{|c|c|c|c|}
\hline & $\mathrm{E}$ & $\mathrm{E}+\mathrm{ZPE}$ & $\mathrm{G}$ \\
\hline $\mathrm{H}_{2}$ & -1.166523 & -1.156529 & -1.168018 \\
\hline NCMe & -132.661485 & -132.616177 & -132.639110 \\
\hline alkyne & -525.113694 & -524.955830 & -524.992630 \\
\hline cat1 & -685.161034 & -684.935983 & -684.987786 \\
\hline cat2 & -552.470812 & -552.293005 & -552.337376 \\
\hline A1a & -553.647753 & -553.453110 & -553.498742 \\
\hline A1b & -946.076607 & -945.770668 & -945.823145 \\
\hline A2a & -946.099326 & -945.791928 & -945.843000 \\
\hline$A 2 b$ & -946.096873 & -945.789844 & -945.842159 \\
\hline A3a-TS & -946.082056 & -945.777893 & -945.830166 \\
\hline A3b-TS & -946.078358 & -945.774142 & -945.826399 \\
\hline A4a & -946.096006 & -945.786654 & -945.837439 \\
\hline$A 4 b$ & -946.090864 & -945.781225 & -945.831472 \\
\hline A5a-TS ${ }_{\text {trans }}$ & -946.095256 & -945.787743 & -945.839170 \\
\hline A5a-TS ${ }_{\text {cis }}$ & -946.092783 & -945.785246 & -945.837410 \\
\hline A6atrans & -946.105331 & -945.796511 & -945.849010 \\
\hline A6acis & -946.106864 & -945.798144 & -945.849121 \\
\hline
\end{tabular}




\begin{tabular}{|c|c|c|c|}
\hline B1-TS & -946.095352 & -945.78753 & -945.838006 \\
\hline B1 & -946.154941 & -945.841074 & -945.892540 \\
\hline C1-TS & -946.100506 & -945.793035 & -945.845047 \\
\hline C1 & -946.104068 & -945.795031 & -945.845672 \\
\hline C2-TS1 & -946.100967 & -945.794298 & -945.845488 \\
\hline C2-TS2 & -946.102606 & -945.794929 & -945.845558 \\
\hline $\mathrm{C} 2$ & -946.131400 & -945.818037 & -945.867640 \\
\hline C3 & -1078.822368 & -1078.462065 & -1078.519246 \\
\hline C3-TSa & -1078.795498 & -1078.435686 & -1078.492233 \\
\hline D1 & -947.308340 & -946.977952 & -947.028332 \\
\hline D1-TS & -947.297400 & -946.970438 & -947.022073 \\
\hline D2 & -947.297439 & -946.969582 & -947.021264 \\
\hline D2-TS & -947.2970897 & -946.969530 & -947.020709 \\
\hline D3 & -947.307706 & -946.975761 & -947.027515 \\
\hline D4 & -947.330580 & -946.997832 & -947.048849 \\
\hline D4-TS & -947.316355 & -946.988690 & -947.039419 \\
\hline D5 & -947.328080 & -946.997319 & -947.048603 \\
\hline C3-TSb & -1078.763617 & -1078.407191 & -1078.464565 \\
\hline C3-TS2a & -946.075589 & -945.766902 & -945.819597 \\
\hline C3-TS2b & -946.111375 & -945.798106 & -945.846727 \\
\hline C4-TSa & -1078.770876 & -1078.415770 & -1078.474418 \\
\hline C4-TS2a & -946.101216 & -945.792758 & -945.842063 \\
\hline C4a & -1078.842579 & -1078.480756 & -1078.536470 \\
\hline E1 & -1077.612709 & -1077.275047 & -1077.332045 \\
\hline E1-TS & -1077.592963 & -1077.255622 & -1077.311919 \\
\hline E2 & -1077.601371 & -1077.264115 & -1077.323497 \\
\hline E3 & -944.907606 & -944.617499 & -944.670015 \\
\hline E4a & -946.087217 & -945.779500 & -945.832063 \\
\hline
\end{tabular}




\begin{tabular}{|c|c|c|c|}
\hline E4b & -946.088661 & -945.779674 & -945.830597 \\
\hline E4-TSa & -946.069068 & -945.764953 & -945.817674 \\
\hline E4-TSb & -946.071711 & -945.767557 & -945.818165 \\
\hline E5b & -946.082097 & -945.773625 & -945.824401 \\
\hline E5-TSb & -946.086596 & -945.779354 & -945.829198 \\
\hline E6b & -946.155079 & -945.841240 & -945.891626 \\
\hline F1 & -813.409508 & -813.148119 & -813.193729 \\
\hline F1-TSa & -946.072129 & -945.764595 & -945.815412 \\
\hline F1-TSb & -813.406960 & -813.145617 & -813.190551 \\
\hline F2 & -946.098872 & -945.791183 & -945.844170 \\
\hline F2-TS & -946.089712 & -945.782424 & -945.832400 \\
\hline F3 & -946.124279 & -945.811376 & -945.860268 \\
\hline F3-TS & -946.117502 & -945.804823 & -945.853944 \\
\hline
\end{tabular}

Table S4. Absolute electronic energies, thermal corrections to energies and free energies at 298K (in Hartree) of the critical steps for hydrogenation of alkyne Me-CC-SiPhMe 2 catalyzed by $\left[\mathrm{CpRu}\left(\mathrm{NCM}_{3}\right)_{3}\right]^{+}$calculated at the SMD B3LYP method.

\begin{tabular}{|l|c|c|c|}
\hline alkyne(SiPhMe $)$ & -716.701032 & -716.489019 & -716.531967 \\
\hline C3(SiPhMe $)$ & -1270.410847 & -1269.995899 & -1270.057244 \\
\hline C3-TSa(SiPhMe $)$ & -1137.711563 & -1137.345090 & -1137.399038 \\
\hline C3-TS(SiPhMe $)$ & -1270.383413 & -1269.969327 & -1270.030338 \\
\hline D1-TS(SiPhMe $)$ & -1138.883866 & -1138.502505 & -1138.561496 \\
\hline D2-TS(SiPhMe $)$ & -1138.884772 & -1138.503471 & -1138.560477 \\
\hline D4-TS(SiPhMe $)$ & -1138.904496 & -1138.522878 & -1138.579548 \\
\hline B1-TS(SiPhMe 2$)$ & -1137.682723 & -1137.320151 & -1137.374798 \\
\hline
\end{tabular}


Table S5. Absolute electronic energies, thermal corrections to energies and free energies at 298K (in Hartree) of the critical steps for hydrogenation of alkyne Me-CC-TMS catalyzed by $\left[\mathrm{CpRu}(\text { pyridine) }]^{+}\right.$and $\left[\mathrm{CpRu}(\text { dabco })\left(\mathrm{NCM}_{3}\right)_{2}\right]^{+}$calculated at the SMD B3LYP method.

\begin{tabular}{|l|c|c|c|}
\hline dabco & -345.080007 & -344.896889 & -344.927049 \\
\hline cat(dabco) & -897.589916 & -897.225847 & -897.277515 \\
\hline C3-TSa(dabco) & -1350.115784 & -1349.606017 & -1349.657907 \\
\hline D2-TS(dabco) & -1351.306297 & -1350.786204 & -1350.842379 \\
\hline B1-TS(dabco) & -1350.101957 & -1349.599768 & -1349.657096 \\
\hline
\end{tabular}

\section{Cartesian Coordinates ( $)$}

alkyne

$$
G=-524.993018 \quad Z P E=-524.955692
$$

$\begin{array}{lrrc}\mathrm{C} & 0.977001 & -0.001215 & -0.001723 \\ \mathrm{C} & 2.197012 & -0.001769 & -0.001751 \\ \mathrm{Si} & -0.866046 & -0.001603 & -0.001412 \\ \mathrm{C} & -1.472259 & 0.983206 & 1.475956 \\ \mathrm{H} & -2.571326 & 0.998235 & 1.498898 \\ \mathrm{H} & -1.124237 & 0.547022 & 2.421868 \\ \mathrm{H} & -1.124551 & 2.024208 & 1.436780 \\ \mathrm{C} & -1.471059 & -1.773891 & 0.112326 \\ \mathrm{H} & -2.570051 & -1.803009 & 0.113204 \\ \mathrm{H} & -1.121686 & -2.373928 & -0.738617 \\ \mathrm{H} & -1.123084 & -2.260618 & 1.033272 \\ \mathrm{C} & -1.471970 & 0.785555 & -1.593052 \\ \mathrm{H} & -2.570974 & 0.799000 & -1.618231 \\ \mathrm{H} & -1.122861 & 1.822509 & -1.688185 \\ \mathrm{H} & -1.124222 & 0.230886 & -2.474860 \\ \mathrm{C} & 3.648667 & -0.002234 & -0.002279 \\ \mathrm{H} & 4.046340 & 0.897641 & -0.489752 \\ \mathrm{H} & 4.045526 & -0.874527 & -0.538189\end{array}$


$\begin{array}{llll}\mathrm{H} & 4.046257 & -0.030439 & 1.020794\end{array}$

alkyne(SiPhMe2)

$G=-716.531967 \quad Z P E=-716.489019$

$\begin{array}{lrrr}\mathrm{C} & 2.032235 & -0.289697 & -0.130986 \\ \mathrm{C} & 2.894644 & -1.130012 & -0.327156 \\ \mathrm{Si} & 0.728154 & 0.970864 & 0.176784 \\ \mathrm{C} & 0.884716 & 2.351784 & -1.077627 \\ \mathrm{H} & 0.132971 & 3.133502 & -0.900275 \\ \mathrm{H} & 1.874297 & 2.822315 & -1.001982 \\ \mathrm{H} & 0.764324 & 1.990428 & -2.107421 \\ \mathrm{C} & 0.945691 & 1.634747 & 1.916636 \\ \mathrm{H} & 0.186290 & 2.398505 & 2.134854 \\ \mathrm{H} & 0.846149 & 0.838030 & 2.666298 \\ \mathrm{H} & 1.934647 & 2.095746 & 2.043714 \\ \mathrm{C} & -0.943687 & 0.122404 & 0.025769 \\ \mathrm{C} & -1.946420 & 0.608106 & -0.823164 \\ \mathrm{C} & -1.228095 & -1.016776 & 0.793989 \\ \mathrm{C} & -3.189225 & -0.016979 & -0.902758 \\ \mathrm{H} & -1.758818 & 1.490191 & -1.437900 \\ \mathrm{C} & -2.466174 & -1.646713 & 0.719428 \\ \mathrm{H} & -0.463859 & -1.425311 & 1.459886 \\ \mathrm{C} & -3.450130 & -1.145381 & -0.130950 \\ \mathrm{H} & -3.954834 & 0.376859 & -1.570339 \\ \mathrm{H} & -2.665822 & -2.531226 & 1.323322 \\ \mathrm{H} & -4.420351 & -1.636821 & -0.192206 \\ \mathrm{C} & 3.920951 & -2.128167 & -0.564104 \\ \mathrm{H} & 3.592420 & -3.121388 & -0.230869 \\ \mathrm{H} & 4.165981 & -2.198995 & -1.632009 \\ \mathrm{H} & 4.845411 & -1.883816 & -0.024517\end{array}$

cat1

$G=-684.987786 \quad Z P E=-684.935983$

$\begin{array}{llll}\mathrm{Ru} & -0.556064 & -0.001780 & -0.003555\end{array}$ 


$\begin{array}{lccc}\mathrm{C} & 1.424094 & -2.584137 & 0.447177 \\ \mathrm{~N} & 0.737220 & -1.665029 & 0.287583 \\ \mathrm{C} & 2.280175 & -3.730937 & 0.646903 \\ \mathrm{H} & 3.326232 & -3.410846 & 0.708209 \\ \mathrm{H} & 2.010131 & -4.244141 & 1.576723 \\ \mathrm{H} & 2.171308 & -4.430444 & -0.189507 \\ \mathrm{C} & 1.432536 & 1.625160 & 2.044119 \\ \mathrm{~N} & 0.762182 & 1.032670 & 1.308148 \\ \mathrm{C} & 2.262825 & 2.368998 & 2.963658 \\ \mathrm{H} & 2.164009 & 1.958554 & 3.974837 \\ \mathrm{H} & 3.312477 & 2.307813 & 2.655806 \\ \mathrm{H} & 1.956711 & 3.421030 & 2.975948 \\ \mathrm{C} & 1.493268 & 0.874609 & -2.409925 \\ \mathrm{~N} & 0.804035 & 0.551361 & -1.536593 \\ \mathrm{C} & 2.345837 & 1.279722 & -3.503708 \\ \mathrm{H} & 2.199250 & 2.344527 & -3.717563 \\ \mathrm{H} & 3.396894 & 1.110791 & -3.244621 \\ \mathrm{H} & 2.102889 & 0.700868 & -4.401943 \\ \mathrm{C} & -2.351048 & -0.846618 & -0.874560 \\ \mathrm{H} & -2.381705 & -1.633500 & -1.620200 \\ \mathrm{C} & -2.387322 & -1.019669 & 0.551715 \\ \mathrm{C} & -2.319166 & 0.560342 & -1.126069 \\ \mathrm{H} & -2.386959 & -1.970451 & 1.075708 \\ \mathrm{C} & -2.362533 & 0.261400 & 1.162332 \\ \mathrm{H} & -2.244183 & 1.023533 & -2.105516 \\ \mathrm{C} & -2.314826 & 1.253278 & 0.118244 \\ \mathrm{H} & -2.357585 & 0.458009 & 2.229627 \\ \mathrm{H} & -2.300121 & 2.329192 & 0.254420\end{array}$

cat2

$G=-552.337376 \quad Z P E=-552.293005$

$\begin{array}{cccc}\mathrm{Ru} & 0.407103 & -0.051496 & 0.210339 \\ \mathrm{C} & -2.112510 & -2.154520 & -0.027867 \\ \mathrm{~N} & -1.219950 & -1.417996 & 0.038006 \\ \mathrm{C} & -3.226261 & -3.068843 & -0.106209 \\ \mathrm{H} & -3.665623 & -3.200859 & 0.889186\end{array}$




$\begin{array}{llll}\mathrm{H} & -3.990859 & -2.666735 & -0.780484 \\ \mathrm{H} & -2.891888 & -4.042105 & -0.481684 \\ \mathrm{C} & -1.694570 & 2.453543 & 0.013920 \\ \mathrm{~N} & -0.993466 & 1.535331 & 0.110104 \\ \mathrm{C} & -2.559390 & 3.601732 & -0.113808 \\ \mathrm{H} & -3.122817 & 3.538524 & -1.051634 \\ \mathrm{H} & -3.265381 & 3.634764 & 0.723606 \\ \mathrm{H} & -1.963079 & 4.521005 & -0.117000 \\ \mathrm{C} & 2.012322 & -1.360259 & -0.389004 \\ \mathrm{H} & 1.889558 & -2.430430 & -0.519084 \\ \mathrm{C} & 1.758378 & -0.353762 & -1.383083 \\ \mathrm{C} & 2.373209 & -0.699603 & 0.812976 \\ \mathrm{H} & 1.453655 & -0.528576 & -2.409817 \\ \mathrm{C} & 2.025141 & 0.927806 & -0.786717 \\ \mathrm{H} & 2.569957 & -1.179636 & 1.766647 \\ \mathrm{C} & 2.388402 & 0.720145 & 0.569972 \\ \mathrm{H} & 1.905087 & 1.889028 & -1.276568 \\ \mathrm{H} & 2.626427 & 1.488931 & 1.297054\end{array}$

\section{$\mathrm{CNCH} 3$}

$G=-132.63911 \quad Z P E=-132.616176$

$\begin{array}{llll}\mathrm{N} & -0.000000 & -0.000000 & 1.432792\end{array}$

C $\quad-0.000000 \quad-0.000000 \quad 0.271596$

$\begin{array}{llll}\mathrm{H} & 0.000000 & 1.028400 & -1.554288\end{array}$

$\begin{array}{llll}\text { C } & -0.000000 & -0.000000 & -1.176254\end{array}$

$\mathrm{H} \quad 0.890620 \quad-0.514200 \quad-1.554288$

$\mathrm{H} \quad-0.890620 \quad-0.514200 \quad-1.554288$

$\mathrm{H} 2$

$G=-1.168018 \quad Z P E=-1.15653$

$\begin{array}{llll}\mathrm{H} & 0.000000 & 0.000000 & 0.371602\end{array}$

$\begin{array}{llll}\mathrm{H} & 0.000000 & 0.000000 & -0.371602\end{array}$ 


\section{A1a}

$G=-553.498743 \quad Z P E=-553.45311$

$\begin{array}{cccc}\mathrm{C} & 1.495077 & 0.015870 & 1.534831 \\ \mathrm{C} & 1.945747 & 1.121939 & 0.735064 \\ \mathrm{C} & 2.537348 & 0.610836 & -0.446206 \\ \mathrm{C} & 2.490909 & -0.827768 & -0.378473 \\ \mathrm{C} & 1.869381 & -1.186313 & 0.843252 \\ \mathrm{Ru} & 0.448823 & -0.034499 & -0.331728 \\ \mathrm{~N} & -1.042732 & 1.452863 & -0.158472 \\ \mathrm{C} & -1.807381 & 2.305445 & 0.014522 \\ \mathrm{~N} & -1.141167 & -1.405746 & -0.105432 \\ \mathrm{C} & -2.002274 & -2.165130 & 0.046965 \\ \mathrm{H} & 1.652436 & -2.198774 & 1.169630 \\ \mathrm{H} & 1.030989 & 0.076650 & 2.513157 \\ \mathrm{H} & 1.797532 & 2.172269 & 0.966464 \\ \mathrm{H} & 2.949571 & 1.197807 & -1.259923 \\ \mathrm{H} & 2.862620 & -1.513966 & -1.131828 \\ \mathrm{H} & 0.061743 & 0.281627 & -2.207494 \\ \mathrm{H} & 0.061555 & -0.516739 & -2.162088 \\ \mathrm{C} & -3.076426 & -3.113126 & 0.229484 \\ \mathrm{H} & -2.963841 & -3.628982 & 1.189470 \\ \mathrm{H} & -4.040495 & -2.592953 & 0.214484 \\ \mathrm{H} & -3.060427 & -3.855443 & -0.576814 \\ \mathrm{C} & -2.754613 & 3.373811 & 0.231272 \\ \mathrm{H} & -3.660972 & 3.198561 & -0.358802 \\ \mathrm{H} & -3.025033 & 3.424066 & 1.291973 \\ \mathrm{H} & -2.313229 & 4.330655 & -0.069127\end{array}$

A1b

$G=-945.823145 \quad Z P E=-945.770669$

$\begin{array}{llll}\mathrm{C} & 1.956585 & -0.927611 & -1.773487 \\ \mathrm{C} & 1.609231 & -1.919715 & -0.800127 \\ \mathrm{C} & 2.366295 & -1.654571 & 0.381323 \\ \mathrm{C} & 3.175594 & -0.496289 & 0.134557\end{array}$




$\begin{array}{cccc}\mathrm{C} & 2.913016 & -0.046866 & -1.190065 \\ \mathrm{Ru} & 1.006053 & 0.012315 & 0.027897 \\ \mathrm{~N} & 0.426360 & 2.022658 & 0.027869 \\ \mathrm{C} & 0.071229 & 3.122652 & 0.049037 \\ \mathrm{H} & 0.901108 & -2.730935 & -0.933801 \\ \mathrm{H} & 2.345058 & -2.239165 & 1.294767 \\ \mathrm{H} & 3.871918 & -0.042268 & 0.830701 \\ \mathrm{H} & 3.351723 & 0.823022 & -1.666912 \\ \mathrm{H} & 1.566293 & -0.860384 & -2.783159 \\ \mathrm{H} & -0.070805 & 0.135124 & -1.185819 \\ \mathrm{H} & 1.390588 & 0.567358 & 1.513451 \\ \mathrm{C} & -0.381565 & 4.493328 & 0.073967 \\ \mathrm{H} & -0.503210 & 4.825974 & 1.111034 \\ \mathrm{H} & 0.348738 & 5.140157 & -0.424975 \\ \mathrm{H} & -1.344823 & 4.574915 & -0.442244 \\ \mathrm{C} & -0.415537 & -0.631939 & 1.777072 \\ \mathrm{C} & -1.102683 & -0.549795 & 0.736393 \\ \mathrm{C} & -0.011469 & -0.872394 & 3.160140 \\ \mathrm{H} & -0.852881 & -1.310701 & 3.712444 \\ \mathrm{H} & 0.836045 & -1.565920 & 3.218773 \\ \mathrm{H} & 0.283996 & 0.059491 & 3.657869 \\ \mathrm{Si} & -2.605554 & -0.599423 & -0.371722 \\ \mathrm{C} & -2.290047 & -1.816240 & -1.759175 \\ \mathrm{H} & -2.029056 & -2.809736 & -1.370068 \\ \mathrm{H} & -3.199639 & -1.928632 & -2.366314 \\ \mathrm{H} & -1.484664 & -1.484745 & -2.428896 \\ \mathrm{C} & -2.903941 & 1.128628 & -1.029974 \\ \mathrm{H} & -3.853614 & 1.163836 & -1.582179 \\ \mathrm{H} & -2.967920 & 1.862159 & -0.214620 \\ \mathrm{H} & -2.106812 & 1.443939 & -1.717406 \\ \mathrm{H} & -4.029140 & -1.171456 & 0.703411 \\ \mathrm{H} & -4.192993 & -0.491489 & 1.550068 \\ \mathrm{H} & -1.211011 & 0.121911 \\ \mathrm{H} & -2.175912 & 1.108518\end{array}$

A2a

$G=-945.843 \quad Z P E=-945.791928$ 


$\begin{array}{cccc}\mathrm{C} & -2.752279 & -1.422633 & -0.043600 \\ \mathrm{C} & -1.989876 & -1.501863 & 1.164870 \\ \mathrm{C} & -0.793746 & -2.246187 & 0.875630 \\ \mathrm{C} & -0.796941 & -2.574206 & -0.502040 \\ \mathrm{C} & -2.015201 & -2.054948 & -1.073010 \\ \mathrm{Ru} & -0.794253 & -0.398705 & -0.268929 \\ \mathrm{~N} & -1.535770 & 1.567301 & -0.411110 \\ \mathrm{C} & -1.961503 & 2.641551 & -0.492760 \\ \mathrm{C} & 1.202352 & 0.371712 & 0.627804 \\ \mathrm{C} & 0.362513 & 0.609259 & 1.515984 \\ \mathrm{H} & -2.296446 & -1.131612 & 2.137274 \\ \mathrm{H} & 0.003477 & -2.464972 & 1.580472 \\ \mathrm{H} & -0.025788 & -3.123256 & -1.032098 \\ \mathrm{H} & -2.312985 & -2.131999 & -2.113529 \\ \mathrm{H} & -3.697689 & -0.903536 & -0.166109 \\ \mathrm{H} & 0.396021 & -0.040970 & -1.686754 \\ \mathrm{H} & -0.331544 & 0.041561 & -2.040794 \\ \mathrm{Si} & 2.872796 & 0.206523 & -0.184399 \\ \mathrm{C} & -0.342006 & 1.015640 & 2.725177 \\ \mathrm{H} & 0.314729 & 1.639300 & 3.346624 \\ \mathrm{H} & -1.244782 & 1.594604 & 2.492329 \\ \mathrm{H} & -0.642641 & 0.144254 & 3.321857 \\ \mathrm{C} & 2.958372 & 1.376332 & -1.645788 \\ \mathrm{H} & 3.978939 & 1.384786 & -2.053995 \\ \mathrm{H} & 2.282403 & 1.083823 & -2.460786 \\ \mathrm{H} & 2.710774 & 2.405484 & -1.353010 \\ \mathrm{C} & 3.079928 & -1.573696 & -0.730947 \\ \mathrm{H} & 4.121631 & -1.764521 & -1.024838 \\ \mathrm{H} & 2.830484 & -2.274367 & 0.078147 \\ \mathrm{H} & 2.444184 & -1.808558 & -1.595629 \\ \mathrm{C} & 4.141516 & 0.670505 & 1.113327 \\ \mathrm{H} & 4.004088 & 1.704647 & 1.456389 \\ \mathrm{H} & 4.077306 & 0.013405 & 1.990744 \\ \mathrm{H} & 5.158742 & 0.585395 & 0.705875 \\ \mathrm{C} & -2.493100 & 3.979744 & -0.601285 \\ \mathrm{H} & -3.163257 & 4.186819 & 0.240390 \\ \mathrm{H} & -1.675300 & 4.709189 & -0.593734\end{array}$


$\begin{array}{llll}\mathrm{H} & -3.054166 & 4.083864 & -1.536771\end{array}$

\section{A2b}

$G=-945.842159 \quad Z P E=-945.789845$

$\begin{array}{cccc}\mathrm{C} & 2.317116 & 0.209351 & -1.623676 \\ \mathrm{C} & 1.155081 & -0.610313 & -1.817509 \\ \mathrm{C} & 1.296517 & -1.761547 & -0.973280 \\ \mathrm{C} & 2.502667 & -1.639363 & -0.232978 \\ \mathrm{C} & 3.131952 & -0.414303 & -0.647664 \\ \mathrm{Ru} & 1.165523 & 0.030807 & 0.248317 \\ \mathrm{~N} & 0.324046 & 1.957574 & 0.233222 \\ \mathrm{C} & -0.111263 & 3.030660 & 0.206412 \\ \mathrm{C} & -0.420701 & -0.851481 & 1.835038 \\ \mathrm{C} & -1.004477 & -0.610744 & 0.763102 \\ \mathrm{H} & 0.353738 & -0.425807 & -2.525836 \\ \mathrm{H} & 0.568118 & -2.561254 & -0.870893 \\ \mathrm{H} & 2.891124 & -2.346791 & 0.491526 \\ \mathrm{H} & 4.059227 & -0.012783 & -0.250978 \\ \mathrm{H} & 2.512556 & 1.162153 & -2.105273 \\ \mathrm{H} & 1.534487 & 0.448704 & 2.058417 \\ \mathrm{H} & 2.153659 & 0.772667 & 1.644345 \\ \mathrm{C} & -0.030633 & -1.264886 & 3.178444 \\ \mathrm{H} & -0.862552 & -1.797461 & 3.659082 \\ \mathrm{H} & 0.837946 & -1.935511 & 3.153786 \\ \mathrm{H} & 0.229301 & -0.401158 & 3.804624 \\ \mathrm{Hi} & -2.448222 & -0.591677 & -0.420163 \\ \mathrm{H} & -2.279197 & -2.028682 & -1.611520 \\ \mathrm{H} & -2.159685 & -2.981396 & -1.077457 \\ \mathrm{H} & -3.193836 & -2.104654 & -2.217937 \\ \mathrm{H} & -1.435213 & -1.915923 & -2.304586 \\ \mathrm{H} & -2.554352 & 1.036899 & -1.335088 \\ \mathrm{H} & -3.392201 & 0.997760 & -2.046759 \\ \mathrm{H} & -1.643474 & 1.259356 & -1.906259 \\ \mathrm{H} & & -0.639626 & \\ \mathrm{H} & -0.025374 & 1.383795\end{array}$




$\begin{array}{lrrc}\mathrm{H} & -4.876151 & -0.868225 & 0.039246 \\ \mathrm{H} & -3.905458 & -1.784387 & 1.209159 \\ \mathrm{C} & -0.658319 & 4.365743 & 0.165892 \\ \mathrm{H} & -1.242123 & 4.502369 & -0.751604 \\ \mathrm{H} & -1.310519 & 4.528267 & 1.031487 \\ \mathrm{H} & 0.152422 & 5.103426 & 0.187411\end{array}$

\section{A3a-TS}

$G=-945.830166 \quad Z P E=-945.777893$

$\begin{array}{lccc}\mathrm{H} & 0.595551 & -0.018256 & -1.038276 \\ \mathrm{C} & 1.250231 & 0.144583 & 0.351013 \\ \mathrm{Ru} & -0.925437 & -0.302261 & -0.259590 \\ \mathrm{C} & 0.427355 & 0.263861 & 1.315217 \\ \mathrm{C} & -1.568512 & -1.994296 & 1.027226 \\ \mathrm{C} & -2.620543 & -1.038322 & 0.926908 \\ \mathrm{C} & -3.020015 & -0.981815 & -0.454485 \\ \mathrm{C} & -2.213859 & -1.895288 & -1.178547 \\ \mathrm{C} & -1.292471 & -2.506735 & -0.272868 \\ \mathrm{H} & -1.023958 & -2.250230 & 1.931412 \\ \mathrm{H} & -3.065253 & -0.480228 & 1.744328 \\ \mathrm{H} & -3.801171 & -0.352533 & -0.867926 \\ \mathrm{H} & -2.250608 & -2.059683 & -2.250394 \\ \mathrm{H} & -0.540310 & -3.246660 & -0.524769 \\ \mathrm{C} & -1.477502 & 2.893312 & -0.355195 \\ \mathrm{~N} & -1.257786 & 1.756563 & -0.341482 \\ \mathrm{C} & -1.758079 & 4.309766 & -0.376726 \\ \mathrm{H} & -0.865016 & 4.862352 & -0.689178 \\ \mathrm{H} & -2.570949 & 4.519475 & -1.080828 \\ \mathrm{H} & -2.055228 & 4.648548 & 0.622080 \\ \mathrm{C} & 0.103423 & 0.577536 & 2.708478 \\ \mathrm{H} & -0.599604 & 1.420958 & 2.754112 \\ \mathrm{H} & -0.377894 & -0.273108 & 3.208102 \\ \mathrm{H} & 1.009878 & 0.846863 & 3.267556 \\ \mathrm{Si} & 3.031339 & -0.076721 & -0.219637 \\ \mathrm{H} & 3.156633 & -1.849755 & -0.805653 \\ & 4.168573 & -2.083434 & -1.163198\end{array}$




$\begin{array}{lrrr}\mathrm{H} & 2.911066 & -2.554911 & -0.000060 \\ \mathrm{H} & 2.460576 & -2.031822 & -1.637646 \\ \mathrm{C} & 4.059603 & 0.291881 & 1.298235 \\ \mathrm{H} & 5.133200 & 0.204072 & 1.083210 \\ \mathrm{H} & 3.873598 & 1.313067 & 1.657653 \\ \mathrm{H} & 3.822781 & -0.402881 & 2.115218 \\ \mathrm{C} & 3.339232 & 1.115223 & -1.626768 \\ \mathrm{H} & 4.369305 & 1.027369 & -1.998347 \\ \mathrm{H} & 2.666524 & 0.908684 & -2.471591 \\ \mathrm{H} & 3.182482 & 2.156954 & -1.317141 \\ \mathrm{H} & -0.271512 & -0.056669 & -1.798084\end{array}$

\section{A3b-TS}

$$
G=-945.826398 \quad Z P E=-945.774141
$$

$\begin{array}{lccc}\mathrm{H} & 0.948496 & 0.211952 & 1.994595 \\ \mathrm{C} & -0.523762 & -0.344860 & 1.875029 \\ \mathrm{Ru} & 1.160275 & 0.056279 & 0.322603 \\ \mathrm{C} & -0.925645 & -0.485304 & 0.678894 \\ \mathrm{C} & 1.271898 & -1.676568 & -1.140212 \\ \mathrm{C} & 1.652831 & -0.478799 & -1.788700 \\ \mathrm{C} & 2.811907 & 0.038670 & -1.114177 \\ \mathrm{C} & 3.132723 & -0.866512 & -0.058497 \\ \mathrm{C} & 2.163659 & -1.910880 & -0.050112 \\ \mathrm{H} & 0.411680 & -2.289491 & -1.392207 \\ \mathrm{H} & 1.154256 & -0.023787 & -2.638527 \\ \mathrm{H} & 3.367297 & 0.928476 & -1.389790 \\ \mathrm{H} & 3.953169 & -0.758838 & 0.643218 \\ \mathrm{H} & 2.129209 & -2.749801 & 0.636367 \\ \mathrm{C} & -0.126967 & 2.989735 & -0.181890 \\ \mathrm{~N} & 0.342002 & 1.951113 & 0.022427 \\ \mathrm{C} & -0.713720 & 4.282639 & -0.446114 \\ \mathrm{H} & -0.981285 & 4.771970 & 0.497120 \\ \mathrm{H} & 0.001837 & 4.914787 & -0.983740 \\ \mathrm{H} & -1.616533 & 4.164600 & -1.056266 \\ \mathrm{H} & 1.952560 & 0.846912 & 1.536293 \\ \mathrm{C} & -0.748793 & -0.402820 & 3.331745\end{array}$




$\begin{array}{lrrr}\mathrm{H} & -0.555720 & 0.565899 & 3.809448 \\ \mathrm{H} & -1.792441 & -0.685541 & 3.517706 \\ \mathrm{H} & -0.094102 & -1.145503 & 3.804907 \\ \mathrm{Si} & -2.369148 & -0.728040 & -0.490777 \\ \mathrm{C} & -1.910744 & -0.185739 & -2.222465 \\ \mathrm{H} & -1.155355 & -0.843837 & -2.672734 \\ \mathrm{H} & -2.802620 & -0.222008 & -2.864502 \\ \mathrm{H} & -1.529321 & 0.844305 & -2.244302 \\ \mathrm{C} & -2.817739 & -2.546571 & -0.464692 \\ \mathrm{H} & -1.985804 & -3.169499 & -0.821336 \\ \mathrm{H} & -3.080500 & -2.881283 & 0.547582 \\ \mathrm{H} & -3.680951 & -2.738212 & -1.117384 \\ \mathrm{C} & -3.739637 & 0.349934 & 0.196474 \\ \mathrm{H} & -3.440055 & 1.407741 & 0.189217 \\ \mathrm{H} & -4.657050 & 0.255563 & -0.400985 \\ \mathrm{H} & -3.980881 & 0.076543 & 1.233008\end{array}$

\section{A4a}

$G=-945.837439 \quad Z P E=-945.786654$

$\begin{array}{llll}\mathrm{H} & 0.686477 & -1.011950 & -0.941587 \\ \mathrm{C} & 1.192208 & -0.682728 & 0.031345 \\ \mathrm{Ru} & -1.150941 & -0.182318 & -0.331938 \\ \mathrm{C} & 0.408777 & -0.290674 & 1.011666 \\ \mathrm{C} & -2.161418 & -1.670715 & 1.044383 \\ \mathrm{C} & -2.821967 & -0.411283 & 1.059433 \\ \mathrm{C} & -3.303736 & -0.159273 & -0.277087 \\ \mathrm{C} & -2.946712 & -1.281095 & -1.086367 \\ \mathrm{C} & -2.220493 & -2.198239 & -0.277747 \\ \mathrm{H} & -1.661986 & -2.140405 & 1.885659 \\ \mathrm{H} & -2.953621 & 0.240248 & 1.917201 \\ \mathrm{H} & -3.875655 & 0.706020 & -0.595250 \\ \mathrm{H} & -3.164498 & -1.399571 & -2.141905 \\ \mathrm{H} & -1.778012 & -3.130742 & -0.611681 \\ \mathrm{C} & -0.520771 & 2.978662 & -0.164740 \\ \mathrm{~N} & -0.738690 & 1.842605 & -0.204991 \\ \mathrm{C} & -0.251906 & 4.396868 & -0.127955\end{array}$




$\begin{array}{lccc}\mathrm{H} & 0.680093 & 4.587911 & 0.415640 \\ \mathrm{H} & -0.155417 & 4.783986 & -1.148568 \\ \mathrm{H} & -1.071863 & 4.920687 & 0.375790 \\ \mathrm{H} & -1.114344 & 0.250873 & -1.910942 \\ \mathrm{Si} & 3.067488 & -0.495064 & -0.214238 \\ \mathrm{C} & 0.475873 & 0.134275 & 2.416878 \\ \mathrm{H} & 0.096256 & 1.159701 & 2.527794 \\ \mathrm{H} & -0.158956 & -0.508866 & 3.042508 \\ \mathrm{H} & 1.499524 & 0.086869 & 2.812281 \\ \mathrm{C} & 3.873078 & -0.145065 & 1.438787 \\ \mathrm{H} & 4.962565 & -0.082965 & 1.304370 \\ \mathrm{H} & 3.540968 & 0.809154 & 1.868956 \\ \mathrm{H} & 3.680526 & -0.942168 & 2.169357 \\ \mathrm{C} & 3.257956 & 0.951510 & -1.391002 \\ \mathrm{H} & 4.309086 & 1.107295 & -1.670914 \\ \mathrm{H} & 2.685224 & 0.791623 & -2.315370 \\ \mathrm{H} & 2.893345 & 1.878798 & -0.926973 \\ \mathrm{C} & 3.717171 & -2.086297 & -0.956931 \\ \mathrm{H} & 3.227429 & -2.316449 & -1.912986 \\ \mathrm{H} & 4.797360 & -2.014018 & -1.146150 \\ \mathrm{H} & 3.554547 & -2.937067 & -0.281666\end{array}$

A4b

$G=-945.831472 \quad Z P E=-945.781225$

$\begin{array}{lccc}\mathrm{H} & 0.537851 & -0.230698 & 2.166279 \\ \mathrm{C} & -0.581585 & -0.459895 & 1.873050 \\ \mathrm{Ru} & 1.188006 & 0.055230 & 0.379063 \\ \mathrm{C} & -0.842317 & -0.507953 & 0.594845 \\ \mathrm{C} & 1.399356 & -1.793880 & -0.912358 \\ \mathrm{C} & 1.538467 & -0.602566 & -1.678107 \\ \mathrm{C} & 2.681348 & 0.109832 & -1.165700 \\ \mathrm{C} & 3.242282 & -0.671322 & -0.107574 \\ \mathrm{C} & 2.430594 & -1.825409 & 0.067055 \\ \mathrm{H} & 0.614647 & -2.535992 & -1.019639 \\ \mathrm{H} & 0.915070 & -0.289277 & -2.509205 \\ \mathrm{H} & 3.075486 & 1.044084 & -1.551350\end{array}$




$\begin{array}{lccc}\mathrm{H} & 4.117060 & -0.414311 & 0.478968 \\ \mathrm{H} & 2.568403 & -2.593467 & 0.820620 \\ \mathrm{C} & -0.104800 & 2.976401 & -0.080939 \\ \mathrm{~N} & 0.350305 & 1.930279 & 0.111416 \\ \mathrm{C} & -0.684197 & 4.273712 & -0.337956 \\ \mathrm{H} & -1.177839 & 4.271686 & -1.316667 \\ \mathrm{H} & -1.424485 & 4.511776 & 0.433935 \\ \mathrm{H} & 0.096508 & 5.042727 & -0.332370 \\ \mathrm{H} & 2.153503 & 0.977698 & 1.311729 \\ \mathrm{Si} & -2.287604 & -0.679303 & -0.582189 \\ \mathrm{C} & -1.351255 & -0.598485 & 3.147024 \\ \mathrm{H} & -1.311610 & 0.332768 & 3.726985 \\ \mathrm{H} & -2.398267 & -0.838252 & 2.932567 \\ \mathrm{H} & -0.926559 & -1.391633 & 3.775600 \\ \mathrm{C} & -2.032033 & -2.140119 & -1.731235 \\ \mathrm{H} & -1.864791 & -3.074574 & -1.178495 \\ \mathrm{H} & -2.938321 & -2.278074 & -2.338640 \\ \mathrm{H} & -1.194880 & -1.996412 & -2.427601 \\ \mathrm{C} & -3.841607 & -0.943175 & 0.437043 \\ \mathrm{H} & -4.025395 & -0.109577 & 1.128346 \\ \mathrm{H} & -4.712990 & -1.016397 & -0.230065 \\ \mathrm{H} & -3.798917 & -1.871779 & 1.022091 \\ \mathrm{C} & -2.442970 & 0.903976 & -1.576555 \\ \mathrm{H} & -1.527034 & 1.138977 & -2.135687 \\ \mathrm{H} & -3.259320 & 0.802601 & -2.306319 \\ & -2.683767 & 1.759614 & -0.930696\end{array}$

A5a-TS $_{\text {cis }}$

$G=-945.83741 \quad Z P E=-945.785245$

$\begin{array}{lccc}\mathrm{H} & 0.767170 & 0.487105 & -1.042486 \\ \mathrm{C} & 1.257804 & 0.413238 & -0.012069 \\ \mathrm{Ru} & -1.015542 & -0.273403 & -0.274176 \\ \mathrm{C} & 0.477304 & 0.256249 & 1.036285 \\ \mathrm{C} & -1.325552 & -2.138036 & 0.903117 \\ \mathrm{C} & -2.417547 & -1.250580 & 1.116491 \\ \mathrm{C} & -3.062960 & -1.049885 & -0.152820\end{array}$




$\begin{array}{lccc}\mathrm{C} & -2.394648 & -1.854274 & -1.119281 \\ \mathrm{C} & -1.297091 & -2.495660 & -0.480730 \\ \mathrm{H} & -0.618622 & -2.480795 & 1.651888 \\ \mathrm{H} & -2.713881 & -0.801845 & 2.058932 \\ \mathrm{H} & -3.931057 & -0.424874 & -0.334836 \\ \mathrm{H} & -2.646359 & -1.920910 & -2.171862 \\ \mathrm{H} & -0.575412 & -3.150779 & -0.957081 \\ \mathrm{C} & -1.738445 & 2.881687 & -0.186917 \\ \mathrm{~N} & -1.451717 & 1.760706 & -0.216139 \\ \mathrm{C} & -2.101724 & 4.279051 & -0.157718 \\ \mathrm{H} & -1.348636 & 4.849647 & 0.397027 \\ \mathrm{H} & -2.164548 & 4.669631 & -1.179529 \\ \mathrm{H} & -3.074065 & 4.402472 & 0.332211 \\ \mathrm{H} & -1.019466 & 0.037263 & -1.878227 \\ \mathrm{Si} & 3.106884 & 0.031229 & -0.242901 \\ \mathrm{C} & 0.571248 & 0.263913 & 2.503267 \\ \mathrm{H} & -0.155321 & 0.979458 & 2.914130 \\ \mathrm{H} & 0.324878 & -0.717772 & 2.929350 \\ \mathrm{H} & 1.571955 & 0.561138 & 2.844771 \\ \mathrm{C} & 3.937182 & -0.062885 & 1.430231 \\ \mathrm{H} & 5.006675 & -0.278202 & 1.294743 \\ \mathrm{H} & 3.861803 & 0.884515 & 1.980199 \\ \mathrm{H} & 3.515899 & -0.862105 & 2.054559 \\ \mathrm{C} & 3.851680 & 1.375017 & -1.311508 \\ \mathrm{H} & 4.906864 & 1.159328 & -1.529908 \\ \mathrm{H} & 3.322764 & 1.456122 & -2.271233 \\ \mathrm{H} & 3.805560 & 2.354242 & -0.816705 \\ \mathrm{H} & 3.109035 & -1.622085 & -1.127508 \\ \mathrm{H} & 2.539035 & -1.563281 & -2.066037 \\ \mathrm{H} & -128717 & -1.947486 & -1.375564 \\ \mathrm{H} & -2.399810 & -0.502069\end{array}$

\section{A5a-TS trans}

$$
G=-945.83917 \quad Z P E=-945.787744
$$

$\begin{array}{llll}\mathrm{H} & 0.636123 & -1.578258 & -0.677919 \\ \mathrm{C} & 1.122459 & -0.991677 & 0.139617\end{array}$




\begin{tabular}{|c|c|c|c|}
\hline $\mathrm{Ru}$ & -1.117496 & -0.181186 & -0.3 \\
\hline C & 0.374381 & -0.377737 & 1.046993 \\
\hline C & -2.303847 & -1.501096 & 1.098946 \\
\hline C & -2.873434 & -0.202103 & 1.017517 \\
\hline C & -3.283043 & 0.004629 & -0.350792 \\
\hline C & -2.974975 & -1.177781 & -1.082682 \\
\hline C & -2.341821 & -2.095848 & -0.197088 \\
\hline $\mathrm{H}$ & -1.875543 & -1.955337 & 1.987226 \\
\hline $\mathrm{H}$ & -2.991762 & 0.504279 & 1.832808 \\
\hline $\mathrm{H}$ & -3.770790 & 0.892219 & -0.739649 \\
\hline $\mathrm{H}$ & -3.155889 & -1.338021 & -2.139707 \\
\hline $\mathrm{H}$ & -1.963375 & -3.078307 & -0.458806 \\
\hline $\mathrm{C}$ & -0.282263 & 2.940164 & -0.211405 \\
\hline $\mathrm{N}$ & -0.565655 & 1.818542 & -0.257404 \\
\hline $\mathrm{C}$ & 0.078197 & 4.337656 & -0.157808 \\
\hline $\mathrm{H}$ & -0.478333 & 4.839382 & 0.641648 \\
\hline $\mathrm{H}$ & 1.151695 & 4.437278 & 0.040488 \\
\hline $\mathrm{H}$ & -0.153643 & 4.822237 & -1.112847 \\
\hline $\mathrm{H}$ & -0.831310 & 0.091872 & -1.909432 \\
\hline $\mathrm{Si}$ & 2.958789 & -0.591221 & -0.207055 \\
\hline C & 0.564143 & 0.233952 & 2.367940 \\
\hline $\mathrm{H}$ & 0.239513 & 1.282900 & 2.375876 \\
\hline $\mathrm{H}$ & -0.057116 & -0.297187 & 3.104607 \\
\hline $\mathrm{H}$ & 1.607707 & 0.164911 & 2.706979 \\
\hline C & 4.019369 & -1.484632 & 1.050503 \\
\hline $\mathrm{H}$ & 5.083394 & -1.275924 & 0.870955 \\
\hline $\mathrm{H}$ & 3.787294 & -1.167650 & 2.076135 \\
\hline $\mathrm{H}$ & 3.876746 & -2.571786 & 0.993968 \\
\hline C & 3.153624 & 1.270785 & -0.101380 \\
\hline $\mathrm{H}$ & 4.199398 & 1.567307 & -0.263574 \\
\hline $\mathrm{H}$ & 2.546593 & 1.757018 & -0.878881 \\
\hline $\mathrm{H}$ & 2.842279 & 1.666580 & 0.875553 \\
\hline $\mathrm{C}$ & 3.257183 & -1.180574 & -1.958284 \\
\hline $\mathrm{H}$ & 2.530807 & -0.723524 & -2.646184 \\
\hline $\mathrm{H}$ & 4.261454 & -0.903477 & -2.306261 \\
\hline $\mathrm{H}$ & 3.161296 & -2.271474 & -2.042183 \\
\hline
\end{tabular}




$$
G=-945.849121 \quad Z P E=-945.798144
$$

$\begin{array}{lccc}\mathrm{H} & 0.709181 & 2.009190 & 0.071798 \\ \mathrm{C} & 0.980272 & 0.965275 & 0.283062 \\ \mathrm{Ru} & -0.781443 & -0.272697 & -0.103636 \\ \mathrm{C} & 0.352191 & 0.321116 & 1.326727 \\ \mathrm{C} & -0.478633 & -2.410792 & 0.509691 \\ \mathrm{C} & -1.810241 & -2.102517 & 0.909117 \\ \mathrm{C} & -2.543357 & -1.785325 & -0.266510 \\ \mathrm{C} & -1.692482 & -1.929307 & -1.392209 \\ \mathrm{C} & -0.394776 & -2.299183 & -0.914305 \\ \mathrm{H} & 0.336908 & -2.696146 & 1.168144 \\ \mathrm{H} & -2.195055 & -2.096394 & 1.923110 \\ \mathrm{H} & -3.578475 & -1.459354 & -0.295861 \\ \mathrm{H} & -1.970432 & -1.770930 & -2.428190 \\ \mathrm{H} & 0.474528 & -2.521006 & -1.525006 \\ \mathrm{C} & -2.516734 & 2.460378 & -0.126556 \\ \mathrm{~N} & -1.892542 & 1.485605 & -0.099713 \\ \mathrm{C} & -3.297125 & 3.674239 & -0.156989 \\ \mathrm{H} & -3.078745 & 4.279667 & 0.729911 \\ \mathrm{H} & -3.055534 & 4.254008 & -1.054965 \\ \mathrm{H} & -4.365236 & 3.430112 & -0.165916 \\ \mathrm{H} & -0.274779 & 0.331139 & -1.506475 \\ \mathrm{Si} & 2.726459 & 0.496784 & -0.320714 \\ \mathrm{C} & 0.622491 & -0.014209 & 2.723523 \\ \mathrm{H} & 0.503790 & 0.909490 & 3.313079 \\ \mathrm{H} & -0.042633 & -0.778678 & 3.136868 \\ \mathrm{H} & 1.676107 & -0.313152 & 2.847049 \\ \mathrm{C} & 3.117888 & -1.269357 & 0.171259 \\ \mathrm{H} & 4.144812 & -1.518445 & -0.131626 \\ \mathrm{H} & 3.055861 & -1.425811 & 1.257391 \\ \mathrm{H} & 2.449747 & -1.990581 & -0.318432 \\ \mathrm{H} & 3.912169 & 1.688720 & 0.513805 \\ \mathrm{H} & 3.949412 & 1.491960 & 0.208422 \\ \mathrm{H} & 3.684849 & 1.590940 & 1.607754\end{array}$




$\begin{array}{llll}\mathrm{C} & 2.739262 & 0.696451 & -2.183354 \\ \mathrm{H} & 2.352187 & 1.680396 & -2.483022 \\ \mathrm{H} & 3.758751 & 0.602208 & -2.582905 \\ \mathrm{H} & 2.118316 & -0.069791 & -2.668131\end{array}$

A6atrans

$G=-945.84901 \quad Z P E=-945.796511$

$\begin{array}{llll}\text { C } & 0.325373 & 0.856549 & 1.103278\end{array}$

$\begin{array}{llll}\mathrm{Ru} & -0.687678 & -0.370416 & 0.044992\end{array}$

$\begin{array}{llll}\text { C } & 1.326276 & -0.052731 & 0.804210\end{array}$

$\begin{array}{llll}\text { C } & 0.215275 & 2.177391 & 1.718968\end{array}$

$\mathrm{H} \quad 1.537239 \quad-0.829651 \quad 1.552445$

$\mathrm{H} \quad-0.780500 \quad 2.623162 \quad 1.641406$

$\mathrm{H} \quad 0.962632 \quad 2.855246 \quad 1.275510$

$\begin{array}{llll}\mathrm{H} & 0.490117 & 2.086357 & 2.781502\end{array}$

C $\quad \begin{array}{llll}-2.308325 & -1.830604 & 0.736073\end{array}$

C $\quad-2.220221 \quad-1.886133 \quad-0.706902$

C $\quad-0.953077 \quad-2.397184 \quad-1.051865$

$\begin{array}{llll}\text { C } & -0.214225 & -2.567172 & 0.156769\end{array}$

$\begin{array}{llll}\text { C } & -1.083939 & -2.277333 & 1.264198\end{array}$

$\mathrm{H} \quad-3.006760 \quad-1.603855 \quad-1.398860$

$\mathrm{H} \quad-0.571461 \quad-2.549806 \quad-2.055536$

$\begin{array}{llll}\mathrm{H} & 0.801206 & -2.943077 & 0.228717\end{array}$

$\mathrm{H} \quad-0.813912 \quad-2.335740 \quad 2.313611$

$\mathrm{H} \quad-3.170380 \quad-1.494569 \quad 1.302791$

$\begin{array}{llll}\mathrm{N} & -2.019731 & 1.205969 & -0.252415\end{array}$

C $\quad \begin{array}{llll}-2.780209 & 2.051126 & -0.467728\end{array}$

$\begin{array}{llll}\text { Si } & 2.823568 & 0.366939 & -0.297076\end{array}$

C $\quad 2.424351 \quad 1.803451 \quad-1.431936$

$\mathrm{H} \quad 2.031380 \quad 2.668908 \quad-0.880313$

$\mathrm{H} \quad 3.338475 \quad 2.132861 \quad-1.946145$

$\mathrm{H} \quad 1.691193 \quad 1.530730 \quad-2.202543$

C $\quad 3.260595 \quad-1.175183 \quad-1.267758$

$\mathrm{H} \quad 4.169179 \quad-1.015069 \quad-1.864863$

$\mathrm{H} \quad 3.449746 \quad-2.028257 \quad-0.601231$

$\mathrm{H} \quad 2.453090 \quad-1.452869 \quad-1.959787$ 


$\begin{array}{lrrr}\mathrm{C} & 4.196897 & 0.840154 & 0.893246 \\ \mathrm{H} & 4.446809 & 0.013688 & 1.572583 \\ \mathrm{H} & 5.113184 & 1.115852 & 0.352632 \\ \mathrm{H} & 3.905180 & 1.702450 & 1.509990 \\ \mathrm{C} & -3.726454 & 3.106866 & -0.738035 \\ \mathrm{H} & -3.190667 & 4.025223 & -1.003218 \\ \mathrm{H} & -4.373994 & 2.820403 & -1.574332 \\ \mathrm{H} & -4.344661 & 3.295346 & 0.146636 \\ \mathrm{H} & -0.045021 & 0.206868 & -1.319818\end{array}$

\section{B1-TS}

$G=-945.838006 \quad Z P E=-945.78753$

$\begin{array}{lccc}\mathrm{H} & -1.310838 & 0.510531 & -1.924021 \\ \mathrm{C} & -1.182372 & 0.589631 & -0.833314 \\ \mathrm{Ru} & 0.665018 & -0.300013 & -0.047798 \\ \mathrm{C} & -0.126980 & 1.404939 & -0.445187 \\ \mathrm{C} & -0.258809 & -2.226747 & -0.670506 \\ \mathrm{C} & 1.043609 & -2.214017 & -1.250782 \\ \mathrm{C} & 1.968519 & -2.157851 & -0.178326 \\ \mathrm{C} & 1.262106 & -2.198397 & 1.062328 \\ \mathrm{C} & -0.124153 & -2.237510 & 0.756688 \\ \mathrm{H} & -1.196262 & -2.269610 & -1.215039 \\ \mathrm{H} & 1.281104 & -2.207498 & -2.308111 \\ \mathrm{H} & 3.045260 & -2.064769 & -0.283164 \\ \mathrm{H} & 1.704550 & -2.181390 & 2.051528 \\ \mathrm{H} & -0.939048 & -2.270864 & 1.472712 \\ \mathrm{C} & 3.487163 & 1.313095 & 0.160808 \\ \mathrm{~N} & 2.456139 & 0.793431 & 0.078424 \\ \mathrm{C} & 4.773380 & 1.959074 & 0.265036 \\ \mathrm{H} & 4.668150 & 3.029346 & 0.054347 \\ \mathrm{H} & 5.174226 & 1.833683 & 1.277058 \\ \mathrm{H} & 5.472994 & 1.521110 & -0.455701 \\ \mathrm{H} & 0.136904 & 0.724632 & 1.158233 \\ \mathrm{Si} & -2.822613 & 0.456350 & 0.119013 \\ \mathrm{C} & 0.181280 & 2.837696 & -0.421265 \\ \mathrm{H} & 1.154951 & 3.071336 & 0.018469\end{array}$




$\begin{array}{lrrr}\mathrm{H} & 0.150165 & 3.208508 & -1.456845 \\ \mathrm{H} & -0.607426 & 3.379374 & 0.123690 \\ \mathrm{C} & -2.568193 & 0.804871 & 1.942535 \\ \mathrm{H} & -3.544580 & 0.836770 & 2.447208 \\ \mathrm{H} & -1.961488 & 0.040506 & 2.445619 \\ \mathrm{H} & -2.087265 & 1.780166 & 2.105737 \\ \mathrm{C} & -3.615982 & -1.218147 & -0.168438 \\ \mathrm{H} & -4.650066 & -1.208585 & 0.204664 \\ \mathrm{H} & -3.659840 & -1.460232 & -1.239744 \\ \mathrm{H} & -3.090056 & -2.033456 & 0.346058 \\ \mathrm{C} & -3.892383 & 1.802456 & -0.641987 \\ \mathrm{H} & -4.060898 & 1.627092 & -1.713581 \\ \mathrm{H} & -4.876283 & 1.844987 & -0.153557 \\ \mathrm{H} & -3.424539 & 2.791476 & -0.533121\end{array}$

\section{B1}

$G=-945.89254 \quad Z P E=-945.841074$

$\begin{array}{lccc}\mathrm{H} & 1.548970 & -0.673058 & 1.811885 \\ \mathrm{C} & 1.296129 & 0.166309 & 1.153034 \\ \mathrm{Ru} & -0.494648 & -0.450367 & -0.088333 \\ \mathrm{C} & 0.284787 & 1.013940 & 1.575560 \\ \mathrm{C} & -0.833606 & -2.167201 & 1.120001 \\ \mathrm{C} & -2.040579 & -1.807696 & 0.435278 \\ \mathrm{C} & -1.817558 & -1.972645 & -0.975044 \\ \mathrm{C} & -0.482109 & -2.420459 & -1.144294 \\ \mathrm{C} & 0.137388 & -2.534383 & 0.134957 \\ \mathrm{H} & -0.683466 & -2.153158 & 2.194918 \\ \mathrm{H} & -2.959073 & -1.470525 & 0.906050 \\ \mathrm{H} & -2.531749 & -1.776752 & -1.767372 \\ \mathrm{H} & 0.016294 & -2.571497 & -2.097847 \\ \mathrm{H} & 1.157420 & -2.851925 & 0.325143 \\ \mathrm{C} & -2.520556 & 2.062329 & -0.590873 \\ \mathrm{~N} & -1.767125 & 1.203675 & -0.395866 \\ \mathrm{C} & -3.454086 & 3.135115 & -0.838175 \\ \mathrm{H} & -3.161583 & 4.026313 & -0.271844 \\ \mathrm{H} & -3.466888 & 3.378502 & -1.906535\end{array}$




$\begin{array}{lrrr}\mathrm{H} & -4.461476 & 2.831788 & -0.531382 \\ \mathrm{H} & 0.180981 & 1.984382 & 1.078727 \\ \mathrm{Si} & 2.447812 & 0.618266 & -0.269649 \\ \mathrm{C} & -0.466063 & 0.884409 & 2.863116 \\ \mathrm{H} & -1.541872 & 1.064748 & 2.726409 \\ \mathrm{H} & -0.332306 & -0.106190 & 3.315949 \\ \mathrm{H} & -0.109041 & 1.635125 & 3.584669 \\ \mathrm{C} & 1.314104 & 1.182648 & -1.676604 \\ \mathrm{H} & 1.889737 & 1.217809 & -2.613714 \\ \mathrm{H} & 0.467998 & 0.499195 & -1.912804 \\ \mathrm{H} & 0.894586 & 2.182658 & -1.502576 \\ \mathrm{C} & 3.432375 & -0.888507 & -0.794363 \\ \mathrm{H} & 4.257537 & -0.601843 & -1.461132 \\ \mathrm{H} & 3.873195 & -1.404231 & 0.070396 \\ \mathrm{H} & 2.808288 & -1.612958 & -1.335723 \\ \mathrm{C} & 3.577858 & 2.034305 & 0.215843 \\ \mathrm{H} & 4.253615 & 1.750256 & 1.033849 \\ \mathrm{H} & 4.198052 & 2.351223 & -0.634723 \\ \mathrm{H} & 2.997302 & 2.905854 & 0.549817\end{array}$

\section{C1-TS}

$G=-945.845047 \quad Z P E=-945.793035$

$\begin{array}{lccc}\mathrm{C} & 0.287748 & -0.427678 & 1.347263 \\ \mathrm{Ru} & -0.915904 & -0.180604 & -0.129370 \\ \mathrm{C} & 1.002052 & -1.114407 & 0.396552 \\ \mathrm{C} & 0.365318 & -0.014678 & 2.741405 \\ \mathrm{H} & 0.921461 & -2.212180 & 0.402706 \\ \mathrm{H} & -0.541339 & 0.484812 & 3.098965 \\ \mathrm{H} & 1.208425 & 0.696359 & 2.812310 \\ \mathrm{H} & 0.630963 & -0.861373 & 3.391410 \\ \mathrm{C} & -3.139369 & -0.035735 & 0.470176 \\ \mathrm{C} & -3.015273 & -0.027712 & -0.968489 \\ \mathrm{C} & -2.491853 & -1.276835 & -1.375495 \\ \mathrm{C} & -2.192239 & -2.024508 & -0.197096 \\ \mathrm{C} & -2.654949 & -1.272216 & 0.935465\end{array}$




$\begin{array}{lrrr}\mathrm{H} & -3.312949 & 0.784945 & -1.623236 \\ \mathrm{H} & -2.287171 & -1.586910 & -2.394290 \\ \mathrm{H} & -1.772439 & -3.025175 & -0.169230 \\ \mathrm{H} & -2.586997 & -1.586969 & 1.972532 \\ \mathrm{H} & -3.537334 & 0.770914 & 1.076940 \\ \mathrm{~N} & -0.447213 & 1.839789 & -0.155185 \\ \mathrm{C} & -0.217679 & 2.974550 & -0.171183 \\ \mathrm{Si} & 2.697375 & -0.564647 & -0.291482 \\ \mathrm{C} & 2.841956 & 1.302159 & -0.352719 \\ \mathrm{H} & 2.622288 & 1.774555 & 0.615023 \\ \mathrm{H} & 3.873153 & 1.573906 & -0.620924 \\ \mathrm{H} & 2.177390 & 1.733459 & -1.112854 \\ \mathrm{C} & 2.858426 & -1.317753 & -1.998109 \\ \mathrm{H} & 3.872122 & -1.175194 & -2.397799 \\ \mathrm{H} & 2.659644 & -2.398318 & -1.974574 \\ \mathrm{H} & 2.152046 & -0.861051 & -2.704820 \\ \mathrm{C} & 3.962469 & -1.284940 & 0.892995 \\ \mathrm{H} & 3.885647 & -2.379514 & 0.948228 \\ \mathrm{H} & 4.985732 & -1.039292 & 0.575536 \\ \mathrm{H} & 3.827201 & -0.887713 & 1.909271 \\ \mathrm{C} & 0.107986 & 4.380773 & -0.199270 \\ \mathrm{H} & 1.190525 & 4.502049 & -0.327758 \\ \mathrm{H} & -0.404876 & 4.868544 & -1.035703 \\ \mathrm{H} & -0.197802 & 4.862202 & 0.736231 \\ & 0.013773 & -0.231069 & -1.447606\end{array}$

\section{C1}

$G=-945.845672 \quad Z P E=-945.795032$

$\begin{array}{lccc}\mathrm{C} & -0.011232 & -1.218024 & 0.945548 \\ \mathrm{Ru} & -0.990353 & -0.012484 & -0.177354 \\ \mathrm{C} & 0.763221 & -1.314796 & -0.190630 \\ \mathrm{C} & -0.174115 & -1.907189 & 2.223836 \\ \mathrm{H} & 0.652248 & -2.248854 & -0.763529 \\ \mathrm{H} & -1.063746 & -1.600908 & 2.783237 \\ \mathrm{H} & 0.717579 & -1.690171 & 2.834556 \\ \mathrm{H} & -0.174984 & -2.997118 & 2.066864\end{array}$




$\begin{array}{lccc}\mathrm{C} & -3.051118 & 0.042679 & 0.903408 \\ \mathrm{C} & -3.059247 & 1.047702 & -0.105425 \\ \mathrm{C} & -2.897604 & 0.431553 & -1.370864 \\ \mathrm{C} & -2.727045 & -0.973250 & -1.154824 \\ \mathrm{C} & -2.839517 & -1.205439 & 0.254416 \\ \mathrm{H} & -3.152863 & 2.115215 & 0.069393 \\ \mathrm{H} & -2.875555 & 0.937198 & -2.329676 \\ \mathrm{H} & -2.610512 & -1.731500 & -1.921592 \\ \mathrm{H} & -2.783171 & -2.176793 & 0.736447 \\ \mathrm{H} & -3.175359 & 0.204210 & 1.968693 \\ \mathrm{~N} & 0.169739 & 1.679028 & 0.211533 \\ \mathrm{C} & 0.778514 & 2.640484 & 0.425516 \\ \mathrm{Si} & 2.549676 & -0.643583 & -0.342903 \\ \mathrm{C} & 3.076544 & 0.136563 & 1.283603 \\ \mathrm{H} & 3.308999 & -0.643692 & 2.022384 \\ \mathrm{H} & 3.993081 & 0.727396 & 1.141351 \\ \mathrm{H} & 2.318147 & 0.795740 & 1.724727 \\ \mathrm{C} & 2.734695 & 0.520329 & -1.800191 \\ \mathrm{H} & 3.801173 & 0.728247 & -1.971023 \\ \mathrm{H} & 2.338819 & 0.061328 & -2.716870 \\ \mathrm{H} & 2.223533 & 1.480634 & -1.658409 \\ \mathrm{C} & 3.583970 & -2.176517 & -0.665727 \\ \mathrm{H} & 3.316716 & -2.643247 & -1.624082 \\ \mathrm{H} & 4.652482 & -1.921906 & -0.708553 \\ \mathrm{H} & 3.454501 & -2.930163 & 0.123367 \\ \mathrm{H} & 1.590654 & 3.802340 & 0.695189 \\ & 2.643386 & 3.560514 & 0.501863 \\ \mathrm{H} & 1.290978 & 4.633454 & 0.047259 \\ \mathrm{H} & -0.269846 & 0.081310 & -1.608136\end{array}$

\section{C2-TS1}

$G=-945.845488 \quad Z P E=-945.794298$

$\begin{array}{llll}\mathrm{H} & 0.679751 & -2.183461 & -0.899186 \\ \mathrm{C} & 0.786984 & -1.276303 & -0.285932 \\ \mathrm{Ru} & -1.013768 & -0.022718 & -0.138173\end{array}$ 


\begin{tabular}{|c|c|c|c|}
\hline $\mathrm{C}$ & 0.031794 & -1.233117 & 504 \\
\hline $\mathrm{C}$ & -2.874081 & -1.207661 & 0.294402 \\
\hline$C$ & -3.072294 & 0.064184 & 0.888332 \\
\hline $\mathrm{C}$ & -3.057738 & 1.028407 & -0.168790 \\
\hline $\mathrm{C}$ & -2.911454 & 0.360397 & -1.406992 \\
\hline $\mathrm{C}$ & -2.738551 & -1.029389 & -1.122404 \\
\hline $\mathrm{H}$ & -2.823817 & -2.158723 & 0.815686 \\
\hline $\mathrm{H}$ & -3.209737 & 0.274294 & 1.943305 \\
\hline $\mathrm{H}$ & -3.141543 & 2.103062 & -0.035806 \\
\hline $\mathrm{H}$ & -2.876136 & 0.822898 & -2.386803 \\
\hline $\mathrm{H}$ & -2.604422 & -1.820536 & -1.852906 \\
\hline $\mathrm{C}$ & 0.710446 & 2.673306 & 0.424366 \\
\hline $\mathrm{N}$ & 0.107220 & 1.703928 & 0.229113 \\
\hline C & 1.511917 & 3.847420 & 0.672327 \\
\hline $\mathrm{H}$ & 1.311604 & 4.238286 & 1.676267 \\
\hline $\mathrm{H}$ & 2.574046 & 3.582134 & 0.597210 \\
\hline $\mathrm{H}$ & 1.285482 & 4.623869 & -0.066591 \\
\hline $\mathrm{H}$ & -0.003210 & -0.142871 & -1.420327 \\
\hline $\mathrm{Si}$ & 2.589557 & -0.609878 & -0.354466 \\
\hline $\mathrm{C}$ & -0.034266 & -1.990668 & 2.134367 \\
\hline $\mathrm{H}$ & -0.875889 & -1.704452 & 2.773744 \\
\hline $\mathrm{H}$ & -0.070712 & -3.070451 & 1.919744 \\
\hline $\mathrm{H}$ & 0.903394 & -1.823037 & 2.688933 \\
\hline $\mathrm{C}$ & 3.617274 & -2.153531 & -0.632241 \\
\hline $\mathrm{H}$ & 4.690185 & -1.915436 & -0.619794 \\
\hline $\mathrm{H}$ & 3.435307 & -2.901773 & 0.152133 \\
\hline $\mathrm{H}$ & 3.392203 & -2.619600 & -1.601371 \\
\hline $\mathrm{C}$ & 3.043456 & 0.162605 & 1.295628 \\
\hline $\mathrm{H}$ & 3.956396 & 0.766133 & 1.189272 \\
\hline $\mathrm{H}$ & 2.260412 & 0.809426 & 1.711291 \\
\hline $\mathrm{H}$ & 3.259808 & -0.618241 & 2.038431 \\
\hline $\mathrm{C}$ & 2.801058 & 0.571564 & -1.791431 \\
\hline $\mathrm{H}$ & 2.246378 & 1.509136 & -1.656774 \\
\hline $\mathrm{H}$ & 3.863826 & 0.824556 & -1.915079 \\
\hline $\mathrm{H}$ & 2.460852 & 0.111905 & -2.72985 \\
\hline
\end{tabular}




\section{C2-TS2}

$$
G=-945.845558 \quad Z P E=-945.794929
$$

$\begin{array}{lccc}\mathrm{H} & 0.056344 & 0.551209 & -1.180318 \\ \mathrm{C} & 0.995573 & 0.982266 & 0.209500 \\ \mathrm{Ru} & -0.789828 & -0.280326 & -0.044835 \\ \mathrm{C} & 0.363468 & 0.380861 & 1.302593 \\ \mathrm{C} & -0.522750 & -2.424705 & 0.582936 \\ \mathrm{C} & -1.872849 & -2.098515 & 0.871663 \\ \mathrm{C} & -2.501917 & -1.784222 & -0.372819 \\ \mathrm{C} & -1.577608 & -1.978756 & -1.428806 \\ \mathrm{C} & -0.328332 & -2.327082 & -0.835208 \\ \mathrm{H} & 0.238552 & -2.703266 & 1.306061 \\ \mathrm{H} & -2.343131 & -2.080305 & 1.848562 \\ \mathrm{H} & -3.528275 & -1.447014 & -0.485729 \\ \mathrm{H} & -1.768574 & -1.835759 & -2.486255 \\ \mathrm{H} & 0.590308 & -2.550262 & -1.368952 \\ \mathrm{C} & -2.631240 & 2.383706 & -0.184944 \\ \mathrm{~N} & -1.965724 & 1.438038 & -0.121298 \\ \mathrm{C} & -3.463459 & 3.560451 & -0.263529 \\ \mathrm{H} & -4.295083 & 3.481324 & 0.445766 \\ \mathrm{H} & -2.872090 & 4.450431 & -0.020391 \\ \mathrm{H} & -3.864878 & 3.667113 & -1.277520 \\ \mathrm{H} & 0.774243 & 2.048758 & 0.048391 \\ \mathrm{Si} & 2.761562 & 0.500098 & -0.350188 \\ \mathrm{C} & 0.664169 & 0.170505 & 2.718347 \\ \mathrm{H} & -0.013780 & -0.539894 & 3.203381 \\ \mathrm{H} & 1.709717 & -0.152736 & 2.848616 \\ \mathrm{H} & 0.581744 & 1.141490 & 3.233267 \\ \mathrm{C} & 3.170079 & -1.231938 & 0.237624 \\ \mathrm{H} & 4.215358 & -1.465382 & -0.009894 \\ \mathrm{H} & 3.064014 & -1.338799 & 1.326259 \\ \mathrm{H} & 2.544639 & -1.994969 & -0.244146 \\ \mathrm{H} & 3.890455 & 1.758904 & 0.460945 \\ \mathrm{H} & 3.941430 & 1.574255 & 0.198740 \\ \mathrm{H} & 2.643192 & 2.782735 & 0.148919 \\ \mathrm{H} & 1.714713 & 1.556546\end{array}$




$\begin{array}{llll}\mathrm{C} & 2.802438 & 0.615827 & -2.218517 \\ \mathrm{H} & 2.472495 & 1.605276 & -2.565001 \\ \mathrm{H} & 3.818753 & 0.450796 & -2.602170 \\ \mathrm{H} & 2.146458 & -0.136798 & -2.678383\end{array}$

\section{C2}

$G=-945.86764 \quad Z P E=-945.818036$

$\begin{array}{lccc}\mathrm{H} & -1.515177 & -2.021298 & 1.045214 \\ \mathrm{C} & -1.289548 & -1.011152 & 0.658905 \\ \mathrm{Ru} & 1.181881 & -0.207137 & 0.128397 \\ \mathrm{C} & -0.366825 & -1.082569 & -0.483070 \\ \mathrm{C} & 2.657928 & -1.437746 & -0.837619 \\ \mathrm{C} & 3.079198 & -0.065706 & -0.843492 \\ \mathrm{C} & 3.446052 & 0.297344 & 0.511337 \\ \mathrm{C} & 3.118839 & -0.770126 & 1.338725 \\ \mathrm{C} & 2.588760 & -1.844622 & 0.524171 \\ \mathrm{H} & 2.410575 & -2.032600 & -1.710461 \\ \mathrm{H} & 3.234832 & 0.546448 & -1.726400 \\ \mathrm{H} & 3.794746 & 1.273704 & 0.831347 \\ \mathrm{H} & 3.176836 & -0.779798 & 2.422732 \\ \mathrm{H} & 2.307051 & -2.826537 & 0.890113 \\ \mathrm{C} & 0.286910 & 2.893124 & -0.287370 \\ \mathrm{~N} & 0.558675 & 1.774811 & -0.151465 \\ \mathrm{C} & -0.082987 & 4.277298 & -0.460341 \\ \mathrm{H} & 0.776079 & 4.852808 & -0.823280 \\ \mathrm{H} & -0.898377 & 4.353003 & -1.189603 \\ \mathrm{H} & -0.416062 & 4.698737 & 0.495055 \\ \mathrm{H} & -0.886810 & -0.398284 & 1.500261 \\ \mathrm{Si} & -3.023814 & -0.227985 & 0.277675 \\ \mathrm{C} & -0.722998 & -1.883118 & -1.671909 \\ \mathrm{H} & 0.154781 & -2.189769 & -2.250904 \\ \mathrm{H} & -1.332851 & -2.764788 & -1.419920 \\ \mathrm{H} & -1.332188 & -1.251438 & -2.341082 \\ \mathrm{C} & -4.012143 & -1.386757 & -0.815579 \\ \mathrm{H} & -5.057153 & -1.045501 & -0.845896 \\ \mathrm{H} & -3.647562 & -1.414326 & -1.850405\end{array}$




$\begin{array}{lccc}\mathrm{H} & -4.013361 & -2.414084 & -0.425967 \\ \mathrm{C} & -2.816384 & 1.447241 & -0.536710 \\ \mathrm{H} & -3.801813 & 1.842754 & -0.821908 \\ \mathrm{H} & -2.359006 & 2.166801 & 0.155882 \\ \mathrm{H} & -2.201251 & 1.398778 & -1.446030 \\ \mathrm{C} & -3.850200 & -0.057898 & 1.950203 \\ \mathrm{H} & -3.276096 & 0.596232 & 2.620679 \\ \mathrm{H} & -4.854791 & 0.374261 & 1.841061 \\ \mathrm{H} & -3.960439 & -1.033985 & 2.442384\end{array}$

\section{C3}

$G=-1078.519247 \quad Z P E=-1078.462065$

$\begin{array}{lccc}\mathrm{H} & -1.212902 & 1.465878 & -0.836076 \\ \mathrm{C} & -1.448572 & 0.484695 & -1.265853 \\ \mathrm{Ru} & 1.106871 & -0.317074 & 0.110142 \\ \mathrm{C} & -0.387379 & -0.500393 & -1.129319 \\ \mathrm{C} & 1.488286 & -2.476295 & 0.259727 \\ \mathrm{C} & 2.626941 & -1.873717 & -0.346937 \\ \mathrm{C} & 3.269090 & -1.059928 & 0.659552 \\ \mathrm{C} & 2.508380 & -1.103328 & 1.827571 \\ \mathrm{C} & 1.354119 & -1.937885 & 1.572886 \\ \mathrm{H} & 0.819513 & -3.195205 & -0.201593 \\ \mathrm{H} & 3.018762 & -2.086742 & -1.336507 \\ \mathrm{H} & 4.155583 & -0.453097 & 0.499399 \\ \mathrm{H} & 2.701788 & -0.550202 & 2.740919 \\ \mathrm{H} & 0.593616 & -2.207236 & 2.299594 \\ \mathrm{C} & 2.115797 & 2.278835 & -1.557984 \\ \mathrm{~N} & 1.731424 & 1.356406 & -0.971409 \\ \mathrm{C} & 2.601980 & 3.423660 & -2.293149 \\ \mathrm{H} & 3.629056 & 3.242053 & -2.629634 \\ \mathrm{H} & 1.968786 & 3.603700 & -3.169092 \\ \mathrm{H} & 2.589832 & 4.314559 & -1.655314 \\ \mathrm{H} & -1.795404 & 0.602520 & -2.306296 \\ \mathrm{Si} & -3.059979 & -0.123116 & -0.352631 \\ \mathrm{C} & -0.548290 & -1.693626 & -2.021823\end{array}$




$\begin{array}{lccc}\mathrm{H} & 0.412103 & -2.103820 & -2.354586 \\ \mathrm{H} & -1.053208 & -2.499992 & -1.463101 \\ \mathrm{H} & -1.167868 & -1.476959 & -2.905136 \\ \mathrm{C} & -2.684307 & -1.215999 & 1.124936 \\ \mathrm{H} & -3.632538 & -1.540392 & 1.578243 \\ \mathrm{H} & -2.131711 & -2.122872 & 0.840938 \\ \mathrm{H} & -2.106397 & -0.693825 & 1.898603 \\ \mathrm{C} & -4.105946 & -1.078378 & -1.581992 \\ \mathrm{H} & -5.077641 & -1.329012 & -1.132602 \\ \mathrm{H} & -4.301045 & -0.491914 & -2.490347 \\ \mathrm{H} & -3.631897 & -2.022299 & -1.883638 \\ \mathrm{C} & -3.934406 & 1.445133 & 0.188781 \\ \mathrm{H} & -4.121000 & 2.113446 & -0.663060 \\ \mathrm{H} & -4.904116 & 1.212596 & 0.650736 \\ \mathrm{H} & -3.336435 & 1.999328 & 0.926466 \\ \mathrm{C} & -0.522597 & 1.722478 & 2.024854 \\ \mathrm{~N} & 0.012725 & 1.005911 & 1.286483 \\ \mathrm{C} & -1.208922 & 2.602292 & 2.944064 \\ \mathrm{H} & -0.538346 & 2.893059 & 3.760792 \\ \mathrm{H} & -1.542849 & 3.506434 & 2.422163 \\ \mathrm{H} & -2.081990 & 2.091415 & 3.367940\end{array}$

\section{C3-TSa}

$$
G=-1078.492234 \quad Z P E=-1078.435686
$$

$\begin{array}{llll}\mathrm{H} & 1.865795 & -3.165477 & -0.022791 \\ \mathrm{C} & 1.382700 & -2.253863 & -0.398720 \\ \mathrm{Ru} & -1.004998 & -0.260474 & -0.204174 \\ \mathrm{C} & 0.783629 & -1.340248 & 0.435962 \\ \mathrm{C} & -1.887139 & -2.243974 & -0.412654 \\ \mathrm{C} & -2.821997 & -1.394417 & 0.235678 \\ \mathrm{C} & -3.170799 & -0.329688 & -0.662434 \\ \mathrm{C} & -2.414930 & -0.526487 & -1.854534 \\ \mathrm{C} & -1.620150 & -1.706348 & -1.719301 \\ \mathrm{H} & -1.437800 & -3.135992 & 0.014199 \\ \mathrm{H} & -3.197606 & -1.522497 & 1.246727 \\ \mathrm{H} & -3.879260 & 0.469050 & -0.471308\end{array}$




\begin{tabular}{|c|c|c|c|}
\hline $\mathrm{H}$ & -2.413825 & 0.134450 & -2.716806 \\
\hline $\mathrm{H}$ & -0.980105 & -2.140444 & -2.480105 \\
\hline C & -1.028149 & 1.359416 & 2.628804 \\
\hline $\mathrm{N}$ & -0.960722 & 0.781373 & 1.625812 \\
\hline $\mathrm{C}$ & -1.097967 & 2.080915 & 3.879584 \\
\hline $\mathrm{H}$ & -1.313576 & 1.389603 & 4.702046 \\
\hline $\mathrm{H}$ & -0.141991 & 2.579708 & 4.077323 \\
\hline $\mathrm{H}$ & -1.890006 & 2.837332 & 3.835990 \\
\hline $\mathrm{H}$ & 1.376414 & -2.144466 & -1.485931 \\
\hline Si & 2.437974 & -0.218790 & -0.110532 \\
\hline $\mathrm{C}$ & 0.853048 & -1.685492 & 1.910453 \\
\hline $\mathrm{H}$ & 1.023708 & -0.808522 & 2.546616 \\
\hline $\mathrm{H}$ & -0.116346 & -2.105057 & 2.218195 \\
\hline 1 & 1.625566 & -2.437363 & 2.137641 \\
\hline C & 2.510344 & 0.187414 & -1.940627 \\
\hline $\mathrm{H}$ & 3.227340 & -0.482047 & -2.435703 \\
\hline $\mathrm{H}$ & 1.544791 & 0.095471 & -2.451108 \\
\hline $\mathrm{H}$ & 2.867741 & 1.218391 & -2.078884 \\
\hline $\mathrm{C}$ & 4.037685 & -1.075030 & 0.395250 \\
\hline $\mathrm{H}$ & 4.864547 & -0.349262 & 0.368979 \\
\hline $\mathrm{H}$ & 3.984431 & -1.465788 & 1.421459 \\
\hline $\mathrm{H}$ & 4.299511 & -1.907345 & -0.271995 \\
\hline $\mathrm{C}$ & 2.352546 & 1.280681 & 1.019880 \\
\hline $\mathrm{H}$ & 2.502489 & 0.988445 & 2.068180 \\
\hline $\mathrm{H}$ & 3.197509 & 1.930833 & 0.741678 \\
\hline $\mathrm{H}$ & 1.426723 & 1.856771 & 0.942751 \\
\hline $\mathrm{C}$ & 0.179950 & 2.486091 & -1.506369 \\
\hline $\mathrm{N}$ & -0.181111 & 1.495346 & -1.023515 \\
\hline $\mathrm{C}$ & 0.683616 & 3.703529 & -2.099568 \\
\hline $\mathrm{H}$ & 1.574378 & 4.037799 & -1.553249 \\
\hline $\mathrm{H}$ & 0.955768 & 3.526037 & -3.146642 \\
\hline $\mathrm{H}$ & -0.075183 & 4.493364 & -2.05890 \\
\hline
\end{tabular}

\section{C3-TSb}

$G=-1078.464565 \quad Z P E=-1078.407191$

$\begin{array}{llll}\mathrm{H} & 0.631668 & 0.803071 & 1.373190\end{array}$ 


\begin{tabular}{|c|c|c|c|}
\hline $\mathrm{C}$ & 1.588112 & -0.284815 & 1.663455 \\
\hline $\mathrm{Ru}$ & -0.998934 & -0.390874 & -0.230625 \\
\hline C & 0.193734 & -0.305215 & 1.534731 \\
\hline C & -0.694298 & -2.546170 & -0.249608 \\
\hline $\mathrm{C}$ & -2.049293 & -2.258703 & 0.116334 \\
\hline $\mathrm{C}$ & -2.665267 & -1.618446 & -1.015853 \\
\hline C & -1.707369 & -1.494659 & -2.046359 \\
\hline C & -0.468625 & -2.047559 & -1.562085 \\
\hline $\mathrm{H}$ & 0.050043 & -3.024776 & 0.381724 \\
\hline $\mathrm{H}$ & -2.543913 & -2.539756 & 1.040310 \\
\hline $\mathrm{H}$ & -3.687073 & -1.252229 & -1.051217 \\
\hline $\mathrm{H}$ & -1.863789 & -1.033962 & -3.016223 \\
\hline $\mathrm{H}$ & 0.461521 & -2.109061 & -2.117544 \\
\hline $\mathrm{C}$ & -2.923924 & 1.941354 & 1.010893 \\
\hline $\mathrm{N}$ & -2.204647 & 1.129764 & 0.599135 \\
\hline $\mathrm{C}$ & -3.826335 & 2.949457 & 1.519963 \\
\hline $\mathrm{H}$ & -3.261501 & 3.747258 & 2.014938 \\
\hline $\mathrm{H}$ & -4.407055 & 3.384338 & 0.698568 \\
\hline $\mathrm{H}$ & -4.517579 & 2.504049 & 2.244299 \\
\hline $\mathrm{H}$ & 1.954417 & -0.412702 & 2.700136 \\
\hline $\mathrm{Si}$ & 3.069005 & -0.036378 & 0.470208 \\
\hline $\mathrm{C}$ & -0.570585 & -0.556421 & 2.810926 \\
\hline $\mathrm{H}$ & -1.522700 & -0.015633 & 2.844116 \\
\hline $\mathrm{H}$ & -0.811222 & -1.628690 & 2.850993 \\
\hline $\mathrm{H}$ & 0.007446 & -0.317215 & 3.717262 \\
\hline $\mathrm{C}$ & 3.432074 & 1.805725 & 0.408552 \\
\hline $\mathrm{H}$ & 4.236875 & 2.019730 & -0.308984 \\
\hline $\mathrm{H}$ & 2.552938 & 2.397779 & 0.120568 \\
\hline $\mathrm{H}$ & 3.763212 & 2.166794 & 1.392220 \\
\hline $\mathrm{C}$ & 2.849539 & -0.787071 & -1.229295 \\
\hline $\mathrm{H}$ & 3.790713 & -0.667791 & -1.786288 \\
\hline $\mathrm{H}$ & 2.647188 & -1.864808 & -1.158764 \\
\hline $\mathrm{H}$ & 2.046432 & -0.321119 & -1.811614 \\
\hline $\mathrm{C}$ & 4.454504 & -0.936012 & 1.359692 \\
\hline $\mathrm{H}$ & 4.219086 & -1.999170 & 1.506795 \\
\hline $\mathrm{H}$ & 5.385226 & -0.883037 & 0.777981 \\
\hline $\mathrm{H}$ & 4.653726 & -0.497212 & 2.347107 \\
\hline$C$ & 0.623521 & 2.010606 & -1.732356 \\
\hline
\end{tabular}




$\begin{array}{llll}\mathrm{N} & 0.066418 & 1.185124 & -1.135892 \\ \mathrm{C} & 1.352995 & 3.004968 & -2.486661 \\ \mathrm{H} & 0.811165 & 3.257575 & -3.405324 \\ \mathrm{H} & 1.483246 & 3.916090 & -1.891658 \\ \mathrm{H} & 2.341894 & 2.611774 & -2.754648\end{array}$

\section{C3-TS2b}

$G=-945.819597 \quad Z P E=-945.766902$

$\begin{array}{lccc}\mathrm{H} & 0.848080 & 0.854253 & -0.978632 \\ \mathrm{C} & 1.582139 & 0.303495 & 0.125245 \\ \mathrm{Ru} & -1.314442 & -0.216414 & 0.102364 \\ \mathrm{C} & 0.623100 & -0.316656 & -0.685841 \\ \mathrm{C} & -2.208005 & -1.881076 & -0.875317 \\ \mathrm{C} & -3.207428 & -0.871504 & -0.628980 \\ \mathrm{C} & -3.383879 & -0.747521 & 0.785460 \\ \mathrm{C} & -2.430313 & -1.593737 & 1.401749 \\ \mathrm{C} & -1.717576 & -2.322115 & 0.387063 \\ \mathrm{H} & -1.888002 & -2.231735 & -1.851524 \\ \mathrm{H} & -3.762052 & -0.333976 & -1.392075 \\ \mathrm{H} & -4.052501 & -0.060005 & 1.291937 \\ \mathrm{H} & -2.247824 & -1.666115 & 2.470014 \\ \mathrm{H} & -0.964169 & -3.083294 & 0.561364 \\ \mathrm{C} & -1.575016 & 3.045684 & -0.119906 \\ \mathrm{~N} & -1.438638 & 1.895849 & -0.044653 \\ \mathrm{C} & -1.765708 & 4.473031 & -0.219300 \\ \mathrm{H} & -2.568643 & 4.795122 & 0.453479 \\ \mathrm{H} & -2.030545 & 4.742377 & -1.248276 \\ \mathrm{H} & -0.842093 & 4.995285 & 0.054634 \\ \mathrm{H} & 1.181640 & 1.059811 & 0.821241 \\ \mathrm{Si} & 3.487921 & 0.021217 & 0.229642 \\ \mathrm{C} & 1.057395 & -1.311955 & -1.722084 \\ \mathrm{H} & 0.399283 & -1.290536 & -2.598262 \\ \mathrm{H} & 0.979156 & -2.318638 & -1.286945 \\ \mathrm{H} & 2.093279 & -1.178601 & -2.067789 \\ \mathrm{C} & 4.251965 & 0.798497 & -1.295880 \\ \mathrm{H} & 5.342029 & 0.659995 & -1.282912 \\ & & & \\ \mathrm{H} & & \\ \mathrm{H} & & \\ \mathrm{H} & & \end{array}$




$\begin{array}{llll}\mathrm{H} & 4.055653 & 1.878553 & -1.330217 \\ \mathrm{H} & 3.872640 & 0.356256 & -2.226582 \\ \mathrm{C} & 4.024403 & 0.918466 & 1.782550 \\ \mathrm{H} & 5.107030 & 0.815621 & 1.938419 \\ \mathrm{H} & 3.524035 & 0.519712 & 2.675475 \\ \mathrm{H} & 3.799321 & 1.992214 & 1.722386 \\ \mathrm{C} & 3.830278 & -1.817489 & 0.350808 \\ \mathrm{H} & 3.150672 & -2.308911 & 1.060378 \\ \mathrm{H} & 4.855532 & -1.980817 & 0.711546 \\ \mathrm{H} & 3.734376 & -2.325044 & -0.617497\end{array}$

\section{C3-TS2a}

$G=-945.846727 \quad Z P E=-945.798106$

$\begin{array}{lccc}\mathrm{H} & 2.153172 & -2.028669 & 2.110994 \\ \mathrm{C} & 1.536249 & -1.734160 & 1.252821 \\ \mathrm{Ru} & -0.760214 & -0.255366 & -0.173435 \\ \mathrm{C} & 0.890351 & -0.528678 & 1.184447 \\ \mathrm{C} & -1.791936 & -1.751695 & 0.987074 \\ \mathrm{C} & -2.525597 & -0.517773 & 0.983501 \\ \mathrm{C} & -2.954311 & -0.271111 & -0.372308 \\ \mathrm{C} & -2.430722 & -1.299784 & -1.183814 \\ \mathrm{C} & -1.696725 & -2.221252 & -0.350637 \\ \mathrm{H} & -1.338142 & -2.213781 & 1.859077 \\ \mathrm{H} & -2.778578 & 0.076840 & 1.855983 \\ \mathrm{H} & -3.529457 & 0.584096 & -0.712253 \\ \mathrm{H} & -2.526128 & -1.367462 & -2.263379 \\ \mathrm{H} & -1.197096 & -3.125724 & -0.683407 \\ \mathrm{C} & -0.677447 & 2.994751 & -0.110775 \\ \mathrm{~N} & -0.584071 & 1.838232 & -0.128589 \\ \mathrm{C} & -0.775551 & 4.435869 & -0.093548 \\ \mathrm{H} & -1.355475 & 4.762976 & 0.776932 \\ \mathrm{H} & 0.225454 & 4.879361 & -0.039954 \\ \mathrm{H} & -1.272542 & 4.789805 & -1.004005 \\ \mathrm{H} & 1.438466 & -2.491428 & 0.468370 \\ \mathrm{Si} & 2.180730 & -0.219640 & -0.384686 \\ \mathrm{C} & 0.982743 & 0.377377 & 2.383376\end{array}$




$\begin{array}{lccc}\mathrm{H} & 0.983010 & 1.440459 & 2.117835 \\ \mathrm{H} & 0.086051 & 0.206795 & 2.998821 \\ \mathrm{H} & 1.860499 & 0.167108 & 3.014533 \\ \mathrm{C} & 3.780946 & -1.197760 & -0.542797 \\ \mathrm{H} & 4.358117 & -0.781467 & -1.383138 \\ \mathrm{H} & 4.402116 & -1.109704 & 0.359087 \\ \mathrm{H} & 3.619054 & -2.264198 & -0.745300 \\ \mathrm{C} & 2.738967 & 1.537579 & -0.073292 \\ \mathrm{H} & 3.439914 & 1.794012 & -0.881871 \\ \mathrm{H} & 1.937991 & 2.282633 & -0.062298 \\ \mathrm{H} & 3.296882 & 1.603895 & 0.870535 \\ \mathrm{C} & 1.244332 & -0.505896 & -1.992455 \\ \mathrm{H} & 0.178824 & -0.216437 & -2.133332 \\ \mathrm{H} & 1.768409 & 0.107502 & -2.742655 \\ \mathrm{H} & 1.329365 & -1.557988 & -2.293289\end{array}$

\section{C4-TSa}

$$
G=-1078.474418 \quad Z P E=-1078.41577
$$

$\begin{array}{lccc}\mathrm{H} & -1.282005 & 0.302078 & 1.514220 \\ \mathrm{C} & -0.960313 & 1.240944 & 1.046249 \\ \mathrm{Ru} & 1.503636 & -0.098704 & -0.397430 \\ \mathrm{C} & 0.294143 & 1.142065 & 0.364288 \\ \mathrm{C} & 0.438802 & -1.113160 & -2.060507 \\ \mathrm{C} & 1.820665 & -1.441352 & -2.290264 \\ \mathrm{C} & 2.279627 & -2.146605 & -1.173825 \\ \mathrm{C} & 1.178146 & -2.323388 & -0.252131 \\ \mathrm{C} & 0.030985 & -1.737130 & -0.835755 \\ \mathrm{H} & -0.216784 & -0.599501 & -2.756484 \\ \mathrm{H} & 2.415383 & -1.132095 & -3.141978 \\ \mathrm{H} & 3.297142 & -2.483809 & -1.006862 \\ \mathrm{H} & 1.217904 & -2.863741 & 0.686590 \\ \mathrm{H} & -0.964694 & -1.708955 & -0.396675 \\ \mathrm{C} & 3.858481 & 0.274401 & 1.804465 \\ \mathrm{~N} & 2.996567 & 0.165118 & 1.038193 \\ \mathrm{C} & 4.939637 & 0.405395 & 2.760074 \\ \mathrm{H} & 5.307570 & -0.585588 & 3.047940\end{array}$




$\begin{array}{lrcc}\mathrm{H} & 5.767794 & 0.973839 & 2.322689 \\ \mathrm{H} & 4.590420 & 0.923500 & 3.659924 \\ \mathrm{H} & -0.934961 & 2.052601 & 1.793185 \\ \mathrm{Si} & -2.413925 & 1.858247 & -0.108974 \\ \mathrm{C} & 1.147422 & 2.045979 & -0.346145 \\ \mathrm{H} & 2.169363 & 1.255979 & -1.110296 \\ \mathrm{H} & 0.761705 & 2.606727 & -1.203690 \\ \mathrm{H} & 1.880579 & 2.623639 & 0.227828 \\ \mathrm{C} & -2.471175 & 0.789221 & -1.645322 \\ \mathrm{H} & -3.320715 & 1.073609 & -2.279582 \\ \mathrm{H} & -1.561617 & 0.908774 & -2.249519 \\ \mathrm{H} & -2.587611 & -0.269035 & -1.374868 \\ \mathrm{C} & -2.099376 & 3.661026 & -0.528498 \\ \mathrm{H} & -2.932062 & 4.053109 & -1.127413 \\ \mathrm{H} & -2.035223 & 4.276003 & 0.378795 \\ \mathrm{H} & -1.182590 & 3.822532 & -1.109459 \\ \mathrm{C} & -3.945628 & 1.690301 & 0.953132 \\ \mathrm{H} & -3.869247 & 2.298862 & 1.863354 \\ \mathrm{H} & -4.834593 & 2.026322 & 0.403617 \\ \mathrm{H} & -4.102088 & 0.645612 & 1.247099 \\ \mathrm{C} & -3.888520 & -2.459565 & 1.092490 \\ \mathrm{~N} & -3.044052 & -1.720179 & 0.796801 \\ \mathrm{C} & -4.946918 & -3.381380 & 1.462316 \\ \mathrm{H} & -4.681105 & -4.402674 & 1.169324 \\ \mathrm{H} & -5.109819 & -3.358971 & 2.545063 \\ & -5.881270 & -3.106404 & 0.961390\end{array}$

\section{C4-TS2a}

$$
G=-945.842063 \quad Z P E=-945.792759
$$

$\begin{array}{llcc}\mathrm{H} & 1.422689 & 1.078205 & -1.769321 \\ \mathrm{C} & 1.549935 & 1.317084 & -0.704610 \\ \mathrm{Ru} & -1.024196 & -0.275360 & 0.274315 \\ \mathrm{C} & 0.436405 & 0.957200 & 0.115868 \\ \mathrm{C} & -0.063610 & -2.291599 & 0.347857 \\ \mathrm{C} & -1.402194 & -2.498219 & 0.815696 \\ \mathrm{C} & -2.282228 & -2.143568 & -0.221545\end{array}$




$\begin{array}{lccc}\mathrm{C} & -1.498767 & -1.771605 & -1.374778 \\ \mathrm{C} & -0.137304 & -1.903227 & -1.031543 \\ \mathrm{H} & 0.843012 & -2.507138 & 0.905133 \\ \mathrm{H} & -1.682660 & -2.806582 & 1.817031 \\ \mathrm{H} & -3.366063 & -2.134424 & -0.166778 \\ \mathrm{H} & -1.893714 & -1.471773 & -2.339562 \\ \mathrm{H} & 0.708921 & -1.696390 & -1.679452 \\ \mathrm{C} & -3.370871 & 1.884017 & -0.307280 \\ \mathrm{~N} & -2.501847 & 1.145598 & -0.105393 \\ \mathrm{C} & -4.458411 & 2.798801 & -0.561395 \\ \mathrm{H} & -5.174520 & 2.772746 & 0.267944 \\ \mathrm{H} & -4.071832 & 3.818580 & -0.666663 \\ \mathrm{H} & -4.973803 & 2.517573 & -1.486699 \\ \mathrm{H} & 1.794306 & 2.384536 & -0.577421 \\ \mathrm{Si} & 3.234656 & 0.480682 & -0.153615 \\ \mathrm{C} & 0.008667 & 1.226910 & 1.430236 \\ \mathrm{H} & -1.325883 & 0.099726 & 1.825570 \\ \mathrm{H} & 0.640260 & 0.954641 & 2.280333 \\ \mathrm{H} & -0.605587 & 2.115645 & 1.608223 \\ \mathrm{C} & 3.361650 & -1.244506 & -0.867244 \\ \mathrm{H} & 4.388902 & -1.612161 & -0.728495 \\ \mathrm{H} & 2.692072 & -1.961237 & -0.373253 \\ \mathrm{H} & 3.155357 & -1.259261 & -1.946112 \\ \mathrm{H} & 3.347681 & 0.445925 & 1.712868 \\ \mathrm{H} & 4.346826 & 0.097591 & 2.011330 \\ \mathrm{H} & 3.201542 & 1.439221 & 2.158315 \\ \mathrm{H} & 2.618029 & -0.244901 & 2.158201 \\ \mathrm{H} & 4.532837 & 1.601788 & -0.903337 \\ \mathrm{H} & 4.504890 & 2.603784 & -0.454367 \\ \mathrm{H} & & 1.190440 & -0.743677 \\ \mathrm{H} & -1.9875615 & 1.714270 & -1.98615\end{array}$

\section{C4a}

$G=-1078.536471 \quad Z P E=-1078.480756$

$\mathrm{H} \quad 0.490080 \quad-0.141851 \quad-2.646030$

C $\quad-0.240273 \quad-0.490006 \quad-1.909306$ 


\begin{tabular}{|c|c|c|c|}
\hline $\mathrm{Ru}$ & 0.763190 & -0.499014 & 0.108621 \\
\hline Si & -2.719003 & 0.020206 & -0.498694 \\
\hline$C$ & -0.995042 & 0.427696 & -1.183547 \\
\hline$C$ & 1.512107 & -2.510168 & -0.301784 \\
\hline C & 2.359891 & -1.943837 & 0.700892 \\
\hline C & 1.570162 & -1.637090 & 1.832062 \\
\hline $\mathrm{C}$ & 0.214054 & -2.042985 & 1.559707 \\
\hline C & 0.192081 & -2.599703 & 0.254279 \\
\hline $\mathrm{H}$ & 1.822001 & -2.865066 & -1.278975 \\
\hline 1 & 3.412850 & -1.707340 & 0.581550 \\
\hline $\mathrm{H}$ & 1.919530 & -1.149602 & 2.736729 \\
\hline $\mathrm{H}$ & -0.626866 & -1.967302 & 2.241687 \\
\hline $\mathrm{H}$ & -0.679031 & -3.005919 & -0.249124 \\
\hline $\mathrm{C}$ & 3.028298 & 1.399324 & -1.267963 \\
\hline $\mathrm{N}$ & 2.151656 & 0.792127 & -0.814534 \\
\hline $\mathrm{C}$ & 4.118693 & 2.156258 & -1.838497 \\
\hline $\mathrm{H}$ & 4.499572 & 1.645765 & -2.730271 \\
\hline $\mathrm{H}$ & 3.774381 & 3.157049 & -2.122105 \\
\hline $\mathrm{H}$ & 4.931107 & 2.252418 & -1.109504 \\
\hline C & -0.789182 & 1.899362 & -1.456973 \\
\hline $\mathrm{H}$ & -1.075626 & 2.537078 & -0.609191 \\
\hline $\mathrm{H}$ & 0.247114 & 2.139648 & -1.727266 \\
\hline $\mathrm{H}$ & -1.423254 & 2.199426 & -2.306097 \\
\hline $\mathrm{H}$ & -0.583759 & -1.514249 & -2.063312 \\
\hline $\mathrm{C}$ & -3.096521 & -1.820203 & -0.429901 \\
\hline $\mathrm{H}$ & -2.768099 & -2.360616 & -1.328237 \\
\hline $\mathrm{H}$ & -2.666023 & -2.314091 & 0.451109 \\
\hline $\mathrm{H}$ & -4.187649 & -1.943731 & -0.364131 \\
\hline $\mathrm{C}$ & -3.935436 & 0.822291 & -1.697624 \\
\hline $\mathrm{H}$ & -3.869203 & 1.919091 & -1.676345 \\
\hline $\mathrm{H}$ & -3.765382 & 0.496856 & -2.733734 \\
\hline $\mathrm{H}$ & -4.967479 & 0.552458 & -1.429977 \\
\hline $\mathrm{C}$ & -3.028725 & 0.775466 & 1.196485 \\
\hline $\mathrm{H}$ & -2.399123 & 0.334024 & 1.980670 \\
\hline $\mathrm{H}$ & -2.871167 & 1.863452 & 1.199676 \\
\hline $\mathrm{H}$ & -4.078376 & 0.600125 & 1.475635 \\
\hline $\mathrm{C}$ & 0.023718 & 2.015037 & 2.053015 \\
\hline $\mathrm{N}$ & 0.263117 & 1.155065 & 1.315274 \\
\hline
\end{tabular}




$\begin{array}{lrrr}\mathrm{C} & -0.319654 & 3.085942 & 2.959701 \\ \mathrm{H} & -1.159617 & 2.778396 & 3.593868 \\ \mathrm{H} & 0.535039 & 3.337842 & 3.597190 \\ \mathrm{H} & -0.613775 & 3.975243 & 2.390105\end{array}$

D1

$G=-947.028333 \quad Z P E=-946.977952$

\begin{tabular}{|c|c|c|c|}
\hline $\mathrm{H}$ & 1.590341 & -1.677743 & -0.910496 \\
\hline C & 1.755668 & -0.612227 & -1.107374 \\
\hline $\mathrm{Ru}$ & -1.211432 & -0.345684 & -0.477032 \\
\hline C & 0.552023 & 0.193143 & -1.182090 \\
\hline C & -0.461467 & -2.011476 & 0.784289 \\
\hline C & -1.291272 & -1.154940 & 1.563275 \\
\hline$ᄃ$ & -2.647327 & -1.292662 & 1.083918 \\
\hline C & -2.616252 & -2.142371 & -0.026430 \\
\hline$C$ & -1.257409 & -2.570237 & -0.250545 \\
\hline $\mathrm{H}$ & 0.600134 & -2.173109 & 0.941169 \\
\hline $\mathrm{H}$ & -0.979323 & -0.582352 & 2.431682 \\
\hline $\mathrm{H}$ & -3.517619 & -0.778234 & 1.477064 \\
\hline $\mathrm{H}$ & -3.462197 & -2.392315 & -0.659778 \\
\hline $\mathrm{H}$ & -0.929195 & -3.274885 & -1.007581 \\
\hline C & -1.836640 & 2.689739 & 0.447896 \\
\hline $\mathrm{N}$ & -1.578264 & 1.632160 & 0.050534 \\
\hline C & -2.165027 & 4.007977 & 0.939272 \\
\hline $\mathrm{H}$ & -1.494951 & 4.280894 & 1.762151 \\
\hline $\mathrm{H}$ & -2.059929 & 4.745285 & 0.135293 \\
\hline $\mathrm{H}$ & -3.198816 & 4.025539 & 1.302214 \\
\hline $\mathrm{H}$ & 2.397581 & -0.497749 & -1.997470 \\
\hline Si & 2.947571 & 0.003691 & 0.312491 \\
\hline C & 0.744899 & 1.525100 & -1.829613 \\
\hline $\mathrm{H}$ & -0.164525 & 1.897256 & -2.315239 \\
\hline $\mathrm{H}$ & 0.986103 & 2.254932 & -1.036957 \\
\hline 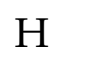 & 1.578659 & 1.538868 & -2.547176 \\
\hline C & 1.997459 & 0.469323 & 1.859914 \\
\hline 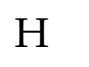 & 2.696247 & 0.885495 & 2.600163 \\
\hline 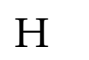 & 1.231585 & 1.233426 & 1.665228 \\
\hline
\end{tabular}




$\begin{array}{lrrr}\mathrm{H} & 1.507377 & -0.396863 & 2.324642 \\ \mathrm{C} & 3.924853 & 1.477079 & -0.308189 \\ \mathrm{H} & 4.712344 & 1.724692 & 0.418248 \\ \mathrm{H} & 4.415866 & 1.268797 & -1.268621 \\ \mathrm{H} & 3.302844 & 2.373371 & -0.433120 \\ \mathrm{C} & 4.066946 & -1.465525 & 0.621464 \\ \mathrm{H} & 4.573816 & -1.781699 & -0.300606 \\ \mathrm{H} & 4.841925 & -1.219699 & 1.360559 \\ \mathrm{H} & 3.500490 & -2.325823 & 1.004856 \\ \mathrm{H} & -1.498741 & -0.247782 & -2.278016 \\ \mathrm{H} & -2.143875 & 0.175454 & -1.982119\end{array}$

\section{D1-TS}

$$
G=-947.020259 \quad Z P E=-946.970401
$$

$\begin{array}{lccc}\mathrm{H} & 1.329703 & -1.890808 & -0.732536 \\ \mathrm{C} & 1.627098 & -0.888020 & -1.057632 \\ \mathrm{Ru} & -1.276770 & -0.215991 & -0.477712 \\ \mathrm{C} & 0.553111 & 0.056815 & -1.221558 \\ \mathrm{C} & -0.826264 & -1.890160 & 1.099058 \\ \mathrm{C} & -1.808870 & -1.040350 & 1.660446 \\ \mathrm{C} & -2.975864 & -1.079449 & 0.840582 \\ \mathrm{C} & -2.692337 & -1.930452 & -0.259082 \\ \mathrm{C} & -1.348895 & -2.417312 & -0.115771 \\ \mathrm{H} & 0.163248 & -2.074388 & 1.506151 \\ \mathrm{H} & -1.689399 & -0.447918 & 2.563284 \\ \mathrm{H} & -3.904710 & -0.550658 & 1.022881 \\ \mathrm{H} & -3.371035 & -2.180524 & -1.067850 \\ \mathrm{H} & -0.858312 & -3.125720 & -0.775732 \\ \mathrm{C} & -1.231222 & 2.877104 & 0.466938 \\ \mathrm{~N} & -1.196354 & 1.781643 & 0.094261 \\ \mathrm{C} & -1.277548 & 4.244480 & 0.929259 \\ \mathrm{H} & -0.695472 & 4.349395 & 1.851602 \\ \mathrm{H} & -0.859992 & 4.910399 & 0.165713 \\ \mathrm{H} & -2.315315 & 4.536406 & 1.125907 \\ \mathrm{H} & 2.296014 & -0.946211 & -1.932839 \\ \mathrm{Si} & 2.876947 & -0.253166 & 0.320440\end{array}$




$\begin{array}{lrrr}\mathrm{C} & 0.904708 & 1.295352 & -1.970038 \\ \mathrm{H} & 0.038827 & 1.754646 & -2.457718 \\ \mathrm{H} & 1.281275 & 2.036190 & -1.243695 \\ \mathrm{H} & 1.707013 & 1.131507 & -2.704157 \\ \mathrm{C} & 1.978662 & 0.563333 & 1.746590 \\ \mathrm{H} & 2.715436 & 0.876865 & 2.500407 \\ \mathrm{H} & 1.430813 & 1.461419 & 1.428990 \\ \mathrm{H} & 1.267744 & -0.112418 & 2.240716 \\ \mathrm{C} & 4.073280 & 0.947944 & -0.474910 \\ \mathrm{H} & 4.886535 & 1.173258 & 0.230028 \\ \mathrm{H} & 4.528571 & 0.523439 & -1.380231 \\ \mathrm{H} & 3.602359 & 1.901866 & -0.746002 \\ \mathrm{C} & 3.756427 & -1.815955 & 0.855521 \\ \mathrm{H} & 4.217082 & -2.323249 & -0.003230 \\ \mathrm{H} & 4.554504 & -1.582689 & 1.573909 \\ \mathrm{H} & 3.068083 & -2.523953 & 1.336906 \\ \mathrm{H} & -1.254726 & -0.380353 & -2.091709 \\ \mathrm{H} & -2.256846 & 0.519954 & -1.533864\end{array}$

D2

$G=-947.0213 \quad Z P E=-946.9696$

$\mathrm{H} \quad 1.351127 \quad-1.87316 \quad-0.75192$

$\begin{array}{llll}\text { C } & 1.641601 & -0.86429 & -1.06511\end{array}$

$\mathrm{Ru} \quad-1.2691 \quad-0.23057 \quad-0.48444$

$\begin{array}{llll}\text { C } & 0.56024 & 0.072275 & -1.22218\end{array}$

$\begin{array}{llll}\text { C } & -0.812 & -1.90311 & 1.10282\end{array}$

$\begin{array}{llll}\text { C } & -1.79018 & -1.04832 & 1.66137\end{array}$

$\begin{array}{llll}\text { C } & -2.95947 & -1.08583 & 0.841392\end{array}$

$\begin{array}{llll}C & -2.68093 & -1.94158 & -0.255\end{array}$

$\begin{array}{llll}\text { C } & -1.33554 & -2.42708 & -0.11249\end{array}$

H $\quad 0.178829 \quad-2.0889 \quad 1.50619$

$\mathrm{H} \quad-1.66876 \quad-0.45484 \quad 2.563303$

$\mathrm{H} \quad-3.88698 \quad-0.55504 \quad 1.024808$

$\mathrm{H} \quad-3.36138 \quad-2.1954 \quad-1.06093$

$\mathrm{H} \quad-0.84582 \quad-3.13642 \quad-0.77218$

$\begin{array}{llll}\text { C } & -1.27936 & 2.863981 & 0.463012\end{array}$ 


$\begin{array}{llll}\mathrm{N} & -1.22049 & 1.771368 & 0.085261 \\ \mathrm{C} & -1.357 & 4.227122 & 0.932983 \\ \mathrm{H} & -0.77538 & 4.340873 & 1.854555 \\ \mathrm{H} & -0.95775 & 4.907938 & 0.172815 \\ \mathrm{H} & -2.40094 & 4.492631 & 1.134466 \\ \mathrm{H} & 2.314503 & -0.90719 & -1.93817 \\ \mathrm{Si} & 2.881781 & -0.23134 & 0.323402 \\ \mathrm{C} & 0.897828 & 1.322571 & -1.95598 \\ \mathrm{H} & 0.028784 & 1.766135 & -2.45246 \\ \mathrm{H} & 1.248283 & 2.066043 & -1.21954 \\ \mathrm{H} & 1.713141 & 1.180869 & -2.68021 \\ \mathrm{C} & 1.968773 & 0.509953 & 1.781151 \\ \mathrm{H} & 2.702125 & 0.899781 & 2.502082 \\ \mathrm{H} & 1.322856 & 1.348538 & 1.485181 \\ \mathrm{H} & 1.347572 & -0.22513 & 2.310166 \\ \mathrm{C} & 4.032846 & 1.032414 & -0.44112 \\ \mathrm{H} & 4.852512 & 1.247323 & 0.259628 \\ \mathrm{H} & 4.484451 & 0.659158 & -1.37052 \\ \mathrm{H} & 3.537195 & 1.986377 & -0.66351 \\ \mathrm{C} & 3.814815 & -1.78201 & 0.801553 \\ \mathrm{H} & 4.277359 & -2.25306 & -0.07666 \\ \mathrm{H} & 4.617371 & -1.54672 & 1.514195 \\ \mathrm{H} & 3.154233 & -2.52296 & 1.272361 \\ \mathrm{H} & -1.15083 & -0.49596 & -2.07635 \\ \mathrm{H} & -2.25325 & 0.486756 & -1.54089\end{array}$

\section{D2-TS}

$$
G=-947.02001 \quad Z P E=-946.96953
$$

$\begin{array}{lrrr}\text { H } & 1.365845 & -1.858485 & -0.771197 \\ \mathrm{C} & 1.651623 & -0.844574 & -1.072200 \\ \mathrm{Ru} & -1.263690 & -0.238653 & -0.480243 \\ \mathrm{C} & 0.565931 & 0.089838 & -1.214276 \\ \mathrm{C} & -0.807653 & -1.912816 & 1.110181 \\ \mathrm{C} & -1.804814 & -1.070476 & 1.654891 \\ \mathrm{C} & -2.955504 & -1.111902 & 0.810530 \\ \mathrm{C} & -2.648122 & -1.963146 & -0.285143\end{array}$




$\begin{array}{lrrr}\mathrm{C} & -1.303600 & -2.438767 & -0.115944 \\ \mathrm{H} & 0.177927 & -2.089622 & 1.530401 \\ \mathrm{H} & -1.705994 & -0.479754 & 2.561280 \\ \mathrm{H} & -3.891676 & -0.591373 & 0.978652 \\ \mathrm{H} & -3.312305 & -2.222696 & -1.102619 \\ \mathrm{H} & -0.793636 & -3.139121 & -0.769909 \\ \mathrm{C} & -1.320157 & 2.856275 & 0.459521 \\ \mathrm{~N} & -1.242683 & 1.765125 & 0.080783 \\ \mathrm{C} & -1.422217 & 4.217461 & 0.930559 \\ \mathrm{H} & -0.845195 & 4.340562 & 1.853810 \\ \mathrm{H} & -1.033113 & 4.905991 & 0.172105 \\ \mathrm{H} & -2.471236 & 4.464262 & 1.129576 \\ \mathrm{H} & 2.323167 & -0.873652 & -1.946788 \\ \mathrm{Si} & 2.886835 & -0.215882 & 0.322429 \\ \mathrm{C} & 0.899898 & 1.356010 & -1.922863 \\ \mathrm{H} & 0.029944 & 1.806432 & -2.411199 \\ \mathrm{H} & 1.249285 & 2.085619 & -1.172500 \\ \mathrm{H} & 1.714719 & 1.229692 & -2.650493 \\ \mathrm{C} & 1.968434 & 0.526191 & 1.776575 \\ \mathrm{H} & 2.699258 & 0.902361 & 2.507233 \\ \mathrm{H} & 1.335371 & 1.374393 & 1.480387 \\ \mathrm{H} & 1.332822 & -0.204981 & 2.293749 \\ \mathrm{C} & 4.043409 & 1.046500 & -0.436464 \\ \mathrm{H} & 4.861756 & 1.258381 & 0.266723 \\ \mathrm{H} & 4.496614 & 0.673415 & -1.365172 \\ \mathrm{H} & 3.550712 & 2.001948 & -0.658977 \\ \mathrm{C} & 3.816050 & -1.767952 & 0.803173 \\ \mathrm{H} & 4.280021 & -2.239454 & -0.074064 \\ \mathrm{H} & 4.617361 & -1.533595 & 1.517518 \\ \mathrm{H} & 3.153576 & -2.508137 & 1.272438 \\ \mathrm{H} & -1.052148 & -0.468577 & -2.071708 \\ \mathrm{H} & -2.297282 & 0.437730 & -1.509293\end{array}$

D3

$$
\begin{array}{cccc}
G=-947.0275 & \multicolumn{2}{c}{Z P E=-946.9758} \\
H & 1.614317 & -1.47632 & -1.15419 \\
C & 1.787511 & -0.39196 & -1.17495
\end{array}
$$




\begin{tabular}{|c|c|c|c|}
\hline $\mathrm{Ru}$ & -1.23007 & -0.38685 & -0.50562 \\
\hline $\mathrm{C}$ & 0.518837 & 0.381831 & -1.23595 \\
\hline $\mathrm{C}$ & -0.34999 & -2.05503 & 0.821079 \\
\hline $\mathrm{C}$ & -1.2274 & -1.20864 & 1.548368 \\
\hline $\mathrm{C}$ & -2.54265 & -1.33717 & 0.974658 \\
\hline $\mathrm{C}$ & -2.45253 & -2.26187 & -0.10164 \\
\hline $\mathrm{C}$ & -1.09489 & -2.68104 & -0.2138 \\
\hline $\mathrm{H}$ & 0.712526 & -2.18078 & 1.002076 \\
\hline $\mathrm{H}$ & -0.96713 & -0.58828 & 2.400337 \\
\hline $\mathrm{H}$ & -3.44188 & -0.8417 & 1.324412 \\
\hline $\mathrm{H}$ & -3.26789 & -2.57606 & -0.74362 \\
\hline $\mathrm{H}$ & -0.70179 & -3.36355 & -0.96062 \\
\hline $\mathrm{C}$ & -2.08007 & 2.557974 & 0.433578 \\
\hline $\mathrm{N}$ & -1.72702 & 1.535493 & 0.020792 \\
\hline $\mathrm{C}$ & -2.51764 & 3.839423 & 0.935113 \\
\hline $\mathrm{H}$ & -3.59526 & 3.815901 & 1.132426 \\
\hline $\mathrm{H}$ & -1.99036 & 4.080162 & 1.864984 \\
\hline $\mathrm{H}$ & -2.30902 & 4.620549 & 0.195511 \\
\hline $\mathrm{H}$ & 2.396308 & -0.17989 & -2.0742 \\
\hline $\mathrm{Si}$ & 2.969627 & 0.067414 & 0.274592 \\
\hline $\mathrm{C}$ & 0.720181 & 1.848543 & -1.48761 \\
\hline $\mathrm{H}$ & -0.18228 & 2.345683 & -1.85539 \\
\hline $\mathrm{H}$ & 1.02933 & 2.353699 & -0.55978 \\
\hline $\mathrm{H}$ & 1.529026 & 2.008463 & -2.21792 \\
\hline $\mathrm{C}$ & 2.039434 & 0.462727 & 1.860452 \\
\hline $\mathrm{H}$ & 2.742181 & 0.889068 & 2.59138 \\
\hline $\mathrm{H}$ & 1.240367 & 1.202783 & 1.707366 \\
\hline $\mathrm{H}$ & 1.595906 & -0.42978 & 2.322751 \\
\hline $\mathrm{C}$ & 4.018908 & 1.548007 & -0.20948 \\
\hline $\mathrm{H}$ & 4.827158 & 1.690429 & 0.522448 \\
\hline $\mathrm{H}$ & 4.487792 & 1.409607 & -1.19387 \\
\hline $\mathrm{H}$ & 3.440406 & 2.481056 & -0.24178 \\
\hline C & 4.069966 & -1.43485 & 0.512323 \\
\hline $\mathrm{H}$ & 4.583806 & -1.70122 & -0.42216 \\
\hline $\mathrm{H}$ & 4.842399 & -1.24495 & 1.270609 \\
\hline $\mathrm{H}$ & 3.495509 & -2.31394 & 0.837362 \\
\hline $\mathrm{H}$ & -0.2435 & -0.0809 & -2.04199 \\
\hline $\mathrm{H}$ & -2.53836 & -0.19854 & -1.42173 \\
\hline
\end{tabular}


D4

$G=-947.048849 \quad Z P E=-946.997832$

$\begin{array}{lccc}\mathrm{Ru} & 1.090413 & -0.057540 & -0.286904 \\ \mathrm{C} & -0.430833 & -0.578536 & 1.133918 \\ \mathrm{C} & -0.238853 & -1.648877 & 2.169746 \\ \mathrm{H} & 0.389788 & -1.308015 & 3.001378 \\ \mathrm{H} & 0.196318 & -2.564229 & 1.747237 \\ \mathrm{C} & -1.063541 & -0.988178 & -0.136407 \\ \mathrm{H} & -0.951851 & -2.063311 & -0.335594 \\ \mathrm{H} & -0.695330 & -0.420894 & -1.072389 \\ \mathrm{C} & 2.723140 & -1.075621 & -1.350215 \\ \mathrm{C} & 2.354253 & -1.912534 & -0.255490 \\ \mathrm{C} & 2.645561 & -1.216144 & 0.955042 \\ \mathrm{C} & 3.129401 & 0.068987 & 0.621091 \\ \mathrm{C} & 3.183326 & 0.170921 & -0.812293 \\ \mathrm{C} & 0.140403 & 2.980536 & 0.287063 \\ \mathrm{~N} & 0.454244 & 1.878562 & 0.120747 \\ \mathrm{C} & -0.269535 & 4.352582 & 0.472006 \\ \mathrm{H} & 0.253169 & 4.996330 & -0.244522 \\ \mathrm{H} & -1.349876 & 4.440891 & 0.305986 \\ \mathrm{H} & -0.034385 & 4.686482 & 1.488513 \\ \mathrm{H} & 0.816750 & 0.523361 & -1.779688 \\ \mathrm{H} & -0.797950 & 0.359069 & 1.567264 \\ \mathrm{H} & -1.217423 & -1.920058 & 2.599832 \\ \mathrm{Si} & -2.914520 & -0.451861 & -0.288580 \\ \mathrm{C} & -3.808320 & -1.183852 & 1.183923 \\ \mathrm{H} & -4.890342 & -1.007295 & 1.105197 \\ \mathrm{H} & -3.655550 & -2.269707 & 1.251714 \\ \mathrm{H} & -3.468308 & -0.731164 & 2.125677 \\ \mathrm{H} & -2.979076 & 1.419871 & -0.281481 \\ \mathrm{H} & -2.637998 & 1.837242 & 0.675658 \\ \mathrm{H} & -2.362149 & 1.844278 & -1.086391 \\ \mathrm{H} & -3.453826 & -2.248256 & -1.927960\end{array}$




$\begin{array}{cccc}\mathrm{H} & -4.601669 & -0.904786 & -2.058738 \\ \mathrm{H} & -2.980623 & -0.753522 & -2.761776 \\ \mathrm{H} & 3.400873 & 0.852546 & 1.321021 \\ \mathrm{H} & 2.474617 & -1.585641 & 1.959759 \\ \mathrm{H} & 3.555479 & 1.019147 & -1.376131 \\ \mathrm{H} & 2.659375 & -1.336349 & -2.400754 \\ \mathrm{H} & 1.945599 & -2.915626 & -0.330069\end{array}$

\section{D4-TS}

$$
G=-947.039419 \quad Z P E=-946.98869
$$

$\begin{array}{lccc}\mathrm{Ru} & 0.985374 & -0.094546 & -0.266785 \\ \mathrm{C} & -0.424246 & -0.492060 & 1.432052 \\ \mathrm{C} & 0.052115 & -1.369111 & 2.552331 \\ \mathrm{H} & 0.783089 & -0.869034 & 3.198840 \\ \mathrm{H} & 0.481808 & -2.312435 & 2.193098 \\ \mathrm{C} & -1.037457 & -1.039247 & 0.274350 \\ \mathrm{H} & -0.877960 & -2.120551 & 0.149670 \\ \mathrm{H} & -0.480381 & -0.429636 & -1.122316 \\ \mathrm{C} & 2.430349 & -1.420903 & -1.247163 \\ \mathrm{C} & 2.299029 & -1.824173 & 0.110997 \\ \mathrm{C} & 2.763375 & -0.749495 & 0.937699 \\ \mathrm{C} & 3.174999 & 0.307747 & 0.086832 \\ \mathrm{C} & 2.966882 & -0.088910 & -1.264174 \\ \mathrm{C} & 0.091108 & 2.984961 & 0.212235 \\ \mathrm{~N} & 0.374161 & 1.874725 & 0.043918 \\ \mathrm{C} & -0.290091 & 4.363091 & 0.414521 \\ \mathrm{H} & 0.240982 & 5.009014 & -0.293574 \\ \mathrm{H} & -1.369422 & 4.473365 & 0.254874 \\ \mathrm{H} & -0.046611 & 4.676186 & 1.436057 \\ \mathrm{H} & 0.163694 & 0.118704 & -1.746590 \\ \mathrm{H} & -0.775638 & 0.492033 & 1.759754 \\ \mathrm{H} & -0.811961 & -1.625826 & 3.186143 \\ \mathrm{Si} & -2.772811 & -0.497087 & -0.308833 \\ \mathrm{C} & -3.929854 & -1.321975 & 0.915359 \\ \mathrm{H} & -4.981901 & -1.114527 & 0.675781 \\ \mathrm{H} & -3.796768 & -2.412658 & 0.919080\end{array}$




$\begin{array}{lrrc}\mathrm{H} & -3.740355 & -0.958513 & 1.935694 \\ \mathrm{C} & -2.955730 & 1.367250 & -0.268541 \\ \mathrm{H} & -2.743205 & 1.784967 & 0.725098 \\ \mathrm{H} & -2.298994 & 1.853768 & -1.003114 \\ \mathrm{H} & -3.990561 & 1.636911 & -0.523492 \\ \mathrm{C} & -3.021668 & -1.148637 & -2.049232 \\ \mathrm{H} & -2.797135 & -2.223016 & -2.109119 \\ \mathrm{H} & -4.060575 & -1.009934 & -2.378540 \\ \mathrm{H} & -2.377792 & -0.629331 & -2.773588 \\ \mathrm{H} & 1.924561 & -2.783245 & 0.454358 \\ \mathrm{H} & 2.170511 & -2.015176 & -2.116936 \\ \mathrm{H} & 3.192127 & 0.502494 & -2.144588 \\ \mathrm{H} & 3.527788 & 1.281317 & 0.412989 \\ \mathrm{H} & 2.795592 & -0.739937 & 2.021587\end{array}$

\section{D5}

$G=-947.048603 \quad Z P E=-946.997319$

$\begin{array}{lccc}\mathrm{Ru} & 0.771416 & -0.353720 & -0.256303 \\ \mathrm{C} & -0.523906 & 0.464134 & 1.523955 \\ \mathrm{C} & 0.084911 & 0.207427 & 2.866100 \\ \mathrm{H} & 1.032951 & 0.745053 & 2.998257 \\ \mathrm{H} & 0.247771 & -0.859900 & 3.058888 \\ \mathrm{C} & -1.293307 & -0.436352 & 0.798609 \\ \mathrm{H} & -1.341977 & -1.454814 & 1.208235 \\ \mathrm{H} & -0.531972 & -0.187157 & -1.584761 \\ \mathrm{C} & 1.206838 & -2.481322 & -0.608351 \\ \mathrm{C} & 1.303088 & -2.202783 & 0.780776 \\ \mathrm{C} & 2.338347 & -1.233212 & 0.979545 \\ \mathrm{C} & 2.920581 & -0.963880 & -0.302408 \\ \mathrm{C} & 2.224909 & -1.713073 & -1.276599 \\ \mathrm{C} & 1.621563 & 2.793297 & -0.328449 \\ \mathrm{~N} & 1.251643 & 1.695668 & -0.311439 \\ \mathrm{C} & 2.084945 & 4.161147 & -0.346308 \\ \mathrm{H} & 2.074620 & 4.546549 & -1.371724 \\ \mathrm{H} & 1.435006 & 4.786248 & 0.276056 \\ \mathrm{H} & 3.108061 & 4.213230 & 0.042455\end{array}$




$\begin{array}{lrcc}\mathrm{H} & 0.138727 & 0.107378 & -1.941310 \\ \mathrm{H} & -0.629160 & 1.525011 & 1.278680 \\ \mathrm{H} & -0.601008 & 0.581887 & 3.642129 \\ \mathrm{Si} & -2.792694 & 0.061699 & -0.236014 \\ \mathrm{C} & -4.273058 & -0.096230 & 0.913319 \\ \mathrm{H} & -5.202374 & 0.189267 & 0.400276 \\ \mathrm{H} & -4.396047 & -1.127114 & 1.273558 \\ \mathrm{H} & -4.163564 & 0.553130 & 1.793042 \\ \mathrm{C} & -2.634941 & 1.837756 & -0.827266 \\ \mathrm{H} & -2.592092 & 2.543302 & 0.014236 \\ \mathrm{H} & -1.746508 & 2.001448 & -1.452545 \\ \mathrm{H} & -3.514051 & 2.107689 & -1.429635 \\ \mathrm{C} & -3.010089 & -1.123872 & -1.681174 \\ \mathrm{H} & -2.741074 & -2.150676 & -1.391665 \\ \mathrm{H} & -4.059229 & -1.138912 & -2.008572 \\ \mathrm{H} & -2.402056 & -0.855873 & -2.556131 \\ \mathrm{H} & 0.672972 & -2.629269 & 1.555086 \\ \mathrm{H} & 0.512649 & -3.170415 & -1.078045 \\ \mathrm{H} & 2.408670 & -1.692655 & -2.345790 \\ \mathrm{H} & 3.711304 & -0.246880 & -0.500569 \\ \mathrm{H} & 2.673001 & -0.834550 & 1.930965\end{array}$

E1

$G=-1077.332044 \quad Z P E=-1077.275047$

$\begin{array}{lccc}\mathrm{Ru} & 0.837063 & -0.387311 & -0.306279 \\ \mathrm{C} & 2.535023 & 1.952970 & 1.205866 \\ \mathrm{~N} & 1.922463 & 1.110770 & 0.697663 \\ \mathrm{C} & 3.300153 & 3.000984 & 1.841568 \\ \mathrm{H} & 3.693345 & 3.690198 & 1.085790 \\ \mathrm{H} & 2.663474 & 3.560862 & 2.535711 \\ \mathrm{H} & 4.138975 & 2.568620 & 2.398334 \\ \mathrm{C} & -0.637400 & 2.176994 & -1.688735 \\ \mathrm{~N} & -0.131344 & 1.276008 & -1.164620 \\ \mathrm{C} & -1.305449 & 3.286060 & -2.330502 \\ \mathrm{H} & -2.334486 & 3.002593 & -2.582948 \\ \mathrm{H} & -1.331205 & 4.149764 & -1.656378\end{array}$




$\begin{array}{lccc}\mathrm{H} & -0.778447 & 3.568355 & -3.248637 \\ \mathrm{C} & 1.548284 & -2.451779 & -0.131734 \\ \mathrm{H} & 1.636183 & -2.988964 & 0.806933 \\ \mathrm{C} & 0.388958 & -2.416907 & -0.980694 \\ \mathrm{C} & 2.561760 & -1.669460 & -0.748955 \\ \mathrm{H} & -0.559295 & -2.906691 & -0.781723 \\ \mathrm{C} & 0.696551 & -1.609129 & -2.105178 \\ \mathrm{H} & 3.546547 & -1.471647 & -0.336059 \\ \mathrm{C} & 2.043481 & -1.125735 & -1.966651 \\ \mathrm{H} & 0.012204 & -1.358709 & -2.910534 \\ \mathrm{H} & 2.583053 & -0.508694 & -2.677115 \\ \mathrm{C} & -0.056652 & -0.624021 & 1.865371 \\ \mathrm{C} & -1.013497 & -0.380908 & 1.106247 \\ \mathrm{Si} & -2.783417 & -0.130365 & 0.580197 \\ \mathrm{C} & -3.042723 & -0.608742 & -1.212541 \\ \mathrm{H} & -4.108675 & -0.499768 & -1.461147 \\ \mathrm{H} & -2.473407 & 0.024978 & -1.904707 \\ \mathrm{H} & -2.770676 & -1.655945 & -1.402917 \\ \mathrm{C} & -3.815713 & -1.247107 & 1.678720 \\ \mathrm{H} & -3.655589 & -1.028256 & 2.742930 \\ \mathrm{H} & -4.886029 & -1.114876 & 1.465848 \\ \mathrm{H} & -3.571841 & -2.305253 & 1.512170 \\ \mathrm{C} & -3.239679 & 1.666560 & 0.861626 \\ \mathrm{H} & -4.277189 & 1.848438 & 0.546812 \\ \mathrm{H} & -3.162168 & 1.934135 & 1.923986 \\ \mathrm{H} & -2.592375 & 2.350741 & 0.296666 \\ \mathrm{H} & 0.816874 & -0.907883 & 2.997641 \\ \mathrm{H} & 1.580437 & -0.129387 & 3.119222 \\ & 0.230168 & -0.955785 & 3.925092 \\ \mathrm{H} & 1.336401 & -1.866734 & 2.872581 \\ \mathrm{H} & & & \\ \mathrm{H} & & \end{array}$

\section{E1-TS}

$G=-1077.311919 \quad Z P E=-1077.255622$

$\begin{array}{lcll}\mathrm{Ru} & 1.046728 & -0.281661 & -0.203626 \\ \mathrm{C} & 1.470233 & 1.001731 & 2.769862 \\ \mathrm{~N} & 1.310544 & 0.558887 & 1.711212\end{array}$




\begin{tabular}{|c|c|c|c|}
\hline C & 1.676134 & 1.548781 & 4.091405 \\
\hline $\mathrm{H}$ & 1.992153 & 0.756052 & 4.778742 \\
\hline $\mathrm{H}$ & 2.452247 & 2.322108 & 4.063119 \\
\hline & 0.746432 & 1.991821 & 4.465078 \\
\hline C & 0.167452 & 2.724647 & -1.144190 \\
\hline . & 0.449999 & 1.660489 & -0.781881 \\
\hline $\mathrm{C}$ & -0.225746 & 4.042985 & -1.585485 \\
\hline $\mathrm{H}$ & -0.960125 & 3.962185 & -2.395639 \\
\hline Н & -0.678356 & 4.593364 & -0.752417 \\
\hline $\mathrm{H}$ & 0.644789 & 4.600355 & -1.949009 \\
\hline C & 2.121093 & -2.174974 & -0.193996 \\
\hline r & 2.111524 & -2.899255 & 0.613192 \\
\hline $\mathrm{C}$ & 1.233858 & -2.141747 & -1.317804 \\
\hline C & 3.094504 & -1.135488 & -0.393296 \\
\hline H & 0.378710 & -2.793585 & -1.470160 \\
\hline $\mathrm{C}$ & 1.615044 & -1.063187 & -2.162601 \\
\hline $\mathrm{H}$ & 3.894476 & -0.880569 & 0.295144 \\
\hline C & 2.792596 & -0.456635 & -1.592614 \\
\hline $\mathrm{H}$ & 1.132669 & -0.778206 & -3.092244 \\
\hline $\mathrm{H}$ & 3.326242 & 0.398354 & -1.994801 \\
\hline$C$ & -1.721394 & -1.518385 & 1.002473 \\
\hline$c$ & -0.755828 & -0.902910 & 0.489486 \\
\hline $\mathrm{Si}$ & -2.575754 & -0.012670 & -0.212443 \\
\hline C & -4.319124 & -0.708414 & -0.072576 \\
\hline $\mathrm{H}$ & -4.977637 & -0.005898 & -0.606432 \\
\hline $\mathrm{H}$ & -4.423031 & -1.686924 & -0.559148 \\
\hline 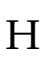 & -4.687052 & -0.785523 & 0.957604 \\
\hline C & -2.544443 & 1.640804 & 0.664250 \\
\hline $\mathrm{H}$ & -1.532547 & 1.960922 & 0.938995 \\
\hline 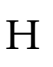 & -2.998627 & 2.422361 & 0.037245 \\
\hline 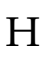 & -3.140060 & 1.571791 & 1.585673 \\
\hline $\mathrm{C}$ & -2.199183 & -0.016627 & -2.046420 \\
\hline $\mathrm{H}$ & -3.057838 & 0.409156 & -2.586589 \\
\hline 11 & -1.301087 & 0.548782 & -2.315642 \\
\hline $\mathrm{H}$ & -2.072568 & -1.051033 & -2.397767 \\
\hline C & -2.553862 & -2.434793 & 1.790252 \\
\hline $\mathrm{H}$ & -1.891481 & -3.105743 & 2.352427 \\
\hline & -3.183125 & -1.900094 & 2.512221 \\
\hline
\end{tabular}


H $\quad-3.202986 \quad-3.050309 \quad 1.156356$

E2

$G=-1077.323497 \quad Z P E=-1077.264116$

$\begin{array}{llll}\text { C } & -2.174450 & -2.076432 & 1.256524\end{array}$

C $\quad-0.748356 \quad-2.295609 \quad 1.169273$

$\begin{array}{llll}\text { C } & -0.430947 & -2.531987 & -0.202095\end{array}$

$\begin{array}{llll}\text { C } & -1.614594 & -2.327309 & -0.970148\end{array}$

$\begin{array}{llll}\text { C } & -2.692874 & -2.090177 & -0.040411\end{array}$

$\mathrm{Ru} \quad-1.033367 \quad-0.446790 \quad 0.027556$

$\mathrm{N} \quad-0.835334 \quad 0.984143 \quad 1.538728$

$\begin{array}{llll}\text { C } & -0.712989 & 1.742024 & 2.405476\end{array}$

$\mathrm{N} \quad-2.140477 \quad 1.020425 \quad-0.985636$

$\begin{array}{llll}\text { C } & -2.768682 & 1.818448 & -1.540775\end{array}$

$\mathrm{H} \quad-3.721376 \quad-1.877882 \quad-0.316438$

$\mathrm{H} \quad-2.728146 \quad-1.866820 \quad 2.165928$

$\mathrm{H} \quad-0.066220 \quad-2.380563 \quad 2.009039$

$\mathrm{H} \quad 0.553600 \quad-2.764884 \quad-0.596831$

$\mathrm{H} \quad-1.713995 \quad-2.447450 \quad-2.043385$

$\begin{array}{llll}\text { C } & -3.549765 & 2.814090 & -2.236381\end{array}$

$\mathrm{H} \quad-4.508668 \quad 2.962286 \quad-1.727437$

$\mathrm{H} \quad-3.006050 \quad 3.765113 \quad-2.256997$

$\mathrm{H} \quad-3.738259 \quad 2.490637 \quad-3.266150$

$\begin{array}{llll}\text { C } & -0.547627 & 2.677056 & 3.493696\end{array}$

$\mathrm{H} \quad 0.400406 \quad 2.484901 \quad 4.009439$

$\mathrm{H} \quad-0.541369 \quad 3.703114 \quad 3.110091$

$\mathrm{H} \quad-1.370158 \quad 2.570971 \quad 4.209900$

$\begin{array}{llll}\text { C } & 0.576382 & 0.122974 & -0.741133\end{array}$

$\begin{array}{llll}\text { Si } & 3.215587 & 0.082256 & -0.081352\end{array}$

$\begin{array}{llll}\text { C } & 4.128730 & -1.407077 & -0.765097\end{array}$

$\mathrm{H} \quad 5.027818 \quad-1.611123 \quad-0.165880$

$\mathrm{H} \quad 3.503510 \quad-2.310425 \quad-0.744043$

$\mathrm{H} \quad 4.453937 \quad-1.246809 \quad-1.802127$

C $\quad 2.648621 \quad-0.254210 \quad 1.670848$

$\begin{array}{llll}\mathrm{H} & 3.524283 & -0.438888 & 2.309498\end{array}$

$\begin{array}{llll}\mathrm{H} & 2.097861 & 0.594778 & 2.097455\end{array}$ 


$\begin{array}{lrrr}\mathrm{H} & 2.003996 & -1.142077 & 1.729180 \\ \mathrm{C} & 4.308926 & 1.603506 & -0.160421 \\ \mathrm{H} & 4.705388 & 1.761469 & -1.172742 \\ \mathrm{H} & 3.769943 & 2.512361 & 0.139224 \\ \mathrm{H} & 5.168125 & 1.485069 & 0.514999 \\ \mathrm{C} & 1.735752 & 0.416460 & -1.243782 \\ \mathrm{C} & 2.029038 & 0.948537 & -2.627476 \\ \mathrm{H} & 2.792873 & 0.338037 & -3.128546 \\ \mathrm{H} & 1.132311 & 0.957407 & -3.258053 \\ \mathrm{H} & 2.417829 & 1.974225 & -2.569908\end{array}$

\section{E3}

$G=-944.670015 \quad Z P E=-944.617499$

$\begin{array}{lccc}\mathrm{Ru} & 1.135048 & -0.361388 & -0.279158 \\ \mathrm{C} & 2.935643 & 2.360711 & 0.043785 \\ \mathrm{~N} & 2.274488 & 1.417571 & -0.076447 \\ \mathrm{C} & 3.756833 & 3.536908 & 0.196500 \\ \mathrm{H} & 4.795600 & 3.305155 & -0.063537 \\ \mathrm{H} & 3.391992 & 4.333197 & -0.461848 \\ \mathrm{H} & 3.716395 & 3.886228 & 1.234385 \\ \mathrm{C} & 1.791475 & -1.550715 & 1.371919 \\ \mathrm{H} & 1.953365 & -1.140008 & 2.363045 \\ \mathrm{C} & 0.554154 & -2.078465 & 0.868352 \\ \mathrm{C} & 2.802391 & -1.778974 & 0.370352 \\ \mathrm{H} & -0.386917 & -2.102902 & 1.408679 \\ \mathrm{C} & 0.776581 & -2.501873 & -0.470971 \\ \mathrm{H} & 3.838328 & -1.464230 & 0.441379 \\ \mathrm{C} & 2.185004 & -2.334741 & -0.754262 \\ \mathrm{H} & 0.045052 & -2.944589 & -1.138653 \\ \mathrm{H} & 2.662503 & -2.542645 & -1.706310 \\ \mathrm{C} & -1.637629 & 1.186513 & -0.116634 \\ \mathrm{C} & -0.492592 & 0.571688 & -0.161265 \\ \mathrm{Si} & -3.205064 & 0.084843 & 0.056421 \\ \mathrm{C} & -2.886106 & -1.641004 & -0.590899 \\ \mathrm{H} & -3.831008 & -2.202421 & -0.614914 \\ \mathrm{H} & -2.489731 & -1.618887 & -1.614980\end{array}$




$\begin{array}{llll}\mathrm{H} & -2.187634 & -2.205720 & 0.039735 \\ \mathrm{C} & -3.627034 & 0.078818 & 1.881365 \\ \mathrm{H} & -3.745413 & 1.099933 & 2.269539 \\ \mathrm{H} & -4.568800 & -0.457384 & 2.063860 \\ \mathrm{H} & -2.839199 & -0.414629 & 2.467361 \\ \mathrm{C} & -4.538051 & 0.927793 & -0.953249 \\ \mathrm{H} & -5.459559 & 0.329088 & -0.919638 \\ \mathrm{H} & -4.778693 & 1.926248 & -0.564905 \\ \mathrm{H} & -4.248976 & 1.030651 & -2.007784 \\ \mathrm{C} & -1.822276 & 2.683990 & -0.139611 \\ \mathrm{H} & -0.863739 & 3.214934 & -0.106258 \\ \mathrm{H} & -2.356789 & 2.986244 & -1.050193 \\ \mathrm{H} & -2.429772 & 3.008127 & 0.716929\end{array}$

E4a

$G=-945.832063 \quad Z P E=-945.7795$

$\begin{array}{lccc}\mathrm{Ru} & -1.115467 & -0.362750 & -0.290299 \\ \mathrm{C} & -2.599836 & 2.527890 & -0.260899 \\ \mathrm{~N} & -2.051974 & 1.509605 & -0.309308 \\ \mathrm{C} & -3.283046 & 3.798023 & -0.199766 \\ \mathrm{H} & -2.977216 & 4.342839 & 0.700607 \\ \mathrm{H} & -3.035200 & 4.398661 & -1.082067 \\ \mathrm{H} & -4.366556 & 3.638300 & -0.169750 \\ \mathrm{C} & -1.110400 & -2.567442 & -0.266890 \\ \mathrm{H} & -0.523963 & -3.166797 & -0.955868 \\ \mathrm{C} & -0.678665 & -2.084393 & 0.997247 \\ \mathrm{C} & -2.502301 & -2.217558 & -0.427695 \\ \mathrm{H} & 0.311278 & -2.209428 & 1.425165 \\ \mathrm{C} & -1.753320 & -1.332710 & 1.568153 \\ \mathrm{H} & -3.121063 & -2.455322 & -1.286296 \\ \mathrm{C} & -2.887044 & -1.468431 & 0.689599 \\ \mathrm{H} & -1.756080 & -0.851800 & 2.540088 \\ \mathrm{H} & -3.852433 & -0.993728 & 0.836722 \\ \mathrm{C} & 1.647471 & 0.927029 & 0.627608 \\ \mathrm{C} & 0.511603 & 0.465354 & 0.224436 \\ \mathrm{H} & -1.143999 & -0.043036 & -2.117072\end{array}$




$\begin{array}{lrrr}\mathrm{H} & -0.345956 & -0.144322 & -1.953587 \\ \mathrm{Si} & 3.138983 & 0.070178 & -0.232949 \\ \mathrm{C} & 1.949002 & 1.989227 & 1.655370 \\ \mathrm{H} & 2.386443 & 2.874040 & 1.174144 \\ \mathrm{H} & 1.045175 & 2.299406 & 2.191682 \\ \mathrm{H} & 2.677074 & 1.623776 & 2.392149 \\ \mathrm{C} & 3.932546 & -1.036861 & 1.053413 \\ \mathrm{H} & 3.246904 & -1.834887 & 1.370006 \\ \mathrm{H} & 4.832482 & -1.515567 & 0.641211 \\ \mathrm{H} & 4.236359 & -0.477272 & 1.948451 \\ \mathrm{C} & 4.291436 & 1.457469 & -0.739388 \\ \mathrm{H} & 4.678744 & 2.001406 & 0.132535 \\ \mathrm{H} & 5.153940 & 1.048336 & -1.284443 \\ \mathrm{H} & 3.792347 & 2.180849 & -1.398154 \\ \mathrm{C} & 2.604015 & -0.933168 & -1.719240 \\ \mathrm{H} & 1.948945 & -1.771546 & -1.445572 \\ \mathrm{H} & 2.086807 & -0.320376 & -2.469985 \\ \mathrm{H} & 3.495808 & -1.358836 & -2.202075\end{array}$

\section{E4b}

$G=-945.830597 \quad Z P E=-945.779673$

$\begin{array}{lrrr}\mathrm{Ru} & -1.388574 & -0.103128 & -0.439021 \\ \mathrm{C} & -0.325380 & 2.959550 & -0.230761 \\ \mathrm{~N} & -0.733463 & 1.881686 & -0.340409 \\ \mathrm{C} & 0.188677 & 4.301489 & -0.088606 \\ \mathrm{H} & 0.911094 & 4.337479 & 0.735074 \\ \mathrm{H} & 0.688875 & 4.609633 & -1.013607 \\ \mathrm{H} & -0.630153 & 4.997735 & 0.123952 \\ \mathrm{C} & -2.647873 & -1.886642 & -0.054088 \\ \mathrm{H} & -2.776724 & -2.698655 & -0.761344 \\ \mathrm{C} & -1.639427 & -1.790593 & 0.940397 \\ \mathrm{C} & -3.542420 & -0.766424 & 0.119630 \\ \mathrm{H} & -0.822723 & -2.489693 & 1.090866 \\ \mathrm{C} & -1.834528 & -0.561181 & 1.644879 \\ \mathrm{H} & -4.411254 & -0.554629 & -0.495448 \\ \mathrm{C} & -3.057495 & 0.032208 & 1.158279\end{array}$




$\begin{array}{cccc}\mathrm{H} & -1.242535 & -0.200491 & 2.479825 \\ \mathrm{H} & -3.479966 & 0.973745 & 1.493676 \\ \mathrm{C} & 1.651702 & -0.895327 & -0.953839 \\ \mathrm{C} & 0.411732 & -0.580040 & -0.782491 \\ \mathrm{Si} & 2.782811 & -0.309485 & 0.485097 \\ \mathrm{C} & 1.803209 & 0.039372 & 2.040395 \\ \mathrm{H} & 2.494415 & 0.335903 & 2.842517 \\ \mathrm{H} & 1.081396 & 0.856500 & 1.907976 \\ \mathrm{H} & 1.261912 & -0.851470 & 2.386489 \\ \mathrm{C} & 3.997753 & -1.706998 & 0.770970 \\ \mathrm{H} & 4.631372 & -1.885788 & -0.107905 \\ \mathrm{H} & 4.660702 & -1.457728 & 1.611785 \\ \mathrm{H} & 3.486304 & -2.646888 & 1.019173 \\ \mathrm{C} & 3.656172 & 1.235498 & -0.114780 \\ \mathrm{H} & 4.383690 & 1.582397 & 0.632936 \\ \mathrm{H} & 4.202843 & 1.058349 & -1.051082 \\ \mathrm{H} & 2.940521 & 2.050859 & -0.291291 \\ \mathrm{C} & 2.303646 & -1.595033 & -2.121137 \\ \mathrm{H} & 1.600154 & -1.735165 & -2.949695 \\ \mathrm{H} & 3.160948 & -1.017024 & -2.491911 \\ \mathrm{H} & 2.680185 & -2.580419 & -1.816585 \\ \mathrm{H} & -1.869734 & 0.499674 & -2.120627 \\ \mathrm{H} & -1.473810 & -0.200999 & -2.289428\end{array}$

\section{E4-TSa}

$G=-945.818165 \quad Z P E=-945.767557$

$\begin{array}{lccc}\mathrm{Ru} & -1.380514 & -0.062242 & -0.427245 \\ \mathrm{C} & -0.125697 & 2.914840 & -0.156242 \\ \mathrm{~N} & -0.581914 & 1.855925 & -0.246851 \\ \mathrm{C} & 0.453246 & 4.236689 & -0.042463 \\ \mathrm{H} & 0.972669 & 4.341038 & 0.916816 \\ \mathrm{H} & 1.168399 & 4.404692 & -0.855473 \\ \mathrm{H} & -0.330304 & 5.000095 & -0.101692 \\ \mathrm{C} & -3.035557 & -1.518285 & -0.317002 \\ \mathrm{H} & -3.376134 & -2.059695 & -1.192832 \\ \mathrm{C} & -1.998198 & -1.932846 & 0.572065\end{array}$




$\begin{array}{lrrr}\mathrm{C} & -3.583880 & -0.290345 & 0.179742 \\ \mathrm{H} & -1.405614 & -2.836507 & 0.481459 \\ \mathrm{C} & -1.882907 & -0.945464 & 1.599807 \\ \mathrm{H} & -4.392704 & 0.273238 & -0.270575 \\ \mathrm{C} & -2.869768 & 0.054559 & 1.350075 \\ \mathrm{H} & -1.206881 & -0.973342 & 2.446374 \\ \mathrm{H} & -3.020399 & 0.953595 & 1.938789 \\ \mathrm{C} & 1.665424 & -0.866632 & -1.010017 \\ \mathrm{C} & 0.439645 & -0.589582 & -0.763799 \\ \mathrm{Si} & 2.767892 & -0.415367 & 0.522992 \\ \mathrm{C} & 1.743974 & -0.260929 & 2.082277 \\ \mathrm{H} & 2.400527 & -0.043188 & 2.935001 \\ \mathrm{H} & 0.999725 & 0.542711 & 2.017174 \\ \mathrm{H} & 1.219350 & -1.200680 & 2.300961 \\ \mathrm{C} & 4.010201 & -1.806156 & 0.668865 \\ \mathrm{H} & 4.636009 & -1.893876 & -0.227911 \\ \mathrm{H} & 4.680493 & -1.612305 & 1.516973 \\ \mathrm{H} & 3.524460 & -2.774059 & 0.844622 \\ \mathrm{C} & 3.572819 & 1.217193 & 0.073246 \\ \mathrm{H} & 4.260239 & 1.538710 & 0.866995 \\ \mathrm{H} & 4.152578 & 1.151434 & -0.855961 \\ \mathrm{H} & 2.813435 & 2.001569 & -0.054834 \\ \mathrm{C} & 2.362946 & -1.375046 & -2.244305 \\ \mathrm{H} & 1.669843 & -1.461512 & -3.087198 \\ \mathrm{H} & 3.176408 & -0.696944 & -2.532950 \\ \mathrm{H} & 2.805052 & -2.360980 & -2.055439 \\ & -1.939084 & 0.683717 & -1.769235 \\ -1.256029 & -0.443376 & -2.009727\end{array}$

\section{E4-TSb}

$G=-945.817674 \quad Z P E=-945.764953$

$\begin{array}{llll}\mathrm{Ru} & -1.171409 & -0.288812 & -0.237008 \\ \mathrm{C} & -1.986886 & 2.851077 & -0.234471 \\ \mathrm{~N} & -1.683566 & 1.735208 & -0.247535 \\ \mathrm{C} & -2.367299 & 4.243348 & -0.211462 \\ \mathrm{H} & -3.162342 & 4.400036 & 0.525908\end{array}$




$\begin{array}{lrrr}\mathrm{H} & -1.502461 & 4.860274 & 0.057404 \\ \mathrm{H} & -2.730412 & 4.549507 & -1.199071 \\ \mathrm{C} & -1.760862 & -2.381904 & -0.622661 \\ \mathrm{H} & -1.389243 & -2.919361 & -1.488696 \\ \mathrm{C} & -1.130114 & -2.313814 & 0.657585 \\ \mathrm{C} & -2.984359 & -1.644270 & -0.547944 \\ \mathrm{H} & -0.192279 & -2.786375 & 0.931524 \\ \mathrm{C} & -1.966459 & -1.539046 & 1.519875 \\ \mathrm{H} & -3.699809 & -1.509400 & -1.351311 \\ \mathrm{C} & -3.095356 & -1.121684 & 0.766924 \\ \mathrm{H} & -1.772056 & -1.302665 & 2.560276 \\ \mathrm{H} & -3.893455 & -0.479457 & 1.125931 \\ \mathrm{C} & 1.726321 & 0.499944 & 0.869461 \\ \mathrm{C} & 0.531769 & 0.242128 & 0.460622 \\ \mathrm{H} & -1.334874 & 0.036226 & -1.808658 \\ \mathrm{H} & 0.133497 & -0.217225 & -1.219942 \\ \mathrm{Si} & 3.141534 & -0.097332 & -0.308606 \\ \mathrm{C} & 3.688458 & 1.428740 & -1.242264 \\ \mathrm{H} & 2.885001 & 1.791288 & -1.898634 \\ \mathrm{H} & 3.964079 & 2.245677 & -0.561811 \\ \mathrm{H} & 4.561914 & 1.208329 & -1.871790 \\ \mathrm{C} & 4.478450 & -0.753793 & 0.824502 \\ \mathrm{H} & 5.303732 & -1.153748 & 0.218102 \\ \mathrm{H} & 4.893643 & 0.027840 & 1.473965 \\ \mathrm{H} & 4.110574 & -1.569463 & 1.461360 \\ \mathrm{H} & 2.561045 & -1.439729 & -1.476281 \\ \mathrm{H} & 3.439527 & -1.869996 & -1.979055 \\ \mathrm{H} & 2.049200 & -2.258608 & -0.952009 \\ \mathrm{H} & 1.892637 & -1.063888 & -2.262540 \\ \mathrm{H} & 2.658894 & 0.526854 & 2.806579 \\ \mathrm{H} & 2.140764 & 1.218072 & 2.129531 \\ \mathrm{H} & 2.033832 & 1.896526 \\ \mathrm{H} & 1.639836 & 2.657028\end{array}$

\section{E5b}

$G=-945.824401 \quad Z P E=-945.773625$ 


\begin{tabular}{|c|c|c|c|}
\hline $\mathrm{Ru}$ & 1.421420 & -0.067648 & 0.469651 \\
\hline $\mathrm{C}$ & 0.332224 & 2.936500 & 0.063621 \\
\hline $\mathrm{N}$ & 0.714611 & 1.858006 & 0.233647 \\
\hline C & -0.147484 & 4.281142 & -0.151371 \\
\hline $\mathrm{H}$ & 0.692580 & 4.945790 & -0.381332 \\
\hline$H$ & -0.856821 & 4.295296 & -0.986803 \\
\hline $\mathrm{I}$ & -0.652453 & 4.644212 & 0.750730 \\
\hline h & 2.545737 & -2.010301 & 0.028197 \\
\hline $\mathrm{H}$ & 2.574614 & -2.851745 & 0.712668 \\
\hline $\mathrm{C}$ & 1.577353 & -1.798179 & -0.994016 \\
\hline $\mathrm{C}$ & 3.465744 & -0.923920 & 0.006273 \\
\hline $\mathrm{H}$ & 0.738464 & -2.449609 & -1.216233 \\
\hline $\mathrm{C}$ & 1.870289 & -0.560930 & -1.628767 \\
\hline $\mathrm{H}$ & 4.319237 & -0.802494 & 0.663632 \\
\hline C & 3.041786 & -0.007592 & -0.997325 \\
\hline $\mathrm{H}$ & 1.317116 & -0.106872 & -2.444348 \\
\hline $\mathrm{H}$ & 3.533759 & 0.920049 & -1.269023 \\
\hline C & -1.696670 & -0.631252 & 1.076996 \\
\hline C & -0.396174 & -0.470071 & 1.026137 \\
\hline Si & -2.745075 & -0.423280 & -0.499982 \\
\hline $\mathrm{C}$ & -1.669971 & -0.249647 & -2.023462 \\
\hline $\mathrm{H}$ & -2.309018 & -0.046506 & -2.894967 \\
\hline $\mathrm{H}$ & -0.960508 & 0.583935 & -1.930614 \\
\hline $\mathrm{H}$ & -1.102539 & -1.164792 & -2.239687 \\
\hline $\mathrm{C}$ & -3.869155 & -1.919488 & -0.640404 \\
\hline $\mathrm{H}$ & -4.597834 & -1.953262 & 0.181207 \\
\hline $\mathrm{H}$ & -4.436053 & -1.883124 & -1.581439 \\
\hline $\mathrm{H}$ & -3.302475 & -2.860493 & -0.629265 \\
\hline $\mathrm{C}$ & -3.770961 & 1.132957 & -0.270836 \\
\hline $\mathrm{H}$ & -4.374291 & 1.323255 & -1.170123 \\
\hline $\mathrm{H}$ & -4.463682 & 1.051167 & 0.577675 \\
\hline $\mathrm{H}$ & -3.137818 & 2.015277 & -0.103259 \\
\hline $\mathrm{C}$ & -2.435173 & -0.930774 & 2.360757 \\
\hline $\mathrm{H}$ & -1.770499 & -0.919673 & 3.234324 \\
\hline $\mathrm{H}$ & -3.235977 & -0.196919 & 2.530542 \\
\hline $\mathrm{H}$ & -2.913209 & -1.919134 & 2.308184 \\
\hline $\mathrm{H}$ & 2.390909 & 0.718266 & 1.493169 \\
\hline $\mathrm{H}$ & 0.347510 & -0.536892 & 1.959752 \\
\hline
\end{tabular}




\section{E5-TSb}

$$
G=-945.829198 \quad Z P E=-945.779354
$$

$\begin{array}{lccc}\mathrm{Ru} & -1.202127 & -0.264117 & 0.268534 \\ \mathrm{C} & -2.141241 & 2.803675 & -0.194862 \\ \mathrm{~N} & -1.808832 & 1.712412 & 0.004031 \\ \mathrm{C} & -2.559597 & 4.161928 & -0.443825 \\ \mathrm{H} & -1.749783 & 4.854629 & -0.189948 \\ \mathrm{H} & -2.812515 & 4.283446 & -1.503285 \\ \mathrm{H} & -3.440243 & 4.400939 & 0.162681 \\ \mathrm{C} & -0.926702 & -2.120773 & -0.795332 \\ \mathrm{H} & 0.019540 & -2.345058 & -1.277382 \\ \mathrm{C} & -1.296696 & -2.453388 & 0.529001 \\ \mathrm{C} & -2.017383 & -1.375883 & -1.375427 \\ \mathrm{H} & -0.689208 & -2.990235 & 1.249906 \\ \mathrm{C} & -2.662982 & -2.024171 & 0.727015 \\ \mathrm{H} & -2.055240 & -0.986632 & -2.387273 \\ \mathrm{C} & -3.108270 & -1.388372 & -0.433157 \\ \mathrm{H} & -3.217693 & -2.113027 & 1.656211 \\ \mathrm{H} & -4.072126 & -0.910764 & -0.573545 \\ \mathrm{C} & 1.868492 & 0.405387 & 1.033946 \\ \mathrm{C} & 0.552038 & 0.272788 & 1.000038 \\ \mathrm{Si} & 2.984531 & 0.038615 & -0.459745 \\ \mathrm{C} & 2.088967 & 0.206677 & -2.102142 \\ \mathrm{H} & 2.830166 & 0.120250 & -2.910932 \\ \mathrm{H} & 1.338177 & -0.577171 & -2.273115 \\ \mathrm{H} & 1.598314 & 1.183321 & -2.216082 \\ \mathrm{C} & 4.430199 & 1.237026 & -0.418725 \\ \mathrm{H} & 5.039509 & 1.111704 & 0.486686 \\ \mathrm{H} & 5.087366 & 1.061468 & -1.282395 \\ \mathrm{H} & 4.100101 & 2.284029 & -0.460917 \\ \mathrm{H} & 3.609290 & -1.721887 & -0.251694 \\ \mathrm{H} & 4.285756 & -1.998675 & -1.072774 \\ \mathrm{H} & 2.16448493657 & -1.843859 & 0.688970 \\ \mathrm{H} & -2.442709 & -0.244547 \\ \mathrm{H} & 0.776286 & 2.320695\end{array}$




$\begin{array}{rrrr}\mathrm{H} & 1.872701 & 0.843288 & 3.168012 \\ \mathrm{H} & 3.343081 & 0.038348 & 2.577900 \\ \mathrm{H} & 3.077947 & 1.745781 & 2.223056 \\ \mathrm{H} & -0.020708 & 0.206634 & -0.697443 \\ \mathrm{H} & -0.133535 & 0.492204 & 1.895735\end{array}$

E6b

$G=-945.891626 \quad Z P E=-945.84124$

$\begin{array}{lccc}\mathrm{H} & -0.390179 & -2.196170 & 1.808995 \\ \mathrm{C} & 0.119770 & -1.852098 & 0.902758 \\ \mathrm{Ru} & -1.047089 & -0.089643 & 0.158667 \\ \mathrm{Si} & 2.384580 & -0.695934 & -0.360433 \\ \mathrm{C} & 0.994884 & -0.772351 & 0.938008 \\ \mathrm{C} & -2.590622 & -1.594637 & -0.050183 \\ \mathrm{C} & -3.074967 & -0.519798 & 0.749597 \\ \mathrm{C} & -3.070550 & 0.674633 & -0.046642 \\ \mathrm{C} & -2.551142 & 0.342648 & -1.324975 \\ \mathrm{C} & -2.227270 & -1.059006 & -1.326620 \\ \mathrm{H} & -2.483820 & -2.626829 & 0.263889 \\ \mathrm{H} & -3.379035 & -0.586036 & 1.789419 \\ \mathrm{H} & -3.358151 & 1.663821 & 0.293824 \\ \mathrm{H} & -2.359453 & 1.034852 & -2.138673 \\ \mathrm{H} & -1.792113 & -1.608883 & -2.155387 \\ \mathrm{C} & 0.399537 & 2.865501 & -0.034917 \\ \mathrm{~N} & -0.034667 & 1.791143 & -0.027480 \\ \mathrm{C} & 0.966096 & 4.191514 & -0.026798 \\ \mathrm{H} & 0.525491 & 4.775378 & 0.789439 \\ \mathrm{H} & 2.050602 & 4.128655 & 0.122143 \\ \mathrm{H} & 0.759914 & 4.695787 & -0.977443 \\ \mathrm{C} & 1.220325 & -0.063890 & 2.257123 \\ \mathrm{H} & 1.375066 & 1.016396 & 2.137834 \\ \mathrm{H} & 0.385415 & -0.214759 & 2.958092 \\ \mathrm{H} & 2.126936 & -0.460256 & 2.744167 \\ \mathrm{H} & 0.142066 & -2.578856 & 0.085566 \\ \mathrm{C} & 1.714390 & -1.021586 & -2.085511 \\ \mathrm{H} & 1.176512 & -1.977415 & -2.155559\end{array}$




$\begin{array}{llll}\mathrm{H} & 1.033348 & -0.220640 & -2.409686 \\ \mathrm{H} & 2.542983 & -1.060127 & -2.808443 \\ \mathrm{C} & 3.588860 & -2.061172 & 0.115777 \\ \mathrm{H} & 3.957823 & -1.938965 & 1.144246 \\ \mathrm{H} & 3.108190 & -3.047871 & 0.048751 \\ \mathrm{H} & 4.463190 & -2.069998 & -0.551298 \\ \mathrm{C} & 3.277922 & 0.956694 & -0.344797 \\ \mathrm{H} & 2.706121 & 1.731083 & -0.873944 \\ \mathrm{H} & 3.494850 & 1.319691 & 0.669439 \\ \mathrm{H} & 4.241337 & 0.851764 & -0.865211\end{array}$

\section{F1}

$G=-813.19373 \quad Z P E=-813.14812$

$\begin{array}{lccc}\mathrm{H} & -1.362494 & 0.910089 & -1.887256 \\ \mathrm{C} & -1.097476 & 0.707966 & -0.839452 \\ \mathrm{Ru} & 0.700569 & -0.166804 & -0.061437 \\ \mathrm{C} & -0.220948 & 1.532503 & -0.198827 \\ \mathrm{C} & 2.637246 & 0.787203 & -0.375073 \\ \mathrm{C} & 2.658126 & 0.421153 & 0.995411 \\ \mathrm{C} & 2.567129 & -1.000893 & 1.062226 \\ \mathrm{C} & 2.519516 & -1.525408 & -0.248845 \\ \mathrm{C} & 2.527038 & -0.417287 & -1.161730 \\ \mathrm{H} & 2.697594 & 1.793523 & -0.777514 \\ \mathrm{H} & 2.716217 & 1.096597 & 1.842130 \\ \mathrm{H} & 2.494016 & -1.581504 & 1.976738 \\ \mathrm{H} & 2.459317 & -2.573525 & -0.517951 \\ \mathrm{H} & 2.542337 & -0.473381 & -2.244622 \\ \mathrm{H} & 0.113451 & -1.284552 & -1.066273 \\ \mathrm{Si} & -2.456439 & -0.323532 & 0.051326 \\ \mathrm{C} & 0.014031 & 2.962388 & -0.011219 \\ \mathrm{H} & 0.994892 & 3.202047 & 0.411187 \\ \mathrm{H} & -0.126285 & 3.491919 & -0.966515 \\ \mathrm{H} & -0.765535 & 3.347787 & 0.665085 \\ \mathrm{C} & -3.858841 & 0.861365 & 0.412254 \\ \mathrm{H} & -4.669154 & 0.346991 & 0.948518 \\ \mathrm{H} & -3.519452 & 1.697773 & 1.038984\end{array}$




$\begin{array}{lrrr}\mathrm{H} & -4.282069 & 1.278900 & -0.510949 \\ \mathrm{C} & -1.714568 & -0.975203 & 1.651180 \\ \mathrm{H} & -2.398685 & -1.709884 & 2.100346 \\ \mathrm{H} & -0.755161 & -1.507859 & 1.518134 \\ \mathrm{H} & -1.562028 & -0.174988 & 2.387502 \\ \mathrm{C} & -2.982583 & -1.693936 & -1.102762 \\ \mathrm{H} & -2.191391 & -2.444595 & -1.228604 \\ \mathrm{H} & -3.872069 & -2.204229 & -0.707524 \\ \mathrm{H} & -3.240414 & -1.301364 & -2.095720\end{array}$

\section{F1-TSa}

$G=-945.815412 \quad Z P E=-945.764595$

$\begin{array}{lccc}\mathrm{H} & 0.858161 & -1.682680 & 2.083546 \\ \mathrm{C} & 0.562472 & -0.778612 & 1.548642 \\ \mathrm{Ru} & -0.748516 & -0.087321 & -0.111212 \\ \mathrm{C} & -0.256160 & 0.162881 & 1.888790 \\ \mathrm{C} & -2.806225 & -0.624314 & 0.486329 \\ \mathrm{C} & -2.834583 & 0.696063 & -0.050326 \\ \mathrm{C} & -2.447253 & 0.613298 & -1.427518 \\ \mathrm{C} & -2.189867 & -0.742108 & -1.735396 \\ \mathrm{C} & -2.374662 & -1.516289 & -0.543142 \\ \mathrm{H} & -3.041319 & -0.908039 & 1.507780 \\ \mathrm{H} & -3.116958 & 1.601636 & 0.476794 \\ \mathrm{H} & -2.350097 & 1.449243 & -2.112720 \\ \mathrm{H} & -1.860376 & -1.123222 & -2.695948 \\ \mathrm{H} & -2.265851 & -2.591974 & -0.455530 \\ \mathrm{C} & 1.116597 & 2.555243 & -0.500986 \\ \mathrm{~N} & 0.495577 & 1.586599 & -0.365138 \\ \mathrm{C} & 1.919505 & 3.741043 & -0.683853 \\ \mathrm{H} & 2.975950 & 3.460165 & -0.769727 \\ \mathrm{H} & 1.614804 & 4.264241 & -1.597270 \\ \mathrm{H} & 1.798378 & 4.416276 & 0.170359 \\ \mathrm{H} & 0.267481 & -0.934891 & -1.059561 \\ \mathrm{Si} & 1.957462 & -1.069911 & -0.083470 \\ \mathrm{C} & -0.856546 & 0.974706 & 2.949506 \\ \mathrm{H} & -1.946195 & 0.843820 & 2.983121\end{array}$




$\begin{array}{lccc}\mathrm{H} & -0.432663 & 0.733000 & 3.932960 \\ \mathrm{H} & -0.672560 & 2.040164 & 2.747179 \\ \mathrm{C} & 3.277645 & -0.215035 & 0.958125 \\ \mathrm{H} & 4.261756 & -0.408937 & 0.504681 \\ \mathrm{H} & 3.128754 & 0.873208 & 0.977141 \\ \mathrm{H} & 3.292534 & -0.572749 & 1.995273 \\ \mathrm{C} & 2.459083 & -0.616951 & -1.856866 \\ \mathrm{H} & 3.531802 & -0.841828 & -1.966038 \\ \mathrm{H} & 1.911058 & -1.205265 & -2.605292 \\ \mathrm{H} & 2.314361 & 0.446166 & -2.087402 \\ \mathrm{C} & 2.033106 & -2.939557 & 0.039411 \\ \mathrm{H} & 1.117642 & -3.406084 & -0.350163 \\ \mathrm{H} & 2.874265 & -3.306358 & -0.565691 \\ \mathrm{H} & 2.181620 & -3.293402 & 1.068090\end{array}$

\section{F1-TSb}

$G=-813.190551 \quad Z P E=-813.145617$

$\begin{array}{lccc}\mathrm{H} & -1.665272 & 1.159534 & -1.860783 \\ \mathrm{C} & -0.996439 & 1.027403 & -1.010285 \\ \mathrm{Ru} & 0.648152 & 0.105860 & -0.368184 \\ \mathrm{C} & -0.263487 & 1.851281 & -0.251146 \\ \mathrm{C} & 2.660255 & 0.486568 & 0.511929 \\ \mathrm{C} & 1.936141 & -0.256847 & 1.466724 \\ \mathrm{C} & 1.602878 & -1.526394 & 0.877094 \\ \mathrm{C} & 2.174422 & -1.589528 & -0.409610 \\ \mathrm{C} & 2.771740 & -0.319418 & -0.676689 \\ \mathrm{H} & 3.039281 & 1.496391 & 0.626649 \\ \mathrm{H} & 1.633774 & 0.075134 & 2.454668 \\ \mathrm{H} & 1.012420 & -2.305451 & 1.349814 \\ \mathrm{H} & 2.101735 & -2.418569 & -1.104209 \\ \mathrm{H} & 3.275465 & -0.033250 & -1.593685 \\ \mathrm{H} & 0.269941 & -0.687490 & -1.722165 \\ \mathrm{Si} & -2.008611 & -0.549833 & 0.118148 \\ \mathrm{C} & -0.204857 & 3.197647 & 0.314470 \\ \mathrm{H} & 0.802901 & 3.485679 & 0.631834 \\ \mathrm{H} & -0.603201 & 3.939577 & -0.391517\end{array}$




$\begin{array}{lccc}\mathrm{H} & -0.859512 & 3.221894 & 1.200779 \\ \mathrm{C} & -3.622527 & 0.358592 & -0.187381 \\ \mathrm{H} & -4.412258 & -0.184884 & 0.353694 \\ \mathrm{H} & -3.598087 & 1.386077 & 0.199349 \\ \mathrm{H} & -3.898905 & 0.390175 & -1.248599 \\ \mathrm{C} & -1.721515 & -0.546742 & 1.967223 \\ \mathrm{H} & -0.914445 & -1.211768 & 2.294228 \\ \mathrm{H} & -1.504140 & 0.467414 & 2.330276 \\ \mathrm{H} & -2.651691 & -0.876519 & 2.455063 \\ \mathrm{C} & -2.041323 & -2.213489 & -0.722882 \\ \mathrm{H} & -1.172119 & -2.834122 & -0.472953 \\ \mathrm{H} & -2.951426 & -2.751830 & -0.419322 \\ \mathrm{H} & -2.074311 & -2.092597 & -1.813769\end{array}$

F2

$G=-945.84417 \quad Z P E=-945.791183$

$\begin{array}{lccc}\mathrm{H} & 0.002112 & 0.073818 & 2.943092 \\ \mathrm{C} & -0.713057 & 0.360195 & 2.190441 \\ \mathrm{Ru} & -0.650855 & -0.317884 & 0.052860 \\ \mathrm{C} & -1.693931 & 0.937776 & 1.698161 \\ \mathrm{C} & -2.578504 & -1.317139 & -0.116766 \\ \mathrm{C} & -2.315767 & -0.577880 & -1.314125 \\ \mathrm{C} & -1.148957 & -1.146656 & -1.932468 \\ \mathrm{C} & -0.696327 & -2.196198 & -1.092214 \\ \mathrm{C} & -1.573364 & -2.313294 & 0.037005 \\ \mathrm{H} & -3.386606 & -1.123367 & 0.582278 \\ \mathrm{H} & -2.911176 & 0.244660 & -1.698584 \\ \mathrm{H} & -0.684574 & -0.819526 & -2.857125 \\ \mathrm{H} & 0.201230 & -2.788261 & -1.251746 \\ \mathrm{H} & -1.501998 & -3.042719 & 0.835951 \\ \mathrm{C} & 0.368883 & 2.635798 & -0.861564 \\ \mathrm{~N} & 0.030231 & 1.582724 & -0.514793 \\ \mathrm{C} & 0.822391 & 3.937658 & -1.292803 \\ \mathrm{H} & 0.282856 & 4.246649 & -2.195107 \\ \mathrm{H} & 0.650012 & 4.677096 & -0.502946 \\ \mathrm{H} & 1.895283 & 3.899795 & -1.515998\end{array}$




$\begin{array}{lccc}\mathrm{H} & 0.994072 & -0.714900 & 0.708172 \\ \mathrm{Si} & 2.481951 & -0.509648 & 0.303551 \\ \mathrm{C} & -2.855026 & 1.764739 & 1.391729 \\ \mathrm{H} & -3.724975 & 1.157901 & 1.109388 \\ \mathrm{H} & -3.131482 & 2.362917 & 2.270610 \\ \mathrm{H} & -2.641426 & 2.452959 & 0.563203 \\ \mathrm{C} & 3.035080 & 0.988639 & 1.271052 \\ \mathrm{H} & 4.110896 & 1.159113 & 1.124155 \\ \mathrm{H} & 2.506956 & 1.898148 & 0.956171 \\ \mathrm{H} & 2.865821 & 0.851724 & 2.347304 \\ \mathrm{C} & 2.713965 & -0.345062 & -1.542423 \\ \mathrm{H} & 3.789204 & -0.298541 & -1.768792 \\ \mathrm{H} & 2.307786 & -1.215729 & -2.074332 \\ \mathrm{H} & 2.244636 & 0.558645 & -1.950885 \\ \mathrm{C} & 3.235427 & -2.100366 & 0.934002 \\ \mathrm{H} & 2.824007 & -2.971367 & 0.406018 \\ \mathrm{H} & 4.322042 & -2.096127 & 0.768819 \\ \mathrm{H} & 3.059150 & -2.239270 & 2.008192\end{array}$

\section{F2-TS}

$\mathrm{G}=-945.8324 \quad \mathrm{ZPE}=-945.782424$

$\begin{array}{lccc}\mathrm{H} & 0.406247 & -0.337285 & 1.448419 \\ \mathrm{C} & -0.786598 & 0.507388 & 2.194455 \\ \mathrm{Ru} & -0.536301 & -0.239547 & 0.068360 \\ \mathrm{Si} & 1.994196 & -0.568256 & 0.321354 \\ \mathrm{C} & -1.709712 & 0.961322 & 1.470356 \\ \mathrm{C} & -2.343483 & -1.514157 & -0.261501 \\ \mathrm{C} & -2.146911 & -0.689288 & -1.408261 \\ \mathrm{C} & -0.880136 & -1.041349 & -1.975897 \\ \mathrm{C} & -0.315761 & -2.081042 & -1.188448 \\ \mathrm{C} & -1.209607 & -2.361184 & -0.107388 \\ \mathrm{H} & -3.189830 & -1.469228 & 0.417654 \\ \mathrm{H} & -2.832324 & 0.061392 & -1.787640 \\ \mathrm{H} & -0.425730 & -0.592776 & -2.854269 \\ \mathrm{H} & 0.622739 & -2.587761 & -1.388372 \\ \mathrm{H} & -1.061740 & -3.103329 & 0.670272\end{array}$




$\begin{array}{lrrr}\mathrm{C} & 0.525422 & 2.699078 & -0.788662 \\ \mathrm{~N} & 0.171542 & 1.644271 & -0.464810 \\ \mathrm{C} & 0.997977 & 4.002607 & -1.193153 \\ \mathrm{H} & 0.450463 & 4.785324 & -0.656319 \\ \mathrm{H} & 2.066109 & 4.095921 & -0.964337 \\ \mathrm{H} & 0.848720 & 4.138717 & -2.270181 \\ \mathrm{C} & -2.910424 & 1.730302 & 1.139733 \\ \mathrm{H} & -2.712761 & 2.417698 & 0.306445 \\ \mathrm{H} & -3.728077 & 1.065829 & 0.831446 \\ \mathrm{H} & -3.249736 & 2.316308 & 2.003838 \\ \mathrm{H} & -0.295496 & 0.373687 & 3.147101 \\ \mathrm{C} & 2.697288 & -0.357625 & -1.410822 \\ \mathrm{H} & 2.376381 & -1.152731 & -2.095907 \\ \mathrm{H} & 2.420511 & 0.607566 & -1.854429 \\ \mathrm{H} & 3.796110 & -0.395934 & -1.351103 \\ \mathrm{C} & 2.402535 & -2.273671 & 0.999163 \\ \mathrm{H} & 1.967058 & -2.416568 & 1.998162 \\ \mathrm{H} & 2.049236 & -3.092512 & 0.359116 \\ \mathrm{H} & 3.493955 & -2.375951 & 1.095215 \\ \mathrm{C} & 2.779313 & 0.708189 & 1.460660 \\ \mathrm{H} & 2.516571 & 0.519848 & 2.511367 \\ \mathrm{H} & 3.874982 & 0.646172 & 1.379892 \\ \mathrm{H} & 2.484901 & 1.737946 & 1.219094 \\ & & & \end{array}$

F3

$G=-945.860268 \quad Z P E=-945.811376$

$\begin{array}{lccc}\mathrm{H} & 1.834885 & 0.055855 & 2.543312 \\ \mathrm{C} & 0.930631 & 0.511565 & 2.131093 \\ \mathrm{Ru} & 0.460867 & -0.453113 & 0.269007 \\ \mathrm{Si} & -0.338697 & 1.783947 & -0.440673 \\ \mathrm{C} & -0.248129 & -0.229351 & 2.011002 \\ \mathrm{C} & 2.431584 & -1.579398 & 0.208021 \\ \mathrm{C} & 1.563045 & -2.227719 & -0.693779 \\ \mathrm{C} & 1.238071 & -1.288623 & -1.736168 \\ \mathrm{C} & 1.943544 & -0.091182 & -1.503894 \\ \mathrm{C} & 2.623429 & -0.232747 & -0.257109\end{array}$




$\begin{array}{lccc}\mathrm{H} & 2.838234 & -1.995039 & 1.124456 \\ \mathrm{H} & 1.185785 & -3.241307 & -0.612609 \\ \mathrm{H} & 0.563590 & -1.475730 & -2.566692 \\ \mathrm{H} & 1.941911 & 0.789713 & -2.135660 \\ \mathrm{H} & 3.255342 & 0.516613 & 0.209077 \\ \mathrm{C} & -2.514985 & -1.388015 & -0.624781 \\ \mathrm{~N} & -1.472209 & -1.034596 & -0.266142 \\ \mathrm{C} & -3.815894 & -1.819589 & -1.076920 \\ \mathrm{H} & -3.935159 & -2.896353 & -0.912517 \\ \mathrm{H} & -4.596674 & -1.285313 & -0.523948 \\ \mathrm{H} & -3.927804 & -1.604555 & -2.145794 \\ \mathrm{C} & -1.480487 & -0.404080 & 2.781475 \\ \mathrm{H} & -2.286913 & -0.892453 & 2.225855 \\ \mathrm{H} & -1.246969 & -1.013151 & 3.668974 \\ \mathrm{H} & -1.826208 & 0.567837 & 3.166615 \\ \mathrm{H} & 0.883161 & 1.599675 & 2.263644 \\ \mathrm{C} & -1.014224 & 1.694362 & -2.198005 \\ \mathrm{H} & -0.260186 & 1.378446 & -2.930863 \\ \mathrm{H} & -1.870194 & 1.010583 & -2.275616 \\ \mathrm{H} & -1.365150 & 2.695138 & -2.494883 \\ \mathrm{C} & 1.038863 & 3.068540 & -0.376501 \\ \mathrm{H} & 1.563753 & 3.081742 & 0.588211 \\ \mathrm{H} & 1.787307 & 2.924917 & -1.167265 \\ \mathrm{H} & 0.596150 & 4.066309 & -0.521923 \\ \mathrm{H} & -1.738198 & 2.379009 & 0.671357 \\ \mathrm{H} & -1.412467 & 2.550024 & 1.706549 \\ & -2.115235 & 3.339220 & 0.286042 \\ -2.581585 & 1.675081 & 0.686404 \\ & & & \\ \mathrm{H} & & \end{array}$

\section{F3-TS}

$G=-945.853944 \quad Z P E=-945.804823$

$\begin{array}{lllc}\mathrm{H} & 0.171202 & -0.228831 & 3.255113 \\ \mathrm{C} & 0.692686 & -0.231655 & 2.293705 \\ \mathrm{Ru} & -0.332372 & -0.524537 & 0.280566 \\ \mathrm{Si} & 1.803604 & 0.778445 & -0.589516 \\ \mathrm{C} & 0.543577 & 0.847111 & 1.439947\end{array}$




$\begin{array}{lccc}\mathrm{C} & -0.741938 & -2.646446 & 0.797227 \\ \mathrm{C} & -1.785222 & -2.193565 & -0.058051 \\ \mathrm{C} & -1.199445 & -1.875596 & -1.329799 \\ \mathrm{C} & 0.191420 & -2.125141 & -1.248395 \\ \mathrm{C} & 0.495946 & -2.577608 & 0.077806 \\ \mathrm{H} & -0.862671 & -2.955789 & 1.828099 \\ \mathrm{H} & -2.833398 & -2.114546 & 0.199511 \\ \mathrm{H} & -1.733228 & -1.502387 & -2.195036 \\ \mathrm{H} & 0.902571 & -1.975197 & -2.050773 \\ \mathrm{H} & 1.465303 & -2.900203 & 0.435273 \\ \mathrm{C} & -2.627246 & 1.681596 & -0.378085 \\ \mathrm{~N} & -1.734180 & 0.990019 & -0.115885 \\ \mathrm{C} & -3.749663 & 2.544666 & -0.699453 \\ \mathrm{H} & -4.649153 & 2.206949 & -0.172949 \\ \mathrm{H} & -3.532702 & 3.574589 & -0.395685 \\ \mathrm{H} & -3.943784 & 2.530961 & -1.777428 \\ \mathrm{C} & 0.148312 & 2.233479 & 1.823283 \\ \mathrm{H} & -0.298393 & 2.802427 & 1.004659 \\ \mathrm{H} & -0.567962 & 2.183303 & 2.656672 \\ \mathrm{H} & 1.021185 & 2.785485 & 2.192480 \\ \mathrm{H} & 1.480204 & -0.974836 & 2.200735 \\ \mathrm{H} & 1.079472 & 1.165497 & -2.298542 \\ \mathrm{H} & 0.603953 & 0.318473 & -2.800904 \\ \mathrm{H} & 0.336370 & 1.967360 & -2.207668 \\ \mathrm{H} & 1.893815 & 1.538854 & -2.935485 \\ \mathrm{H} & 3.167168 & -0.529061 & -0.530008 \\ \mathrm{H} & 3.360974 & -0.844691 & 0.503354 \\ \mathrm{H} & 2.962447 & -1.423389 & -1.127443 \\ \mathrm{H} & 3.094189 & -0.076660 & -0.906707 \\ \mathrm{H} & 2.395618410 & 2.397810 & -0.062271 \\ \mathrm{H} & 2.314424 & 0.896910 \\ \mathrm{H} & 2.626860 & -0.831320 \\ \mathrm{H} & 3.251574 & -0.016079\end{array}$

\section{C3(SiPhMe $)$}

$G=-1270.057244 \quad Z P E=-1269.9959$ 


\begin{tabular}{|c|c|c|c|}
\hline $\mathrm{H}$ & 0.072618 & 1.454733 & 1.198984 \\
\hline C & 0.698557 & 0.571371 & 1.022803 \\
\hline$P_{11}$ & -1.841371 & -0.532131 & -0.162475 \\
\hline C & -0.033280 & -0.599990 & 0.562668 \\
\hline 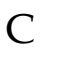 & -1.600910 & -2.344770 & -1.391196 \\
\hline C & -2.543320 & -2.635453 & -0.362926 \\
\hline C & -3.723764 & -1.852261 & -0.632923 \\
\hline C & -3.495515 & -1.055406 & -1.756908 \\
\hline C & -2.144246 & -1.308044 & -2.203689 \\
\hline $\mathrm{H}$ & -0.626201 & -2.803981 & -1.519734 \\
\hline$\Pi$ & -2.447327 & -3.396312 & 0.405128 \\
\hline $\mathrm{H}$ & -4.615575 & -1.835149 & -0.012865 \\
\hline $\mathrm{H}$ & -4.178090 & -0.324141 & -2.176914 \\
\hline $\mathrm{H}$ & -1.672129 & -0.868566 & -3.077423 \\
\hline $\mathrm{C}$ & -2.948494 & 0.487333 & 2.707984 \\
\hline$T$ & -2.519370 & 0.129343 & 1.692928 \\
\hline 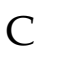 & -3.492553 & 0.925485 & 3.972691 \\
\hline $\mathrm{H}$ & -3.715514 & 0.058117 & 4.604334 \\
\hline $\mathrm{H}$ & -2.770027 & 1.566102 & 4.490393 \\
\hline $\mathrm{H}$ & -4.416010 & 1.491854 & 3.807219 \\
\hline $\mathrm{H}$ & 1.318238 & 0.368734 & 1.912511 \\
\hline S & 2.016157 & 1.109472 & -0.304109 \\
\hline$C$ & 0.742013 & -1.876524 & 0.674674 \\
\hline $\mathrm{H}$ & 0.104678 & -2.758827 & 0.794418 \\
\hline $\mathrm{H}$ & 1.321030 & -2.028289 & -0.253173 \\
\hline 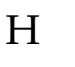 & 1.472782 & -1.848620 & 1.496955 \\
\hline C & 1.460809 & 0.772796 & -2.061441 \\
\hline $\mathrm{H}$ & 2.240042 & 1.100494 & -2.764470 \\
\hline 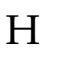 & 1.268007 & -0.292315 & -2.251129 \\
\hline $\mathrm{H}$ & 0.542929 & 1.324944 & -2.303578 \\
\hline C & -1.533801 & 2.589041 & -0.961185 \\
\hline $\mathrm{N}$ & -1.603201 & 1.478854 & -0.632645 \\
\hline C & -1.421925 & 3.966250 & -1.386251 \\
\hline $\mathrm{H}$ & -2.361630 & 4.299018 & -1.841694 \\
\hline 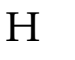 & -1.197782 & 4.609527 & -0.527624 \\
\hline 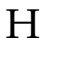 & -0.616731 & 4.063958 & -2.124811 \\
\hline & 2.250264 & 2.944923 & -0.005710 \\
\hline
\end{tabular}




$\begin{array}{lrrr}\mathrm{H} & 2.534051 & 3.147604 & 1.036287 \\ \mathrm{H} & 3.034917 & 3.356354 & -0.654921 \\ \mathrm{H} & 1.319049 & 3.493400 & -0.210501 \\ \mathrm{C} & 3.601969 & 0.166069 & 0.054252 \\ \mathrm{C} & 4.253666 & 0.319951 & 1.287856 \\ \mathrm{C} & 4.174411 & -0.704141 & -0.883544 \\ \mathrm{C} & 5.427675 & -0.368497 & 1.575840 \\ \mathrm{C} & 5.349223 & -1.397988 & -0.601856 \\ \mathrm{C} & 5.976626 & -1.231363 & 0.629134 \\ \mathrm{H} & 3.838639 & 0.991628 & 2.043636 \\ \mathrm{H} & 3.697507 & -0.847838 & -1.854618 \\ \mathrm{H} & 5.916744 & -0.233374 & 2.539704 \\ \mathrm{H} & 5.775072 & -2.070757 & -1.345204 \\ \mathrm{H} & 6.894578 & -1.773374 & 0.852398\end{array}$

\section{C3-TSa(SiPhMe2)}

$G=-1137.399038 \quad Z P E=-1137.34509$

$\mathrm{H} \quad-2.146743 \quad-1.971403 \quad-1.522679$

C $\quad-1.357530 \quad-1.655614 \quad-0.829400$

$\mathrm{Ru} \quad 1.287147 \quad-0.316644 \quad 0.006222$

C $\quad-0.554952 \quad-0.570784 \quad-1.069863$

$\begin{array}{llll}\text { C } & 2.021464 & -2.022741 & -1.122022\end{array}$

C $\quad 2.997077 \quad-0.981476 \quad-1.155735$

$\begin{array}{llll}C & 3.457385 & -0.749939 & 0.185871\end{array}$

C $\quad 2.720290 \quad-1.615196 \quad 1.035014$

$\begin{array}{llll}\text { C } & 1.831568 & -2.414708 & 0.239703\end{array}$

$\mathrm{H} \quad 1.489298 \quad-2.418700 \quad-1.982251$

$\mathrm{H} \quad 3.336146 \quad-0.460629 \quad-2.046390$

$\mathrm{H} \quad 4.205953 \quad-0.027822 \quad 0.493750$

$\begin{array}{llll}\mathrm{H} & 2.782261 & -1.639468 & 2.119439\end{array}$

$\mathrm{H} \quad 1.172354 \quad-3.196331 \quad 0.604043$

$\begin{array}{llll}\text { C } & 1.650714 & 2.722034 & -1.121413\end{array}$

N $1.417929 \quad 1.674395 \quad-0.680074$

$\begin{array}{llll}\text { C } & 1.929960 & 4.029564 & -1.670477\end{array}$

$\mathrm{H} \quad 2.274623 \quad 3.936664 \quad-2.706618$ 


$\begin{array}{lccc}\mathrm{H} & 1.023433 & 4.645319 & -1.652266 \\ \mathrm{H} & 2.708560 & 4.526978 & -1.081130 \\ \mathrm{H} & -1.211849 & -2.306306 & 0.037603 \\ \mathrm{Si} & -1.542439 & 0.125459 & 0.615582 \\ \mathrm{C} & -0.754292 & 0.153845 & -2.373767 \\ \mathrm{H} & -0.666375 & 1.241527 & -2.278546 \\ \mathrm{H} & 0.045486 & -0.165589 & -3.059452 \\ \mathrm{H} & -1.717793 & -0.084785 & -2.850337 \\ \mathrm{C} & -1.922424 & 1.877540 & 0.094112 \\ \mathrm{H} & -1.054008 & 2.460253 & -0.229159 \\ \mathrm{H} & -2.663960 & 1.873887 & -0.716619 \\ \mathrm{H} & -2.383907 & 2.383533 & 0.954783 \\ \mathrm{C} & -3.171730 & -0.594455 & 1.211973 \\ \mathrm{H} & -3.102073 & -1.630410 & 1.565761 \\ \mathrm{H} & -3.538850 & 0.023637 & 2.045717 \\ \mathrm{H} & -3.926316 & -0.558404 & 0.413757 \\ \mathrm{C} & -0.235498 & -0.032371 & 1.944529 \\ \mathrm{C} & 0.671945 & 1.018281 & 2.211110 \\ \mathrm{C} & -0.216022 & -1.155735 & 2.816403 \\ \mathrm{C} & 1.556509 & 0.945631 & 3.304570 \\ \mathrm{H} & 0.604771 & 1.953034 & 1.657486 \\ \mathrm{C} & 0.640512 & -1.210768 & 3.894604 \\ \mathrm{H} & -0.914344 & -1.977091 & 2.646892 \\ \mathrm{C} & 1.535804 & -0.153081 & 4.139544 \\ \mathrm{H} & 2.231955 & 1.776298 & 3.502553 \\ \mathrm{H} & 0.616467 & -2.066586 & 4.567433 \\ & 2.202539 & -0.197785 & 4.999320\end{array}$

\section{C3-TS(SiPhMe $)$}

$G=-1270.030338 \quad Z P E=-1269.969327$

$\begin{array}{lccc}\mathrm{H} & -1.737377 & -2.151538 & -1.271467 \\ \mathrm{C} & -0.988031 & -1.745163 & -0.579726 \\ \mathrm{Ru} & 1.791951 & -0.405743 & -0.239766 \\ \mathrm{C} & -0.294003 & -0.585283 & -0.843708 \\ \mathrm{C} & 2.087092 & -2.136658 & -1.540913\end{array}$




\begin{tabular}{|c|c|c|c|}
\hline$C$ & 3.077086 & -1.165094 & -1.841478 \\
\hline C & 3.888763 & -0.970409 & -0.671125 \\
\hline & 3.362712 & -1.812618 & 0.348140 \\
\hline & 2.246884 & -2.541760 & -0.172267 \\
\hline & 1.323034 & -2.494403 & -2.225113 \\
\hline & 3.195323 & -0.653675 & -2.792231 \\
\hline & 4.742674 & -0.307267 & -0.583200 \\
\hline & 3.723907 & -1.865397 & 1.371315 \\
\hline 1 & 1.674423 & -3.301506 & 0.349182 \\
\hline$C$ & 1.820879 & 2.688630 & -1.268023 \\
\hline & 1.757751 & 1.593508 & -0.892257 \\
\hline$C$ & 1.876423 & 4.057138 & -1.730361 \\
\hline $\mathrm{H}$ & 2.039227 & 4.083555 & -2.813664 \\
\hline $\mathrm{H}$ & 0.933297 & 4.566972 & -1.500329 \\
\hline 11 & 2.696610 & 4.590069 & -1.236216 \\
\hline $\mathrm{H}$ & -0.783765 & -2.358495 & 0.301568 \\
\hline $\mathrm{Si}$ & -1.527751 & 0.114426 & 0.670533 \\
\hline$C$ & -0.671075 & 0.094839 & -2.144407 \\
\hline $\mathrm{H}$ & -0.612062 & 1.188483 & -2.088933 \\
\hline $\mathrm{H}$ & 0.046133 & -0.215894 & -2.919120 \\
\hline $\mathrm{H}$ & -1.677476 & -0.183868 & -2.496053 \\
\hline $\mathrm{C}$ & -1.271888 & -0.730823 & 2.324019 \\
\hline $\mathrm{H}$ & -2.059631 & -1.471807 & 2.512000 \\
\hline $\mathrm{H}$ & -0.301809 & -1.234923 & 2.403101 \\
\hline $\mathrm{H}$ & -1.329511 & 0.022386 & 3.123573 \\
\hline $\mathrm{C}$ & -1.134550 & 1.949170 & 0.730231 \\
\hline $\mathrm{H}$ & -1.235700 & 2.449070 & -0.240830 \\
\hline $\mathrm{H}$ & -1.858077 & 2.411360 & 1.419365 \\
\hline $\mathrm{H}$ & -0.124412 & 2.132331 & 1.109542 \\
\hline 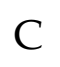 & 1.657221 & 0.799322 & 2.785173 \\
\hline $\mathrm{N}$ & 1.628543 & 0.393498 & 1.698892 \\
\hline C & 1.636574 & 1.298742 & 4.140954 \\
\hline $\mathrm{I}$ & 1.403073 & 0.482993 & 4.835171 \\
\hline $\mathrm{H}$ & 2.609871 & 1.725563 & 4.408189 \\
\hline $\mathrm{H}$ & 0.869235 & 2.076570 & 4.236986 \\
\hline $\mathrm{C}$ & -3.348207 & -0.006496 & 0.169991 \\
\hline$\Omega$ & -4.088897 & -1.173039 & 0.416951 \\
\hline & -4.009255 & 1.062151 & -0.451390 \\
\hline
\end{tabular}




$\begin{array}{lrrr}\mathrm{C} & -5.430850 & -1.268900 & 0.065049 \\ \mathrm{H} & -3.608393 & -2.031522 & 0.892419 \\ \mathrm{C} & -5.351993 & 0.972262 & -0.813680 \\ \mathrm{H} & -3.470951 & 1.987988 & -0.660564 \\ \mathrm{C} & -6.065592 & -0.193270 & -0.553401 \\ \mathrm{H} & -5.984358 & -2.183775 & 0.272848 \\ \mathrm{H} & -5.841840 & 1.816180 & -1.297849 \\ \mathrm{H} & -7.116125 & -0.265339 & -0.831569\end{array}$

\section{D1-TS(SiPhMe2)}

$G=-1138.561495 \quad Z P E=-1138.502505$

$\begin{array}{lccc}\mathrm{H} & 1.386156 & 0.224257 & -1.794995 \\ \mathrm{C} & 0.904914 & 0.013704 & -0.824215 \\ \mathrm{Ru} & -1.883017 & -0.500240 & 0.199987 \\ \mathrm{C} & -0.461601 & 0.474766 & -0.753054 \\ \mathrm{C} & -1.970424 & -2.213953 & -1.333452 \\ \mathrm{C} & -3.256623 & -1.647339 & -1.215709 \\ \mathrm{C} & -3.736868 & -1.881089 & 0.130705 \\ \mathrm{C} & -2.726903 & -2.542952 & 0.839409 \\ \mathrm{C} & -1.594064 & -2.693892 & -0.045038 \\ \mathrm{H} & -1.340589 & -2.204601 & -2.217317 \\ \mathrm{H} & -3.812707 & -1.157788 & -2.009127 \\ \mathrm{H} & -4.701797 & -1.572596 & 0.518890 \\ \mathrm{H} & -2.756433 & -2.843721 & 1.880929 \\ \mathrm{H} & -0.667391 & -3.204270 & 0.196031 \\ \mathrm{C} & -3.800807 & 2.133997 & 0.219886 \\ \mathrm{~N} & -3.068393 & 1.239218 & 0.167998 \\ \mathrm{C} & -4.712220 & 3.251622 & 0.288088 \\ \mathrm{H} & -5.642148 & 3.005370 & -0.236690 \\ \mathrm{H} & -4.261174 & 4.132393 & -0.182350 \\ \mathrm{H} & -4.942884 & 3.482045 & 1.334269 \\ \mathrm{H} & 1.056807 & -1.034144 & -0.530712 \\ \mathrm{Si} & 2.073011 & 0.991984 & 0.405899 \\ \mathrm{C} & -0.757496 & 1.703350 & -1.544284 \\ \mathrm{H} & -1.034057 & 2.526953 & -0.869395\end{array}$




$\begin{array}{lccc}\mathrm{H} & -1.633621 & 1.540441 & -2.186996 \\ \mathrm{H} & 0.085817 & 2.029450 & -2.168790 \\ \mathrm{C} & 2.105741 & 2.802963 & -0.058099 \\ \mathrm{H} & 2.863574 & 3.317762 & 0.549373 \\ \mathrm{H} & 1.140279 & 3.287962 & 0.140488 \\ \mathrm{H} & 2.358473 & 2.968253 & -1.113747 \\ \mathrm{C} & 1.514901 & 0.767994 & 2.174723 \\ \mathrm{H} & 2.282747 & 1.164194 & 2.854661 \\ \mathrm{H} & 1.341028 & -0.283430 & 2.440746 \\ \mathrm{H} & 0.585646 & 1.321573 & 2.365504 \\ \mathrm{H} & -0.605457 & -0.752611 & 1.143574 \\ \mathrm{H} & -1.572118 & 0.087728 & 1.697996 \\ \mathrm{C} & 3.745304 & 0.194027 & 0.120385 \\ \mathrm{C} & 4.279184 & -0.725238 & 1.033940 \\ \mathrm{C} & 4.492550 & 0.493670 & -1.028420 \\ \mathrm{C} & 5.515675 & -1.325452 & 0.809461 \\ \mathrm{H} & 3.726160 & -0.977487 & 1.940426 \\ \mathrm{C} & 5.728758 & -0.102158 & -1.257643 \\ \mathrm{H} & 4.105050 & 1.207475 & -1.758970 \\ \mathrm{C} & 6.241355 & -1.013721 & -0.336870 \\ \mathrm{H} & 5.914964 & -2.035831 & 1.532158 \\ \mathrm{H} & 6.295774 & 0.145827 & -2.153922 \\ \mathrm{H} & 7.209293 & -1.480816 & -0.513314\end{array}$

\section{D2-TS(SiPhMe $)$}

$G=-1138.560477 \quad Z P E=-1138.503471$

$\begin{array}{llll}\mathrm{H} & 1.136813 & -0.292647 & -1.608106 \\ \mathrm{C} & 0.797465 & 0.741223 & -1.492777 \\ \mathrm{Ru} & -1.819642 & -0.585212 & -0.729608 \\ \mathrm{C} & -0.623501 & 0.907183 & -1.300618 \\ \mathrm{C} & -0.561758 & -2.386701 & -1.122094 \\ \mathrm{C} & -0.276577 & -2.062575 & 0.234425 \\ \mathrm{C} & -1.482177 & -2.178997 & 0.968939 \\ \mathrm{C} & -2.515621 & -2.617693 & 0.092647 \\ \mathrm{C} & -1.947883 & -2.746615 & -1.206364\end{array}$




\begin{tabular}{|c|c|c|c|}
\hline $\mathrm{H}$ & 0.150921 & -2.421940 & -1.940060 \\
\hline & 0.687455 & -1.756239 & 0.631969 \\
\hline & -1.604435 & -1.948718 & 2.023557 \\
\hline & -3.545474 & -2.816943 & 0.366939 \\
\hline & -2.462388 & -3.082536 & -2.100370 \\
\hline & -3.309991 & 1.526100 & 1.210907 \\
\hline & -2.752000 & 0.813291 & 0.489564 \\
\hline & -4.004003 & 2.422101 & 2.105694 \\
\hline & -3.299591 & 2.853294 & 2.825959 \\
\hline & -4.470983 & 3.231336 & 1.533161 \\
\hline & -4.783498 & 1.876970 & 2.649729 \\
\hline & 1.208564 & 1.373835 & -2.296906 \\
\hline 51 & 1.730218 & 1.429654 & 0.102223 \\
\hline C & -1.137907 & 2.289127 & -1.517828 \\
\hline$\Pi$ & -2.211624 & 2.315247 & -1.726338 \\
\hline 1 & -0.977625 & 2.870740 & -0.594810 \\
\hline $\mathrm{H}$ & -0.591231 & 2.807587 & -2.318961 \\
\hline & 0.632206 & 1.544643 & 1.614294 \\
\hline $\mathrm{H}$ & 1.247849 & 1.839003 & 2.476626 \\
\hline $\mathrm{H}$ & -0.137024 & 2.319211 & 1.493393 \\
\hline I & 0.128844 & 0.601622 & 1.865555 \\
\hline$c$ & 2.372202 & 3.120843 & -0.375346 \\
\hline $\mathrm{H}$ & 2.913198 & 3.577200 & 0.464951 \\
\hline $\mathrm{H}$ & 3.063321 & 3.061054 & -1.227286 \\
\hline$H$ & 1.553563 & 3.799064 & -0.653479 \\
\hline $\mathrm{H}$ & -1.809107 & -0.099878 & -2.282978 \\
\hline $\mathrm{H}$ & -3.238366 & -0.372469 & -1.452360 \\
\hline$c$ & 3.132863 & 0.222501 & 0.399382 \\
\hline $\mathrm{C}$ & 3.254663 & -0.477997 & 1.607347 \\
\hline$C$ & 4.105241 & 0.008167 & -0.589838 \\
\hline 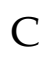 & 4.310855 & -1.360494 & 1.822954 \\
\hline $\mathrm{H}$ & 2.517441 & -0.333531 & 2.398906 \\
\hline 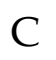 & 5.162178 & -0.871163 & -0.379592 \\
\hline 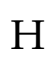 & 4.038900 & 0.538113 & -1.543395 \\
\hline$c$ & 5.265618 & -1.556508 & 0.829615 \\
\hline 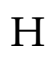 & 4.389413 & -1.893443 & 2.769552 \\
\hline & 5.908813 & -1.022226 & -1.157921 \\
\hline & 6.093007 & -2.244563 & 0.996918 \\
\hline
\end{tabular}




\section{D4-TS(SiPhMe $)$}

$G=-1138.579548 \quad Z P E=-1138.522878$

$\begin{array}{lccc}\mathrm{Ru} & -1.873866 & -0.296677 & 0.204968 \\ \mathrm{C} & -0.068601 & -0.384124 & -1.120839 \\ \mathrm{C} & 0.014968 & -1.488214 & -2.133711 \\ \mathrm{H} & -0.659420 & -1.332998 & -2.984177 \\ \mathrm{H} & -0.182572 & -2.474393 & -1.695441 \\ \mathrm{C} & 0.409532 & -0.565039 & 0.204088 \\ \mathrm{H} & 0.574409 & -1.616194 & 0.482637 \\ \mathrm{H} & -0.626945 & -0.063032 & 1.370901 \\ \mathrm{C} & -3.078265 & -1.887385 & 1.116672 \\ \mathrm{C} & -2.504376 & -2.395685 & -0.081847 \\ \mathrm{C} & -3.040741 & -1.645343 & -1.178699 \\ \mathrm{C} & -3.929147 & -0.676372 & -0.650944 \\ \mathrm{C} & -3.958438 & -0.809627 & 0.767210 \\ \mathrm{C} & -1.896046 & 2.845884 & -0.584640 \\ \mathrm{~N} & -1.837514 & 1.725409 & -0.297902 \\ \mathrm{C} & -1.944188 & 4.247676 & -0.927666 \\ \mathrm{H} & -2.741182 & 4.744931 & -0.363550 \\ \mathrm{H} & -0.985534 & 4.720379 & -0.682550 \\ \mathrm{H} & -2.137586 & 4.367288 & -1.999570 \\ \mathrm{H} & -1.531948 & 0.321088 & 1.753347 \\ \mathrm{H} & 0.022320 & 0.619047 & -1.549668 \\ \mathrm{H} & 1.039874 & -1.512980 & -2.537858 \\ \mathrm{Si} & 1.704815 & 0.590611 & 0.994598 \\ \mathrm{C} & 1.348160 & 2.387486 & 0.607069 \\ \mathrm{H} & 1.254669 & 2.585186 & -0.469193 \\ \mathrm{H} & 0.425462 & 2.718757 & 1.103914 \\ \mathrm{H} & 2.170528 & 3.009449 & 0.987620 \\ \mathrm{C} & 1.703796 & 0.296619 & 2.843140 \\ \mathrm{H} & 1.757608 & -0.773665 & 3.086249 \\ \mathrm{H} & 2.552949 & 0.796113 & 3.328928 \\ \mathrm{H} & 0.787144 & 0.696774 & 3.299803 \\ \mathrm{H} & -1.800829 & -3.219408 & -0.150020 \\ \mathrm{H} & -2.882667 & -2.250656 & 2.120200\end{array}$




$\begin{array}{lccc}\mathrm{H} & -4.554071 & -0.216562 & 1.452037 \\ \mathrm{H} & -4.453457 & 0.080922 & -1.225636 \\ \mathrm{H} & -2.807389 & -1.785074 & -2.228181 \\ \mathrm{C} & 3.325647 & 0.042485 & 0.219803 \\ \mathrm{C} & 4.296227 & -0.658709 & 0.947887 \\ \mathrm{C} & 3.585150 & 0.313276 & -1.132780 \\ \mathrm{C} & 5.483639 & -1.074153 & 0.349494 \\ \mathrm{H} & 4.128350 & -0.884817 & 2.002269 \\ \mathrm{C} & 4.768717 & -0.099102 & -1.736548 \\ \mathrm{H} & 2.849799 & 0.859353 & -1.728895 \\ \mathrm{C} & 5.720464 & -0.794732 & -0.993391 \\ \mathrm{H} & 6.226548 & -1.616029 & 0.933401 \\ \mathrm{H} & 4.951930 & 0.122877 & -2.787098 \\ \mathrm{H} & 6.648297 & -1.118626 & -1.462906\end{array}$

\section{E1-TS(SiPhMe2)}

$\mathrm{G}=-1137.374798 \quad Z P E=-1137.320151$

$\begin{array}{lccc}\mathrm{H} & 0.104030 & 1.429862 & 2.029005 \\ \mathrm{C} & 0.078070 & 1.320134 & 0.935448 \\ \mathrm{Ru} & -1.227413 & -0.301265 & 0.233646 \\ \mathrm{C} & -1.160433 & 1.605023 & 0.354572 \\ \mathrm{C} & -0.615099 & -1.748097 & 1.826969 \\ \mathrm{C} & -1.818011 & -2.202059 & 1.255552 \\ \mathrm{C} & -1.556321 & -2.530239 & -0.128491 \\ \mathrm{C} & -0.196053 & -2.291584 & -0.392549 \\ \mathrm{C} & 0.383240 & -1.746059 & 0.789260 \\ \mathrm{H} & -0.474289 & -1.405237 & 2.846483 \\ \mathrm{H} & -2.777555 & -2.284756 & 1.754775 \\ \mathrm{H} & -2.292897 & -2.886847 & -0.840902 \\ \mathrm{H} & 0.303223 & -2.409411 & -1.348211 \\ \mathrm{H} & 1.423370 & -1.462011 & 0.908489 \\ \mathrm{C} & -4.325531 & 0.045632 & -0.729377 \\ \mathrm{~N} & -3.223335 & -0.025117 & -0.383515 \\ \mathrm{C} & -5.700152 & 0.137519 & -1.159155 \\ \mathrm{H} & -6.200630 & 0.957331 & -0.631877 \\ \mathrm{H} & -5.744630 & 0.323481 & -2.237995\end{array}$




$\begin{array}{lrrr}\mathrm{H} & -6.221410 & -0.799974 & -0.935225 \\ \mathrm{H} & -0.889493 & 0.718661 & -1.076276 \\ \mathrm{Si} & 1.712536 & 1.783725 & 0.062096 \\ \mathrm{C} & 2.571459 & 2.939643 & 1.263735 \\ \mathrm{H} & 3.552036 & 3.255464 & 0.881842 \\ \mathrm{H} & 1.965523 & 3.843299 & 1.419709 \\ \mathrm{H} & 2.726664 & 2.473616 & 2.246435 \\ \mathrm{C} & 1.307576 & 2.675575 & -1.537992 \\ \mathrm{H} & 2.231903 & 2.878452 & -2.096946 \\ \mathrm{H} & 0.640109 & 2.106725 & -2.199791 \\ \mathrm{H} & 0.829107 & 3.642767 & -1.329525 \\ \mathrm{C} & 2.801897 & 0.298371 & -0.302434 \\ \mathrm{C} & 3.724311 & -0.178361 & 0.640966 \\ \mathrm{C} & 2.683629 & -0.398125 & -1.514260 \\ \mathrm{C} & 4.481351 & -1.321711 & 0.395792 \\ \mathrm{H} & 3.854019 & 0.347209 & 1.588942 \\ \mathrm{C} & 3.437652 & -1.541272 & -1.766314 \\ \mathrm{H} & 1.985157 & -0.048523 & -2.277520 \\ \mathrm{C} & 4.332737 & -2.008802 & -0.806670 \\ \mathrm{H} & 5.190213 & -1.676717 & 1.142745 \\ \mathrm{H} & 3.329159 & -2.067907 & -2.713865 \\ \mathrm{H} & 4.921616 & -2.904305 & -1.000215 \\ \mathrm{C} & -1.983654 & 2.789853 & 0.087079 \\ \mathrm{H} & -2.210382 & 3.261369 & 1.055242 \\ \mathrm{H} & -1.410570 & 3.528736 & -0.492533 \\ & -2.920269 & 2.565391 & -0.430738\end{array}$

\section{dabco}

$\begin{array}{lrrr}\mathrm{C} & 0.000000 & 1.375690 & 0.774334 \\ \mathrm{C} & 0.000000 & 1.375690 & -0.774334 \\ \mathrm{H} & -0.886573 & 1.887104 & 1.176103 \\ \mathrm{H} & 0.886573 & 1.887104 & 1.176103 \\ \mathrm{H} & -0.886573 & 1.887104 & -1.176103 \\ \mathrm{H} & 0.886573 & 1.887104 & -1.176103 \\ \mathrm{C} & -1.191383 & -0.687845 & 0.774334 \\ \mathrm{H} & -1.190994 & -1.711347 & 1.176103\end{array}$




$\begin{array}{lrrr}\mathrm{H} & -2.077567 & -0.175758 & 1.176103 \\ \mathrm{C} & -1.191383 & -0.687845 & -0.774334 \\ \mathrm{H} & -1.190994 & -1.711347 & -1.176103 \\ \mathrm{H} & -2.077567 & -0.175758 & -1.176103 \\ \mathrm{C} & 1.191383 & -0.687845 & -0.774334 \\ \mathrm{H} & 1.190994 & -1.711347 & -1.176103 \\ \mathrm{H} & 2.077567 & -0.175758 & -1.176103 \\ \mathrm{C} & 1.191383 & -0.687845 & 0.774334 \\ \mathrm{H} & 1.190994 & -1.711347 & 1.176103 \\ \mathrm{H} & 2.077567 & -0.175758 & 1.176103 \\ \mathrm{~N} & 0.000000 & 0.000000 & 1.280358 \\ \mathrm{~N} & 0.000000 & 0.000000 & -1.280358\end{array}$

cat(dabco)

$\begin{array}{llll}\mathrm{Ru} & 0.947885 & -0.402821 & 0.007054 \\ \mathrm{C} & 1.172576 & 1.853964 & -2.349529 \\ \mathrm{~N} & 0.996511 & 1.104266 & -1.482115 \\ \mathrm{C} & 1.396402 & 2.783894 & -3.433146 \\ \mathrm{H} & 1.259484 & 2.277601 & -4.395420 \\ \mathrm{H} & 0.689452 & 3.618563 & -3.367648 \\ \mathrm{H} & 2.417518 & 3.178621 & -3.382895 \\ \mathrm{C} & 1.122308 & 1.911526 & 2.318698 \\ \mathrm{~N} & 0.966933 & 1.135410 & 1.470653 \\ \mathrm{C} & 1.322202 & 2.875676 & 3.376778 \\ \mathrm{H} & 2.338094 & 3.283419 & 3.324288 \\ \mathrm{H} & 0.604422 & 3.697928 & 3.279446 \\ \mathrm{H} & 1.182745 & 2.396457 & 4.352421 \\ \mathrm{C} & 0.933937 & -2.575000 & 0.112557 \\ \mathrm{H} & 0.018087 & -3.154763 & 0.186816 \\ \mathrm{C} & 1.558450 & -2.161899 & -1.103381 \\ \mathrm{C} & 1.672058 & -2.053801 & 1.212844 \\ \mathrm{H} & 1.241039 & -2.418581 & -2.109086 \\ \mathrm{C} & 2.721773 & -1.394722 & -0.742071 \\ \mathrm{H} & 1.447649 & -2.203650 & 2.264218 \\ \mathrm{C} & 2.793647 & -1.325652 & 0.673160 \\ \mathrm{H} & 3.404417 & -0.912345 & -1.435197 \\ \mathrm{H} & 3.545692 & -0.793907 & 1.247589 \\ \mathrm{~N} & -1.314171 & -0.157854 & -0.017441 \\ \mathrm{C} & -1.876447 & -0.906408 & -1.173532 \\ \mathrm{C} & -1.790754 & 1.247645 & -0.125321\end{array}$




$\begin{array}{llll}\mathrm{C} & -1.879050 & -0.737257 & 1.231577 \\ \mathrm{C} & -3.402712 & -0.681998 & -1.249709 \\ \mathrm{H} & -1.363339 & -0.562231 & -2.080402 \\ \mathrm{H} & -1.629948 & -1.965384 & -1.027576 \\ \mathrm{H} & -1.266825 & 1.847868 & 0.626734 \\ \mathrm{H} & -1.494270 & 1.612301 & -1.115667 \\ \mathrm{C} & -3.321567 & 1.296957 & 0.058550 \\ \mathrm{C} & -3.416949 & -0.818576 & 1.123326 \\ \mathrm{H} & -1.558851 & -0.091140 & 2.059313 \\ \mathrm{H} & -1.431004 & -1.725482 & 1.384875 \\ \mathrm{H} & -3.664457 & -0.031143 & -2.095403 \\ \mathrm{H} & -3.927720 & -1.636309 & -1.390921 \\ \mathrm{~N} & -3.891325 & -0.047312 & -0.025288 \\ \mathrm{H} & -3.589807 & 1.716772 & 1.037942 \\ \mathrm{H} & -3.782852 & 1.930249 & -0.711460 \\ \mathrm{H} & -3.891988 & -0.430415 & 2.034470 \\ \mathrm{H} & -3.747688 & -1.858373 & 0.993583\end{array}$

\section{C3-TSa(dabco)}

$\begin{array}{lrrr}\mathrm{H} & -2.361715 & 2.647798 & -2.327611 \\ \mathrm{C} & -2.106049 & 1.744738 & -1.759087 \\ \mathrm{Ru} & -0.259869 & -0.466180 & -0.665201 \\ \mathrm{C} & -0.845785 & 1.519926 & -1.258779 \\ \mathrm{C} & -0.521606 & -1.105496 & -2.750674 \\ \mathrm{C} & 0.616404 & -1.780645 & -2.222524 \\ \mathrm{C} & 0.200459 & -2.612018 & -1.136376 \\ \mathrm{C} & -1.191190 & -2.396383 & -0.958731 \\ \mathrm{C} & -1.655373 & -1.472591 & -1.957636 \\ \mathrm{H} & -0.500159 & -0.384385 & -3.562316 \\ \mathrm{H} & 1.627357 & -1.674283 & -2.602607 \\ \mathrm{H} & 0.819887 & -3.280306 & -0.547258 \\ \mathrm{H} & -1.807520 & -2.838289 & -0.181896 \\ \mathrm{H} & -2.684642 & -1.156188 & -2.087869 \\ \mathrm{H} & -2.905264 & 1.002225 & -1.692817 \\ \mathrm{Si} & -2.041327 & 1.808244 & 0.474384 \\ \mathrm{C} & 0.182945 & 2.562502 & -1.614550 \\ \mathrm{H} & 0.894875 & 2.766149 & -0.809188 \\ \mathrm{H} & 0.754004 & 2.177502 & -2.473948 \\ \mathrm{H} & -0.260754 & 3.519380 & -1.931868 \\ \mathrm{C} & -0.942056 & 2.975043 & 1.424522\end{array}$




\begin{tabular}{|c|c|c|c|}
\hline 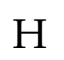 & 0.039168 & 2.553851 & 1.667427 \\
\hline H & -0.777235 & 3.904201 & 0.862405 \\
\hline & -1.445649 & 3.221576 & 2.369208 \\
\hline & -3.772745 & 2.532580 & 0.488731 \\
\hline & -4.505787 & 1.915825 & -0.046306 \\
\hline & -4.106495 & 2.596836 & 1.535870 \\
\hline & -3.793359 & 3.540412 & 0.053975 \\
\hline & -2.032406 & 0.046393 & 1.091809 \\
\hline 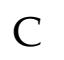 & -0.878857 & -0.488742 & 1.712548 \\
\hline$C$ & -3.216005 & -0.738523 & 1.084216 \\
\hline & -0.933984 & -1.748281 & 2.353130 \\
\hline 1 & -0.055151 & 0.184573 & 1.968731 \\
\hline 0 & -3.259985 & -1.949651 & 1.741279 \\
\hline $\mathrm{H}$ & -4.126481 & -0.340488 & 0.634637 \\
\hline & -2.108112 & -2.468688 & 2.369004 \\
\hline $\mathrm{H}$ & -0.048838 & -2.116244 & 2.871034 \\
\hline $\mathrm{H}$ & -4.188462 & -2.519081 & 1.772097 \\
\hline 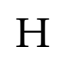 & -2.158516 & -3.435736 & 2.865393 \\
\hline$x$ & 2.073813 & 0.149312 & 0.150981 \\
\hline$C$ & 2.583641 & -1.132374 & 0.708179 \\
\hline C & 2.254923 & 1.175896 & 1.205780 \\
\hline 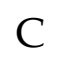 & 2.956258 & 0.542887 & -0.979532 \\
\hline $\mathrm{C}$ & 3.998754 & -0.952344 & 1.296004 \\
\hline 11 & 1.883074 & -1.473368 & 1.480082 \\
\hline 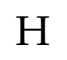 & 2.578506 & -1.873902 & -0.097480 \\
\hline $\mathrm{H}$ & 1.774112 & 2.103858 & 0.879213 \\
\hline $\mathrm{H}$ & 1.757520 & 0.831590 & 2.122286 \\
\hline 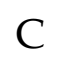 & 3.756075 & 1.400395 & 1.493899 \\
\hline 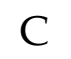 & 4.440253 & 0.440544 & -0.562918 \\
\hline 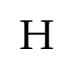 & 2.687556 & 1.566498 & -1.267663 \\
\hline 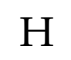 & 2.747951 & -0.107457 & -1.834215 \\
\hline $\mathrm{H}$ & 3.977041 & -0.988698 & 2.394049 \\
\hline T & 4.658710 & -1.753463 & 0.935613 \\
\hline $\mathrm{N}$ & 4.561038 & 0.336861 & 0.893831 \\
\hline $\mathrm{H}$ & 4.097050 & 2.361569 & 1.084575 \\
\hline T & 3.939461 & 1.413402 & 2.576635 \\
\hline & 5.005909 & 1.315229 & -0.909221 \\
\hline & 4.899339 & -0.458286 & -0.996966 \\
\hline
\end{tabular}




\section{D2-TS(dabco)}

\begin{tabular}{|c|c|c|c|}
\hline $\mathrm{H}$ & -2.112943 & 1.149727 & -1.577713 \\
\hline $\mathrm{C}$ & -1.617615 & 0.276359 & -2.020555 \\
\hline $\mathrm{u}$ & 0.855062 & 1.372584 & -0.603684 \\
\hline C & -0.165485 & 0.248634 & -1.882653 \\
\hline $\mathrm{C}$ & -0.652130 & 2.986044 & -0.384688 \\
\hline $\mathrm{C}$ & -0.912712 & 2.208997 & 0.782438 \\
\hline C & 0.230675 & 2.274438 & 1.609235 \\
\hline $\mathrm{C}$ & 1.209263 & 3.086500 & 0.978027 \\
\hline $\mathrm{C}$ & 0.660791 & 3.544059 & -0.255056 \\
\hline $\mathrm{H}$ & -1.345667 & 3.194498 & -1.193440 \\
\hline $\mathrm{H}$ & -1.826471 & 1.664811 & 1.003148 \\
\hline $\mathrm{H}$ & 0.351538 & 1.757912 & 2.556973 \\
\hline $\mathrm{H}$ & 2.188831 & 3.335688 & 1.371878 \\
\hline $\mathrm{H}$ & 1.132260 & 4.228676 & -0.952476 \\
\hline $\mathrm{H}$ & -1.924425 & 0.183836 & -3.078102 \\
\hline Si & -2.616213 & -1.197896 & -1.224722 \\
\hline $\mathrm{C}$ & 0.476740 & -0.575032 & -2.950021 \\
\hline $\mathrm{H}$ & 0.346486 & -0.029977 & -3.900332 \\
\hline $\mathrm{H}$ & 1.549124 & -0.738847 & -2.816036 \\
\hline $\mathrm{H}$ & -0.032619 & -1.541230 & -3.083773 \\
\hline C & -1.829337 & -2.866037 & -1.530959 \\
\hline $\mathrm{H}$ & -2.465902 & -3.646267 & -1.088948 \\
\hline $\mathrm{H}$ & -1.762082 & -3.074567 & -2.607700 \\
\hline $\mathrm{H}$ & -0.826019 & -2.973048 & -1.099764 \\
\hline C & -4.266010 & -1.083147 & -2.104826 \\
\hline$H$ & -5.007592 & -1.745606 & -1.638000 \\
\hline $\mathrm{H}$ & -4.671392 & -0.062167 & -2.092977 \\
\hline $\mathrm{H}$ & -4.159315 & -1.385375 & -3.155960 \\
\hline $\mathrm{H}$ & 1.039533 & 1.648479 & -2.167308 \\
\hline $\mathrm{H}$ & 2.315183 & 1.691179 & -1.172722 \\
\hline $\mathrm{C}$ & -2.840543 & -0.870451 & 0.608116 \\
\hline $\mathrm{C}$ & -2.080720 & -1.527937 & 1.585139 \\
\hline C & -3.783579 & 0.075916 & 1.037858 \\
\hline$C$ & -2.223632 & -1.224305 & 2.937217 \\
\hline 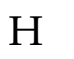 & -1.373771 & -2.305869 & 1.29090 \\
\hline
\end{tabular}




$\begin{array}{lrrr}\mathrm{C} & -3.941868 & 0.375725 & 2.388168 \\ \mathrm{H} & -4.405087 & 0.596091 & 0.305482 \\ \mathrm{C} & -3.150753 & -0.265986 & 3.339029 \\ \mathrm{H} & -1.620076 & -1.745254 & 3.679517 \\ \mathrm{H} & -4.681213 & 1.111839 & 2.700466 \\ \mathrm{H} & -3.268109 & -0.029460 & 4.395563 \\ \mathrm{~N} & 2.171343 & -0.412991 & 0.050371 \\ \mathrm{C} & 2.561250 & -0.145287 & 1.469404 \\ \mathrm{C} & 3.437437 & -0.561372 & -0.726138 \\ \mathrm{C} & 1.454707 & -1.718053 & 0.039725 \\ \mathrm{C} & 3.597867 & -1.188497 & 1.935633 \\ \mathrm{H} & 2.956755 & 0.875274 & 1.525990 \\ \mathrm{H} & 1.640327 & -0.192548 & 2.065505 \\ \mathrm{H} & 3.200403 & -0.587119 & -1.794720 \\ \mathrm{H} & 4.028108 & 0.344887 & -0.540898 \\ \mathrm{C} & 4.183103 & -1.831022 & -0.272071 \\ \mathrm{C} & 2.284418 & -2.787195 & 0.780551 \\ \mathrm{H} & 1.290722 & -1.998804 & -1.006285 \\ \mathrm{H} & 0.478407 & -1.557713 & 0.511602 \\ \mathrm{H} & 4.602099 & -0.746983 & 1.992624 \\ \mathrm{H} & 3.341401 & -1.561917 & 2.936007 \\ \mathrm{~N} & 3.648949 & -2.312165 & 1.002275 \\ \mathrm{H} & 4.077207 & -2.638198 & -1.009844 \\ \mathrm{H} & 5.255541 & -1.623288 & -0.158564 \\ \mathrm{H} & 2.317654 & -3.718378 & 0.199287 \\ & 1.842133 & -3.021437 & 1.758859\end{array}$

\section{B1-TS(dabco)}

$\begin{array}{lrrr}\text { H } & -1.261746 & 0.909260 & -1.869477 \\ \text { C } & -0.960497 & 1.032971 & -0.817224 \\ \text { Ru } & 0.515565 & -0.408202 & -0.167628 \\ \text { C } & 0.372791 & 1.448310 & -0.659427 \\ \text { C } & -0.268327 & -2.151496 & -1.398688 \\ \text { C } & 0.851975 & -2.584777 & -0.665409 \\ \text { C } & 0.538674 & -2.521528 & 0.741681 \\ \text { C } & -0.770249 & -2.019678 & 0.865119 \\ \text { C } & -1.261476 & -1.750134 & -0.449790\end{array}$




\begin{tabular}{|c|c|c|c|}
\hline $\mathrm{H}$ & -0.345689 & -2.078372 & -2.477959 \\
\hline $\mathrm{H}$ & 1.781241 & -2.936186 & -1.100882 \\
\hline $\mathrm{H}$ & 1.185073 & -2.823779 & 1.557056 \\
\hline 5 & -1.302023 & -1.826263 & 1.791030 \\
\hline $\mathrm{H}$ & -2.255096 & -1.384472 & -0.687769 \\
\hline 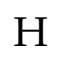 & 0.483433 & 0.898692 & 0.914512 \\
\hline & -2.375030 & 1.655815 & 0.288881 \\
\hline & -2.884956 & 3.289875 & -0.486729 \\
\hline $\mathrm{H}$ & -3.123564 & 3.178303 & -1.553509 \\
\hline $\mathrm{H}$ & -3.772925 & 3.701722 & 0.012811 \\
\hline 1 & -2.079422 & 4.033267 & -0.402513 \\
\hline$\zeta$ & -1.818410 & 1.919023 & 2.056411 \\
\hline $\mathrm{H}$ & -2.647196 & 2.337286 & 2.645725 \\
\hline $\mathrm{H}$ & -1.486253 & 0.997084 & 2.551342 \\
\hline r & -0.990519 & 2.640870 & 2.103046 \\
\hline C & -3.795504 & 0.432519 & 0.194744 \\
\hline$C$ & -4.453909 & 0.222285 & -1.027097 \\
\hline C & -4.185615 & -0.345610 & 1.292989 \\
\hline C & -5.451733 & -0.739549 & -1.153025 \\
\hline $\mathrm{H}$ & -4.178368 & 0.814895 & -1.902928 \\
\hline C & -5.184221 & -1.309913 & 1.174459 \\
\hline $\mathrm{H}$ & -3.701556 & -0.202204 & 2.260952 \\
\hline C & -5.814619 & -1.510992 & -0.050480 \\
\hline 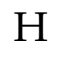 & -5.948897 & -0.888355 & -2.110736 \\
\hline $\mathrm{H}$ & -5.471898 & -1.905240 & 2.040139 \\
\hline $\mathrm{H}$ & -6.593470 & -2.266273 & -0.146333 \\
\hline C & 0.983169 & 2.774332 & -0.876823 \\
\hline $\mathrm{H}$ & 0.587203 & 3.162314 & -1.826494 \\
\hline $\mathrm{H}$ & 0.644394 & 3.471954 & -0.093929 \\
\hline $\mathrm{H}$ & 2.076085 & 2.801033 & -0.914684 \\
\hline $\mathrm{N}$ & 2.790398 & -0.086938 & 0.076624 \\
\hline $\mathrm{C}$ & 3.147665 & 1.024157 & 1.017255 \\
\hline C & 3.481940 & -1.291712 & 0.637152 \\
\hline $\mathrm{C}$ & 3.391679 & 0.168581 & -1.267673 \\
\hline $\mathrm{C}$ & 4.641362 & 1.363066 & 0.877911 \\
\hline $\mathrm{H}$ & 2.899697 & 0.662971 & 2.023756 \\
\hline 11 & 2.524552 & 1.898250 & 0.827066 \\
\hline 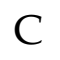 & 4.972719 & -0.977504 & 0.890054 \\
\hline
\end{tabular}




$\begin{array}{lrrr}\mathrm{H} & 3.374403 & -2.106027 & -0.086164 \\ \mathrm{H} & 2.971497 & -1.573288 & 1.563783 \\ \mathrm{H} & 3.035862 & -0.620278 & -1.941245 \\ \mathrm{H} & 2.997416 & 1.120948 & -1.635949 \\ \mathrm{C} & 4.931070 & 0.194547 & -1.168226 \\ \mathrm{H} & 5.082995 & 1.553755 & 1.865335 \\ \mathrm{H} & 4.782680 & 2.268098 & 0.269876 \\ \mathrm{~N} & 5.357492 & 0.266206 & 0.228523 \\ \mathrm{H} & 5.600678 & -1.795979 & 0.513764 \\ \mathrm{H} & 5.175397 & -0.870351 & 1.964343 \\ \mathrm{H} & 5.370616 & -0.709546 & -1.611081 \\ \mathrm{H} & 5.334567 & 1.059868 & -1.710720\end{array}$




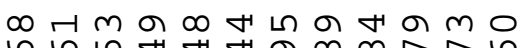

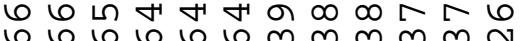

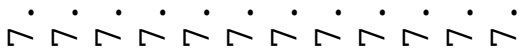
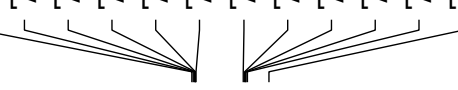

$\mathrm{PhMe}_{2} \mathrm{Si}={ }^{n} \mathrm{Bu}$

1a

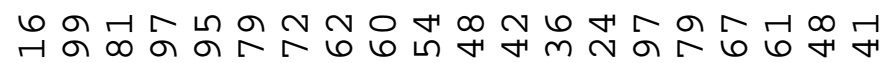
ทำ

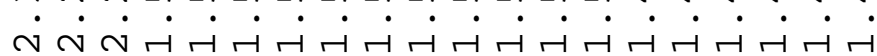

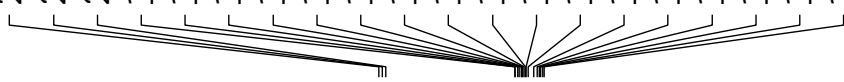

ner

NAME

EXPNO
PROCNO

Date_

INSTRUM

PROBHD

PULPROG

TD

SOLVENT

NS

SWH

FIDRES

AQ

RG

DE

TE

D1

$====$

$\mathrm{SFO1}$

N1

P 1

SF

WDW

SSB

LB
GB

PC fq2-71-1

20180606 18.50 $5 \mathrm{~mm}$ DUL $13 \mathrm{C}-1$ $\mathrm{zg} 30$
65536 $\mathrm{CDCl} 3$ 8

$8012.820 \mathrm{~Hz}$ $0.122266 \mathrm{~Hz}$ $4.0894966 \mathrm{sec}$ 62.93 62.400 usec 6.50 usec $296.1 \mathrm{~K}$ $1.00000000 \mathrm{sec}$

CHANNEL $\mathrm{f} 1$ $1 \mathrm{H}$
14.30 usec 400.1300097

$\mathrm{EM}$
0

$0.30 \mathrm{~Hz}$

0
1.00 
$\mathrm{PhMe}_{2} \mathrm{Si}={ }^{n} \mathrm{Bu}$

1a

\begin{tabular}{|c|c|c|}
\hline 하 & 두 & \\
\hline$\cdot \cdot \cdot \cdot$ & & $\rightarrow$ m 06 \\
\hline$m \sim m \sim$ & $\begin{array}{l}9 \\
0\end{array}$ & $\dot{r}$ \\
\hline નનનન & $\Gamma$ & $\infty<r$ \\
\hline
\end{tabular}

․ $4 \pi$

$m a r$

1

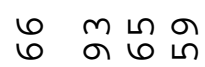

$\circ$ त

11

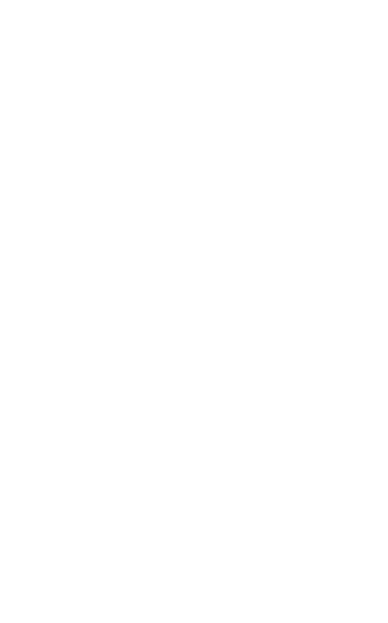

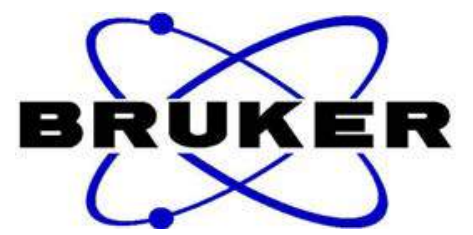

NAME PROCNO

Date

Time

INSTRUM

PROBHD

PULPROG

TD

SOLV
NS
DS

DS

SWH

FIDR
$\mathrm{AQ}$
$\mathrm{RG}$

DW

DE

TE

D11

D11 0

$==$

$\mathrm{SFO}$

P 1

$S I$
$S F$

WDW

$S S B$

LB
GB

$\mathrm{PC}$ fq2-71-1

20180606 18.51 18.51

$5 \mathrm{~mm}$ DUL $13 \mathrm{C}-1$

zgpg 30

$\mathrm{CDCl} 3$ 24

$24038.461 \mathrm{~Hz}$ $0.366798 \mathrm{~Hz}$

$1.3631988 \mathrm{sec}$

196.92

20.800 usec

6.50 usec

$2.00000000 \mathrm{sec}$

1

CHANNEL $\mathrm{f} 1$ $100.6228298 \mathrm{MH}$ $13 \mathrm{C}$

9.60 usec 100.6127736

100

EM

$1.00 \mathrm{~Hz}$

0
1.40

80 


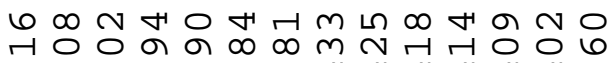
닫의

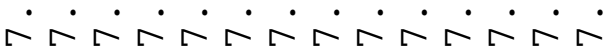

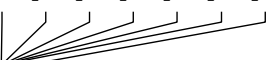

$\mathrm{PhMe}_{2} \mathrm{Si}=\left(\mathrm{CH}_{2}\right)_{7} \mathrm{CH}_{3}$

1b

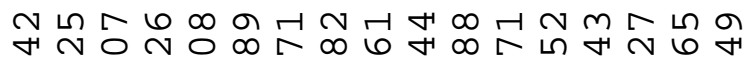
ॠ $\dot{\sim} \dot{\sim} \dot{-} \dot{-} \dot{-} \dot{-} \dot{-} \dot{-} \dot{-} \dot{-} \dot{-} \dot{-} \dot{-} \dot{0}$
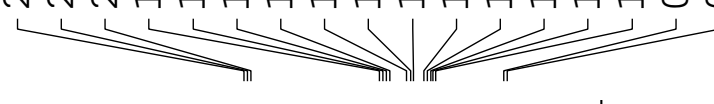

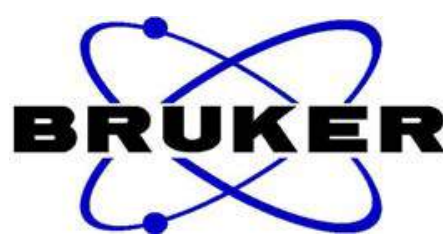

NAME EXPNO PROCNO Date Time

INSTRUM

PROBHD

PULPROG

TD

SOLVENT

NS
DS

SWH

FIDRES

AQ
RG
DW

DE

DE

D1 0

$=====$

NUC1

P1 1

PL1

SFO

SI

WDW

$S S B$

LB

PC
ZT-1029-0929

20170929 23.06 pect $5 \mathrm{~mm}$ РABBO $\mathrm{BB}-$ $\mathrm{zg} 30$
65536 $\mathrm{CDCl} 3$ 5
2

$8223.685 \mathrm{~Hz}$ $0.125483 \mathrm{~Hz}$ $3.9846387 \mathrm{sec}$ 40.3 60.800 usec $295.7 \mathrm{~K}$ $1.00000000 \mathrm{sec}$ CHANNEL $\mathrm{f}$ $1 \mathrm{H}$ 13.60 usec $-1.00 \mathrm{~dB}$ $400.1324710 \mathrm{MHz}$ 32768 $400.1300099 \mathrm{MHz}$ EM 0
$0 . \mathrm{Hz}$ 0.30
0
1.00 1.00

तi 


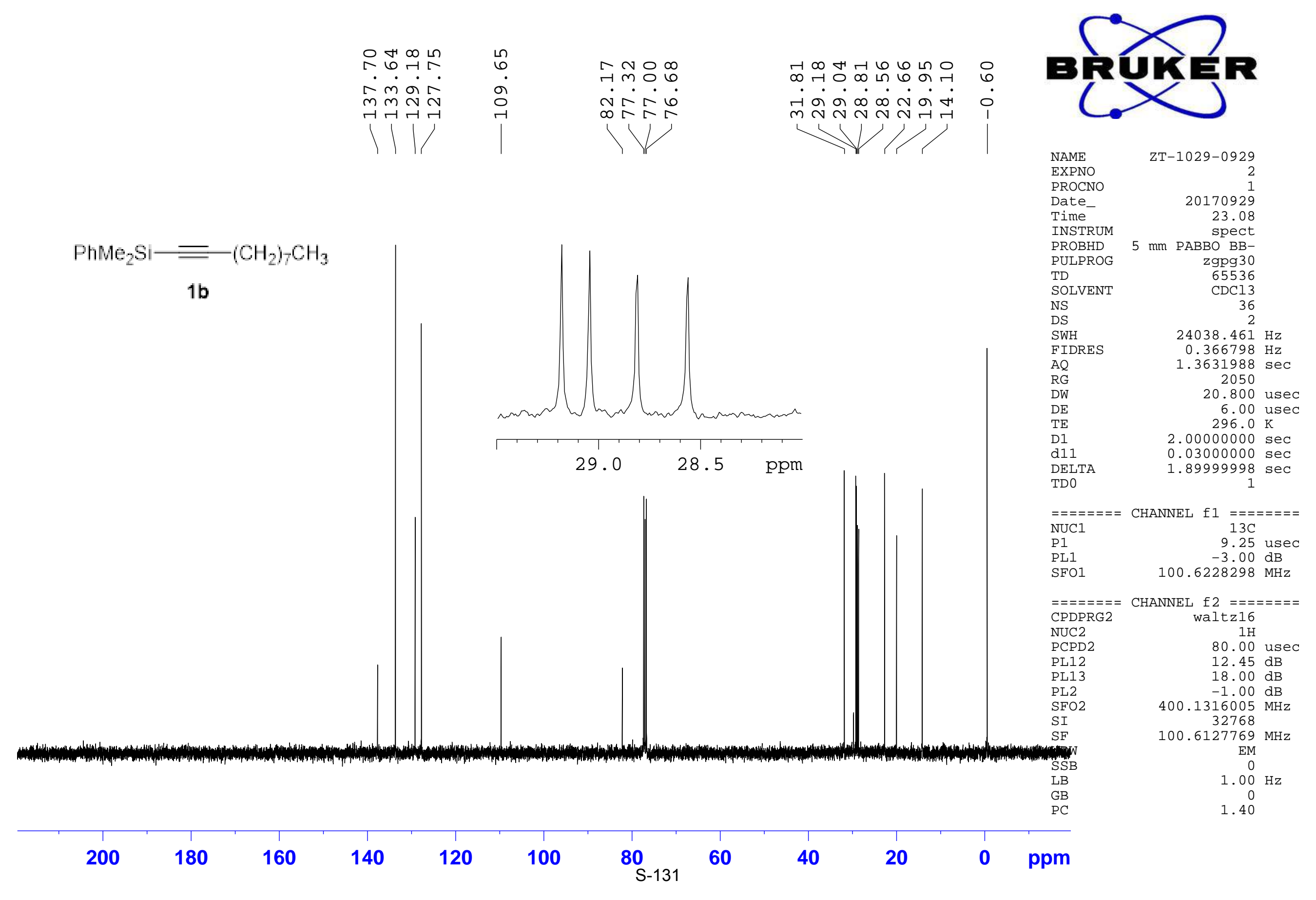




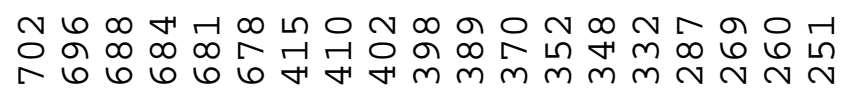

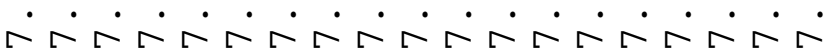

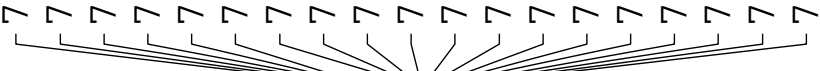

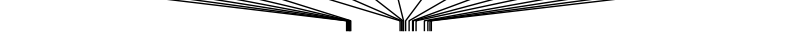

EXPNO

PROCNO

Date_-

Time

INSTRUM

PROBHD

PULPROG

TD

SOLVENT

$\mathrm{PhMe}_{2} \mathrm{Si}=\frac{=}{{ }_{1 \mathrm{c}}}$

NS
DS

SWH

FIDRES

AQ
RG

DW

$\mathrm{DE}$

D1 0

ZT-1037-1014

1
20171014
20.27

$5 \mathrm{~mm}$ PABBO BB-

$===$
NUC1

P1 1

PLI

SF

$\mathrm{SI}$

WDW

SSB

LB

$\mathrm{PC}$

65536
$\mathrm{CDCl} 3$
9

9
2
$8223.685 \mathrm{~Hz}$

$0.125483 \mathrm{~Hz}$

$3.9846387 \mathrm{sec}$ 144

60.800 usec 6.00 usec

$1.00000000 \mathrm{sec}$

CHANNEL $\mathrm{f}$

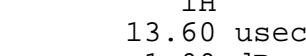
13.60 use $400.1324710 \mathrm{~dB}$ $\begin{array}{r}32768 \\ \hline\end{array}$ $400.1300098 \mathrm{MHz}$

$\mathrm{EM}$
0

$0.30 \mathrm{~Hz}$

0.30
0
1.00

3

2

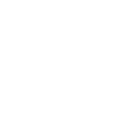

1

$\left|\begin{array}{l}0 \\ 0 \\ 0\end{array}\right|$

ppm 


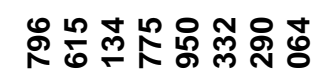

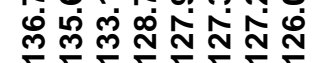

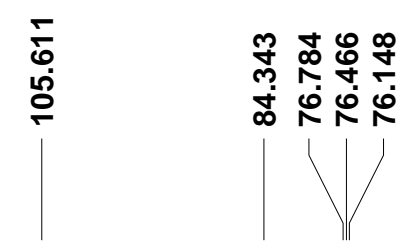

$\mathrm{PhMe}_{2} \mathrm{Si}=\mathrm{Ph}_{\mathrm{Ph}}$

1c

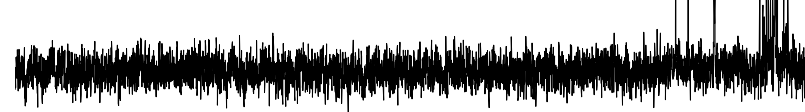

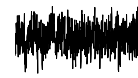

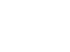

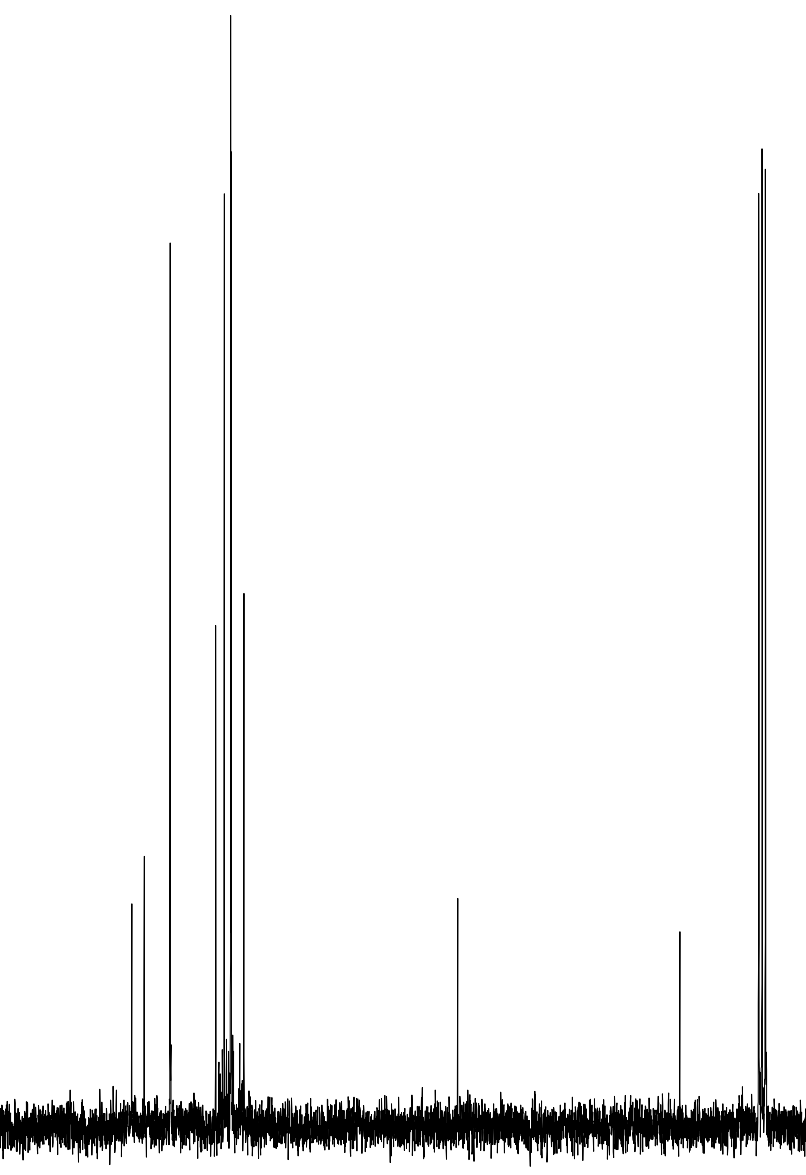

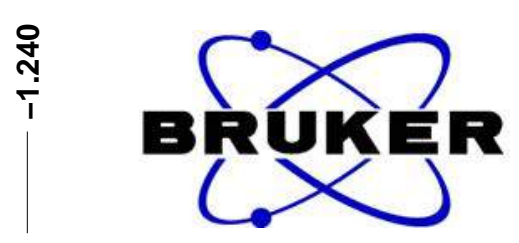

Current Data Parameters

NAME ZT-1029-0929

EXPNO

F2 - Acquisition Parameters

Date 20170929

Time 20170929
22.56

NSTRUM 22.5 PROBHD $5 \mathrm{~mm}$ PABBO BBPULPROG Zgpg30 TD 65536 SOLVENT $\quad \mathrm{CDCl} 3$ NS

DS

SWH $\quad 24038.461 \mathrm{~Hz}$ FIDRES $\quad 0.366798 \mathrm{~Hz}$ AQ $\quad 1.3631988 \mathrm{sec}$

20.800 usec

DE $\quad 6.00$ us

D1 $\quad 2.00000000 \mathrm{sec}$

d11 $0.03000000 \mathrm{sec}$

DELTA $\begin{array}{ll}0.03000000 \mathrm{sec} \\ \mathbf{1 . 8 9 9 9 9 9 9 8} \mathrm{sec}\end{array}$

$\begin{array}{ll}\text { TELTA } & 1.89999998 \mathrm{sec}\end{array}$

$=======$ CHANNEL $\mathrm{f} 1$ ==== NUC1 $13 \mathrm{C}$

P1 $\quad 9.25$ usec

SFO1 $\quad 100.6228298 \mathrm{MHz}$

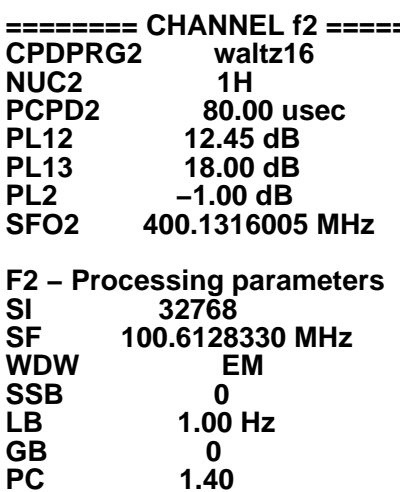

$\begin{array}{lllllllllllllllllllllll}190 & 180 & 170 & 160 & 150 & 140 & 130 & 120 & 110 & 100 & 90 & 80 & 50 & 70 & 60 & 50 & 40 & 30 & 20 & 10 & 0 & \mathrm{ppm}\end{array}$ 


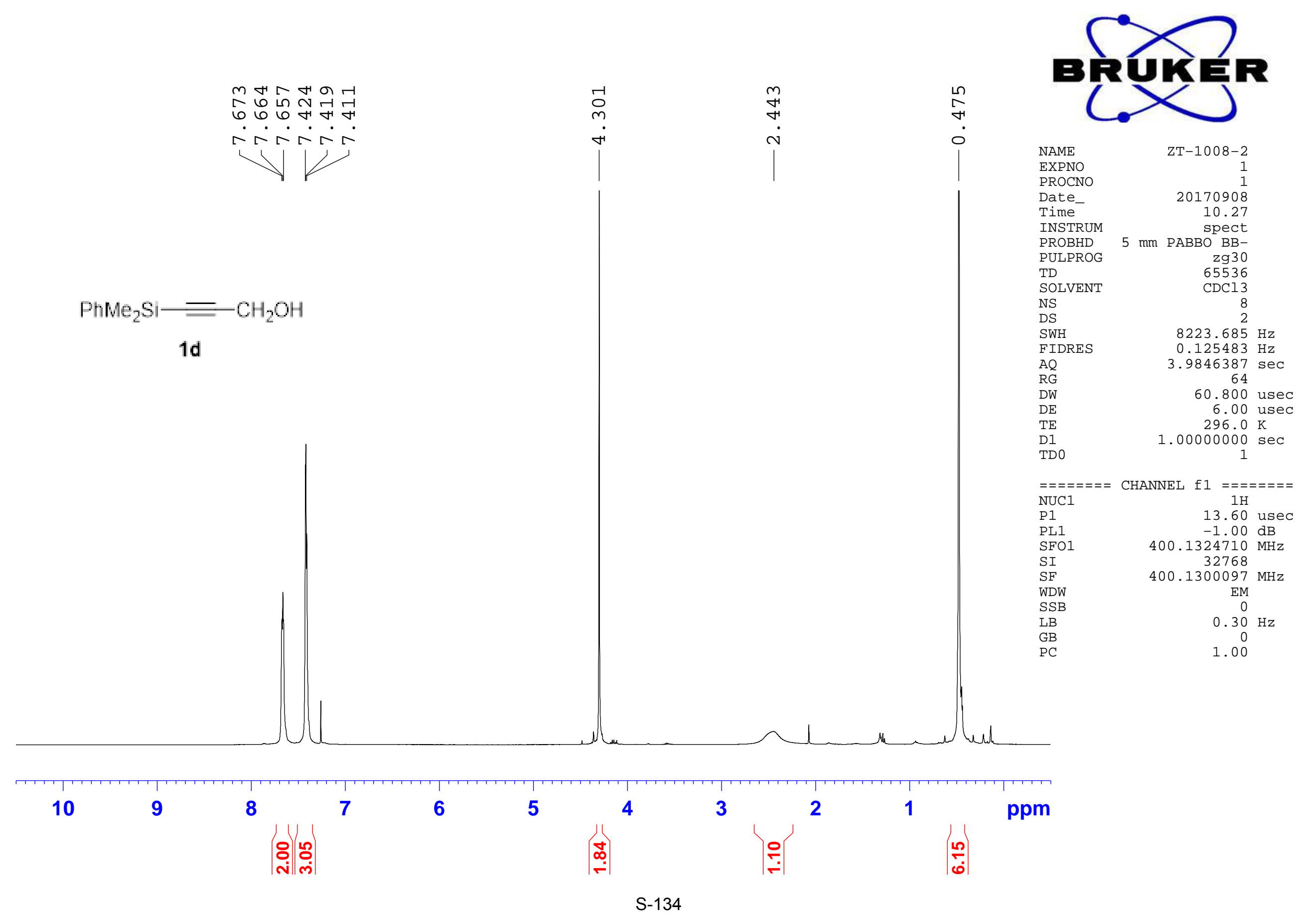




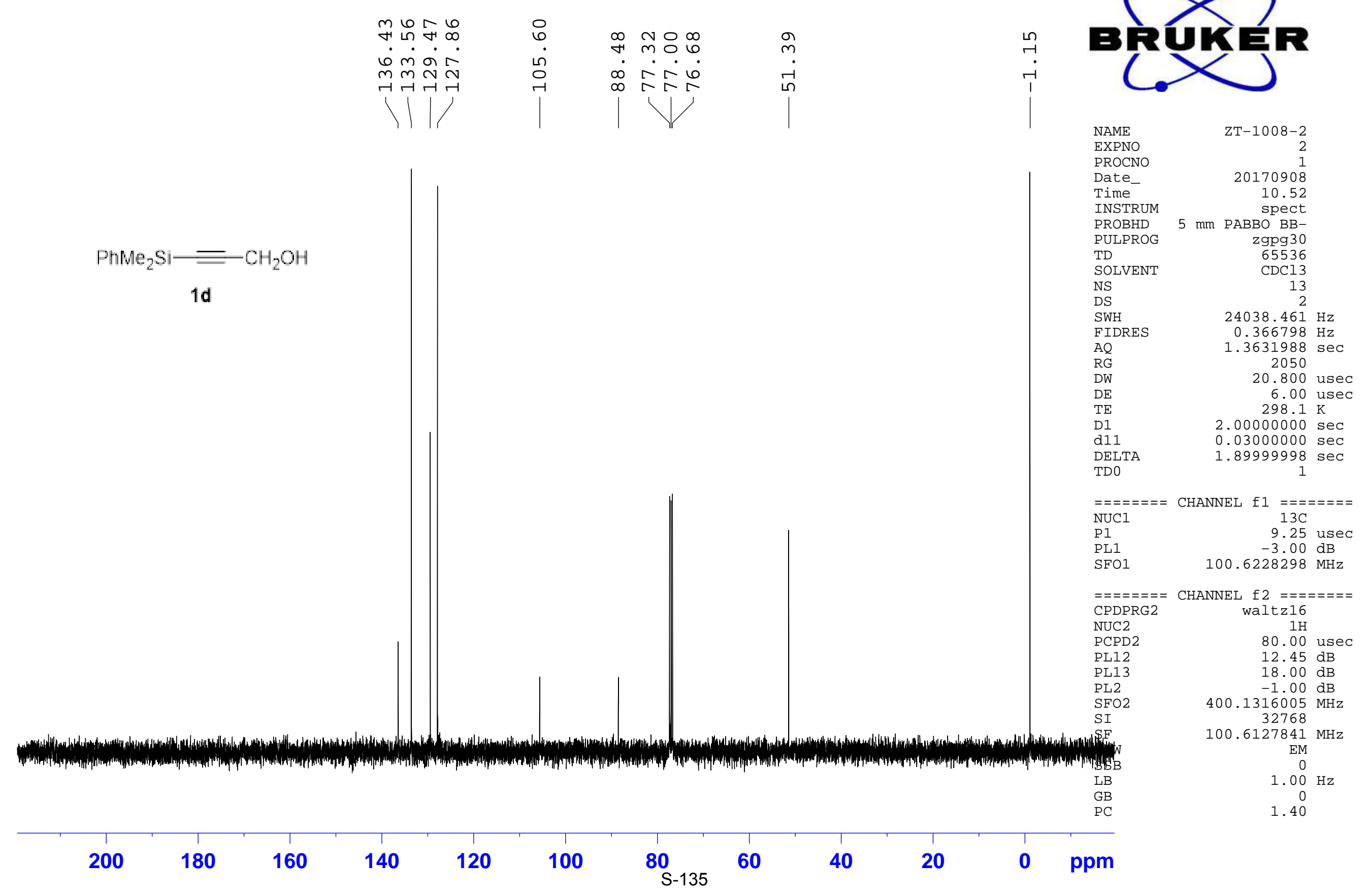




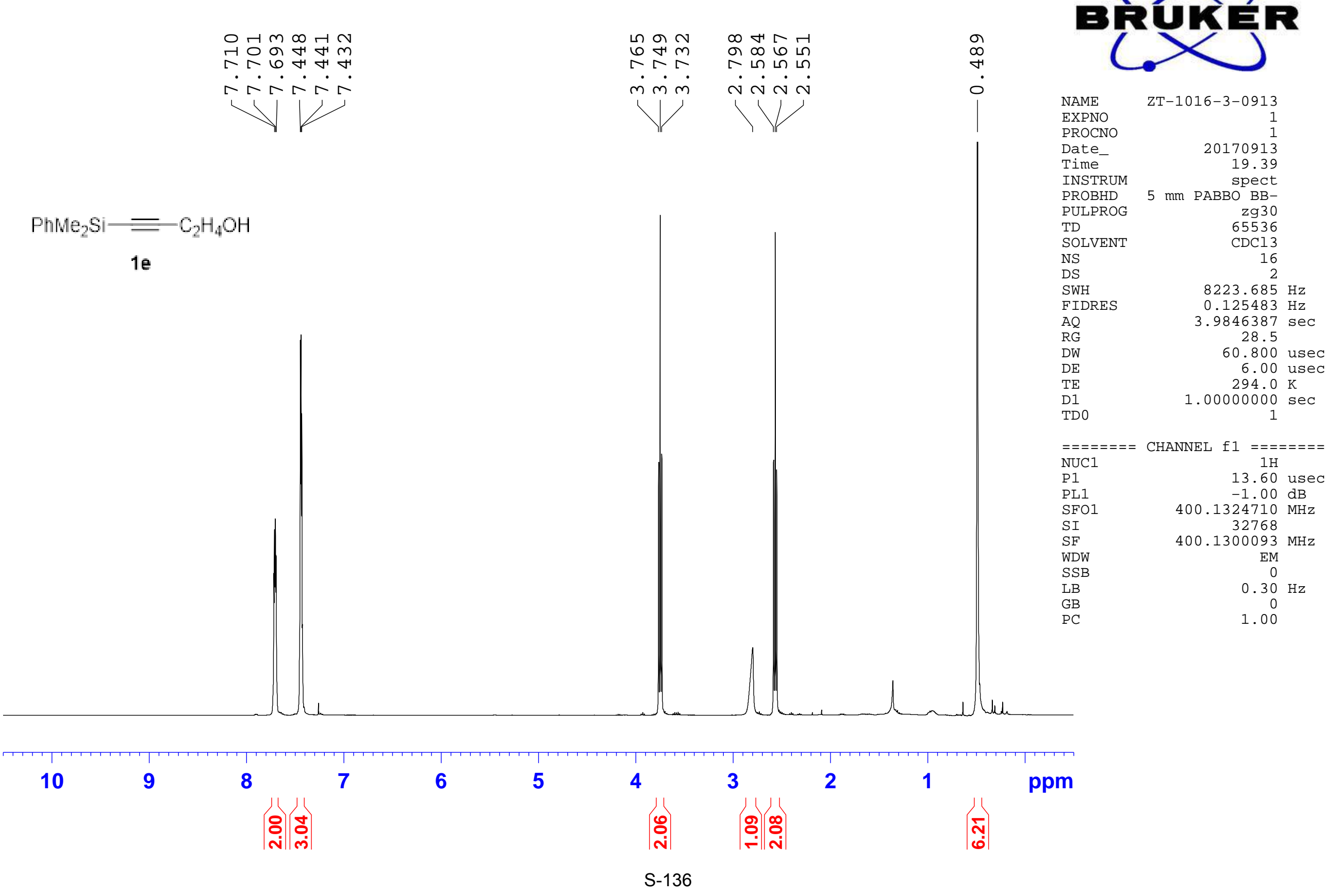




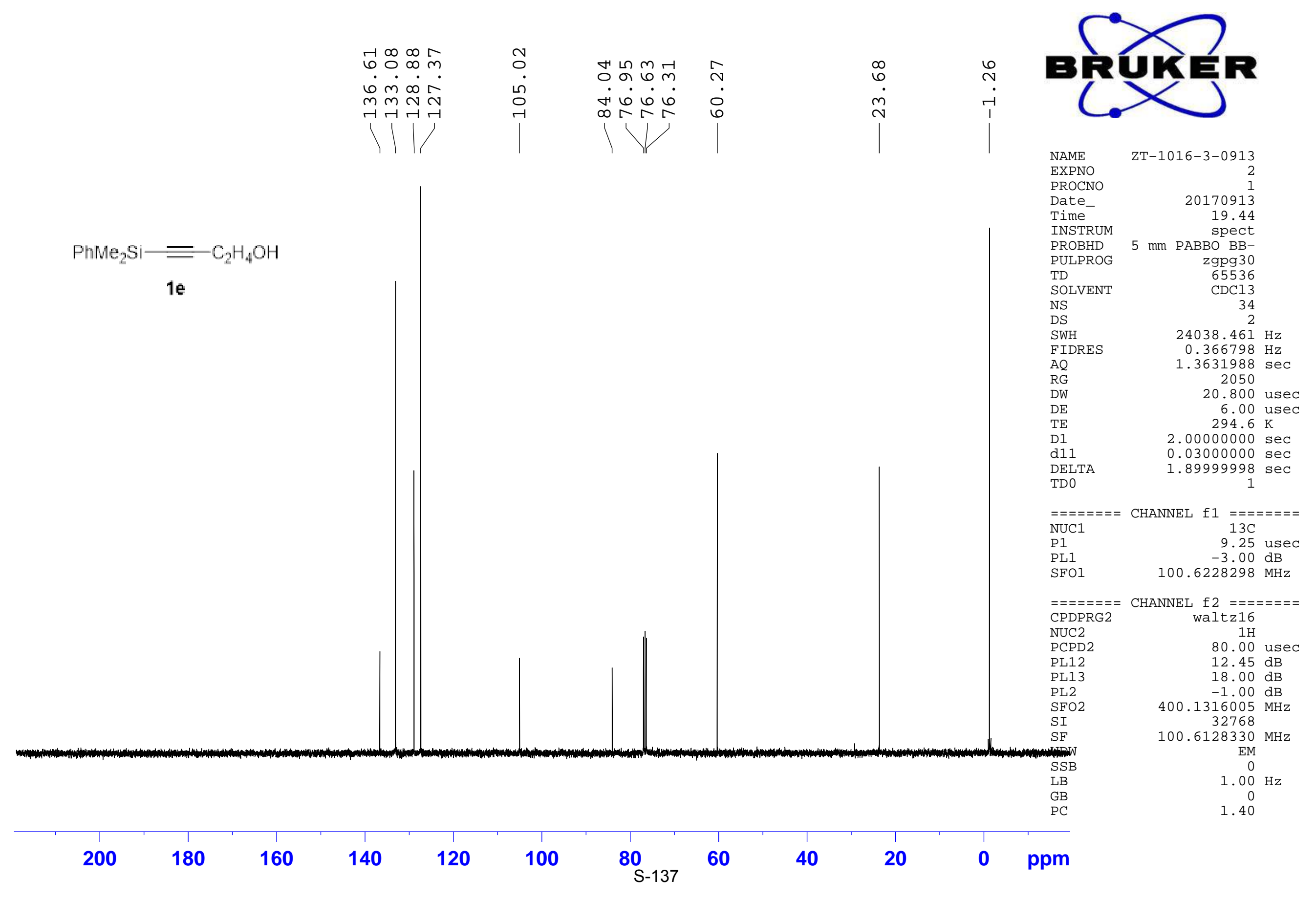




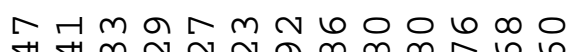
ฟै rarrarrirarir

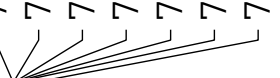

$\mathrm{PhMe}_{2} \mathrm{Si}=\mathrm{C}_{3} \mathrm{H}_{6} \mathrm{OH}$

$1 f$

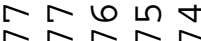
$\dot{m} \dot{m} \dot{m} \dot{m} \dot{m}$

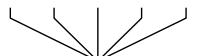

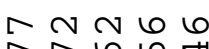

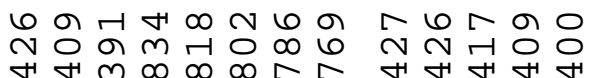
$\sim \sim N-H-1$

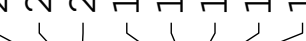
$\dot{\circ} \dot{0} \dot{0} \dot{0}$

NAME

AQ
RG
DW

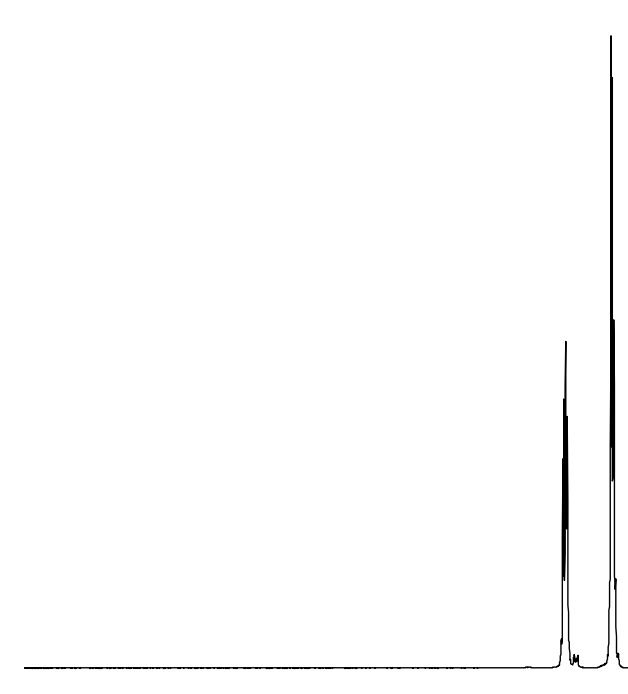

10

9

8

$\left|\begin{array}{cc}8 \\ 0 \\ \text { N่ }\end{array}\right|$

7

6

5

4

$\left(\begin{array}{l}\text { N } \\ \text { N }\end{array}\right.$

$-138$
EXPNO

PROCNO

Date

Time

INSTRUM

PROBHD

PULPROG

TD

SOLVENT

NS

SWH

FIDRES

DW

DE

D1

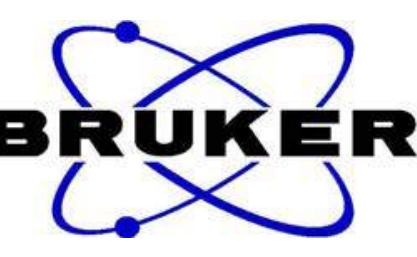

ZT-1038

20170927

23.08

$5 \mathrm{~mm}$ PABBO BB-

$\mathrm{zg} 30$
65536

CDC13

16
2

$8223.685 \mathrm{~Hz}$

$0.125483 \mathrm{~Hz}$

$3.9846387 \mathrm{sec}$

60.800 usec

6.00 use $673.2 \mathrm{~K}$

$1.00000000 \mathrm{sec}$

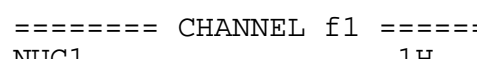

NUC1

P1 1

PL1

SFO1

SF

WDW

SSB

LB

PC

$1 \mathrm{H}$

13.60 usec $400.1324710 \mathrm{dH}$ 400.13247108
32768

$400.1300098 \mathrm{MHz}$

EM

0
$0.30 \mathrm{~Hz}$

0.30
0
1.00 


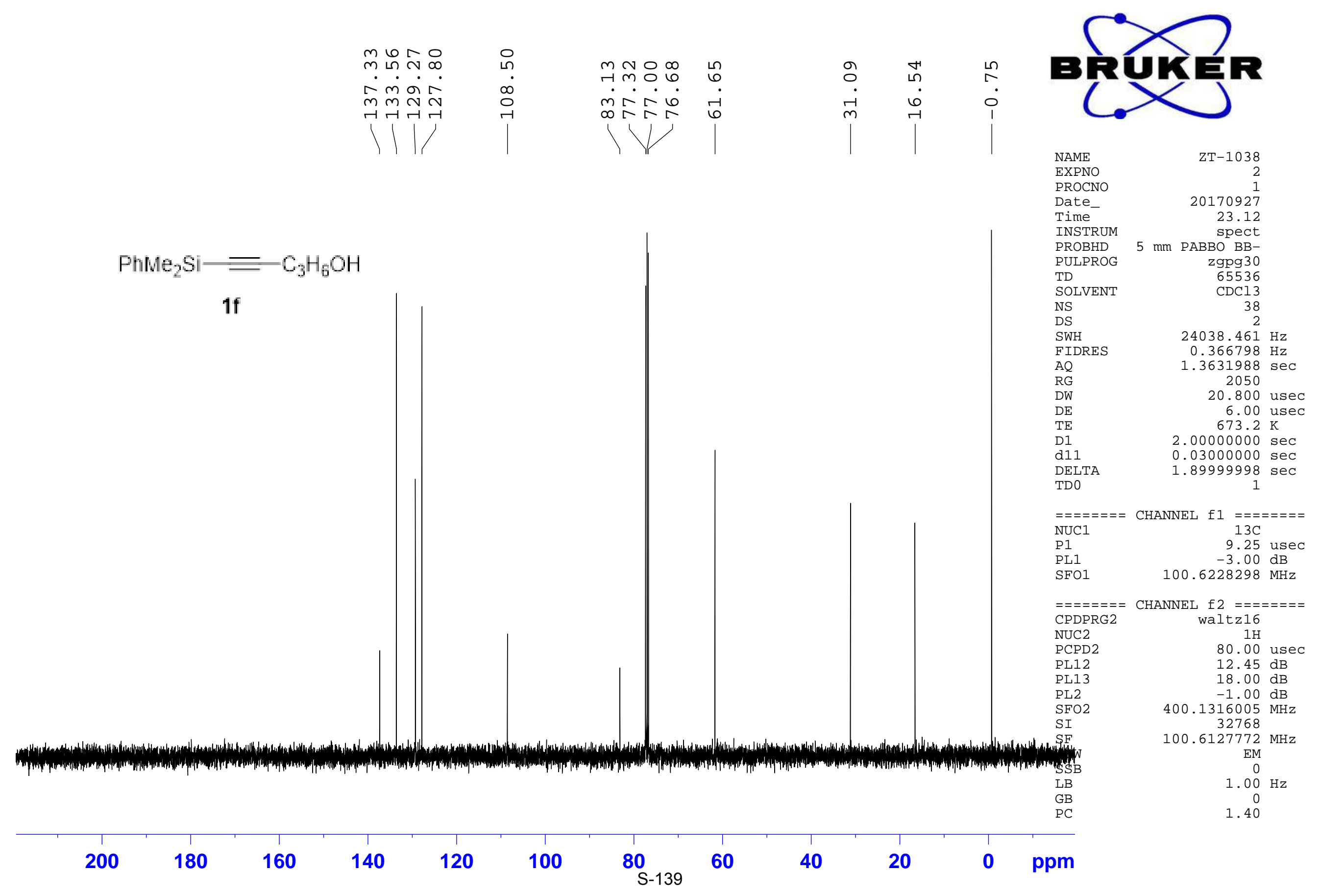




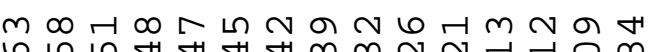

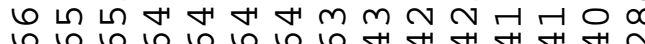

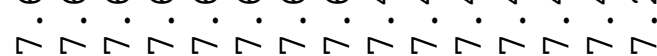

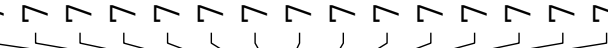

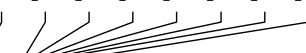

$\mathrm{PhMe}_{2} \mathrm{Si}=\mathrm{CH}_{2} \mathrm{OAC}$

$1 \mathrm{~g}$

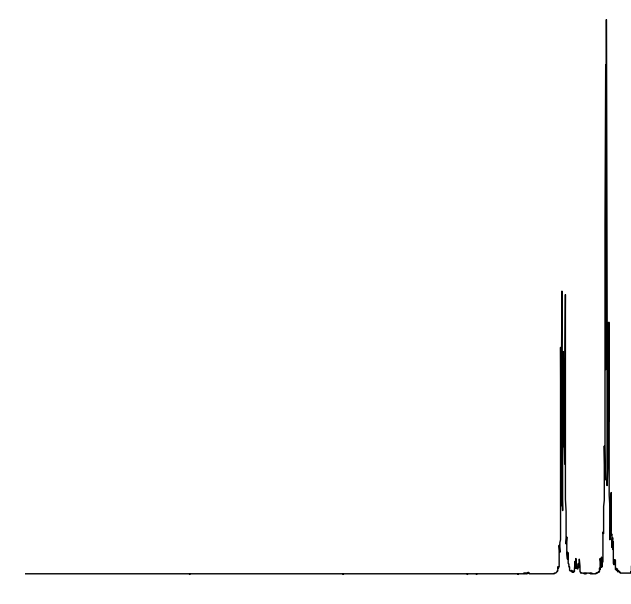

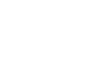
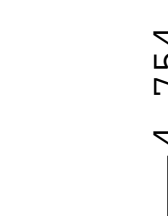

r.

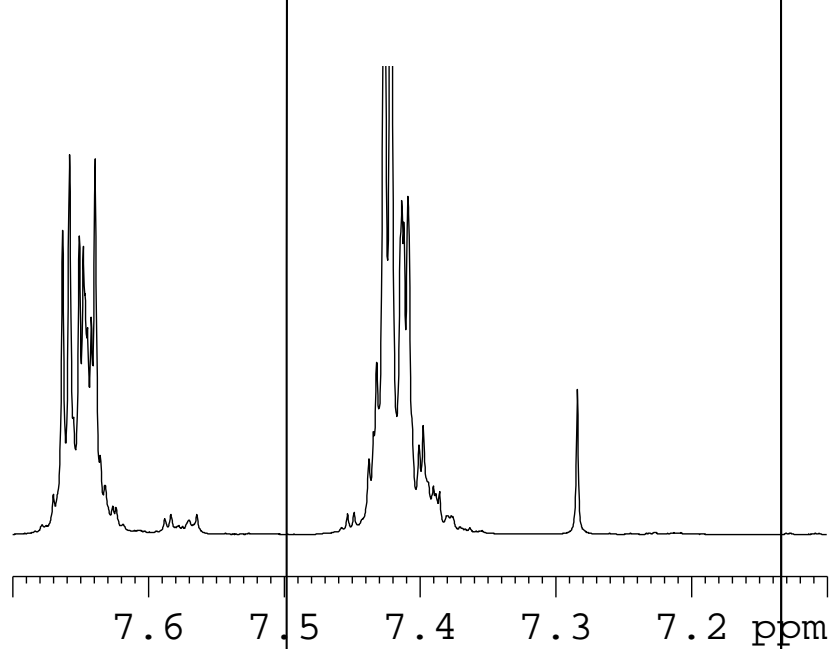

10

9

7

.

4

$\left|\begin{array}{c}8 \\ 8 \\ \text { N|| }\end{array}\right| \begin{gathered}n \\ \text { m. }\end{gathered} \mid$

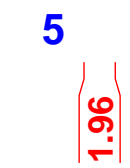

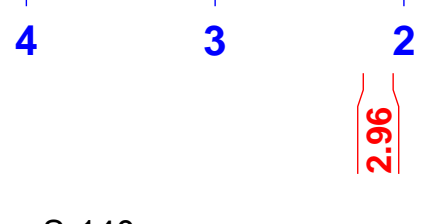

S-140 

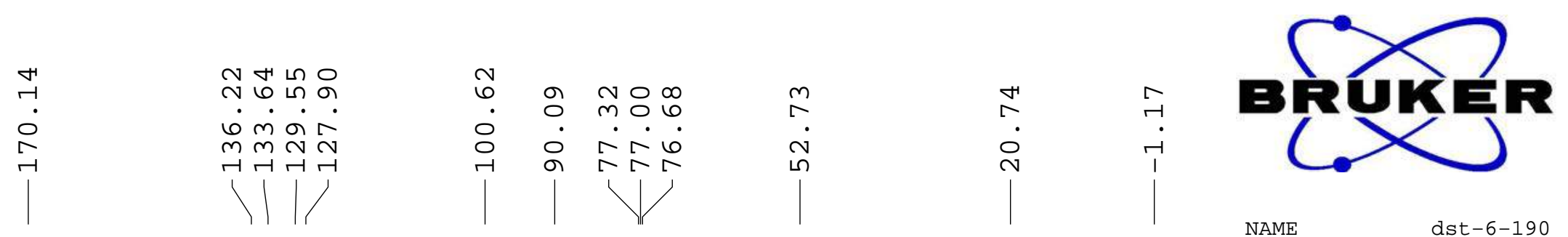

$$
\text { EXPNO }
$$

PROCNO

Date

INSTRUM

PROBHD

PULPRO

$\mathrm{PhMe}_{2} \mathrm{Si}=\mathrm{CH}_{2} \mathrm{OAC}$

$1 \mathrm{~g}$

TD

SOLVENT

NS

DS

FIDRES

$\mathrm{AQ}$

RG

DW

$\mathrm{DE}$

TE

D11

D11

$===$

SEO

Nuc1

P 1

SF

WDW

$\mathrm{SSB}$

LB

$\mathrm{GB}$
$\mathrm{PC}$

dst $-6-190$

20140707

11.51

spect

$5 \mathrm{~mm}$ DUL $13 \mathrm{C}-1$

zgpg 30

CDCl3

40

$24038.461 \mathrm{~Hz}$

$0.366798 \mathrm{~Hz}$

1. $3631988 \mathrm{sec}$

196.92

20.800 usec

6.50 usec

$2.00000000 \mathrm{sec}$

$0.03000000 \mathrm{sec}$

.03000000
1

CHANNEL $\mathrm{f} 1======$ $100.6228298 \mathrm{MHz}$ $13 \mathrm{C}$

9.60 usec

$100.6127747 \mathrm{MHz}$

EM

0
$1.00 \mathrm{~Hz}$

$1.00 \mathrm{~Hz}$
0
1.40 


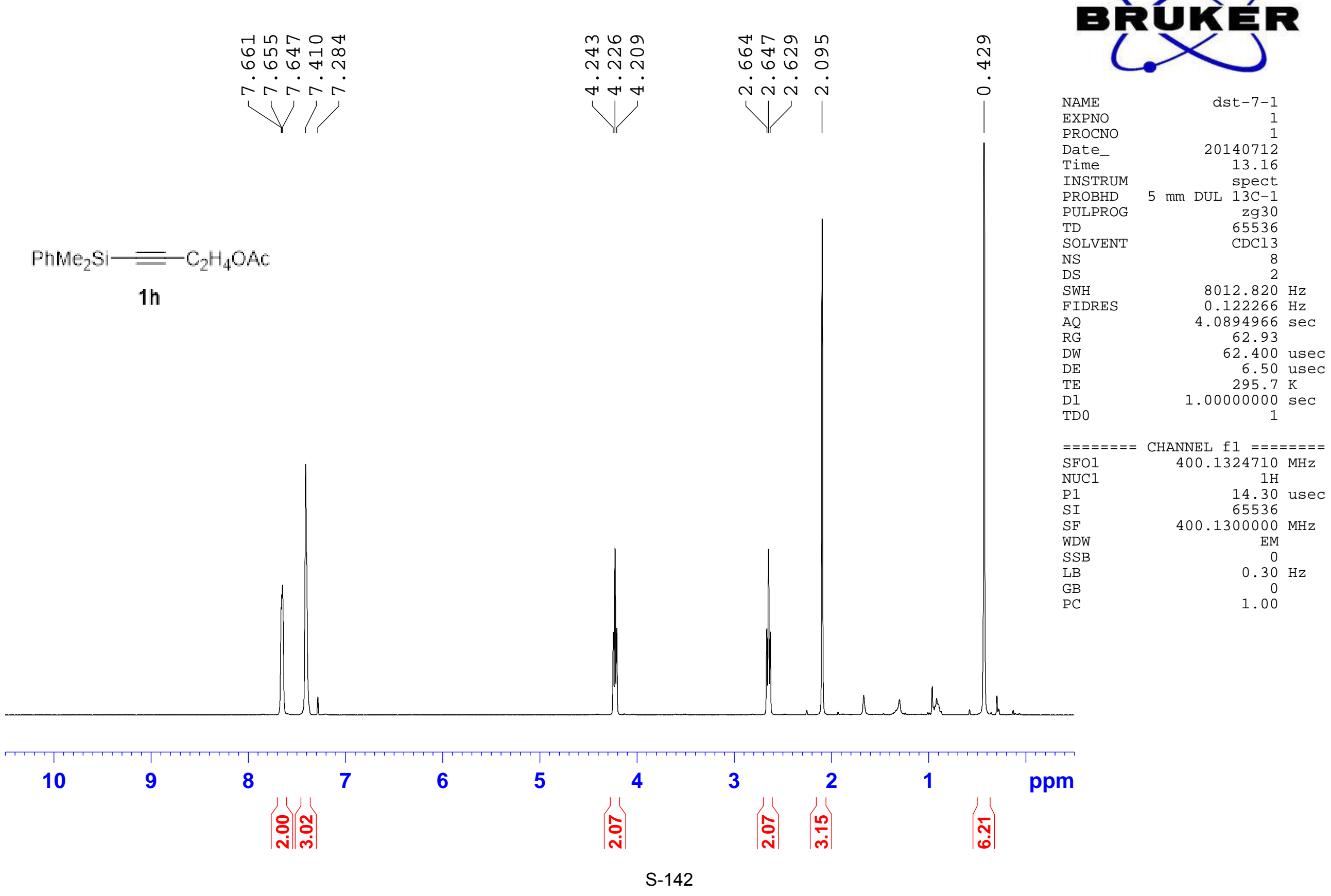




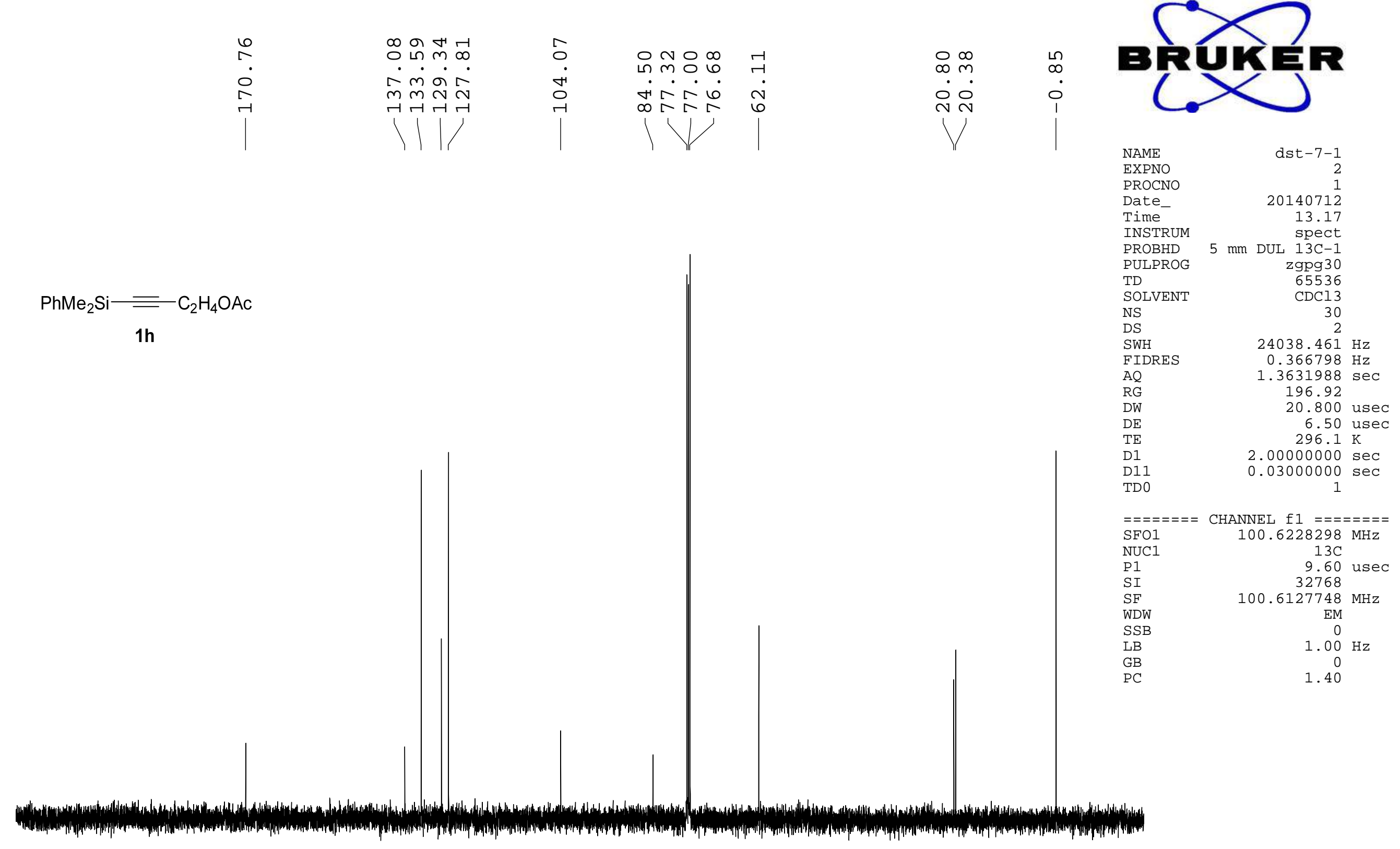

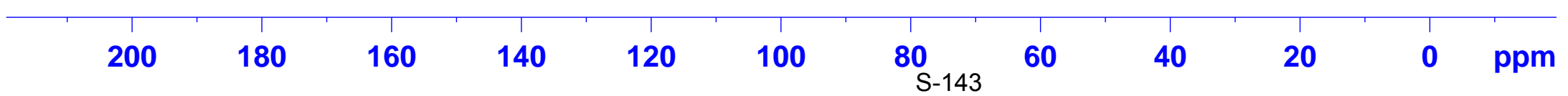


$\operatorname{rom} 2 \pi$

बैं

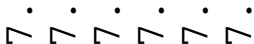

MI

$\mathrm{PhMe}_{2} \mathrm{Si}=\mathrm{C}_{2} \mathrm{H}_{4} \mathrm{OMs}$

$1 \mathrm{i}$ $\infty$ r

$m m m$

$\dot{\nabla} \dot{\nabla} \dot{\gamma}$

II $\begin{array}{llll}\infty & m & 0 & 0 \\ 0 & \infty & 0 & 0\end{array}$

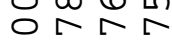

$\dot{m} \dot{\sim} \dot{\sim}$

11

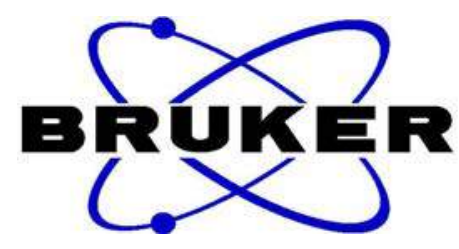

NAME

EXPNO

PROCNO

Date

Time

INSTRUM

PROBHD

PULPROG

TD

SOLVENT

NS

SWH

$\begin{array}{ll}\text { FIDRES } & 8012.820 \mathrm{~Hz} \\ & 0.122266 \mathrm{~Hz}\end{array}$

$\mathrm{AQ} \quad 4.0894966 \mathrm{sec}$

RG

RG

DE

TE

TD0

$=======$ CHANNEL $\mathrm{f} 1 \mathrm{1}=======$

$\mathrm{SFO} 1$
$\mathrm{NUC1}$

P 1

SI

WDW

SSB

LB

LB

PC dst $-6-198$

20140712

13.10

$5 \mathrm{~mm}$ DUL $13 \mathrm{C}-1$

zg30
65536

$\mathrm{CDCl} 3$ 62.93

62.400 usec 6.50 usec $1.00000000 \mathrm{sec}$ $14.3 \mathrm{H}$ 14.30 use $400.1300000 \mathrm{MHz}$

EM

0
$0.30 \mathrm{~Hz}$

0.30
0
1.00 


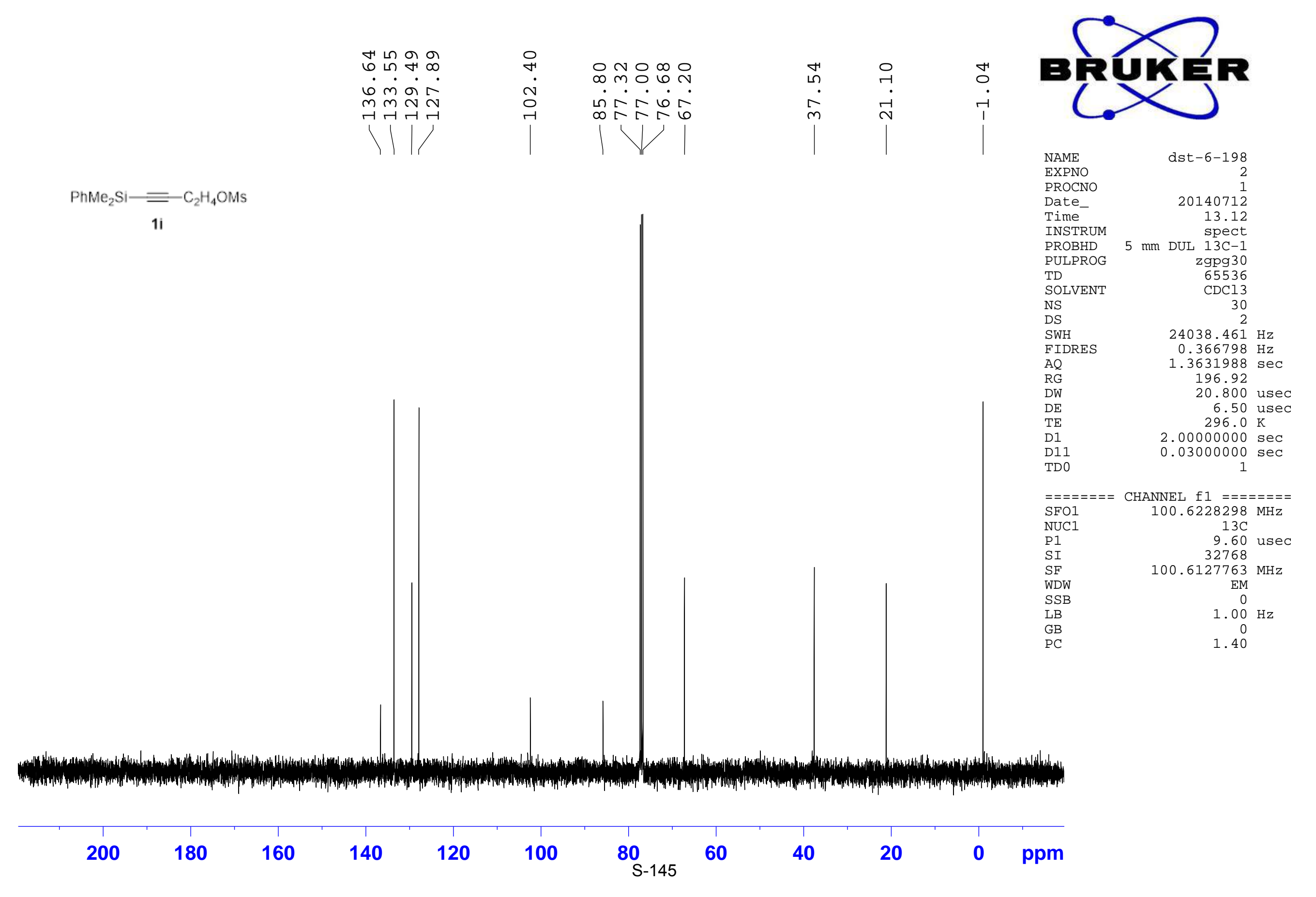




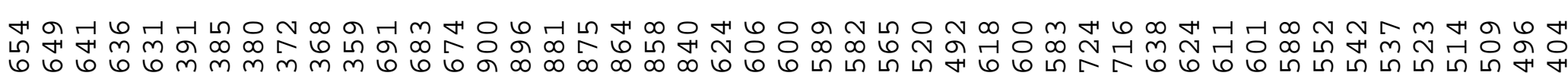

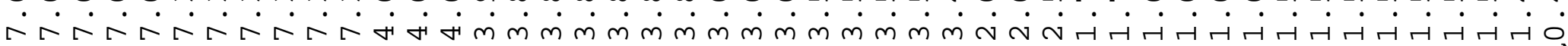

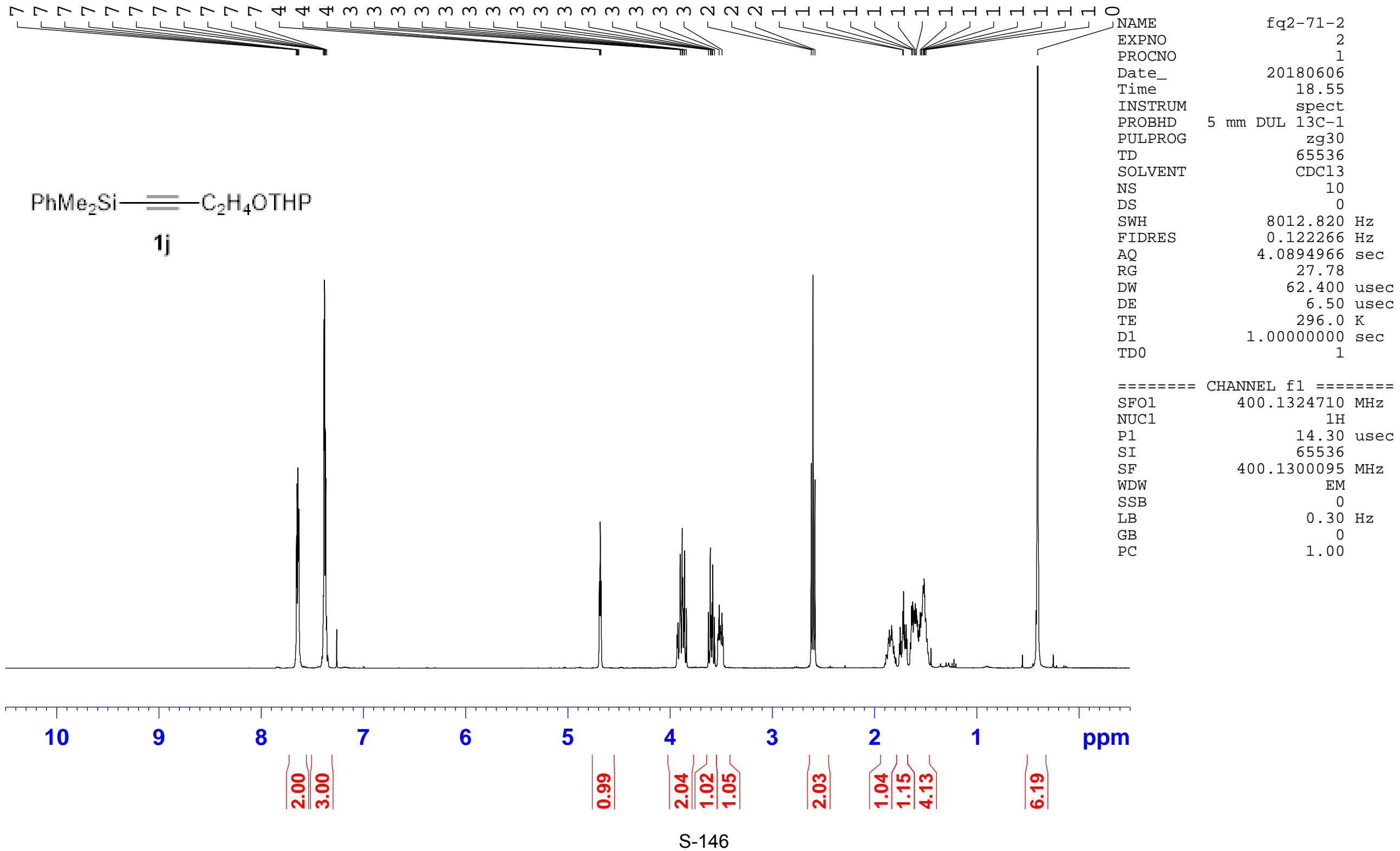


$\mathrm{PhMe}_{2} \mathrm{Si}=\mathrm{C}_{2} \mathrm{H}_{4} \mathrm{OTHP}$

1j

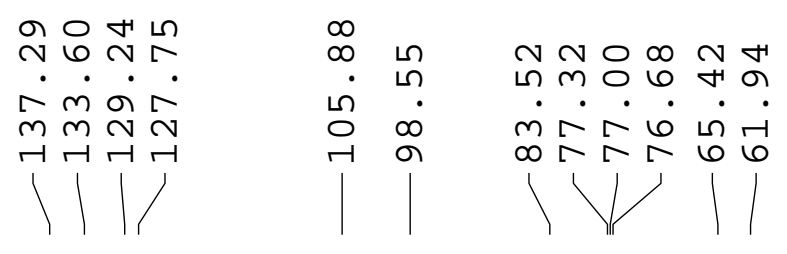

ก

$\hat{m} m \tilde{N}$

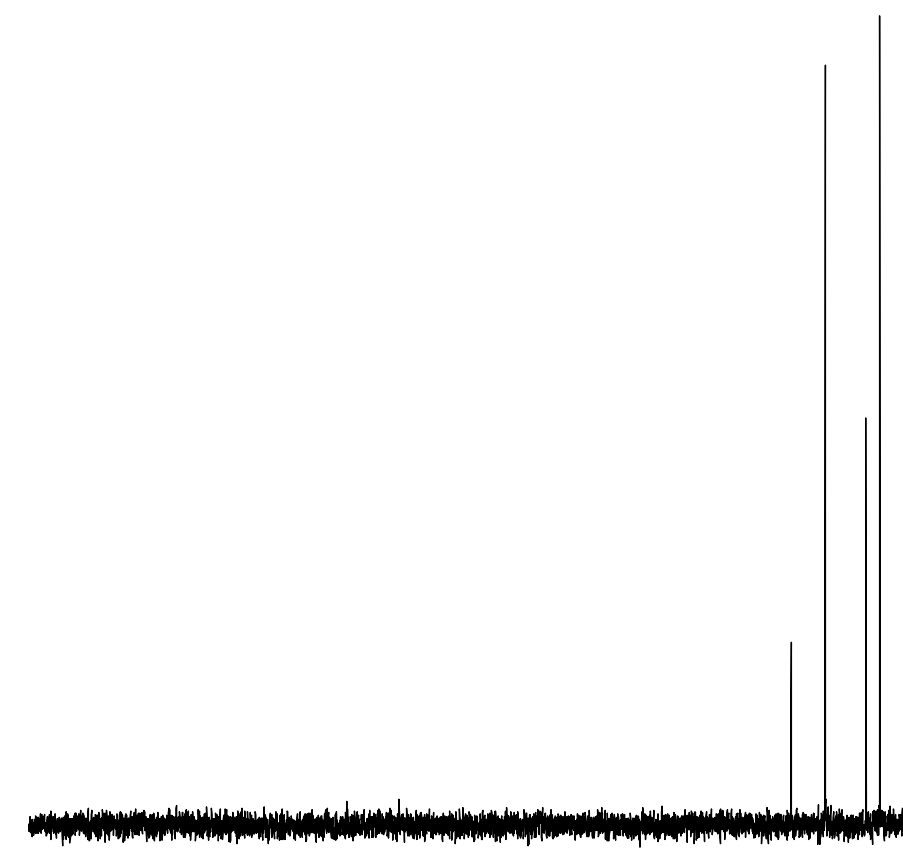

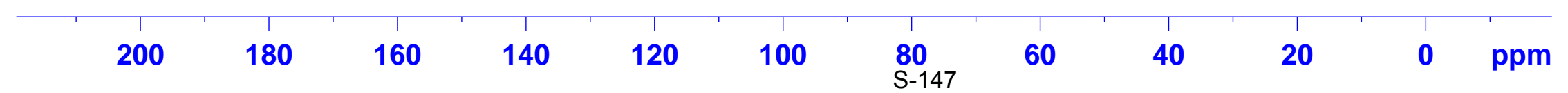

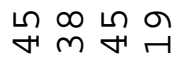

का न

111

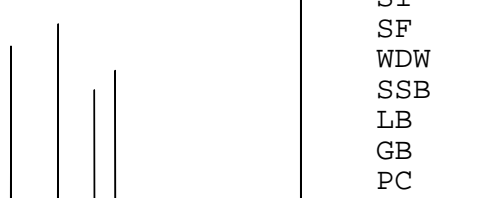

(2)

NAME
EXPNO

PROCNO

Date

INSTRUM

PROBHD

PULPROG

SOLVENT

NS

DS

FIDRES

AQ

RG

DE

TE

D11
TD 0

$===$

$\mathrm{SFO}$

NUC

P1 fq2 $2-71-2$

20180606 18.57 18.57

$5 \mathrm{~mm}$ DUL $13 \mathrm{C}-1$

zgpg 30
65536

$\mathrm{CDCl} 3$ 40

$24038.461 \mathrm{~Hz}$

$0.366798 \mathrm{~Hz}$

$3631988 \mathrm{sec}$

196.92

20.800 usec

6.50 usec

$2.00000000 \mathrm{sec}$

0.03000000

CHANNEL $\mathrm{f} 1======$ $100.6228298 \mathrm{MHz}$ $13 \mathrm{C}$

9.60 usec 100.6127765

$100.6127765 \mathrm{MHz}$

EM

$1.00 \mathrm{~Hz}$

0
1.40 


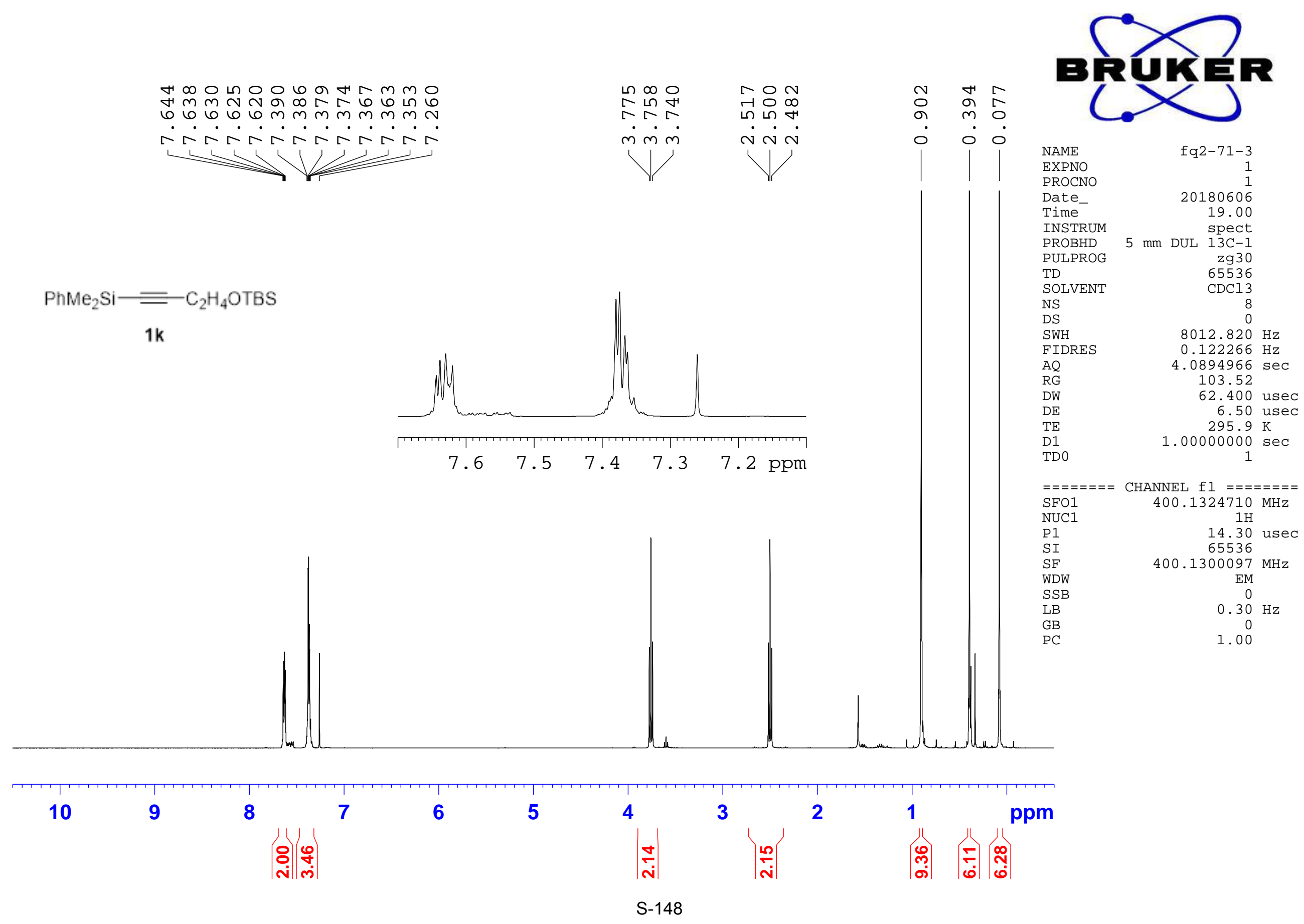




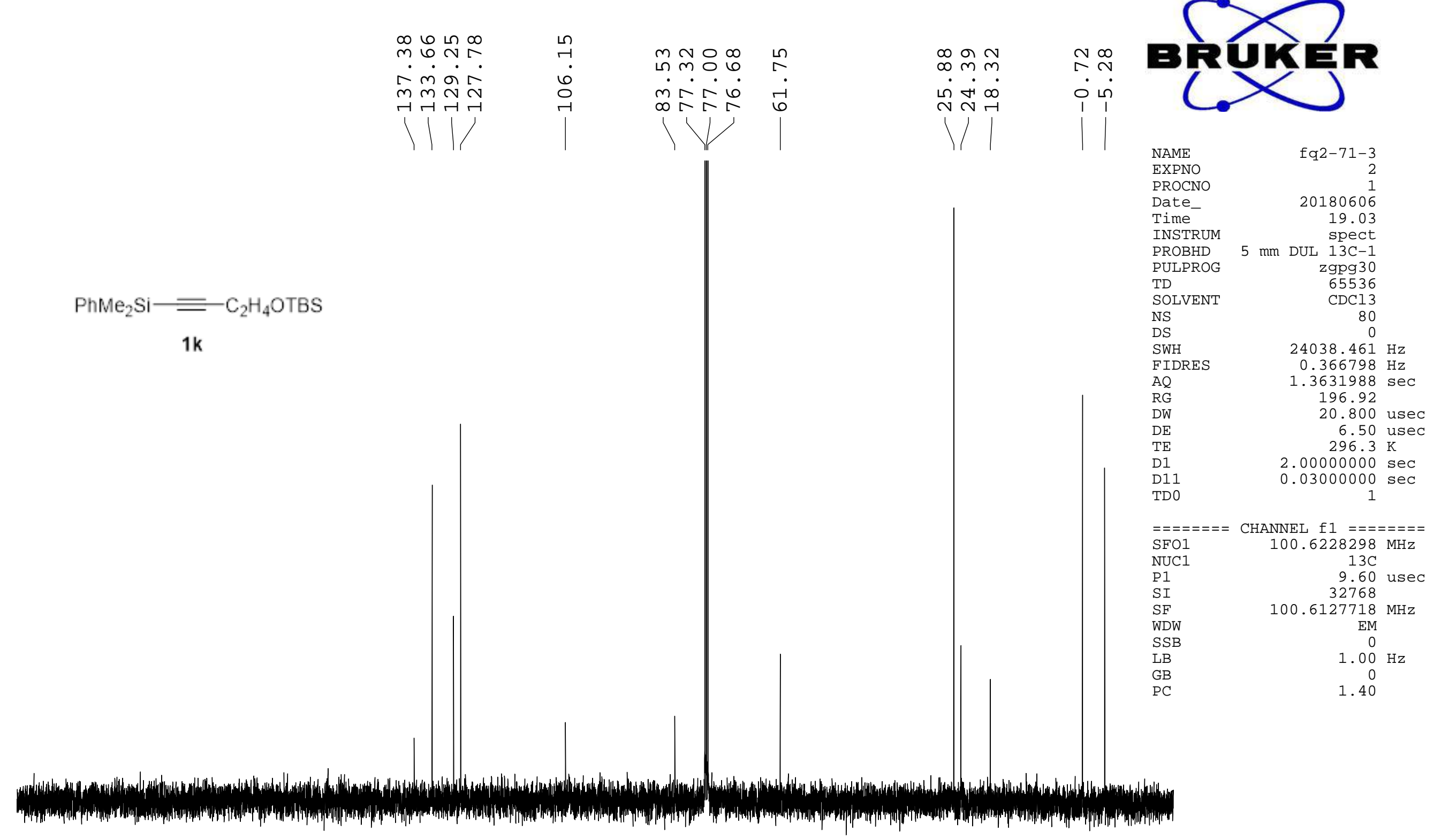

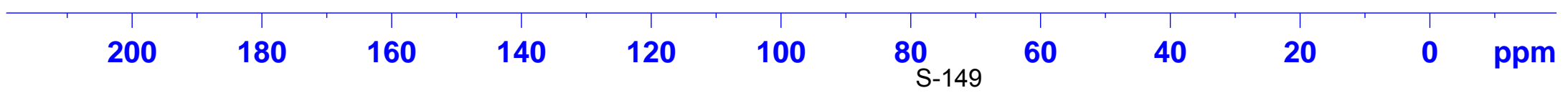


con 0 n

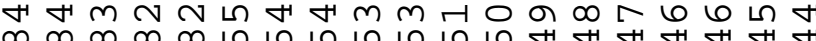

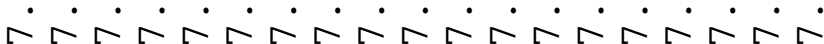

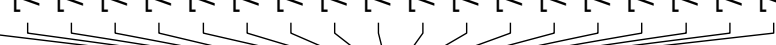

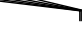

$\mathrm{PhMe}_{2} \mathrm{Si} \equiv \mathrm{CH}_{2} \mathrm{OBn}$

11 a $\infty \circ 6$

तิ

$\dot{\nabla} \dot{\nabla} \dot{\nabla} \dot{\gamma}$

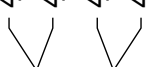

$\stackrel{n}{n}$

(a)

NAME

EXPNO

PROCNO

Date_

Time

PROBHD

PULPROG

TD

SOLVENT

NS

SWH

FIDRES

FID
AQ
RG
DW

DW

DE

D1
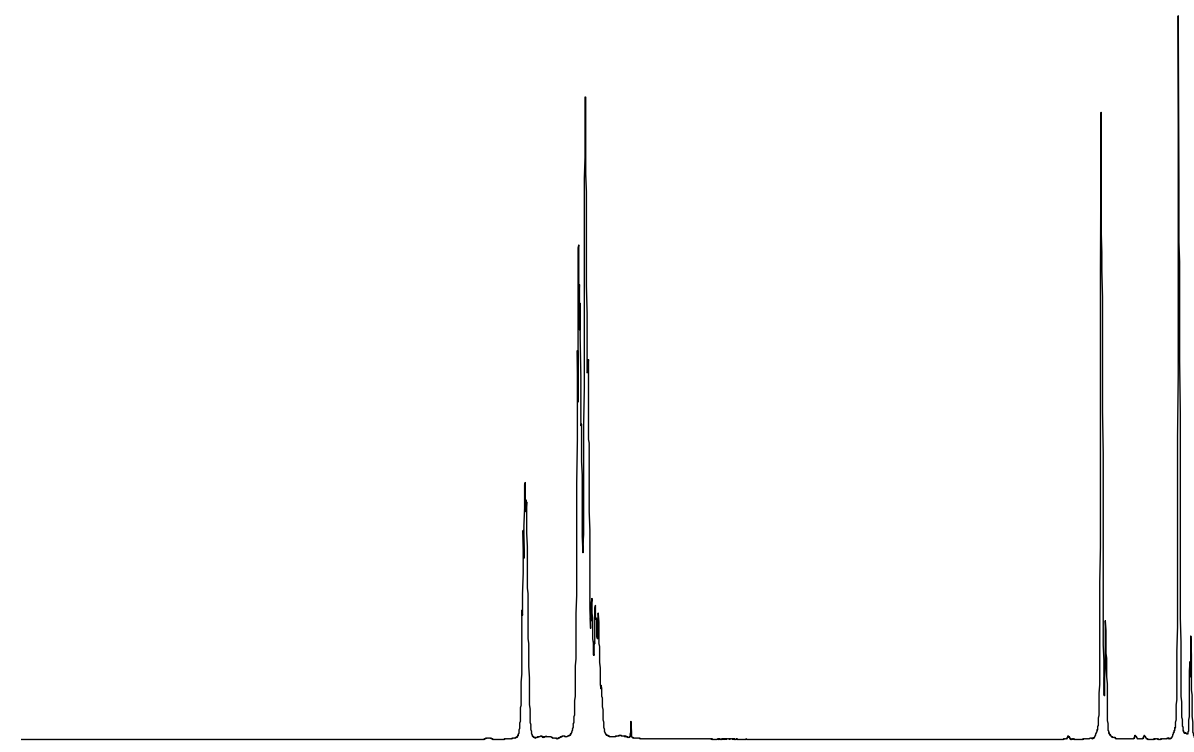

ZT-1017-0914

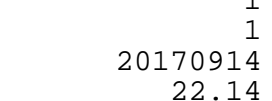
$5 \mathrm{~mm}$ PABBO $\mathrm{\text {BB}}-$

$\mathrm{zg} 30$
65536

$\mathrm{CDCl} 3$
16
2

2
$8223.685 \mathrm{~Hz}$

$3.9846387 \mathrm{sec}$ 28.5

60.800 usec 6.00 usec $1.00000000 \mathrm{sec}$

$\begin{array}{lc}======= & \text { CHANNEL } \mathrm{f} 1 \quad======= \\ \text { NUC1 } & 1 \mathrm{H} \\ \text { P1 } & 13.60 \mathrm{useC} \\ \text { PL1 } & -1.00 \mathrm{~dB} \\ \text { SFO1 } & 400.1324710 \mathrm{MHz} \\ \text { SI } & 32768 \mathrm{HH} \\ \text { SF } & 400.1300054 \mathrm{MHz} \\ \text { WDW } & \text { EM } \\ \text { SSB } & 0 \\ \text { LB } & 0.30 \mathrm{~Hz} \\ \text { GB } & 0 \\ \text { PC } & 1.00\end{array}$


เ $6 m \curvearrowleft \infty \sim \curvearrowleft N$ ก.

- $6 m \sigma \infty r-r$ $m m m \sim \sim \sim \sim$

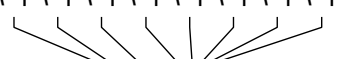

$\mathrm{PhMe}_{2} \mathrm{Si} \equiv \mathrm{CH}_{2} \mathrm{OBn}$

11

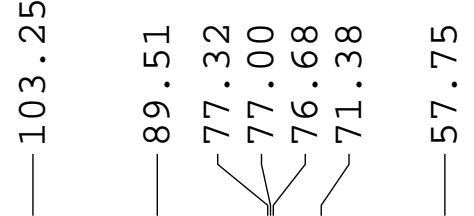

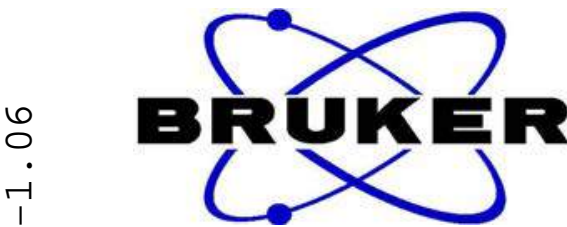

NAME

PROCNO

Date

Time

PROBHD

PULPROG

SOLVENT

NS

DS

FIDRES

AQ

$A Q$
$R G$

DW

DE

D1

d11

DELTA

TDO

$=======$ CHANNEI f1

NUC1

$\mathrm{P} 1$

$\mathrm{PL} 1$
$\mathrm{SFO} 1$ 130

$100.6228298 \mathrm{MHz}$

CHANNET f2 $======$

CPDPRG2 waltz16

$\mathrm{NUC2}$

PL12

$\mathrm{PL} 13$

PL2

$\mathrm{SFO} 2$

$\mathrm{SI}$
$\mathrm{SF}$

ZT-1017-0914

20170914 22.22

$\mathrm{mm}$ PABBO BB-

zgpg 30

CDC13

$24038.461 \mathrm{~Hz}$

$0.366798 \mathrm{~Hz}$

$.3631988 \mathrm{sec}$

2050

0.800 usec

$295.5 \mathrm{~K}$

$2.00000000 \mathrm{sec}$

$000000 \mathrm{sec}$

$1.89999998 \mathrm{sec}$

\section{$=$}

$\mathrm{MHz}$




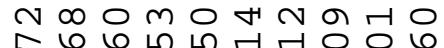

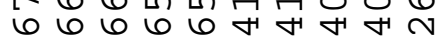

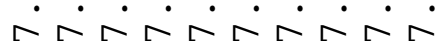

$\longrightarrow$

$\mathrm{PhMe}_{2} \mathrm{Si}=\mathrm{C}_{2} \mathrm{H}_{4} \mathrm{Br}$

$1 \mathrm{~m}$
กั

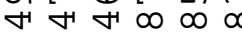

$\dot{m} \dot{m} \dot{m} \dot{\sim}$

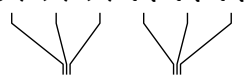

$\infty$

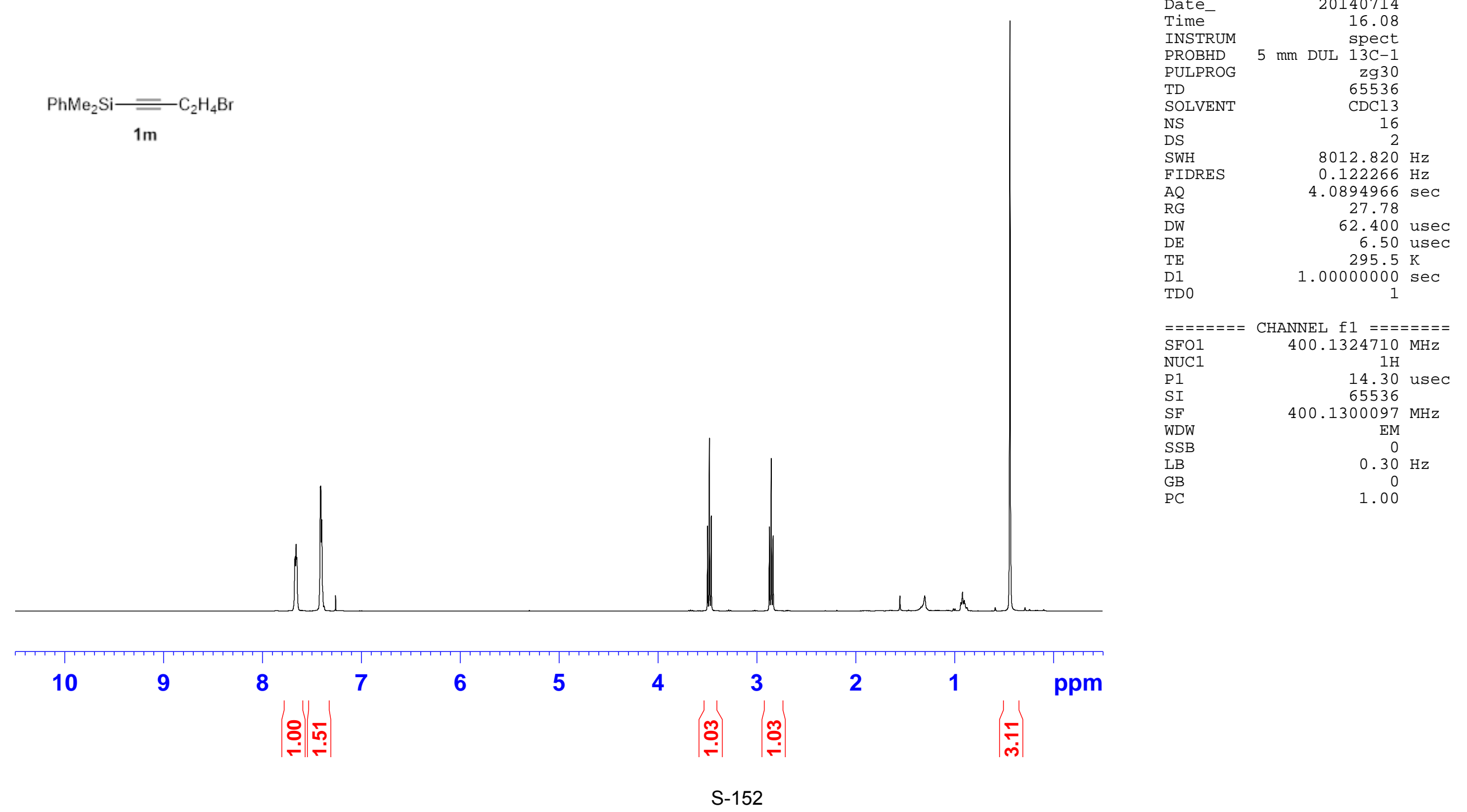

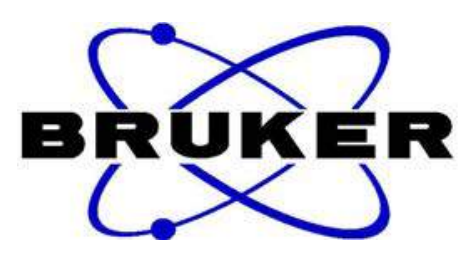

NAME

EXPNO

PROCNO

Time-

NSTRUM

SOLVENT

IDRES

RG

$\mathrm{DW}$

DE

TDO

dst $-7-3$

-3
1

140714

$3 \mathrm{C}-1$

65536 $400.1324710 \mathrm{MHz}$ NUC1 $1 \mathrm{H}$ P1 14.30 usec 400.1300097

WDW EM

$\begin{array}{lr}\mathrm{SSB} & 0 \\ \mathrm{LB} & 0.30 \mathrm{~Hz}\end{array}$

GB

0 
$\mathrm{PhMe}_{2} \mathrm{Si}=\mathrm{C}_{2} \mathrm{H}_{4} \mathrm{Br}$

$1 \mathrm{~m}$
नํํำ

$\dot{\theta} \dot{0} \dot{ }$

$m \sim N$

다 -1

)11

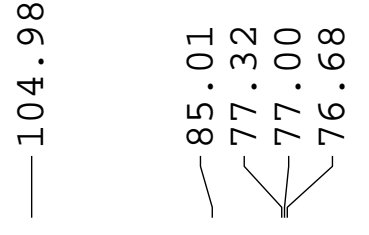

の

$\dot{\sigma} \dot{\pi}$

$\sim \sim$

1

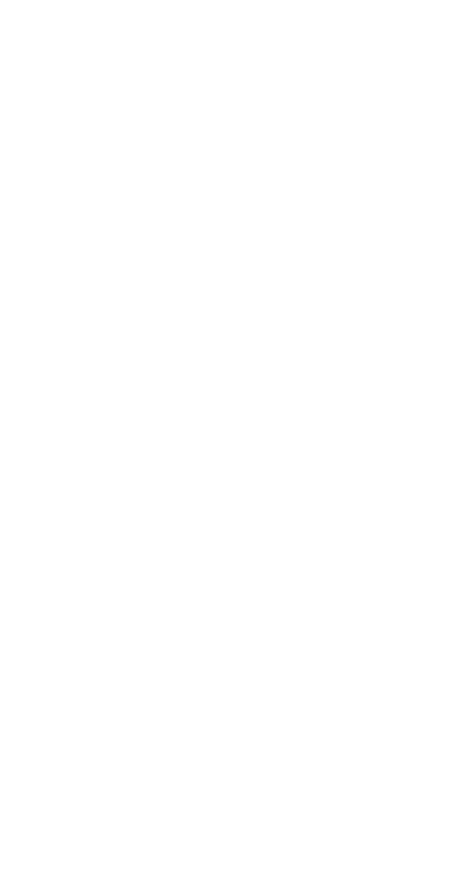

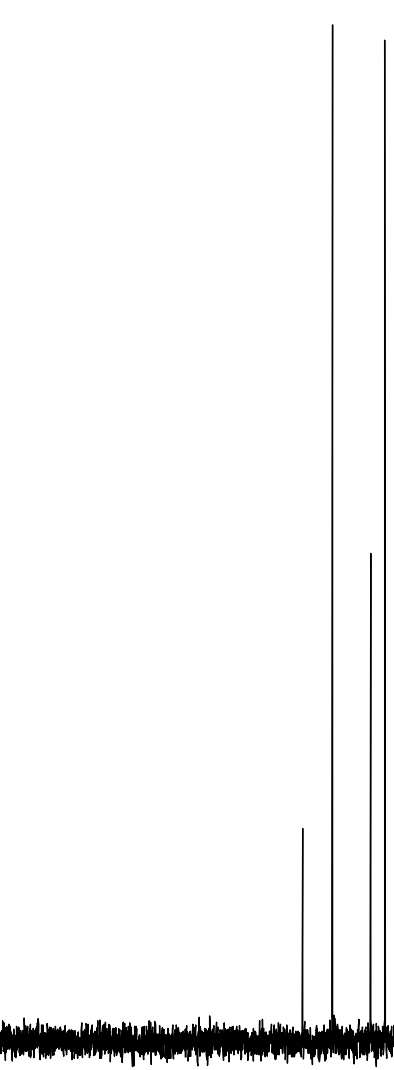

Nist-7-3

Date

INSTRUM

PROBHD

PULPROG

TD

SOLVENT

NS
DS

DS

SWH

AQ
RG
DW

DW

$\mathrm{DE}$

D1

D11

20140714

16.10

$5 \mathrm{~mm}$ DUL $13 \mathrm{C}-1$

zgpg 30

CDCl3

30

$24038.461 \mathrm{~Hz}$

$0.366798 \mathrm{~Hz}$

1. $3631988 \mathrm{sec}$

196.92

20.800 usec

6.50 usec

$2.00000000 \mathrm{sec}$

0.0300000

CHANNEL

$\mathrm{SFO}$

NUC

P 1

SI

WDW

SSB

LB

$\mathrm{PC}$

$100.6228298 \mathrm{MHz}$

$13 \mathrm{C}$

9.60 usec

100.6127773

100.6127

EM

$1.00 \mathrm{~Hz}$

0
1.40

80 


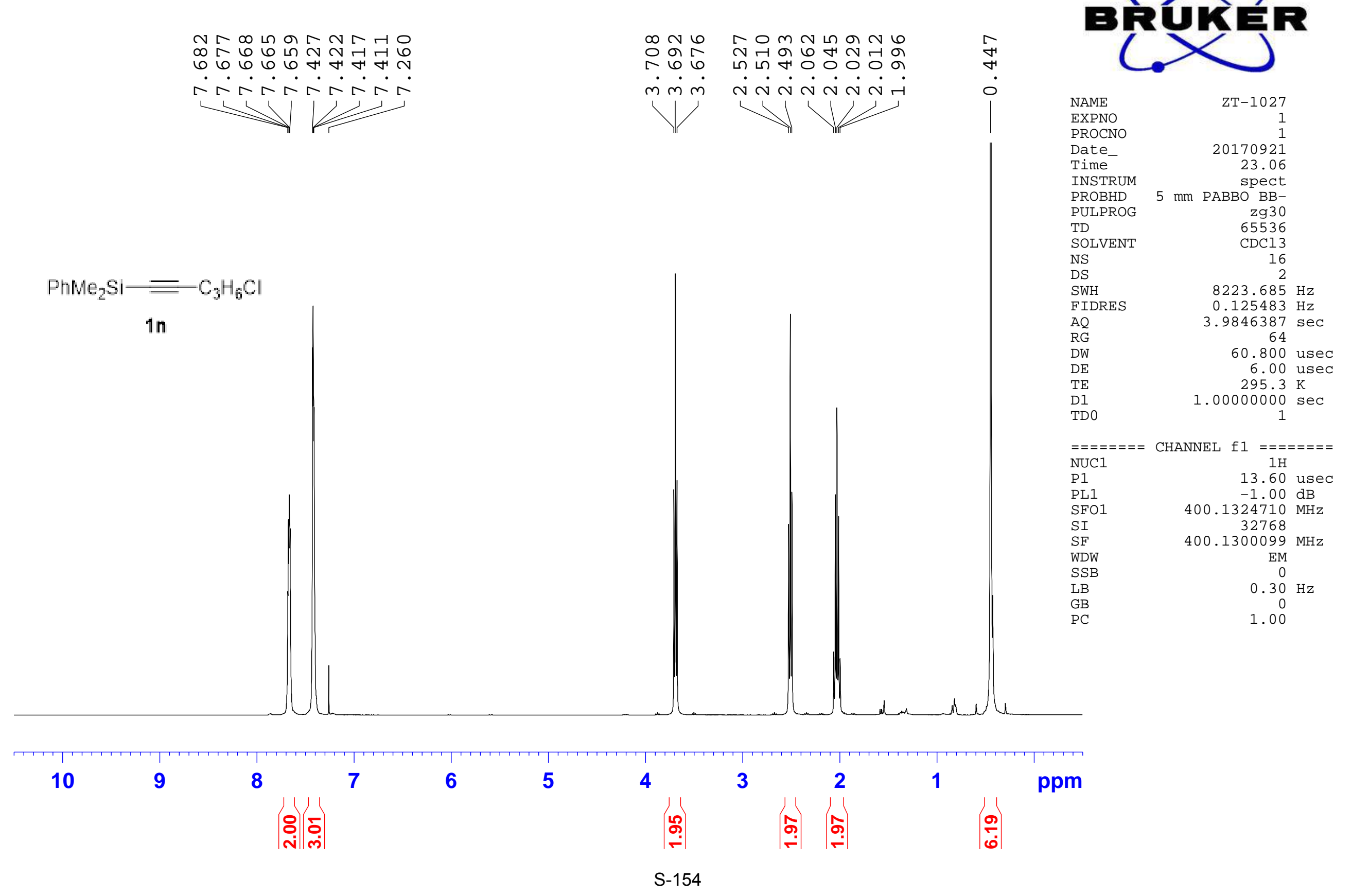




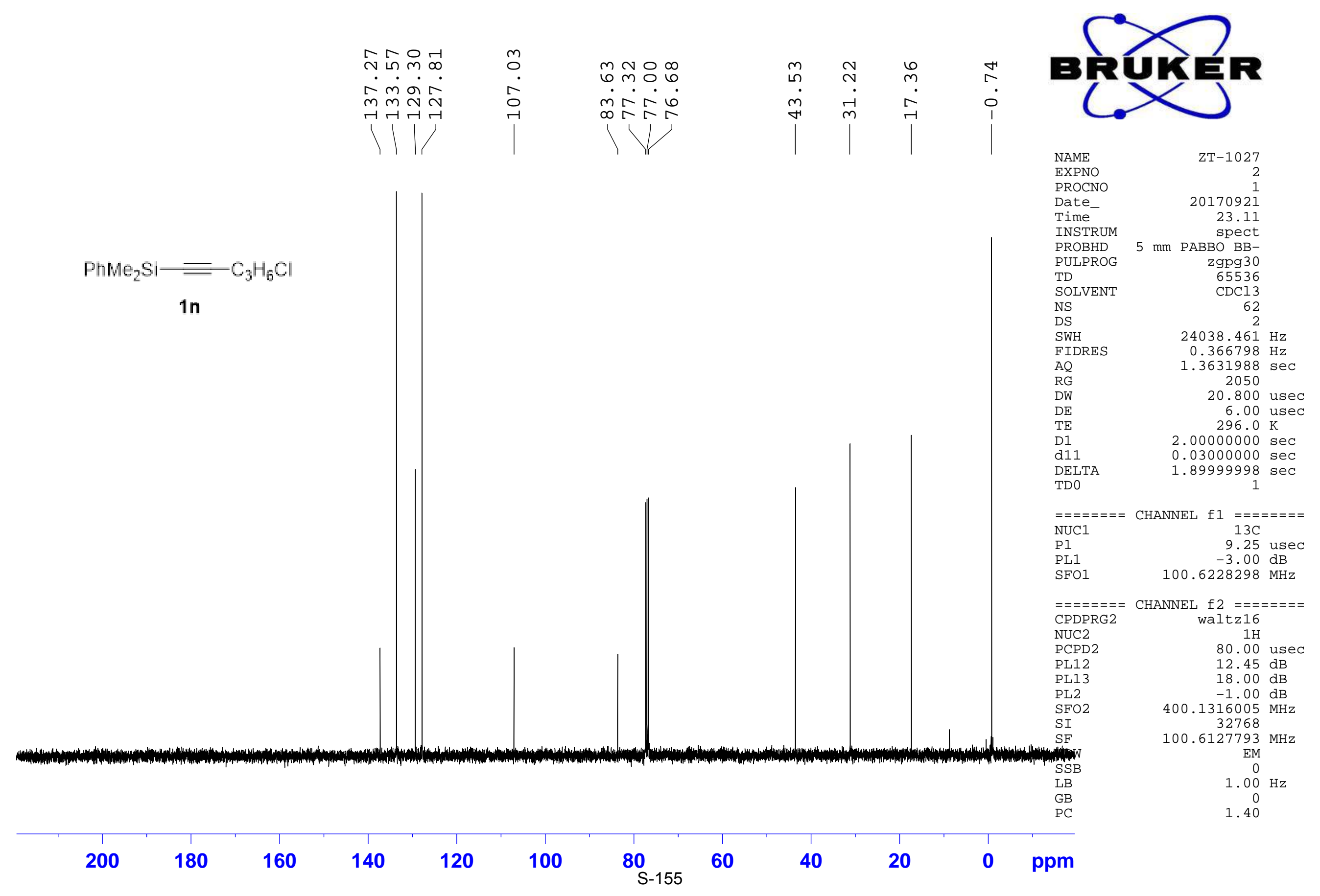




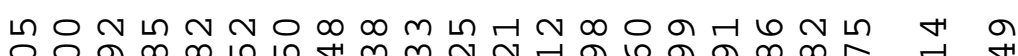

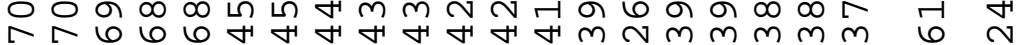

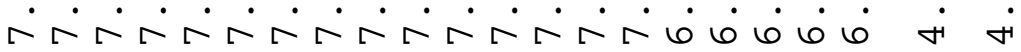

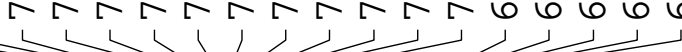

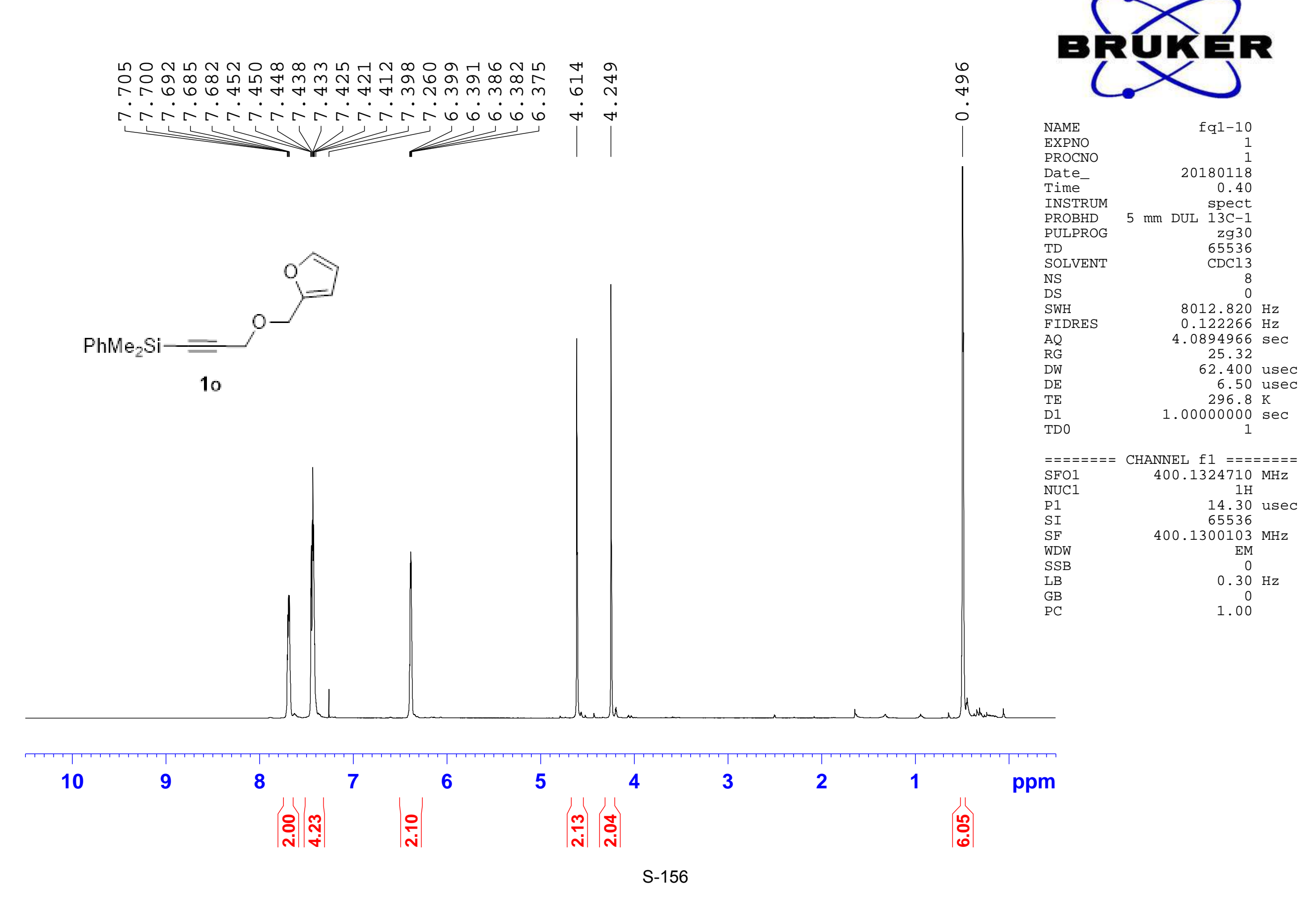

10

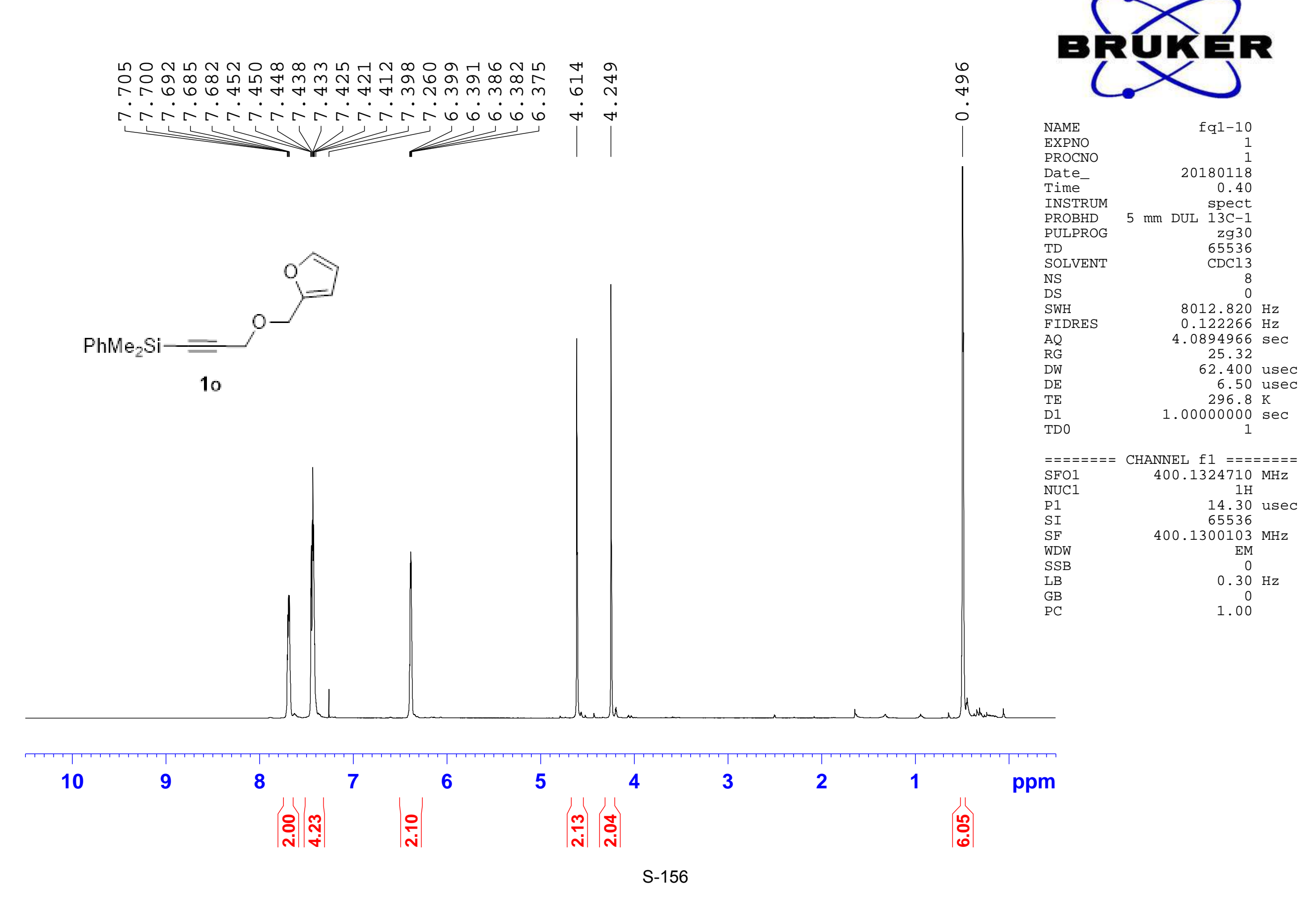

6 


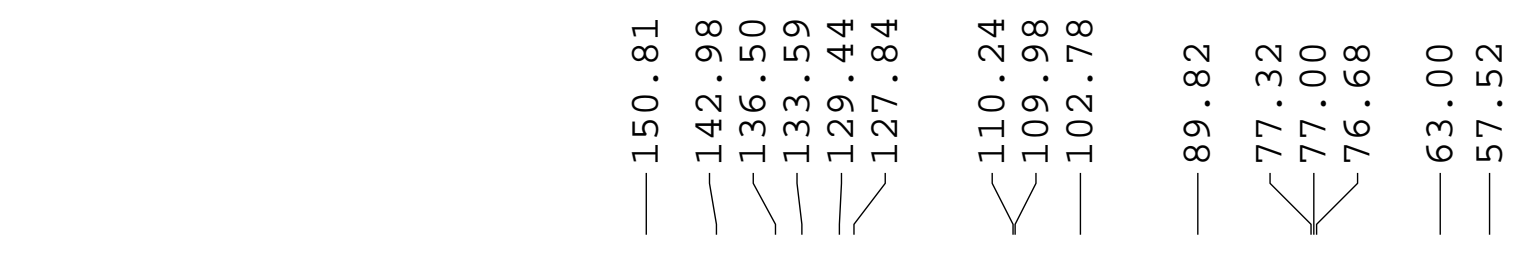

arixen

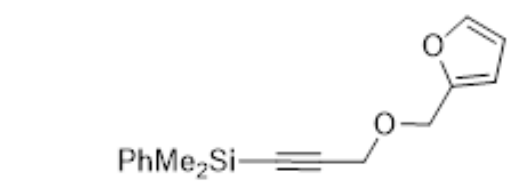

1o

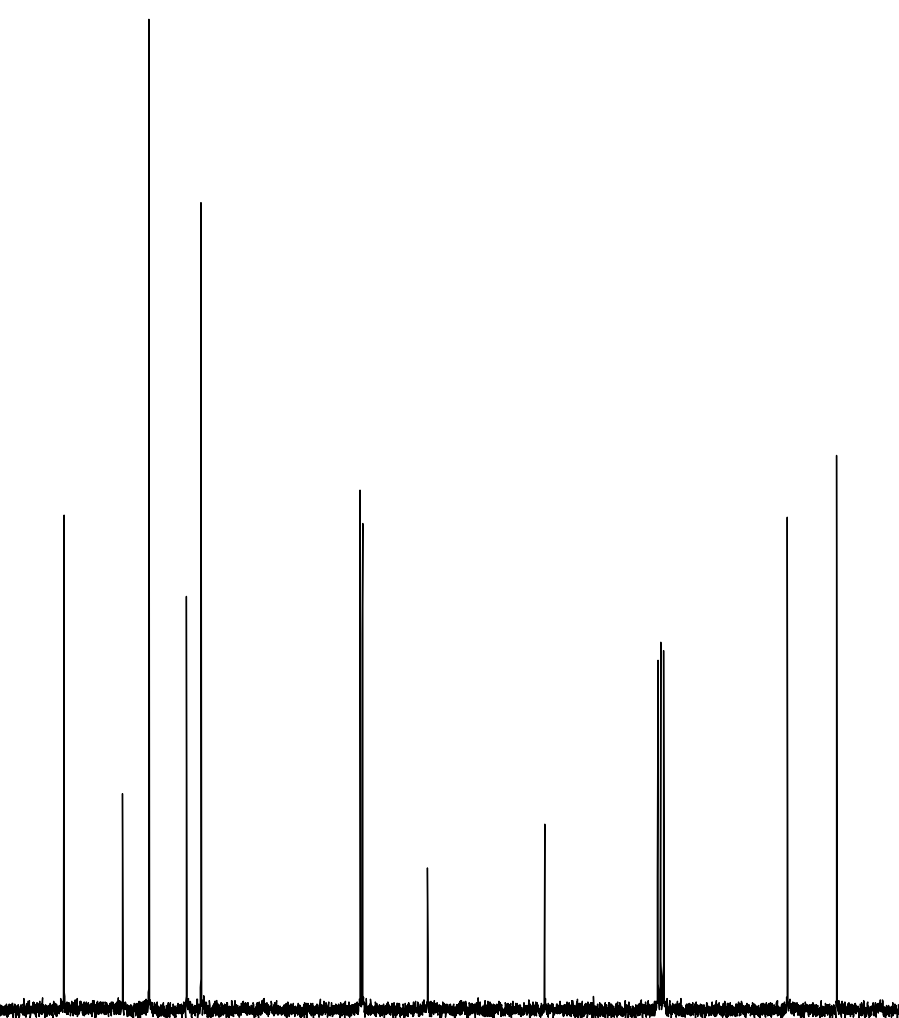

NAME

PROCNO

Date_

INSTRUM

INRTRD

PULPROG

TD

SOLVENT

NS

DS

FIDRES

FIDR
$\mathrm{AQ}$
$\mathrm{RG}$

RG
DW
DE

$\mathrm{DE}$

TE

D1
D11
TD0

$==$

$\mathrm{SFO}$

NUC

P 1

SI

WDW

SSB
LB
GB

GB
PC

fq1-10

20180118

0.45

spect

$5 \mathrm{~mm}$ DUL $13 \mathrm{C}-1$

zgpg 30

CDCl3

97

$24038.461 \mathrm{~Hz}$

$0.366798 \mathrm{~Hz}$

$.3631988 \mathrm{sec}$

196.92

20.800 usec

6.50 usec

$000000 \mathrm{~K}$

2.00000000 sec

$0.03000000 \mathrm{sec}$

CHANNEL $\mathrm{f} 1======$ $100.6228298 \mathrm{MHz}$ $13 \mathrm{C}$

9.60 usec $100.6127839 \mathrm{MHz}$ EM

0
$1.00 \mathrm{~Hz}$

1.00
0

1.40

\begin{tabular}{|cccccccccc|c|c}
\hline 200 & 180 & 160 & 140 & 120 & 100 & 80 & 60 & 40 & 20 & 0 & ppm
\end{tabular}




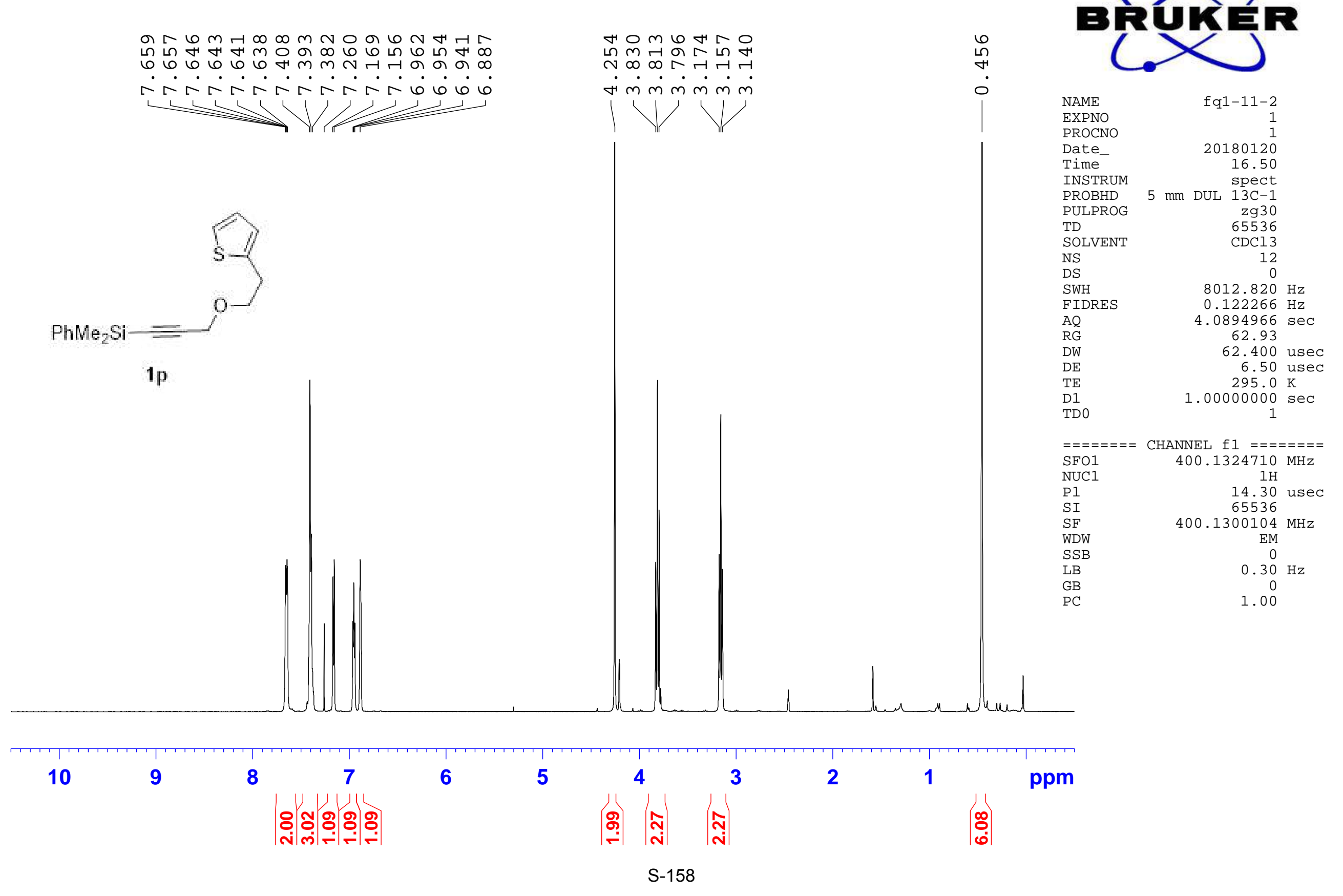




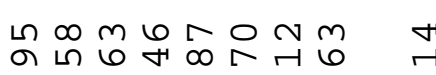

- $\dot{m} \dot{m} \dot{r} \dot{0} \dot{m} \dot{m}$

$\forall m m \sim N \sim N$

11

.

다 m의

के

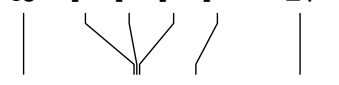

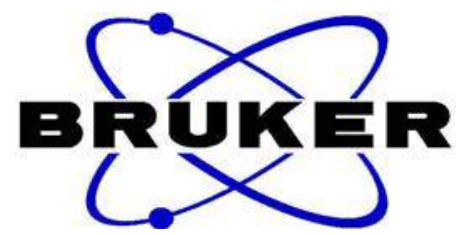

NAME PROCNO

Date

Time

INSTRUM

PROBHD

PULPROG

TD

SOLVENT

NS

DS

SWH
FIDRES

$\mathrm{FIDR}$
$\mathrm{AQ}$
$\mathrm{RG}$

DW

DE

TE

D1 11

TD 0

$===$

$\mathrm{SFO}$

P 1

SI

WDW

$\mathrm{SSB}$

LB

PC fq1-11-2

20180120 16.53 spect

$5 \mathrm{~mm}$ DUL $13 \mathrm{C}-1$

zgpg 30

$\mathrm{CDCl} 3$

$24038.461 \mathrm{~Hz}$

$0.366798 \mathrm{~Hz}$

1. $3631988 \mathrm{sec}$

196.92

20.800 usec

6.50 usec

CHANNEL $\mathrm{f} 1=======$ 9.60 usec 3.60

$100.6127773 \mathrm{MHz}$

EM

$1.00 \mathrm{~Hz}$
$0 \mathrm{H}$

0
1.40 


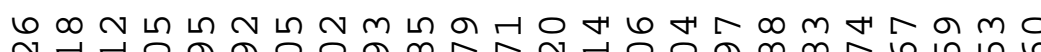
N

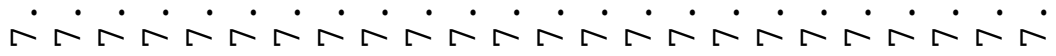

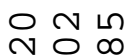
$\stackrel{\infty}{\infty} \infty$

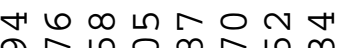

$\dot{m} \dot{m} \dot{m}$ $m m$ ल न न न $\dot{\sim} \dot{\sim} \dot{\sim} \dot{\sim} \dot{-} \dot{H}$
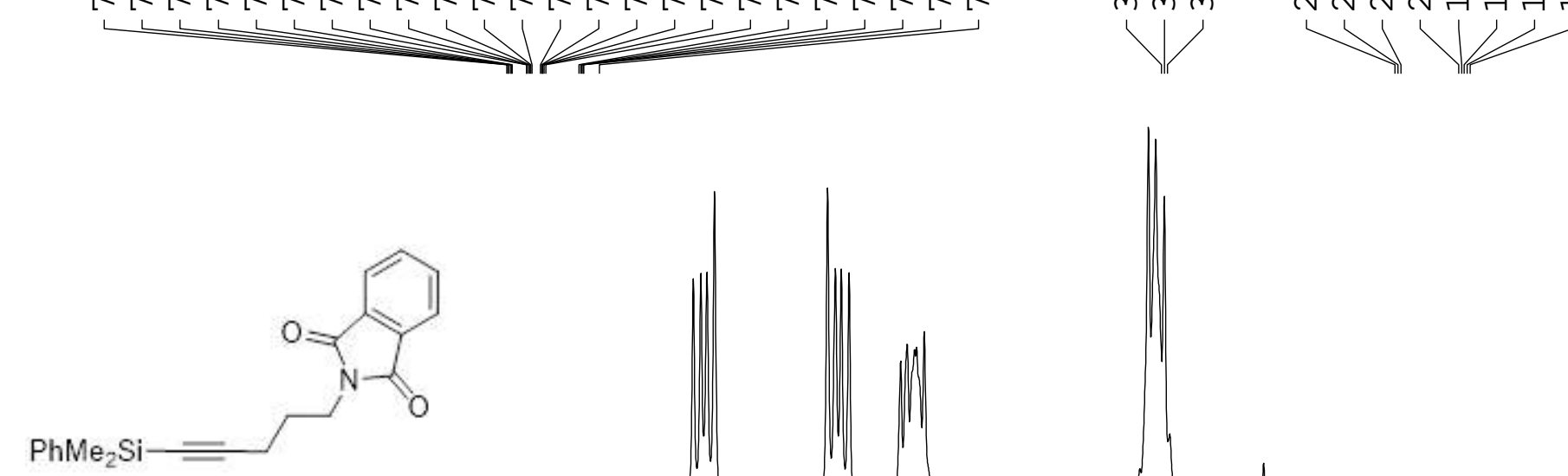

$1 \mathrm{q}$
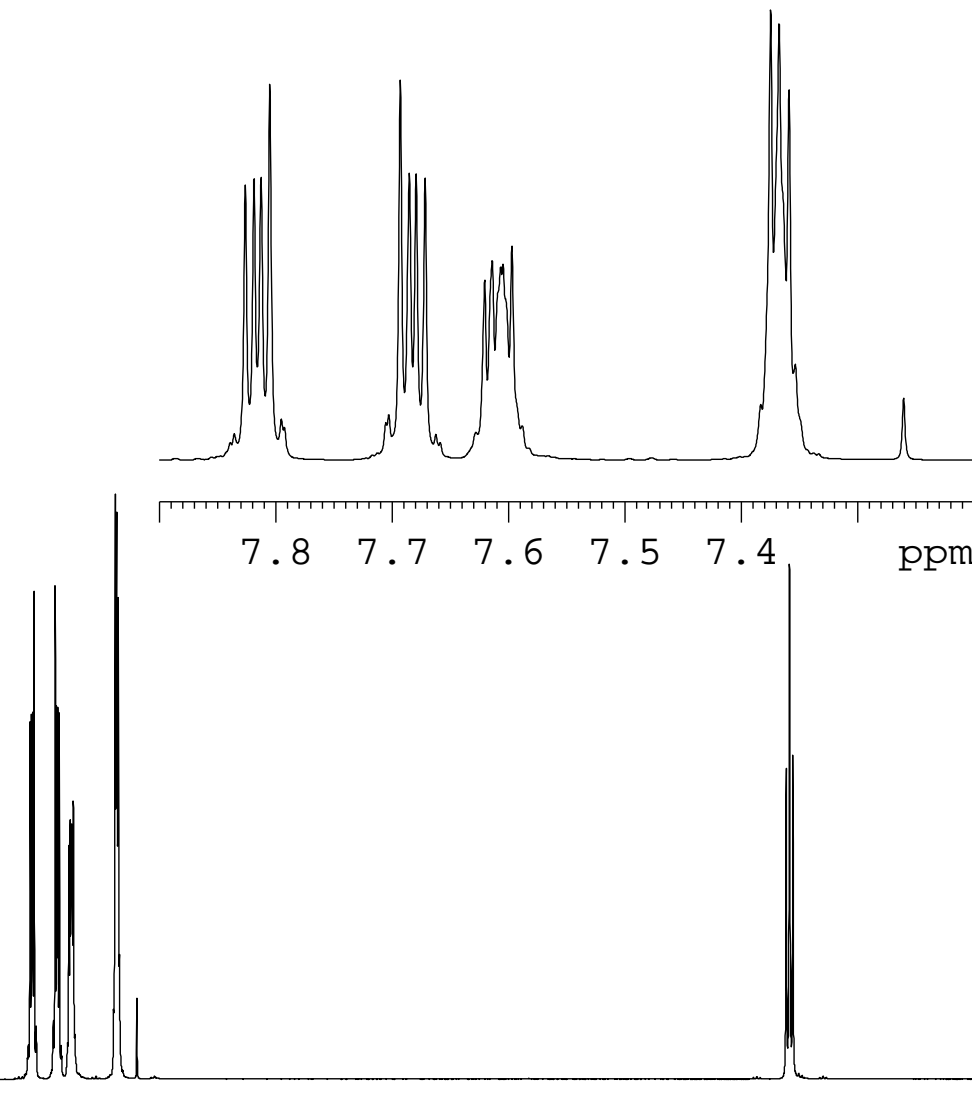

10

9

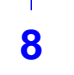

7

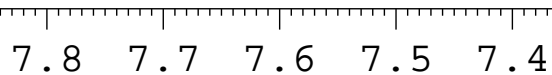

ppm

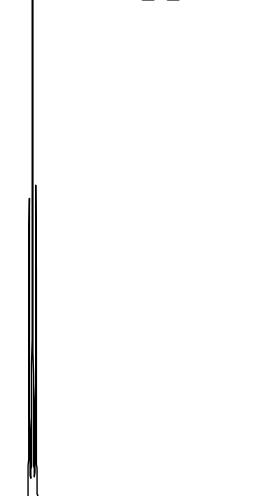

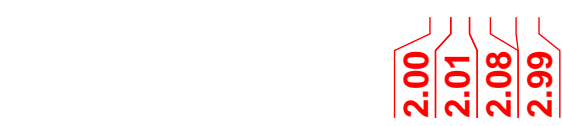

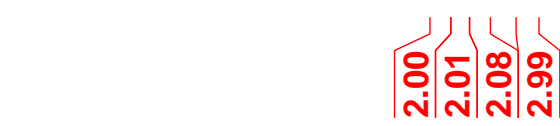

5

4
(o)
Ni

3

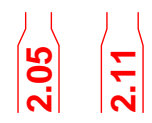

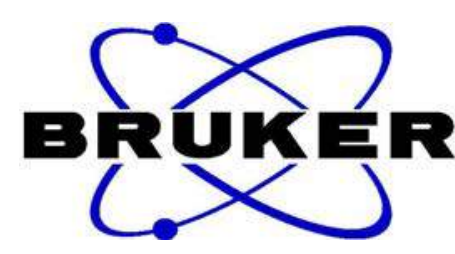

NAME

EXPNO

PROCNO

Date_-

INSTRUM

PROBHD

PULPROG

TD

SOLVENT

NS

SWH

FIDRES

AQ
RG
DW

DE

TE

D1 0

fq2-71-4

20180616

11.03

$5 \mathrm{~mm}$ DUL $13 \mathrm{C}-1$

$13 \mathrm{C}-1$
$\mathrm{zg} 30$

zg30
65536

$\mathrm{CDC} 13$
8

$8012.820 \mathrm{~Hz}$

$0.122266 \mathrm{~Hz}$

$4.0894966 \mathrm{sec}$ 54.81

62.400 usec 6.50 usec $1.00000000 \mathrm{sec}$

$===$ CHANNEL $\mathrm{f} 1 \mathrm{l}=======$

$\mathrm{SFO1}$

NUC

$\mathrm{P} 1$
$\mathrm{SI}$
$\mathrm{SF}$

WDW

SSB

LB

$\mathrm{PC}$

$400.1324710 \mathrm{MHz}$

$1 \mathrm{H}$
14.30 usec

$400.1300096 \mathrm{MHz}$

EM

$0.30 \mathrm{~Hz}$

0
1.00

ppm

S-160 


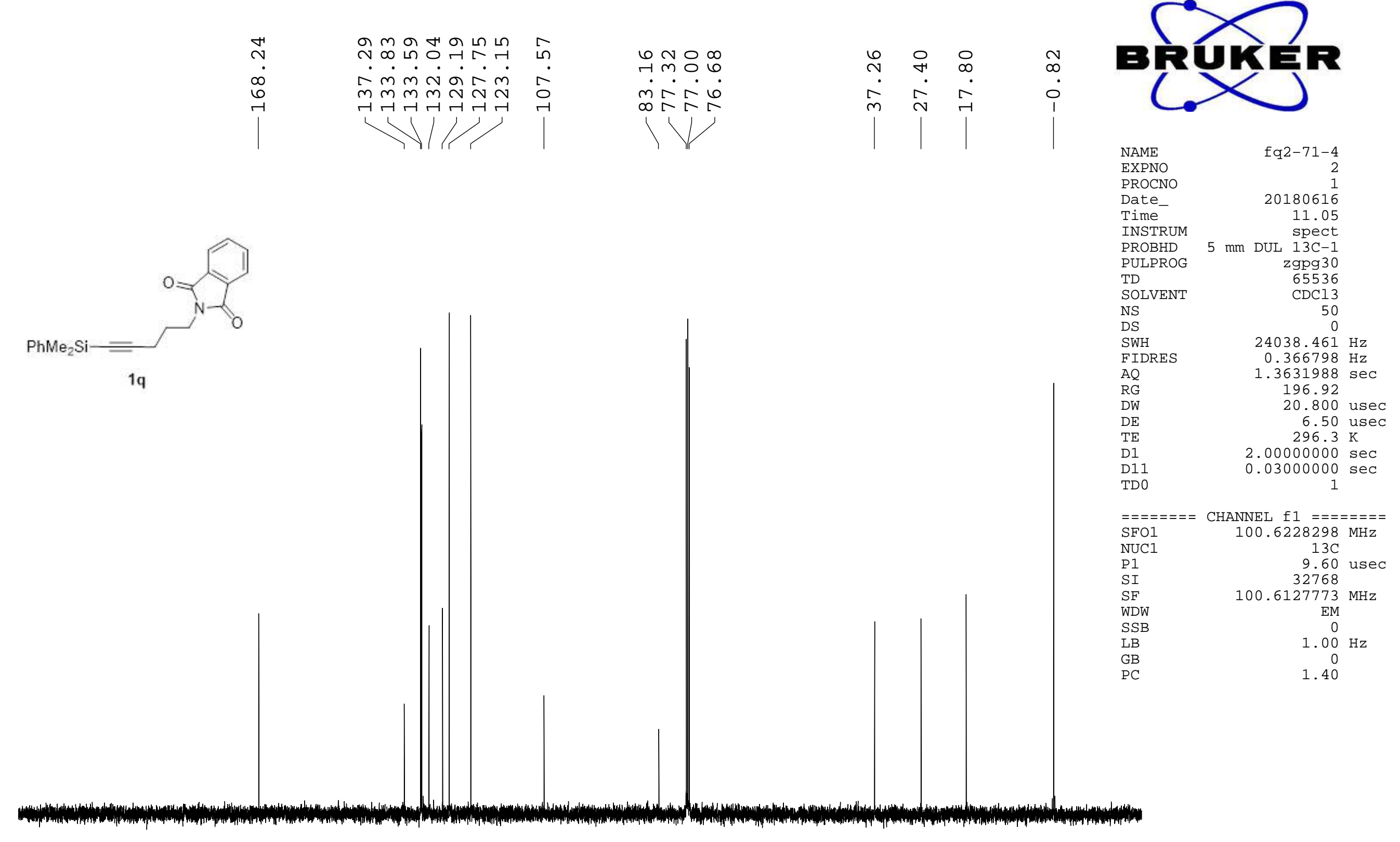




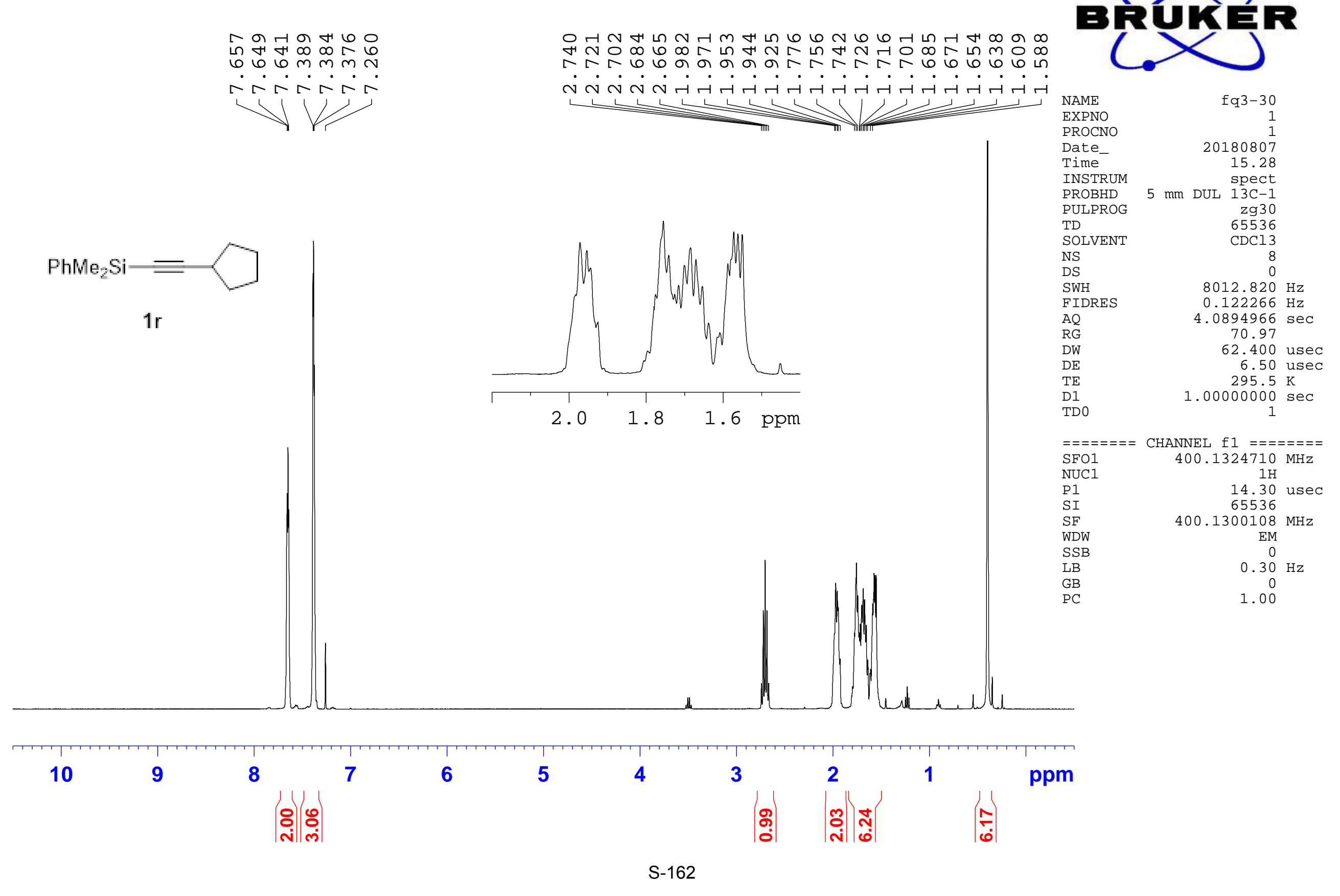




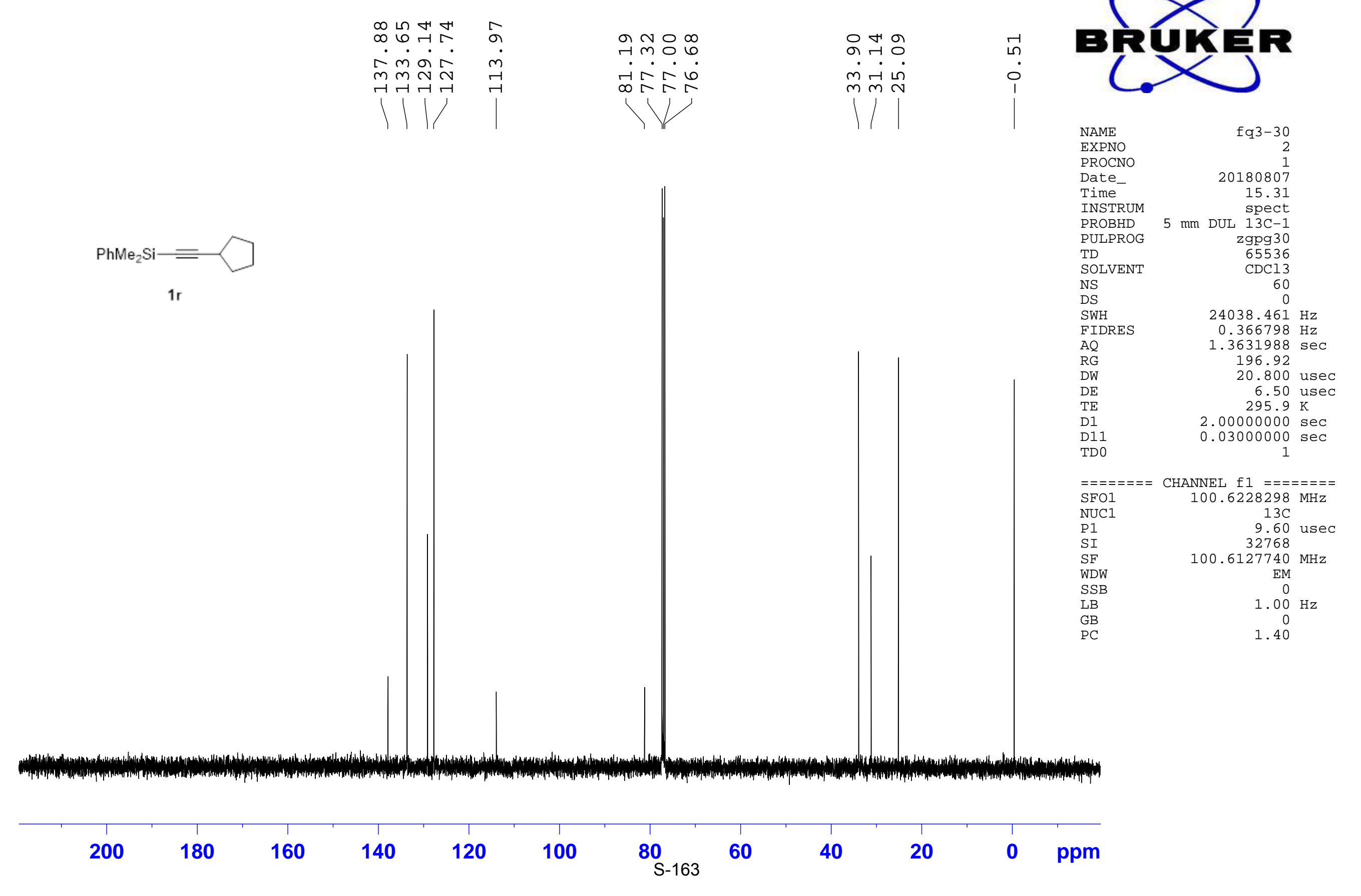




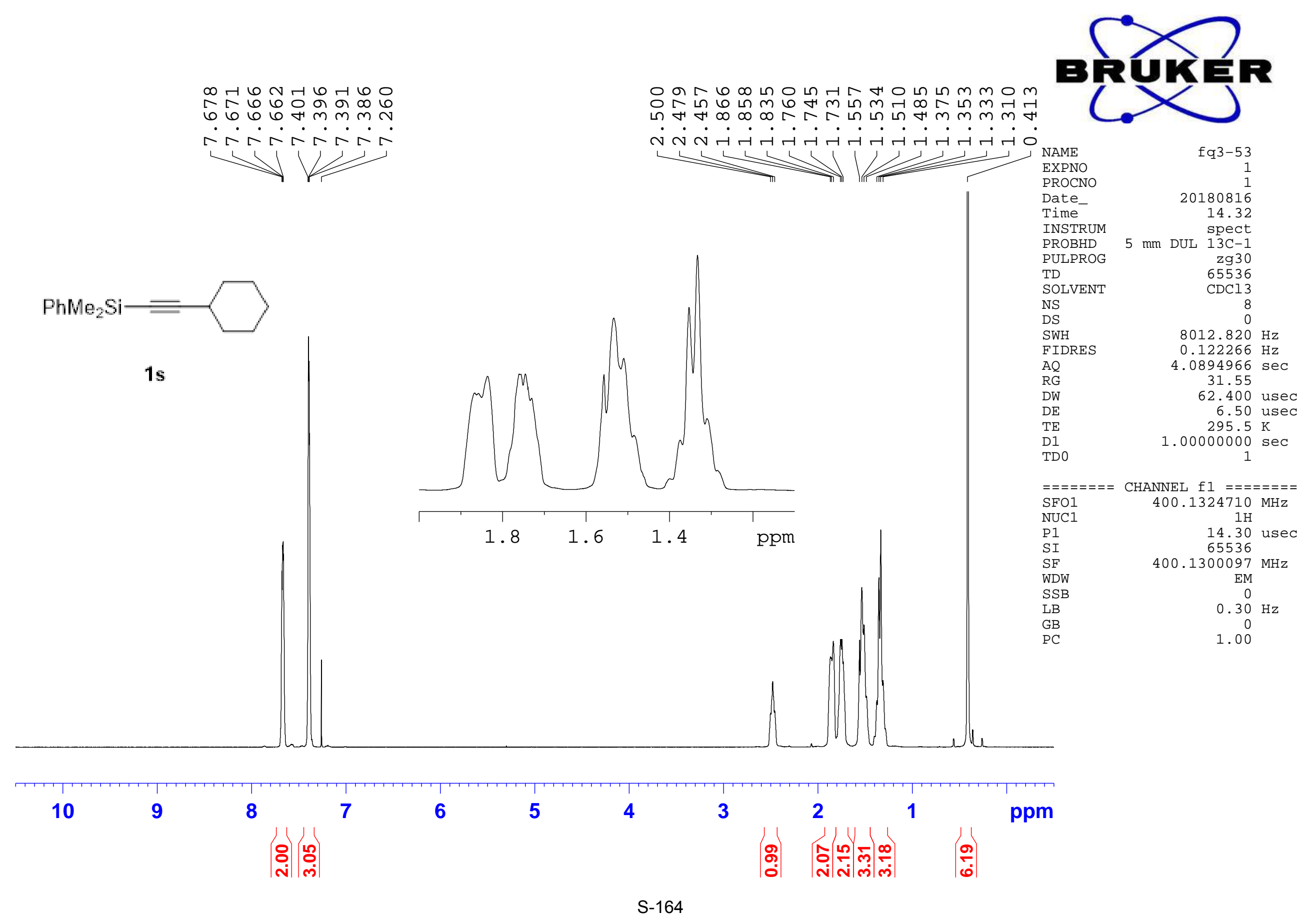




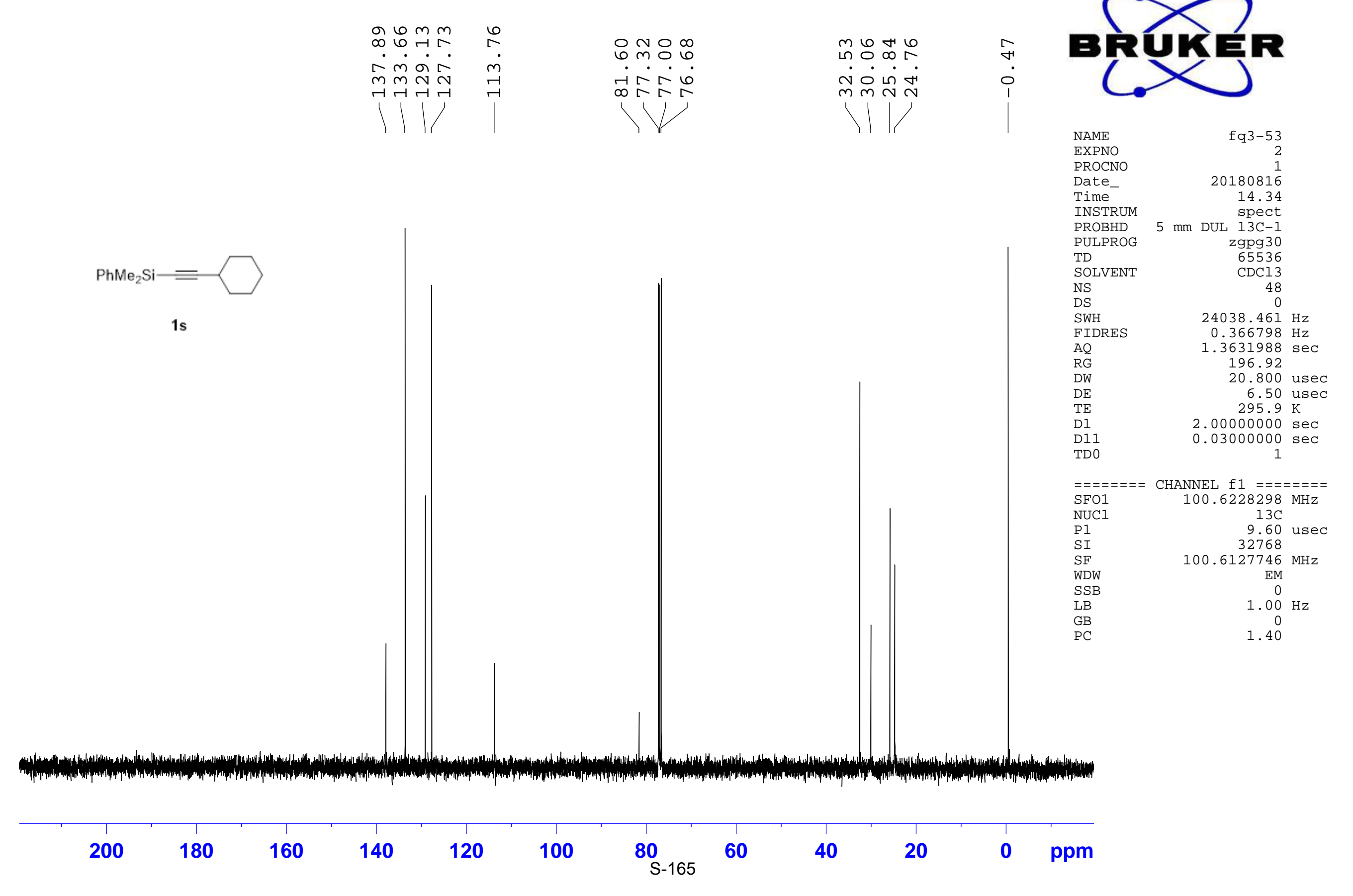




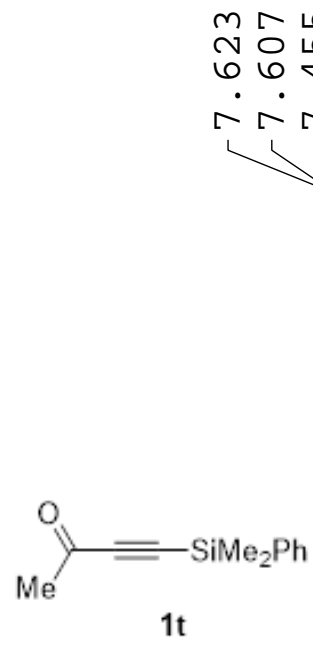

m $N 6 \infty \sigma$

n

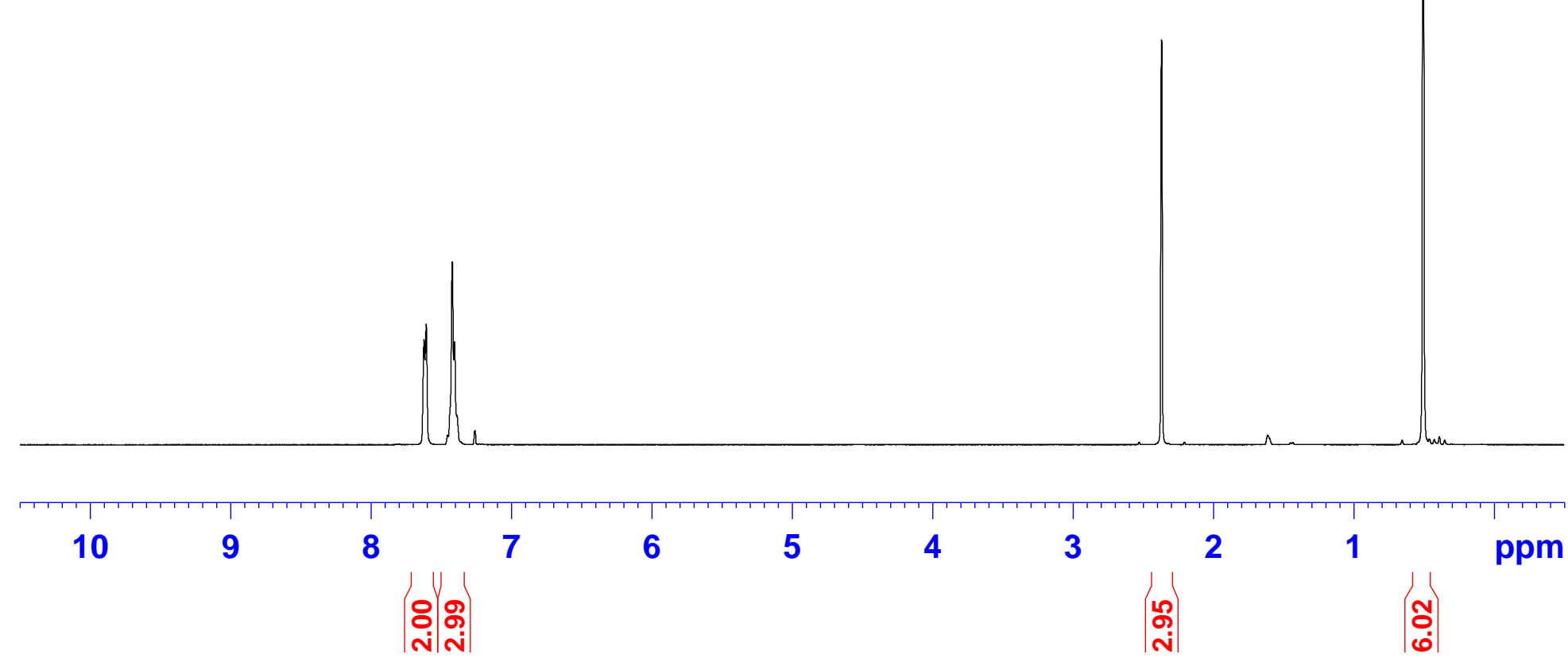

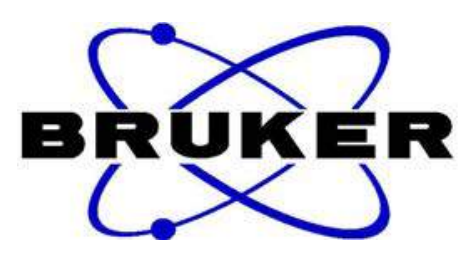

NAME
EXPNO

PROCNO

Date_

Time

INSTRUM

PROBHD

PULPROG

TD

SOLVENT

NS

SWH

FIDRES

$\mathrm{AQ}$
$\mathrm{RG}$

RG

DE

TE

TDO

fq3-101

20180907

80907
19.53

$5 \mathrm{~mm}$ РABBO BB/

$\mathrm{BB} /$
$\mathrm{zg} 30$

$\mathrm{zg} 30$
65536

$\mathrm{CDCl} 3$

4
0

$8012.820 \mathrm{~Hz}$

$0.122266 \mathrm{~Hz}$

$4.0894966 \mathrm{sec}$ 112.31

62.400 usec

6.50 usec

$1.00000000 \mathrm{sec}$

$========$ CHANNEL $\mathrm{f} 1========$

SFO1 $\quad 400.1324710 \mathrm{MHz}$

STC1

P1

SI

SF

WDW

LB

LB

PC
14.50 usec $400.1300105 \mathrm{MHz}$

EM

0
$0.30 \mathrm{~Hz}$

0.30
0
1.00 


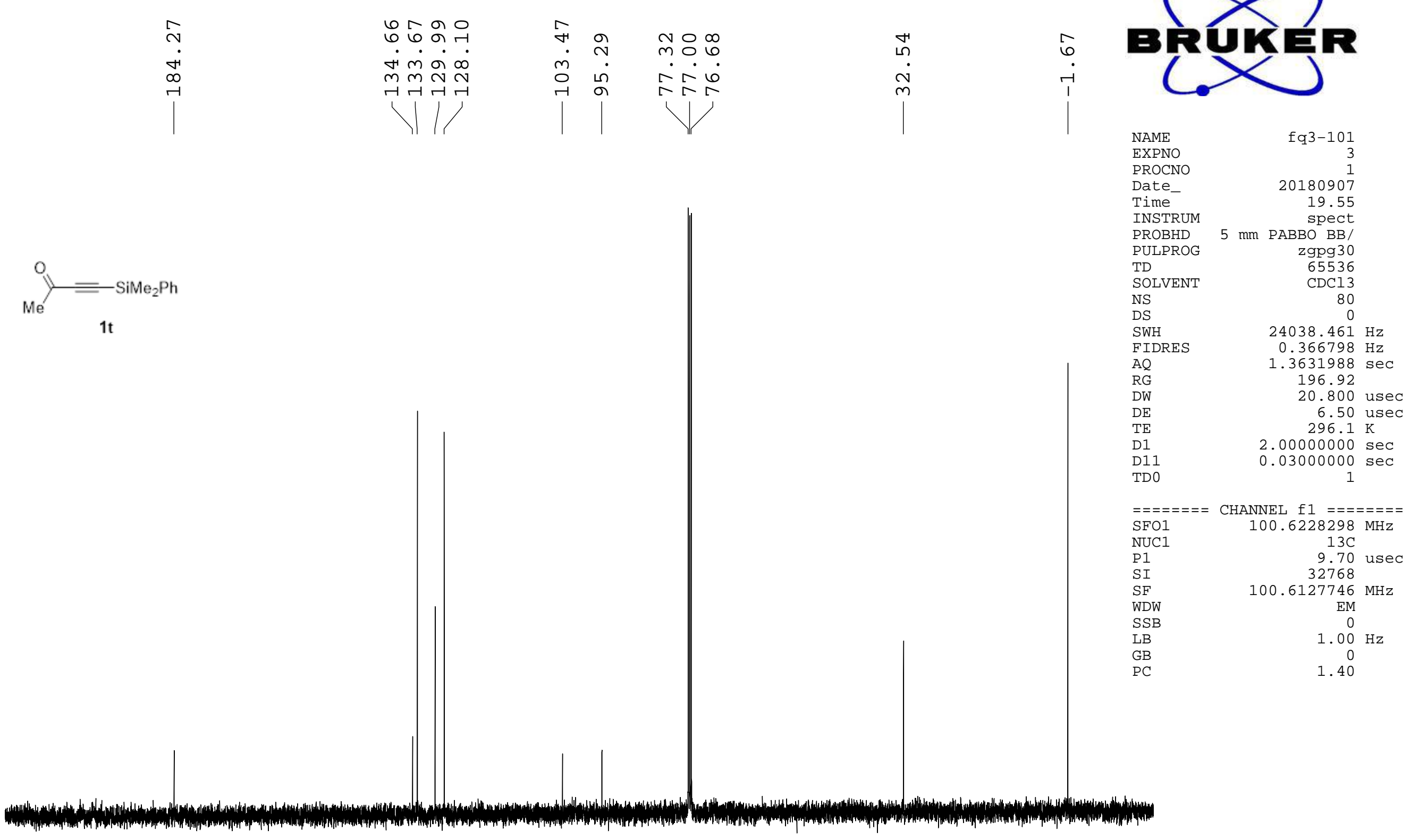



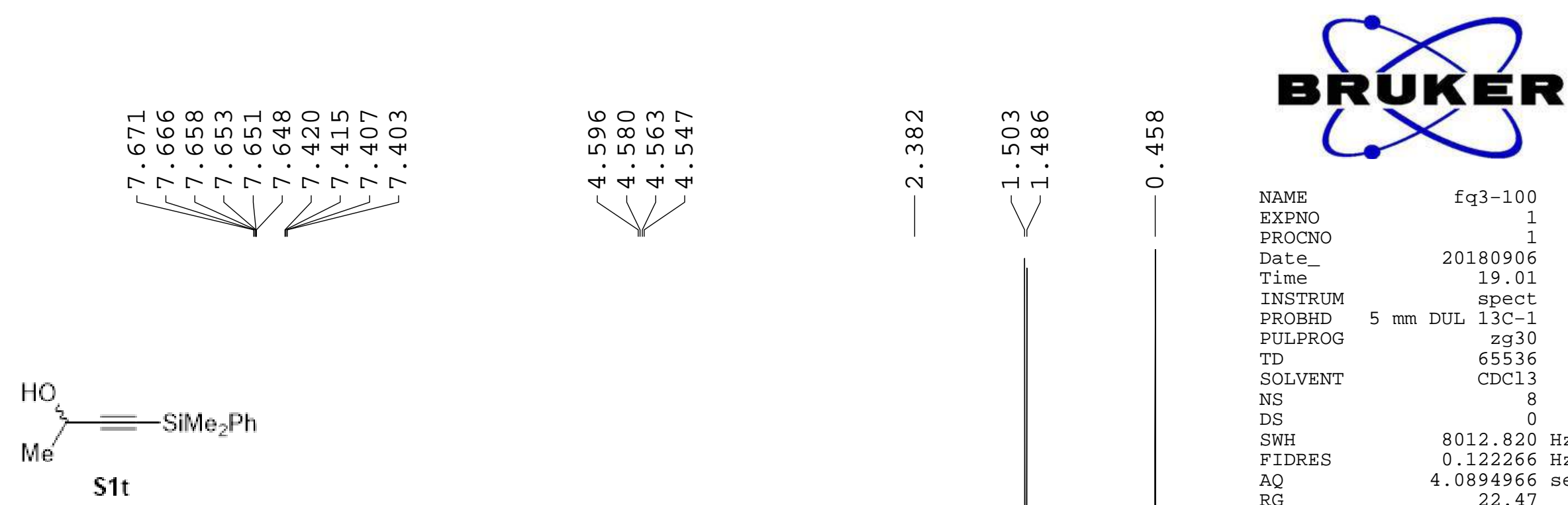

NAME

EXPNO
PROCNO

Date_-

Time

PROBHD

PULPROG

TD

SOLVENT

NS

SWH

FIDRES

AQ
RG
DW

DW

DE
TE
D1

D1

q $3-100$
1
1
20180906

0
1
1
06
01

$5 \mathrm{~mm}$ DUL $13 \mathrm{C}-1$

$\mathrm{DCl} 3$

$========$ CHANNEL $\mathrm{fl}========$

SFO1 $400.1324710 \mathrm{MHz}$

NUC1

P 1

SF

WDW

SSB

LB

GB
PC

$1 \mathrm{H}$
14.30
65536 $400.1300095 \mathrm{MHz}$

EM

$8012.820 \mathrm{~Hz}$

$0.122266 \mathrm{~Hz}$ 22.47

62.400 usec

$295.8 \mathrm{~K}$
$1.00000000 \mathrm{sec}$

0
$0.30 \mathrm{~Hz}$
0
1.00 


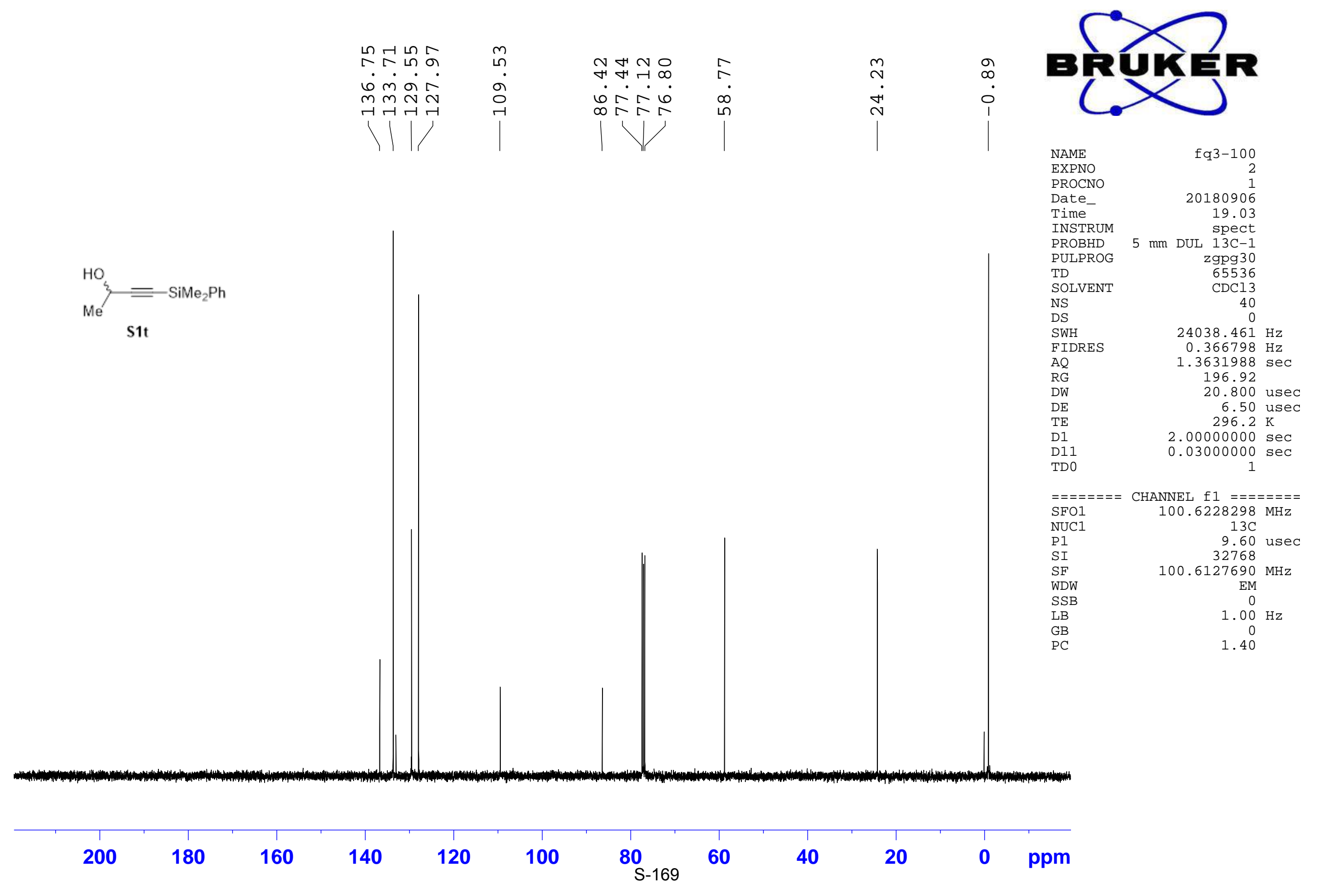


읍 가유

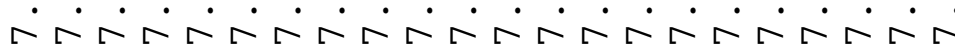

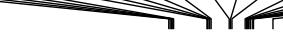

$\mathrm{PhMe}_{2} \mathrm{Si}=\mathrm{Ph}$

1u

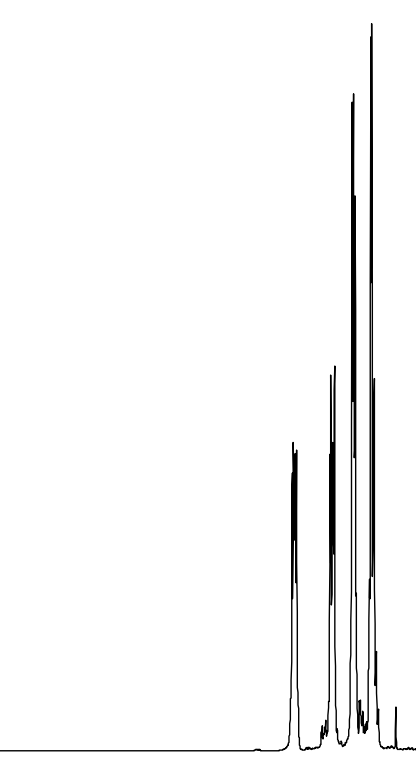

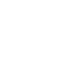

$\begin{array}{lr}\text { NAME } & \text { ZT-1006 } \\ \text { EXPNO } & 1 \\ \text { PROCNO } & 1 \\ \text { Date_- } & 20171027 \\ \text { Time } & 19.02 \\ \text { INSTRUM } & \text { spect } \\ \text { PROBHD } & 5 \mathrm{~mm} \text { PABBO BB- } \\ \text { PULPROG } & \text { zg30 } \\ \text { TD } & 65536 \\ \text { SOLVENT } & \text { CDC13 } \\ \text { NS } & 8 \\ \text { DS } & 2 \\ \text { SWH } & 8223.685 \mathrm{~Hz} \\ \text { FIDRES } & 0.125483 \mathrm{~Hz} \\ \text { AQ } & 3.9846387 \mathrm{sec} \\ \text { RG } & 644 \\ \text { DW } & 60.800 \mathrm{usec} \\ \text { DE } & 6.00 \mathrm{usec} \\ \text { TE } & 294.0 \mathrm{~K} \\ \text { D1 } & 1.0000000 \mathrm{sec} \\ \text { TDO } & 1 \\ & \end{array}$
$========$ CHANNEL $\mathrm{f} 1 \mathrm{l}=======$ NUC1 $1 \mathrm{H}$
$\mathrm{NUC1}$
P1 1

SFO1

SI

SF

WDW

$S S B$
$L B$

LB

PC

13.60 usec
$-1.00 \mathrm{~dB}$
$400.1324710 \mathrm{MHz}$
$32768 \mathrm{HH}$
$400.1300098 \mathrm{MHz}$
$\mathrm{EM}$
0
$0.30 \mathrm{~Hz}$
0
1.00

$10 \quad 98$

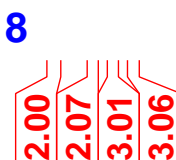

7

6

5

4

3

2

1

1

ppm

ก| ล่|ต่| 


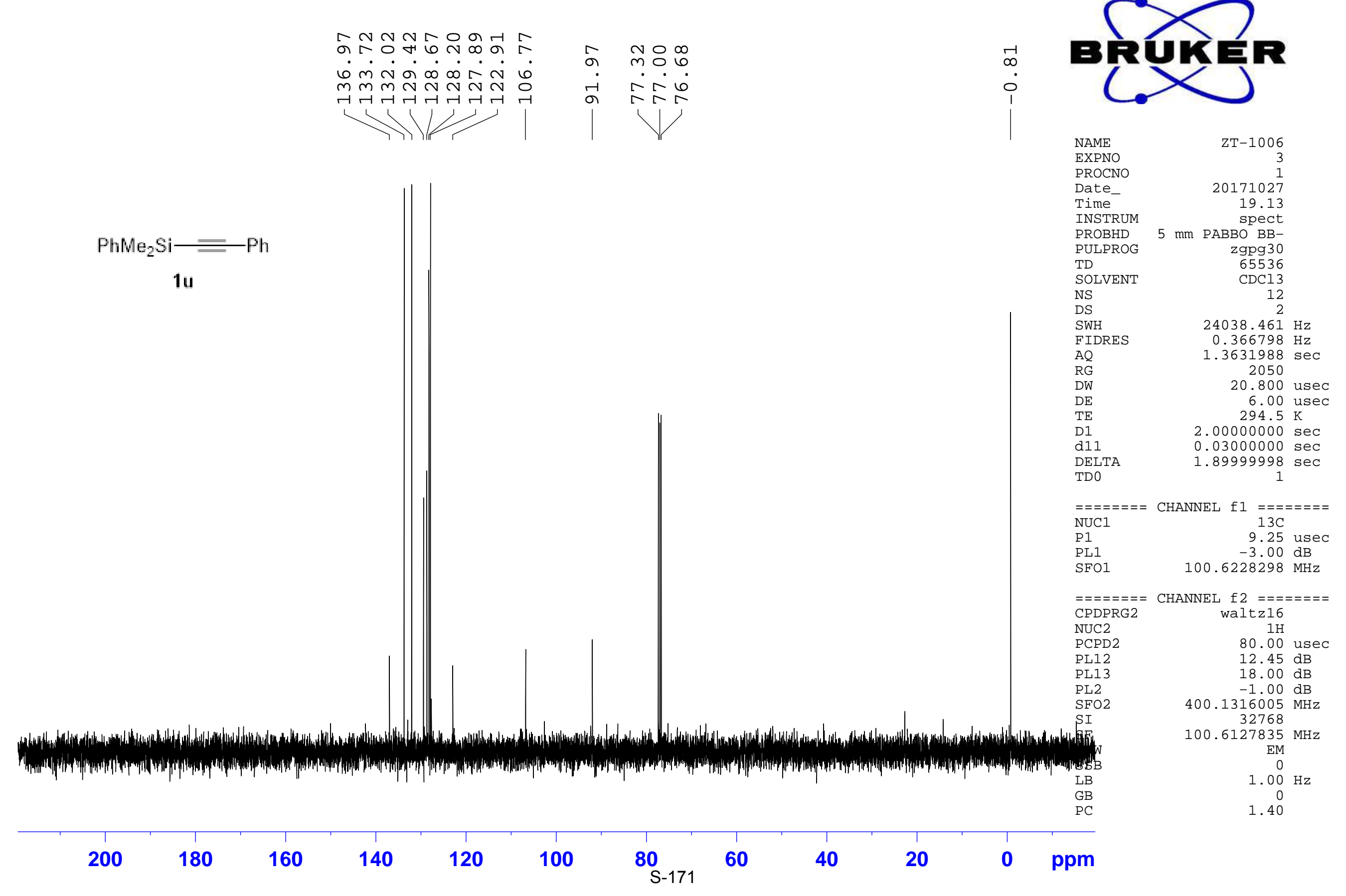




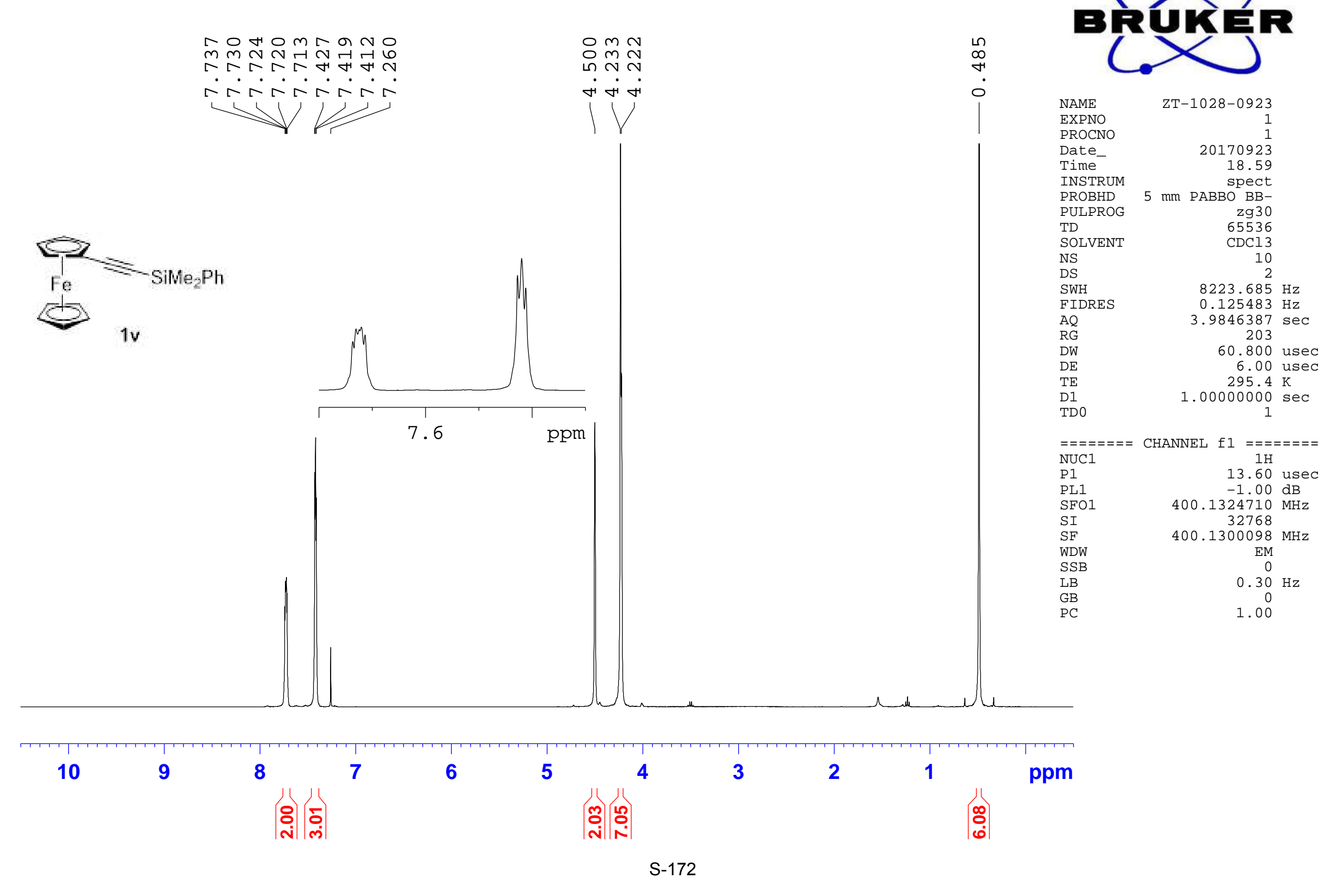




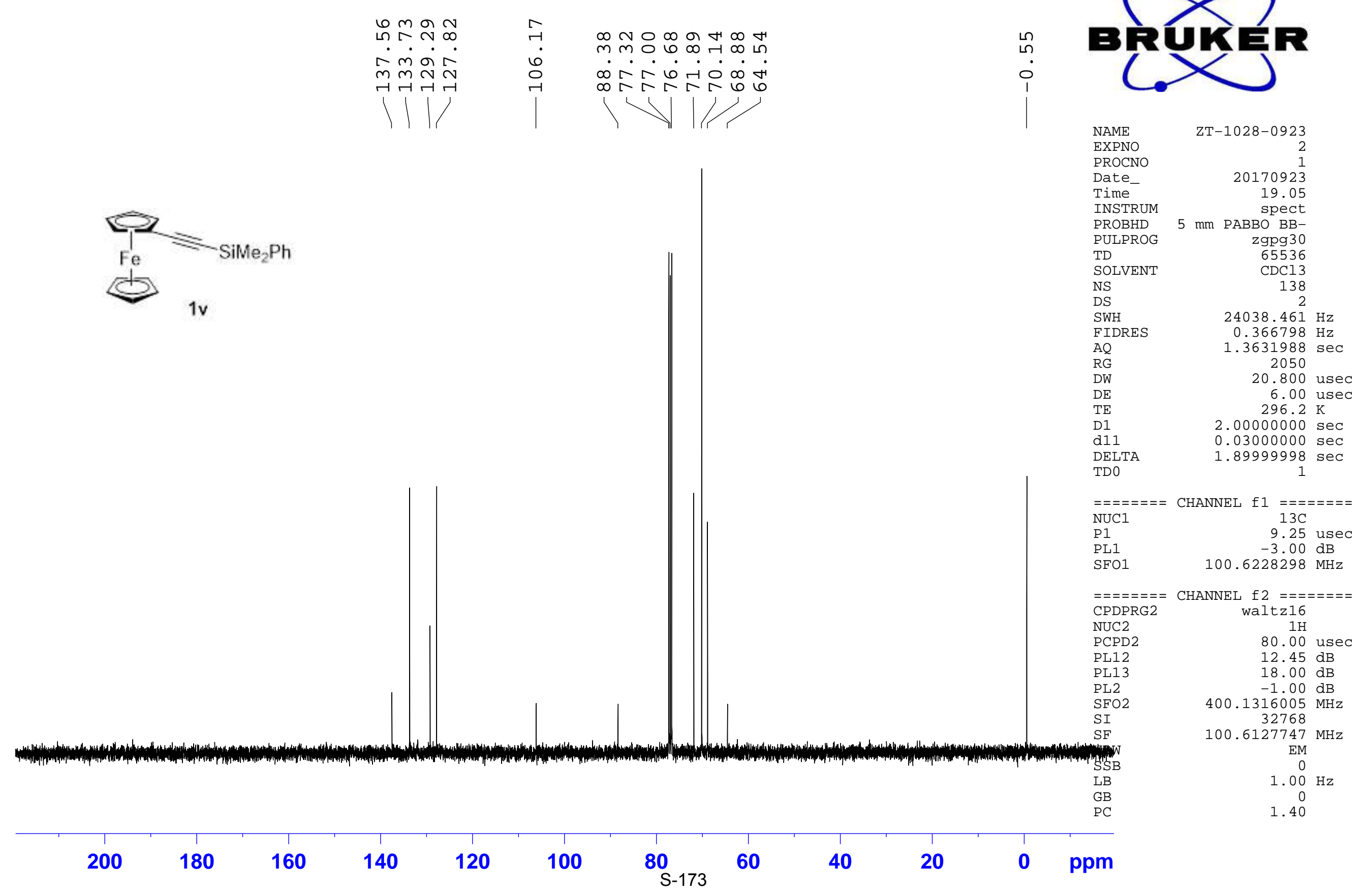




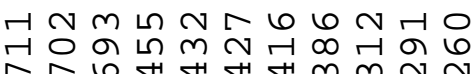

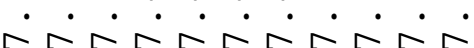
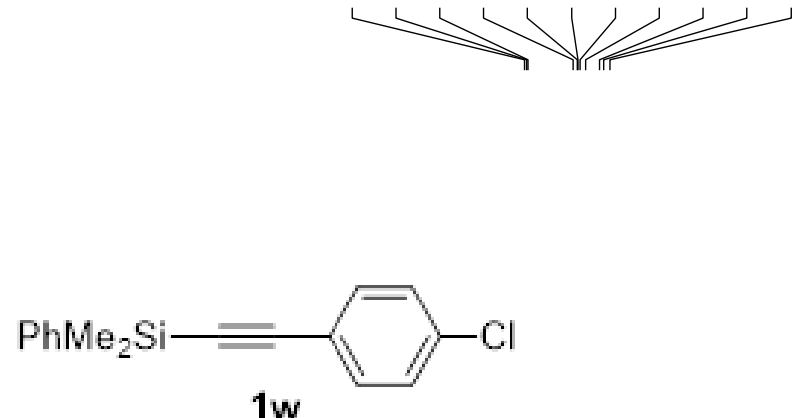

$1 w$
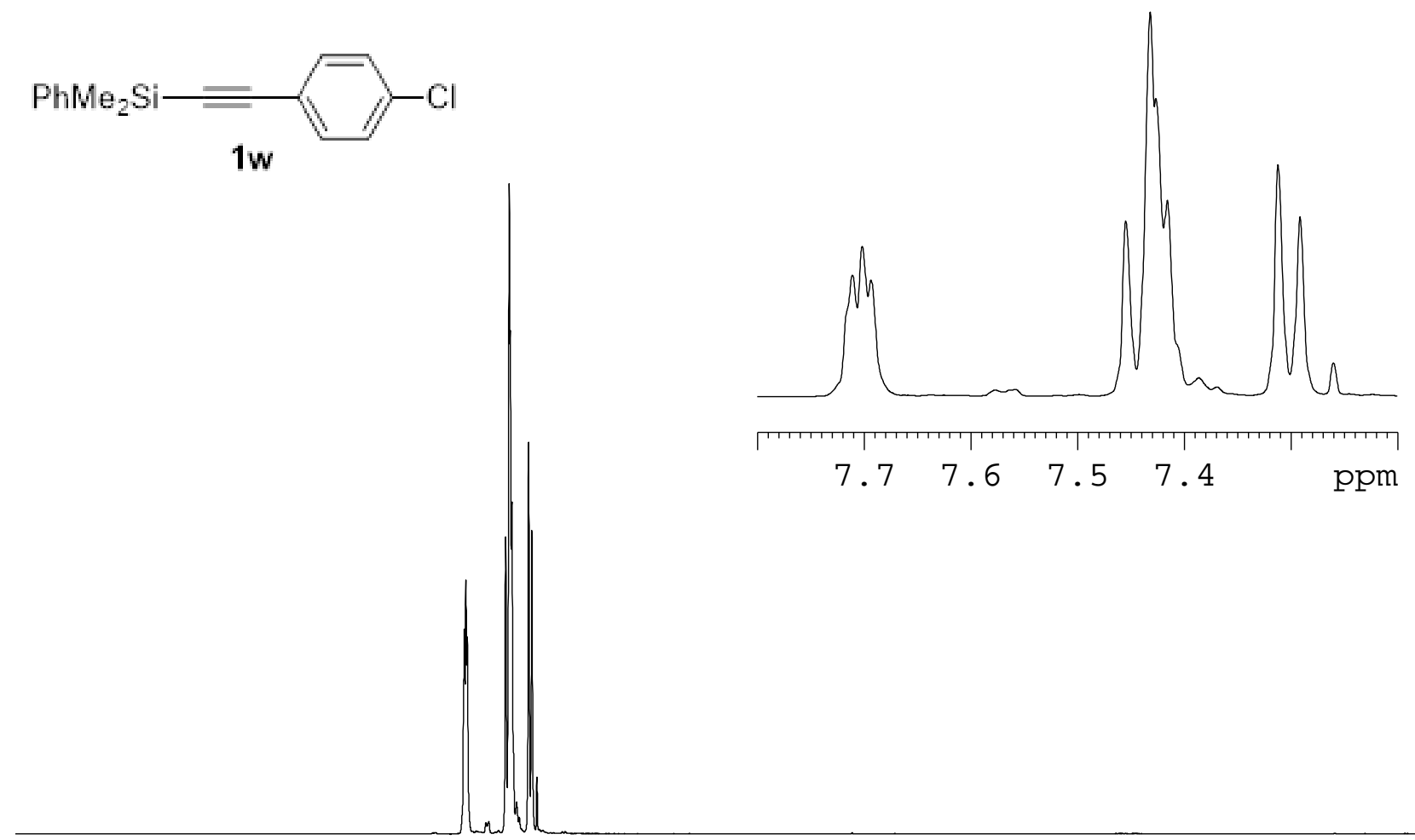

10 9

8

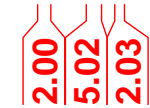

7

6

5

4

3

2

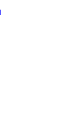

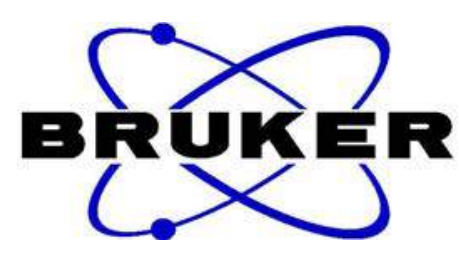

NAME

EXPNO

PROCNO

Date_

Time

INSTRUM

PROBHD

PULPROG

TD

SOLVENT

NS
DS

SWH

FIDRES

AQ

RG

DE

TE

D1

$\mathrm{SFO1}$

NUC1

$\mathrm{P} 1$

SI

SF

WDW

LB

LB
GB

PC fq2 $2-73$

20180727

13.12

$5 \mathrm{~mm}$ DUL $13 \mathrm{C}-1$

$\mathrm{zg} 30$
65536

CDCl3

8
2

$8012.820 \mathrm{~Hz}$

$0.122266 \mathrm{~Hz}$

$4.0894966 \mathrm{sec}$ 82.92

62.400 usec 6.50 usec $295.5 \mathrm{~K}$

CHANNEL $\mathrm{f} 1 \mathrm{=}=======$ $400.1324710 \mathrm{MHz}$ $1 \mathrm{H}$
14.30 usec 400.1300098

$\mathrm{EM}$
0

$0.30 \mathrm{~Hz}$

0
1.00

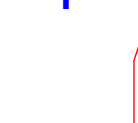

ppm 

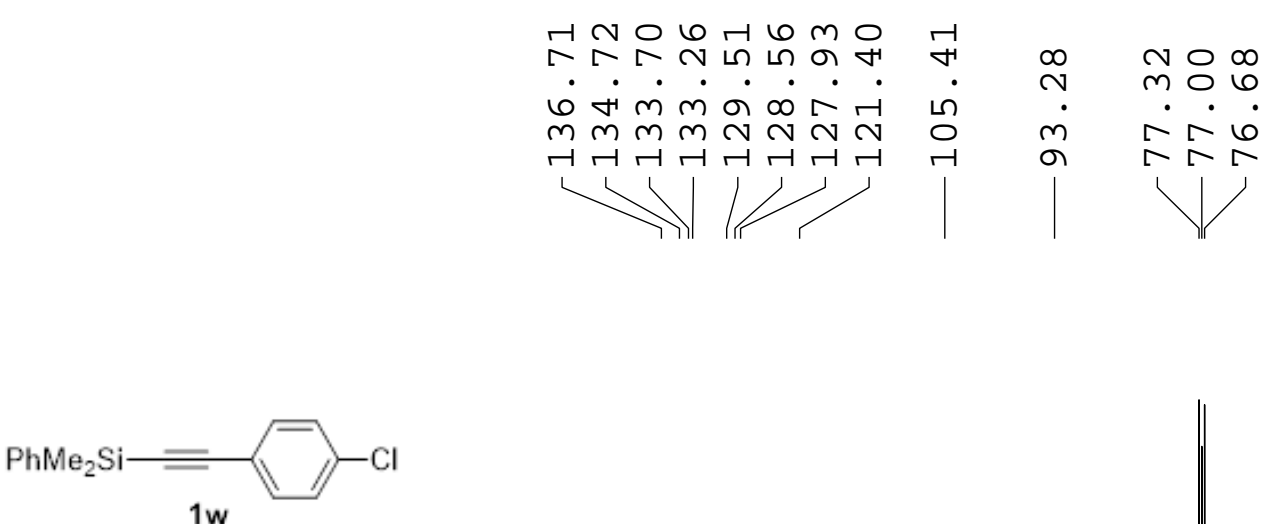

$1 w$

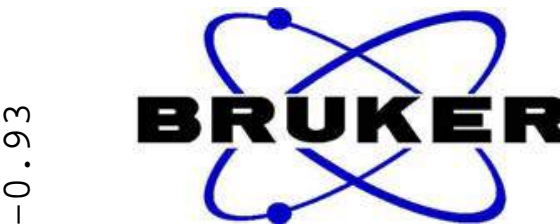

NAME

PROCNO

Dime

INSTRUM

PROBHD

PUIPROG

TD

SOLVENT

NS

DS

FIDRES

FIDR
AQ
RG

DW

DE

D1

D11

D11 0

$===$

$\mathrm{SFO}$

P1

SI
SF

WDW

SSB

LB

$\mathrm{PC}$ fq2 $2-73$

$2018072^{1}$

13.14

spect

$5 \mathrm{~mm}$ DUL $13 \mathrm{C}-1$

zgpg 30

$\mathrm{CDCl}$

$24038.461 \mathrm{~Hz}$

$0.366798 \mathrm{~Hz}$

$1.3631988 \mathrm{sec}$

196.92

20.800 usec

6.50 usec

$2.00000000 \mathrm{sec}$

$0.03000000 \mathrm{sec}$

CHANNEL $f 1$ $100.6228298 \mathrm{MHz}$ $13 \mathrm{C}$

9.60 usec 100.6127748

100

EM

$1.00 \mathrm{~Hz}$

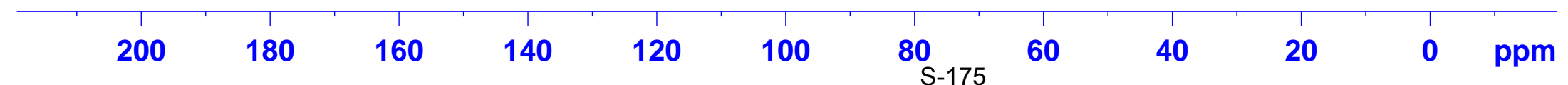




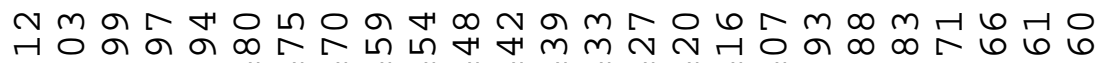
닷의

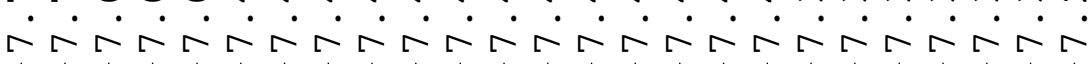

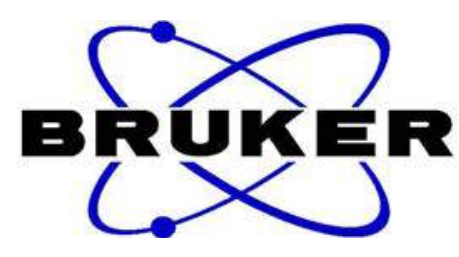

NAME

EXPNO

PROCNO

Date_

Time

INSTRUM

PROBHD

PULPROG

TD

SOLVENT

NS

SWH

FIDRES

$A Q$

RG

DE

TE

D1

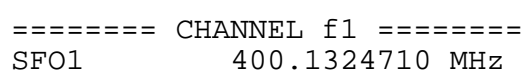

$\mathrm{SFO1}$

NUC

SI

SF

WDW

SSB

LB
GB

PC fq2-150

20180711

7.48

$5 \mathrm{~mm}$ DUL $13 \mathrm{C}-1$

65536

$\mathrm{CDCl} 3$

$8012.820 \mathrm{~Hz}$

$0.122266 \mathrm{~Hz}$

$4.0894966 \mathrm{sec}$ 70.97

62.400 usec 6.50 usec $1.00000000 \mathrm{sec}$

$400.1324710 \mathrm{MHz}$

$1 \mathrm{H}$
14.30 usec $400.1300098 \mathrm{MHz}$

EM

0
$0.30 \mathrm{~Hz}$

0.30
0
1.00
10

9

8

| \begin{tabular}{|c|c|}
0 & \\
0 & N \\
N
\end{tabular} \mid

7

5

4

3

2

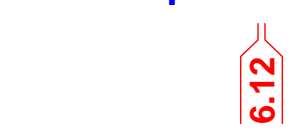

S-176 


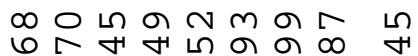

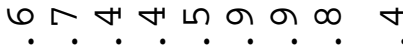

$6 m m-\sigma r N r$

다다 ${ }_{-1}{ }_{-1}$

$\longrightarrow 1$

$\mathrm{PhMe}_{2} \mathrm{Si}=\underset{\text { 1x }}{=} \mathrm{Br}$

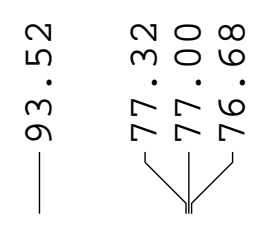

$\therefore 6$

$\sqrt{1}$

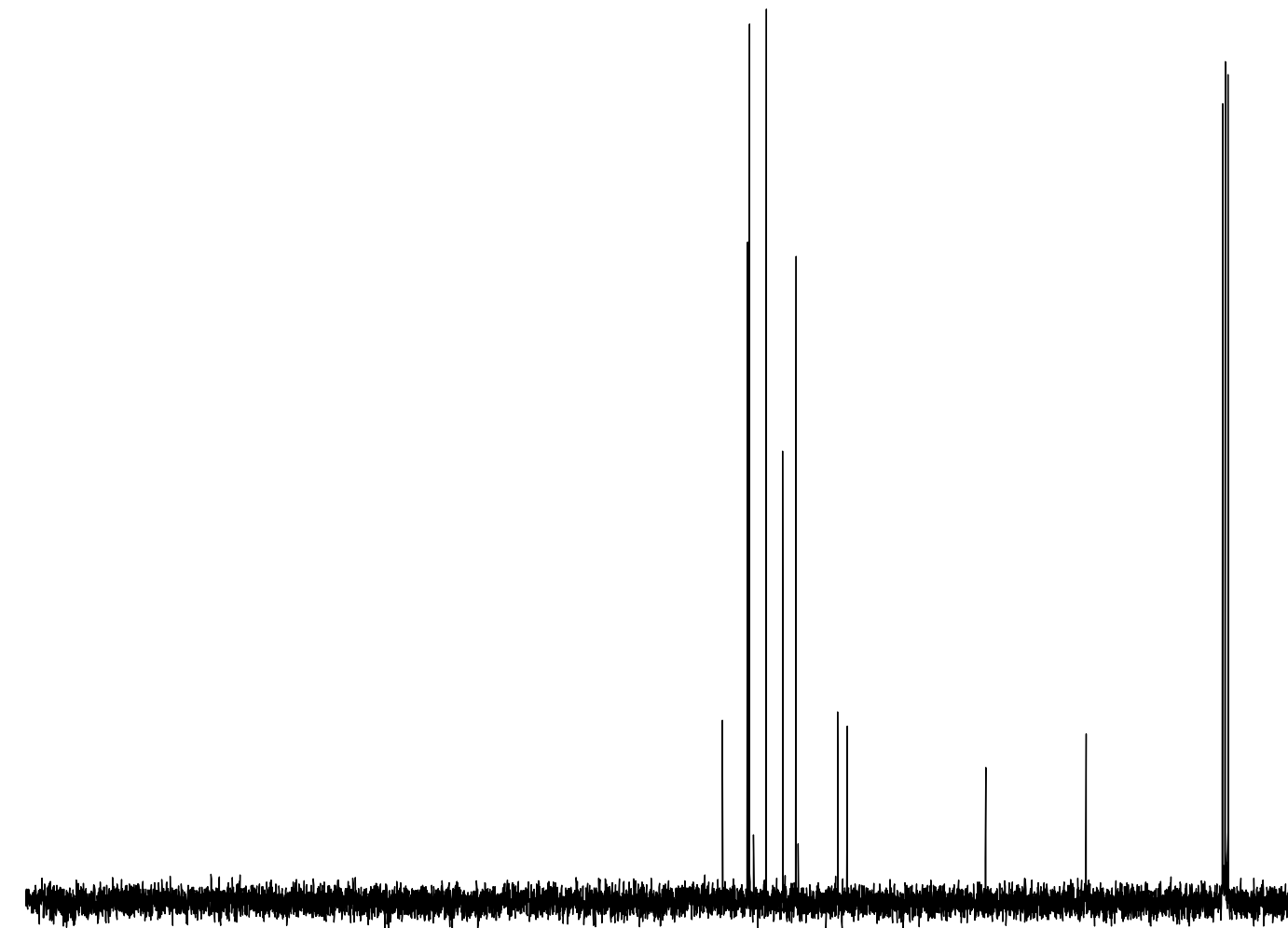

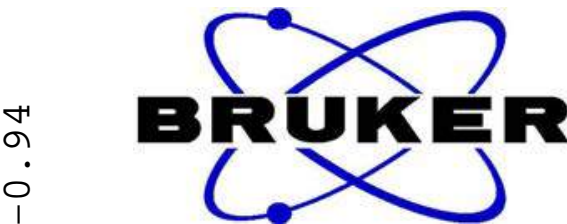

NAME
EXPNO

PROCNO

Date

INSTRUM

PROBHD

PULPROG

TD

SOLVENT

NS

DS

SWH
FIDRES

$\mathrm{AQ}$
$\mathrm{RG}$

DW

DE

TE

D11

$===$

$\mathrm{SFO}$

P1

SI

WDW

SSB

LB

PC fq2-150

20180711 18.00 spect

$\mathrm{mm}$ DUL 13C-1

zgpg30

$\mathrm{CDCl} 3$

$24038.461 \mathrm{~Hz}$ $0.366798 \mathrm{~Hz}$ $.3631988 \mathrm{sec}$ 196.92 20.800 usec $297.4 \mathrm{~K}$ $2.00000000 \mathrm{sec}$ $2.00000000 \mathrm{sec}$ 1 CHANNEL $\mathrm{f} 1========$ $100.6228298 \mathrm{MHz}$ $13 \mathrm{C}$ 9.60 use $100.6127751 \mathrm{MHz}$ EM

$1.00 \mathrm{~Hz}$ 0
1.40

80 

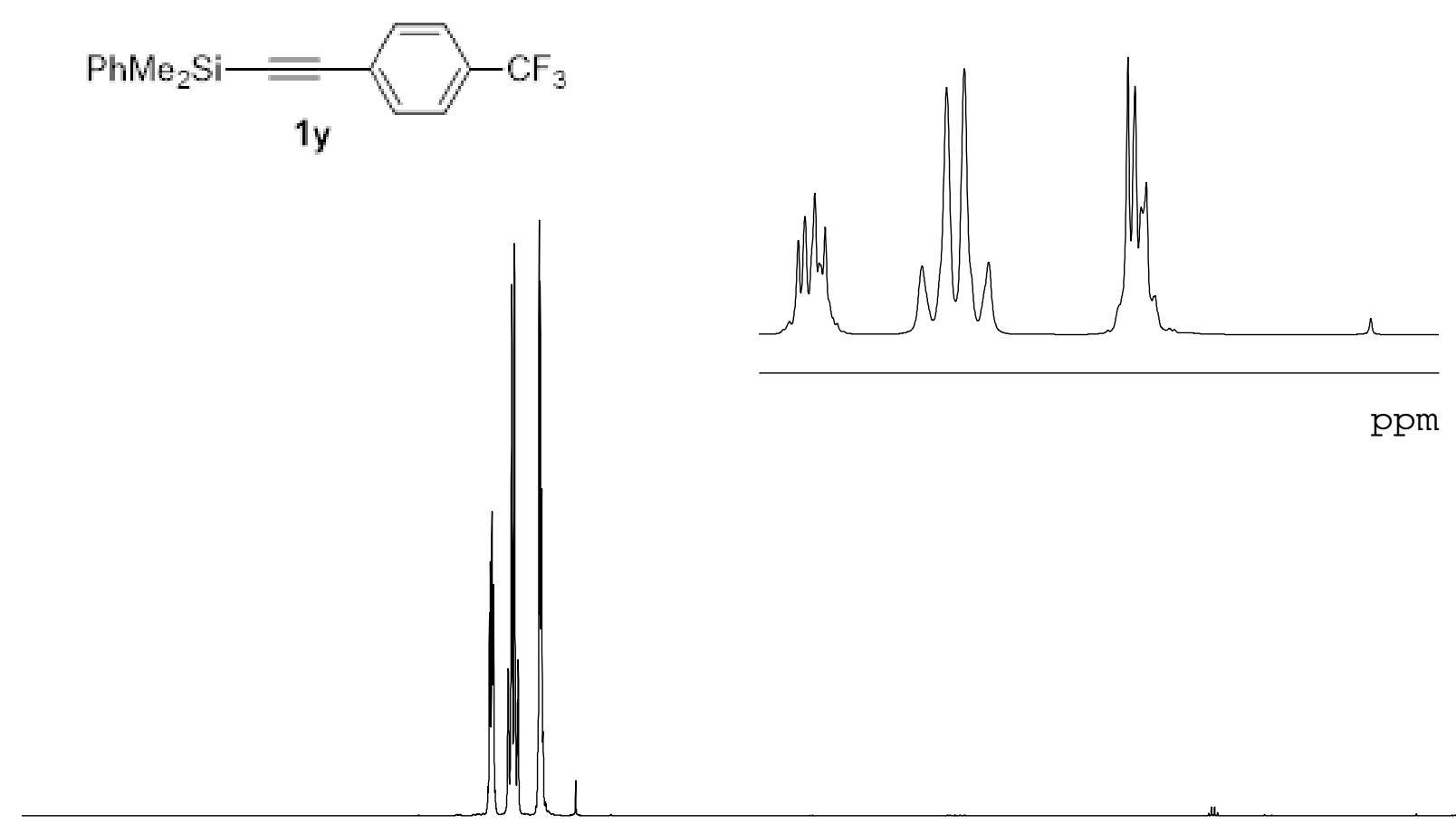

10 9
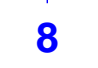

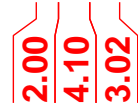

7

6

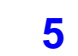

5

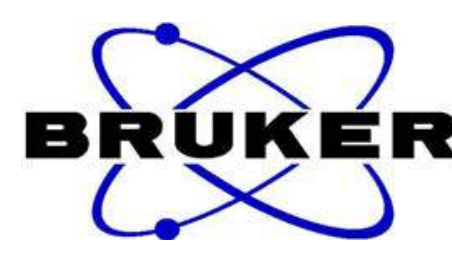

NAME

EXPNO

PROCNO

Date-

Time

INSTRUM

PROBHD

PULPROG

TD

SOLVENT

NS

SWH

FIDRES

$A Q$

RG

DE

TE

D 1 0

$========$ CHANNEL $\mathrm{f} 1 \mathrm{1}=======$

$\mathrm{SFO1}$

NUC1

P 1

SI

WDW

$S S B$

LB

PC fq2-149

20180710

18.33

$5 \mathrm{~mm}$ DUL $13 \mathrm{C}-1$

2930
65536

-13
8

$8012.820 \mathrm{~Hz}$

$0.122266 \mathrm{~Hz}$

$4.0894966 \mathrm{sec}$ 27.78

62.400 usec 6.50 usec $1.00000000 \mathrm{sec}$ 0.1324710

14.30 usec 400.130

EM

$0.30 \mathrm{~Hz}$

0
1.00 


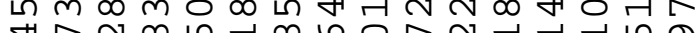

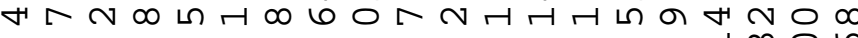

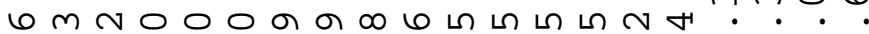
$m m m m m m \sim N \sim \sim N \sim \sim N O N$

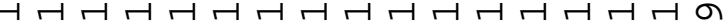

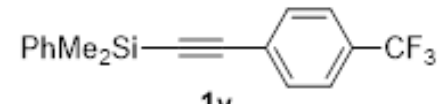

$1 \mathrm{y}$

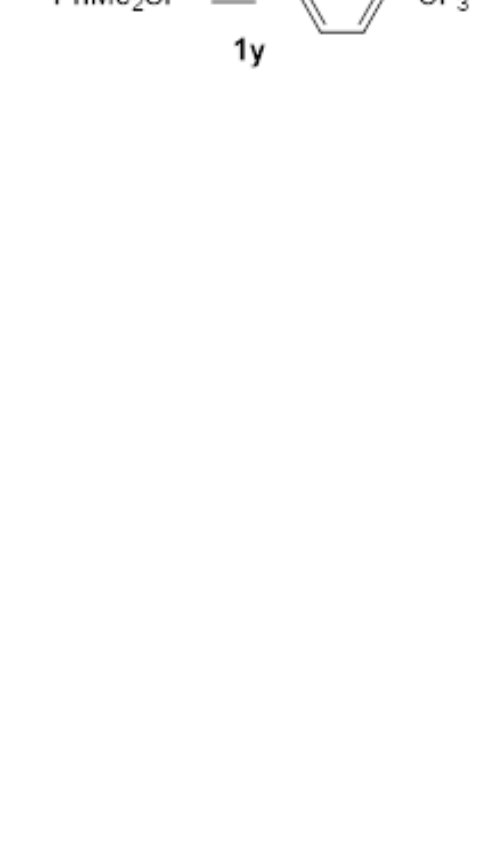

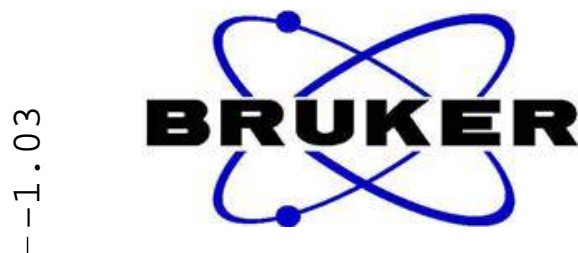

NAME

PROCNO

Date

Time

INSTRUM

INRTR

PUI PROG

SOLVENT

NS

DS

FIDRES

FIDR
AQ
RG
DW

DW

DE

TE

TD 11

$==$

$\mathrm{SFO}$

P 1

SI

WDW

SSB

$\mathrm{LB}$
$\mathrm{GB}$

PC

$5 \mathrm{~mm}$ DUL 13C-1 fq2-149

20180710 18.35 18.35

zgpg 30
65536

$\mathrm{CDCl}$

$24038.461 \mathrm{~Hz}$

$0.366798 \mathrm{~Hz}$

$3631988 \mathrm{sec}$

196.92

20.800 usec

6.50 usec

$2.00000000 \mathrm{sec}$

$0.03000000 \mathrm{sec}$

1

CHANNEL $\mathrm{f} 1=$ $100.6228298 \mathrm{MHz}$ $13 \mathrm{C}$

9.60 usec 32768

$100.6127751 \mathrm{MHz}$

EM

0
$1.00 \mathrm{~Hz}$

1. $00 \mathrm{~Hz}$

1.40

80 


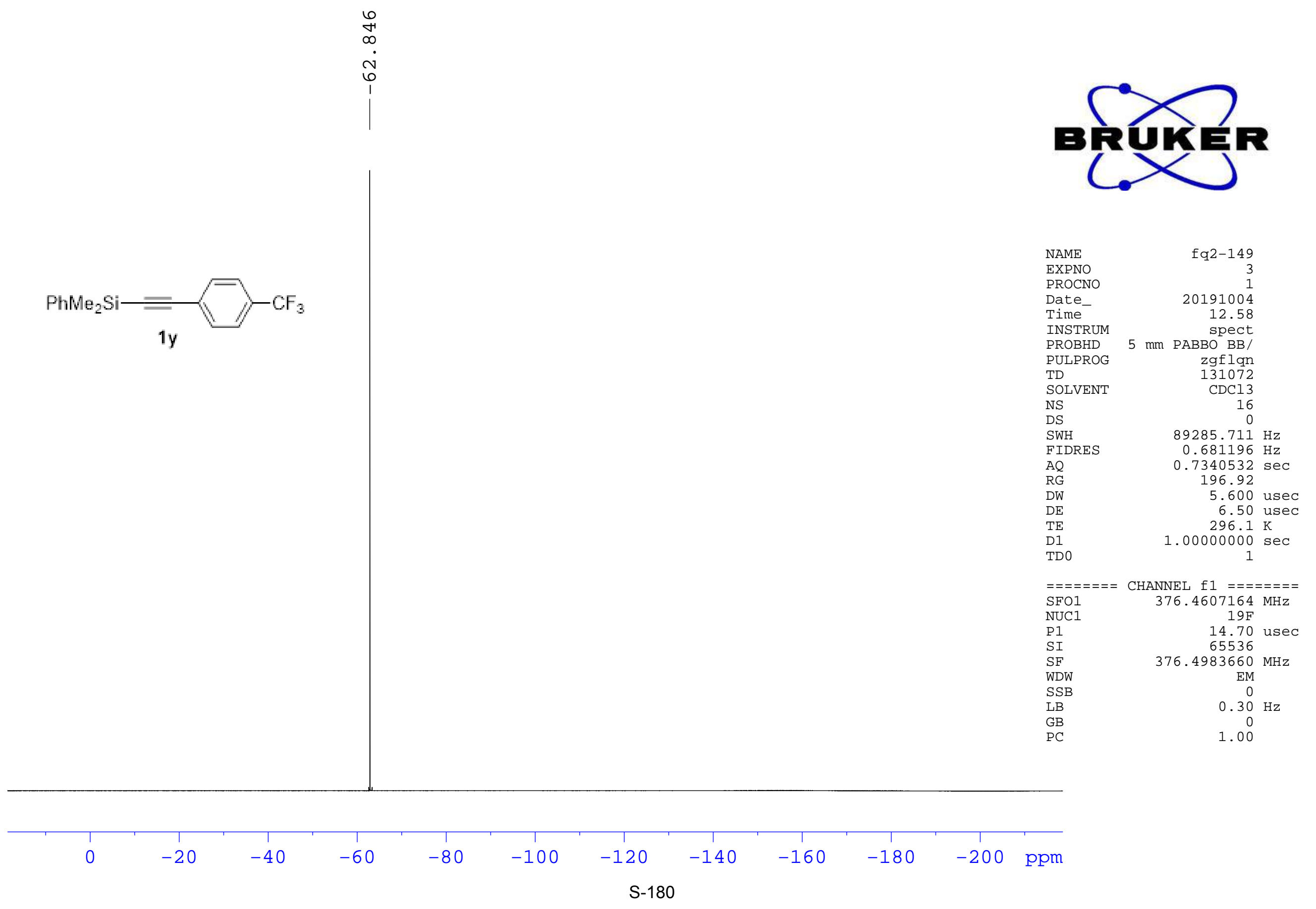



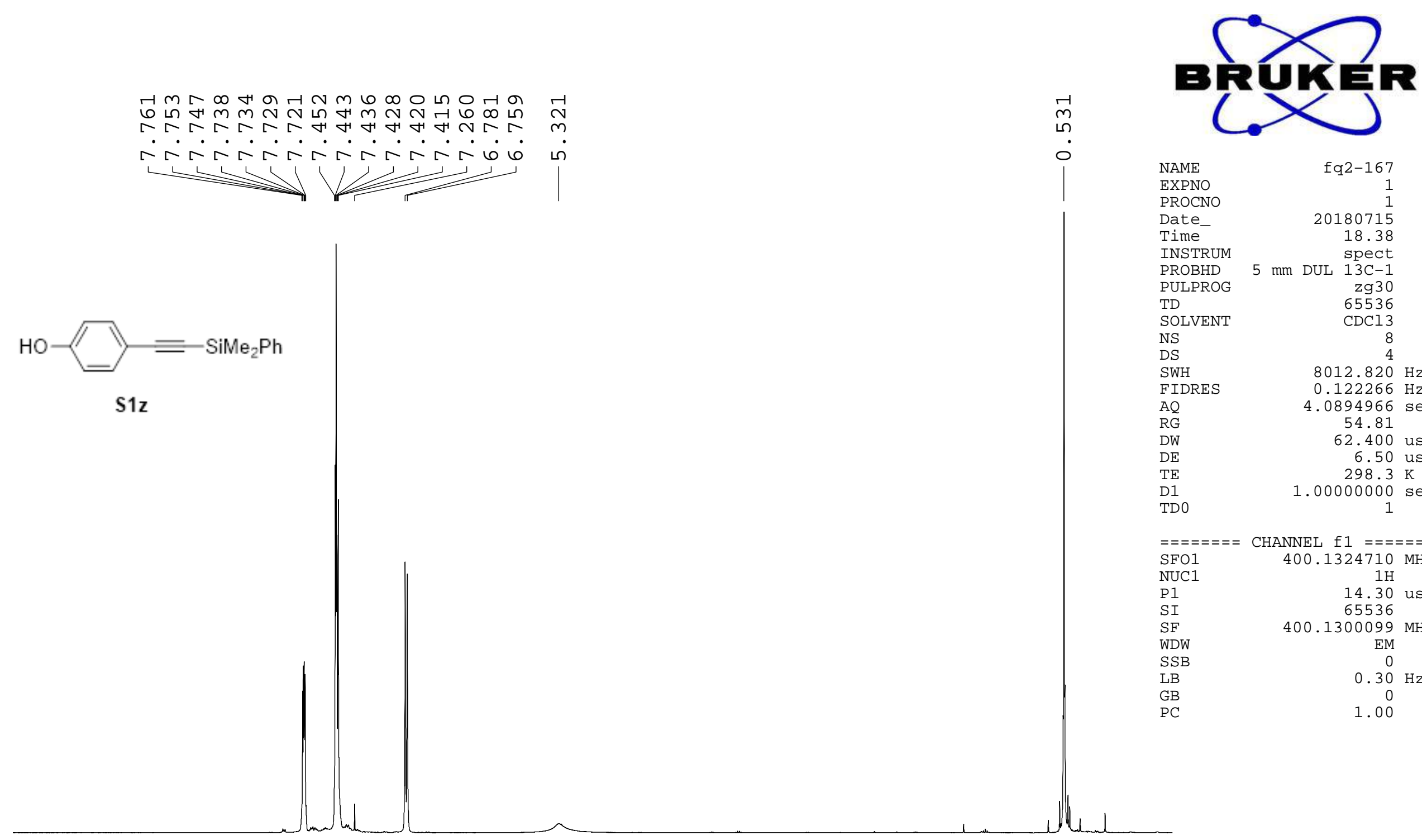

EXPNO

PROCNO

Date

Time

INSTRUM

PROBHD

PULPROG

TD

SOLVENT

NS

SWH

FIDRES

$A Q$

RG

DE

TE

TD 0

fq2-167

18.38

$5 \mathrm{~mm}$ DUL $13 \mathrm{C}-1$

$3 \mathrm{C}-1$

$\mathrm{zg} 30$
65536

$\mathrm{CDCl} 3$
8

$=$ CHANNEL $\mathrm{f} 1======$

SFO1 $400.1324710 \mathrm{MHz}$

NUC1 $1 \mathrm{H}$

$\begin{array}{lr}\text { P1 } & 1 \mathrm{H} \\ \mathrm{SI} & 14.30 \text { usec }\end{array}$

SI $\quad 65536$

SF

WDW EM

$\begin{array}{ll} & 0 \\ \mathrm{LB} & 0.30 \mathrm{~Hz}\end{array}$

LB

$0.30 \mathrm{~Hz}$
0

1.00

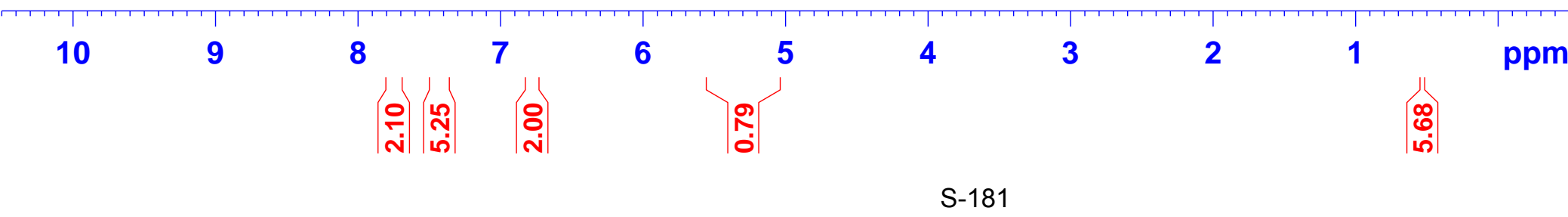




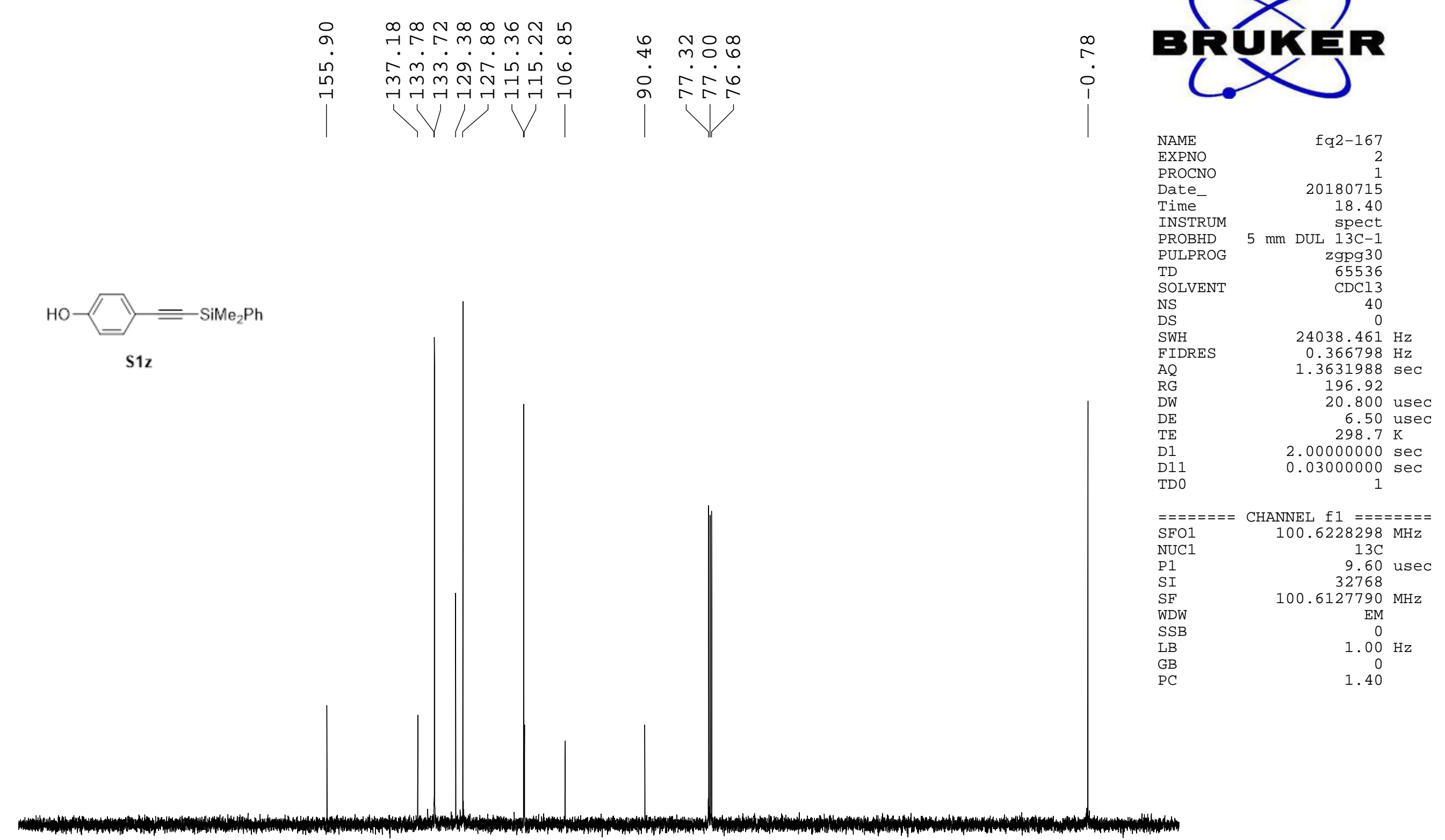

80 
म

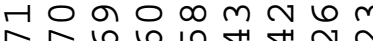

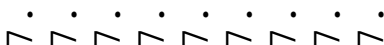

$<<10$

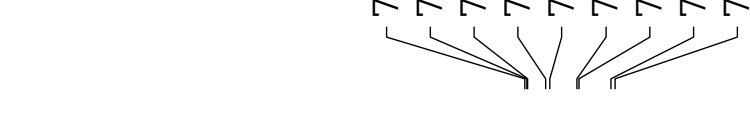

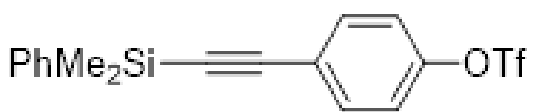

$1 z$

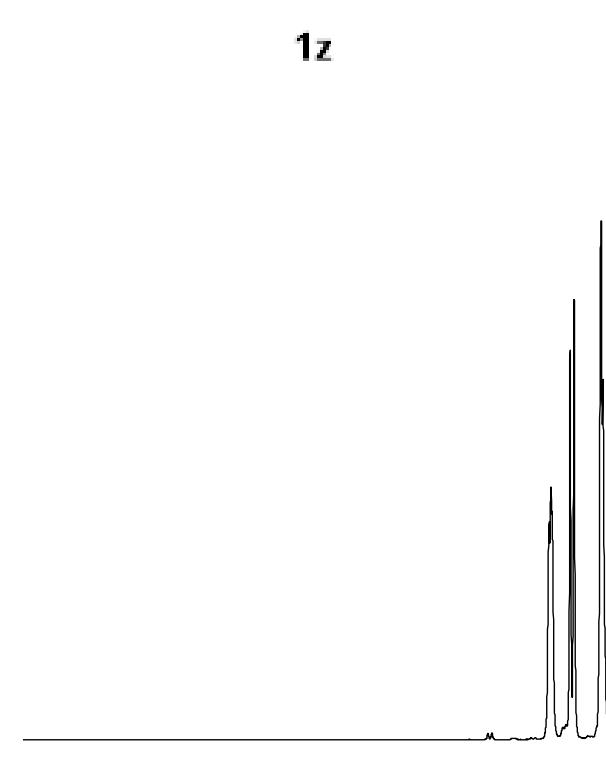
9
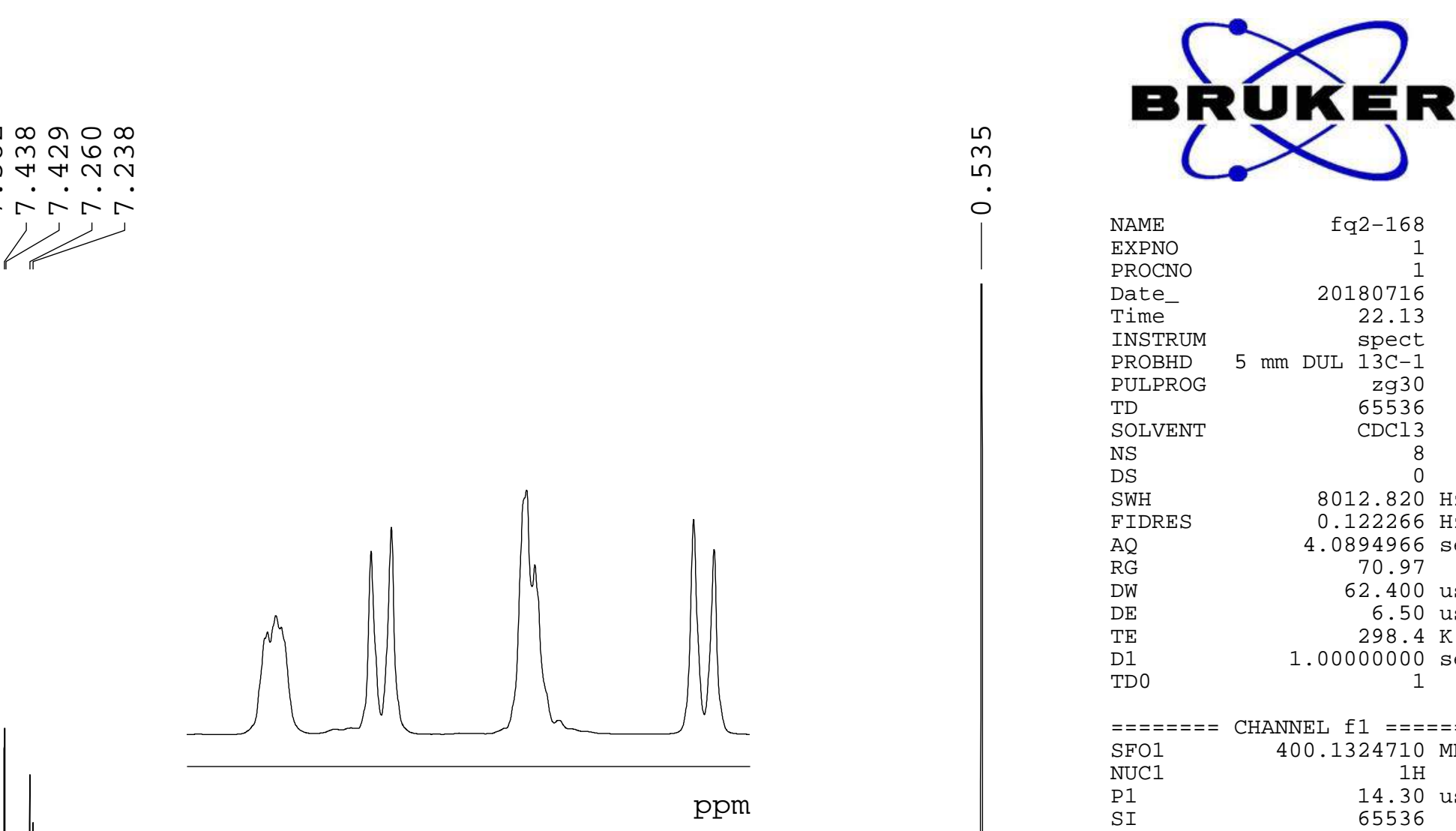

NAME

EXPNO

PROCNO

Date

Time

INSTRUM

PROBHD

PULPROG

TD

SOLVENT

NS

SWH

FIDRES

AQ

RG

DW

DE

D1 0

fq2 $2-168$

68
1
1

20180716

$5 \mathrm{~mm}$ DUL $13 \mathrm{C}-1$

$\mathrm{DCl} 3$
$=======0$ CHANNEI $\mathrm{f} 1=======$
$\mathrm{SFO1}$
$400.1324710 \mathrm{MHz}$

NUC 1

P 1

SI

SF

SSB

LB
GB
PC

$1 \mathrm{H}$
14.30 usec 400.1300084 MHz

$8012.820 \mathrm{~Hz}$

$0.122266 \mathrm{~Hz}$ $\begin{array}{r}70.97 \\ \hline\end{array}$

62.400 usec 6.50 usec $298.4 \mathrm{~K}$

EM

$\mathrm{EM}$
0

$0.30 \mathrm{~Hz}$

0
1.00

융ำ

3

2

1


ॠ

$\dot{0} \dot{\dot{m}} \dot{0} \cdot \dot{0} \cdot \dot{0} 0$

भ $m m \sim \sim \sim$

다다다다다다

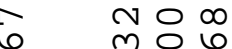

宊

12

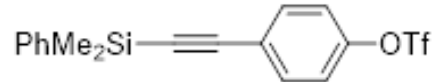

$1 z$

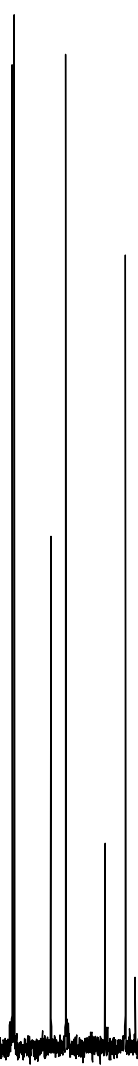

200

180

160

140

120

100 i

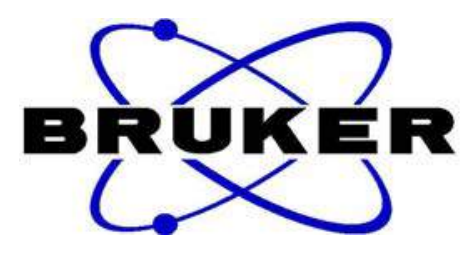

NAME
EXPNO

PROCNO

Date

Time

INSTRUM

PROBHD

PULPROG

TD

SOLVENT

NS

DS

FIDRES

FIDR
AQ
RG
DW

DW

$\mathrm{DE}$

D1

D11

TD 0

$===$

$\mathrm{SFO}$

Noc1

P 1

SI
SF

WDW

SSB
LB

LB

PC

fq2-1 68

20180716

22.15

spect

$5 \mathrm{~mm}$ DUL $13 \mathrm{C}-1$

zgpg 30

$\mathrm{CDCl} 3$

160

$24038.461 \mathrm{~Hz}$

$0.366798 \mathrm{~Hz}$

$1.3631988 \mathrm{sec}$

196.92

20.800 usec

6.50 usec

$.00000000 \mathrm{~K}$

$0.03000000 \mathrm{sec}$

CHANNEL $\mathrm{f} 1=$

$100.6228298 \mathrm{MHz}$

$13 \mathrm{C}$

9.60 usec

100.6127731

$100.6127731 \mathrm{MHz}$

EM

$1.00 \mathrm{~Hz}$

0
1.40 


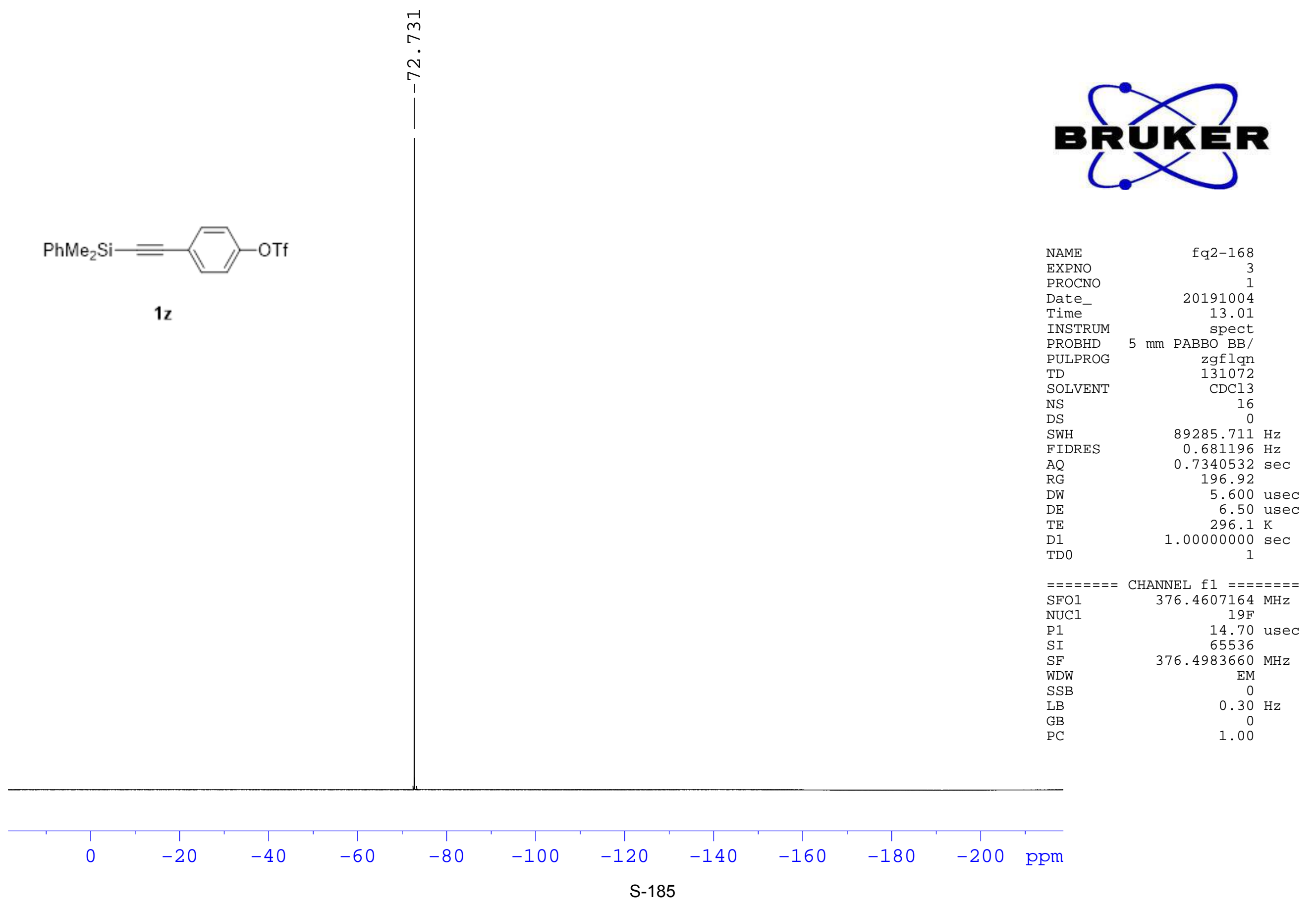




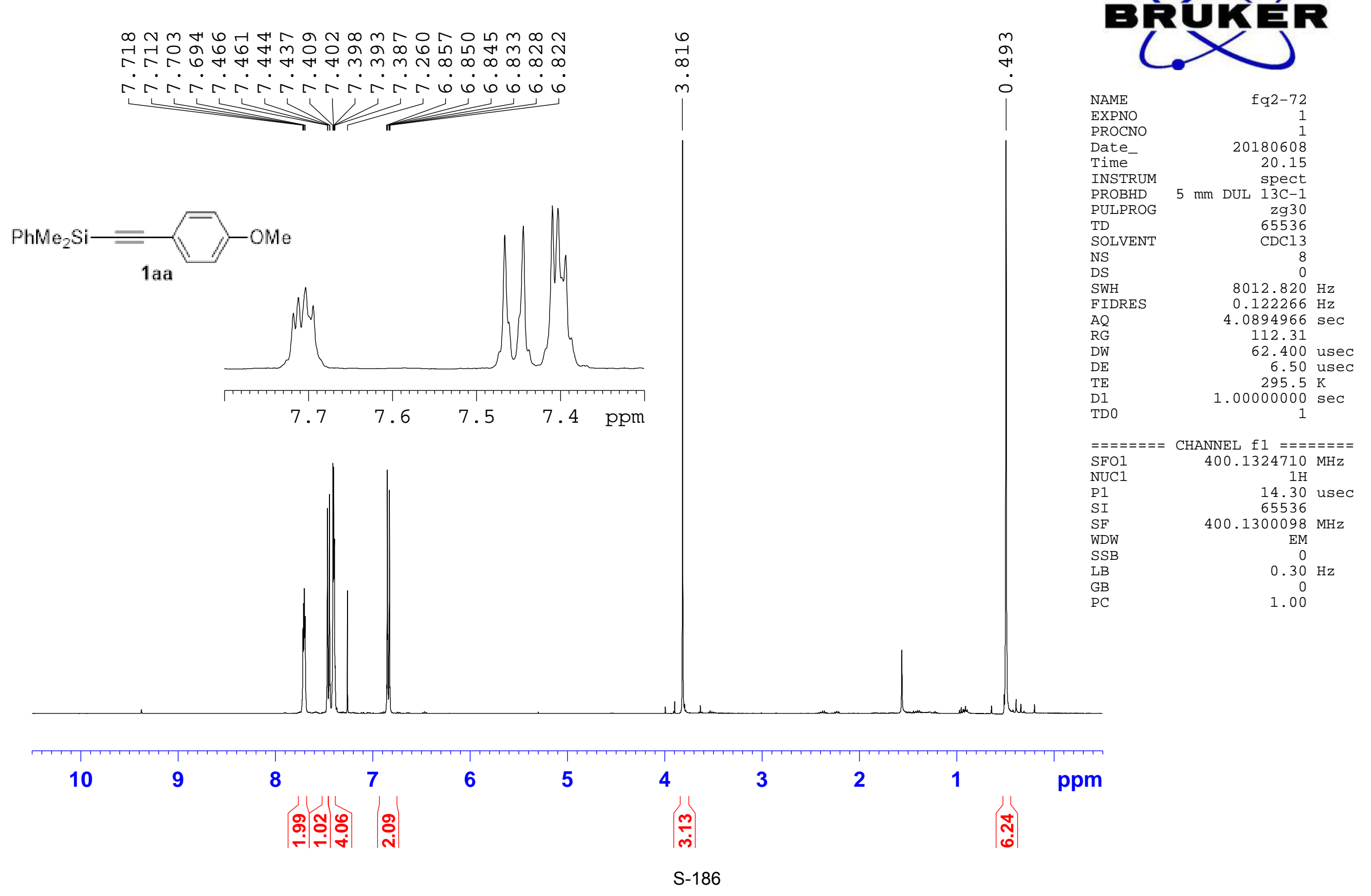




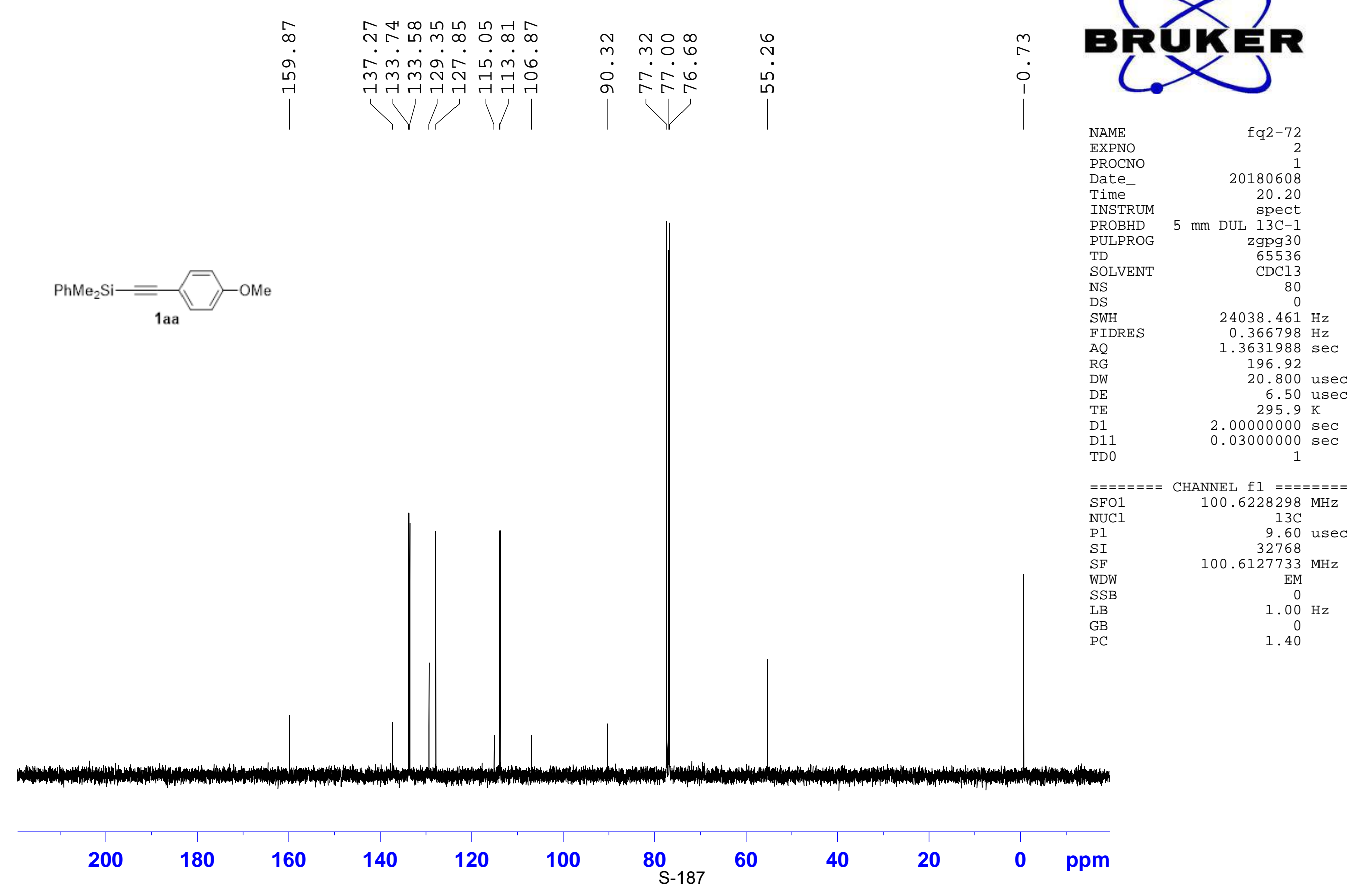




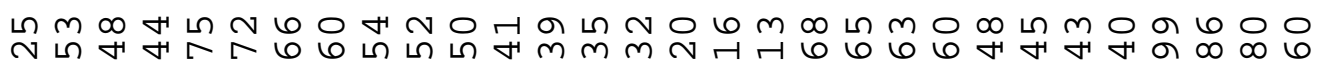
는

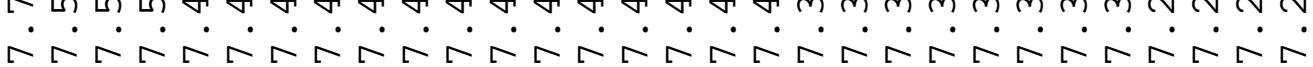

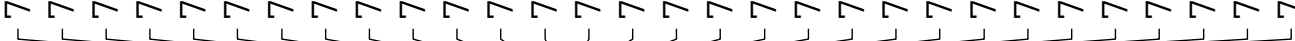

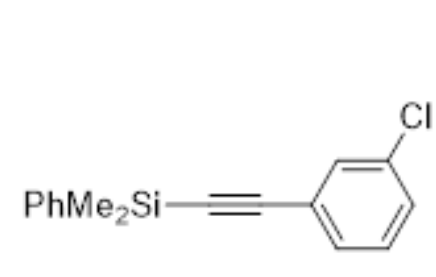

$1 \mathrm{ab}$

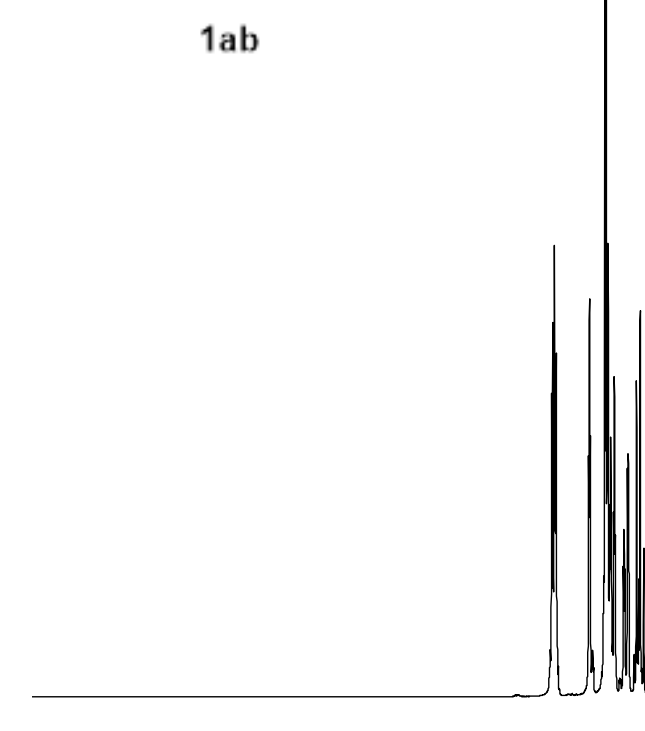

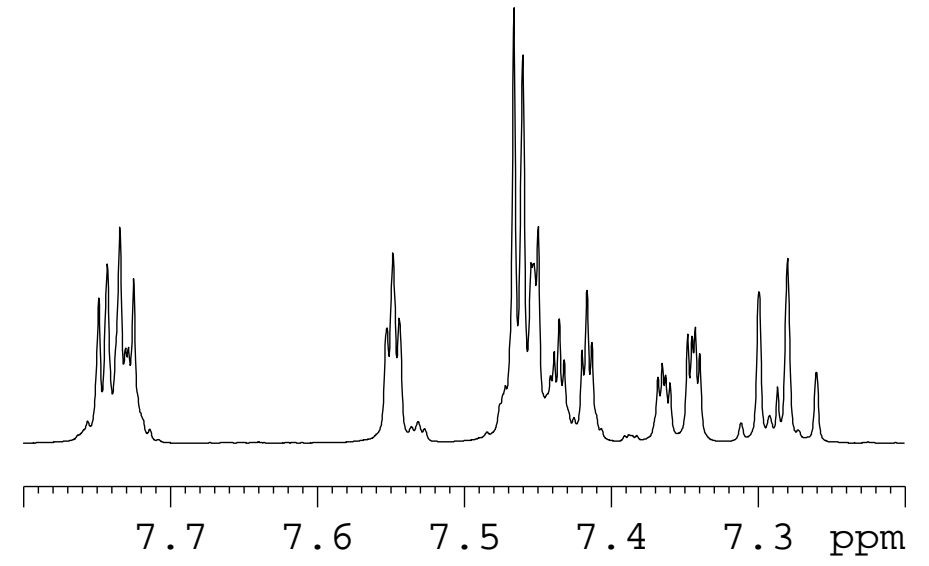

10

9

8

7

5

4

3

2

+1

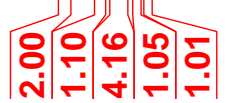

1

)

ppm 


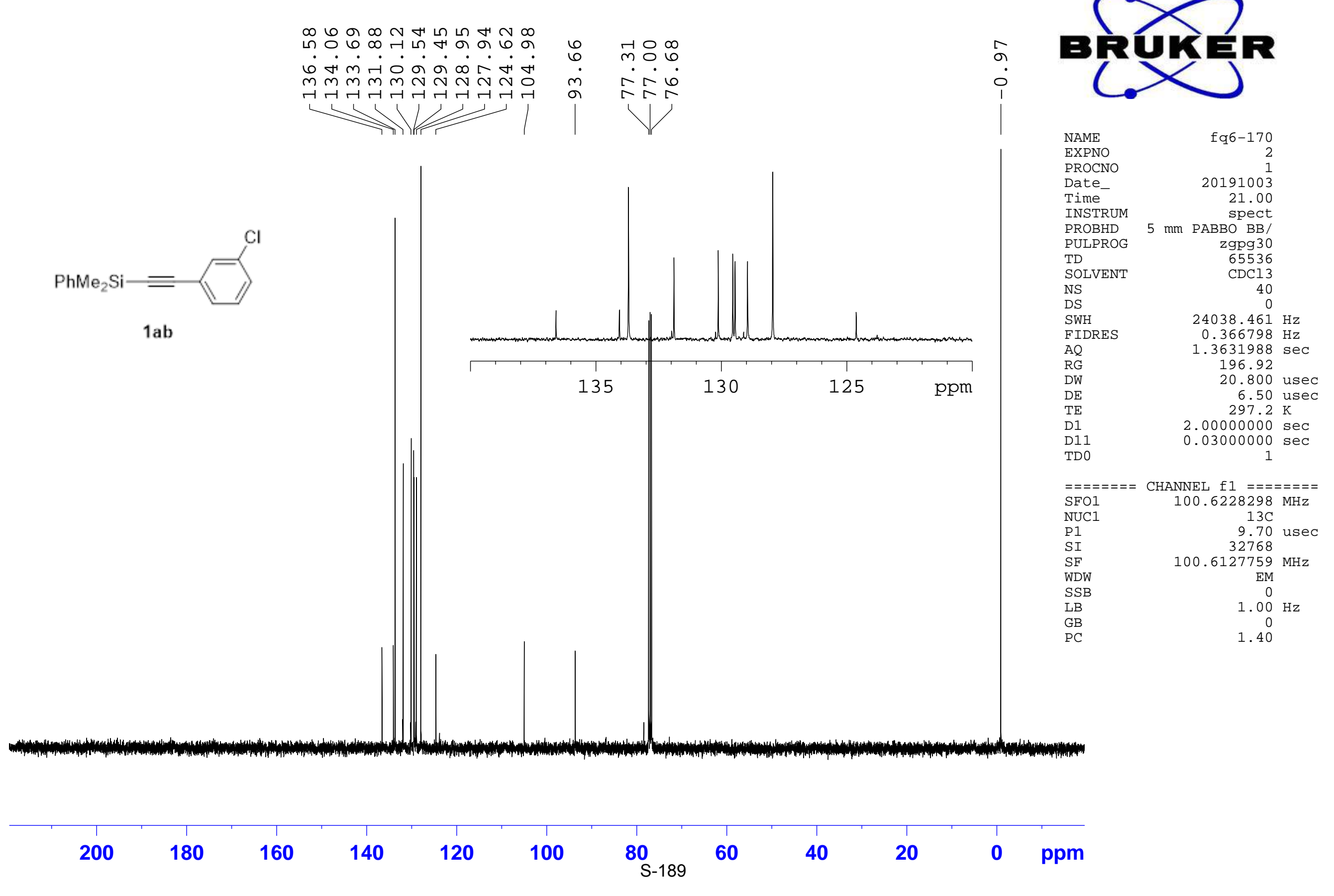




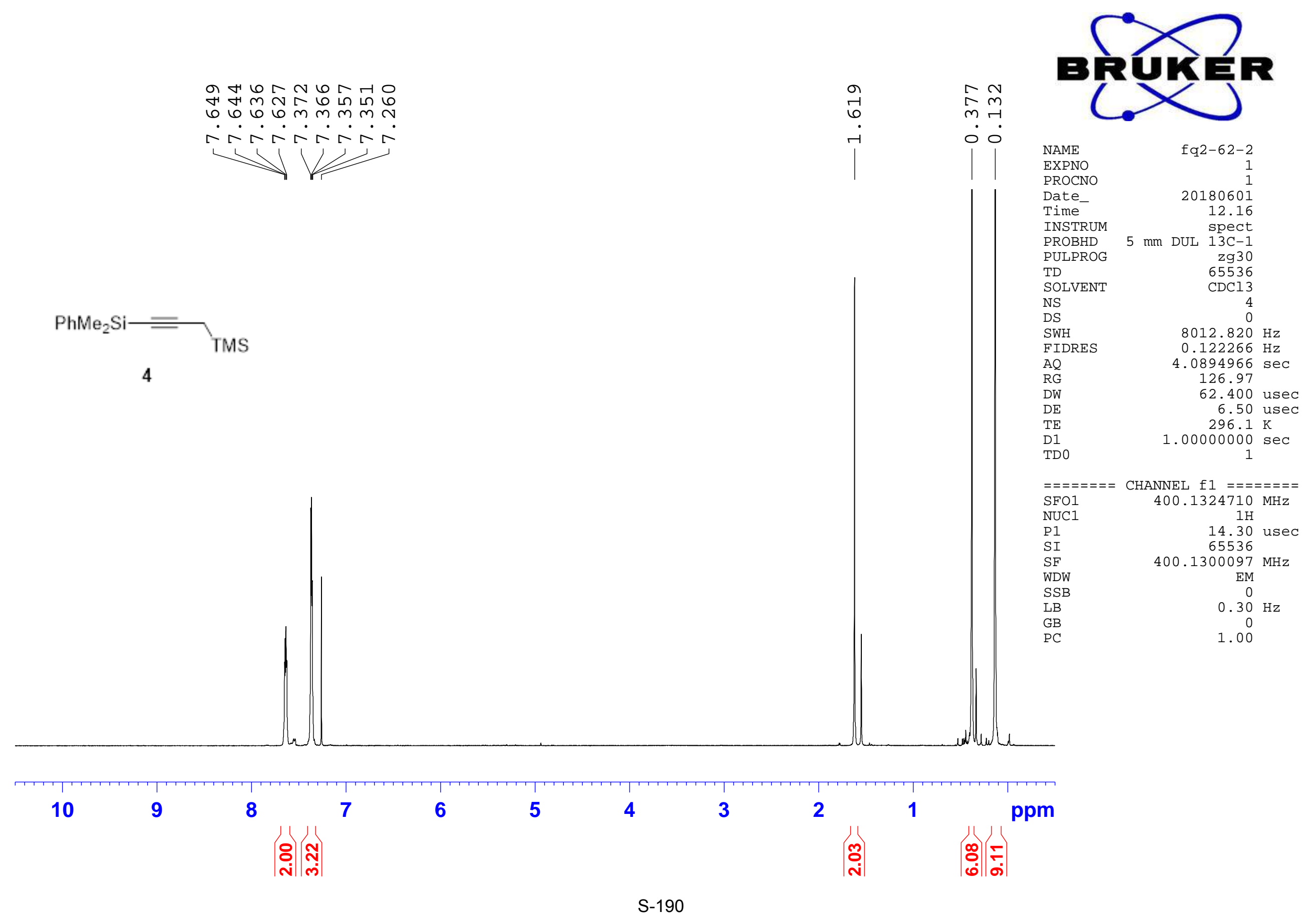




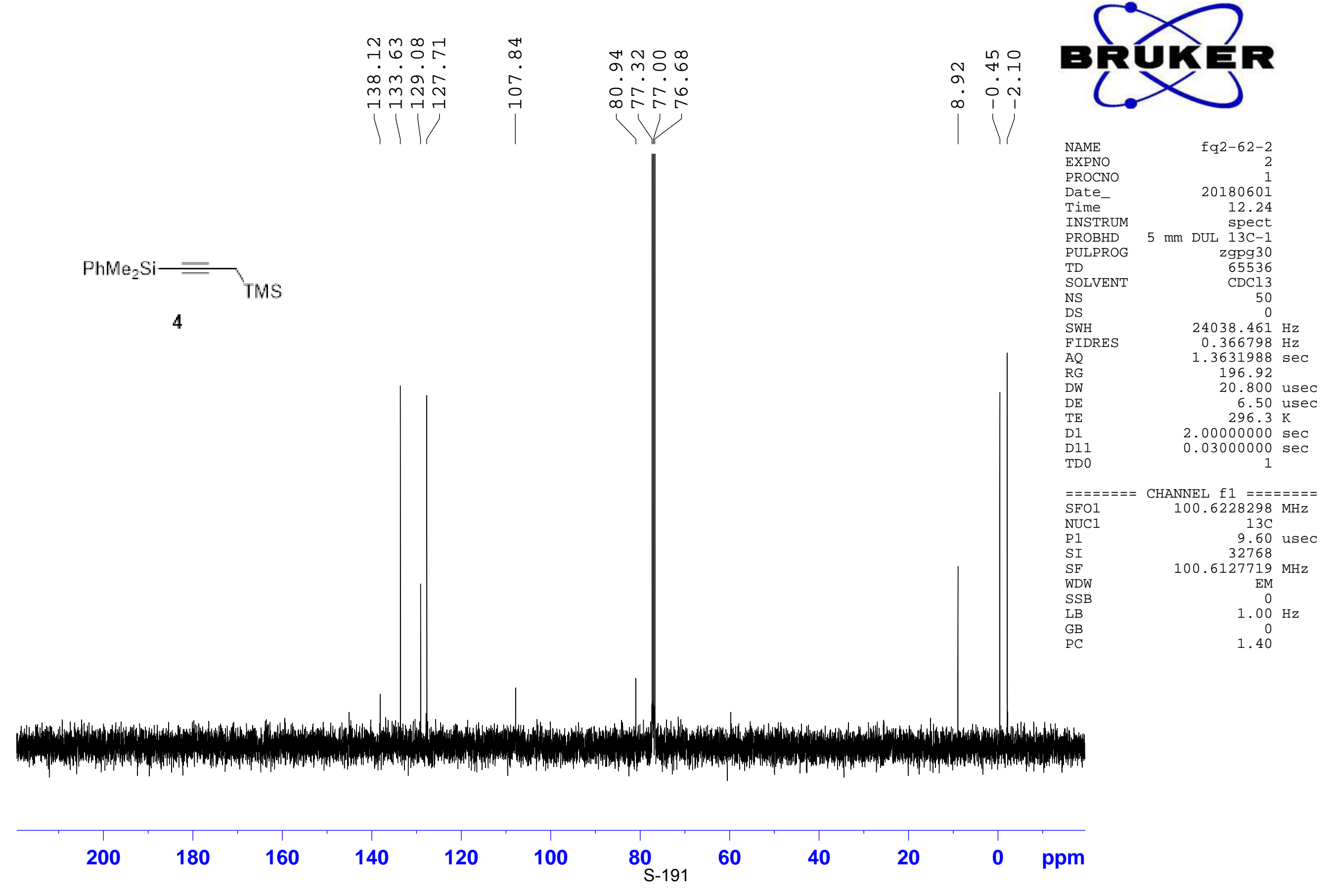


$\infty$ 小

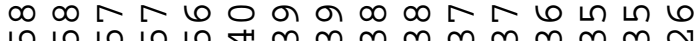

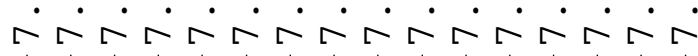

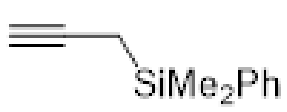

S5
10 9

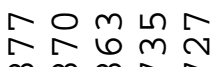
$\infty \infty \infty \Lambda$

$\dot{\vec{r}} \dot{\vec{r}} \dot{\mathrm{r}}$
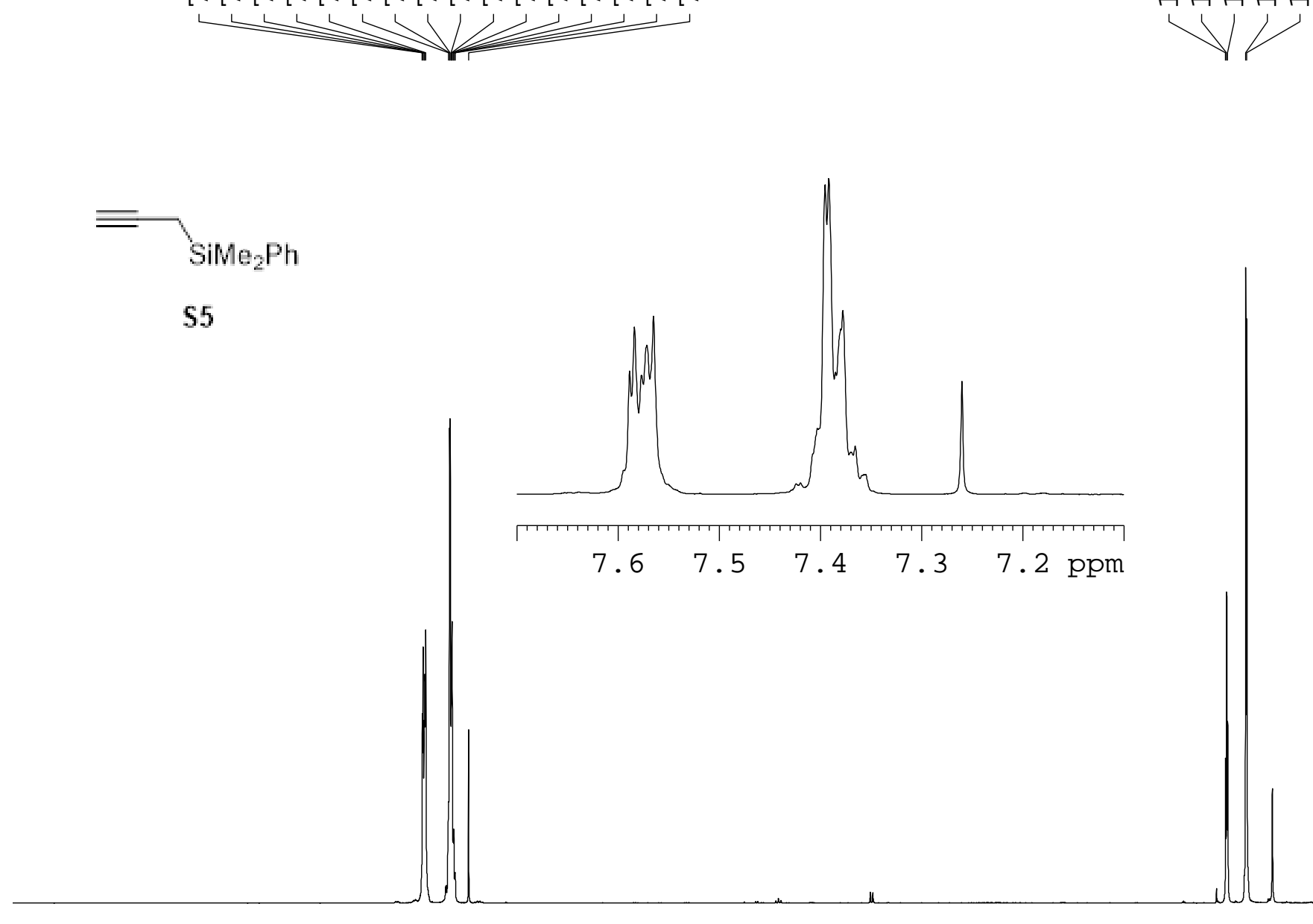

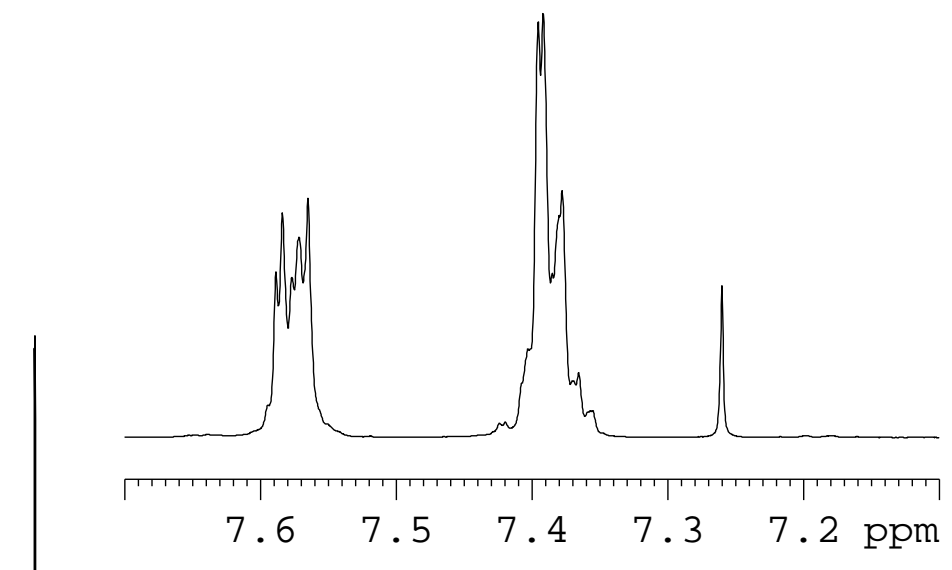

7

6

5

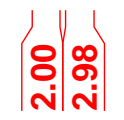

5

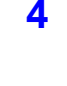

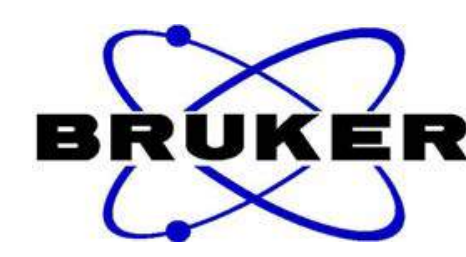

NAME

EXPNO

PROCNO

Date_-

INSTRUM

PROBHD

PULPROG

TD

SOLVENT

NS

SWH

FIDRES

AQ

RG

DE

TE

D1 0

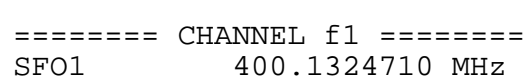

$\mathrm{SFO1}$

NUC1

PI

SF

WDW

SSB

LB
GB

PC fq2 $-67-2$

20180605 12.40 $5 \mathrm{~mm}$ DUL $13 \mathrm{C}-1$ zg30
65536 $\mathrm{CDCl} 3$ 8

$8012.820 \mathrm{~Hz}$ $0.122266 \mathrm{~Hz}$ $4.0894966 \mathrm{sec}$ 126.97 62.400 usec 6.50 usec $296.3 \mathrm{~K}$ $1.00000000 \mathrm{sec}$ $400.1324710 \mathrm{MHz}$ $1 \mathrm{H}$
14.30 usec $400.1300098 \mathrm{MHz}$ EM $0.30 \mathrm{~Hz}$ $0.30 \mathrm{~Hz}$
0 


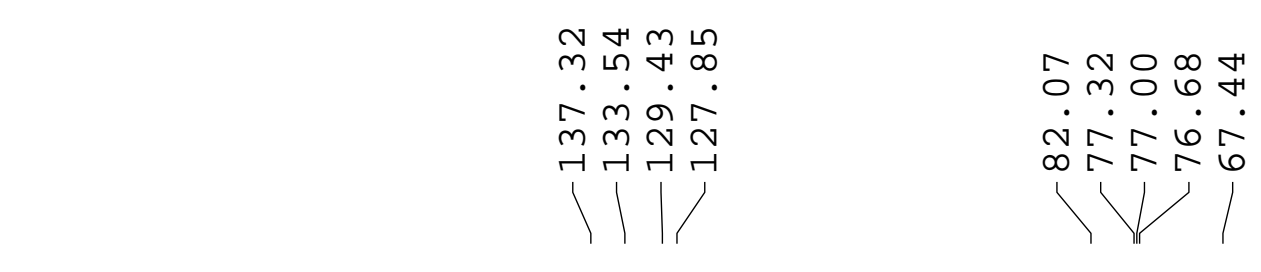

$\because \underset{\text { NAME }}{i}$

EXPNO

PROCNO

Date

Time

PROBHD

PULPROG

20180605

12.42

TD

SOLVENT

NS

DS

FIDRES

$\mathrm{AQ}$

RG

DW

S5

DE

D1

D11

mm DUL $13 \mathrm{C}-1$
m

zgpg 30

CDCl3

50

$24038.461 \mathrm{~Hz}$
$0.366798 \mathrm{~Hz}$

1. $3631988 \mathrm{sec}$

196.92

20.800 usec

6.50 usec

$0.03000000 \mathrm{sec}$

1

$=======$ CHANNEL $\mathrm{f} 1 \mathrm{l}======$

$\mathrm{SFO} 1$

Nuc1

P1

SF

WDW

SSB

LB
GB

$P C$

$100.6228298 \mathrm{MHz}$

$13 \mathrm{C}$

9.60 usec

$100.6127725 \mathrm{MHz}$

EM

0
$1.00 \mathrm{~Hz}$
0

0
1.40 


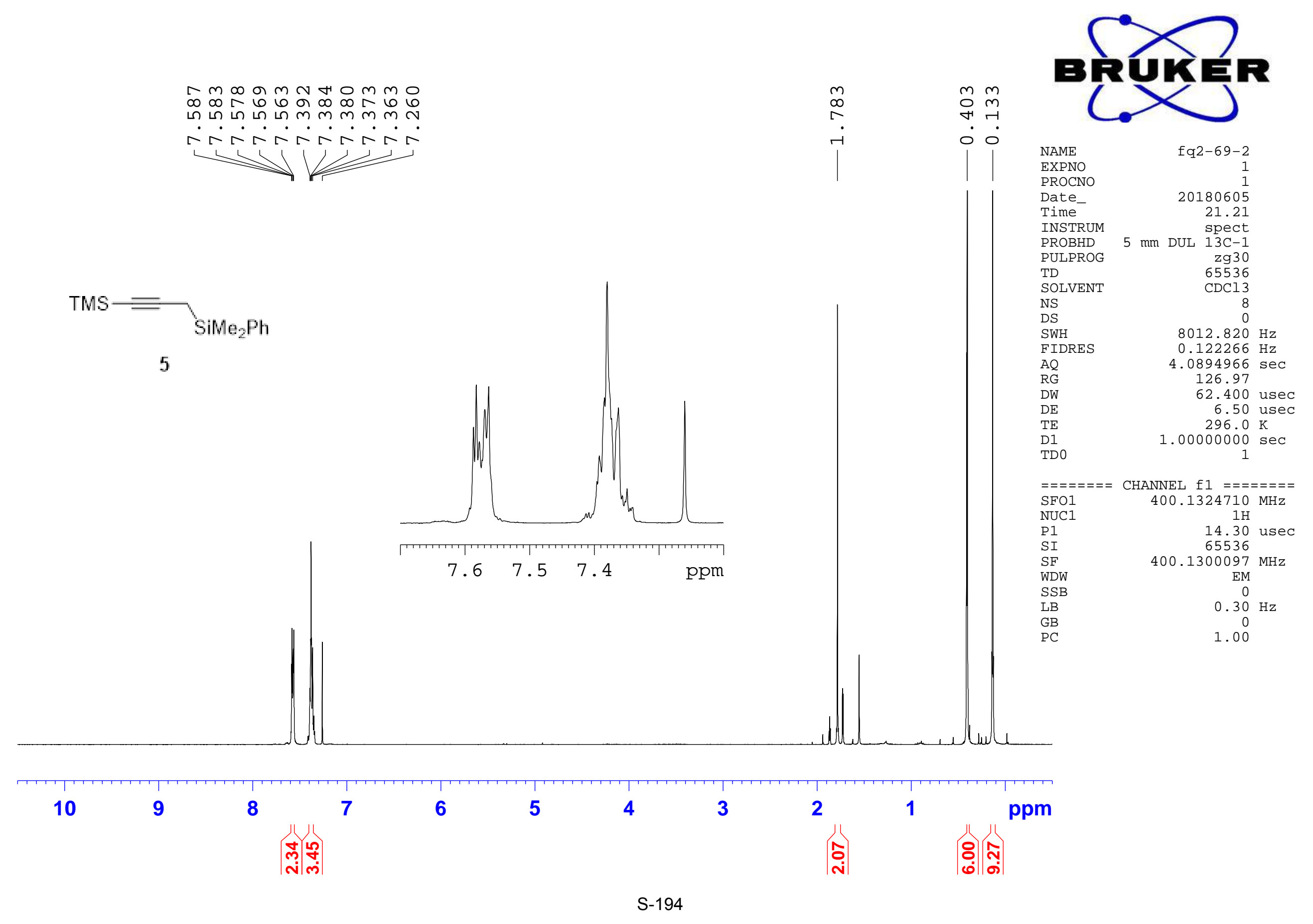


$\underset{\gamma}{\sim} \in \mathbb{R}$

$\therefore \dot{m}$

$m m \sim N$

111

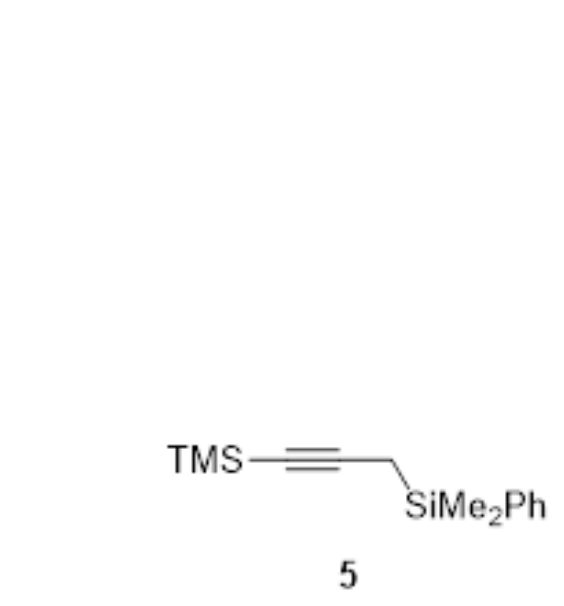

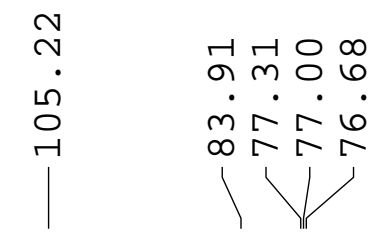

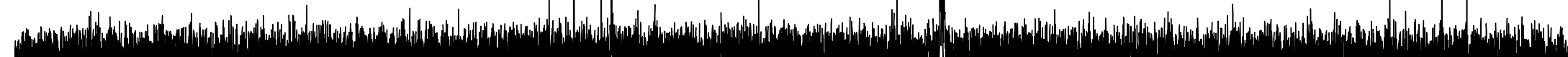

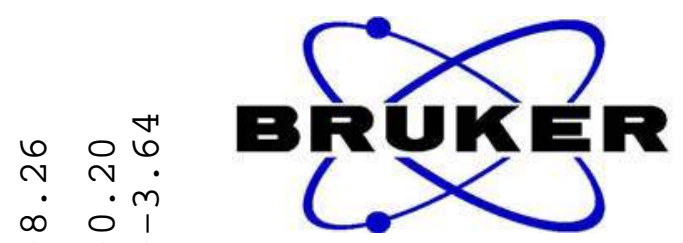

$\infty 01$

$1 \mid$

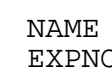

PROCNO

Date

Time

INSTRUM

PROBHD

PULPROG

SOLVENT

NS

DS

FIDRES

$\mathrm{AQ}$

RG

DW

$\mathrm{DE}$

D1

D11

SFO

NEO1

$\mathrm{P} 1$

SI

WDW

SSB

LB

$\mathrm{PC}$

fq2 $2-69-2$

20180605 21.24

spect

$5 \mathrm{~mm}$ DUL 13C-1

zgpg 30

$\mathrm{CDCl} 3$

50

$0.366798 \mathrm{~Hz}$

1. $3631988 \mathrm{sec}$

196.92

20.800 usec

6.50 usec

$0.03000000 \mathrm{sec}$

0.03000000

CHANNEL $\mathrm{f} 1==$

$13 \mathrm{C}$

$13 \mathrm{C}$
$9.60 \mathrm{usec}$

32768

$100.6127721 \mathrm{MHz}$

EM

$1.00 \mathrm{~Hz}$

0
1.40

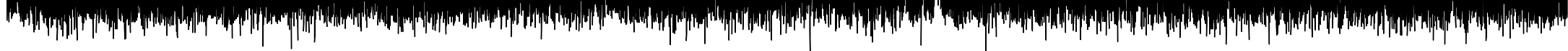

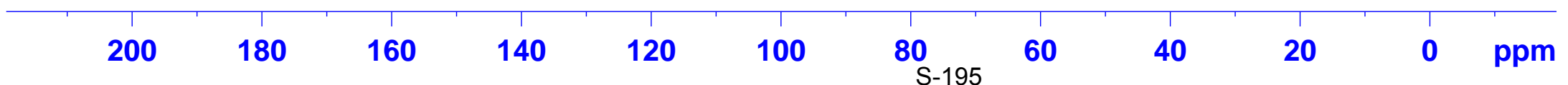




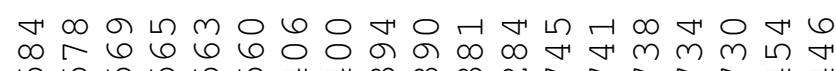

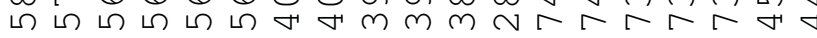
-

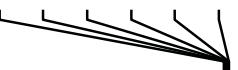
1

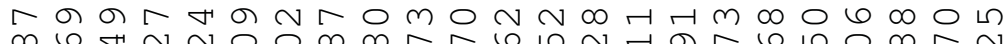

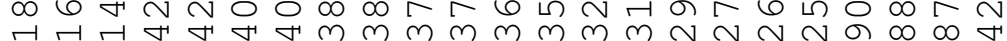
iंن.

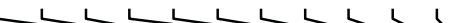
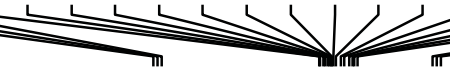

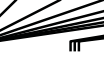

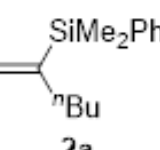

$2 \mathrm{a}$

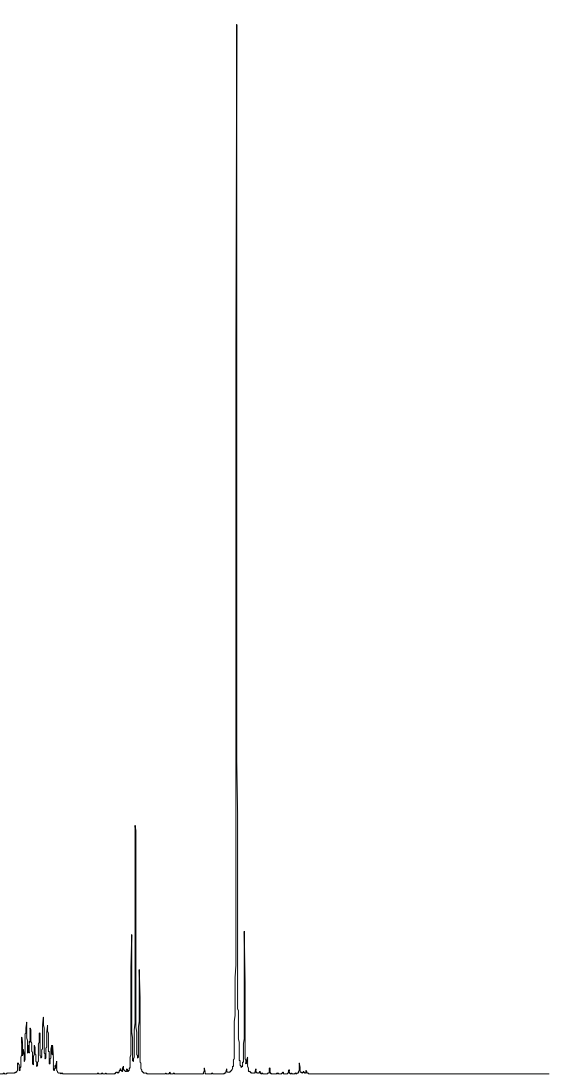

Current Data Parameters
NAME EXPNO
PROCNO

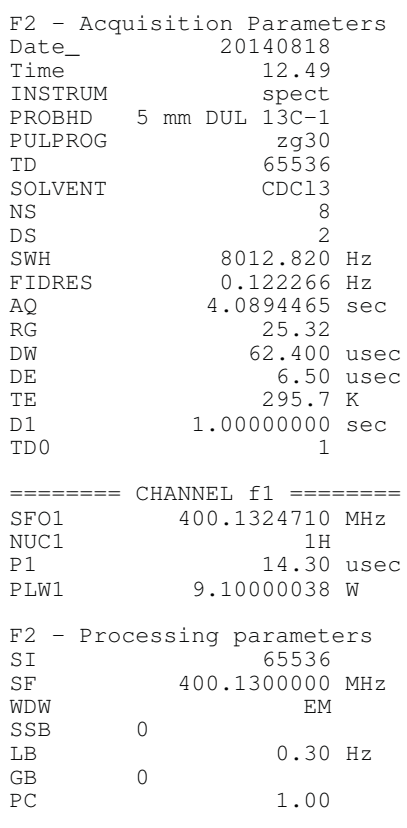

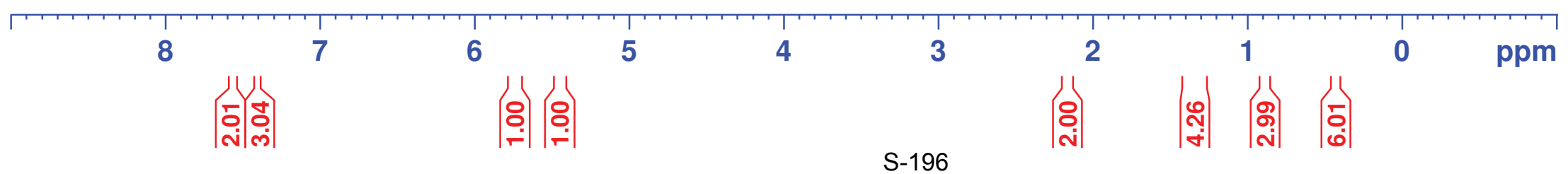




\section{BRUKE}

$=\overbrace{{ }^{n} \mathrm{Bu}}^{\mathrm{SiMe}_{2} \mathrm{Ph}}$
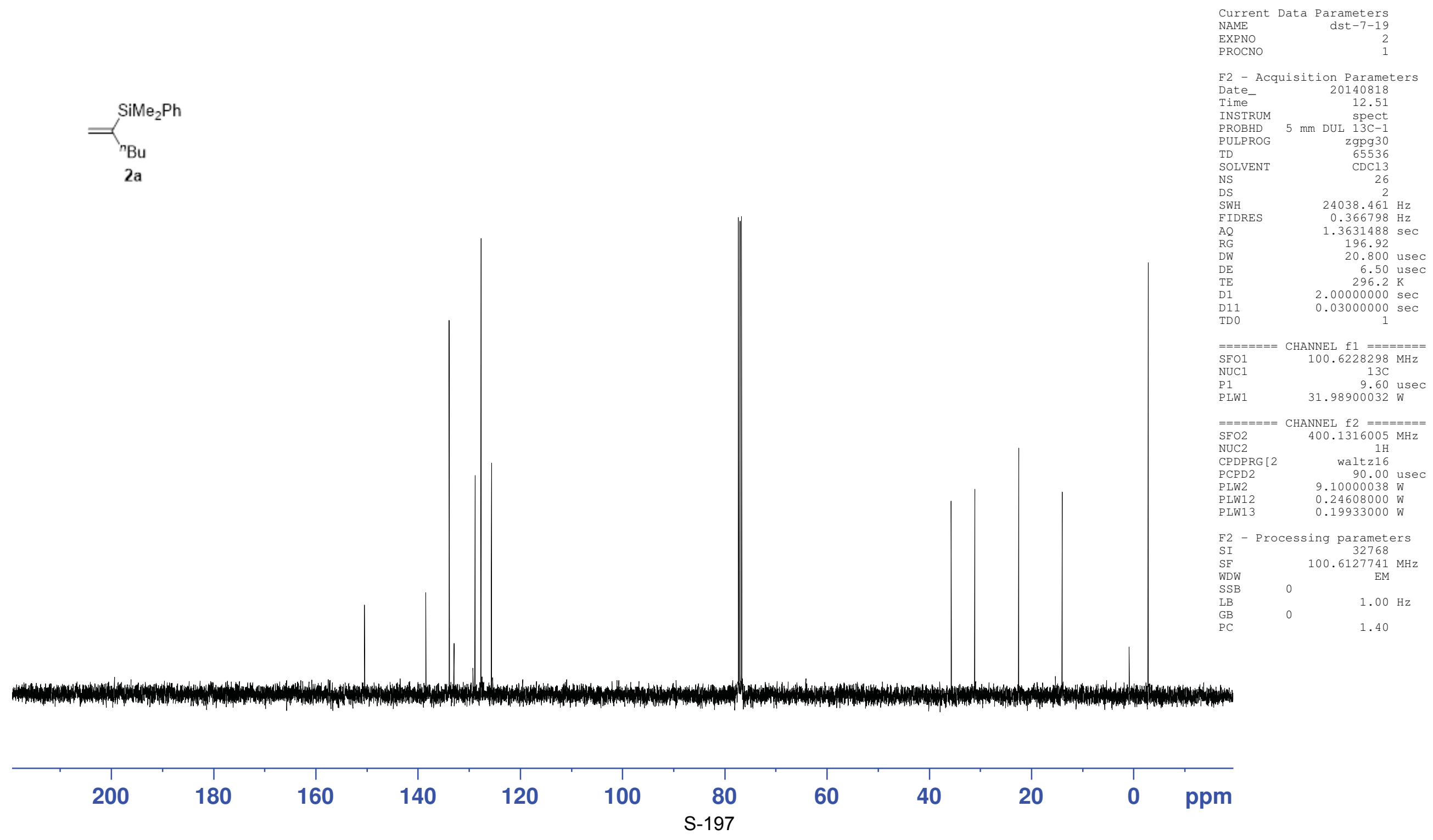
உた เ $\longrightarrow \longrightarrow$

$\mathrm{SiMe}_{2} \mathrm{Ph}$

$\left(\mathrm{CH}_{2}\right)_{7} \mathrm{CH}_{3}$

$2 \mathrm{~b}$

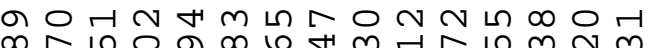
$\infty$ ก . . . . . . . . . . .
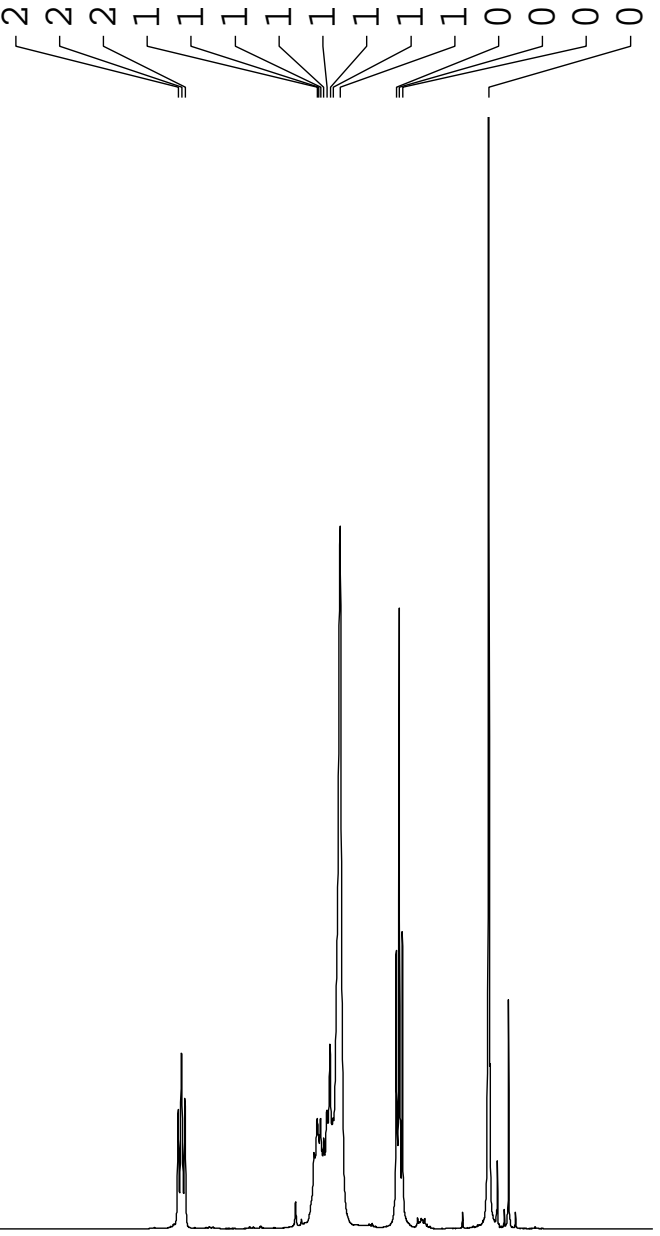

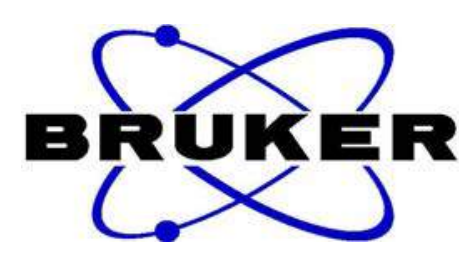

NAME

EXPNO

PROCNO

Date

Time

INSTRUM

PROBHD

PULPROG

TD

SOLVENT

NS
DS

SWH

FIDRES

$A Q$

RG

DE

DE

D1

$\begin{array}{lr}=======\text { CHANNEL } \mathrm{f} 1 \quad======= \\ 1 \mathrm{H} \\ \text { NUC1 } \\ \text { P1 } & 13.60 \mathrm{useC} \\ \text { PL1 } 1.00 \mathrm{~dB} \\ \text { SFO1 } & -1.00 .1324710 \mathrm{MHz} \\ \text { SI } & 32768 \\ \text { SF } & 400.1300097 \mathrm{MHz} \\ \text { WDW } & \mathrm{EM} \\ \text { SSB } & 0 \\ \text { LB } & 0.30 \mathrm{~Hz} \\ \text { GB } & 0 \\ \text { PC } & 1.00\end{array}$

10 9

8

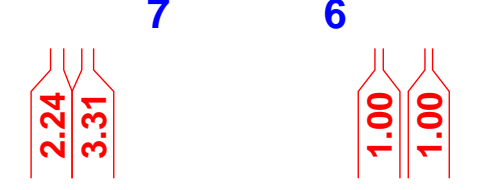

\begin{abstract}
5
\end{abstract}

4

3

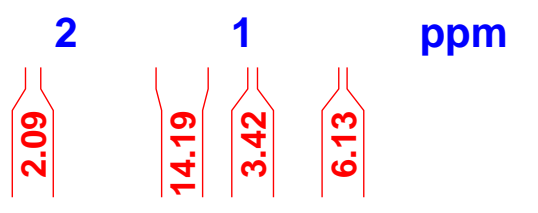




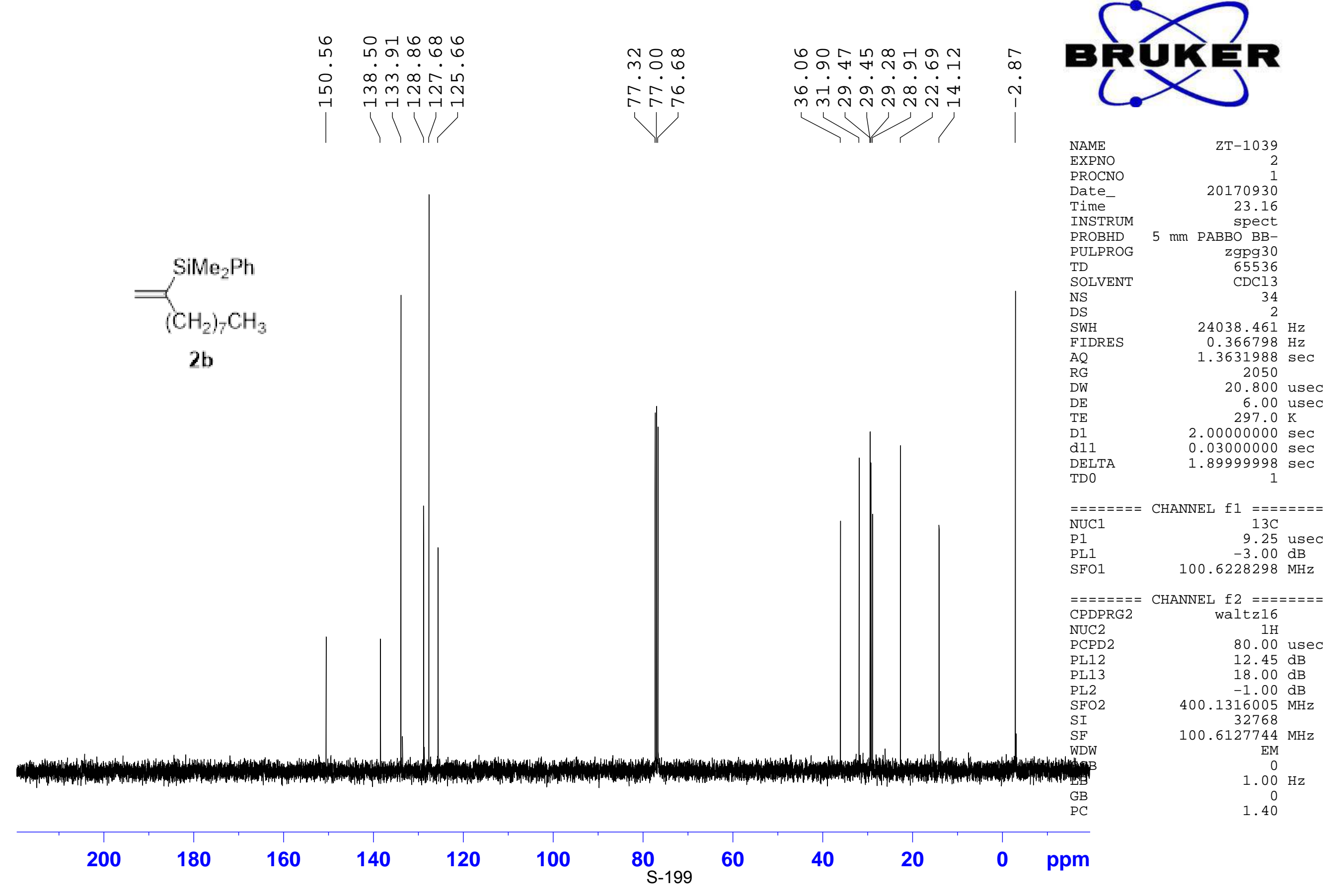


늄 เी

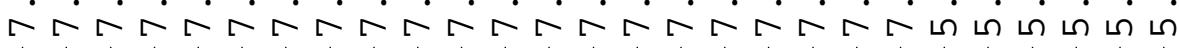

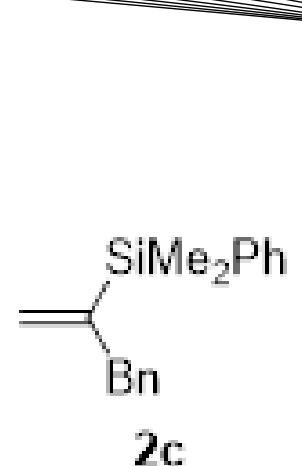

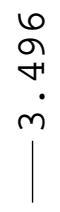
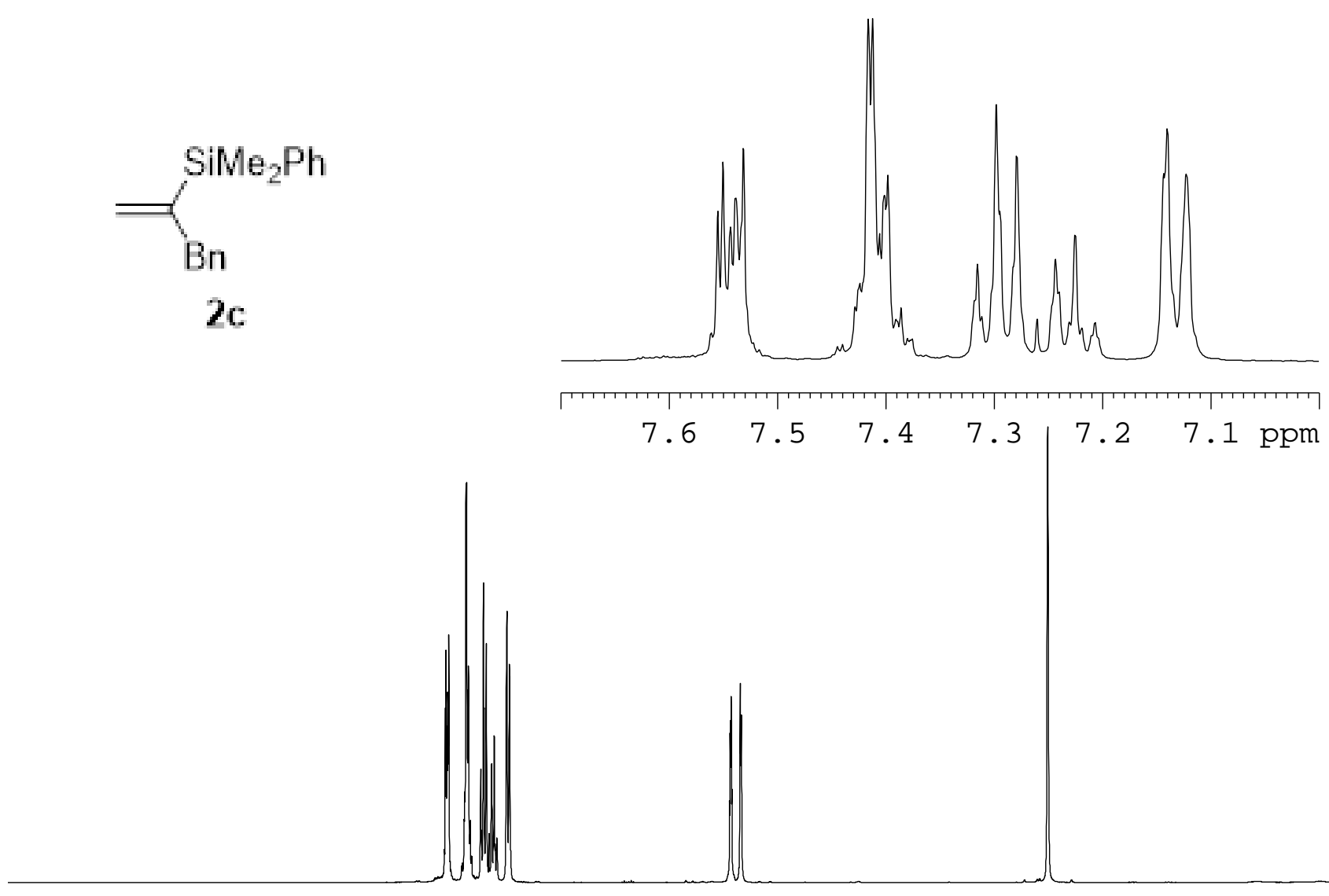

9

8

8

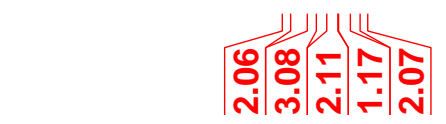

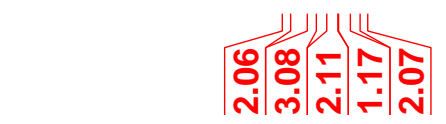

10

6

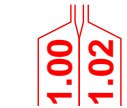

$\stackrel{m}{m}$

○

(2) $(><)$

NAME

EXPNO
PROCNO

Date

Time

INSTRUM

PROBHD

PULPROG

TD

SOLVENT

NS

SWH

FIDRES

$\mathrm{AQ}$
$\mathrm{RG}$

RG

$\mathrm{DE}$

TE

TDO

$\mathrm{ZT}-1040$

20170930

23.23

$5 \mathrm{~mm}$ PABBO $\mathrm{BB}-$

$\mathrm{zg} 30$
65536

CDC13

16
2

$8223.685 \mathrm{~Hz}$

$0.125483 \mathrm{~Hz}$

$3.9846387 \mathrm{sec}$

64

60.800 usec 6.00 usec $1.00000000 \mathrm{sec}$

$=======$ CHANNEL f 1 =e==e===

NUC1

P1

PL1

$\mathrm{SFO} 1$

SI

SF

WDW

SSB

LB
GB

PC

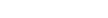

$1 \mathrm{H}$

13.60 usec $-1.00 \mathrm{~dB}$

$400.1324710 \mathrm{MHz}$

$\begin{array}{r}32768 \\ \hline\end{array}$

$400.1300096 \mathrm{MHz}$

EM

$0.30 \mathrm{~Hz}$

0.30
0
1.00

0
1.00 


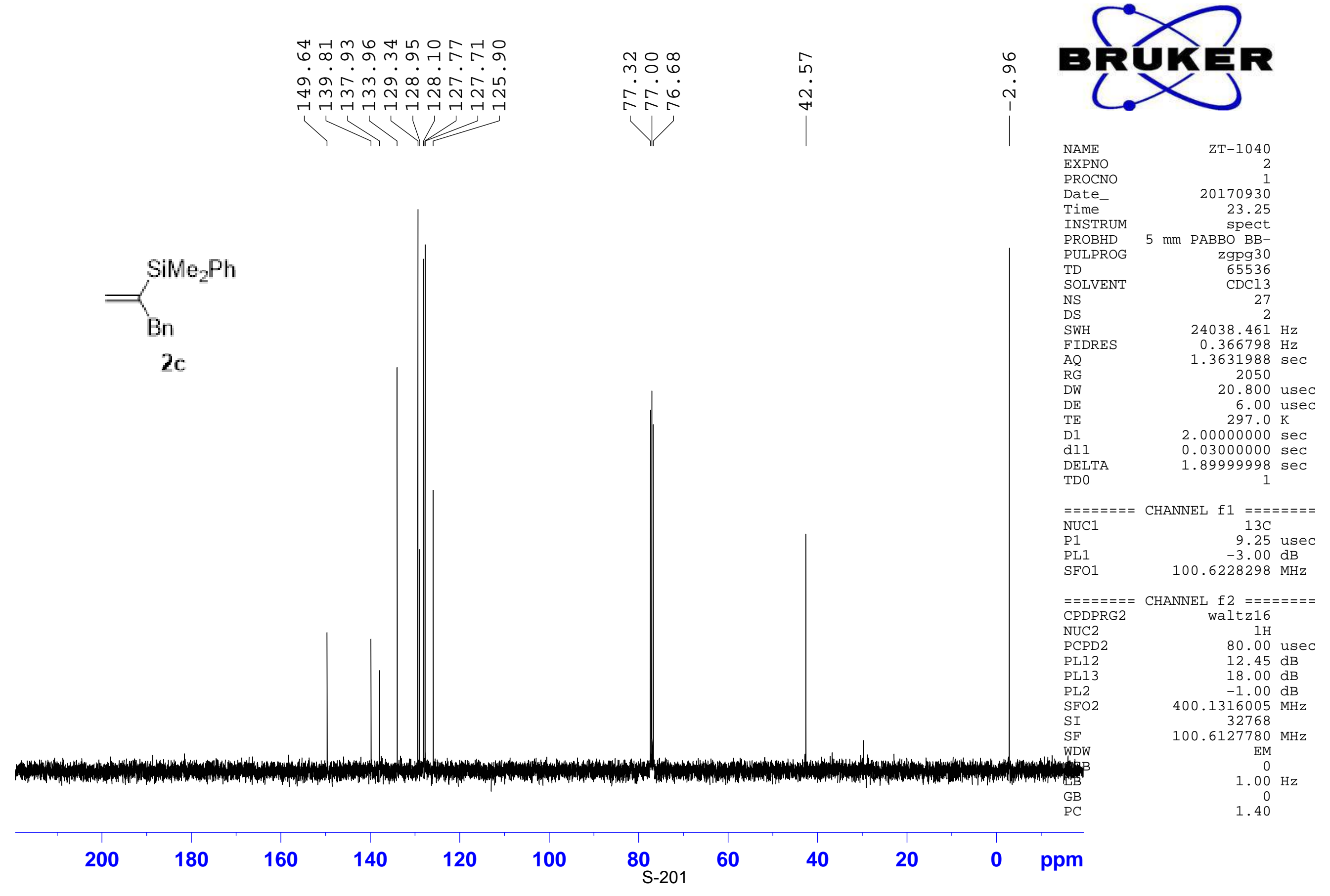




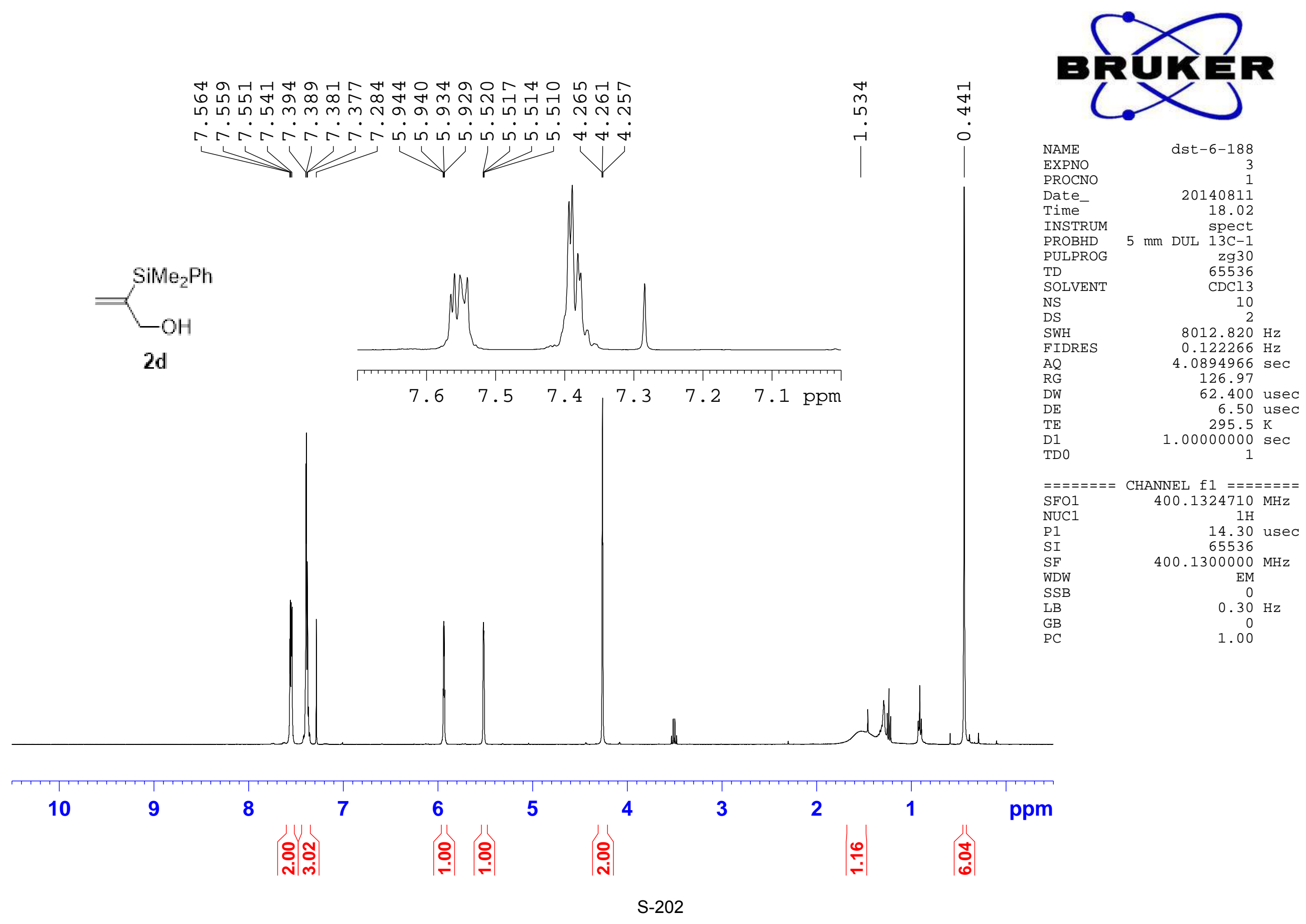




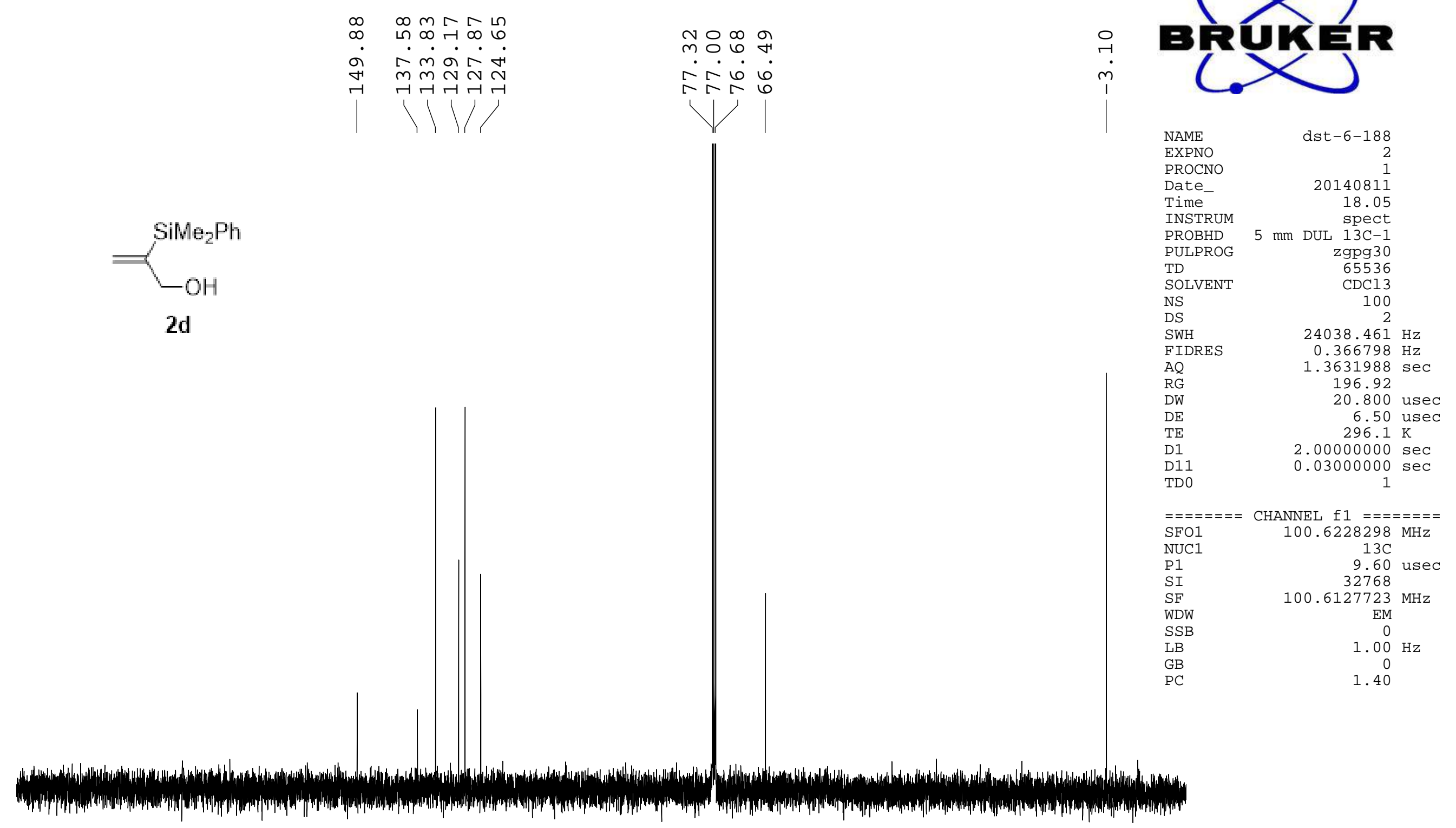




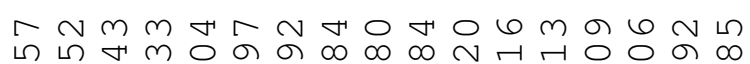

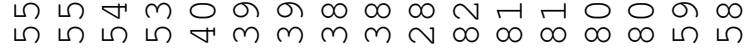

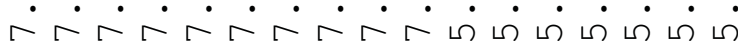

$\longrightarrow$

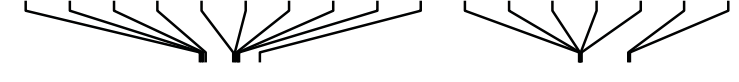

$\underbrace{\mathrm{SiMe}_{2} \mathrm{Ph}}_{\mathrm{OH}}$

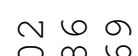

$\circ \infty 6$

.

$m m$

V

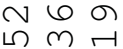

$\forall \ngtr$

$\dot{\sim} \dot{\sim} \dot{\sim}$

11

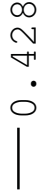

me

Current Data Parameters NAME
EXPNO
PROCNO

F2 - Acquisition Parameters Date_ 20140818

TimePROBHD $5 \mathrm{~mm}$ DUL $\begin{array}{r}\text { spect } \\ 13 \mathrm{C}-1\end{array}$

TD
NS

NS

DS
SWH
FIDRES
AD

$A Q$
RG

RG
DW
DE

DE
TE
D1

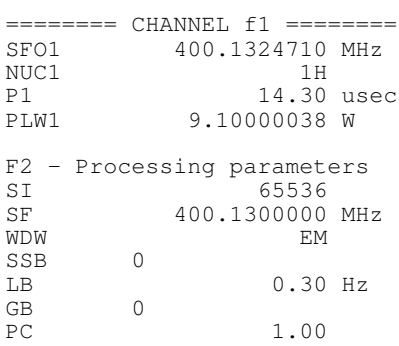

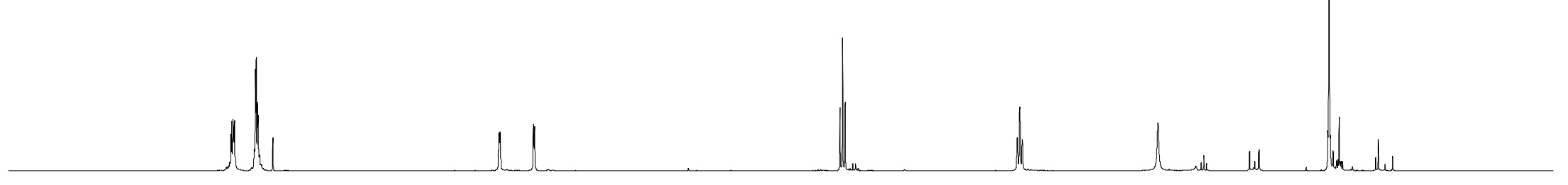

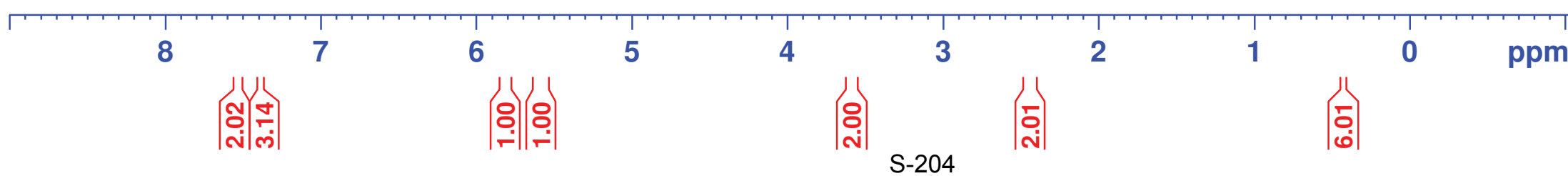




\section{brukER}<smiles>C=C(CCO)[AsH2]c1ccccc1</smiles>

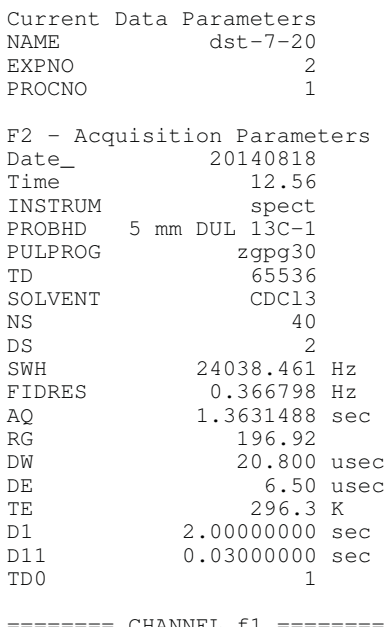

SFO1 $\quad 100.6228298 \mathrm{MHz}$

NUC1 PLW1 $\quad 9.60$ usec $=======$ CHANNEL $f 2=======$
SFO2
$400.1316005 \mathrm{MHz}$ $\begin{array}{lr}\text { NUC2 } & 1 \mathrm{H} \\ \text { CPDPRG [2 waltz16 } & \end{array}$

waltz16
90.00 usec $\begin{array}{ll}\text { PLW2 } & 9.10000038 \mathrm{~W} \\ \text { PLW12 } & 0.24608000 \mathrm{~W}\end{array}$ PLW13 $0.19933000 \mathrm{~W}$ $\begin{array}{lr}\text { F2 } & \text { - Processing parameters } \\ \text { SI } & 32768 \\ \text { SF } & 100.6127745 \mathrm{MHz}\end{array}$ SF
WDW

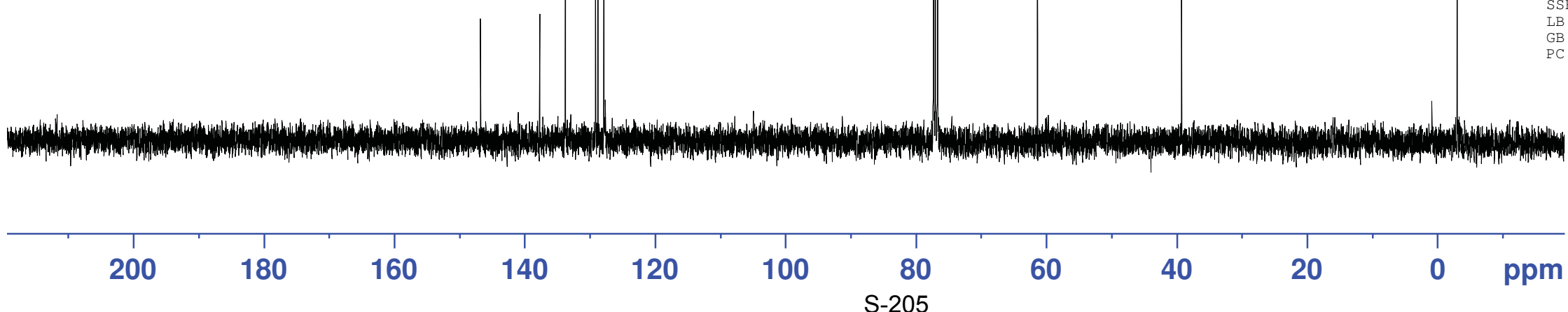
$0 \quad 1.00 \mathrm{~Hz}$ 


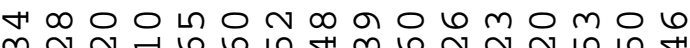
ก $N$ ก

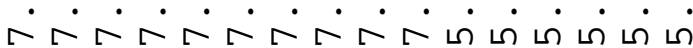
$\longrightarrow 1$

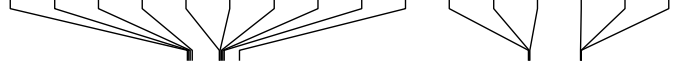

$2 f$

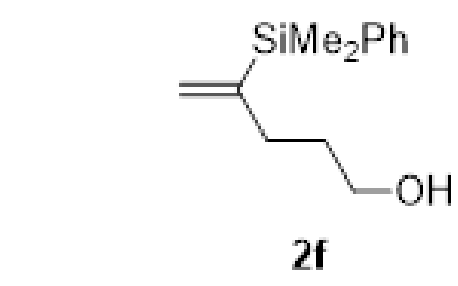

$\infty$ क ศิ N N N $\sim-r-r-r$
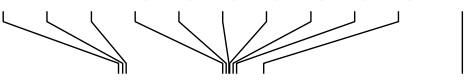

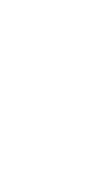

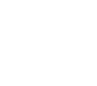

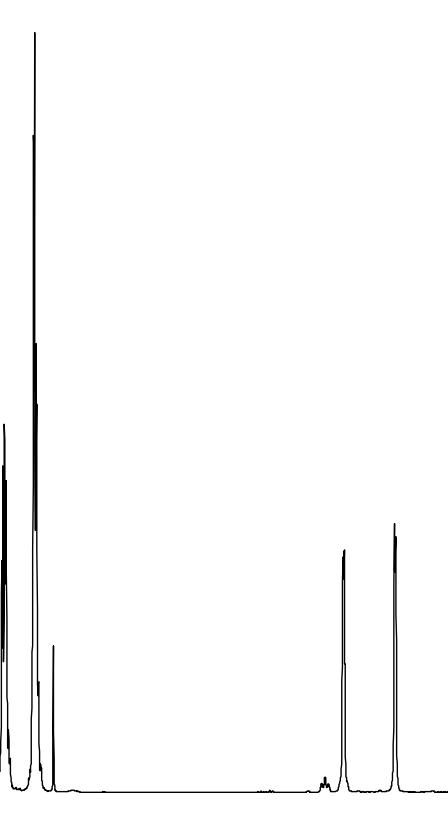

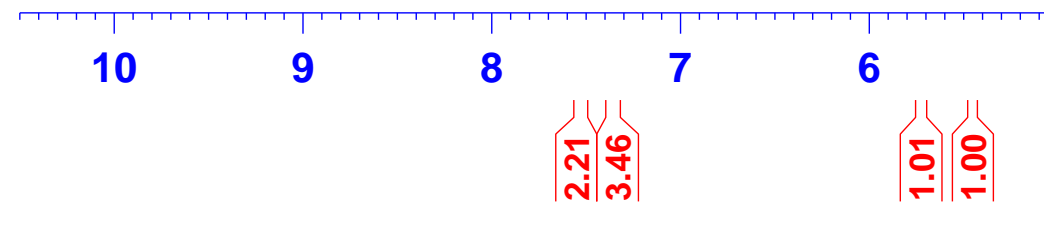

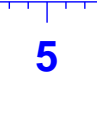



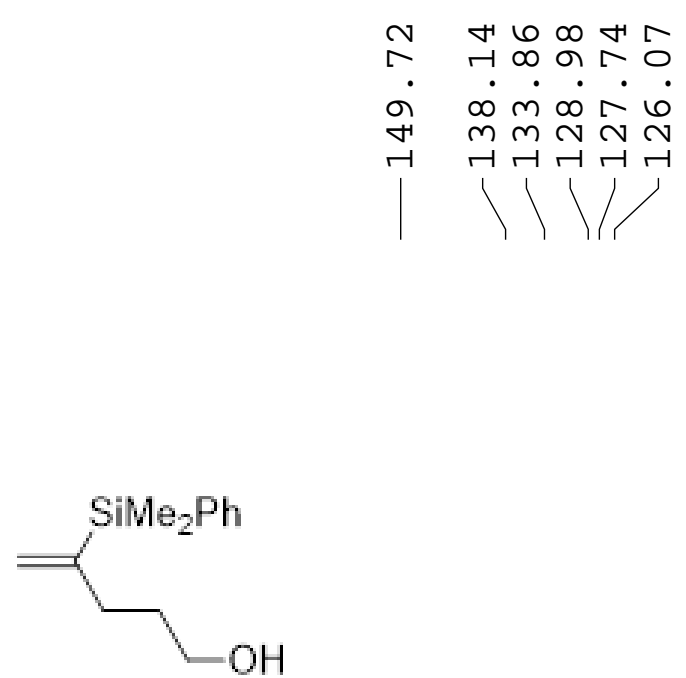

$2 f$
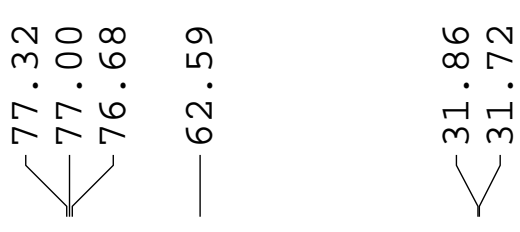

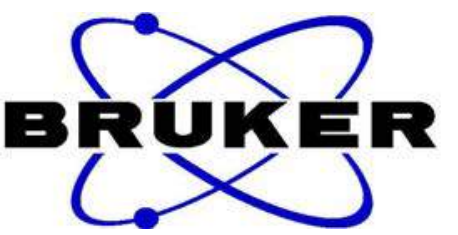

NAME PROCNO

Date

Time

INSTRUM

PROBHD

PULPROG

TD

SOLVENT

NS

DS

FIDRES

AQ

DW

DE

D1

d11

DELIA

(1D)

NUC1

P1
PL1

PL1
SFO1

$======$

CPDPRG

NUC2

PCPD

PL12

PL13

PL2

$\mathrm{SFO} 2$

SI

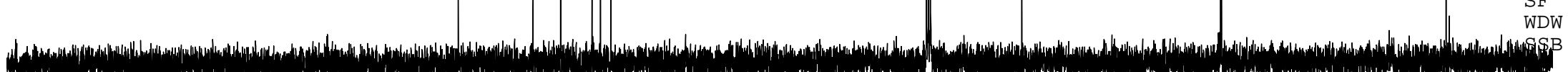

$\mathrm{PC}$
ZT-1049

20171014

13.33

mm $P A B B O$

$\mathrm{zgpg} 30$
65536

$\mathrm{CDCl} 3$

2
$24038.461 \mathrm{~Hz}$
$0.366798 \mathrm{~Hz}$

1. $3631988 \mathrm{sec}$$$
\begin{array}{r}
2050 \\
2058
\end{array}
$$

20.800 usec

6.00 usec

673.2

$2.00000000 \mathrm{sec}$

$0.03000000 \mathrm{sec}$

$1.89999998 \mathrm{sec}$

CHANNEL $\mathrm{f} 1========$
$13 \mathrm{C}$

9.25 us

$100.6228298 \mathrm{MHz}$

CHANNEL $\mathrm{f} 2=======$ waltz16

$1 \mathrm{H}$

80.00 use

$12.45 \mathrm{~dB}$

$18.00 \mathrm{~dB}$

$-1.00 \mathrm{~dB}$

$400.1316005 \mathrm{MHz}$

32768

$100.6127744 \mathrm{MHz}$ EM

0
$1.00 \mathrm{~Hz}$

1.00

1.40 


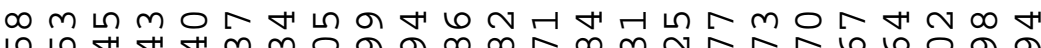
ம

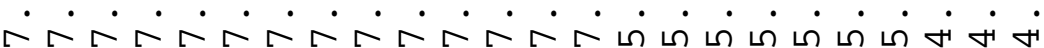

ㄴ.<smiles>C=C(COC(C)=O)Cc1ccccc1</smiles>

$2 \mathrm{~g}$

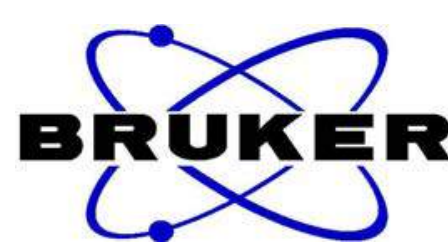

NAME

EXPNO

PROCNO

Date-

Time

INSTRUM

PROBHD

PULPROG

TD

SOLVENT

NS

SWH

FIDRES

AQ

RG

DE

TE

D1

$20140710^{1}$

10.55

$5 \mathrm{~mm}$ DUL $13 \mathrm{C}-1$

$13 \mathrm{C}-1$

$\mathrm{zg} 30$
65536

$\mathrm{CDCl} 3$

8
2

$8012.820 \mathrm{~Hz}$

$0.122266 \mathrm{~Hz}$

$4.0894966 \mathrm{sec}$ 34.77

62.400 usec 6.50 usec $295.8 \mathrm{~K}$

$1.00000000 \mathrm{sec}$

$=======$ CHANNEL $\mathrm{f} 1 \mathrm{l}======$

SFO1 $400.1324710 \mathrm{MHz}$

NUC1

P1

$\mathrm{SI}$
$\mathrm{SF}$
$\mathrm{WDW}$

$S S B$

LB
GB

PC

$1 \mathrm{H}$
14.30 usec $400.1300000 \mathrm{MH}$

EM

0
$0.30 \mathrm{~Hz}$

0.30
0
1.00

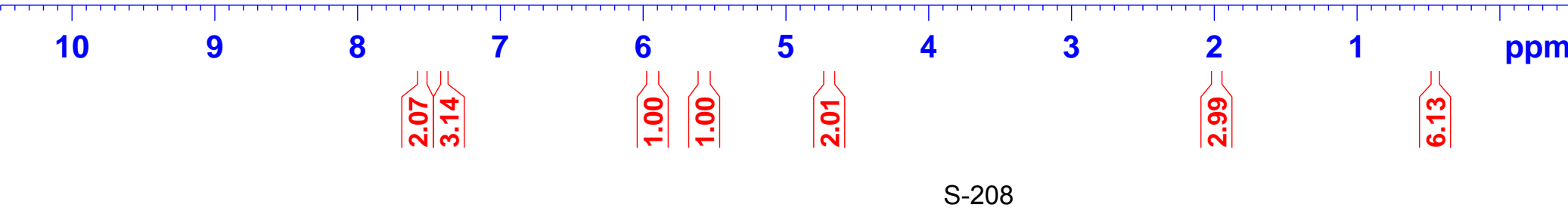



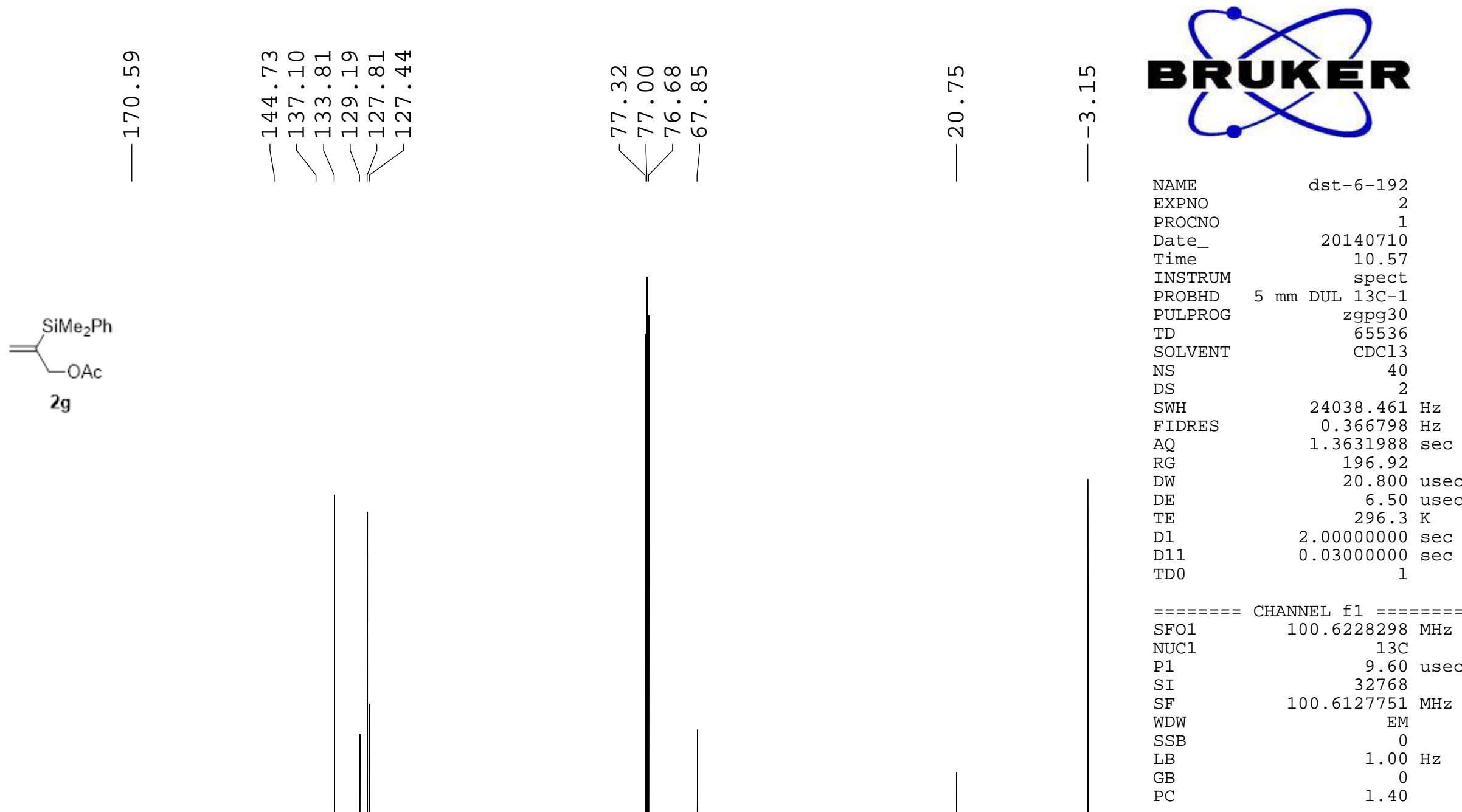

ex
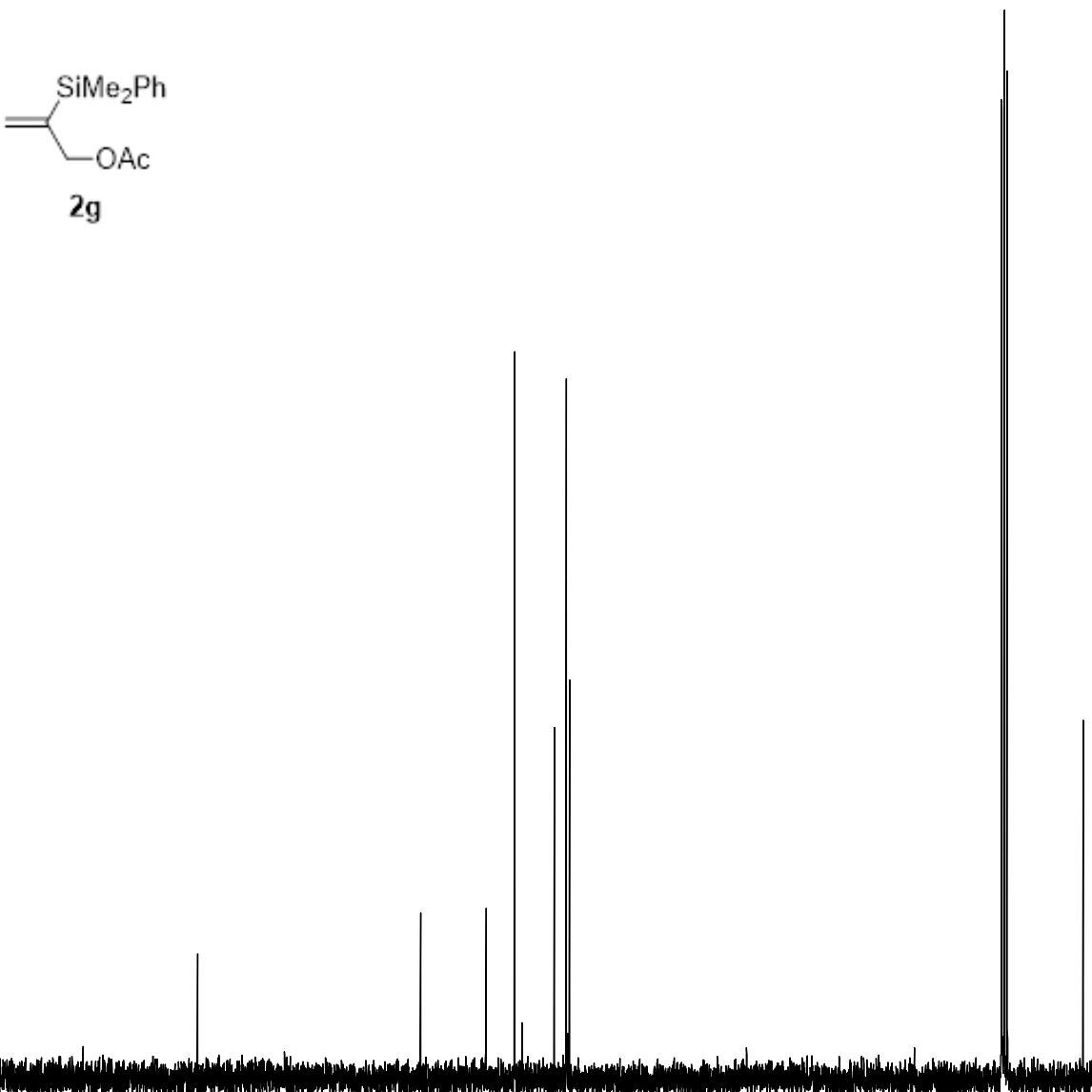

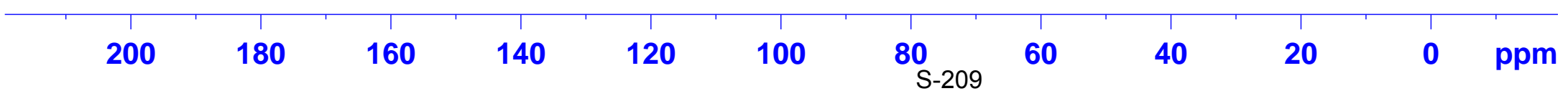




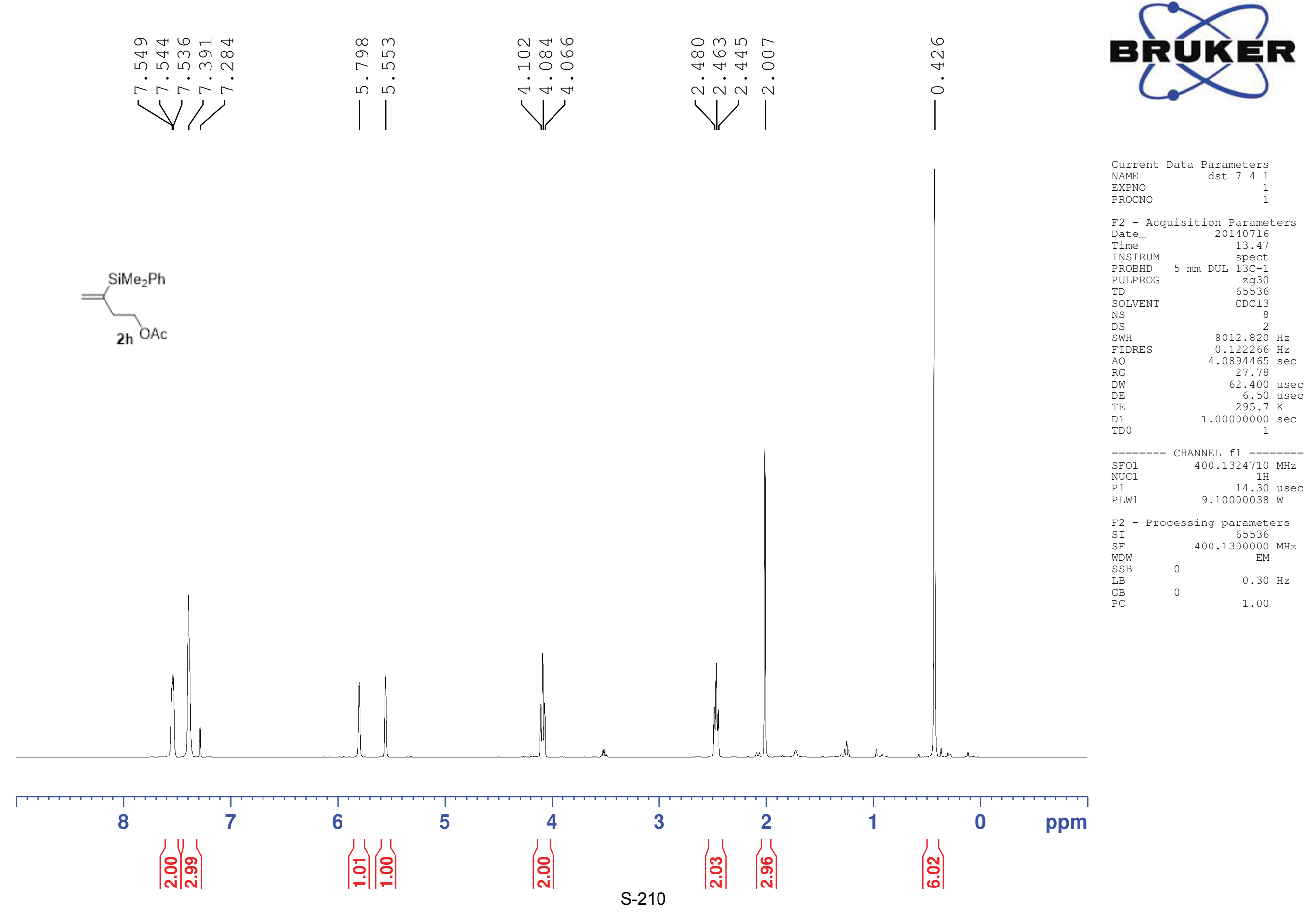




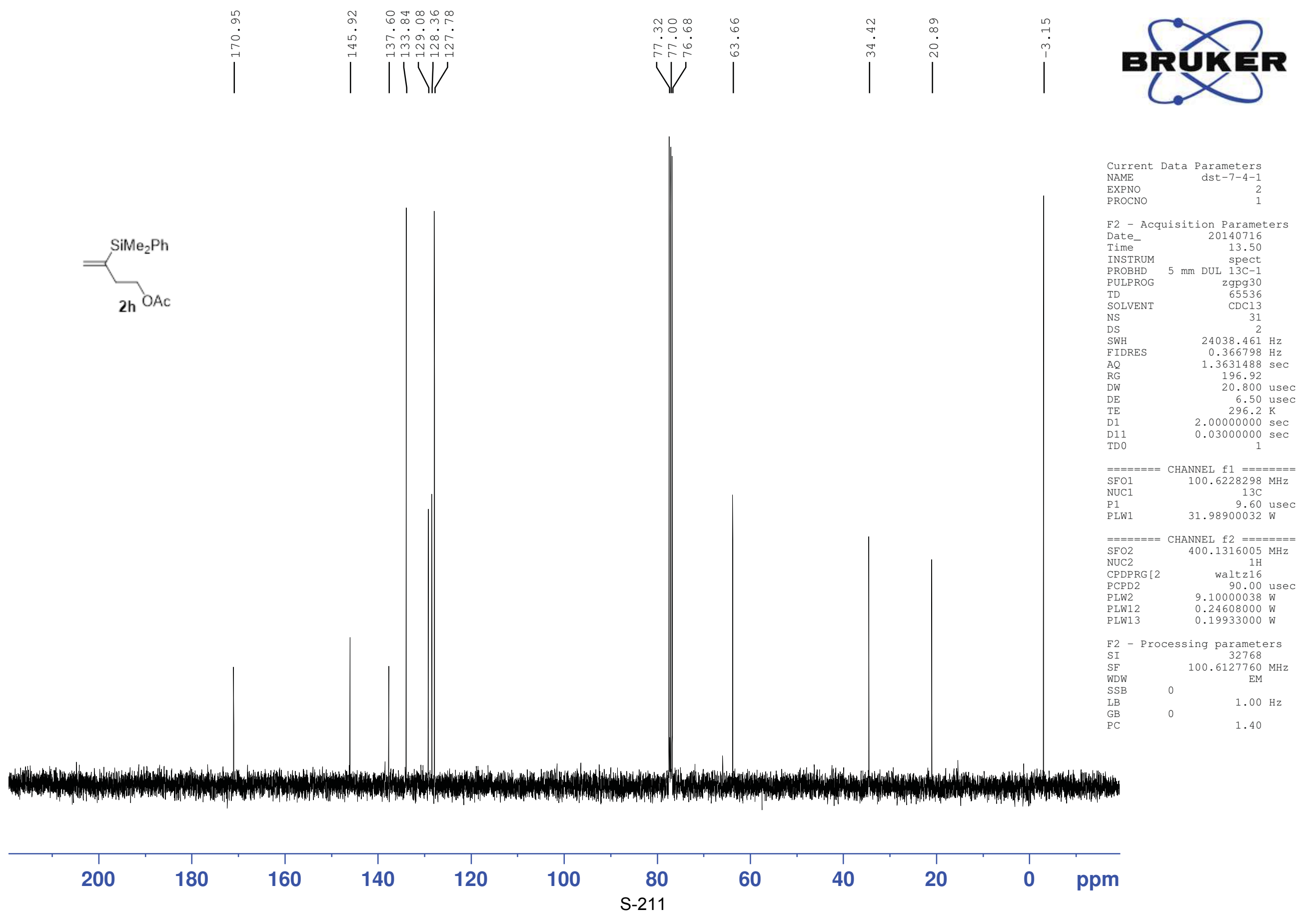




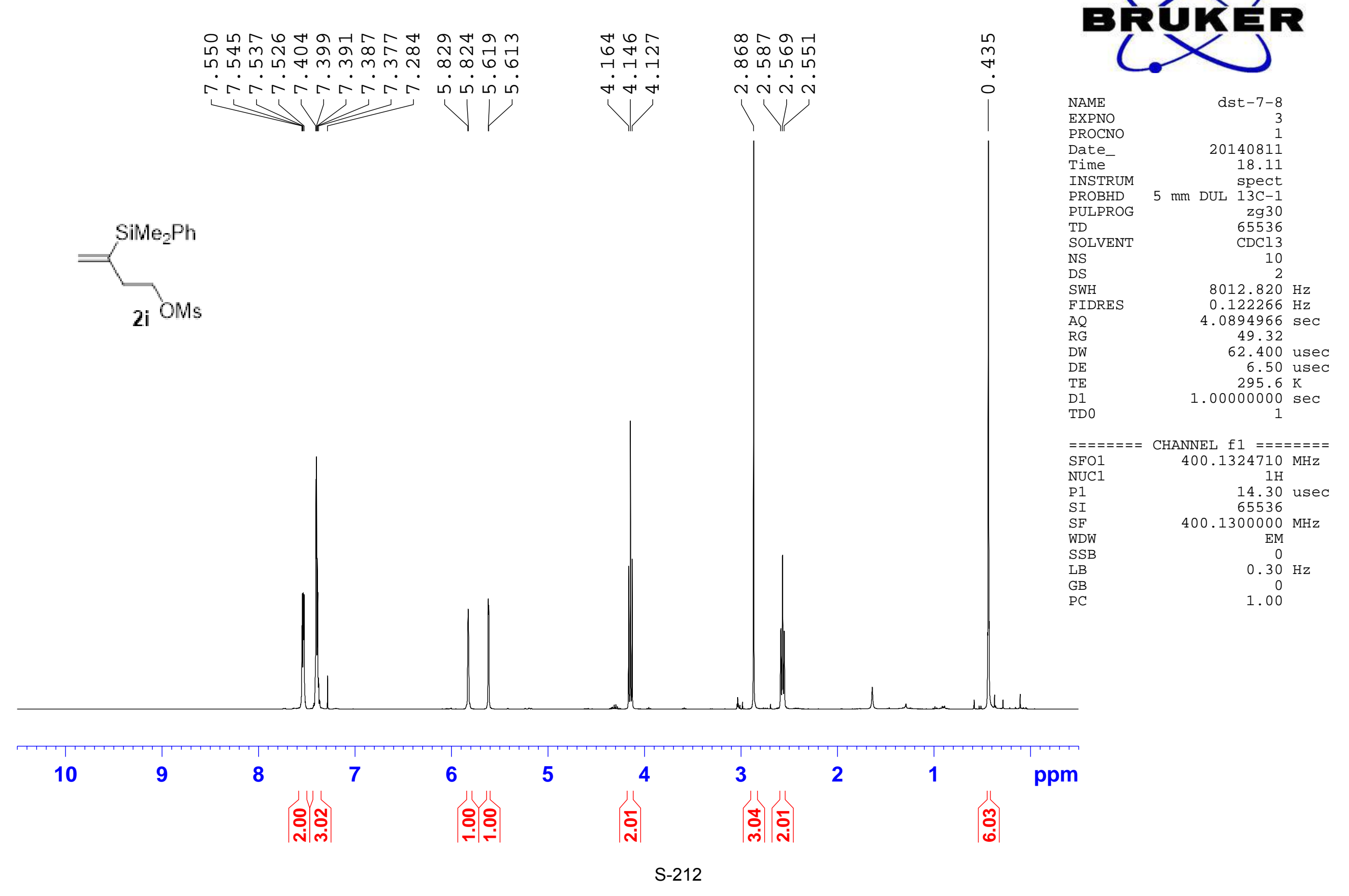



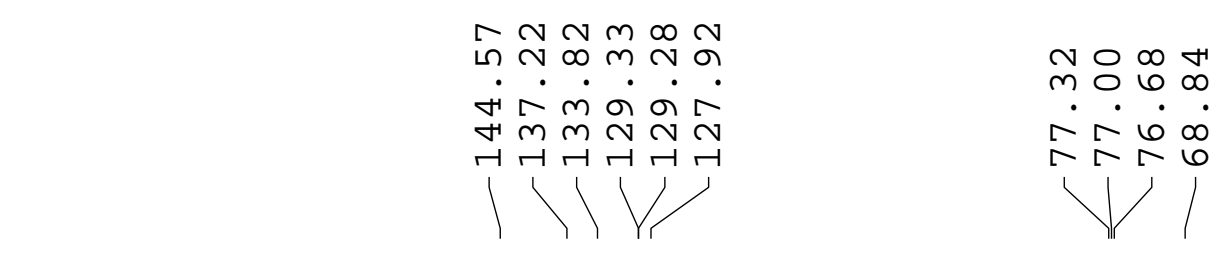

6

$\pi \infty m \sim$

m क

$\therefore \dot{4}$

11

$m \mathrm{~m}$
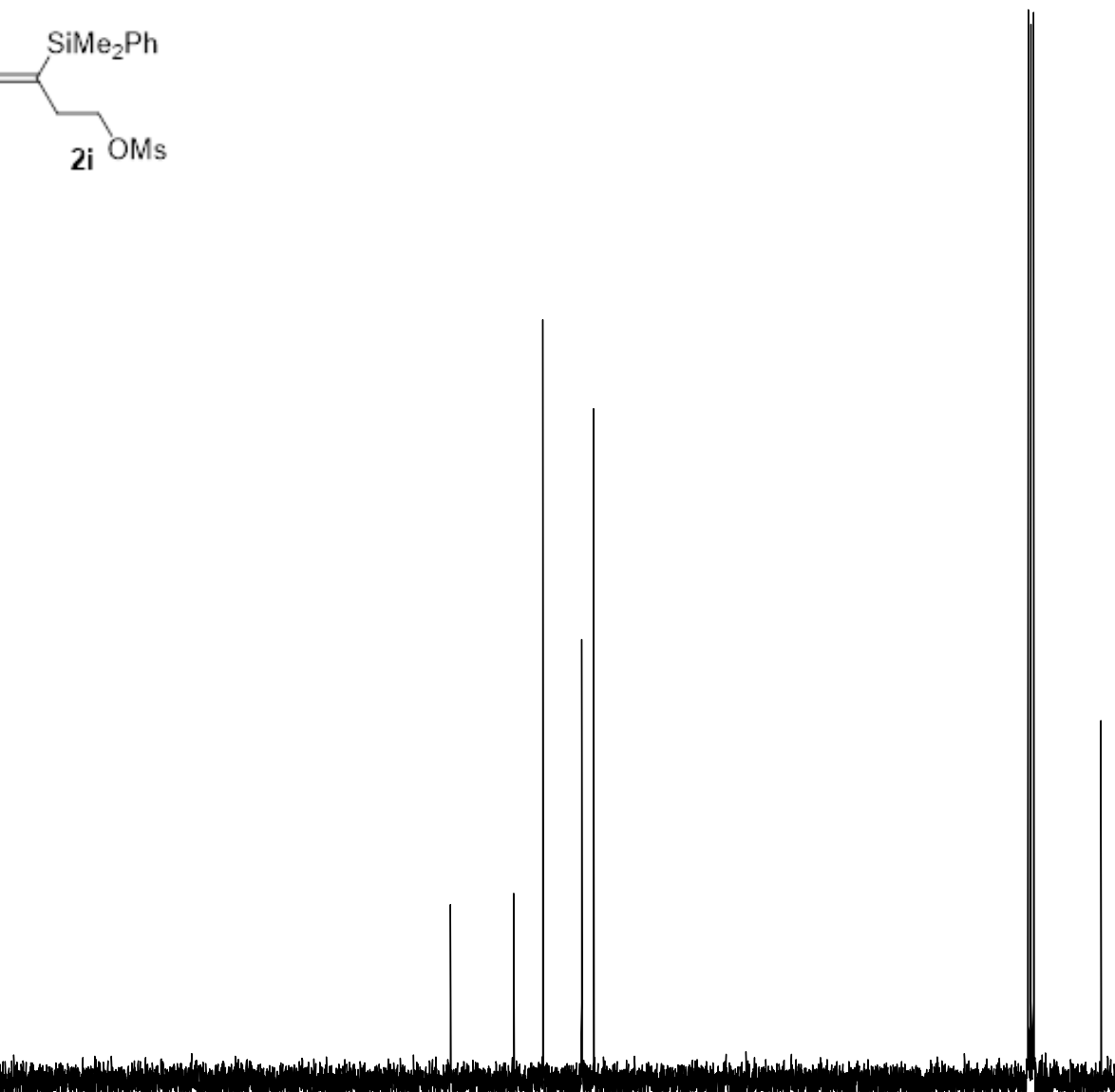

NAME

EXPNO

PROCNO

Date

20140811

INSTRUM

INRTRD

PULPROG

18.14

SOLVENT

NS
DS
SWH

SWH

AQ

RG

DW
DE
TE
D1

D1

D11

$5 \mathrm{~mm}$ DUL $13 \mathrm{C}-1$

$13 \mathrm{C}-1$
$\mathrm{zgpg} 30$

zgpg 30
65536

CDCl 3

$24038.461 \mathrm{~Hz}$

$0.366798 \mathrm{~Hz}$

1. $3631988 \mathrm{sec}$

196.92

20.800 usec

6.50 usec

$2.00000000 \mathrm{sec}$

$0.03000000 \mathrm{sec}$

SFO1 $100.6228298 \mathrm{MHZ}$

SFO1

P 1

SI
SF
WDW
SSB

$\mathrm{SSB}$

LB

GB
PC

$100.6228298 \mathrm{MHz}$
$13 \mathrm{C}$

9.60 usec

$100.6127768 \mathrm{MH}$

EM

0
$1.00 \mathrm{~Hz}$
0

0
1.40

80 


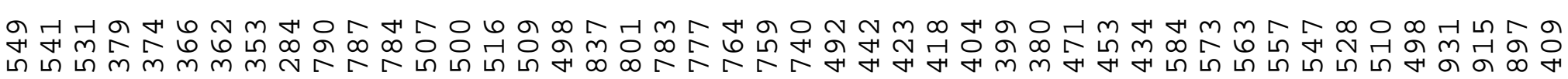

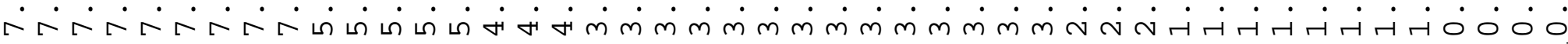

\section{BR

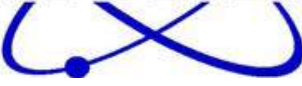

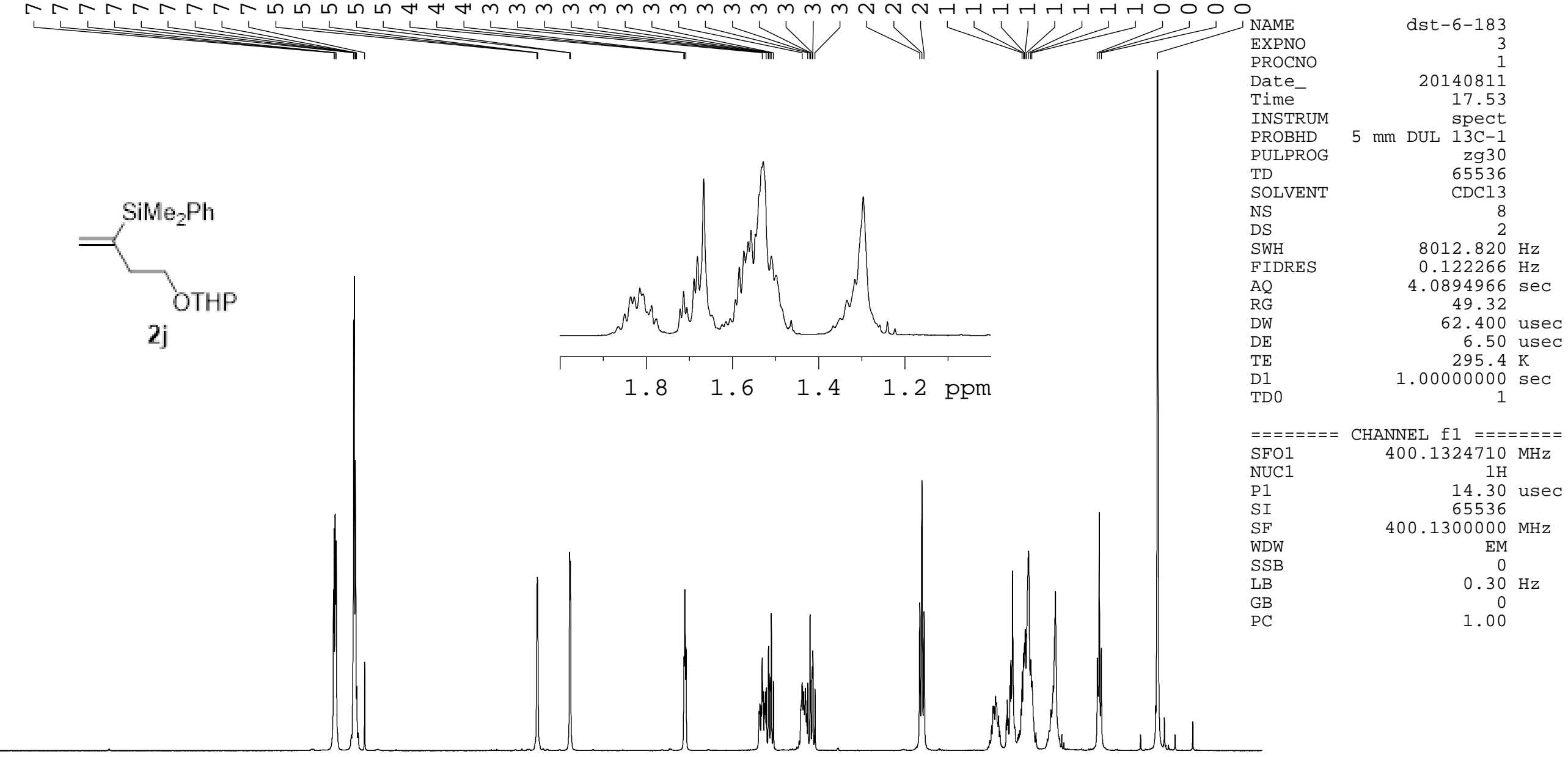

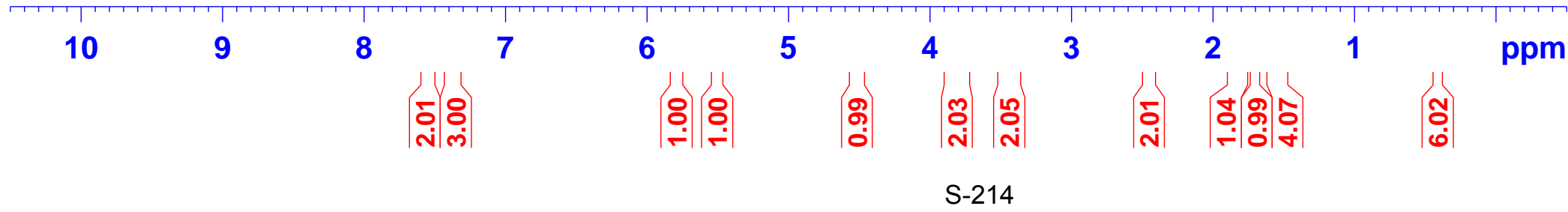




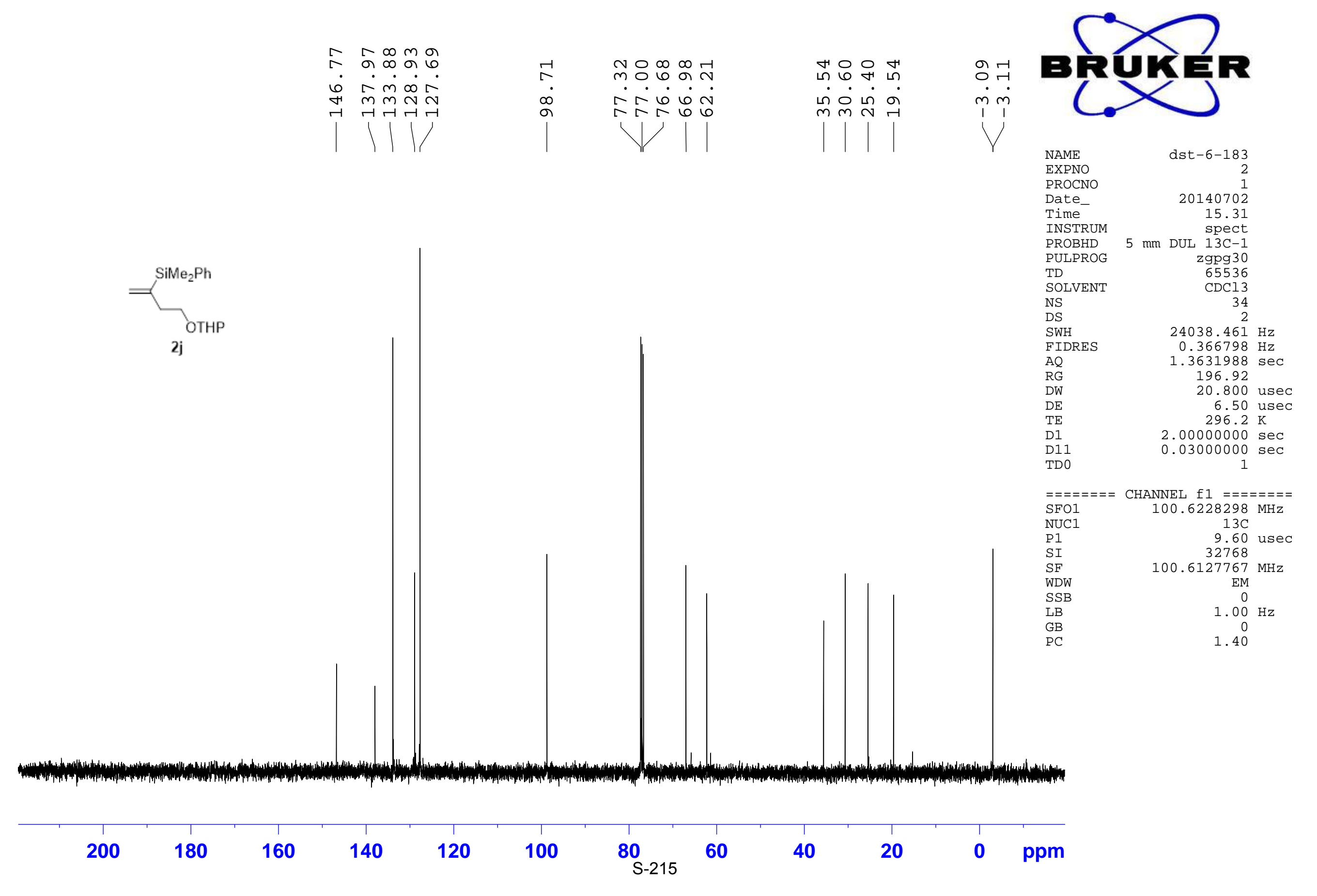




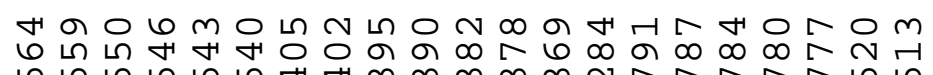
เ

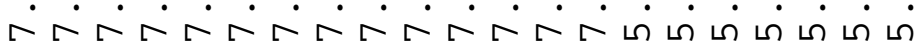
$\begin{array}{lll}\forall & 6 & 6 \\ 0 & \infty & 6\end{array}$ $\begin{array}{lll}0 & \infty & 0 \\ 6 & 1 & 0\end{array}$

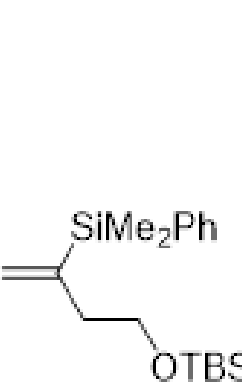

( )

$2 \mathrm{k}$

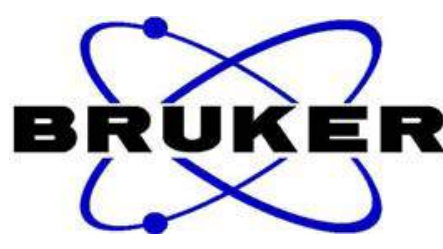

NAME

EXPNO

PROCNO

Date

Time

INSTRUM

PROBHD

PULPROG

TD

SOLVENT

NS

SWH

FIDRES $\quad 0.122266 \mathrm{~Hz}$

$\mathrm{AQ}$

RG

DE

TE

TD0

20140616 10.31

$5 \mathrm{~mm}$ DUL $13 \mathrm{C}-1$ 65536 CDC13

$8012.820 \mathrm{~Hz}$

$4.0894966 \mathrm{sec}$ 25.32

62.400 usec 6.50 usec $296.5 \mathrm{~K}$

$1.00000000 \mathrm{sec}$

SFO1

NUC1

P1

SF

WDW

SSB

LB

LB

$\mathrm{PC}$

$1 \mathrm{H}$
14.30 usec 400.1300000

(

EM

$0.30 \mathrm{~Hz}$

0.30
0

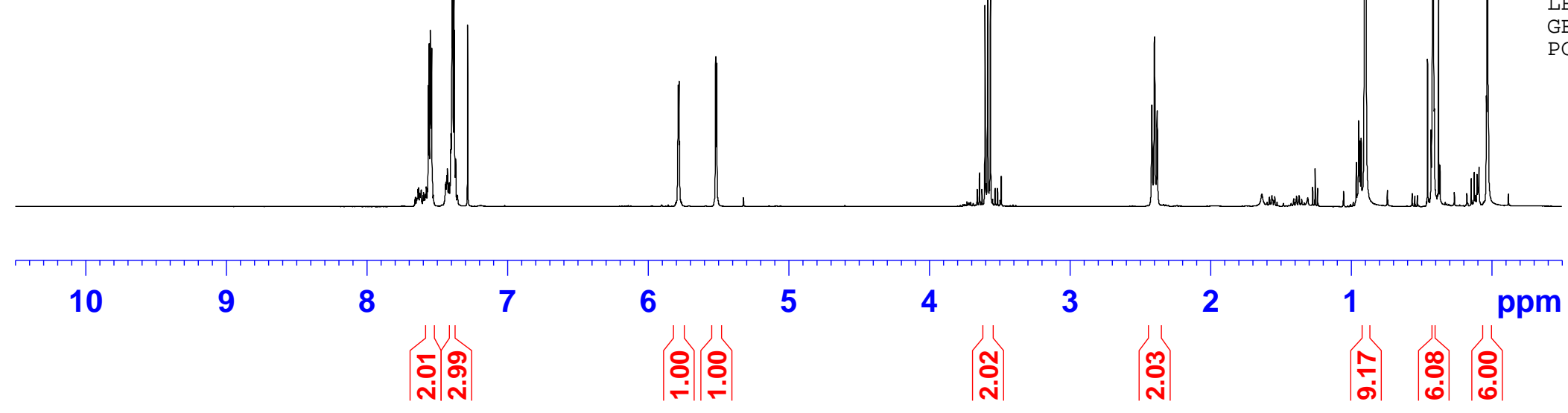

S-216 
மூ

$6 \infty m \infty r$

$\forall m m \sim N$

m 000

두 $\dot{0}$

$1 /$

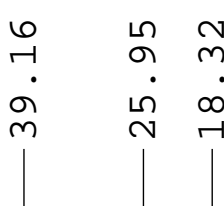

$\stackrel{\ln }{\circ}$

$\dot{m} \dot{1}$

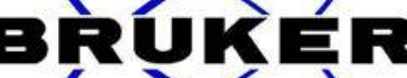

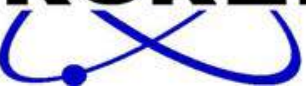

NAME

EXPNO

Date

Time

dst $-6-173$

PROBHD

PULPROG

20140616

10.34

10.34

TD

$\underbrace{\mathrm{SiMe}_{2} \mathrm{Ph}}_{\text {OTBS }}$

SOLVENT

NS

DWH

FIDRES

AQ

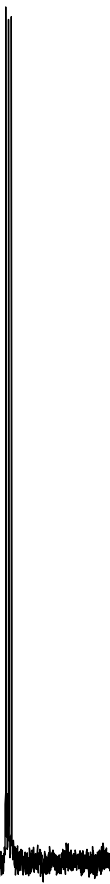

DW

$\mathrm{DE}$

TE

D1

D11

mm DUL 13C-1

zgpg 30
65536

$\mathrm{CDCl} 3$

$24038.461 \mathrm{~Hz}$

$0.366798 \mathrm{~Hz}$

$3631988 \mathrm{sec}$

196.92

20.800 usec

6.50 usec

CHANNET 11

SFO1 $100.6228298 \mathrm{MHz}$

NUC1

NUC1

$100.6228298 \mathrm{MHz}$
$13 \mathrm{C}$

$100.6127731 \mathrm{MHz}$

WDW

SSB

LB
GB

EM

$1.00 \mathrm{~Hz}$

1.00
0

1.40 
の

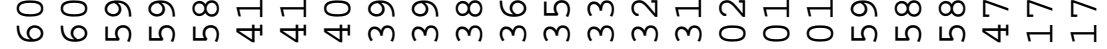

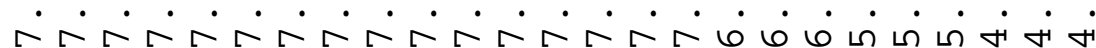
L

i $\underset{a}{a} \mathrm{a}$ $\dot{0} \dot{0}$
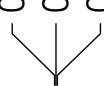<smiles>C=C(COc1ccccc1)[SiH2]c1ccccc1</smiles>

2I
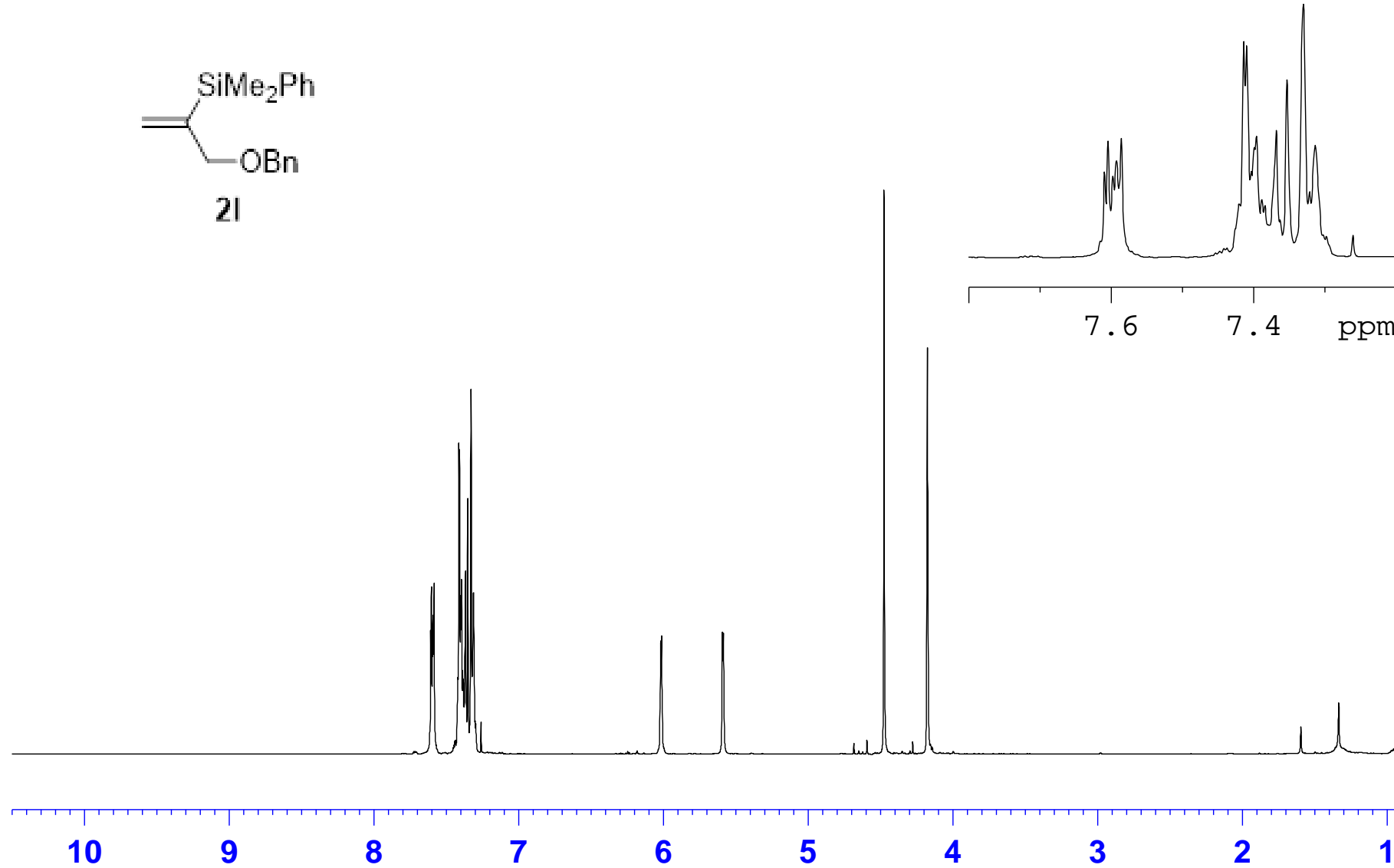

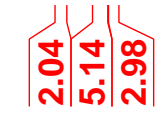

웅
5

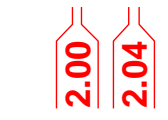

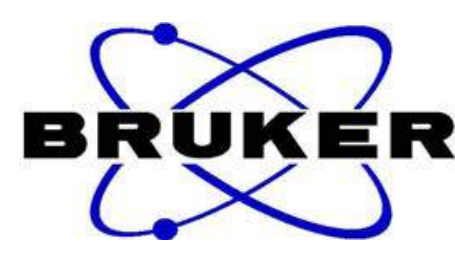

NAME

EXPNO

PROCNO

Date_-

INIme

PROBHD

PULPROG

TD

SOLVENT

NS

SWH

FIDRES

AQ
RG
DW

DE

TE

D1 0

ZT-1023

20170920

19.19

$5 \mathrm{~mm}$ PABBO $\mathrm{BB}-$

zg30
65536

CDCl3

16
2

$8223.685 \mathrm{~Hz}$

$0.125483 \mathrm{~Hz}$

$3.9846387 \mathrm{sec}$

60.800 usec 6.00 usec $1.00000000 \mathrm{sec}$

$========$ CHANNEL f 1 =======
NUC1 $1 \mathrm{H}$

NUC1 13.60 usec P1

SFO1

$\mathrm{SI}$

SF

WDW

SSB

LB
GB

PC

$-1.00 \mathrm{~dB}$

$400.1324710 \mathrm{MHz}$

32768

$400.1300099 \mathrm{MHz}$

EM

0
$0.30 \mathrm{~Hz}$

0.30
0
1.00

1.00 


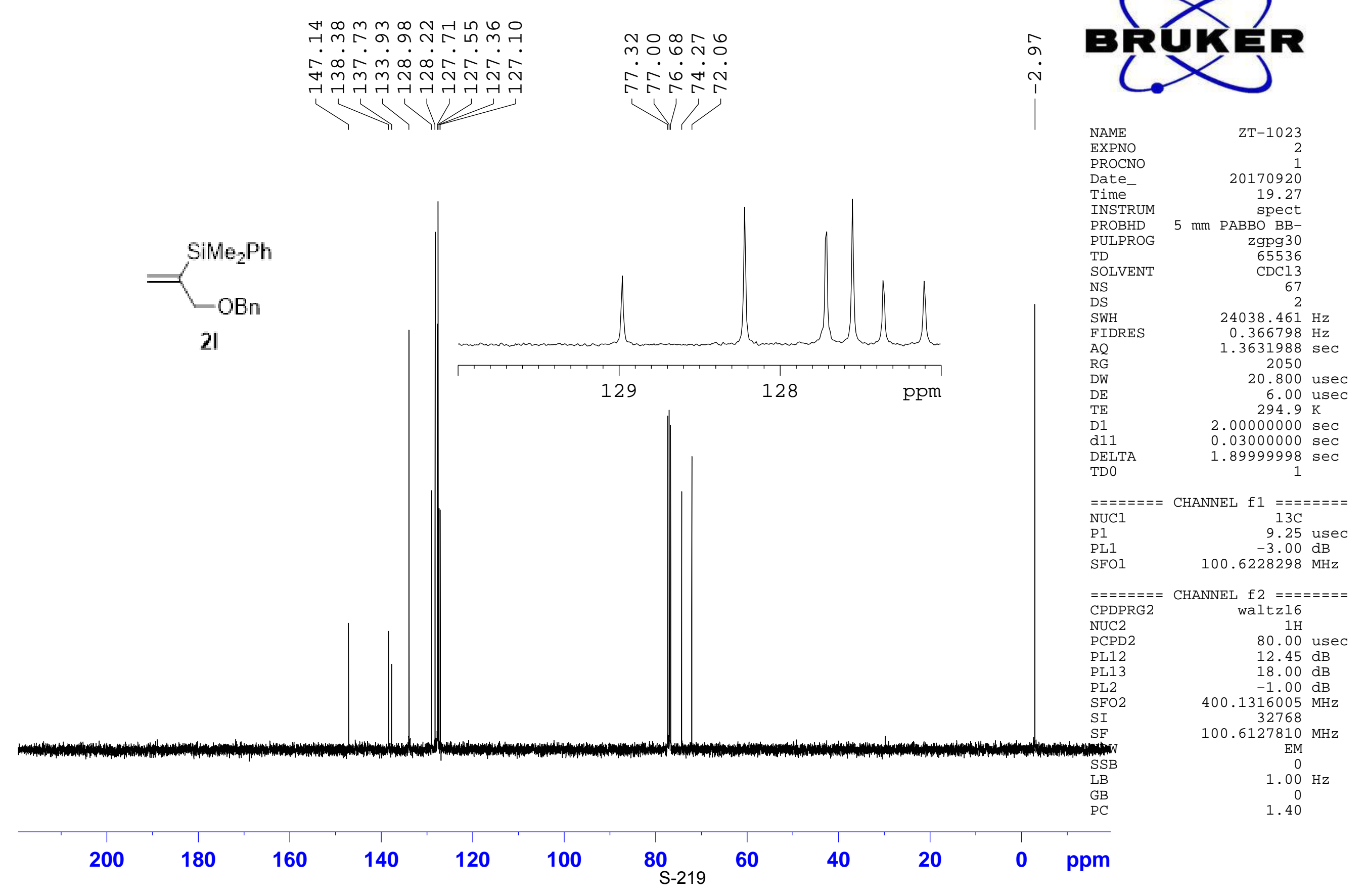


0 ம

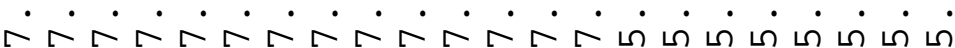

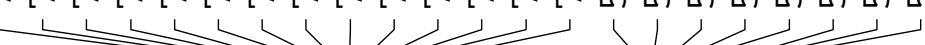

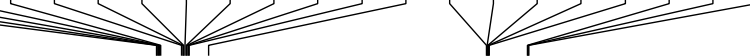

$2 \mathrm{~m}$

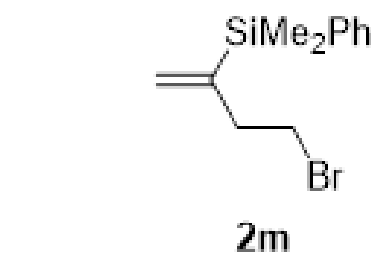

$06 \sim \sim N$ $m m \sim N o$

$\dot{m} \dot{m} \dot{\sim} \dot{\sim}$

111
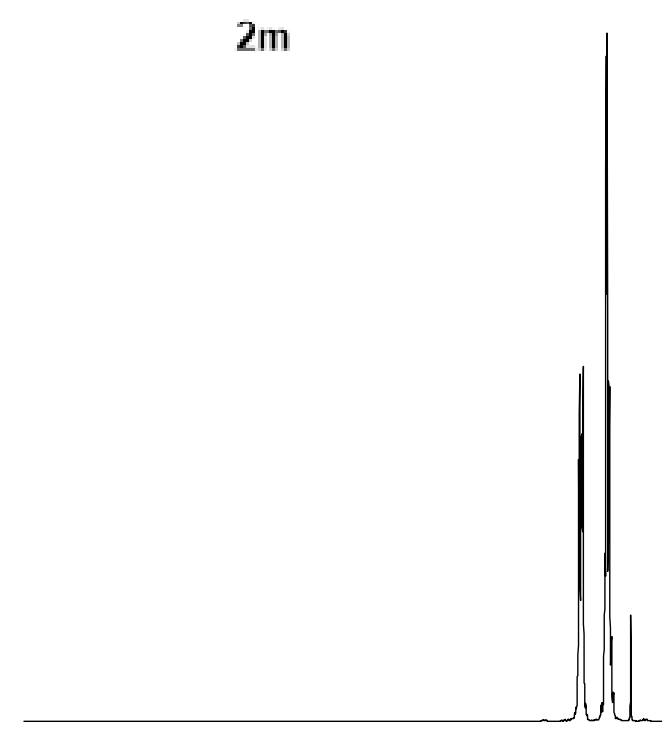

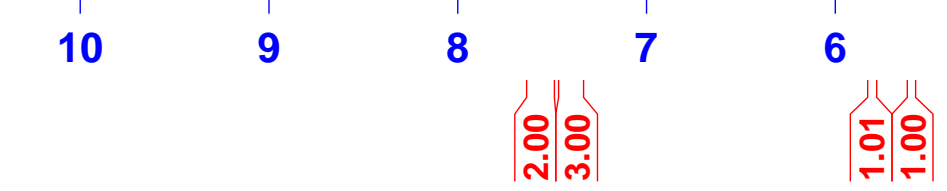

5

5

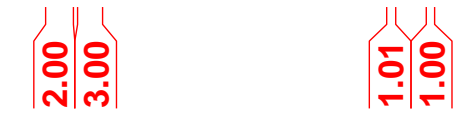

4
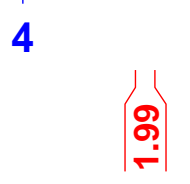

3

(ิ)

2

|

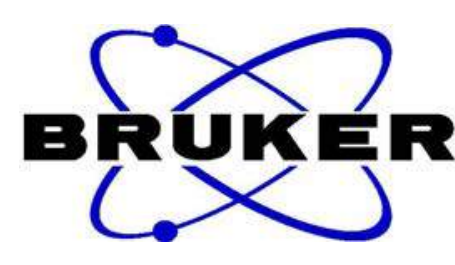

NAME

EXPNO

PROCNO

Date_

Time

INSTRUM

PROBHD

PULPROG

TD

SOLVENT

NS

SWH

FIDRES

FID
AQ
RG
DW

DE

TE

D1

dst-7-11-2

20140719

14.57

$5 \mathrm{~mm}$ DUL $13 \mathrm{C}-1$

zg30
65536

$\mathrm{CDCl} 3$

8
2

$8012.820 \mathrm{~Hz}$

$0.122266 \mathrm{~Hz}$

$4.0894966 \mathrm{sec}$ 31.55

62.400 usec 6.50 usec

$1.00000000 \mathrm{sec}$

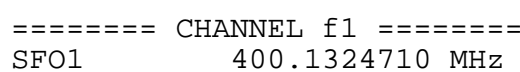

$\mathrm{SFO1}$

NUC1

SI

SF

WDW

SSB

LB
GB

PC

$1 \mathrm{H}$
14.30 usec

$400.1300000 \mathrm{MHz}$

EM

$0.30 \mathrm{~Hz}$

0
1.00 


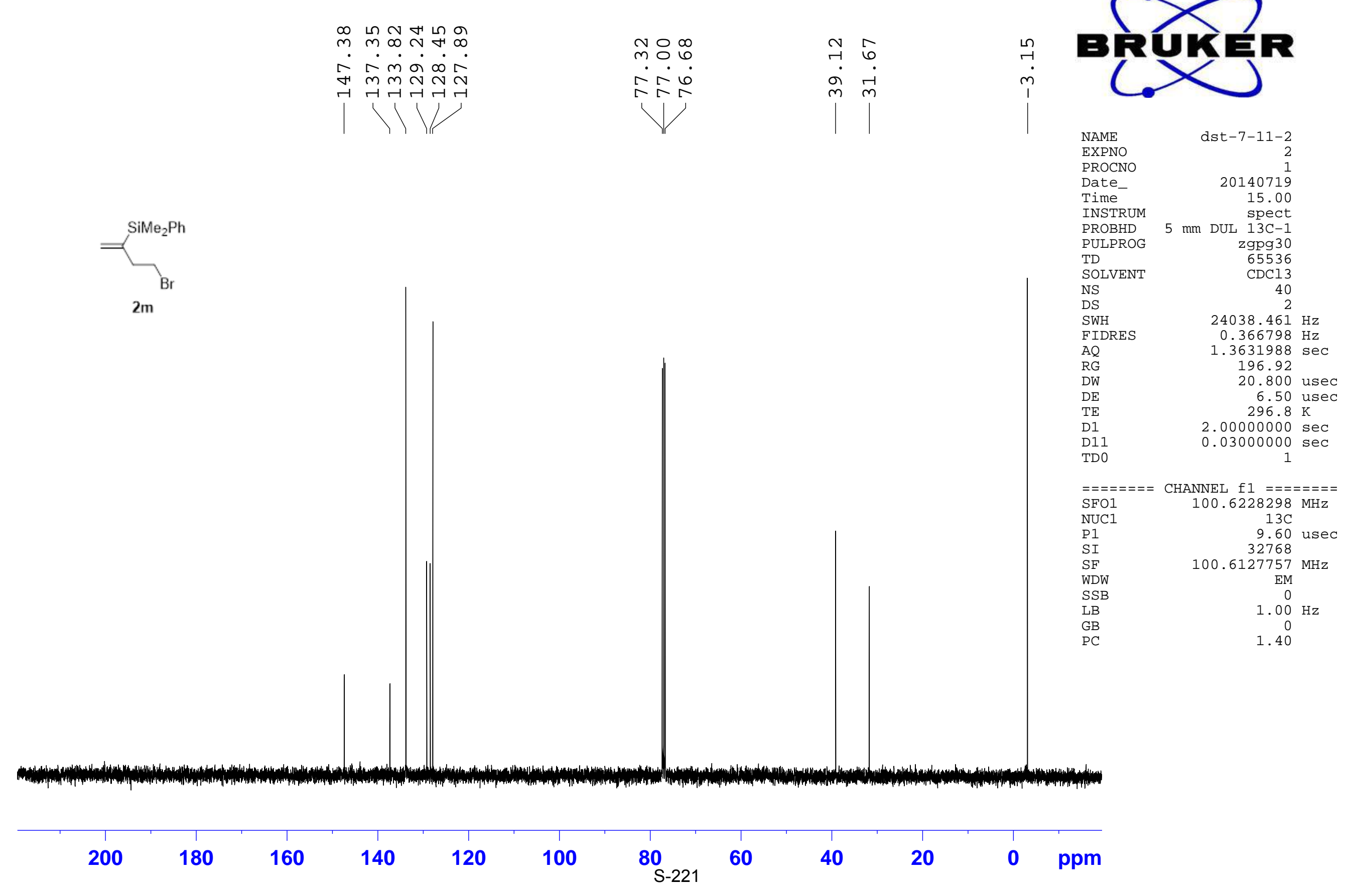


엤 $m$ न

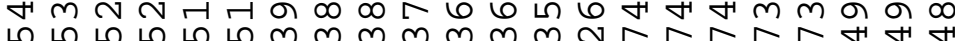

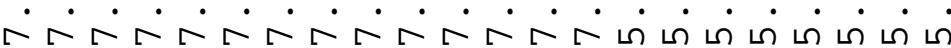
nul

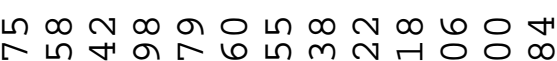
ศ

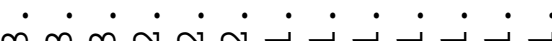

mmm N N

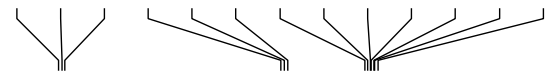

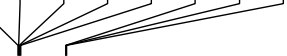

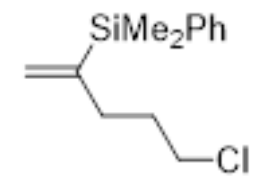

$2 n$
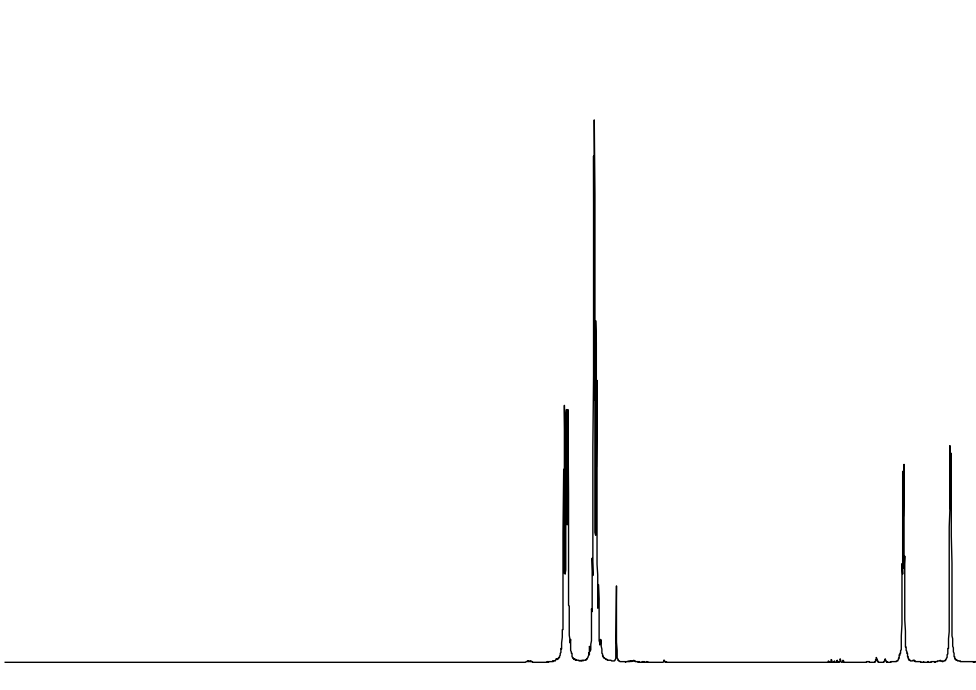

10

9

8

7

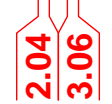

6

5

4

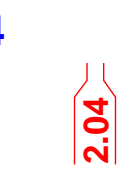

3

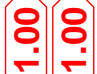

$\left|\begin{array}{c}\mathbf{+} \\ \mathbf{N}\end{array}\right|$

2

$\left|\begin{array}{cc}\infty & \\ 0 \\ \text { Ni }\end{array}\right| \begin{aligned} & 0 \\ & 0 \\ & \text { Ni }\end{aligned} \mid$

1

ppm

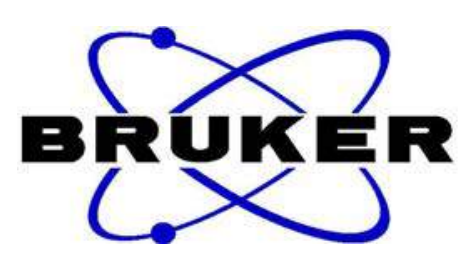

NAME

EXPNO
PROCNO

Date_

INIme

PROBHD

PULPROG

TD

SOLVENT

NS

SWH

FIDRES

AQ
RG

RG

DW

DE

$\mathrm{TE}$
$\mathrm{D} 1$
$\mathrm{TD} 0$

ZT-1032-0929

20170929

22.41

$5 \mathrm{~mm}$ PABBO $\mathrm{BB}-$

zg30
65536

$\mathrm{CDCl} 3$

9
2

$8223.685 \mathrm{~Hz}$

$0.125483 \mathrm{~Hz}$

$3.9846387 \mathrm{sec}$

114

60.800 usec 6.00 usec

$1.00000000 \mathrm{sec}$

\begin{tabular}{|c|c|}
\hline $\begin{array}{l}\text { NUC1 } \\
\text { P1 }\end{array}$ & $1 \mathrm{H}$ \\
\hline PL1 & $\begin{array}{l}13.60 \\
-1.00\end{array}$ \\
\hline
\end{tabular}

P1

$\mathrm{SFO} 1$

$\mathrm{SI}$

SF

SSB

SSB

GB

$\mathrm{PC}$

$400.1324710 \mathrm{MHz}$

400.1300099

EM

0
$0.30 \mathrm{~Hz}$

0.30
0
1.00

0
1.00 


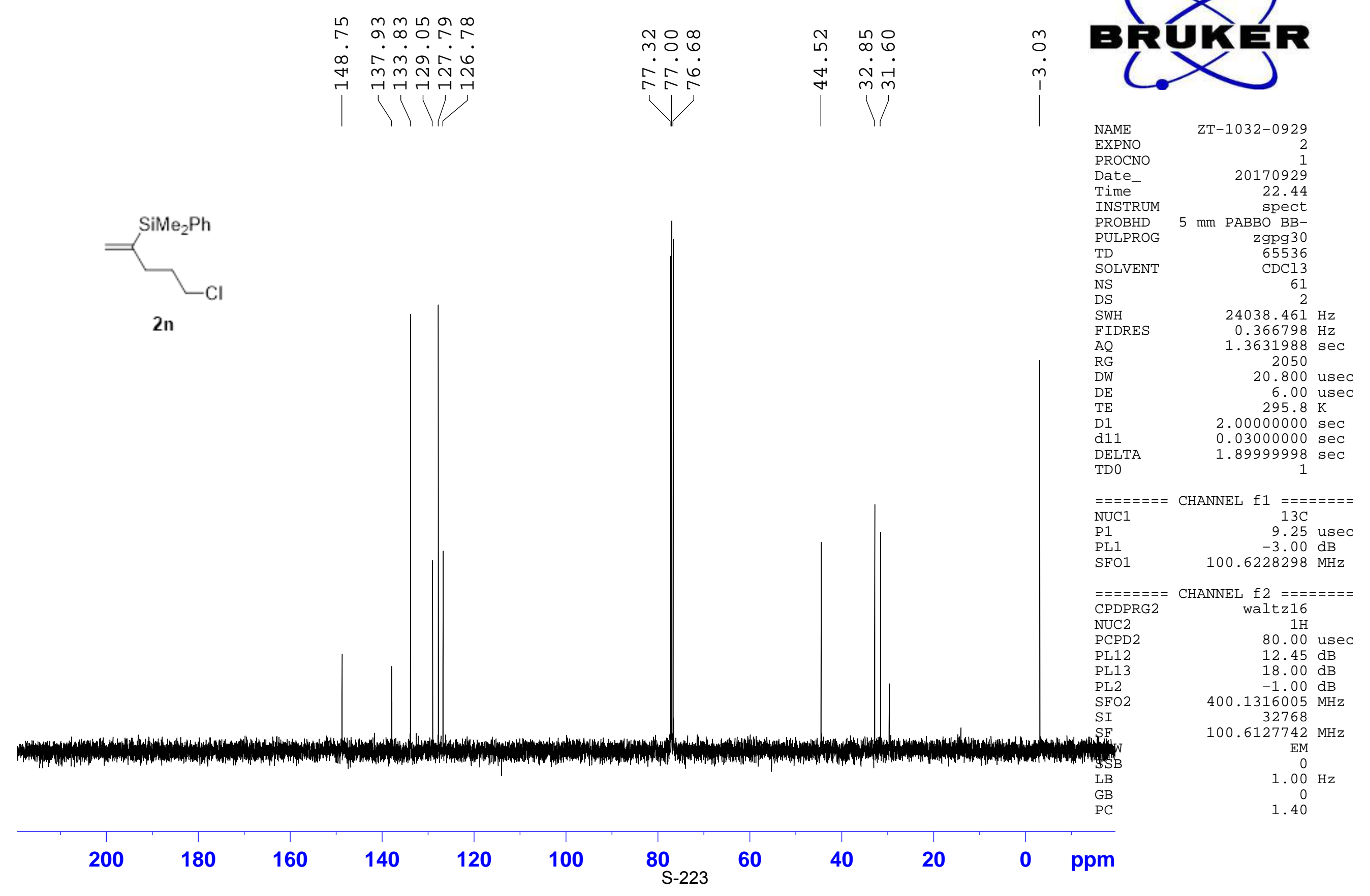




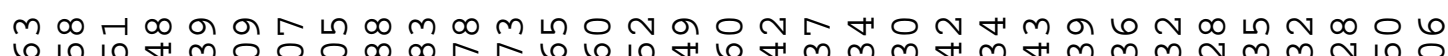

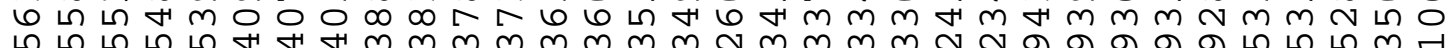

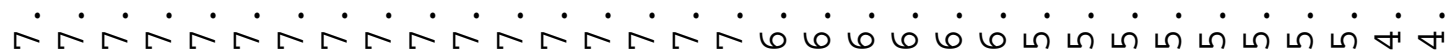

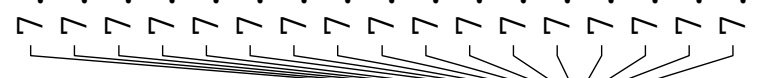
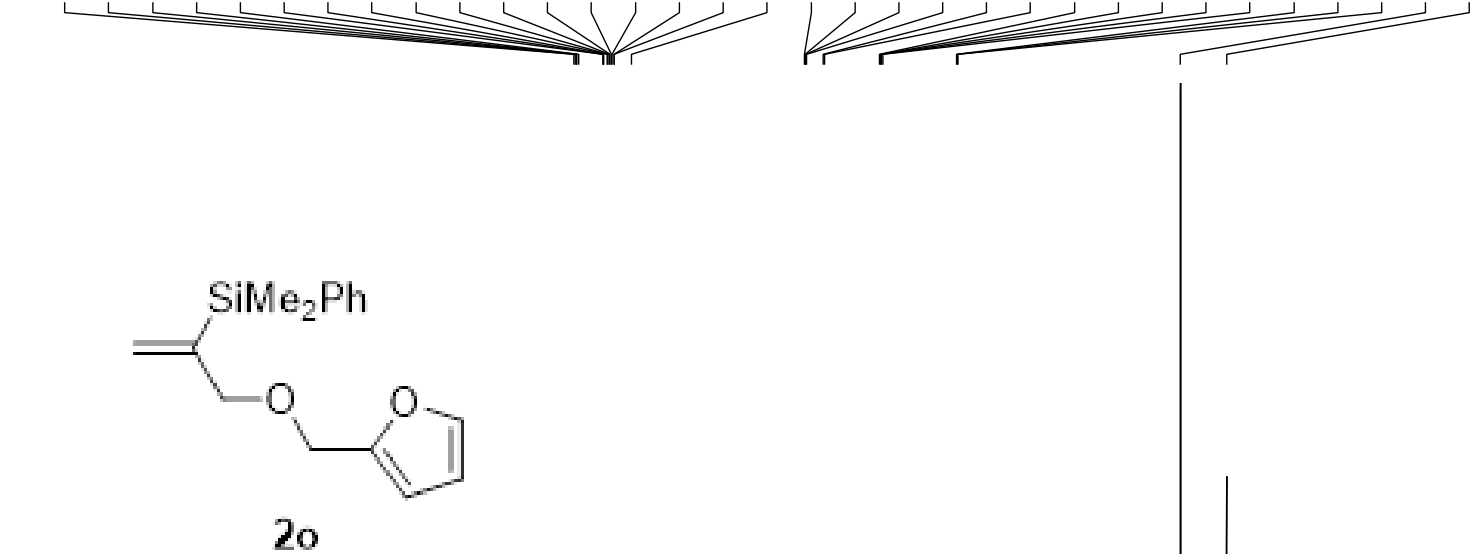

20
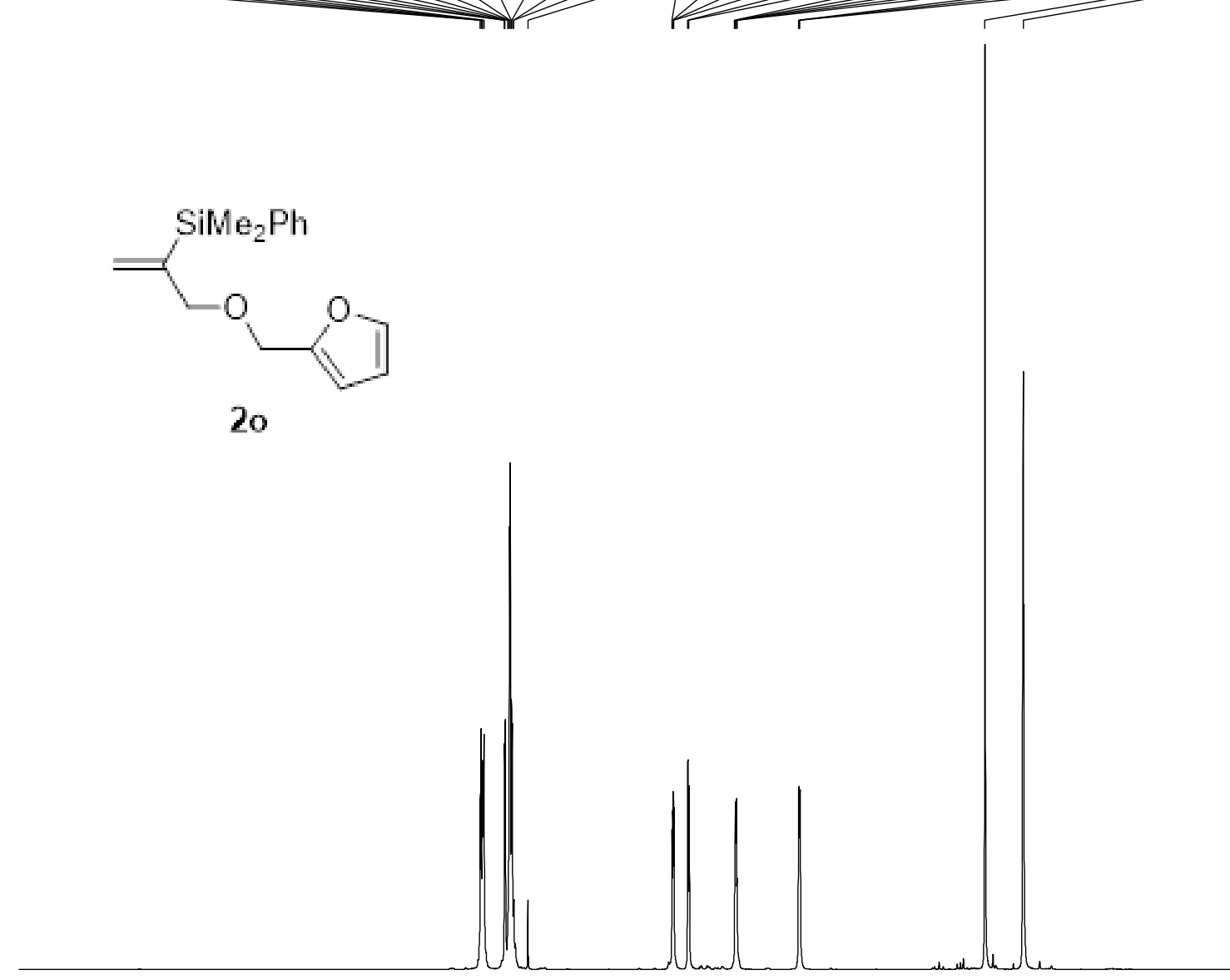
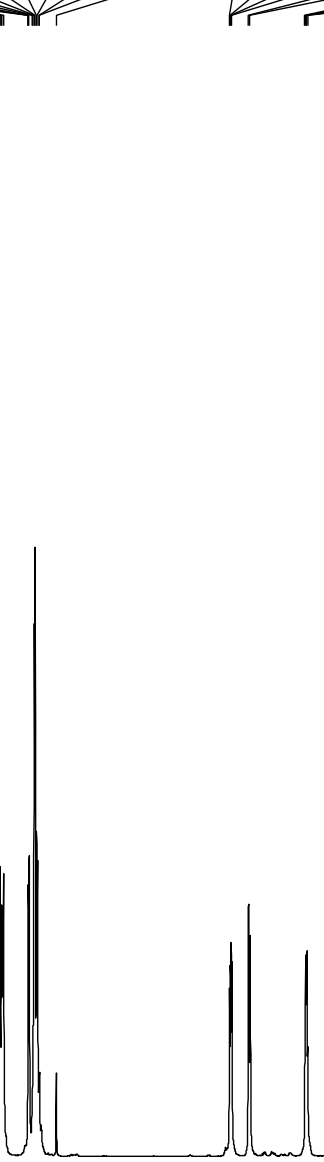

$10 \quad 9 \quad 8$

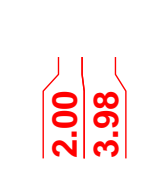

7

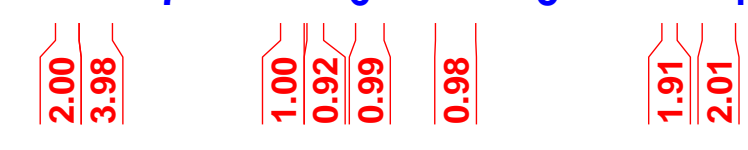

5

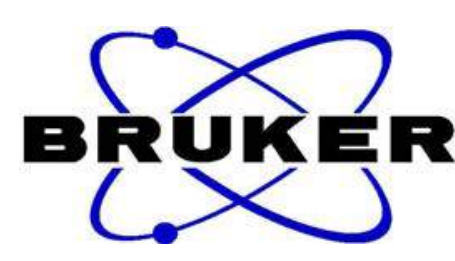

NAME
EXPNO

EXPNO
PROCNO

Date

Time-

INSTRUM

PROBHD

PULPROG

TD

SOLVENT

NS

SWH

FIDRES

$A Q$

RG

DE

TE

D1

$========$ CHANNEL $\mathrm{f} 1 \mathrm{l}========$

$\mathrm{SFO} 1$

NUC1

P 1

SI

WDW

SSB

LB

PC

$400.1324710 \mathrm{MHz}$

$1 \mathrm{H}$
14.30 usec 400.1300097

EM

$0.30 \mathrm{~Hz}$

0
1.00

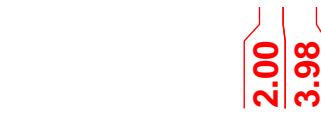

(1)




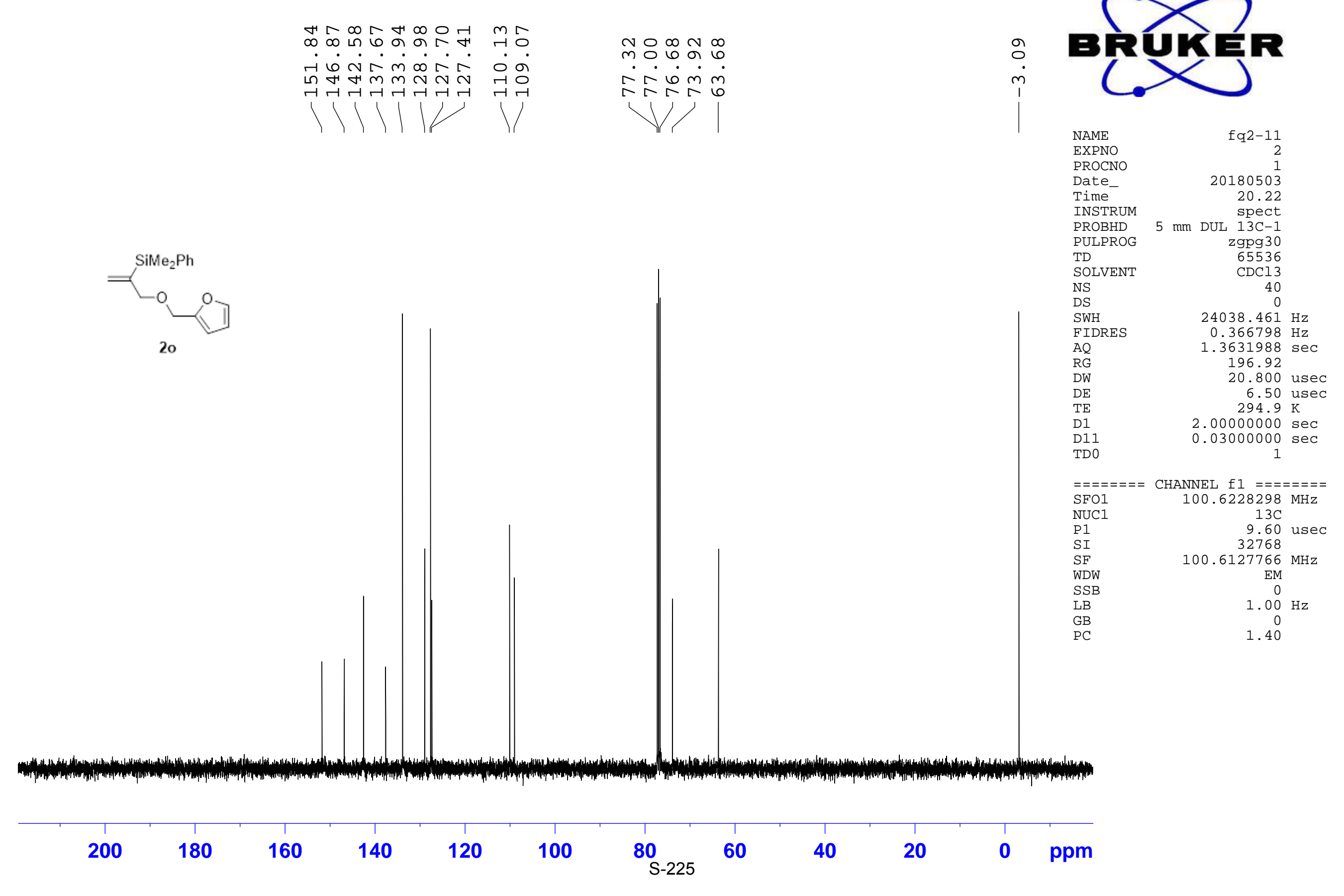




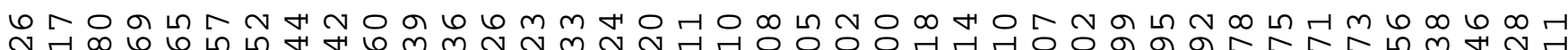
ग 대

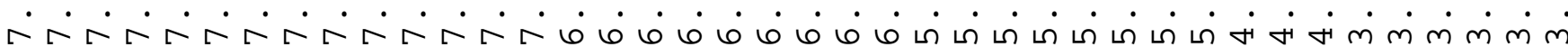

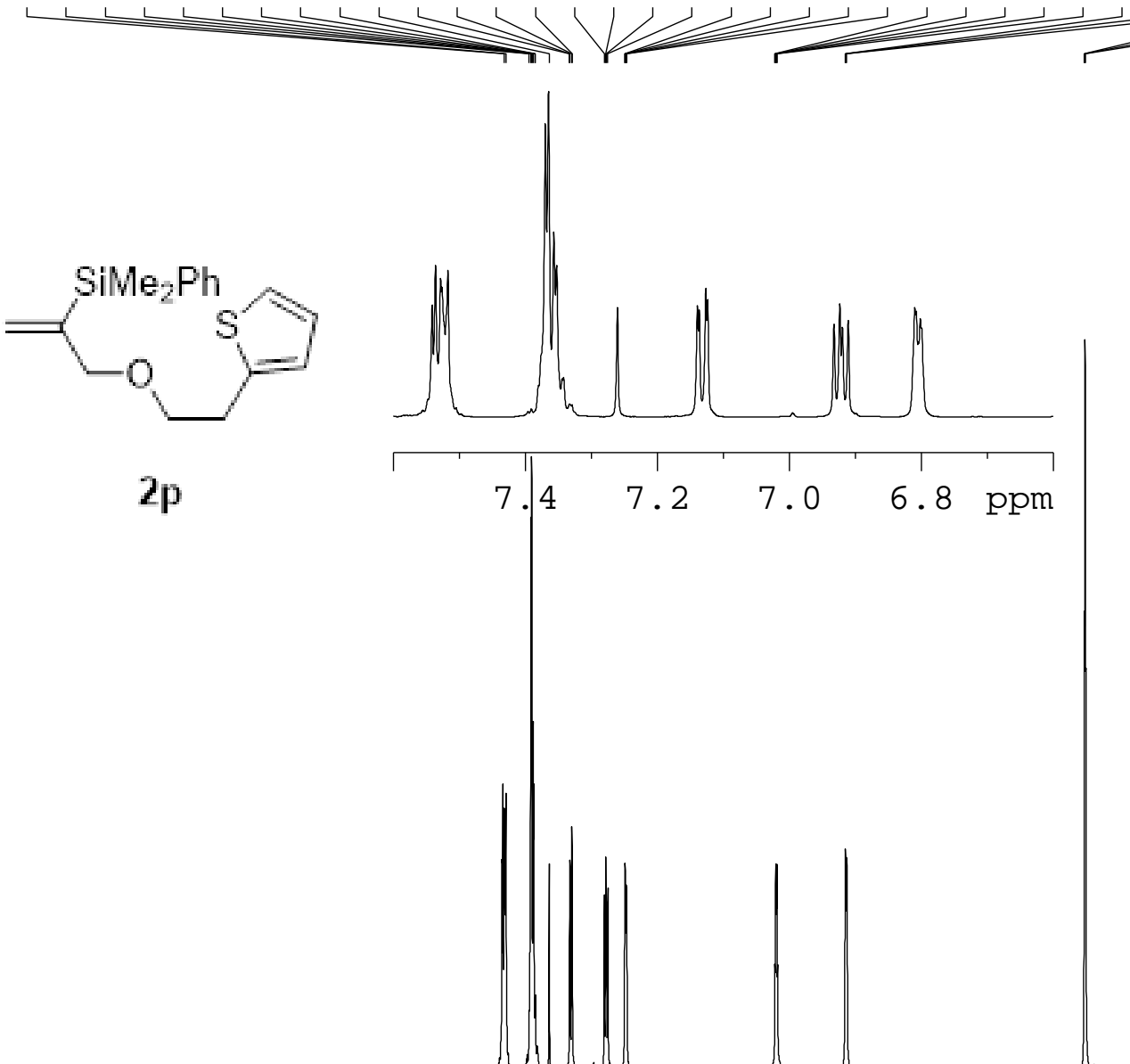

NAME

EXPNO

PROCNO

Date_

Time

INSTRUM

PROBHD

PULPROG

TD

SOLVENT

NS
DS

SWH

FIDRES

AQ

RG

DE

$\mathrm{DE}$
$\mathrm{TE}$
$\mathrm{D} 1$

TD0

$====$

$\mathrm{SFO1}$

PUC1

P1

SF

WDW

SSB

LB
GB

PC

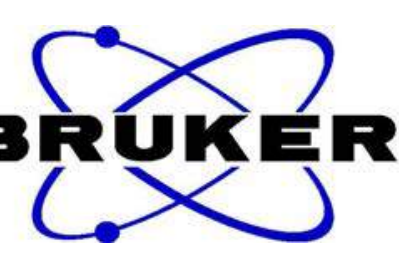

fq2-12-2

20180504 17.16 $5 \mathrm{~mm}$ DUL $13 \mathrm{C}-1$ zg30
65536 $\mathrm{CDCl} 3$ 8

$8012.820 \mathrm{~Hz}$ $0.122266 \mathrm{~Hz}$ $4.0894966 \mathrm{sec}$ 103.52 62.400 usec 6.50 usec $294.8 \mathrm{~K}$ $1.00000000 \mathrm{sec}$

CHANNEL $f 1$ $14 \mathrm{H}$ 14.30 $400.1300096 \mathrm{MHz}$ EM $0.30 \mathrm{~Hz}$ 0.30
0

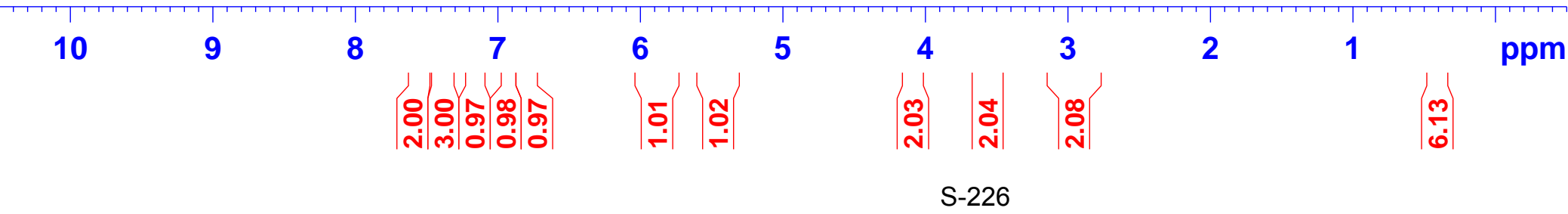




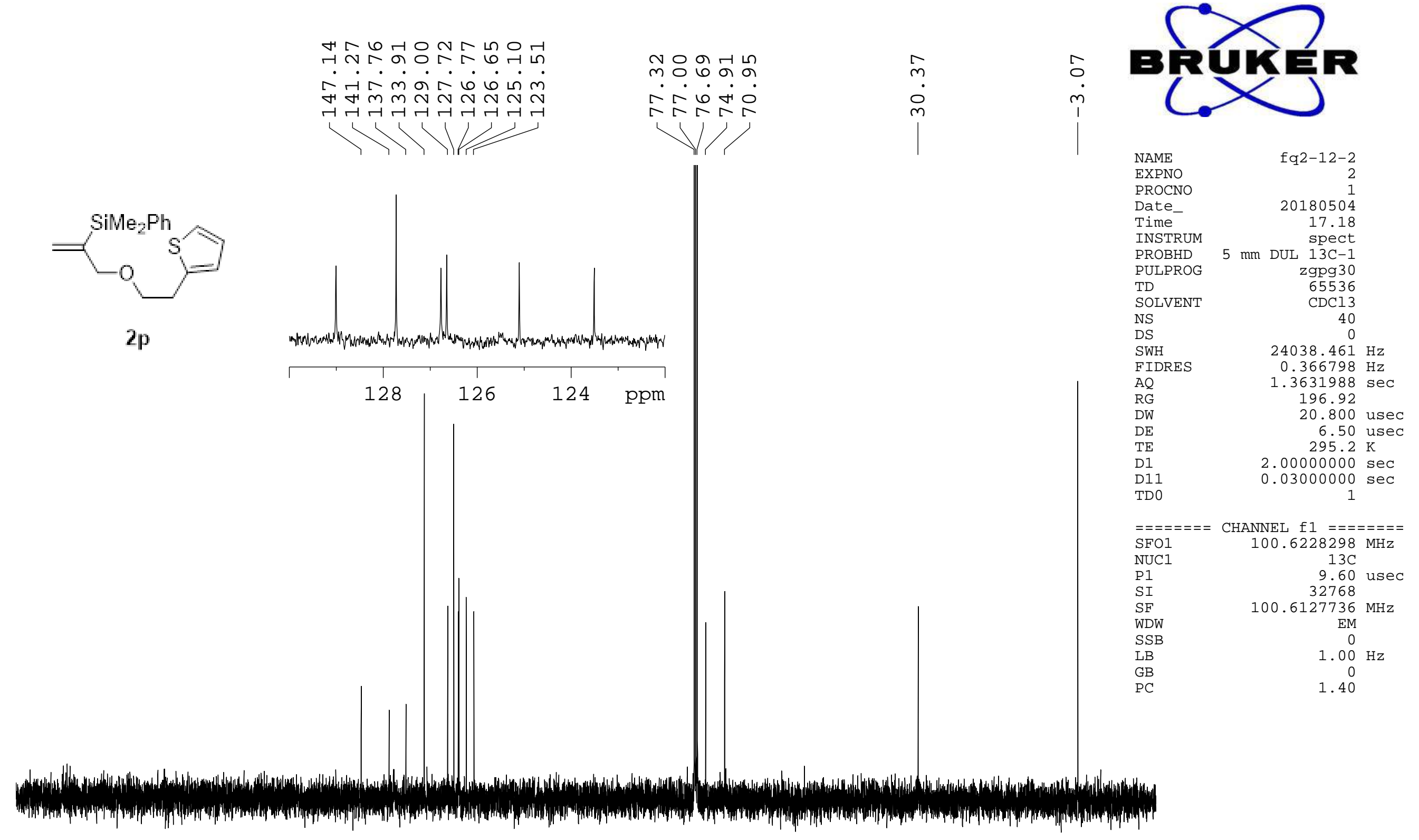


๓ の

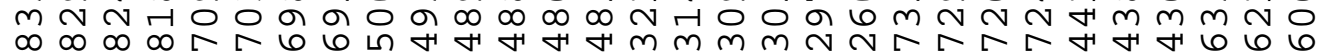

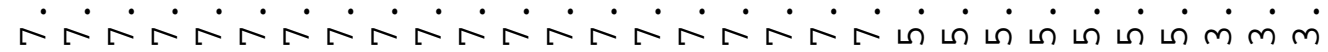
N

II
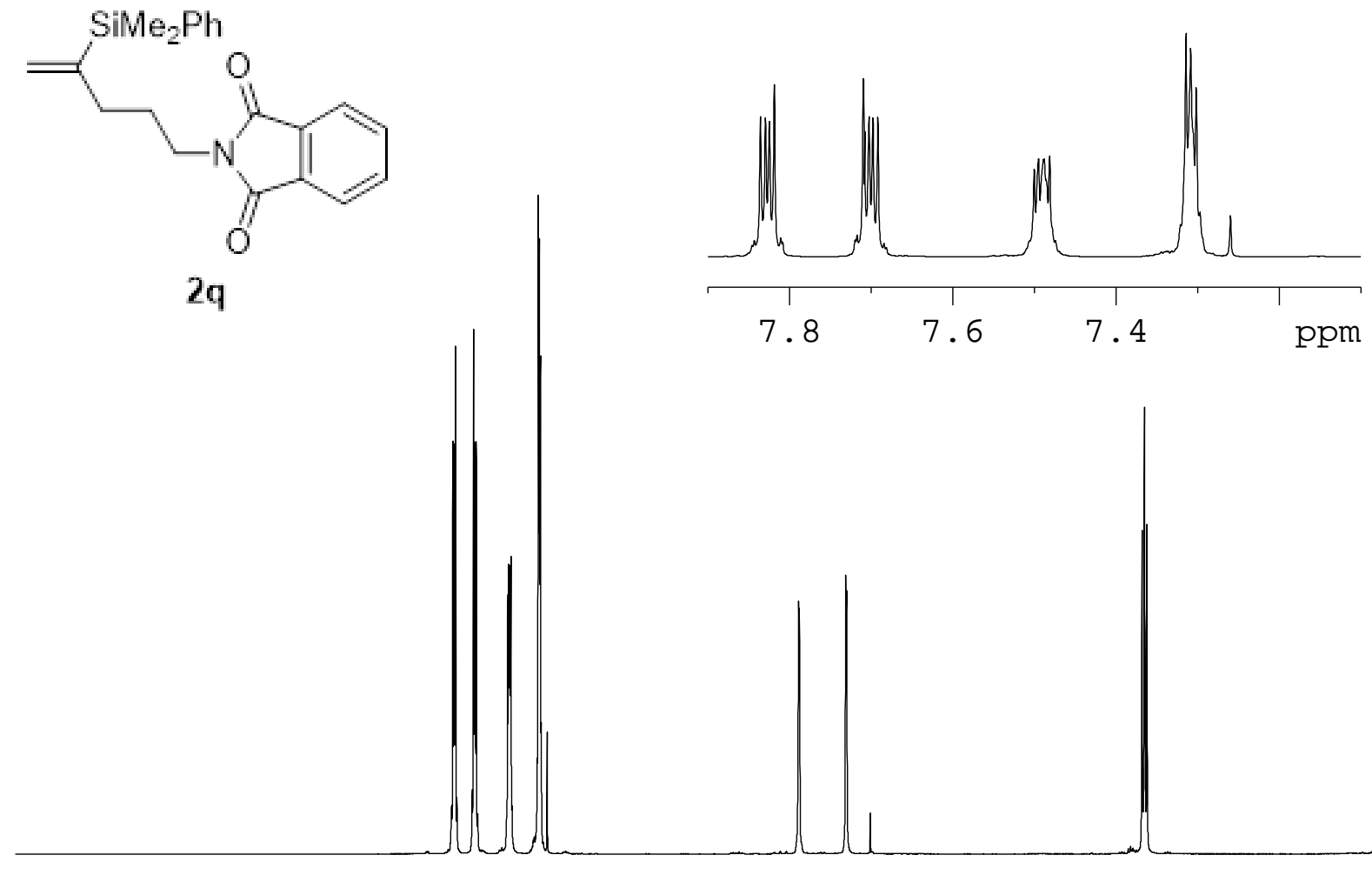

$10 \quad 9$

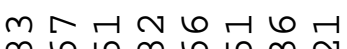

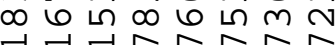
N N $\longrightarrow$

pm
8 80\% ஸे|ci|:
7

|⿱宀⿻三丨口

EXPNO

PROCNO

Date_-

INSTRUM

PROBHD

PULPROG

TD

SOLVENT

NS

SWH

FIDRES

$A Q$

RG

$\mathrm{DE}$

TE

TD0

$===$

$\mathrm{SFO1}$

N1

P1

SF

WDW

$S S B$

LB

$\mathrm{PC}$

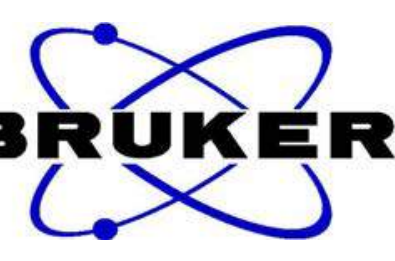

fq2 $2-21-\mathrm{H}$

20171201

15.48

$5 \mathrm{~mm}$ CPРBBO BB

$\mathrm{zg} 30$
65536

CDCl3

8

$10000.000 \mathrm{~Hz}$

$0.152588 \mathrm{~Hz}$

$3.2768500 \mathrm{sec}$ 31.14

50.000 usec 10.00 use

$1.00000000 \mathrm{sec}$

CHANNEL $\mathrm{f} 1$

$500.1330885 \mathrm{MHz}$

$1 \mathrm{H}$

10.71

$500.1300119 \mathrm{MHz}$

EM

$0.30 \mathrm{~Hz}$

0.30
0
1.00 


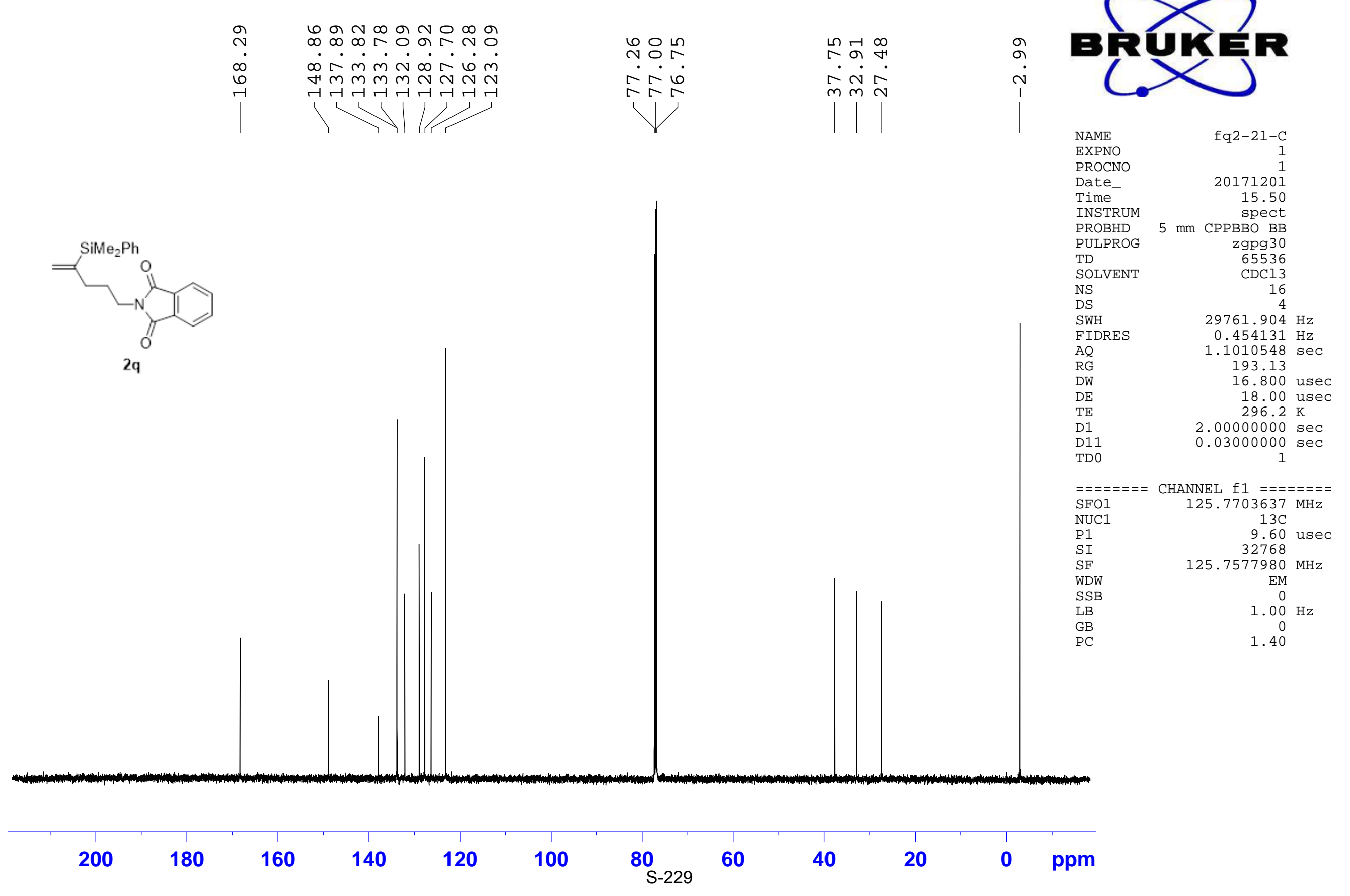


m

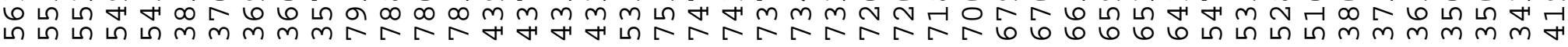

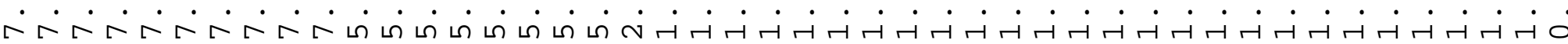
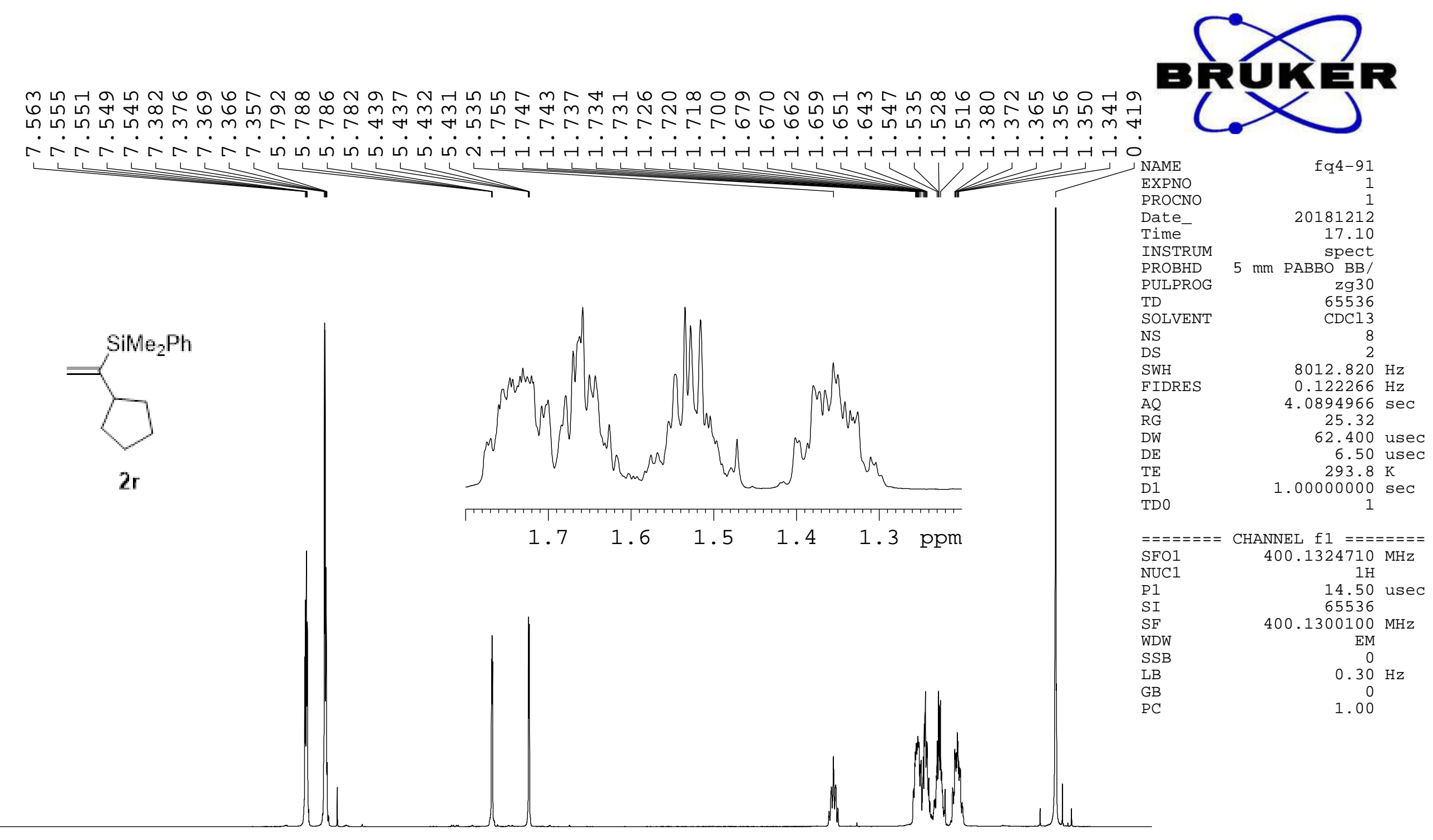

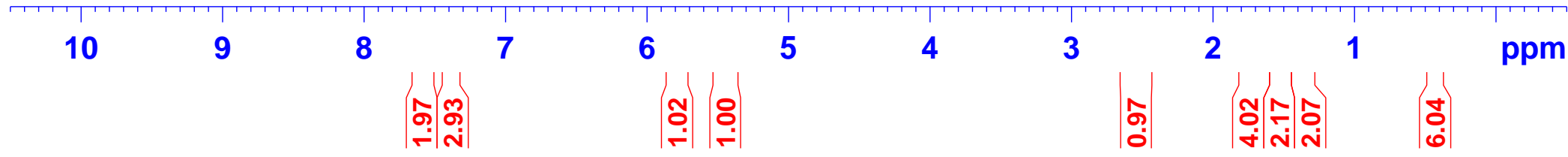




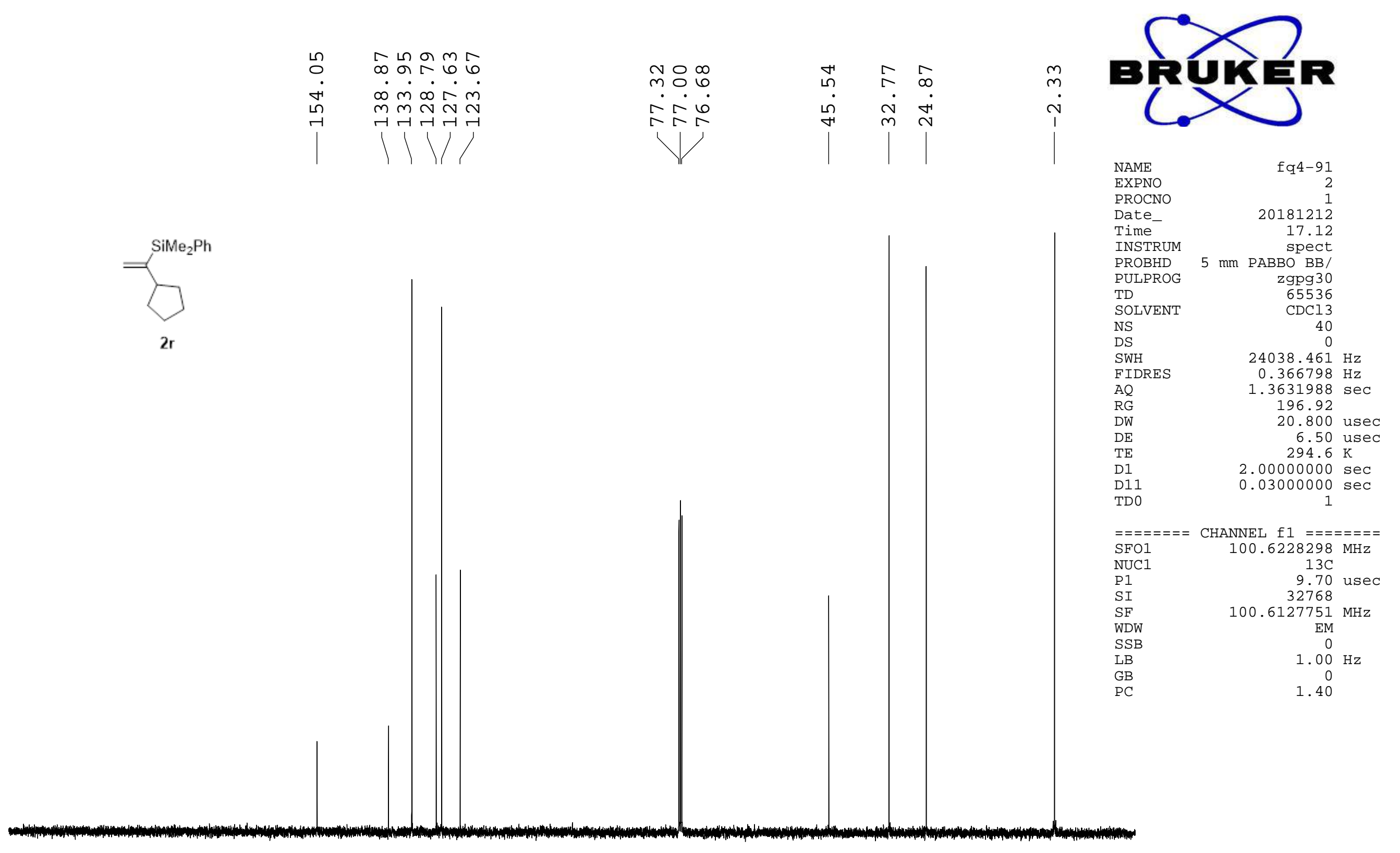

\begin{tabular}{llllllllllll}
\hline 200 & 180 & 160 & 140 & 120 & 100 & 80 & 60 & 40 & 20 & 0 & ppm
\end{tabular}




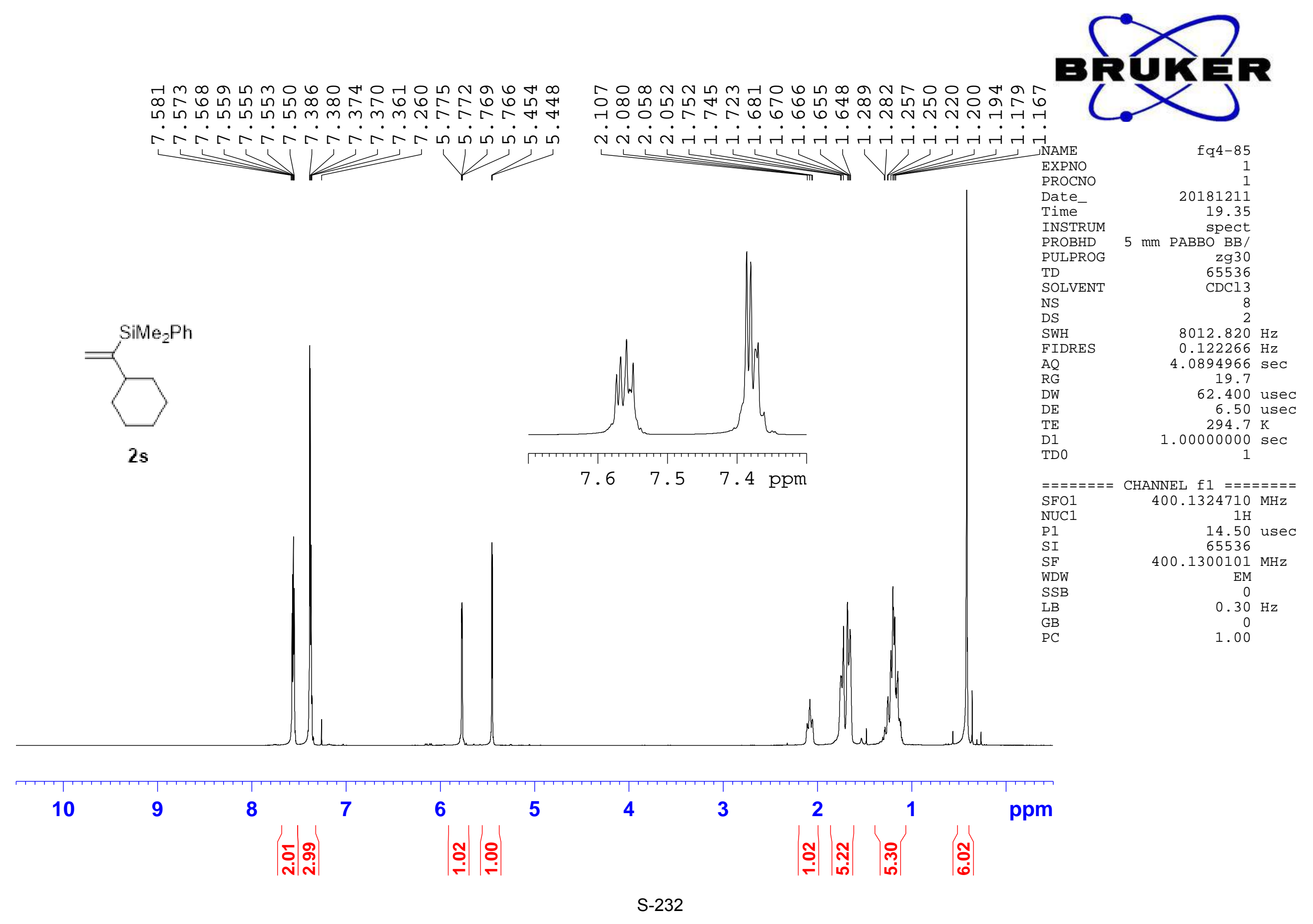




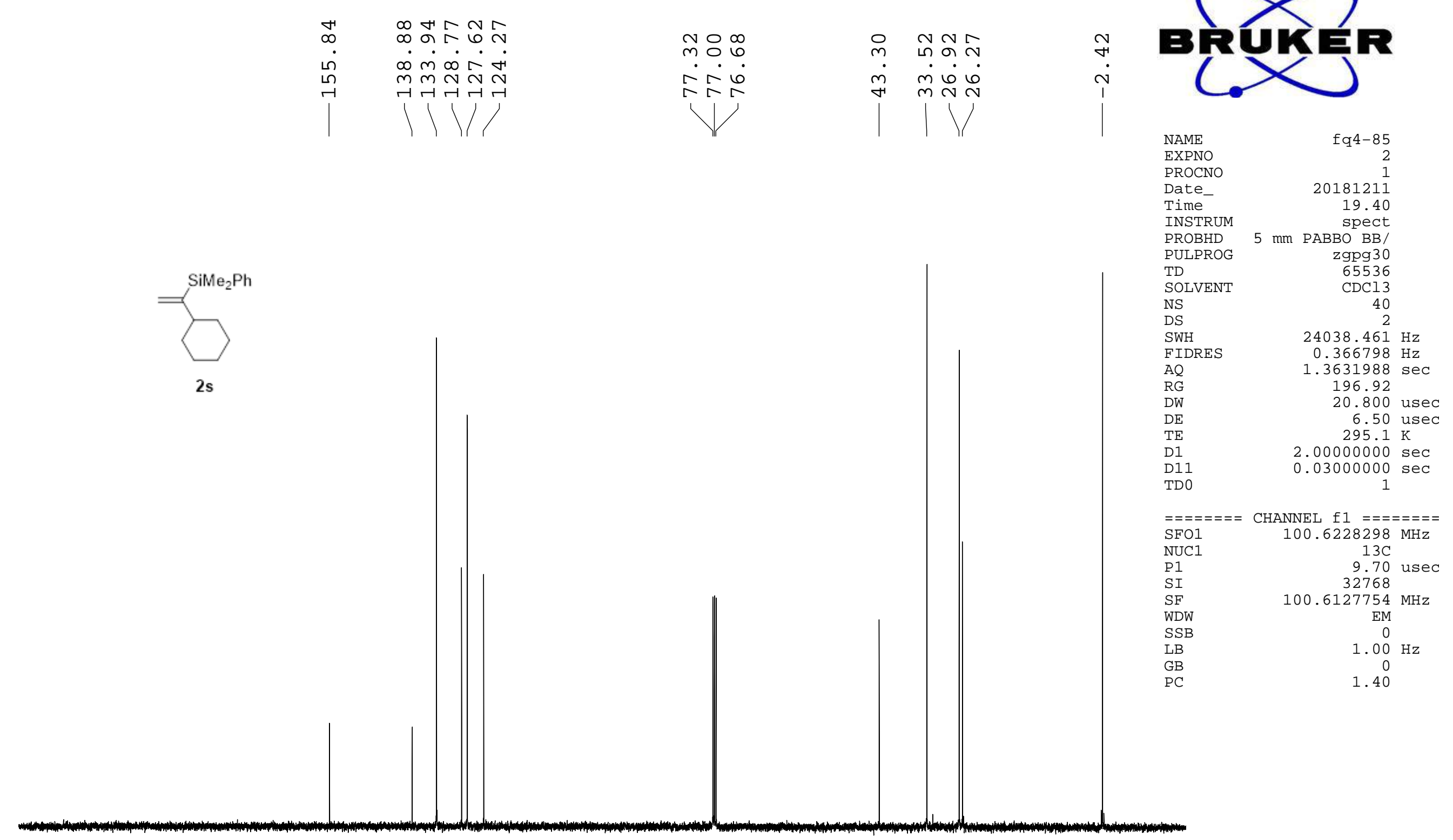

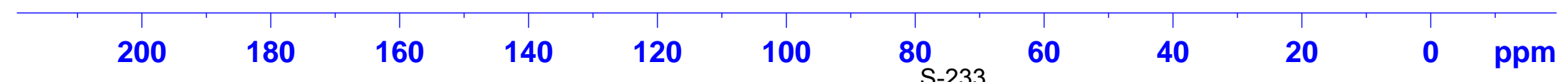


N $⿻ 0060$ 은 เ rarkrhrarkrariber

$\longrightarrow$

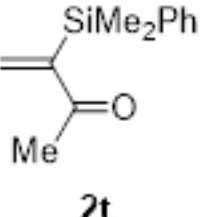

$2 t$

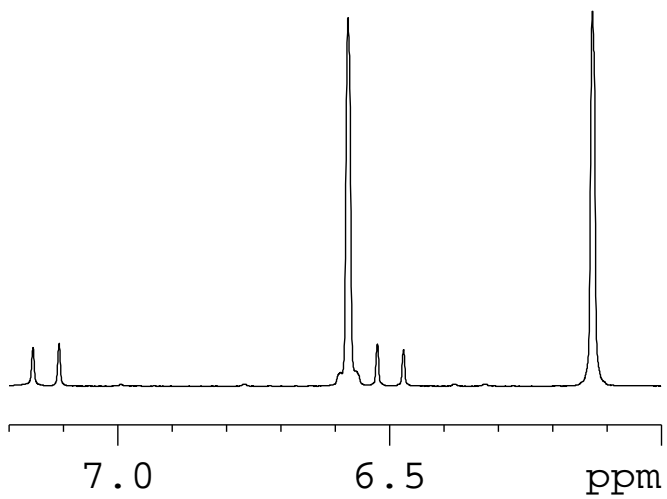

10 9 8

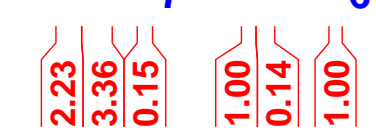

5

4

3

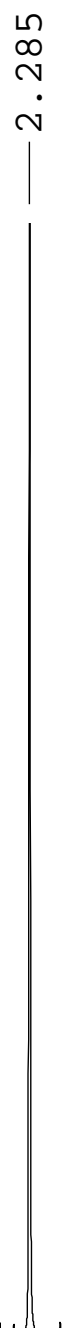

$\underset{\sigma}{\sigma}$

(Ty

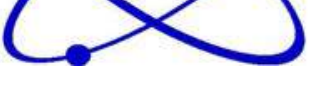

NAME

EXPNO

PROCNO

Date_

Time

INSTRUM

PROBHD

PULPROG

$\begin{array}{lr}\text { PULPROG } & \text { zg30 } \\ \text { TD } & 65536 \\ \text { SOLVENT } & \text { CDC13 }\end{array}$

NS

DS

SWH

FIDRES

AQ

RG

DE

TE

TD0

fq3-175

20181016

81016
11.13

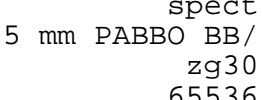

$\mathrm{CDCl} 3$
8

$8012.820 \mathrm{~Hz}$

$0.122266 \mathrm{~Hz}$

$4.0894966 \mathrm{sec}$ 70.97

62.400 usec 6.50 usec $1.00000000 \mathrm{sec}$

$=======$ CHANNEL $\mathrm{f} 1 \mathrm{l}========$
SFO1
$400.1324710 \mathrm{MHz}$

$\mathrm{SFO1}$

P 1

SF

WDW

$S S B$

LB

PC

$1 \mathrm{H}$
14.50 usec

$400.1300101 \mathrm{MHz}$

EM

0
$0.30 \mathrm{~Hz}$

0.30
0
1.00

0
1.00

2

ppm

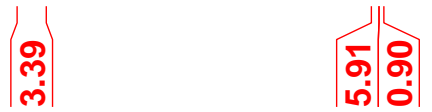




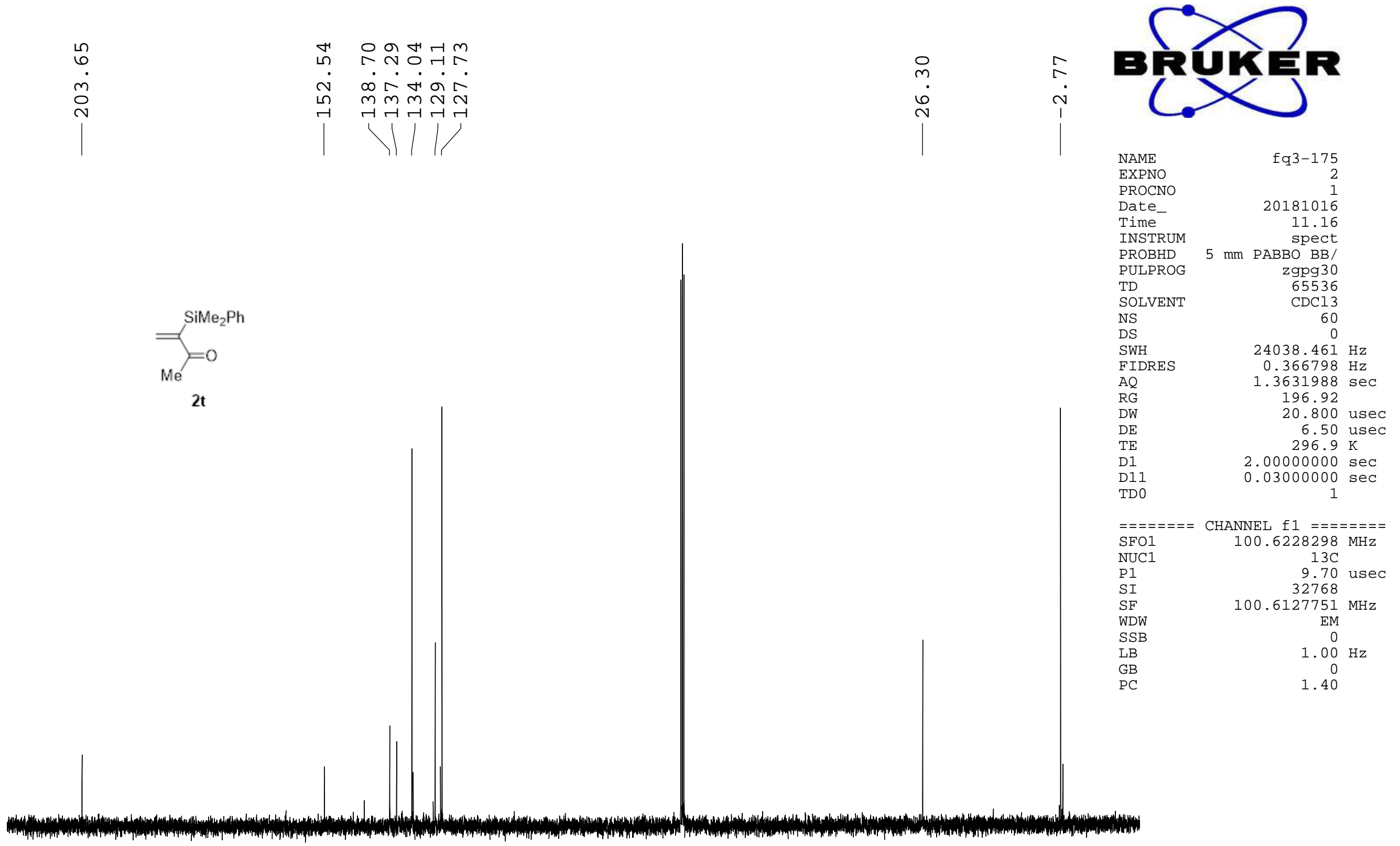




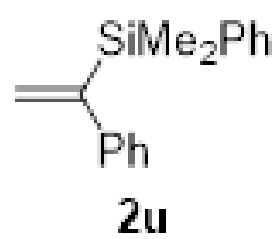
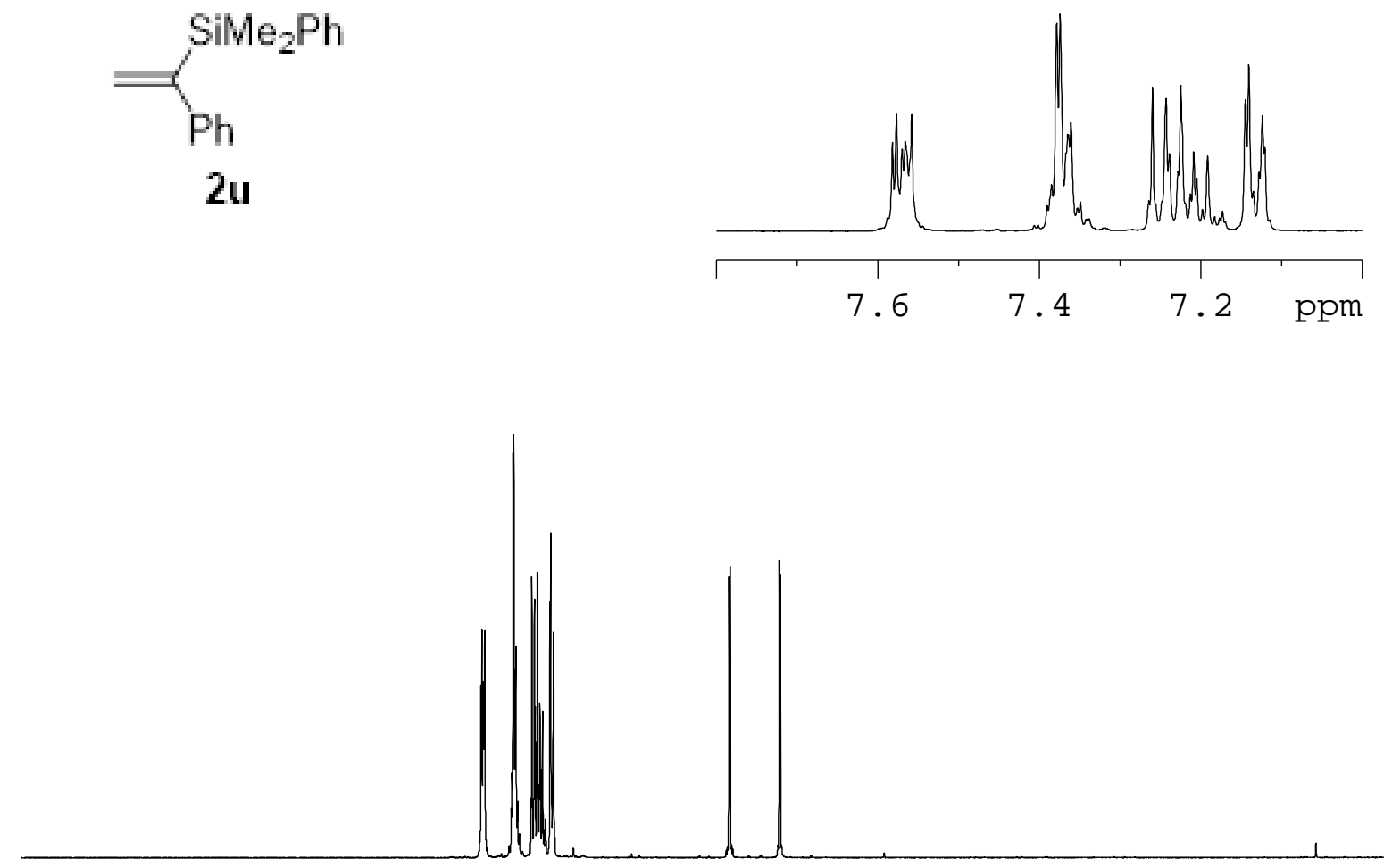

10

9

8

7

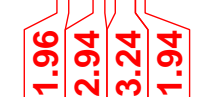

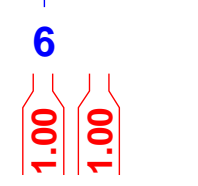

5

4

3

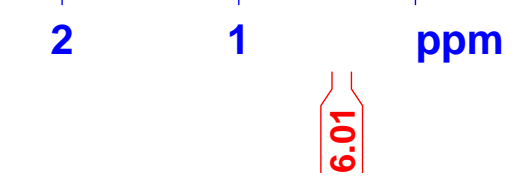




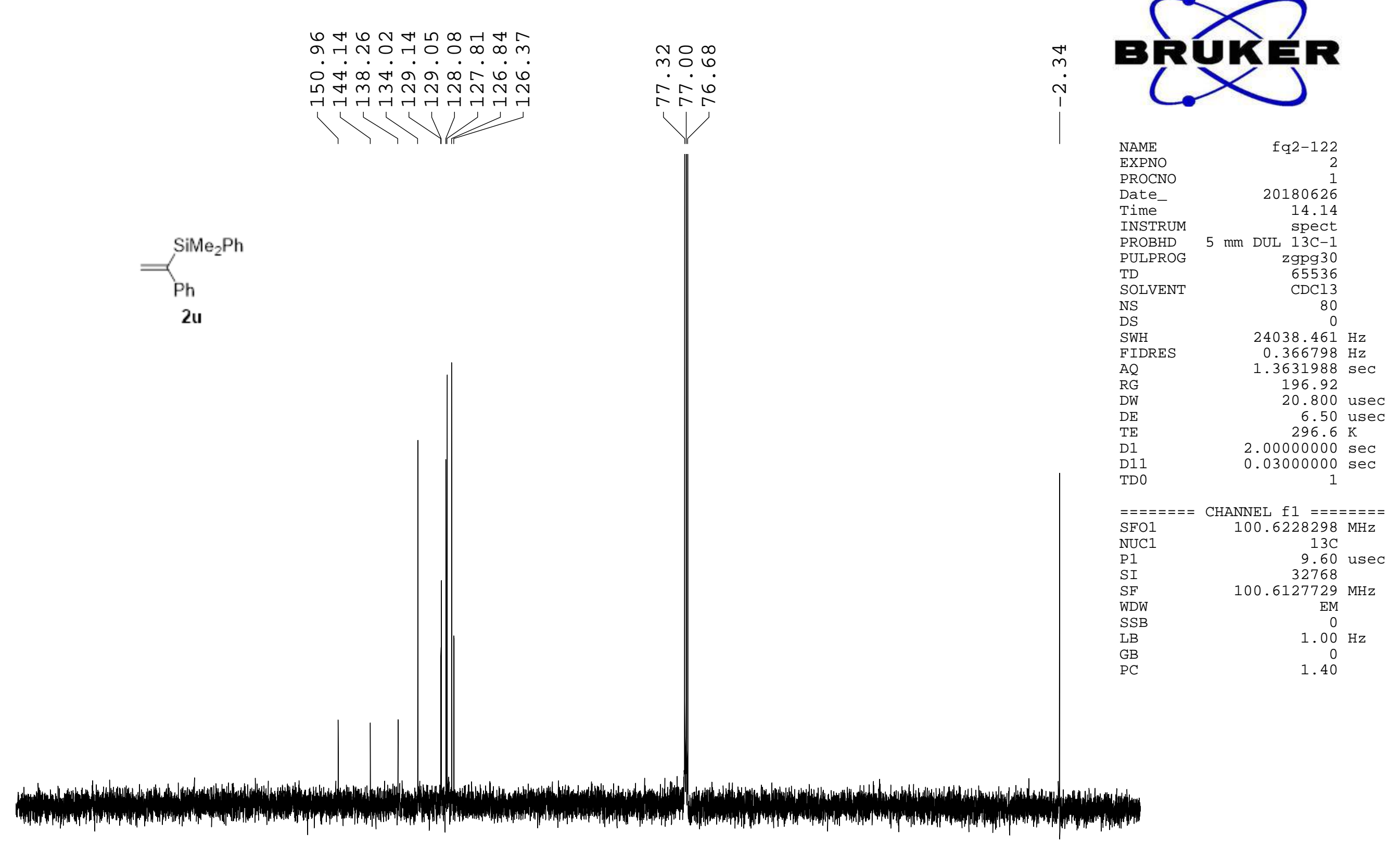

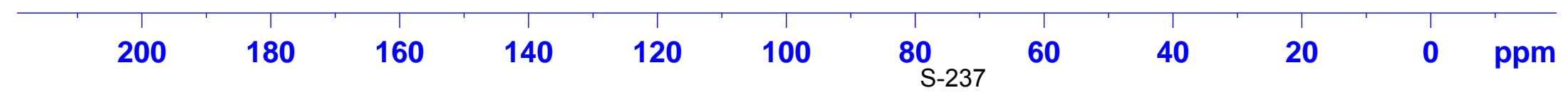



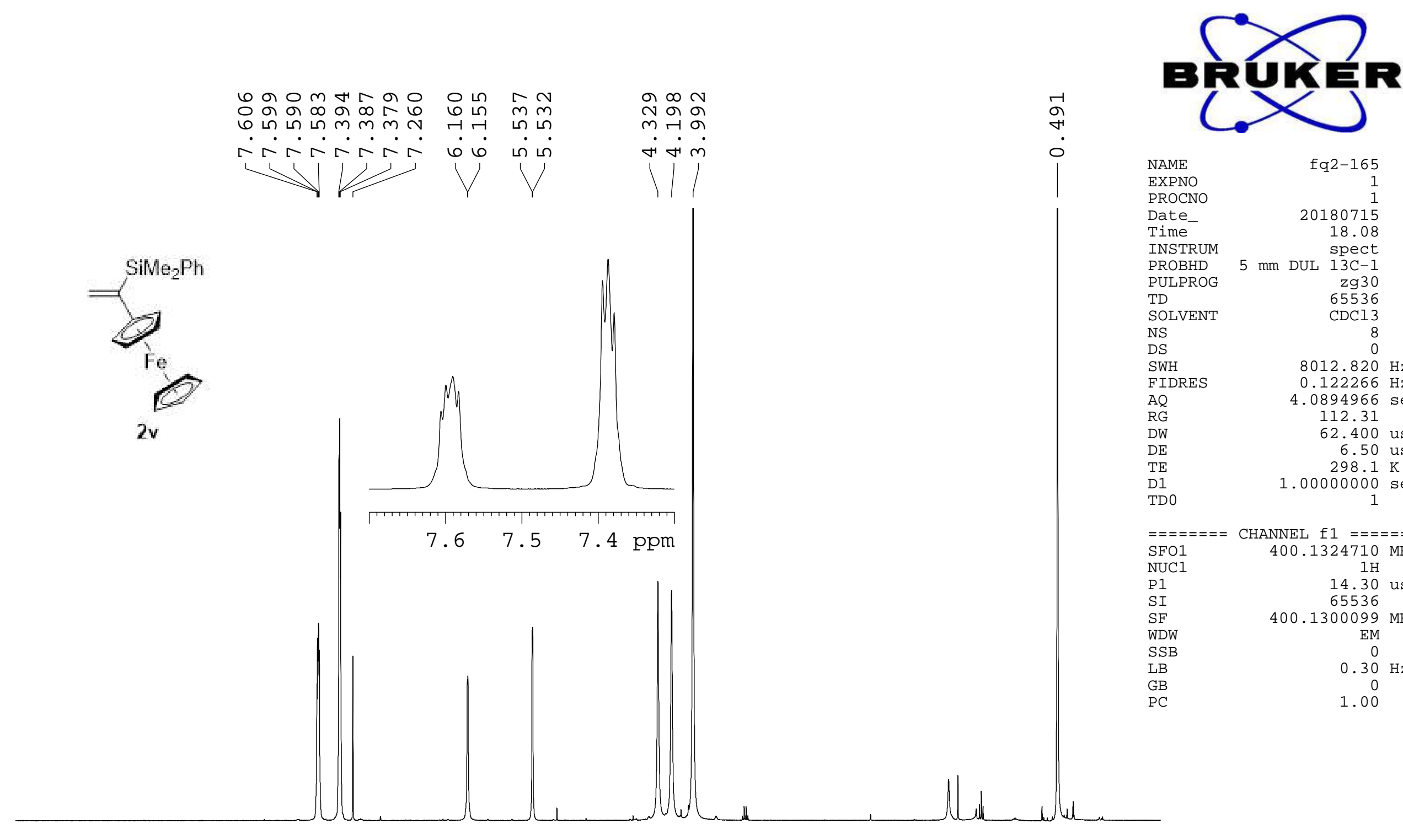

NAME

EXPNO

PROCNO

Date_-

Time

PROBHD

PULPROG

TD

SOLVENT

NS
DS

SWH

FIDRES

AQ
RG
DW

DE

TE

TDO

fq2-165

165
1

20180715

$5 \mathrm{~mm}$ DUL $13 \mathrm{C}-1$

SFO1 CHANNEL

$\mathrm{SFO}$

$400.1324710 \mathrm{MHz}$

P 1

SI

WDW

SSB

LB
GB

GB

14.30 usec

$400.1300099 \mathrm{MHz}$

EM
0
$0.30 \mathrm{~Hz}$
0
0

10

9

8

7

6

5

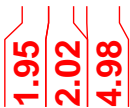

2

(1)

$\left|\begin{array}{l}1 \\ 0 \\ 0\end{array}\right|$

ppm 


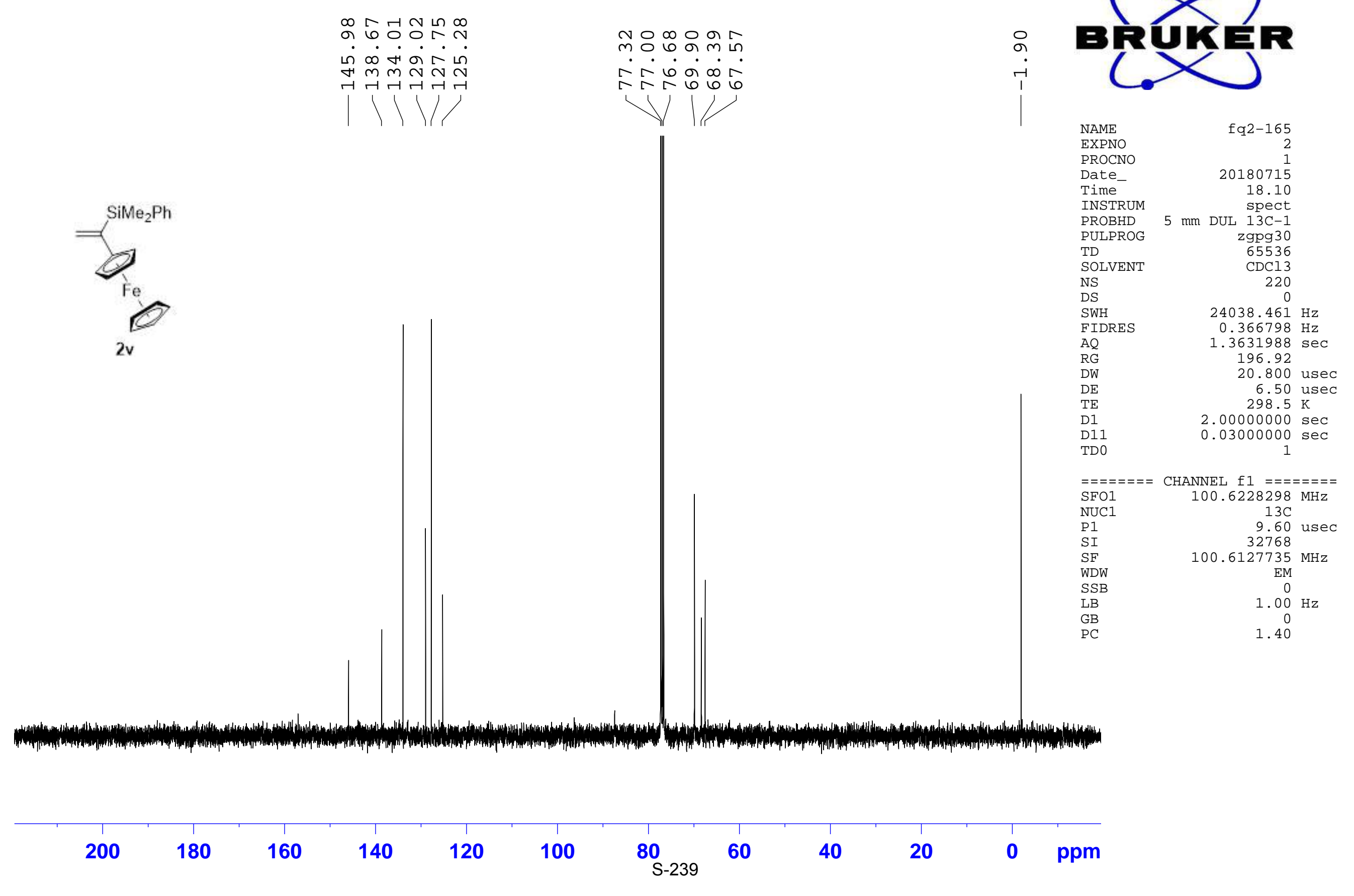




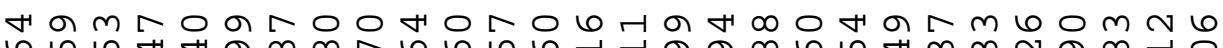
6 约

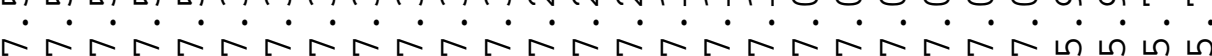

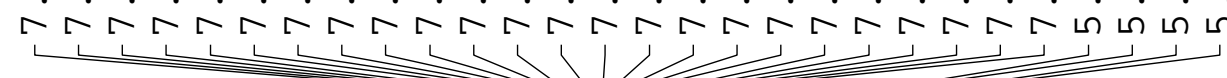

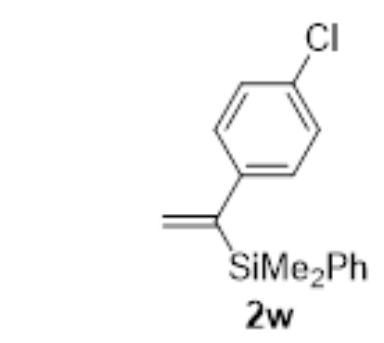

10 9

8

6

5
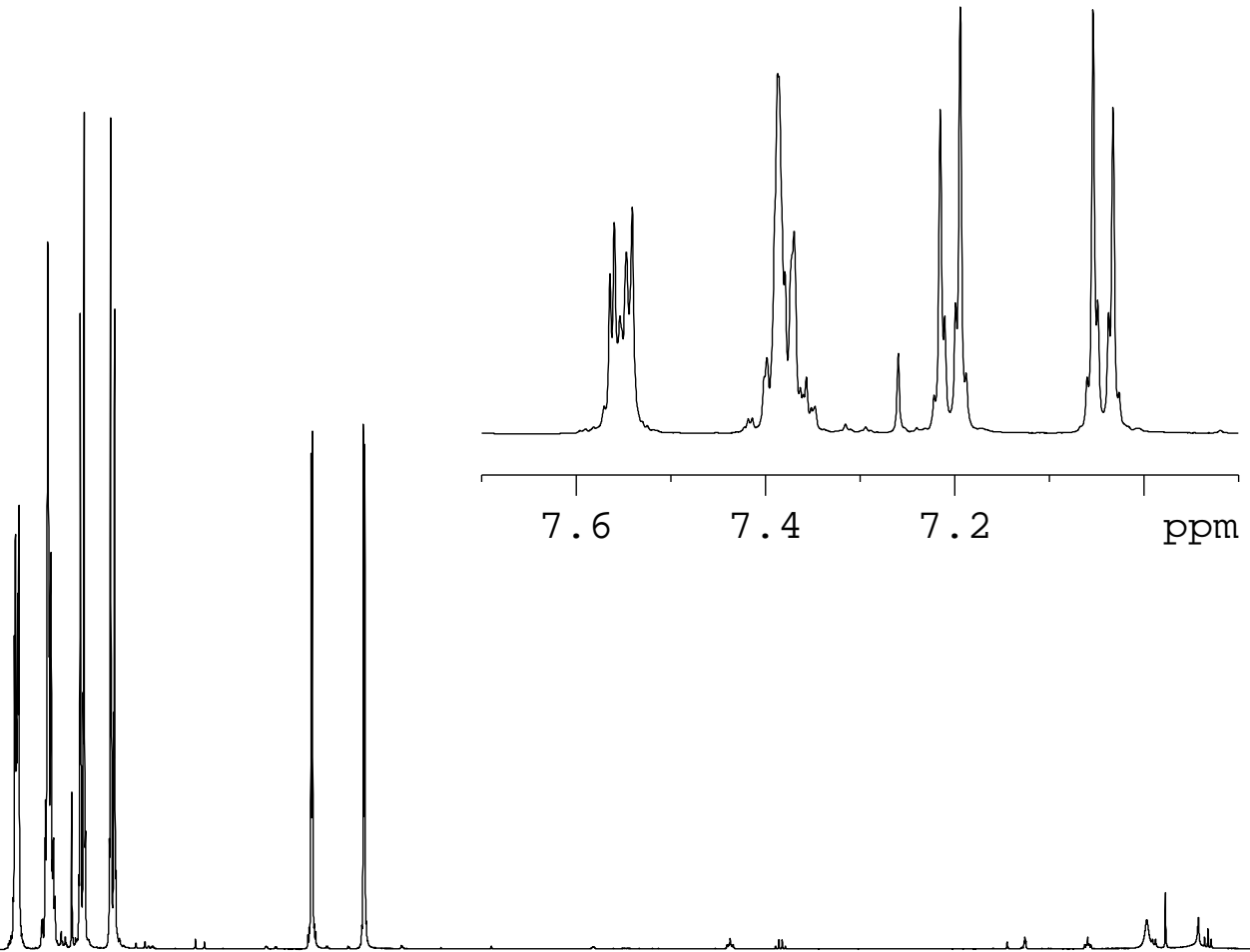

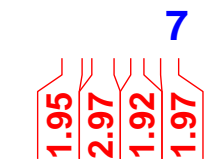

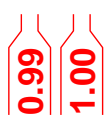

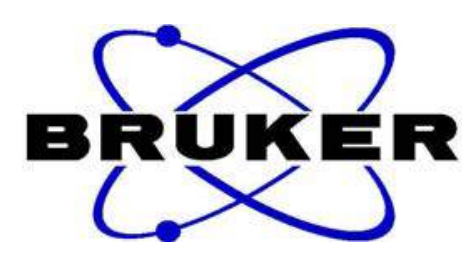

NAME

EXPNO

PROCNO

Date_

Time

INSTRUM

PROBHD

PULPROG

TD

SOLVENT

NS

SWH

FIDRES

AQ
RG
DW

DE

TE

TD0

fq2-148

$20180710^{1}$

18.45

$5 \mathrm{~mm}$ DUL $13 \mathrm{C}-1$

$13 \mathrm{C}-1$

65536

$\mathrm{CDCl} 3$
8

$8012.820 \mathrm{~Hz}$

$0.122266 \mathrm{~Hz}$

$4.0894966 \mathrm{sec}$ 82.92

62.400 usec 6.50 usec $1.00000000 \mathrm{sec}$

$=======$ CHANNEL $\mathrm{f} 1 \mathrm{f}=======$
$\mathrm{SFO1}$
$400.1324710 \mathrm{MHz}$

$\mathrm{SFO1}$

NUC1

P 1

SI

WDW

SSB

LB

GB

14.30 usec

400.1300098

$\mathrm{EM}$
0

$0.30 \mathrm{~Hz}$

0.30
0
1.00 


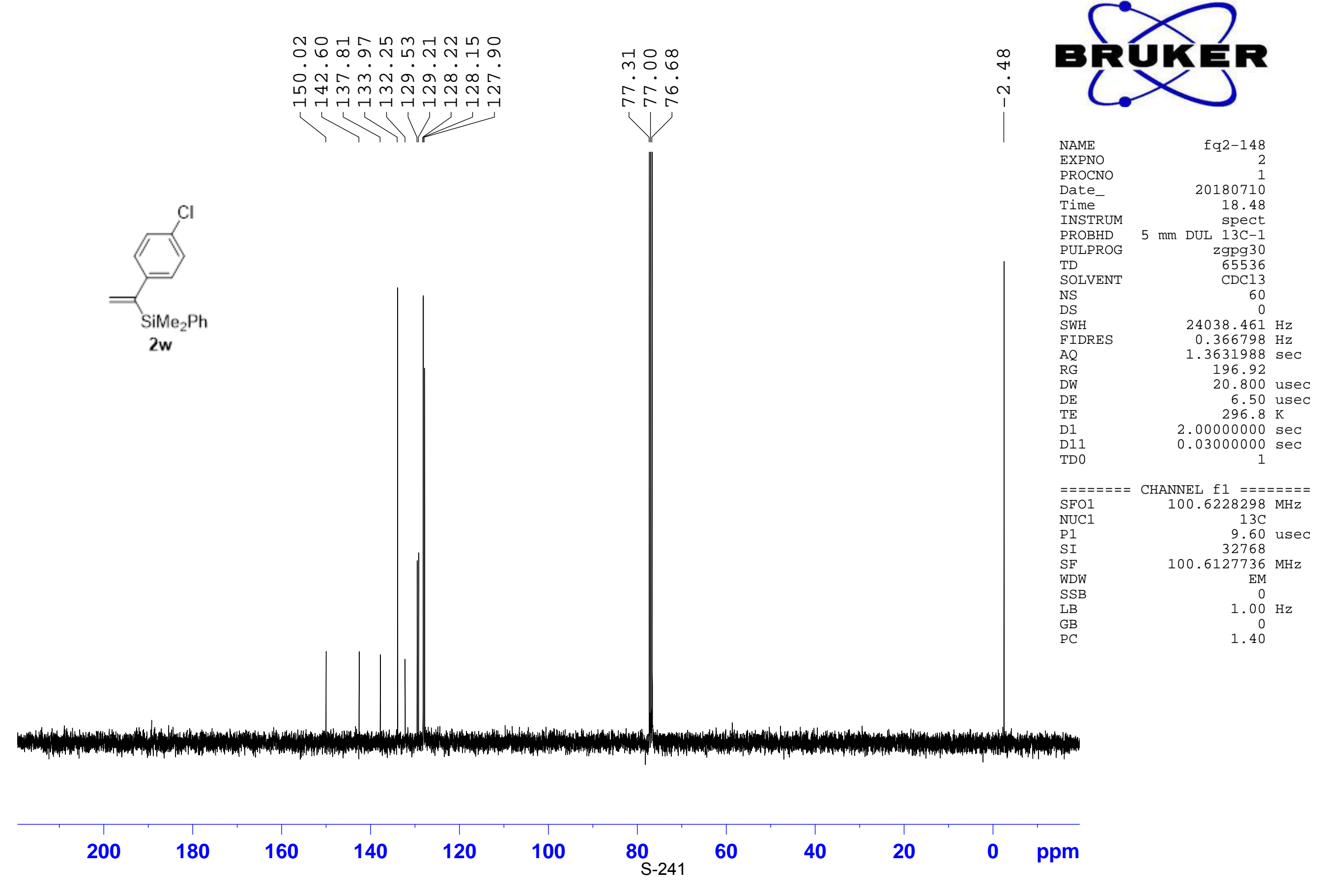




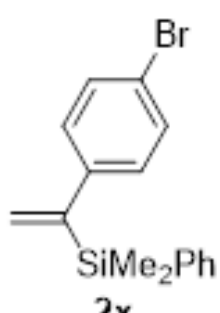

$2 x$
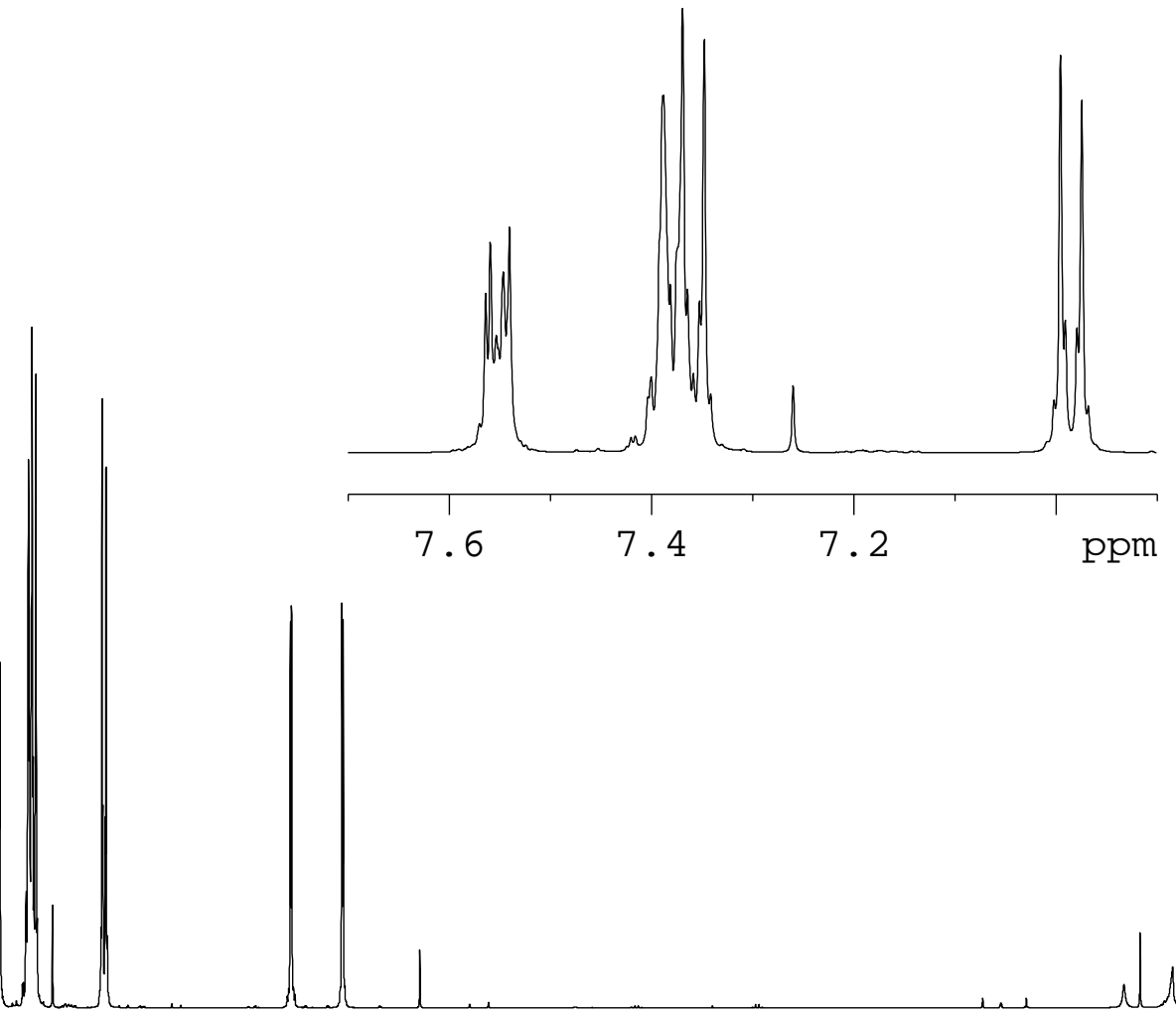

10 9
8
7

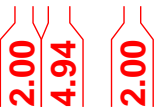

6

กั่

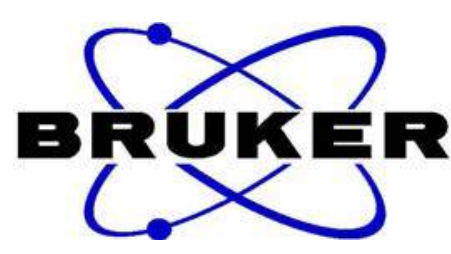

NAME

EXPNO

PROCNO

Date_

Time

INSTRUM

PROBHD

PULPROG

TD

SOLVENT

NS

SWH

FIDRES

$\mathrm{AQ}$
$\mathrm{RG}$

RG

$\mathrm{DE}$

TE

TDO

$==$

SFO1

P 1

SI

WDW

SSB

LB
GB

PC fq2-163

20180715 14.05 $5 \mathrm{~mm}$ DUL $13 \mathrm{C}-1$ $\mathrm{zg} 30$
65536 $\mathrm{CDCl} 3$ 8

$8012.820 \mathrm{~Hz}$ $0.122266 \mathrm{~Hz}$ $4.0894966 \mathrm{sec}$ 70.97

62.400 usec 6.50 usec $1.00000000 \mathrm{~K}$

HANNEL $\mathrm{f}$

$$
\begin{array}{r}
1 \mathrm{H} \\
14.30 \text { usec }
\end{array}
$$

14.30 us

400.1300097

EM

0
$0.30 \mathrm{~Hz}$

0.30
0
1.00 


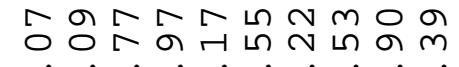

$\dot{m} \dot{m} \dot{m} \dot{m} \dot{\sigma} \dot{\sigma} \dot{0}$

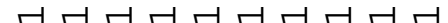

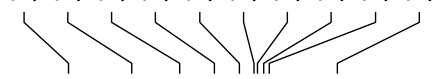

$2 x$

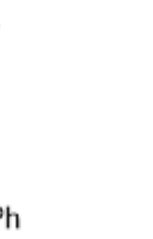

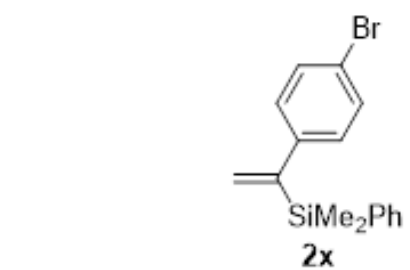

m $\bigcirc 0$

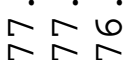

11

.

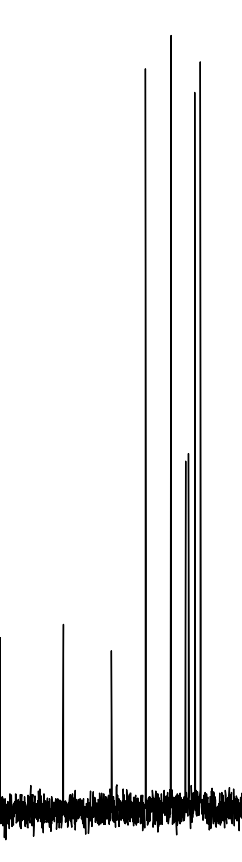

EAME

Date

INSTRUM

PROBHD

PULPROG

TD

SOLVENT

NS

DS

FIDRES

FIDR
AQ
RG
DW

DW

$\mathrm{DE}$

D1

D11

TD0

$==$
$\mathrm{SFO}$

$\mathrm{P} 1$
$\mathrm{SI}$
$\mathrm{SF}$

SF
WDW
SSB

LB
GB

GB
20180715 14.07

$5 \mathrm{~mm}$ DUL $13 \mathrm{C}-1$

zgpg 30

$\mathrm{CDCl} 3$

$24038.461 \mathrm{~Hz}$

$0.366798 \mathrm{~Hz}$

$1.3631988 \mathrm{sec}$

196.92

20.800 usec

6.50 usec

$00000000 \mathrm{sec}$

$0.03000000 \mathrm{sec}$

1

CHANNEL $\mathrm{f} 1======$ $100.6228298 \mathrm{MHz}$ $13 \mathrm{C}$

9.60 usec

$100.6127740 \mathrm{MHz}$

EM

$1.00 \mathrm{~Hz}$

0
1.40 


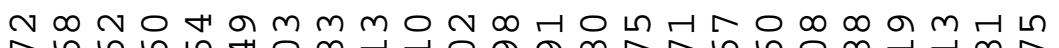

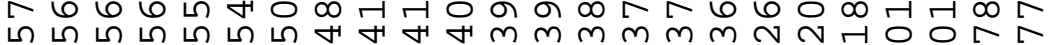

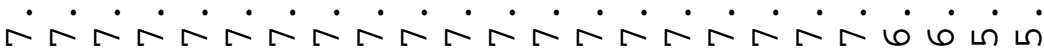
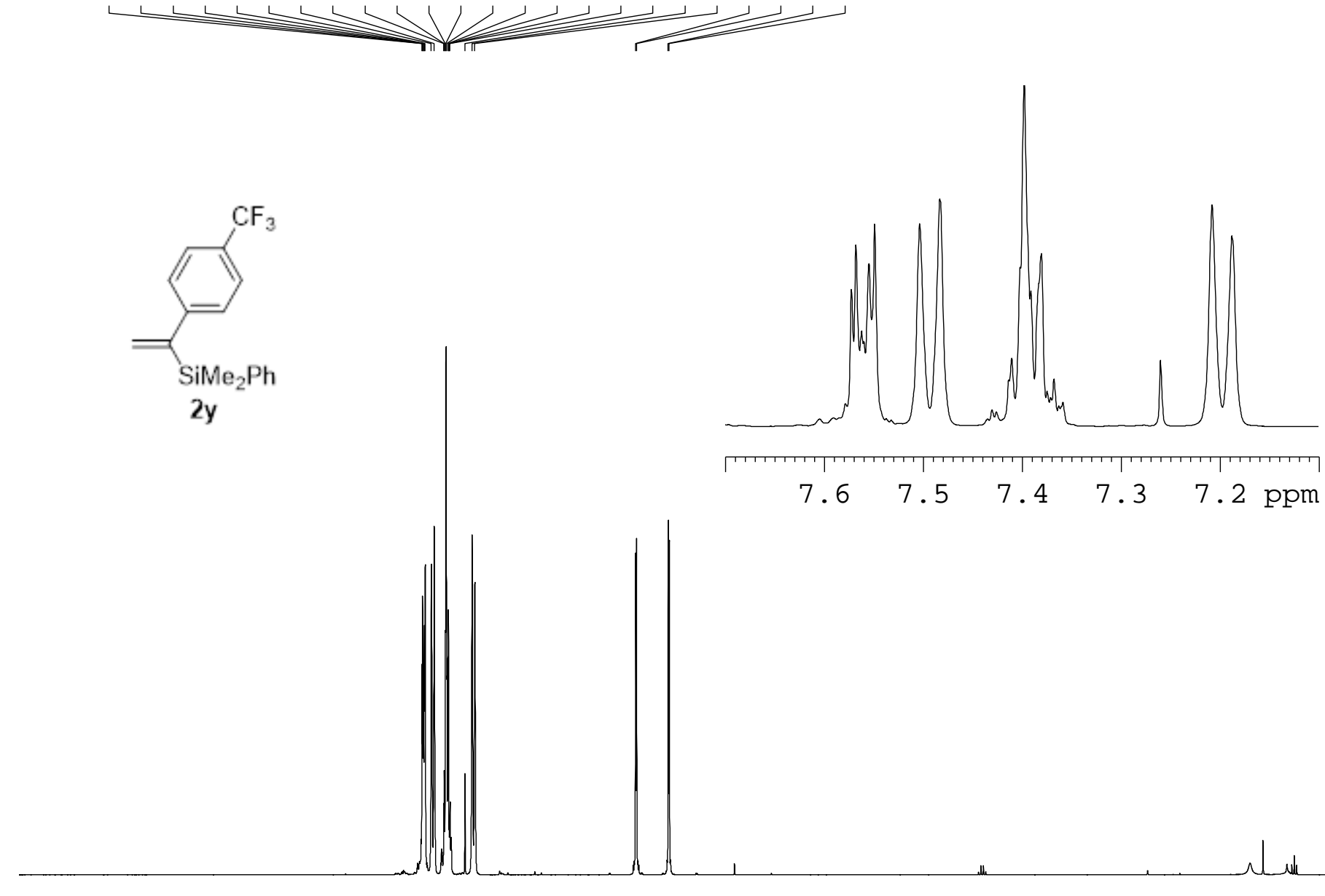

10 9

109

5

4

3

2

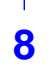

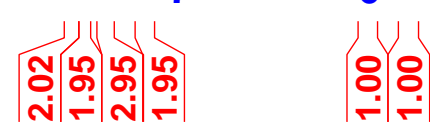

6

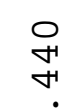

$\dot{0}$

NAME

EXPNO

PROCNO

Date_

Time

INSTRUM

PROBHD

PULPROG

TD

SOLVENT

NS

SWH

FIDRES

$\mathrm{AQ}$
$\mathrm{RG}$

RG

DE

TE

D1 0
$========$ CHANNEL $\mathrm{f}$
$\mathrm{SFO} 1$
NUC1
NNEL $\mathrm{fl}========$
$400.1324710 \mathrm{MHz}$

P 1

SI

SF

SSB

SSB

LB
GB

PC

$1 \mathrm{H}$
14.30 usec $400.1300100 \mathrm{MHz}$

EM

$0.30 \mathrm{~Hz}$

0.30
0
1.00

0
1.00

1

ppm

$\left|\begin{array}{c}6 \\ 0 \\ 1\end{array}\right|$ 
न ॠ

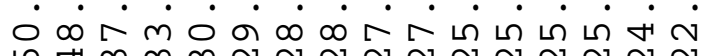

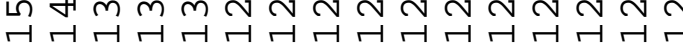
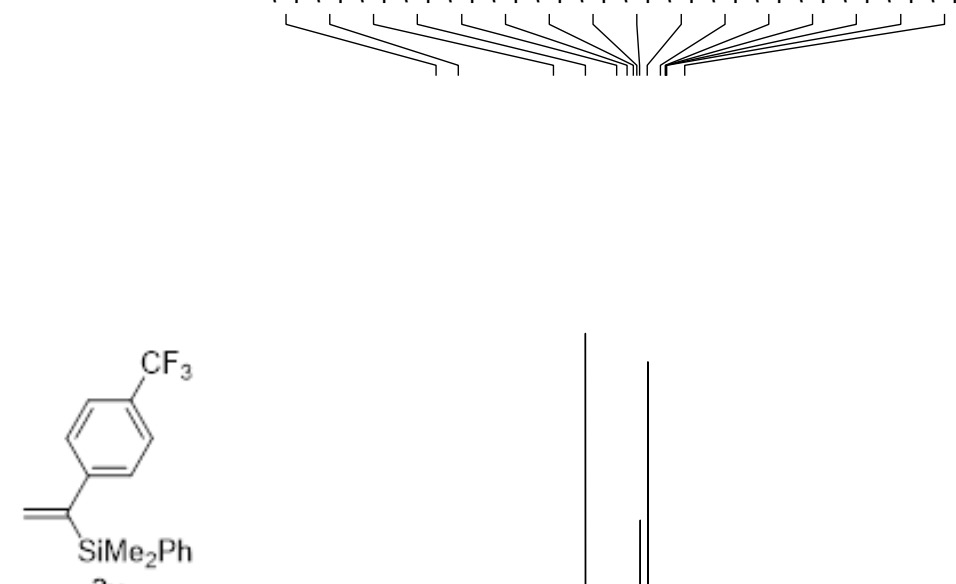

\section{,}

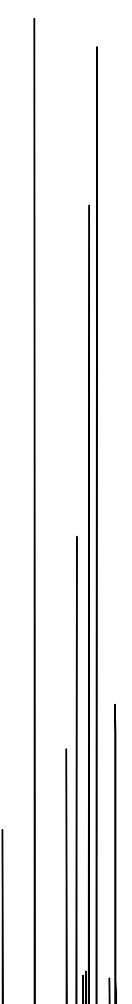

N $\bigcirc 0$

$\cdot \dot{r} \cdot$

r.

$\checkmark$

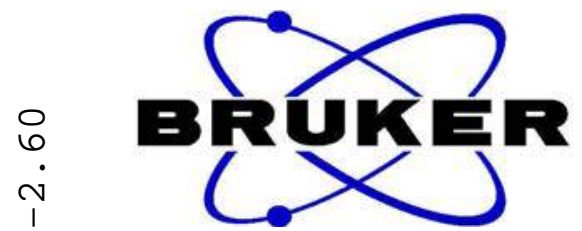

NAME EXPNO

Date

Time

INSTRUM

PROBHD

PULPROG

TD

SOLVENT

NS

DS

FIDRES

AQ
RG
DW

DW

DE

TE

D11

TDO

$===$

$\mathrm{SFO}$

P 1

SF

WDW

SSB
LB
GB

PC fq2-164

20180715

18.28

18.28

$5 \mathrm{~mm}$ DUL $13 \mathrm{C}-1$

zgpg 30

$\mathrm{CDCl} 3$

$24038.461 \mathrm{~Hz}$

$0.366798 \mathrm{~Hz}$

$3631988 \mathrm{sec}$

196.92

20.800 usec

6.50 usec

$2.00000000 \mathrm{sec}$

$0.03000000 \mathrm{sec}$

1

CHANNEL $\mathrm{f} 1=$ $100.6228298 \mathrm{MHz}$ $13 \mathrm{C}$

9.60 usec 100.6127711

$100.6127711 \mathrm{MHz}$

EM

$1.00 \mathrm{~Hz}$

0
1.40

80 


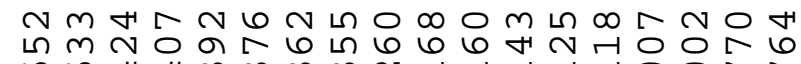

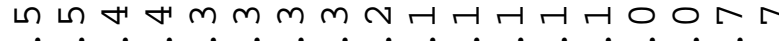

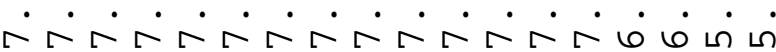

$\longrightarrow$
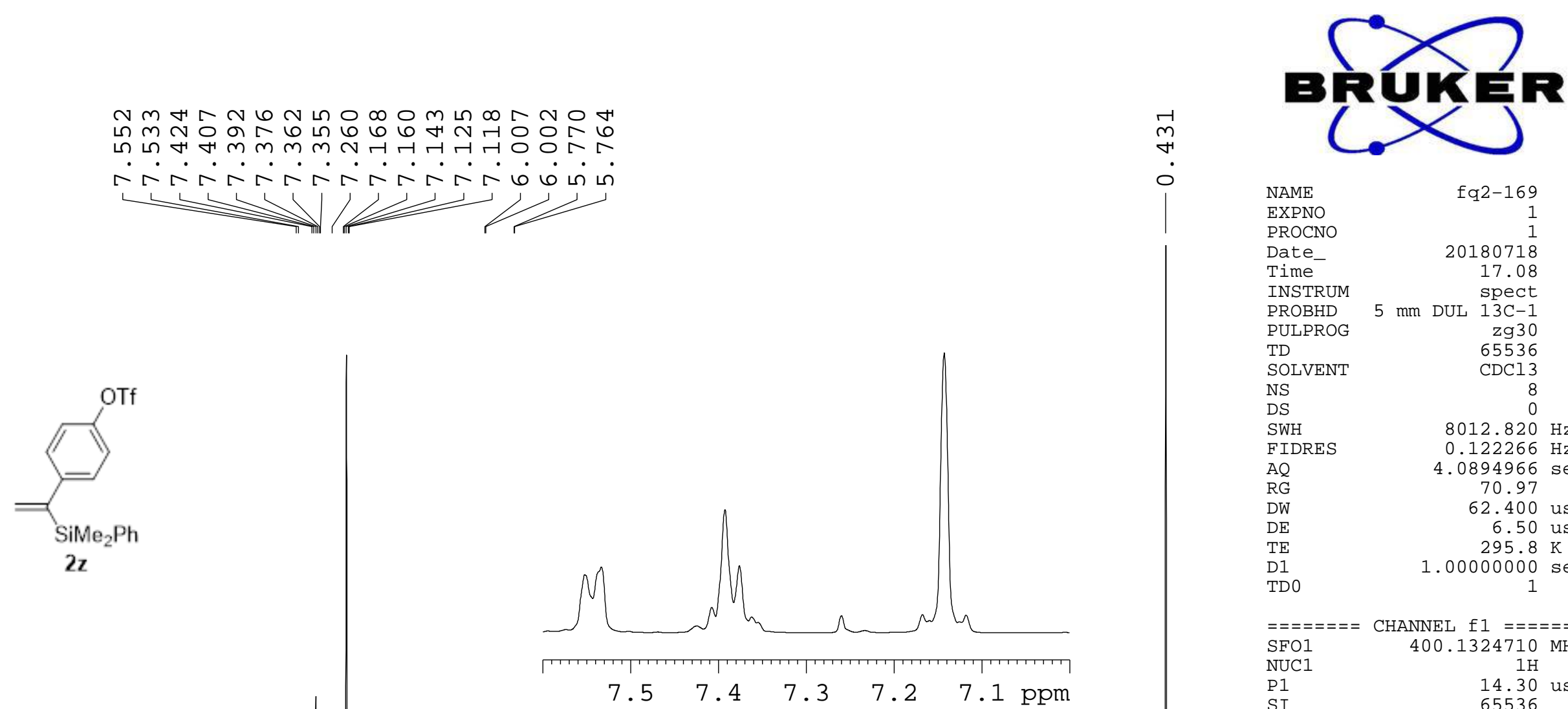

NAME

EXPNO

PROCNO

Date_

Time

INSTRUM

PROBHD

PULPROG

TD

SOLVENT

NS

SWH

FIDRES

$A Q$

RG

DE

TE

TD0

fq2-169

20180718

17.08

$5 \mathrm{~mm}$ DUL $13 \mathrm{C}-1$

$13 \mathrm{C}-1$

$\mathrm{zg} 30$
65536

$\mathrm{CDCl} 3$
8

$8012.820 \mathrm{~Hz}$

$0.122266 \mathrm{~Hz}$

$4.0894966 \mathrm{sec}$ 70.97

62.400 usec 6.50 usec $1.00000000 \mathrm{sec}$

$======$ CHANNEL $\mathrm{f} 1 \mathrm{l}=======$ SFO1 $400.1324710 \mathrm{MHz}$

NUC1

P 1

SI

WDW

SSB

LB

GB

$400.1324710 \mathrm{MHz}$

$1 \mathrm{H}$
14.30 usec $400.1300097 \mathrm{MHz}$

EM

$0.30 \mathrm{~Hz}$

0.30
0

1.00

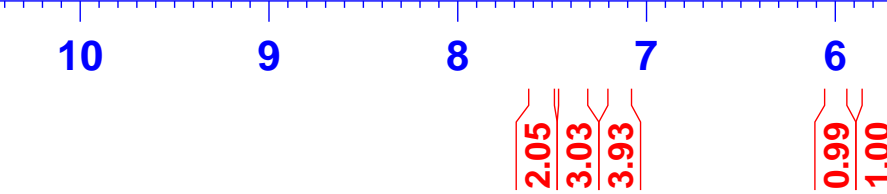

5

4

3

2

1

ppm

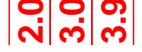

잉요 


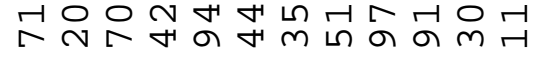

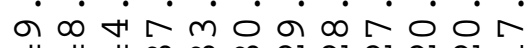

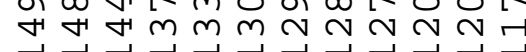

नન न न

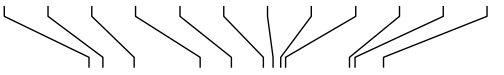

$2 z$

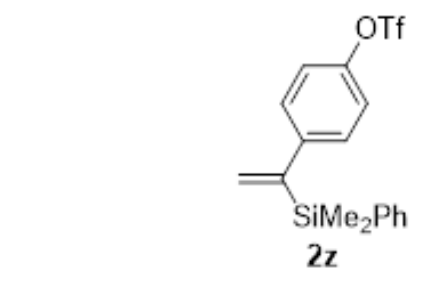

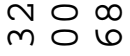

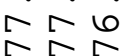

11

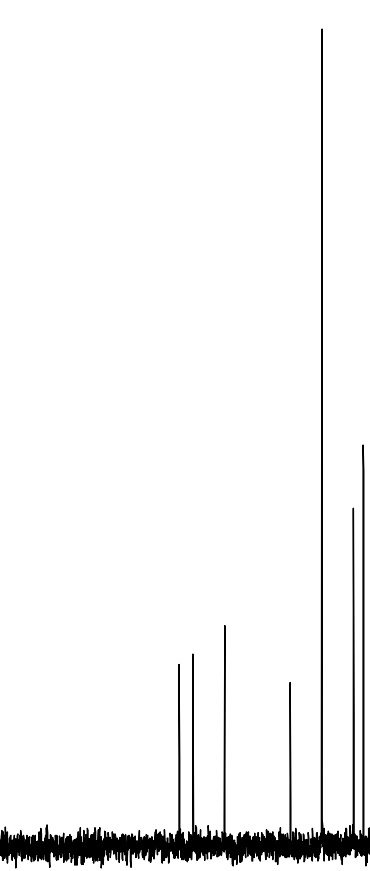

$\underset{i}{i}$

NAME
EXPNO

PROCNO

Date_-

INSTRUM

INRTRD

PULPROG

SOLVENT

NS

DS

FIDRES

FIDR
AQ
RG

DW

DE

$\mathrm{TE}$

D1 11

$===$

$\mathrm{SFO}$

P 1

SF

WDW

SSB

LB

PC fq2-169

20180718

17.11

spect

$5 \mathrm{~mm}$ DUL $13 \mathrm{C}-1$

zgpg 30

$\mathrm{CDCl} 3$

$24038.461 \mathrm{~Hz}$

$0.366798 \mathrm{~Hz}$

1.3631988 sec

196.92

20.800 usec

6.50 usec

$296.2 \mathrm{~K}$

$2.00000000 \mathrm{sec}$

$0.03000000 \mathrm{sec}$

1

CHANNEL $\mathrm{f} 1=$ $100.6228298 \mathrm{MHz}$ $13 \mathrm{C}$

9.60 usec 100.6127732

$100.6127732 \mathrm{MHz}$

EM

0
$1.00 \mathrm{~Hz}$

0
1.40 


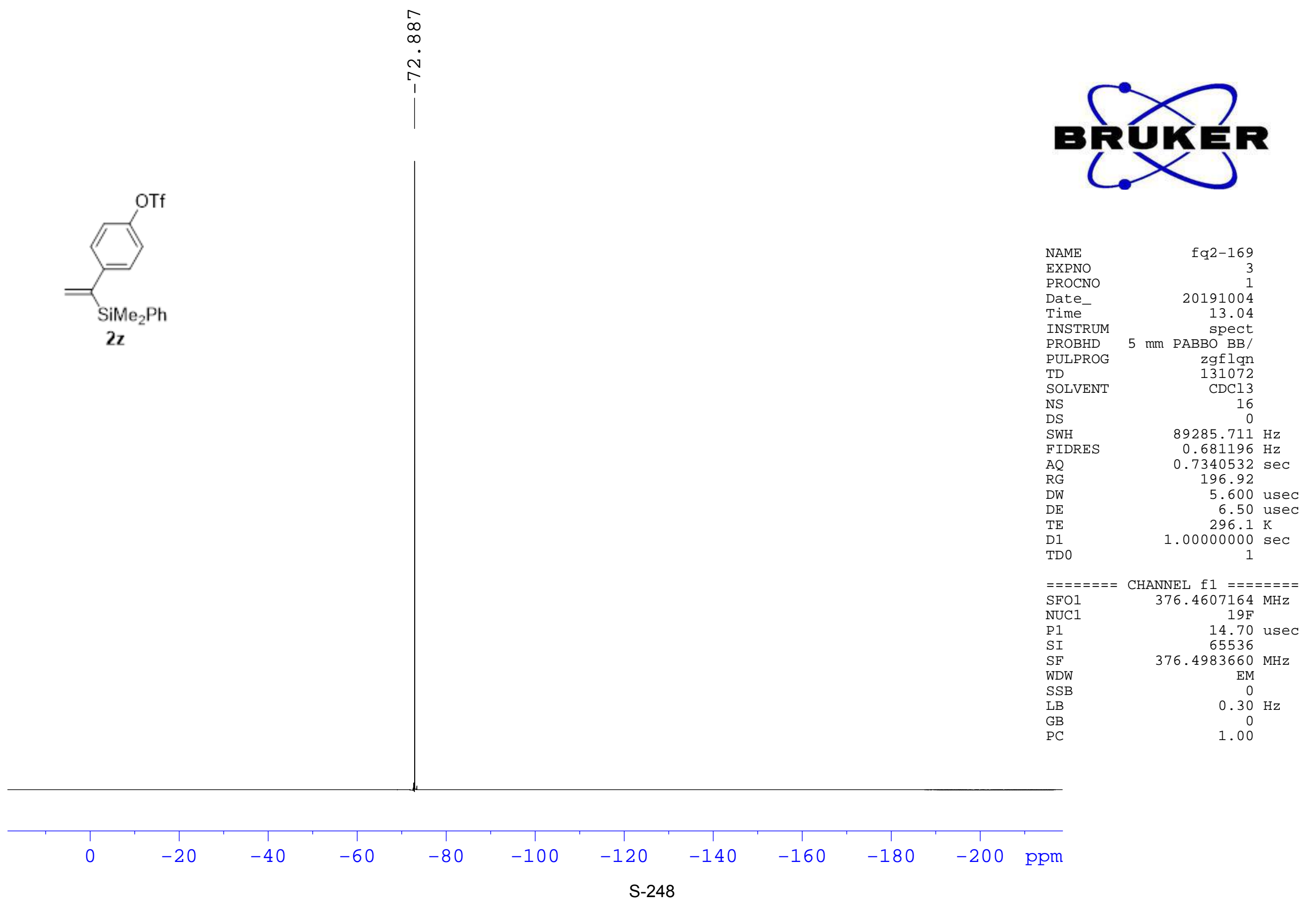




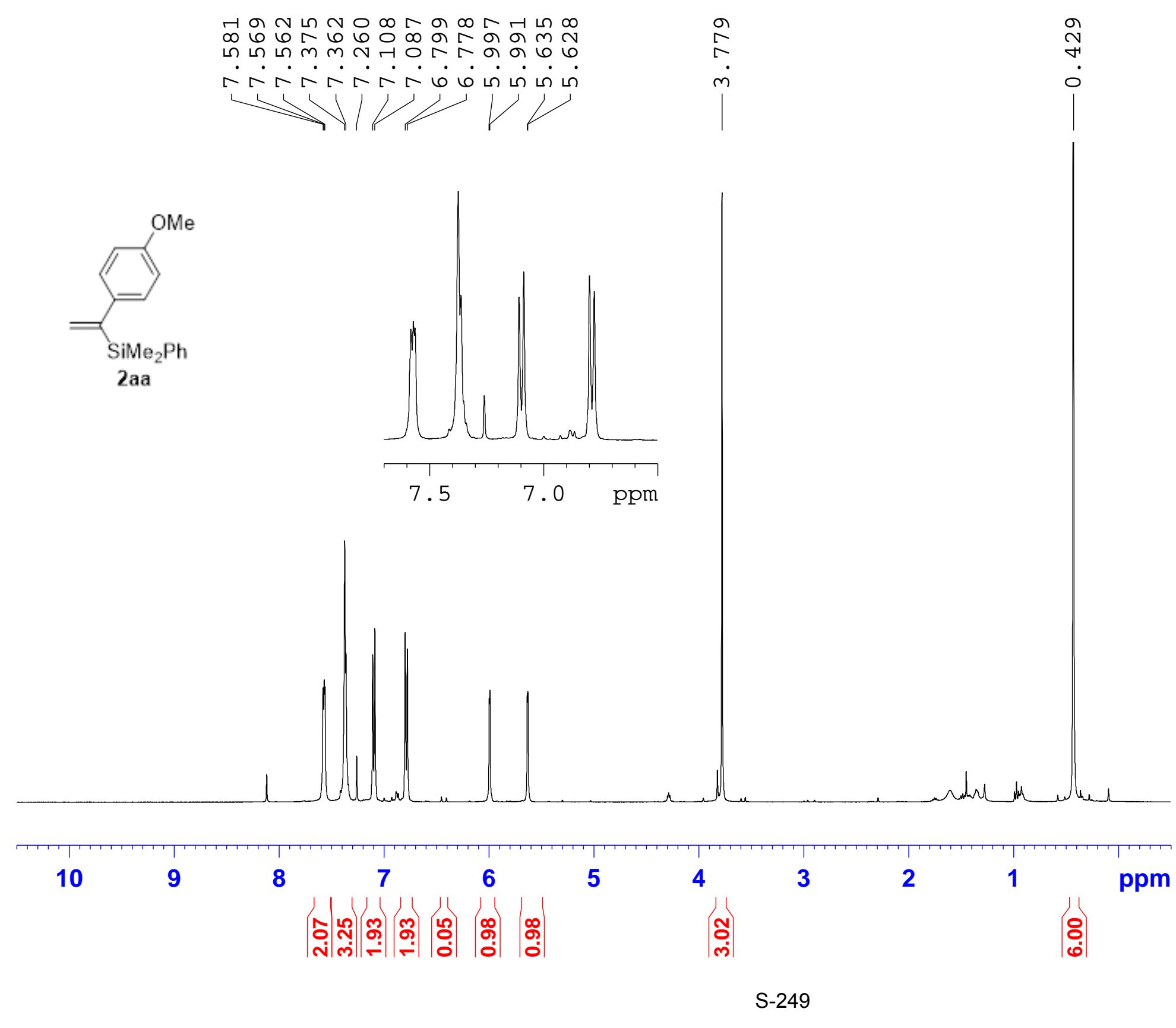

NAME

EXPNO

PROCNO

Date_

Time

PROBHD

PULPROG

TD

SOLVENT

NS

SWH

FIDRES

$\mathrm{AQ}$
$\mathrm{RG}$

RG

DE

TE

TD0

$====$

$\mathrm{SFO1}$

NUC1

SI

SF

WDW

LSB
LB

LB

PC

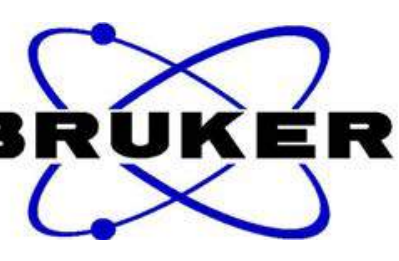

fq2-170

20180718 17.21

$5 \mathrm{~mm}$ DUL $13 \mathrm{C}-1$

65536

$\mathrm{CDCl} 3$ 8
0

$8012.820 \mathrm{~Hz}$

$0.122266 \mathrm{~Hz}$

$4.0894966 \mathrm{sec}$ 88.84

62.400 usec 6.50 usec $295.8 \mathrm{~K}$

$1.00000000 \mathrm{sec}$

CHANNEL $\mathrm{f} 1======$

$400.1324710 \mathrm{MHz}$

$1 \mathrm{H}$
14.30 usec $400.1300099 \mathrm{MHz}$

EM

$0.30 \mathrm{~Hz}$

0
1.00 
m $m$ 읭ㅇㅁ

$\infty \dot{0} \dot{0} \dot{\sigma} \dot{0} \dot{r} \dot{m}$

म $\rightarrow m m m \sim N \sim$

${ }^{-1}$

2aa

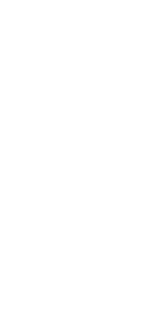

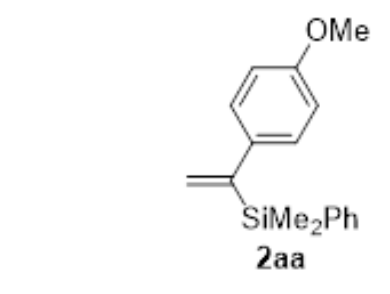

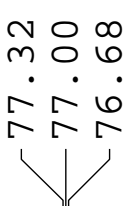

6
ำ
ம

ก)

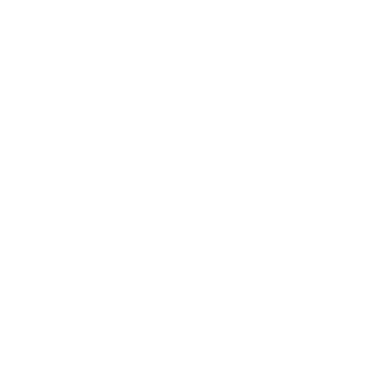

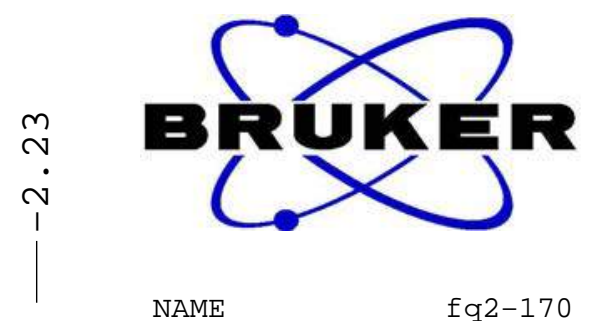

EXPNO

PROCNO

Date_

INSTRUM

PROBHD

PUTPROG

TD

SOLVENT

NS

DS

FIDRES

FIDR
$\mathrm{AQ}$
$\mathrm{RG}$

DW

DE

TE

D1
TD 0

$===$

$\mathrm{SFO}$

P 1

SF

WDW

SSB

LB

PC fq2-170

20180718 17.25

spect

$5 \mathrm{~mm}$ DUL $13 \mathrm{C}-1$

zgpg30

$\mathrm{CDCl} 3$

$24038.461 \mathrm{~Hz}$

$0.366798 \mathrm{~Hz}$

1. $3631988 \mathrm{sec}$

196.92

20.800 usec

6.50 usec

$.000000 \mathrm{~K}$

$0.03000000 \mathrm{sec}$

1

CHANNEL $\mathrm{f} 1=====$ $100.6228298 \mathrm{MHz}$ $13 \mathrm{C}$

9.60 usec

$100.6127737 \mathrm{MHz}$

EM

0
$1.00 \mathrm{~Hz}$

0
1.40

80 
$2 \mathrm{ab}$
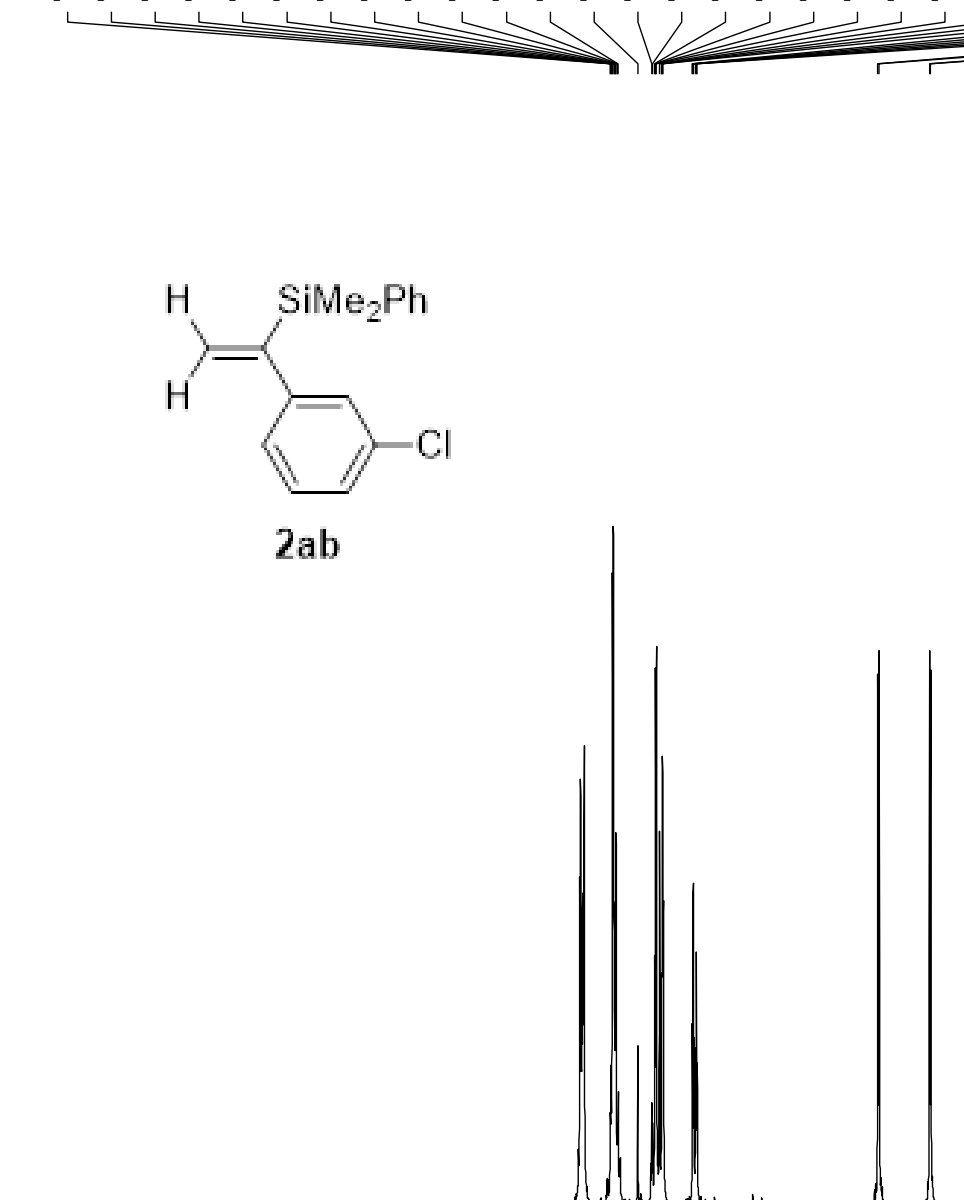

10 9 8 |

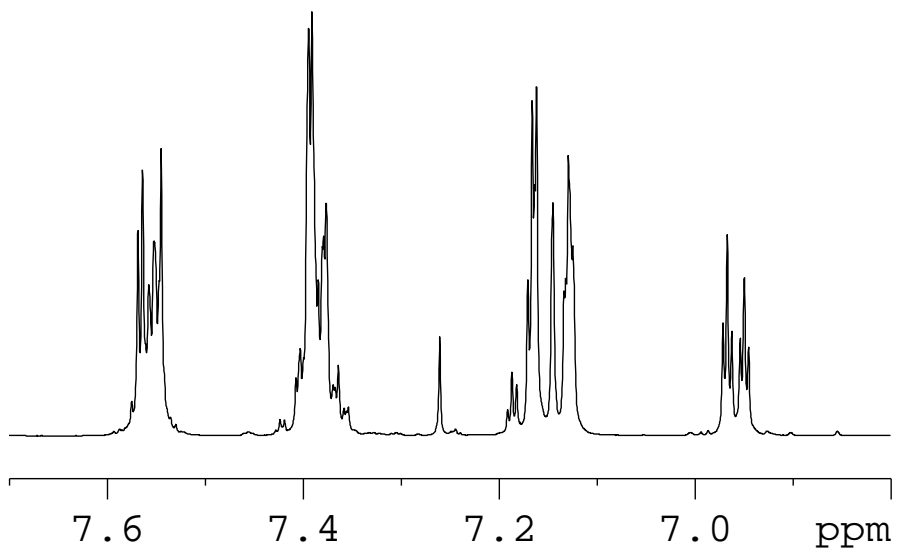

5

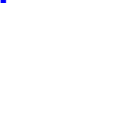

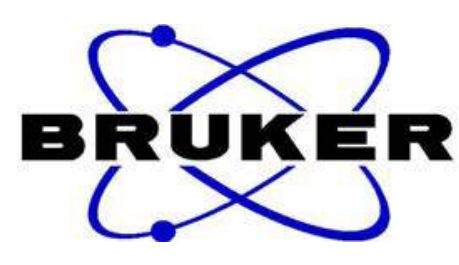

NAME

EXPNO

PROCNO

Date_

Time

INSTRUM

PROBHD

PULPROG

TD

SOLVENT

NS

SWH

FIDRES

$\mathrm{AQ}$
$\mathrm{RG}$

RG

DE

$\mathrm{DE}$
$\mathrm{TE}$
$\mathrm{D} 1$

TD 0

fq6-168

20191005

91005
23.44

$5 \mathrm{~mm}$ PABBO BB/

$\mathrm{zg} 30$
65536

$\mathrm{CDCl} 3$
8

$8012.820 \mathrm{~Hz}$

$0.122266 \mathrm{~Hz}$

$4.0894966 \mathrm{sec}$ 70.97

62.400 usec 6.50 usec $1.00000000 \mathrm{sec}$

SF $1===$ CHANNEL $\mathrm{f} 1$ 1 $========$ SFO1 400.1324710 M

SI

SF

WSB

SSB

LB
GB

PC

$400.1324710 \mathrm{MHz}$

14.50 usec

400.1300099

$\mathrm{EM}$
0

0
$0.30 \mathrm{~Hz}$

0.30
0
1.00 


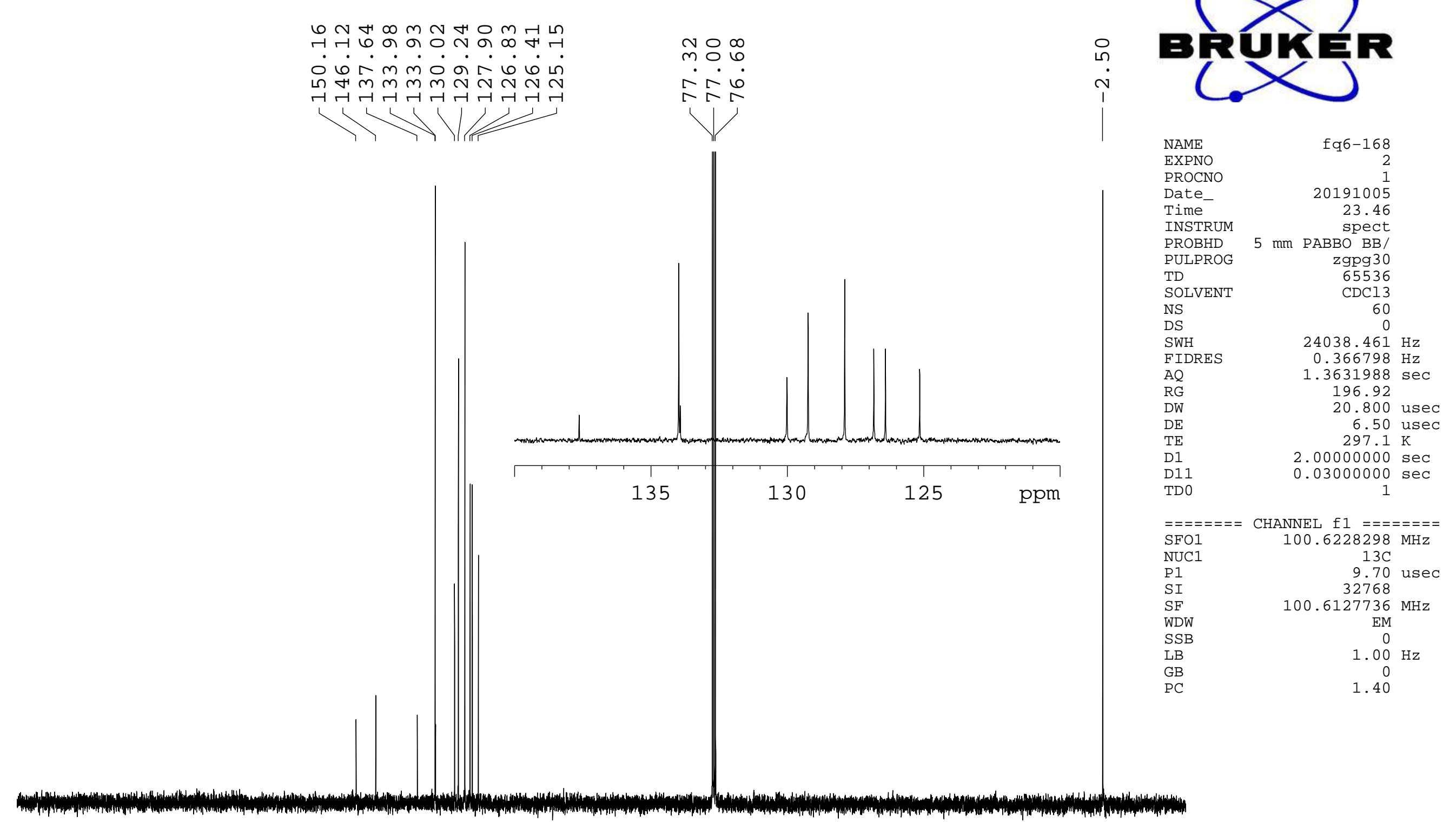

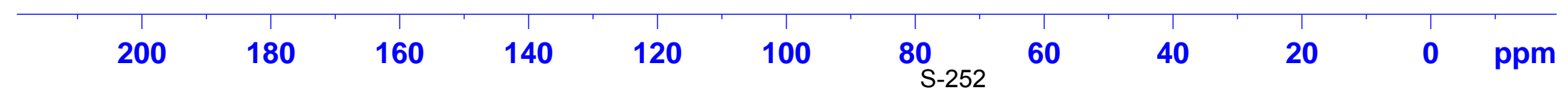


人ூ

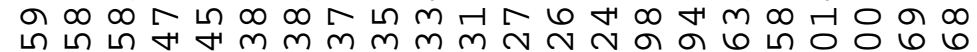
-

ــ
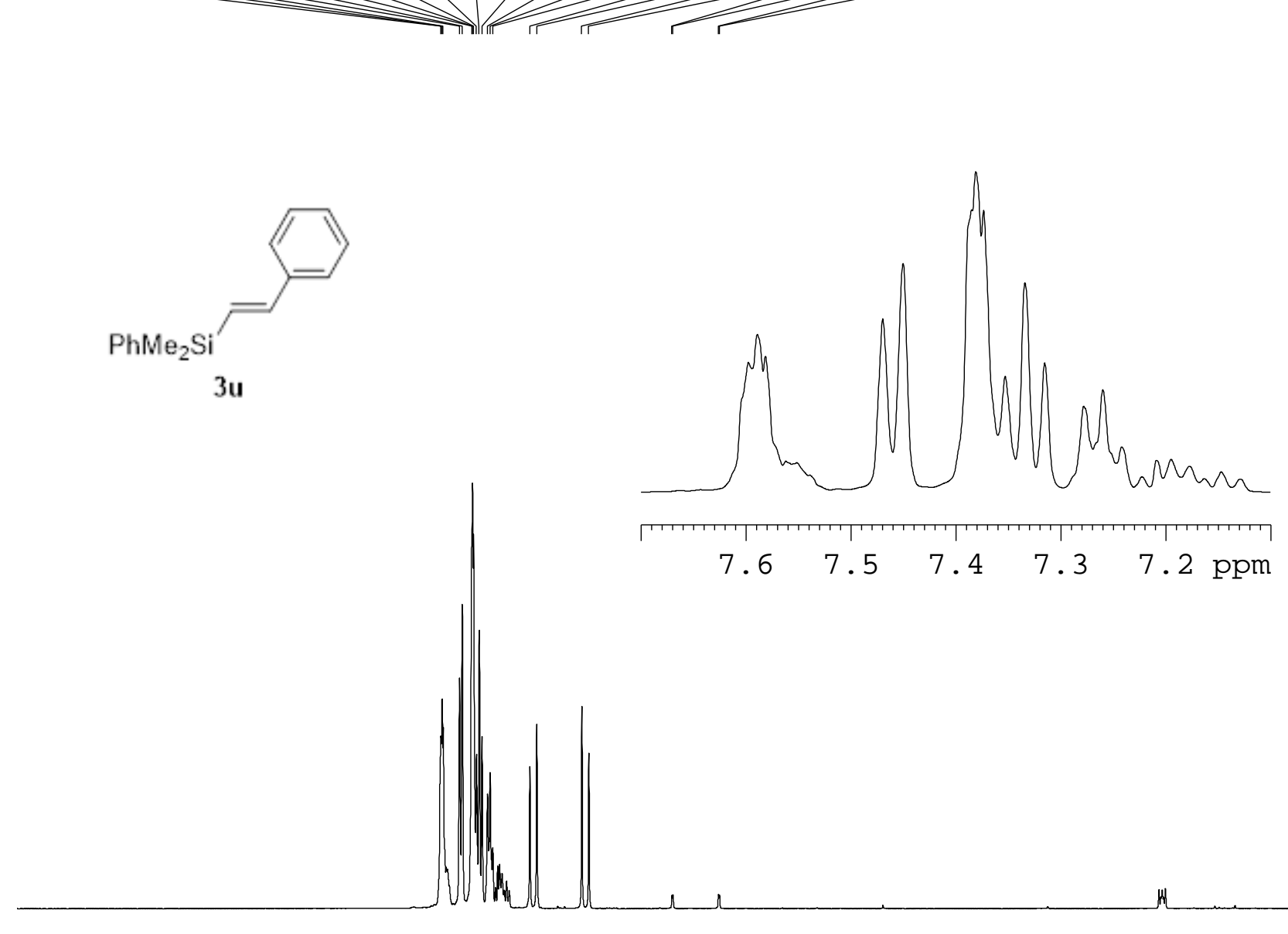

3

2

1

1

ppm

NAME
EXPNO

PROCNO

Date_

Time

INSTRUM

PROBHD

PULPROG

TD

SOLVENT

NS

SWH

FIDRES

$\mathrm{AQ}$
$\mathrm{RG}$

RG

DW

TE

TD 0
$====$
$\mathrm{SFO} 1$
CHANNEL $\mathrm{f} 1$ $400.1324710 \mathrm{MHz}$

NUC1

P 1

SI

SF

WDW

SSB
LB

LB
GB

$\mathrm{PC}$

fq2-182-1

20180720 21.57 pect

$5 \mathrm{~mm}$ DUL $13 \mathrm{C}-1$ zg30
65536 $\mathrm{CDCl} 3$ 8

$8012.820 \mathrm{~Hz}$ $0.122266 \mathrm{~Hz}$ $4.0894966 \mathrm{sec}$ 34.77 62.400 usec 6.50 usec $1.00000000 \mathrm{sec}$ $1 \mathrm{H}$ 14.30 use
65536 $400.1300304 \mathrm{MHz}$

EM

$0.30 \mathrm{~Hz}$

0
1.00 


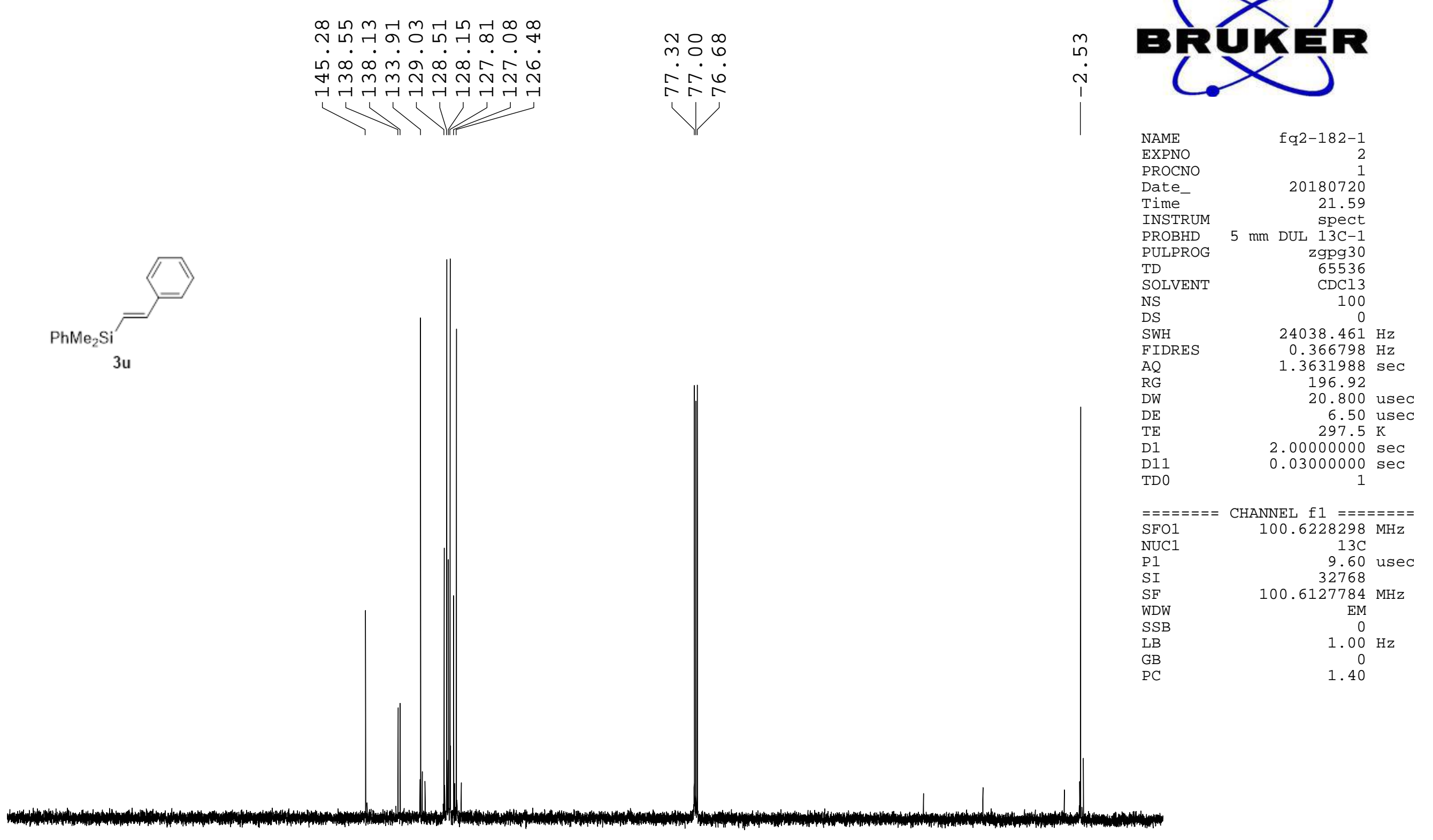

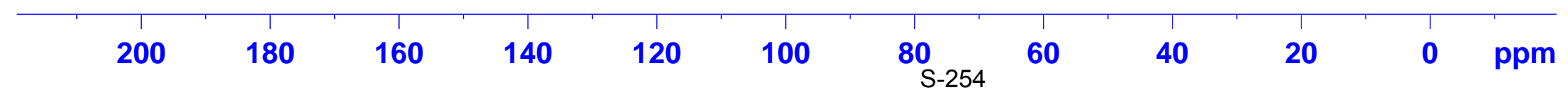




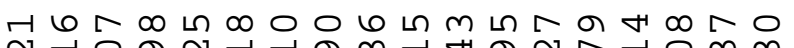

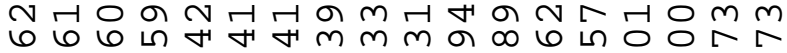

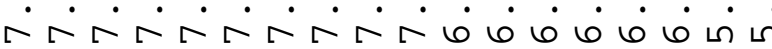

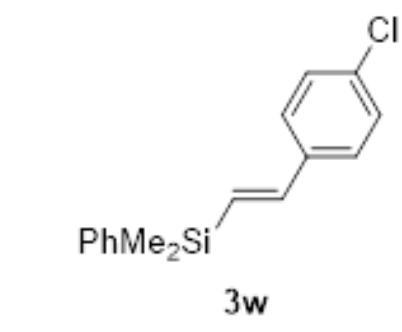

$3 w$

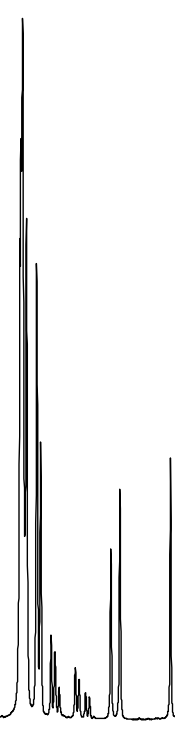

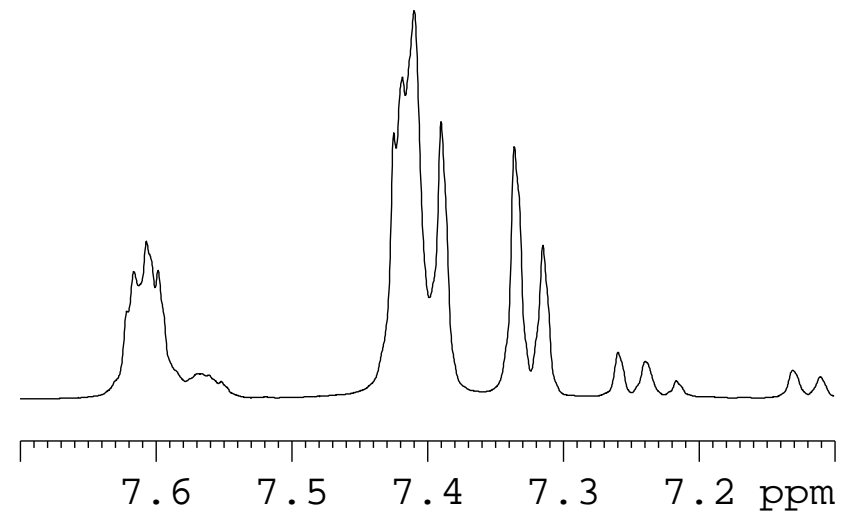

10 9

8
7 6 A 2 ( ก)

$\left|\begin{array}{l|l}0 \\ 0 \\ 0\end{array}\right| \begin{aligned} & 0 \\ & 0 \\ & 0\end{aligned} \mid$

5

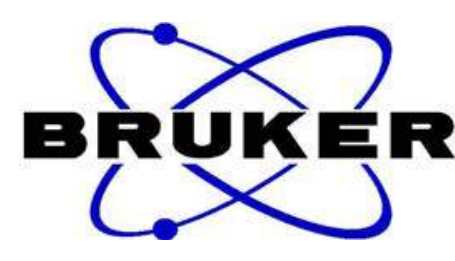

NAME

EXPNO

PROCNO

Date_

Time

INSTRUM

PROBHD

PULPROG

TD

SOLVENT

NS

SWH

FIDRES

$A Q$

RG

DE

TE

D 1

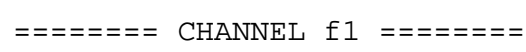

$\mathrm{SFO} 1$

NUC1

NUC1

SI

SF

WDW

SSB

LB

PC

$400.1324710 \mathrm{MHz}$

$1 \mathrm{H}$
14.30 usec $400.1300104 \mathrm{MHz}$

EM

0
$0.30 \mathrm{~Hz}$

0.30
0
1.00

$8012.820 \mathrm{~Hz}$
$0.122266 \mathrm{~Hz}$

$4.0894966 \mathrm{sec}$ 34.77

6.400 usec $295.9 \mathrm{~K}$

$1.00000000 \mathrm{sec}$ 


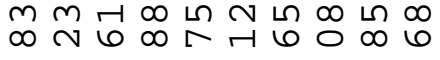

mं $\dot{m} \dot{m} \dot{0} \dot{\infty} \dot{\sim}$ $\forall m m m m \sim N \sim N$ 다다대다

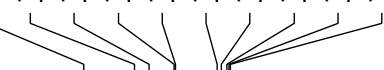

$3 w$
N $\bigcirc \circ$

$\therefore \circ \dot{0}$

กิำ

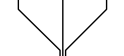

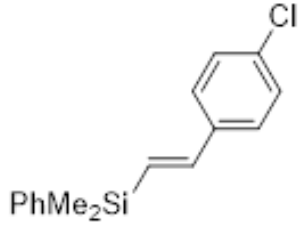

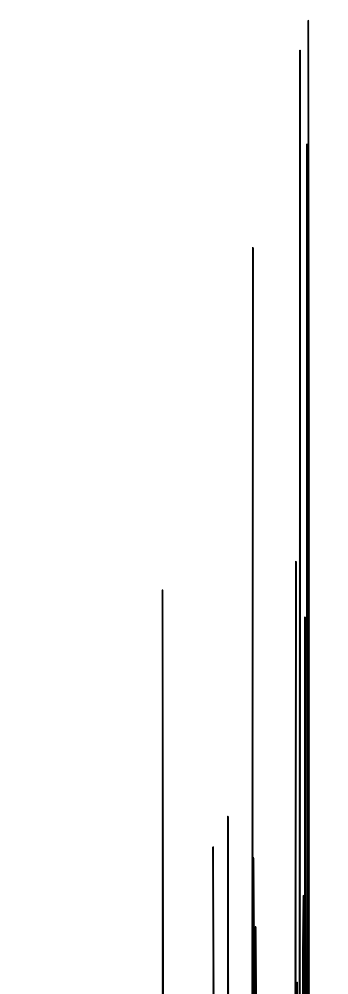

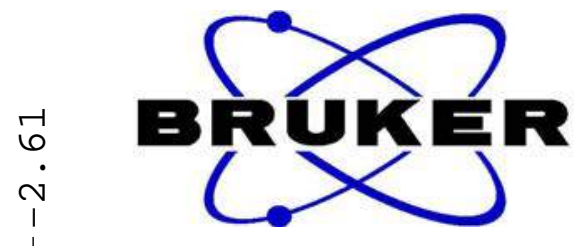

NAME

PROCNO

Date

INSTRUM

PROBHD

PULPROG

TD

SOLVENT

NS

DS

FIDRES

FIDR
$\mathrm{AQ}$
$\mathrm{RG}$

DW

$\mathrm{DE}$

DE

D11

TDO

$===$

$\mathrm{SFO}$

(1)

P 1

SI

WDW

SSB

LB

PC
20180720 20.52 20.52

mm DUL 13C-1

zgpg 30
65536

$\mathrm{CDCl} 3$

$24038.461 \mathrm{~Hz}$

$0.366798 \mathrm{~Hz}$

$3631988 \mathrm{sec}$

196.92

20.800 usec

6.50 usec

$2.00000000 \mathrm{sec}$

0.03000000

CHANNEL $\mathrm{f} 1 \mathrm{l}=======$ $100.6228298 \mathrm{MHz}$ $13 \mathrm{C}$

9.60 usec 100.6127773

$100.6127773 \mathrm{MHz}$

EM

$1.00 \mathrm{~Hz}$

0
1.40 fq2-178

\begin{tabular}{rrrrrrrrrrrr}
\hline 200 & 180 & 160 & 140 & 120 & 100 & 80 & 60 & 40 & 20 & 0 & ppm
\end{tabular}




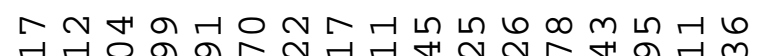

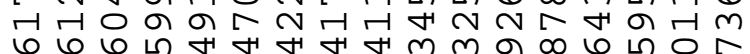

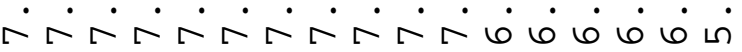

पूरा
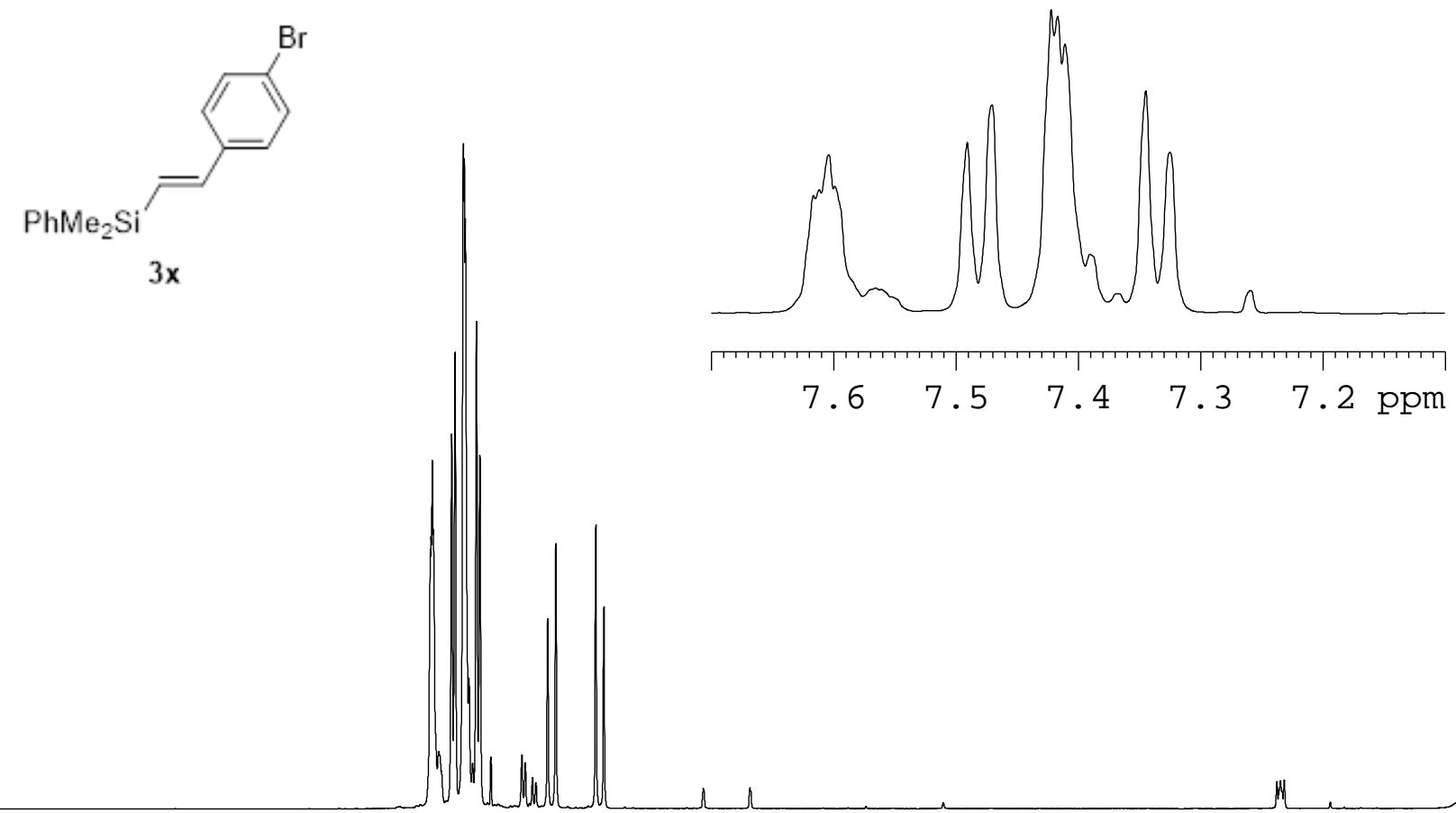

10 9

8 7 6 กู่

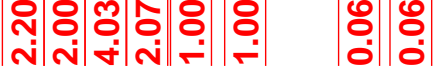




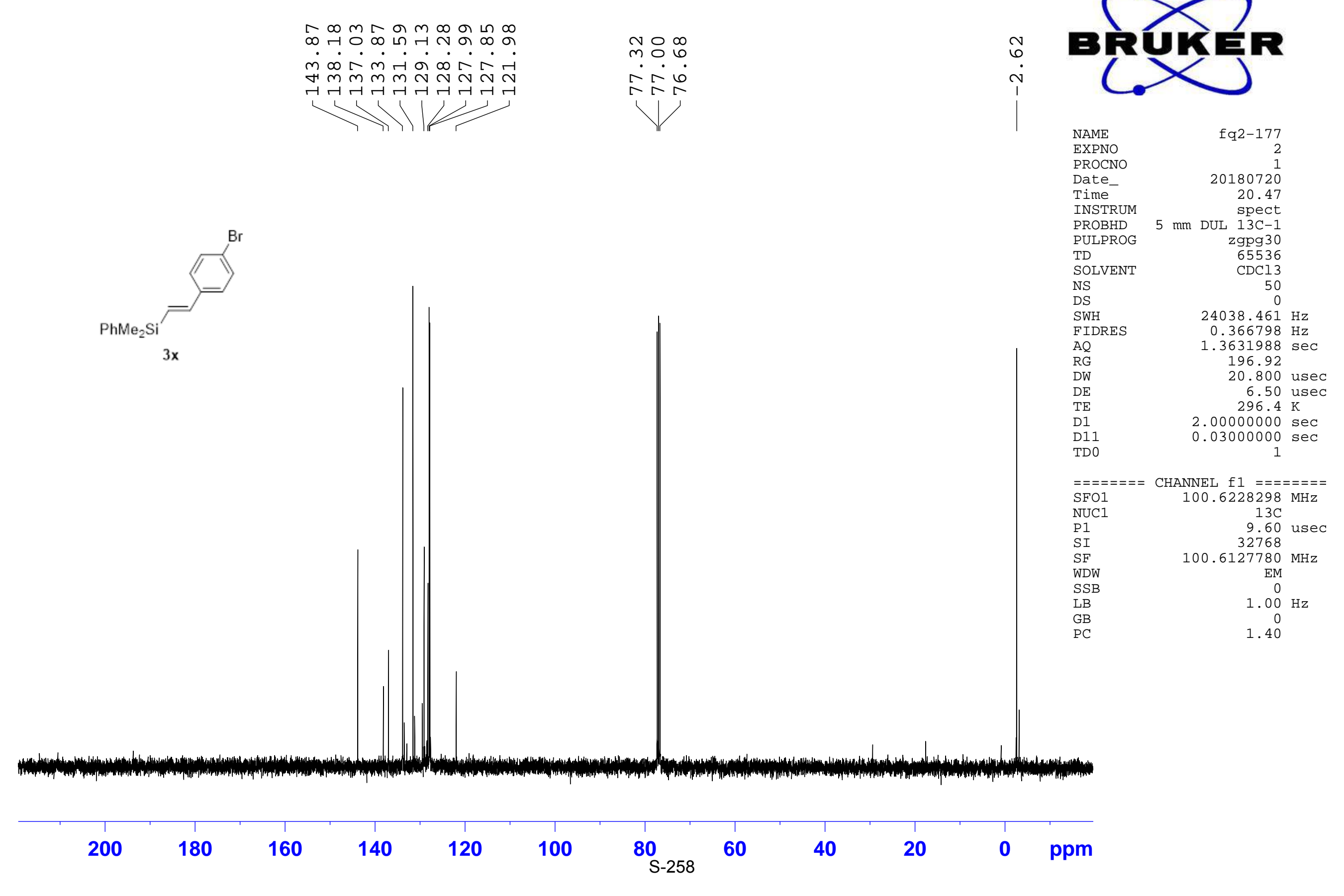




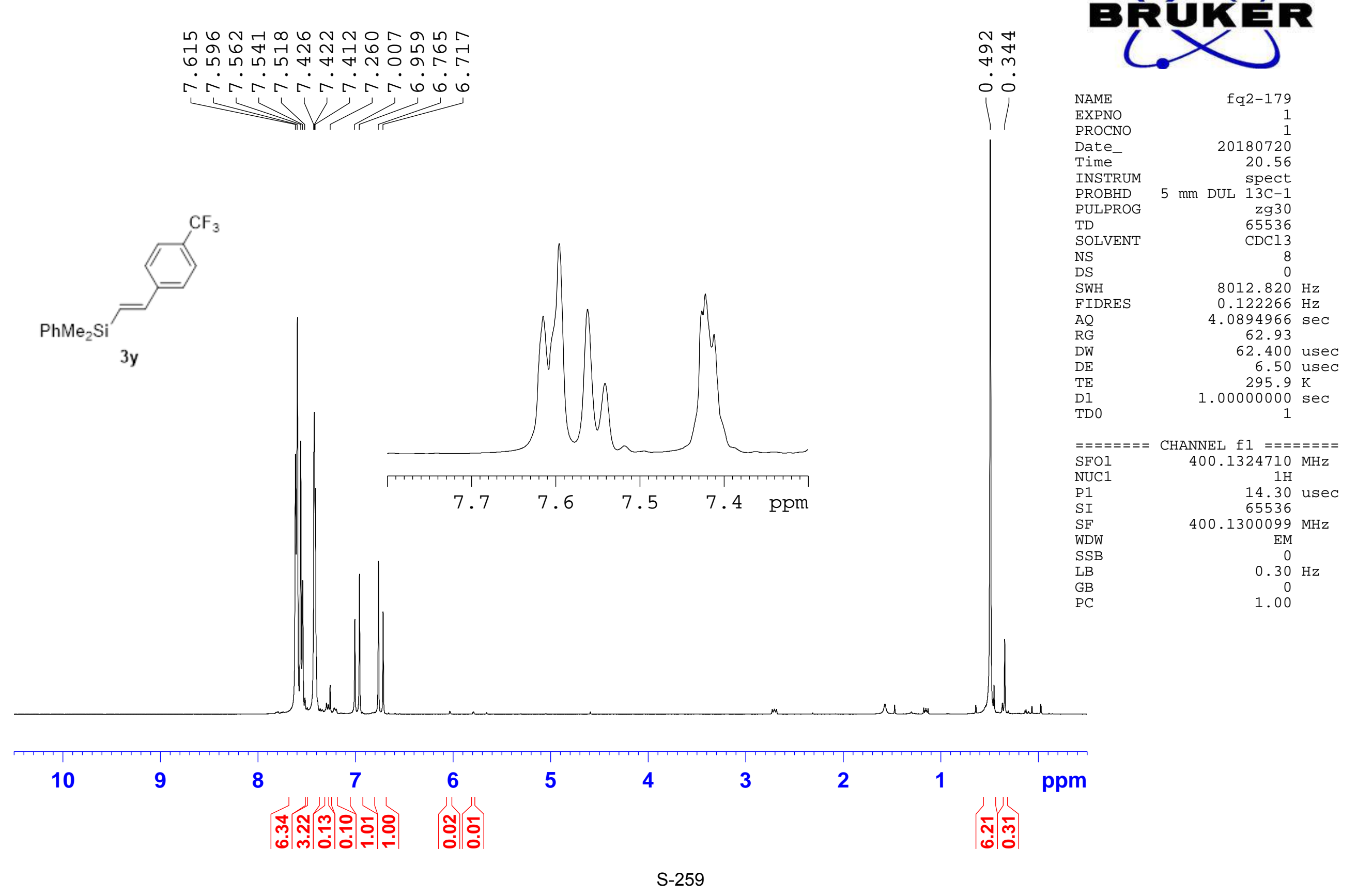




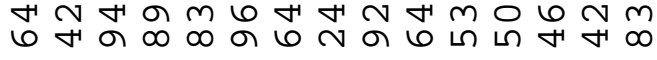

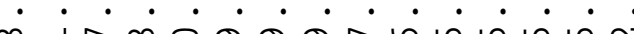
$\forall \forall m m \sim N \sim \sim N \sim N$

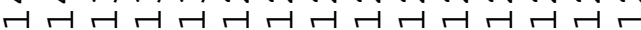

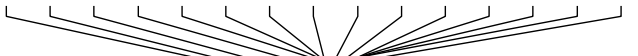

$3 y$

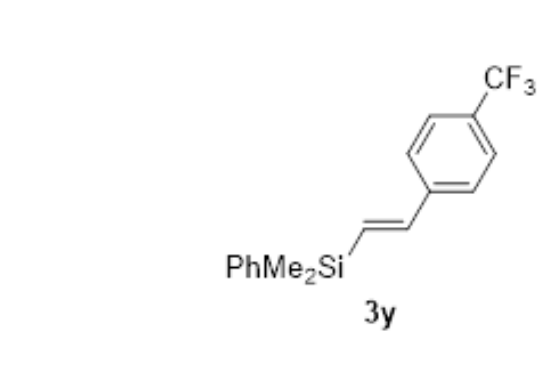

ㄱํㅇ

$\therefore \therefore \dot{0}$

r

11

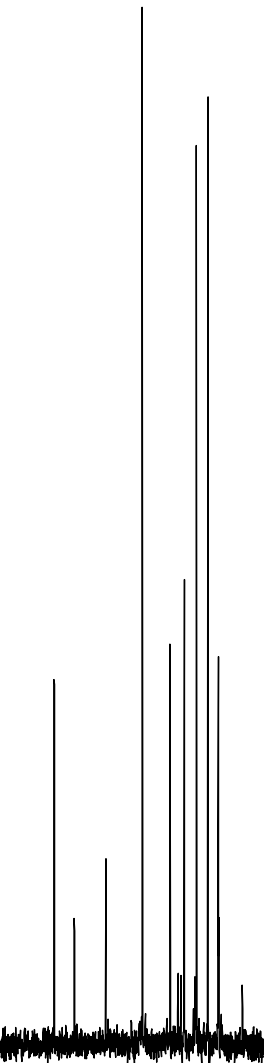

,

NAME
EXPNO

PROCNO

Date

INSTRUM

PROBHD

PULPROG

SOLVENT

NS

DS

FIDRES

FIDR
AQ
RG

DW

DE

D1

D11

DO

$==$
SFO1

Noc1

P 1

SI
SF

WDW

SSB
LB

LB

PC fq2-179

20180720 20.58 20.58

$5 \mathrm{~mm}$ DUL 13C-1

zgpg 30

$\mathrm{CDCl}$

$24038.461 \mathrm{~Hz}$

$0.366798 \mathrm{~Hz}$

$.3631988 \mathrm{sec}$

196.92

20.800 usec

6.50 usec

$0.03000000 \mathrm{sec}$

0.03000000

CHANNEL $\mathrm{f} 1======$ $100.6228298 \mathrm{MHz}$ $13 \mathrm{C}$

9.60 usec 100.6127736

$100.6127736 \mathrm{MHz}$

EM

$1.00 \mathrm{~Hz}$

0
1.40 
ก

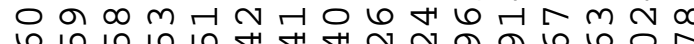

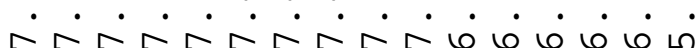

$\cdots$ n

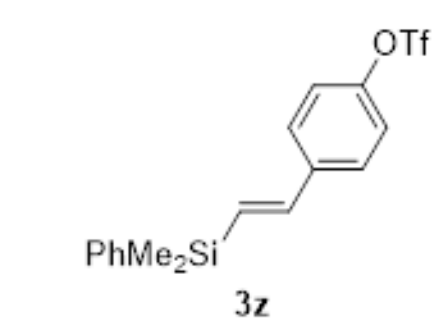

10 9

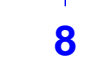

8 7 6 슬 쇼용

ชิ

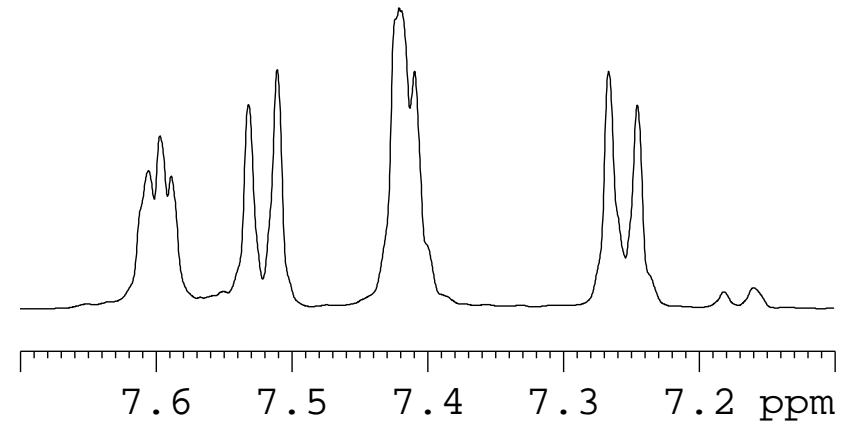
$=C_{-1}^{-1}$

NAME

EXPNO

PROCNO

Date_

Time

INSTRUM

PROBHD

PULPROG

TD

SOLVENT

NS

SWH

FIDRES

$A Q$

RG

DE

TE

TDO

fq2-180

180
1
1

20180720
21.49

$5 \mathrm{~mm}$ DUL $13 \mathrm{C}-1$

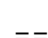

$\mathrm{SFO} 1$

$\mathrm{SFO1}$

NUC1

PI

SF

WDW

SSB

LB

PC

65536
CDC13

8
0
8012.820

$0.122266 \mathrm{~Hz}$

$4.0894966 \mathrm{sec}$ 54.81

62.400 usec 6.50 usec $1.00000000 \mathrm{se}$

CHANNEL $f 1$

NNEL $\mathrm{f} 1 \mathrm{l}=======$ $400.1324710 \mathrm{M}$

$1 \mathrm{H}$

14.30 usec $400.1300105 \mathrm{MHz}$

EM

0
$0.30 \mathrm{~Hz}$

$0.30 \mathrm{~Hz}$
0
1.00

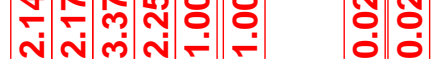




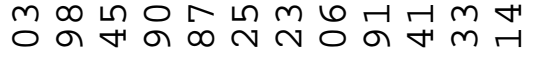

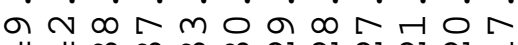

$\forall \forall m m m \sim N \sim \sim$

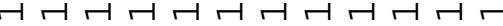

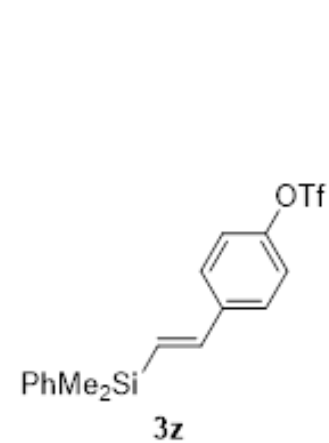

m $\bigcirc 0$

$\therefore \sim 6$

$\backslash 1$

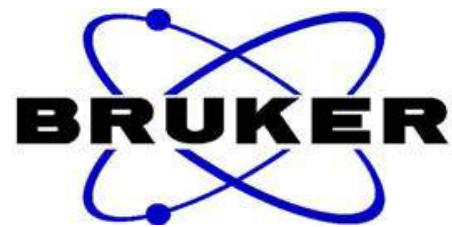

NAME

PROCNO

Date

Time

INSTRUM

PROBHD

PULPROG

TD

NS

DS

FIDH

FIDR
AQ
RG

DW

DE

TE

D1
TD0

$==$

$\mathrm{SFO}$

NUC1

P 1

SF

WDW

SSB
LB

LB

PC

fq2-180

$20180720^{1}$

21.51

spect

$5 \mathrm{~mm}$ DUL $13 \mathrm{C}-1$

zgpg 30

$\mathrm{CDCl} 3$

$24038.461 \mathrm{~Hz}$

$0.366798 \mathrm{~Hz}$

$3631988 \mathrm{sec}$

196.92

20.800 usec

6.50 usec

1

CHANNEL $\mathrm{f} 1$ $100.6228298 \mathrm{MHz}$ $13 \mathrm{C}$

9.60 usec 100.6127748

$100.6127748 \mathrm{MHz}$

EM

$1.00 \mathrm{~Hz}$

0
1.40 


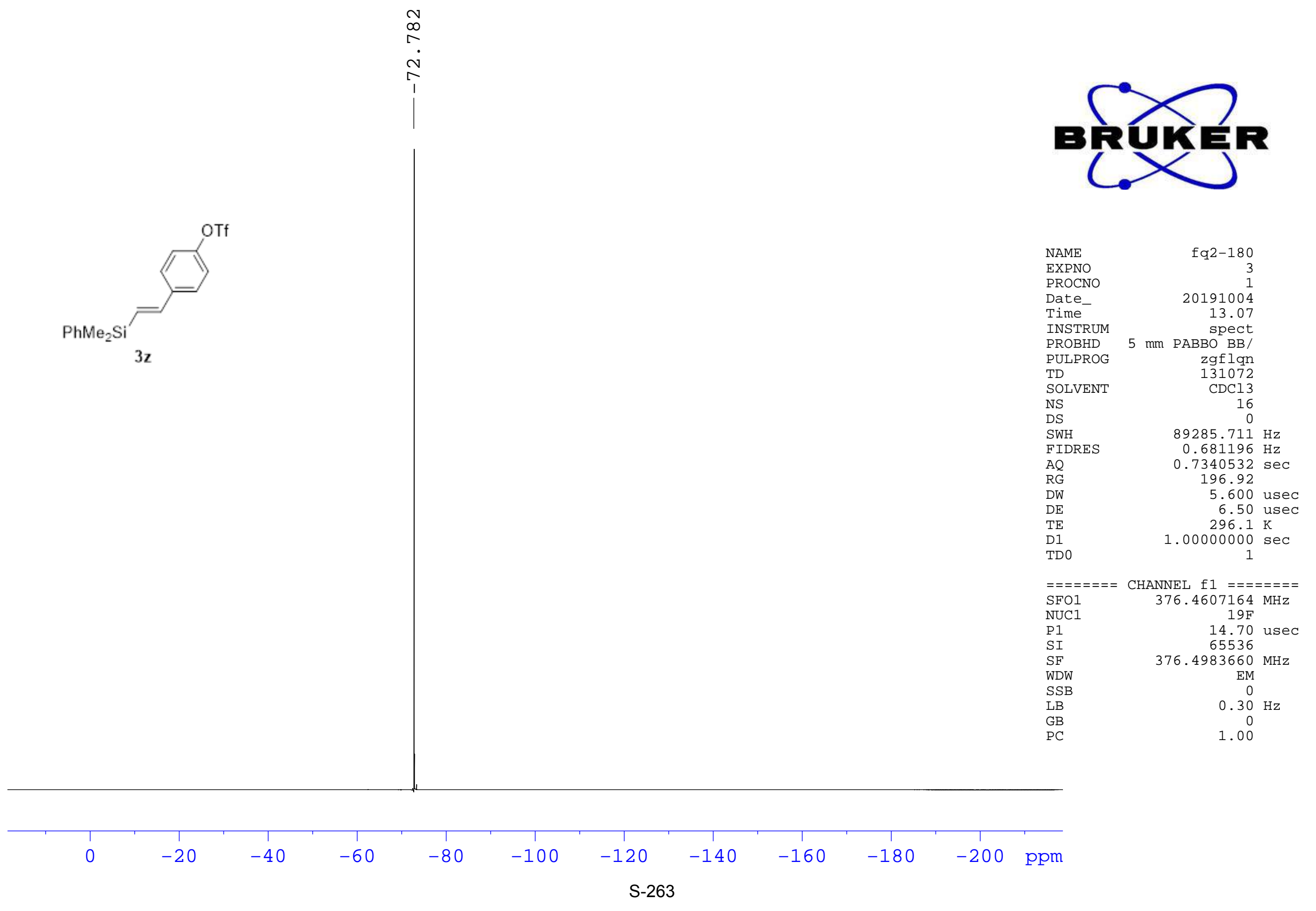


ம

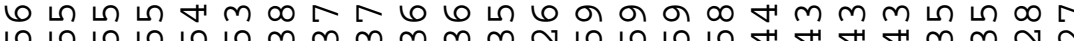

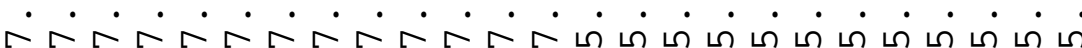

$\infty \begin{array}{llll}\infty & \infty & 6 \\ \infty & \infty & 6 & 0\end{array}$

$\dot{-1} \dot{-1} \dot{-1}$

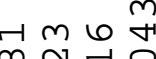
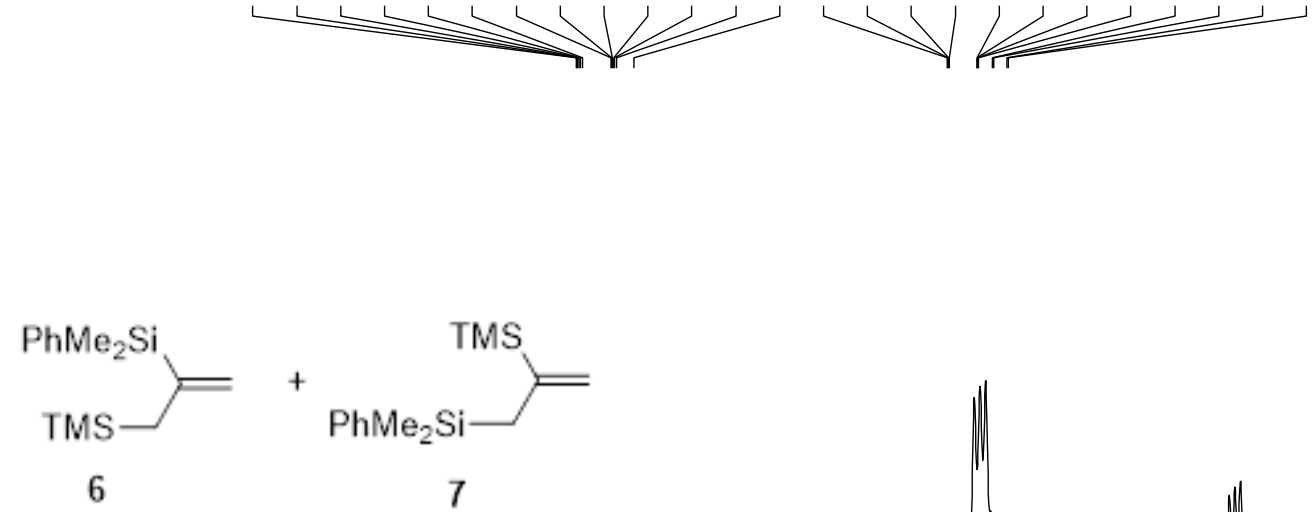

10

9

8

7

$\begin{array}{lll}8 & \infty \\ \text { Ni } & \infty \\ \text { N. }\end{array}$

6

5

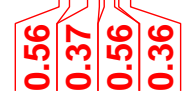

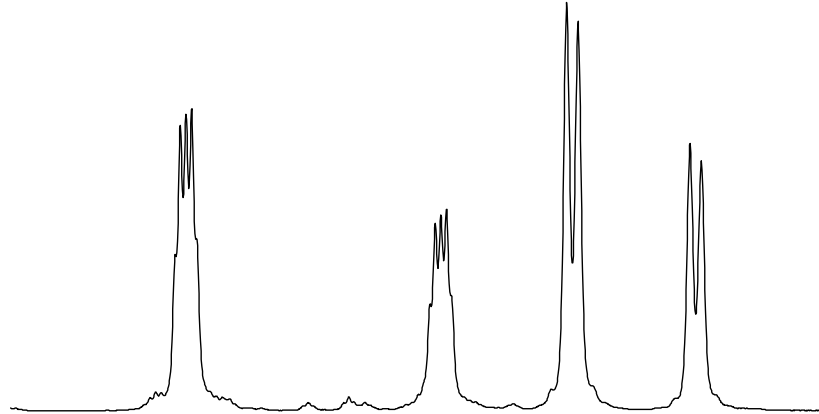

5.6
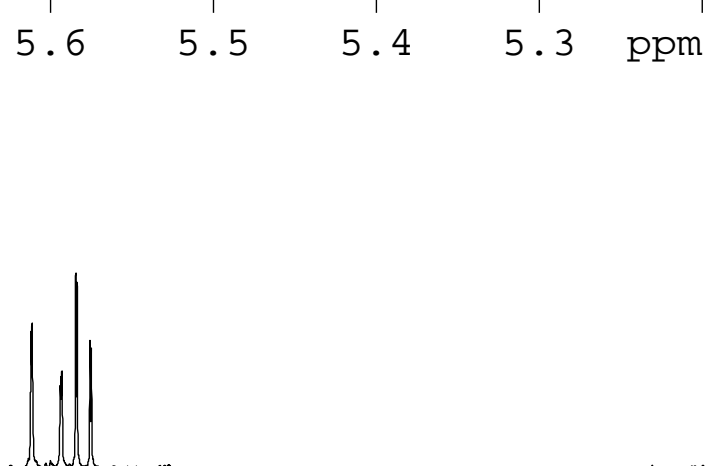

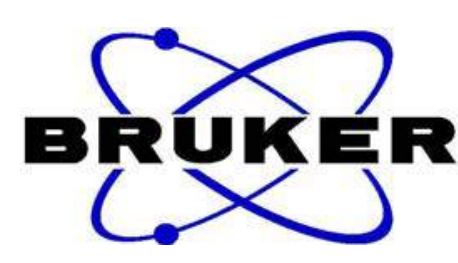

EXPNO

PROCNO

Date_

Time

INSTRUM

PROBHD

PULPROG

TD

SOLVENT

NS

SWH

FIDRES

$\mathrm{A} Q$
$\mathrm{RG}$

RG

DE

TE

D 1

fq2 $2-75-1$

20180608

22.01

$5 \mathrm{~mm}$ DUL $13 \mathrm{C}-1$

65536

$\mathrm{CDCl} 3$

$8012.820 \mathrm{~Hz}$

$0.122266 \mathrm{~Hz}$

$4.0894966 \mathrm{sec}$ 34.77

62.400 usec 6.50 usec $1.00000000 \mathrm{sec}$

$========$ CHANNEL $\mathrm{f} 1========$

SFO1 $400.1324710 \mathrm{MHz}$

NUC1

P1

SI

SF

WDW

SSB
LB

GB

$P C$

400.1324710

14.30 usec

400.1300098

EM
0

$0.30 \mathrm{~Hz}$

0.30
0
1.00 


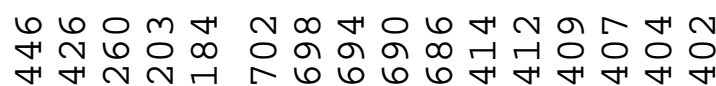

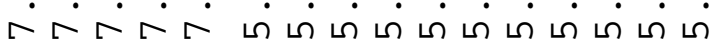
11201

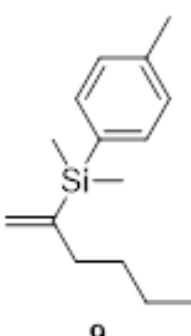

9

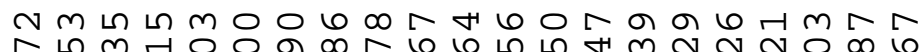

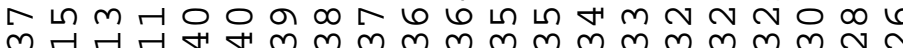

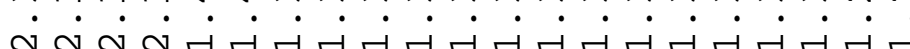
मન ન

\section{NAME} PROCNO Date Time INSTRUM PROBHD PULPROG

TD

SOLVENT

NS

SWH

FIDRES

AQ

DW

$\mathrm{DE}$

TE

TD0

$=======\begin{gathered}\text { CHANNEL } \mathrm{f} 1 \mathrm{f}======== \\ \mathrm{SFO} 1\end{gathered} \quad 400.1324710 \mathrm{MHz}$

$\mathrm{SFO1}$

P1

P1

SF

WDW

SSB

LB

GB

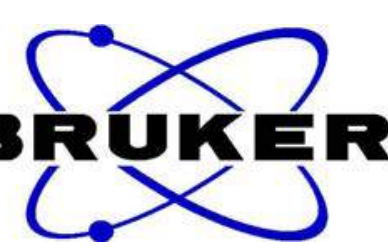

fq6-179-1-1

20191007 22.23 $\mathrm{zg} 30$
65536 $\mathrm{CDCl} 3$ 8
0

$8012.820 \mathrm{~Hz}$ $0.122266 \mathrm{~Hz}$ $4.0894966 \mathrm{sec}$ 31.55 62.400 usec 6.50 usec $296.3 \mathrm{~K}$ $1.00000000 \mathrm{sec}$ $1 \mathrm{H}$
14.50 usec $400.1300098 \mathrm{MHz}$

EM

$0.30 \mathrm{~Hz}$

0.30
0 $5 \mathrm{~mm}$ РABBO BB/ 


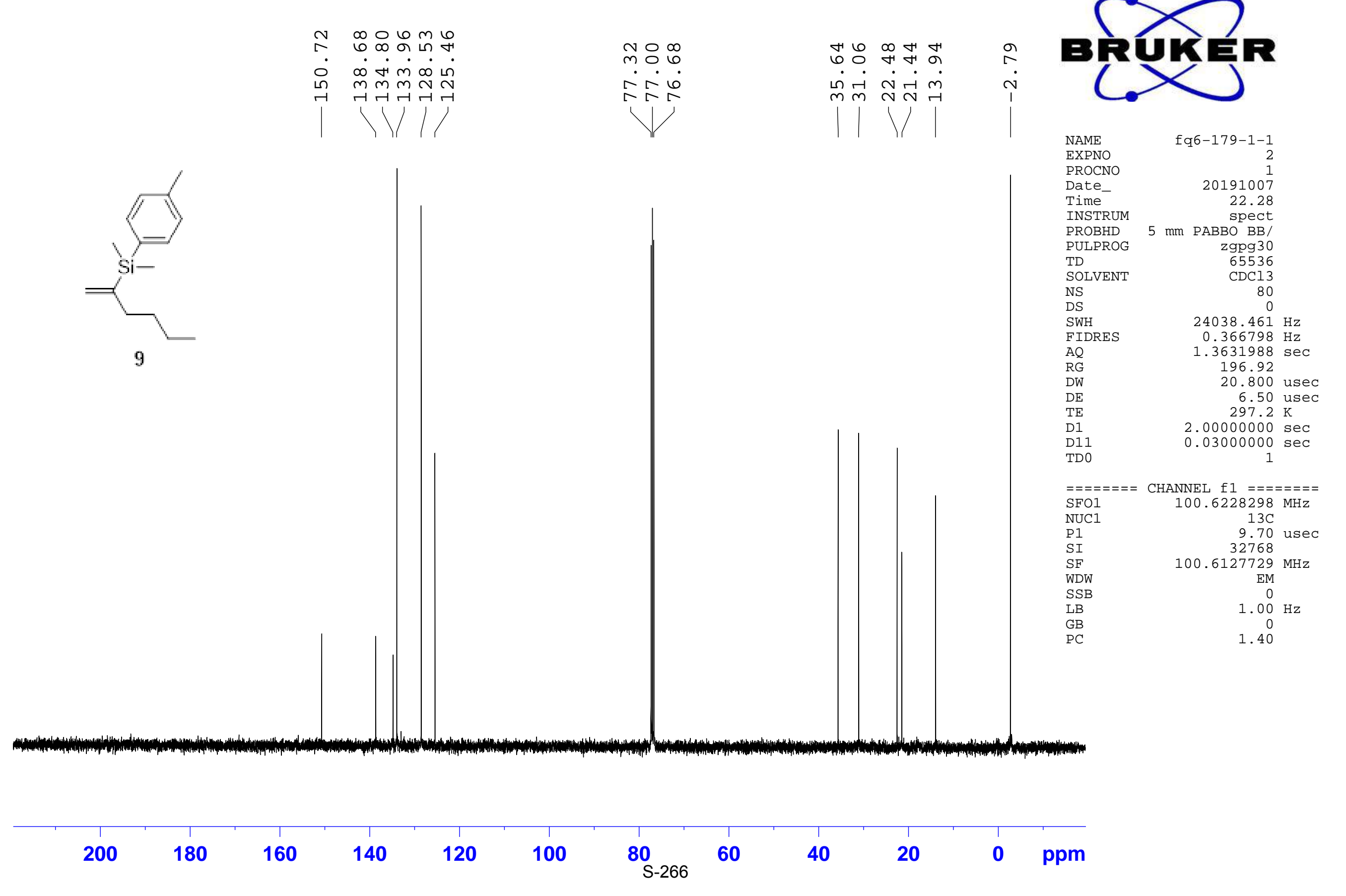

